Supporting Information

\title{
Accessing (Multi)Fluorinated Piperidines Using Heterogeneous Hydrogenation
}

Tobias Wagener, Arne Heusler, Zackaria Nairoukh, Klaus Bergander, Constantin G. Daniliuc, Frank Glorius* glorius@uni-muenster.de

Organisch-Chemisches Institut, Westfälische Wilhelms-Universität Münster, Corrensstraße 40, 48149 Münster, Germany

\section{Table of Contents}

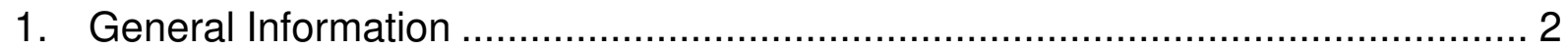

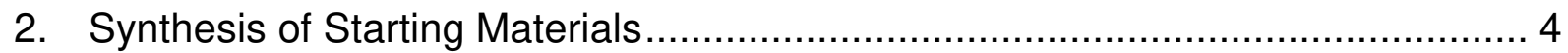

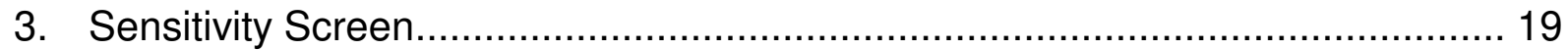

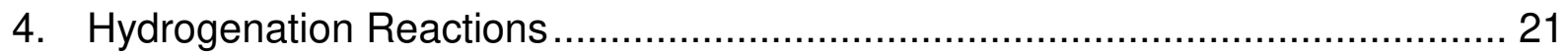

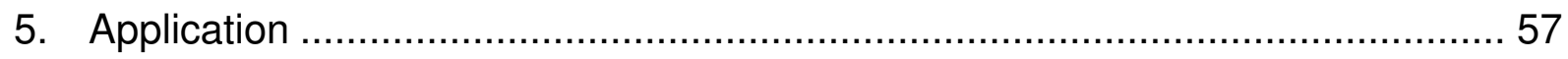

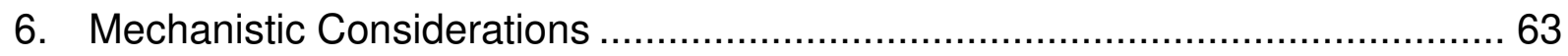

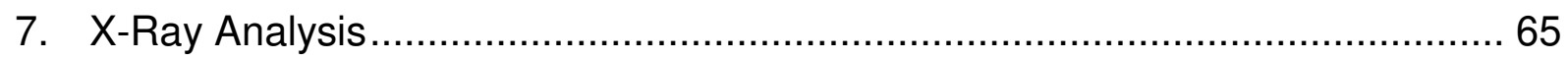

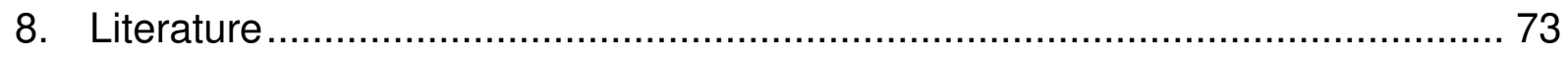

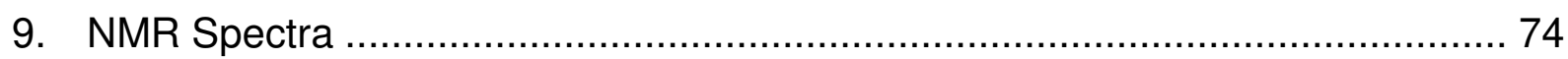




\section{General Information}

Unless otherwise noted, all reactions for starting material synthesis were carried out under an atmosphere of argon in oven-dried glassware. Catalytic hydrogenation reactions were prepared under air without further care of excluding moisture. Reaction temperatures are reported as the temperature of the bath surrounding the vessel unless otherwise stated. The solvents used for starting material synthesis were purified by distillation over the drying agents indicated in parentheses and were transferred under argon: toluene $\left(\mathrm{CaH}_{2}\right)$, dichloromethane $\left(\mathrm{CaH}_{2}\right)$, THF (Na-benzophenone). Ethanol ( $4 \AA$ ) and methanol ( $3 \AA$ ) were purchased as dry solvents from commercial suppliers and stored over molecular sieves. Solvents for hydrogenation reactions (methanol, THF) were purchased as reagent grade solvents $(>99 \%)$ and used as received. All hydrogenation reactions were carried out in Berghof High Pressure Reactors using hydrogen gas.

Commercially available chemicals were obtained from Acros Organics, Aldrich Chemical Co., Strem Chemicals, Alfa Aesar, ABCR, Combi-Blocks, Chempur and TCI Europe and used as received. Heterogeneous catalysts were obtained from Johnson Matthey (Rhodium/C, 5wt\%, Ruthenium/C, 5wt\%), Sigma Aldrich (Palladium hydroxide/C, 20wt\%) Evonik Industries (Palladium/C, 10wt\%), Acros Organics $\left(\mathrm{PtO}_{2}\right)$ or Alfa Aesar (Rhodium $/ \mathrm{Al}_{2} \mathrm{O}_{3}, 5 \mathrm{wt} \%$ ) and used as received. Severel batches of $\mathrm{Pd}(\mathrm{OH})_{2} / \mathrm{C}$ from different vendors have been investigated and gave comparable results. However, a sample of $\mathrm{Pd}(\mathrm{OH})_{2}$ on activated carbon performed slightly worse which underlines the importance of catalyst support.
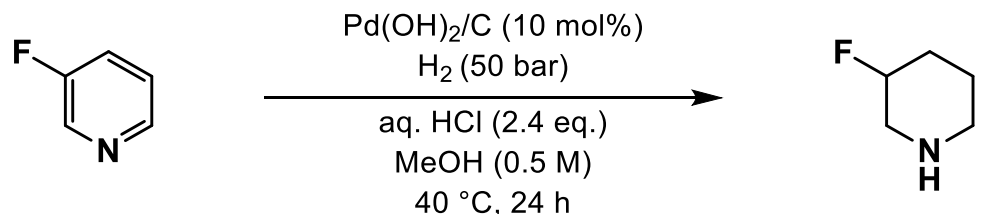

\begin{tabular}{cccc}
\hline Batch & Catalyst & Supplier & Yield \\
\hline 1 & palladium hydroxide on carbon $20 \mathrm{wt} \%$ & Aldrich & $85 \%$ \\
2 & palladium hydroxide on carbon $20 \mathrm{wt} \%$ & Sigma Aldrich & $85 \%$ \\
3 & palladium hydroxide on carbon $20 \mathrm{wt} \%$ & Aldrich & $82 \%$ \\
4 & palladium hydroxide on carbon $20 \mathrm{wt} \%$ & Fluorochem & $86 \%$ \\
5 & palladium hydroxide on activated carbon $20 \mathrm{wt} \%$ & Aldrich & $72 \%$ \\
\hline
\end{tabular}


Analytical thin layer chromatography (TLC) was performed on silica gel 60 F254 aluminum plates (Merck). TLC plates were visualized by exposure to short wave ultraviolet light $(254 \mathrm{~nm}, 366 \mathrm{~nm})$ and were dipped into a solution of $\mathrm{KMnO}_{4}$. Flash chromatography was performed on Acros Organics silica gel (35-70 mesh) under a positive pressure of argon, eluting with the specified solvent system.

GC-MS spectra were recorded on an Agilent Technologies 7890A GC-system with an Agilent 5975C VL MSD or an Agilent 5975 inert Mass Selective Detector (EI) and a HP-5MS column $(0.25 \mathrm{~mm} \times 30 \mathrm{~m}$, film: $0.25 \mu \mathrm{m})$. ESI mass spectra were recorded on a Bruker Daltonics MicroTof spectrometer. ${ }^{1} \mathrm{H}$ and ${ }^{13} \mathrm{C}$ and ${ }^{19} \mathrm{~F}$ NMR spectra were recorded on a Bruker Avance II300 or Avance II400, AgilentDD2 500 or AgilentDD2 600 in the indicated solvents. Chemical shifts $(\delta)$ are given in ppm relative to TMS. The residual solvent signals were used as references and the chemical shifts converted to the TMS scale $\left(\mathrm{CDCl}_{3}: \delta_{\mathrm{H}}=7.26 \mathrm{ppm}, \delta \mathrm{c}=77.16 \mathrm{ppm} ; \mathrm{CD}_{3} \mathrm{OD}: \delta_{\mathrm{H}}=3.31 \mathrm{ppm}, \delta \mathrm{c}=\right.$ 49.0ppm. ${ }^{19} \mathrm{~F}$ NMR spectra are referenced according to the proton resonance of TMS as the primary reference for the unified chemical shift scale (IUPAC recommendation 2001). Broadband ${ }^{19} \mathrm{~F}$-decoupled ${ }^{13} \mathrm{C}$ spectra were recorded using the WURST decoupling method. 


\section{Synthesis of Starting Materials}

\section{General procedure 1 for Suzuki coupling (GP1):}

To a screw-cap flask equipped with a stir bar were added the indicated amounts of $\left[\mathrm{Pd}\left(\mathrm{PPh}_{3}\right)_{4}\right]$ (2.0-5.0 mol\%), $\mathrm{K}_{2} \mathrm{CO}_{3}$ (4.8 equiv), the corresponding phenylboronic acid (1.2 equiv) and pyridine (1.0 equiv, if solid). The flask was evacuated and purged with Argon three times, followed by the addition of THF, water, and pyridine (1.0 equiv, if liquid). The mixture was stirred overnight at the indicated temperature (at least $14 \mathrm{~h}$ ). The mixture was allowed to come to room temperature and the organic phase was separated. The aqueous phase was extracted with EtOAc $(2 x)$ and the combined organic phases were dried over magnesium sulfate, concentrated and purified by column chromatography on silica gel.

\section{General procedure 2 for amide coupling (GP2):}

To a screw-cap flask equipped with a stir bar was added the indicated amount of carboxylic acid (1.0 equiv) followed by the addition of $\mathrm{CH}_{2} \mathrm{Cl}_{2}(0.3 \mathrm{M})$. The suspension was cooled to $0^{\circ} \mathrm{C}$, the indicated amount of oxalyl chloride (1.2 equiv) was added dropwise at $0{ }^{\circ} \mathrm{C}$ followed by the addition of a few drops of dimethylformamide. The suspension turned red and was allowed to come to room temperature. After stirring for three hours at room temperature, the volatiles were removed under vacuum. The remaining solid was suspended in $\mathrm{CH}_{2} \mathrm{Cl}_{2}$ and added to a solution of amine (1.1 equiv) and $\mathrm{NEt}_{3}$ (2.2 equiv) in $\mathrm{CH}_{2} \mathrm{Cl}_{2}(0.2 \mathrm{M})$ at $0{ }^{\circ} \mathrm{C}$. The mixture was allowed to reach room temperature and stirred overnight. $\mathrm{H}_{2} \mathrm{O}$ was added, the phases were separated and the aqueous phase was extracted with $\mathrm{CH}_{2} \mathrm{Cl}_{2}(2 \mathrm{x})$. The combined organic phases were dried over $\mathrm{MgSO}_{4}$ and concentrated. The product was purified using column chromatography on silica gel.

\section{General procedure 3 for the synthesis of imidazo[1,2-a]pyridines (GP3):}

In a screw-cap flask equipped with a stir bar, the indicated amount of aminopyridine $(1.0 \mathrm{eq})$ was dissolved in dry ethanol $(0.3 \mathrm{M})$. The indicated amount of aldehyde / ketone was added and the mixture was stirred at $100^{\circ} \mathrm{C}$ overnight. The mixture was allowed to come to room temperature and saturated aqueous $\mathrm{NaHCO}_{3}$ was added. The aqueous phase was extracted with $\mathrm{CH}_{2} \mathrm{Cl}_{2}(3 \mathrm{x})$, the combined organic phases were dried using magnesium sulfate and concentrated. The compound was purified by column chromatography on silica gel. 


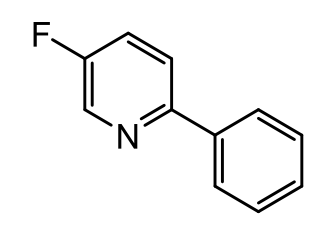

5-Fluoro-2-phenylpyridine: The title compound was prepared according to GP1 from 2-bromo-5-fluoropyridine $(1.0 \mathrm{~g}, 5.7 \mathrm{mmol}, 1.0$ equiv) and phenylboronic acid $(0.9 \mathrm{~g}$, $7.3 \mathrm{mmol}, 1.3$ equiv) using [Pd($\left.\left(\mathrm{PPh}_{3}\right)_{4}\right](200 \mathrm{mg})$ and $\mathrm{Na}_{2} \mathrm{CO}_{3}(4.5 \mathrm{~g}, 42.6 \mathrm{mmol}, 7.5$ equiv) in toluene $(20 \mathrm{ml})$, ethanol $(4.3 \mathrm{ml})$ and $\mathrm{H}_{2} \mathrm{O}(20 \mathrm{ml})$ at $90{ }^{\circ} \mathrm{C}$. The product was isolated after column chromatography (eluent: $n$-pentane, later 98:2, 95:5 $n$ pentane/EtOAc) as a white solid (0.92 g, $5.3 \mathrm{mmol}, 94 \%)$.

${ }^{1} \mathrm{H}$ NMR $(400 \mathrm{MHz}$, Chloroform- $d) \delta 8.55(\mathrm{~d}, J=2.9 \mathrm{~Hz}, 1 \mathrm{H}), 8.00-7.91(\mathrm{~m}, 2 \mathrm{H}), 7.70$ (dd, $J=8.8,4.3 \mathrm{~Hz}, 1 \mathrm{H}), 7.54-7.38(\mathrm{~m}, 4 \mathrm{H}) .{ }^{13} \mathrm{C}\left\{{ }^{1} \mathrm{H}\right\}$ NMR (101 MHz, Chloroform- $d$ ) $\delta 158.9$ (d, $J=256.1 \mathrm{~Hz}$ ), 153.8 (d, $J=3.9 \mathrm{~Hz}$ ), 138.5, 137.8 (d, $J=23.5 \mathrm{~Hz}), 129.0$, 128.9, $126.8,123.5(\mathrm{~d}, J=18.5 \mathrm{~Hz}), 121.3(\mathrm{~d}, J=4.2 \mathrm{~Hz}) .{ }^{19} \mathrm{~F}\left\{{ }^{1} \mathrm{H}\right\}$ NMR $(376 \mathrm{MHz}$, Chloroform- $d$ ) $\delta$-129.8. HRMS (ESI) $\mathrm{m} / \mathrm{z}$ calculated for $\left[\mathrm{C}_{11} \mathrm{H}_{9} \mathrm{FN}\right]\left(\left[\mathrm{M}+\mathrm{H}^{+}\right]\right)$174.0714, found 174.0709 .

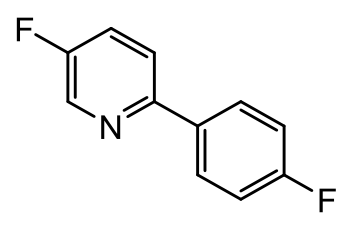

5-Fluoro-2-(4-fluorophenyl)pyridine: The title compound was prepared according to GP1 from 2-bromo-5-fluoropyridine $(2.11 \mathrm{~g}, 12.0 \mathrm{mmol}, 1.2$ equiv) and 4fluorophenylboronic acid (1.40 g, $12.0 \mathrm{mmol}, 1.0$ equiv) using $\left[\mathrm{Pd}\left(\mathrm{PPh}_{3}\right)_{4}\right](416 \mathrm{mg})$ and $\mathrm{K}_{2} \mathrm{CO}_{3}\left(7.0 \mathrm{ml} .2 \mathrm{M}\right.$ aq.) in THF $(50 \mathrm{ml})$ at $90^{\circ} \mathrm{C}$. The product was isolated after column chromatography (eluent: $n$-pentane, later 98:2, 95:5n-pentane/EtOAc) as a white solid (0.88 g, $4.6 \mathrm{mmol}, 46 \%)$.

${ }^{1} \mathrm{H}$ NMR $(400 \mathrm{MHz}$, Chloroform- $d) \delta 8.50(\mathrm{~d}, J=2.9 \mathrm{~Hz}, 1 \mathrm{H}), 7.93-7.85(\mathrm{~m}, 2 \mathrm{H}), 7.61$ $(\mathrm{dd}, J=8.8,4.2 \mathrm{~Hz}, 1 \mathrm{H}), 7.40(\mathrm{td}, J=8.4,2.9 \mathrm{~Hz}, 1 \mathrm{H}), 7.16-7.05(\mathrm{~m}, 2 \mathrm{H}) .{ }^{13} \mathrm{C}\left\{{ }^{1} \mathrm{H}\right\}$ NMR $(101 \mathrm{MHz}$, Chloroform- $d) \delta 163.5(\mathrm{~d}, J=248.5 \mathrm{~Hz}), 158.8(\mathrm{~d}, J=256.2 \mathrm{~Hz}), 152.7$ (d, $J=4.0 \mathrm{~Hz}$ ), $137.8(\mathrm{~d}, J=23.6 \mathrm{~Hz}), 134.6(\mathrm{~d}, J=3.2 \mathrm{~Hz}), 128.6(\mathrm{~d}, J=8.3 \mathrm{~Hz})$, $123.6(\mathrm{~d}, J=18.6 \mathrm{~Hz}), 121.0(\mathrm{~d}, J=4.2 \mathrm{~Hz}), 115.7(\mathrm{~d}, J=21.6 \mathrm{~Hz}) .{ }^{19} \mathrm{~F}\left\{{ }^{1} \mathrm{H}\right\} \operatorname{NMR}(376$ $\mathrm{MHz}$, Chloroform- $d$ ) $\delta-113.1,-129.8$. HRMS (ESI) $\mathrm{m} / \mathrm{z}$ calculated for $\left[\mathrm{C}_{11} \mathrm{H}_{8} \mathrm{~F}_{2} \mathrm{~N}\right]$ $\left(\left[\mathrm{M}+\mathrm{H}^{+}\right]\right)$192.0619, found 192.0618 . 


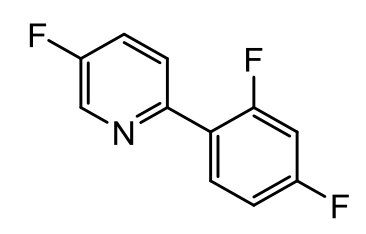

5-Fluoro-2-(2,4,-difluorophenyl)pyridine: The title compound was prepared according to GP1 from 2-bromo-5-fluoropyridine (1.34 g, $7.6 \mathrm{mmol}, 1.2$ equiv) and 2,4difluorophenylboronic acid (1.00 g, $6.3 \mathrm{mmol}, 1.0$ equiv) using [Pd( $\left.\left.\mathrm{PPh}_{3}\right)_{4}\right](260 \mathrm{mg})$ and $\mathrm{K}_{2} \mathrm{CO}_{3}\left(4.0 \mathrm{ml}, 2 \mathrm{M}\right.$ aq.) in $\mathrm{THF}(60 \mathrm{ml})$ at $90^{\circ} \mathrm{C}$. The product was isolated after column chromatography (eluent: $n$-pentane, later 98:2, 95:5 $n$-pentane/EtOAc) as a white solid (0.66 g, $3.2 \mathrm{mmol}, 50 \%)$.

${ }^{1} \mathrm{H}$ NMR $(400 \mathrm{MHz}$, Chloroform-d) $\delta 8.52(\mathrm{~d}, J=3.0 \mathrm{~Hz}, 1 \mathrm{H}), 7.95$ (td, $J=8.9,6.6 \mathrm{~Hz}$, $1 \mathrm{H}$ ), 7.72 (ddd, $J=8.9,4.4,1.9 \mathrm{~Hz}, 1 \mathrm{H}), 7.41$ (td, $J=8.4,3.0 \mathrm{~Hz}, 1 \mathrm{H}), 7.00-6.92(\mathrm{~m}$, $1 \mathrm{H}), 6.87$ (ddd, $J=11.4,8.8,2.5 \mathrm{~Hz}, 1 \mathrm{H}) .{ }^{13} \mathrm{C}\left\{{ }^{1} \mathrm{H}\right\} \mathbf{N M R}(101 \mathrm{MHz}$, Chloroform- $d) \delta$ 163.2 (dd, $J=251.2,12.1 \mathrm{~Hz}$ ), 160.4 (dd, $J=252.2,11.9 \mathrm{~Hz}), 158.7(\mathrm{~d}, J=257.1 \mathrm{~Hz}$ ), 148.7 - $148.6(\mathrm{~m}), 138.0$ (d, $J=23.7 \mathrm{~Hz}$ ), 132.1 (dd, $J=9.7,4.4 \mathrm{~Hz}$ ), 124.9 (dd, $J=$ 10.2, $4.3 \mathrm{~Hz}$ ), 123.2 (d, $J=18.4 \mathrm{~Hz}$ ), 122.8 (dd, $J=11.7,3.9 \mathrm{~Hz}$ ), 112.0 (dd, $J=21.2$, 3.7 Hz), $104.4(\mathrm{dd}, J=27.1,25.4 \mathrm{~Hz}) \cdot{ }^{19} \mathrm{~F}\left\{{ }^{1} \mathrm{H}\right\}$ NMR (376 MHz, Chloroform- $\left.d\right) \delta-109.2$ (d, $J=8.5 \mathrm{~Hz}$ ), $-113.0(\mathrm{~d}, J=8.6 \mathrm{~Hz}),-128.4$. HRMS $(\mathrm{ESI}) \mathrm{m} / \mathrm{z}$ calculated for $\left[\mathrm{C}_{11} \mathrm{H}_{7} \mathrm{~F}_{3} \mathrm{~N}\right]$ $\left(\left[\mathrm{M}+\mathrm{H}^{+}\right]\right) 210.0525$, found 210.0524 .

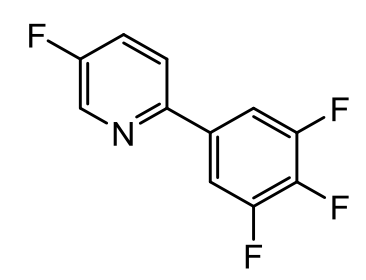

5-Fluoro-2-(3,4,5-trifluorophenyl)pyridine: The title compound was prepared according to GP1 from 2-bromo-5-fluoropyridine $(880.0 \mathrm{mg}, 5.0 \mathrm{mmol}$ ) and 3,4,5trifluorophenylboronic acid $(1.06 \mathrm{~g}, 6.0 \mathrm{mmol})$ using $\left[\mathrm{Pd}\left(\mathrm{PPh}_{3}\right)_{4}\right](116 \mathrm{mg})$ and $\mathrm{K}_{2} \mathrm{CO}_{3}$ (3.32 $\mathrm{g})$ in THF $(16 \mathrm{ml})$ and $\mathrm{H}_{2} \mathrm{O}(12 \mathrm{ml})$ at $80^{\circ} \mathrm{C}$. The product was isolated after column chromatography (eluent: $n$-pentane, later 98:2, 95:5 $n$-pentane/EtOAc) as a white solid (0.99 g, $4.4 \mathrm{mmol}, 87 \%)$. ${ }^{1} \mathrm{H}$ NMR $(599 \mathrm{MHz}$, Chloroform- $d) \delta 8.52$ (d, J = $2.9 \mathrm{~Hz}, 1 \mathrm{H}$ ), 7.64 (ddd, $J=8.8,4.1,0.7 \mathrm{~Hz}, 1 \mathrm{H}), 7.63-7.56(\mathrm{~m}, 2 \mathrm{H}), 7.50-7.47$ (m, $1 \mathrm{H}) .{ }^{13} \mathrm{C}\left\{{ }^{1} \mathrm{H}\right\}$ NMR $(151 \mathrm{MHz}$, Chloroform- $d) \delta 159.4(\mathrm{~d}, J=258.2 \mathrm{~Hz}), 151.7$ (ddd, $J=$ 249.7, 10.1, 4.0 Hz), 150.5 - $150.2(\mathrm{~m}), 140.4$ (dt, $J=253.9,15.5 \mathrm{~Hz}), 138.3(\mathrm{~d}, J=$ $24.0 \mathrm{~Hz}$ ), 134.5 (td, $J=7.6,4.3 \mathrm{~Hz}), 123.9(\mathrm{~d}, J=18.9 \mathrm{~Hz}), 121.1$ (dd, $J=4.5,0.7 \mathrm{~Hz})$, 
110.9 (dd, $J=17.7,4.8 \mathrm{~Hz}) .{ }^{19} \mathrm{~F}$ NMR $(564 \mathrm{MHz}$, Chloroform- $d$ ) $\delta-127.8$ (dd, $J=7.9$, 4.1 Hz, 1F), -133.6--134.52 (m, 2F), -157.0--163.5 (m, 1F). ${ }^{19} \mathrm{~F}\left\{{ }^{1} \mathrm{H}\right\}$ NMR (564 MHz, Chloroform-d) $\delta-127.8$ (s, 1F), -133.78 (d, $J=20.5 \mathrm{~Hz}, 2 \mathrm{~F}),-160.2$ (td, $J=20.4,1.2$ $\mathrm{Hz}, 1 \mathrm{~F})$. HRMS $(\mathrm{ESI}) \mathrm{m} / \mathrm{z}$ calculated for $\left[\mathrm{C}_{11} \mathrm{H}_{6} \mathrm{~F}_{4} \mathrm{~N}\right]\left(\left[\mathrm{M}+\mathrm{H}^{+}\right]\right) 228.0431$, found 228.0425 .

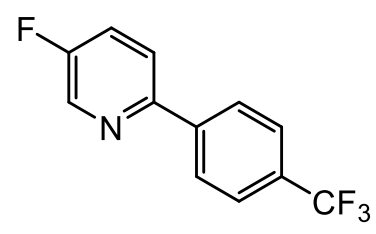

5-Fluoro-2-(4-(trifluoromethyl)phenyl)pyridine: The title compound was prepared according to GP1 from 2-bromo-5-fluoropyridine $(880.0 \mathrm{mg}, 5.0 \mathrm{mmol})$ and 4(trifluoromethyl)phenylboronic acid (1.14 g, $6.0 \mathrm{mmol})$ using [Pd(PPh 3$\left.)_{4}\right](116 \mathrm{mg})$ and $\mathrm{K}_{2} \mathrm{CO}_{3}(3.32 \mathrm{~g})$ in THF $(16 \mathrm{ml})$ and $\mathrm{H}_{2} \mathrm{O}(12 \mathrm{ml})$ at $90{ }^{\circ} \mathrm{C}$. The product was isolated after column chromatography (eluent: $n$-pentane, later 98:2, 95:5 n-pentane/EtOAc) as a white solid (1.02 g, $4.2 \mathrm{mmol}, 85 \%)$. ${ }^{1} \mathrm{H}$ NMR (400 MHz, Chloroform- $d$ ) $\delta 8.57$ (d, J $=2.9 \mathrm{~Hz}, 1 \mathrm{H}), 8.06(\mathrm{~d}, J=8.0 \mathrm{~Hz}, 2 \mathrm{H}), 7.76(\mathrm{dd}, J=8.8,4.2 \mathrm{~Hz}, 1 \mathrm{H}), 7.72(\mathrm{~d}, J=8.1$ $\mathrm{Hz}, 2 \mathrm{H}), 7.50$ (ddd, $J=8.8,8.0,2.9 \mathrm{~Hz}, 1 \mathrm{H}) .{ }^{13} \mathrm{C}\left\{{ }^{1} \mathrm{H}\right\}$ NMR $(101 \mathrm{MHz}$, Chloroform- $d) \delta$ 159.4 (d, $J=257.8 \mathrm{~Hz}$ ), 152.2 (d, J=4.0 Hz), 141.8 (d, J=1.5 Hz), 138.3 (d, J=23.7 Hz), 130.9 (q, $J=32.5 \mathrm{~Hz}$ ), 127.2, 125.9 (q, $J=3.8 \mathrm{~Hz}), 124.3$ (q, J=272.1 Hz), 123.8 (d, $J=18.7 \mathrm{~Hz}), 121.8(\mathrm{~d}, J=4.5 \mathrm{~Hz}) .{ }^{19} \mathrm{~F}\left\{{ }^{1} \mathrm{H}\right\}$ NMR (376 MHz, Chloroform-d) $\delta-62.6$ $(3 \mathrm{~F}),-128.1(1 \mathrm{~F})$. HRMS (ESI) $\mathrm{m} / \mathrm{z}$ calculated for $\left[\mathrm{C}_{12} \mathrm{H}_{8} \mathrm{~F}_{4} \mathrm{~N}\right]\left(\left[\mathrm{M}+\mathrm{H}^{+}\right]\right) 242.0587$, found 242.0591 .

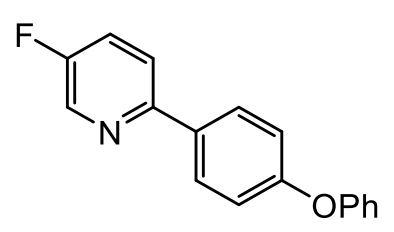

5-Fluoro-2-(4-phenoxyphenyl)pyridine: The title compound was prepared according to GP1 from 2-bromo-5-fluoropyridine (1.06 g, $6.0 \mathrm{mmol})$ and 4(phenoxy)phenylboronic acid (1.54 g, $7.2 \mathrm{mmol})$ using $\left[\mathrm{Pd}\left(\mathrm{PPh}_{3}\right)_{4}\right](139 \mathrm{mg})$ and $\mathrm{K}_{2} \mathrm{CO}_{3}(3.98 \mathrm{~g})$ in THF $(16 \mathrm{ml})$ and $\mathrm{H}_{2} \mathrm{O}(12 \mathrm{ml})$ at $90^{\circ} \mathrm{C}$. The product was isolated after column chromatography (eluent: $n$-pentane, later 95:5 n-pentane/EtOAc) as a white solid in quantitative yield. ${ }^{1} \mathbf{H}$ NMR $(400 \mathrm{MHz}$, Chloroform- $d) \delta 8.53(\mathrm{~d}, J=2.9$ $\mathrm{Hz}, 1 \mathrm{H}), 7.96-7.88(\mathrm{~m}, 2 \mathrm{H}), 7.66(\mathrm{dd}, J=8.8,4.3 \mathrm{~Hz}, 1 \mathrm{H}), 7.43(\mathrm{td}, J=8.4,2.9 \mathrm{~Hz}$, $1 \mathrm{H}), 7.40-7.34(\mathrm{~m}, 2 \mathrm{H}), 7.18-7.04(\mathrm{~m}, 5 \mathrm{H}) .{ }^{13} \mathrm{C}\left\{{ }^{1} \mathrm{H}\right\}$ NMR $(101 \mathrm{MHz}$, Chloroform- $d)$ 
$\delta 158.7(\mathrm{~d}, J=255.8 \mathrm{~Hz}), 158.3,156.9,153.2(\mathrm{~d}, J=3.9 \mathrm{~Hz}), 137.7(\mathrm{~d}, J=23.5 \mathrm{~Hz}$ ), 133.5, 129.9, 128.3, 123.7, 123.6 (d, $J=18.6 \mathrm{~Hz}), 120.9$ (d, $J=4.2 \mathrm{~Hz}), 119.3,118.9$. ${ }^{19} \mathrm{~F}\left\{{ }^{1} \mathrm{H}\right\}$ NMR (376 MHz, Chloroform- $d$ ) $\delta$-130.2. HRMS (ESI) $\mathrm{m} / \mathrm{z}$ calculated for $\left[\mathrm{C}_{17} \mathrm{H}_{13} \mathrm{FNO}\right]\left(\left[\mathrm{M}+\mathrm{H}^{+}\right]\right) 266.0976$, found 266.0974.<smiles>COc1cc(OC)cc(-c2ccc(F)cn2)c1</smiles>

2-(3,5-Dimethoxyphenyl)-5-fluoropyridine: The title compound was prepared according to GP1 from 2-bromo-5-fluoropyridine $(0.51 \mathrm{ml}, 5.0 \mathrm{mmol})$ and 3,5 (dimethoxy)phenylboronic acid $(1.09 \mathrm{~g}, 6.0 \mathrm{mmol})$ using $\left[\mathrm{Pd}\left(\mathrm{PPh}_{3}\right)_{4}\right](116 \mathrm{mg})$ and $\mathrm{K}_{2} \mathrm{CO}_{3}(3.32 \mathrm{~g})$ in THF $(16 \mathrm{ml})$ and $\mathrm{H}_{2} \mathrm{O}(12 \mathrm{ml})$ at $90^{\circ} \mathrm{C}$. The product was isolated after column chromatography (eluent: $n$-pentane, later 95:5, 90:10 n-pentane/EtOAc) as a colorless liquid (1.02 g, $4.4 \mathrm{mmol}, 88 \%) .{ }^{1} \mathrm{H}$ NMR (400 MHz, Chloroform- $d$ ) $\delta 8.53$ $(\mathrm{d}, J=2.9 \mathrm{~Hz}, 1 \mathrm{H}), 7.71-7.67(\mathrm{~m}, 1 \mathrm{H}), 7.45$ (ddd, $J=8.7,8.1,2.9 \mathrm{~Hz}, 1 \mathrm{H}), 7.10$ (d, $J$ $=2.3 \mathrm{~Hz}, 2 \mathrm{H}), 6.52(\mathrm{t}, J=2.3 \mathrm{~Hz}, 1 \mathrm{H}), 3.87(\mathrm{~s}, 6 \mathrm{H}) \cdot{ }^{13} \mathrm{C}\left\{{ }^{1} \mathrm{H}\right\} \mathbf{N M R}(101 \mathrm{MHz}$, Chloroform- $d$ ) $\delta 161.3,159.1$ (d, $J=256.4 \mathrm{~Hz}$ ), 153.6 (d, $J=3.9 \mathrm{~Hz}$ ), 140.6, 137.7 (d, $J=23.6 \mathrm{~Hz}), 123.6(\mathrm{~d}, J=18.6 \mathrm{~Hz}), 121.7(\mathrm{~d}, J=4.3 \mathrm{~Hz}), 105.0,101.3,55.6 .{ }^{19} \mathrm{~F}\left\{{ }^{1} \mathrm{H}\right\}$ NMR (376 MHz, Chloroform- $d$ ) $\delta-129.3$ HRMS (ESI) m/z calculated for $\left[\mathrm{C}_{13} \mathrm{H}_{13} \mathrm{FNO}_{2}\right]$ $\left(\left[\mathrm{M}+\mathrm{H}^{+}\right]\right)$234.0925, found 234.0933 .<smiles>COc1cc(OC)cc(-c2ncccc2F)c1</smiles>

2-(3,5-Dimethoxyphenyl)-3-fluoropyridine: The title compound was prepared according to GP1 from 2-bromo-3-fluoropyridine $(0.51 \mathrm{ml}, 5.0 \mathrm{mmol})$ and 3,5(dimethoxy)phenylboronic acid $(1.09 \mathrm{~g}, 6.0 \mathrm{mmol})$ using $\left[\mathrm{Pd}\left(\mathrm{PPh}_{3}\right)_{4}\right](116 \mathrm{mg})$ and $\mathrm{K}_{2} \mathrm{CO}_{3}(3.32 \mathrm{~g})$ in $\mathrm{THF}(16 \mathrm{ml})$ and $\mathrm{H}_{2} \mathrm{O}(12 \mathrm{ml})$ at $90^{\circ} \mathrm{C}$. The product was isolated after column chromatography (eluent: $n$-pentane, later 95:5, 90:10 $n$-pentane/EtOAc) as a white solid (1.17 g, $5.0 \mathrm{mmol}, 99 \%) .{ }^{1} \mathrm{H}$ NMR $(599 \mathrm{MHz}$, Chloroform-d) $\delta 8.50$ (dt, $J=4.6,1.6 \mathrm{~Hz}, 1 \mathrm{H}$ ), 7.46 (ddd, $J=11.1,8.3,1.4 \mathrm{~Hz}, 1 \mathrm{H}$ ), 7.25 (ddd, $J=8.2,4.5,3.6$ $\mathrm{Hz}, 1 \mathrm{H}), 7.15(\mathrm{dd}, J=2.3,1.4 \mathrm{~Hz}, 2 \mathrm{H}), 6.55(\mathrm{t}, J=2.3 \mathrm{~Hz}, 1 \mathrm{H}), 3.85(\mathrm{~s}, 6 \mathrm{H}) .{ }^{13} \mathrm{C}\left\{{ }^{1} \mathrm{H}\right\}$ 
NMR (151 MHz, Chloroform- $d) \delta 160.9,157.6$ (d, $J=261.0 \mathrm{~Hz}), 146.0$ (d, $J=10.2 \mathrm{~Hz})$, 145.3 (d, J = 5.2 Hz), 137.2 (d, J=5.5 Hz), 124.3 (d, J=21.0 Hz), 123.7 (d, J = 4.0 $\mathrm{Hz}), 106.9$ (d, $J=6.1 \mathrm{~Hz}), 101.9,55.6 .{ }^{19} \mathrm{~F}\left\{{ }^{1} \mathrm{H}\right\}$ NMR (564 MHz, Chloroform- $d$ ) $\delta-121.9$. HRMS (ESI) m/z calculated for $\left[\mathrm{C}_{13} \mathrm{H}_{13} \mathrm{FNO}_{2}\right]\left(\left[\mathrm{M}+\mathrm{H}^{+}\right]\right) 234.0925$, found 234.0921.

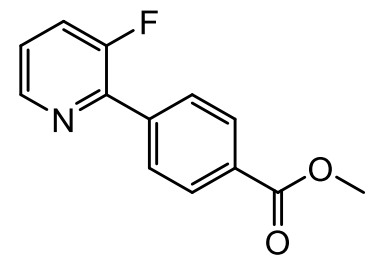

Methyl 4-(3-fluoropyridin-2-yl)benzoate: The title compound was prepared according to GP1 from 2-bromo-3-fluoropyridine $(0.61 \mathrm{ml}, 6.0 \mathrm{mmol})$ and (4(methoxycarbonyl)phenyl)boronic acid (1.30 g, $7.2 \mathrm{mmol})$ using [Pd(PPh 3$\left.)_{4}\right]$ (139 mg) and $\mathrm{K}_{2} \mathrm{CO}_{3}(3.98 \mathrm{~g})$ in THF $(16 \mathrm{ml})$ and $\mathrm{H}_{2} \mathrm{O}(12 \mathrm{ml})$ at $90{ }^{\circ} \mathrm{C}$. The product was isolated after column chromatography (eluent: $n$-pentane, later 95:5 n-pentane/EtOAc) as a white solid (0.67 g, 2.9 mmol, 48\%). ${ }^{1} \mathrm{H}$ NMR (400 MHz, Chloroform- $d$ ) $\delta 8.54-8.47$ $(\mathrm{m}, 1 \mathrm{H}), 8.17-8.10(\mathrm{~m}, 2 \mathrm{H}), 8.09-8.00(\mathrm{~m}, 2 \mathrm{H}), 7.52-7.43(\mathrm{~m}, 1 \mathrm{H}), 7.31-7.22(\mathrm{~m}$, $1 \mathrm{H}), 3.92(\mathrm{~s}, 3 \mathrm{H}) .{ }^{13} \mathrm{C}\left\{{ }^{1} \mathrm{H}\right\}$ NMR (101 MHz, Chloroform- $\left.d\right) \delta 166.8,157.8(\mathrm{~d}, J=261.7$ Hz), 145.6 (d, J=5.2 Hz), 144.9 (d, $J=10.2 \mathrm{~Hz}$ ), 139.6 (d, $J=5.7 \mathrm{~Hz}$ ), 130.5, 129.7, $128.8(\mathrm{~d}, J=6.2 \mathrm{~Hz}), 124.4(\mathrm{~d}, J=15.0 \mathrm{~Hz}), 124.3(\mathrm{~d}, J=1.5 \mathrm{~Hz}), 52.2 .{ }^{19} \mathrm{~F}\left\{{ }^{1} \mathrm{H}\right\}$ NMR (376 MHz, Chloroform- $d$ ) $\delta-122.1$. HRMS (ESI) $\mathrm{m} / \mathrm{z}$ calculated for $\left[\mathrm{C}_{13} \mathrm{H}_{11} \mathrm{FNO}_{2}\right]$ $\left(\left[\mathrm{M}+\mathrm{H}^{+}\right]\right)$232.0768, found 232.0766.<smiles>Fc1cnccc1-c1cc(F)c(F)c(F)c1</smiles>

3-Fluoro-4-(3,4,5-trifluorophenyl)pyridine: The title compound was prepared according to GP1 from 4-chloro-3-fluoropyridine $(0.59 \mathrm{ml}, 6.0 \mathrm{mmol})$ and 3,4,5trifluorophenylboronic acid $(1.27 \mathrm{~g}, 6.6 \mathrm{mmol})$ using $\left[\mathrm{Pd}\left(\mathrm{PPh}_{3}\right)_{4}\right](347 \mathrm{mg})$ and $\mathrm{K}_{2} \mathrm{CO}_{3}$ $(3.98 \mathrm{~g})$ in THF $(16 \mathrm{ml})$ and $\mathrm{H}_{2} \mathrm{O}(12 \mathrm{ml})$ at $90{ }^{\circ} \mathrm{C}$. The product was isolated after column chromatography (eluent: $n$-pentane, later 95:5, 90:10 n-pentane/EtOAc) as a white solid (4.9 mmol, $1.12 \mathrm{~g}, 82 \%)$. ${ }^{1} \mathrm{H}$ NMR (599 MHz, Chloroform- $d$ ) $\delta 8.57$ (d, $J=$ $2.5 \mathrm{~Hz}, 1 \mathrm{H}$ ), 8.50 (dd, $J=5.0,0.9 \mathrm{~Hz}, 1 \mathrm{H}), 7.33(\mathrm{dd}, J=6.7,5.0 \mathrm{~Hz}, 1 \mathrm{H}), 7.28-7.25$ 
(m, 2H). ${ }^{13} \mathrm{C}\left\{{ }^{1} \mathrm{H}\right\}$ NMR $(151 \mathrm{MHz}$, Chloroform- $d) \delta 156.3(\mathrm{~d}, J=258.9 \mathrm{~Hz}), 151.5$ (ddd, $J=251.2,10.1,4.2 \mathrm{~Hz}), 146.5$ (d, $J=5.4 \mathrm{~Hz}), 141.4$ (t, $J=15.2 \mathrm{~Hz}), 139.6$ (d, $J=25.4$ $\mathrm{Hz}), 133.2-132.9(\mathrm{~m}), 129.0-128.7(\mathrm{~m}), 123.6,113.4$ (ddd, $J=17.5,5.0,3.8 \mathrm{~Hz}$ ). ${ }^{19} \mathrm{~F}\left\{{ }^{1} \mathrm{H}\right\}$ NMR $(564 \mathrm{MHz}$, Chloroform- $d) \delta-132.6(1 \mathrm{~F}),-133.0(\mathrm{~d}, J=20.6 \mathrm{~Hz}, 2 \mathrm{~F}),-$ $158.5(\mathrm{t}, J=20.5 \mathrm{~Hz}, 1 \mathrm{~F})$. HRMS (ESI) $\mathrm{m} / \mathrm{z}$ calculated for $\left[\mathrm{C}_{11} \mathrm{H}_{6} \mathrm{~F}_{4} \mathrm{~N}\right]\left(\left[\mathrm{M}+\mathrm{H}^{+}\right]\right)$ 228.0431 , found 228.0425 .

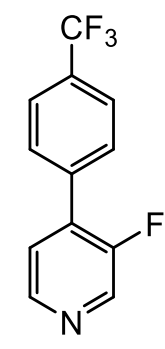

3-Fluoro-4-(4-(trifluoromethyl)phenyl)pyridine: The title compound was prepared according to GP1 from 4-chloro-3-fluoropyridine $(0.59 \mathrm{ml}, 6.0 \mathrm{mmol})$ and 4(trifluoromethyl)phenylboronic acid $(1.37 \mathrm{~g}, 6.6 \mathrm{mmol})$ using $\left[\mathrm{Pd}\left(\mathrm{PPh}_{3}\right)_{4}\right](347 \mathrm{mg})$ and $\mathrm{K}_{2} \mathrm{CO}_{3}(3.98 \mathrm{~g})$ in THF $(16 \mathrm{ml})$ and $\mathrm{H}_{2} \mathrm{O}(12 \mathrm{ml})$ at $90^{\circ} \mathrm{C}$. The product was isolated after column chromatography (eluent: $n$-pentane, later 95:5, 90:10 n-pentane/EtOAc) as a white solid (5.8 mmol, $1.40 \mathrm{~g}, 97 \%) .{ }^{1} \mathrm{H}$ NMR $(400 \mathrm{MHz}$, Chloroform- $d) \delta 8.57$ (d, $J=2.4 \mathrm{~Hz}, 1 \mathrm{H}$ ), $8.51(\mathrm{~d}, J=4.9 \mathrm{~Hz}, 1 \mathrm{H}), 7.81-7.68(\mathrm{~m}, 4 \mathrm{H}), 7.39(\mathrm{dd}, J=6.6,4.9 \mathrm{~Hz}$, 1H). ${ }^{13} \mathrm{C}\left\{{ }^{1} \mathrm{H}\right\}$ NMR $(101 \mathrm{MHz}$, Chloroform- $d) \delta 156.6(\mathrm{~d}, J=258.5 \mathrm{~Hz}), 146.4(\mathrm{~d}, J=$ $5.3 \mathrm{~Hz}$ ), 139.4 (d, $J=25.4 \mathrm{~Hz}$ ), 136.6 (t, $J=1.4 \mathrm{~Hz}$ ), 134.7 (d, $J=10.6 \mathrm{~Hz}), 131.4$ (q, $J=32.8 \mathrm{~Hz}$ ), $129.3(\mathrm{~d}, J=3.3 \mathrm{~Hz}), 125.9(\mathrm{q}, J=3.8 \mathrm{~Hz}), 124.1,124.0$ (q, $J=272.3$ $\mathrm{Hz}$ ). ${ }^{19} \mathrm{~F}\left\{{ }^{1} \mathrm{H}\right\}$ NMR (376 MHz, Chloroform- $d$ ) $\delta-62.9$ (3F), -132.7 (1F). HRMS (ESI) m/z calculated for $\left[\mathrm{C}_{12} \mathrm{H}_{8} \mathrm{~F}_{4} \mathrm{~N}\right]\left(\left[\mathrm{M}+\mathrm{H}^{+}\right]\right) 242.0587$, found 242.0582 .

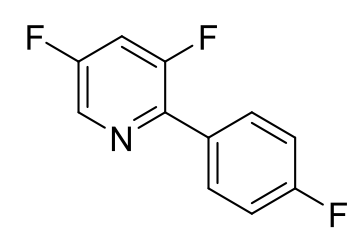

3,5-Difluoro-2-(4-fluorophenyl)pyridine: The title compound was prepared according to GP1 from 2-bromo-3,5-difluoropyridine $(0.39 \mathrm{~g}, 2.0 \mathrm{mmol}$ ) and (4fluorophenyl)boronic acid $(0.34 \mathrm{~g}, 2.4 \mathrm{mmol})$ using $\left[\mathrm{Pd}\left(\mathrm{PPh}_{3}\right)_{4}\right](116 \mathrm{mg})$ and $\mathrm{K}_{2} \mathrm{CO}_{3}$ $(1.33 \mathrm{~g})$ in THF $(8 \mathrm{ml})$ and $\mathrm{H}_{2} \mathrm{O}(6 \mathrm{ml})$ at $80^{\circ} \mathrm{C}$. The product was isolated after column chromatography (eluent: $n$-pentane, later 95:5n-pentane/EtOAc) as a white solid (0.37 g, $1.8 \mathrm{mmol}, 89 \%) .{ }^{1} \mathrm{H}$ NMR (599 MHz, Chloroform- $\left.d\right) \delta 8.45-8.41(\mathrm{~m}, 1 \mathrm{H}), 7.93$ 
(ddt, $J=6.9,5.4,1.6 \mathrm{~Hz}, 2 \mathrm{H}$ ), 7.30 (ddd, $J=10.5,7.9,2.4 \mathrm{~Hz}, 1 \mathrm{H}), 7.20-7.13(\mathrm{~m}$, 2H). ${ }^{13} \mathrm{C}\left\{{ }^{1} \mathrm{H}\right\}$ NMR $(151 \mathrm{MHz}$, Chloroform- $d) \delta 163.5(\mathrm{~d}, J=249.2 \mathrm{~Hz}), 158.3(\mathrm{dd}, J=$ 262.3, $5.1 \mathrm{~Hz}$ ), 156.7 (dd, $J=266.2,5.8 \mathrm{~Hz}$ ), 141.8 (dd, $J=10.4,4.0 \mathrm{~Hz}$ ), 133.9 (dd, $J=22.4,4.7 \mathrm{~Hz}$ ), $130.9(\mathrm{dd}, J=6.0,3.2 \mathrm{~Hz}), 130.6(\mathrm{dd}, J=8.4,6.1 \mathrm{~Hz}), 115.7(\mathrm{~d}, J=$ $21.5 \mathrm{~Hz}$ ), 112.4 (dd, $J=23.6,21.3 \mathrm{~Hz}) .{ }^{19} \mathrm{~F}\left\{{ }^{1} \mathrm{H}\right\} \mathbf{N M R}(564 \mathrm{MHz}$, Chloroform- $d) \delta-$ 112.0, $-118.9(\mathrm{~d}, J=6.3 \mathrm{~Hz}),-125.5(\mathrm{~d}, J=6.5 \mathrm{~Hz})$. HRMS (ESI) $\mathrm{m} / \mathrm{z}$ calculated for $\left[\mathrm{C}_{11} \mathrm{H}_{7} \mathrm{~F}_{3} \mathrm{~N}\right]\left(\left[\mathrm{M}+\mathrm{H}^{+}\right]\right) 210.0525$, found 210.0527 .<smiles>COC(=O)c1ccc(F)cn1</smiles>

Methyl 5-fluoropicolinate: 5-Fluoropicolinic acid (1.41 g, $10.0 \mathrm{mmol}, 1.0$ equiv) was added to a screw-cap flask equipped with a stir bar. $\mathrm{MeOH}(10 \mathrm{ml})$ and $\mathrm{H}_{2} \mathrm{SO}_{4}(1.61 \mathrm{ml}$, $30.0 \mathrm{mmol}, 3.0$ equiv) were added to the flask which was then stirred for $48 \mathrm{~h}$ at room temperature. The mixture was concentrated, diluted with water and basified using sat. aq. $\mathrm{Na}_{2} \mathrm{CO}_{3}$. The aqueous mixture was extracted with $\mathrm{CH}_{2} \mathrm{Cl}_{2}(3 \mathrm{x})$, the combined organic phases were dried over $\mathrm{MgSO}_{4}$ and concentrated. The product was isolated after purification by column chromatography (silical gel, eluent: $n$-pentane/EtOAc 90:10, later 80:20) as a white solid (1.48 g, $9.5 \mathrm{mmol}, 95 \%) .{ }^{1} \mathrm{H}$ NMR $(400 \mathrm{MHz}$, Chloroform-d) $\delta 8.56$ (d, $J=2.8 \mathrm{~Hz}, 1 \mathrm{H}$ ), 8.17 (ddd, $J=8.7,4.5,0.6 \mathrm{~Hz}, 1 \mathrm{H}$ ), 7.52 (ddd, $J=8.7,7.8,2.8 \mathrm{~Hz}, 1 \mathrm{H}), 3.99(\mathrm{~s}, 3 \mathrm{H}) .{ }^{13} \mathrm{C}\left\{{ }^{1} \mathrm{H}\right\} \mathbf{N M R}(101 \mathrm{MHz}$, Chloroform- $d) \delta 164.7$, $161.3(\mathrm{~d}, J=263.8 \mathrm{~Hz}), 144.3(\mathrm{~d}, J=4.0 \mathrm{~Hz}), 138.6$ (d, $J=24.7 \mathrm{~Hz}), 127.1$ (d, $J=5.7$ $\mathrm{Hz}), 123.7(\mathrm{~d}, J=18.5 \mathrm{~Hz}), 53.1 .{ }^{19} \mathrm{~F}\left\{{ }^{1} \mathrm{H}\right\}$ NMR $(376 \mathrm{MHz}$, Chloroform- $d) \delta-119.8$ HRMS $(E S I) \mathrm{m} / \mathrm{z}$ calculated for $\left[\mathrm{C}_{7} \mathrm{H}_{6} \mathrm{FNO}_{2} \mathrm{Na}\right]\left(\left[\mathrm{M}+\mathrm{Na}^{+}\right]\right)$178.0275, found 178.0277.<smiles>COC(=O)c1cncc(F)c1</smiles>

Methyl 5-fluoronicotinate: 5-Fluoronicolinic acid $(1.41 \mathrm{~g}, 10.0 \mathrm{mmol}, 1.0$ equiv) was added to a screw-cap flask equipped with a stir bar. $\mathrm{MeOH}(10 \mathrm{ml})$ and $\mathrm{H}_{2} \mathrm{SO}_{4}(1.61 \mathrm{ml}$, $30.0 \mathrm{mmol}, 3.0$ equiv) were added to the flask which was then stirred for $48 \mathrm{~h}$ at room temperature. The mixture was concentrated, diluted with water and basified using sat. aq. $\mathrm{Na}_{2} \mathrm{CO}_{3}$. The aqueous mixture was extracted with $\mathrm{CH}_{2} \mathrm{Cl}_{2}(3 \mathrm{x})$, the combined organic phases were dried over $\mathrm{MgSO}_{4}$ and concentrated. The product was isolated after purification by column chromatography (silical gel, eluent: $n$-pentane/EtOAc 
90:10, later 80:20) as a white solid in quantitative yield. ${ }^{1} \mathbf{H} \mathbf{~ N M R}(400 \mathrm{MHz}$, Chloroformd) $\delta 9.04-8.94(\mathrm{~m}, 1 \mathrm{H}), 8.65-8.52(\mathrm{~m}, 1 \mathrm{H}), 7.99-7.87(\mathrm{~m}, 1 \mathrm{H}), 3.97-3.86(\mathrm{~m}, 3 \mathrm{H})$. ${ }^{13} \mathrm{C}\left\{{ }^{1} \mathrm{H}\right\}$ NMR $(101 \mathrm{MHz}$, Chloroform- $d) \delta 164.7(\mathrm{~d}, J=2.2 \mathrm{~Hz}), 159.1(\mathrm{~d}, J=258.3 \mathrm{~Hz})$, 146.7 (d, $J=4.3 \mathrm{~Hz}$ ), 142.2 (d, $J=23.2 \mathrm{~Hz}), 127.4$ (d, $J=3.6 \mathrm{~Hz}$ ), 123.6 (d, $J=19.5$ $\mathrm{Hz}), 52.8 .{ }^{19} \mathrm{~F}\left\{{ }^{1} \mathrm{H}\right\}$ NMR $(376 \mathrm{MHz}$, Chloroform- $d) \delta-126.0$. HRMS (ESI) $\mathrm{m} / \mathrm{z}$ calculated for [ $\left.\mathrm{C}_{7} \mathrm{H}_{7} \mathrm{FNO}_{2}\right]\left(\left[\mathrm{M}+\mathrm{Na}^{+}\right]\right)$156.0455, found 156.0456 .<smiles>COC(=O)c1ccncc1F</smiles>

Methyl 3-fluoroisonicotinate: 3-Fluoroisonicolinic acid (1.41 g, $10.0 \mathrm{mmol}, 1.0$ equiv) was added to a screw-cap flask equipped with a stir bar. $\mathrm{MeOH}(10 \mathrm{ml})$ and $\mathrm{H}_{2} \mathrm{SO}_{4}$ (1.61 ml, $30.0 \mathrm{mmol}, 3.0$ equiv) were added to the flask which was then stirred for $48 \mathrm{~h}$ at room temperature. The mixture was concentrated, diluted with water and basified using sat. aq. $\mathrm{Na}_{2} \mathrm{CO}_{3}$. The aqueous mixture was extracted with $\mathrm{CH}_{2} \mathrm{Cl}_{2}(3 \mathrm{x})$, the combined organic phases were dried over $\mathrm{MgSO}_{4}$ and concentrated. The product was isolated after purification by column chromatography (silical gel, eluent: $n$ pentane/EtOAc 90:10, later 80:20) as a colorless liquid in quantitative yield. ${ }^{1} \mathbf{H}$ NMR (400 MHz, Chloroform- $d$ ) $\delta 8.57(\mathrm{~d}, J=2.2 \mathrm{~Hz}, 1 \mathrm{H}), 8.50(\mathrm{~d}, J=4.9 \mathrm{~Hz}, 1 \mathrm{H}), 7.73(\mathrm{t}, J$ $=5.5 \mathrm{~Hz}, 1 \mathrm{H}), 3.94(\mathrm{~s}, 3 \mathrm{H}) \cdot{ }^{13} \mathrm{C}\left\{{ }^{1} \mathrm{H}\right\} \mathbf{N M R}(101 \mathrm{MHz}$, Chloroform- $d) \delta 163.4(\mathrm{~d}, J=3.0$ $\mathrm{Hz}), 157.1$ (d, $J=269.1 \mathrm{~Hz}), 146.0(\mathrm{~d}, J=5.8 \mathrm{~Hz}), 140.5(\mathrm{~d}, J=25.4 \mathrm{~Hz}), 125.3(\mathrm{~d}, J$ $=8.4 \mathrm{~Hz}), 124.4(\mathrm{~d}, J=1.5 \mathrm{~Hz}), 53.0 .{ }^{19} \mathrm{~F}\left\{{ }^{1} \mathrm{H}\right\} \mathrm{NMR}(376 \mathrm{MHz}$, Chloroform- $d) \delta-125.0$. HRMS (ESI) $\mathrm{m} / \mathrm{z}$ calculated for $\left[\mathrm{C}_{7} \mathrm{H}_{7} \mathrm{FNO}_{2}\right]\left(\left[\mathrm{M}+\mathrm{H}^{+}\right]\right) 156.0455$, found 156.0456 .<smiles>O=C(Nc1ccc(F)cc1)c1ccc(F)cn1</smiles>

5-Fluoro- $\mathrm{N}$-(4-fluorophenyl)picolinamide: The title compound was prepared according to GP2 from 5-fluoropicolinic acid $(423 \mathrm{mg}, 3.0 \mathrm{mmol})$, oxalyl chloride $(0.31 \mathrm{ml}, 3.6 \mathrm{mmol})$, 4-fluoroaniline $(0.31 \mathrm{ml}, 3.3 \mathrm{mmol})$, and NEt3 $(0.92 \mathrm{ml}, 6.6 \mathrm{mmol})$. The product was isolated after column chromatography (eluent: 90:10 $n$ pentane/EtOAc) as a white solid (613 mg, $2.6 \mathrm{mmol}, 87 \%) .{ }^{1} \mathrm{H}$ NMR $(400 \mathrm{MHz}$, Chloroform- $d$ ) $\delta 9.77(\mathrm{~s}, 1 \mathrm{H}$ ), $8.41(\mathrm{~d}, J=2.8 \mathrm{~Hz}, 1 \mathrm{H}$ ), 8.31 (dd, $J=8.7,4.6 \mathrm{~Hz}, 1 \mathrm{H}$ ), $7.74-7.67(\mathrm{~m}, 2 \mathrm{H}), 7.57(\mathrm{td}, J=8.3,2.8 \mathrm{~Hz}, 1 \mathrm{H}), 7.10-7.02(\mathrm{~m}, 2 \mathrm{H}) .{ }^{13} \mathrm{C}\left\{{ }^{1} \mathrm{H}\right\}$ NMR 
(101 MHz, Chloroform- $d$ ) $\delta 161.4(\mathrm{~d}, J=261.3 \mathrm{~Hz}), 161.0,159.5(\mathrm{~d}, J=243.7 \mathrm{~Hz}$ ), 146.1 (d, $J=3.8 \mathrm{~Hz}$ ), 136.7 (d, $J=25.5 \mathrm{~Hz}), 133.8(\mathrm{~d}, J=2.8 \mathrm{~Hz}), 124.4$ (d, $J=1.1$ $\mathrm{Hz}), 124.3(\mathrm{~d}, J=11.9 \mathrm{~Hz}), 121.4(\mathrm{~d}, J=7.9 \mathrm{~Hz}), 115.8(\mathrm{~d}, J=22.5 \mathrm{~Hz}) .{ }^{19} \mathrm{~F}\left\{{ }^{1} \mathrm{H}\right\} \mathbf{N M R}$ (376 MHz, Chloroform- $d$ ) $\delta-117.8,-121.5$. HRMS (ESI) $\mathrm{m} / \mathrm{z}$ calculated for $\left[\mathrm{C}_{12} \mathrm{H}_{8} \mathrm{~F}_{2} \mathrm{~N}_{2} \mathrm{ONa}\right]\left(\left[\mathrm{M}+\mathrm{Na}^{+}\right]\right)$257.0467, found 257.0496.<smiles>Cc1cccc(C)c1NC(=O)c1ccc(F)cn1</smiles>

$\mathbf{N}$-(2,6-Dimethylphenyl)-5-fluoropicolinamide: This compound was synthesized in analogy of a literature procedure. ${ }^{1}$ In a microwave flask equipped with a stir bar, 5fluoropicolinic acid (187 mg, $1.30 \mathrm{mmol}, 1.1$ equiv) was suspended in dry acetonitrile (15 ml). 2,6-Xylidine (146 $\mu \mathrm{l}, 1.18 \mathrm{mmol}, 1.0$ equiv) and $\mathrm{PCl}_{3}(114 \mu \mathrm{l}, 1.30 \mathrm{mmol}$, 1.1 equiv) were added and the sealed flask was heated for 10 minutes (hold time) at $150^{\circ} \mathrm{C}$ in a microwave. Caution: During the reaction time, the pressure in the vessel reached up to 8 bar. The flask was cooled to room temperature using compressed air. The mixture was poured into aq. $\mathrm{NaOH}(1 \mathrm{M})$ and extracted with $\mathrm{CH}_{2} \mathrm{Cl}_{2}(3 \mathrm{x})$. The combined organic phases were dried using $\mathrm{MgSO}_{4}$ and concentrated. The product was isolated after purification by column chromatography (silical gel, eluent $n$ pentane/EtOAc 90:10) as a white solid. ${ }^{1} \mathbf{H}$ NMR $(400 \mathrm{MHz}$, Chloroform- $d) \delta 9.27$ (s, $1 \mathrm{H}$ ), 8.47 (d, $J=2.8 \mathrm{~Hz}, 1 \mathrm{H}$ ), 8.34 (dd, $J=8.7,4.6 \mathrm{~Hz}, 1 \mathrm{H}$ ), 7.59 (td, $J=8.3,2.8 \mathrm{~Hz}$, $1 \mathrm{H}), 7.20-7.09(\mathrm{~m}, 3 \mathrm{H}), 2.29(\mathrm{~s}, 6 \mathrm{H}) .{ }^{13} \mathrm{C}\left\{{ }^{1} \mathrm{H}\right\}$ NMR $(101 \mathrm{MHz}$, Chloroform- $d) \delta 161.5$, $161.4(\mathrm{~d}, J=260.9 \mathrm{~Hz}), 146.4(\mathrm{~d}, J=3.9 \mathrm{~Hz}), 136.8$ (d, $J=25.3 \mathrm{~Hz}), 135.5,133.7$, 128.3, 127.4, 124.5 (d, $J=5.6 \mathrm{~Hz}), 124.1(\mathrm{~d}, J=18.5 \mathrm{~Hz}), 18.7 .{ }^{19} \mathrm{~F}\left\{{ }^{1} \mathrm{H}\right\}$ NMR $(376$ $\mathrm{MHz}$, Chloroform- $d$ ) $\delta-122.0$ HRMS (ESI) $\mathrm{m} / \mathrm{z}$ calculated for $\left[\mathrm{C}_{14} \mathrm{H}_{13} \mathrm{FN}_{2} \mathrm{ONa}\right.$ ] ([M+Na+]) 267.0904, found 267.0898.

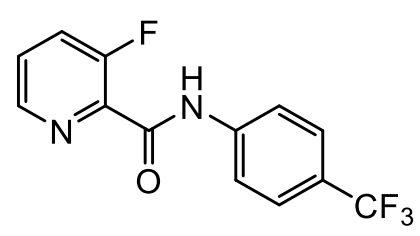

3-Fluoro-N-(4-(trifluoromethyl)phenyl)picolinamide: The title compound was prepared according to GP2 from 3-fluoropicolinic acid $(423 \mathrm{mg}, 3.0 \mathrm{mmol}$ ), oxalyl chloride $(0.31 \mathrm{ml}, 3.6 \mathrm{mmol})$, 4-(trifluoromethyl)aniline $(0.41 \mathrm{ml}, 3.3 \mathrm{mmol})$, and $\mathrm{NEt}_{3}$ $(0.92 \mathrm{ml}, 6.6 \mathrm{mmol})$. The product was isolated after column chromatography (eluent: 
90:10 n-pentane/EtOAc) as a white solid (453 mg, $1.6 \mathrm{mmol}, 53 \%) .{ }^{1} \mathbf{H} \mathbf{~ N M R}(400 \mathrm{MHz}$, Chloroform- $d$ ) $\delta 10.01$ (s, 1H), $8.42-8.34(\mathrm{~m}, 1 \mathrm{H}), 7.85$ (d, J = $8.5 \mathrm{~Hz}, 2 \mathrm{H}), 7.62-$ $7.47(\mathrm{~m}, 4 \mathrm{H}) .{ }^{13} \mathrm{C}\left\{{ }^{1} \mathrm{H}\right\}$ NMR (101 MHz, Chloroform- $\left.d\right) \delta 160.2(\mathrm{~d}, J=6.0 \mathrm{~Hz}), 159.9$ (d, $J=274.4 \mathrm{~Hz}$ ), $143.8(\mathrm{~d}, J=5.6 \mathrm{~Hz}), 140.9-140.5(\mathrm{~m}), 136.8(\mathrm{~d}, J=3.6 \mathrm{~Hz}), 128.9$ (d, $J=5.3 \mathrm{~Hz}$ ), 126.9 (d, $J=19.7 \mathrm{~Hz}$ ), 126.3 (q, $J=3.8 \mathrm{~Hz}$ ), 126.0 (q, $J=32.7 \mathrm{~Hz}), 124.2$ (q, $J=271.5 \mathrm{~Hz})$, 119.3. ${ }^{19} \mathrm{~F}\left\{{ }^{1} \mathrm{H}\right\}$ NMR (376 MHz, Chloroform- $d$ ) $\delta-62.0(3 \mathrm{~F}),-118.1$ $(1 \mathrm{~F})$. HRMS (ESI) $\mathrm{m} / \mathrm{z}$ calculated for $\left[\mathrm{C}_{13} \mathrm{H}_{8} \mathrm{~F}_{4} \mathrm{~N}_{2} \mathrm{ONa}\right]\left(\left[\mathrm{M}+\mathrm{Na}^{+}\right]\right) 307.0465$, found 307.0459.<smiles>Cc1cccc(C)c1NC(=O)c1ncccc1F</smiles>

$\mathbf{N}$-(2,6-Dimethylphenyl)-3-fluoropicolinamide: The title compound was prepared according to GP2 from 3-fluoropicolinic acid (423 mg, $3.0 \mathrm{mmol}$ ), oxalyl chloride (0.31 ml, $3.6 \mathrm{mmol}), 2,6$-xylidine $(0.41 \mathrm{ml}, 3.3 \mathrm{mmol})$, and NEt3 $(0.92 \mathrm{ml}, 6.6 \mathrm{mmol})$. The product was isolated after column chromatography (silica gel, eluent: $n$-pentane, later 90:10, 80:20 $n$-pentane/EtOAc) as a white solid (607 mg, $2.5 \mathrm{mmol}, 83 \%)$. ${ }^{\mathbf{1}} \mathbf{H}$ NMR (400 MHz, Chloroform- d) $\delta 9.27$ (s, 1H), 8.46 (dt, J=4.4, $1.4 \mathrm{~Hz}, 1 \mathrm{H}), 7.65-$ $7.49(\mathrm{~m}, 2 \mathrm{H}), 7.19-7.08(\mathrm{~m}, 3 \mathrm{H}), 2.31(\mathrm{~s}, 6 \mathrm{H}) .{ }^{13} \mathrm{C}\left\{{ }^{1} \mathrm{H}\right\}$ NMR $(101 \mathrm{MHz}$, Chloroformd) $\delta 160.3$ (d, $J=5.9 \mathrm{~Hz}), 159.7$ (d, $J=273.4 \mathrm{~Hz}), 144.0$ (d, $J=5.7 \mathrm{~Hz}), 137.8$ (d, $J=$ 4.3 Hz), 135.5, 133.6, 128.4 (d, $J=5.2 \mathrm{~Hz}$ ), 128.3, 127.3, 126.6 (d, J=19.8 Hz), 18.7. ${ }^{19} \mathrm{~F}\left\{{ }^{1} \mathrm{H}\right\}$ NMR (376 MHz, Chloroform- $d$ ) $\delta$-118.0. HRMS (ESI) m/z calculated for $\left[\mathrm{C}_{14} \mathrm{H}_{13} \mathrm{FN} 2 \mathrm{ONa}\right]\left(\left[\mathrm{M}+\mathrm{H}^{+}\right]\right)$267.0904, found 267.0918.<smiles>O=C(c1ncccc1F)N1CCCCC1</smiles>

(3-Fluoropyridin-2-yl)(piperidin-1-yl)methanone: This compound was synthesized in analogy of a literature procedure. ${ }^{1}$ In a microwave flask equipped with a stir bar, 3fluoropicolinic acid (240 mg, $1.70 \mathrm{mmol}, 1.1$ equiv) was suspended in dry acetonitrile $(15 \mathrm{ml})$. Piperidine (153 $\mu \mathrm{l}, 1.55 \mathrm{mmol}, 1.0$ equiv) and $\mathrm{PCl}_{3}(149 \mu \mathrm{l}, 1.70 \mathrm{mmol}$, 1.1 equiv) were added and the sealed flask was heated for 10 minutes (hold time) at $150^{\circ} \mathrm{C}$ in a microwave. Caution: During the reaction time, the pressure in the vessel reached up to 8 bar. The flask was cooled to room temperature using compressed air. 
The mixture was poured into aq. $\mathrm{NaOH}(1 \mathrm{M})$ and extracted with $\mathrm{CH}_{2} \mathrm{Cl}_{2}(3 \mathrm{x})$. The combined organic phases were dried using $\mathrm{MgSO}_{4}$ and concentrated. The product was isolated after purification by column chromatography (silical gel, eluent EtOAc) as a white solid (243 mg, $1.2 \mathrm{mmol}, 76 \%) .{ }^{1} \mathrm{H}$ NMR $(400 \mathrm{MHz}$, Chloroform- $d$ ) $\delta 8.40$ (dt, $J=$ $4.7,1.6 \mathrm{~Hz}, 1 \mathrm{H}), 7.44(\mathrm{td}, J=8.7,1.3 \mathrm{~Hz}, 1 \mathrm{H}), 7.32(\mathrm{dt}, J=8.7,4.4 \mathrm{~Hz}, 1 \mathrm{H}), 3.74-$ $3.68(\mathrm{~m}, 2 \mathrm{H}), 3.35-3.12(\mathrm{~m}, 2 \mathrm{H}), 1.69-1.61(\mathrm{~m}, 4 \mathrm{H}), 1.55-1.49(\mathrm{~m}, 2 \mathrm{H}) .{ }^{13} \mathrm{C}\left\{{ }^{1} \mathrm{H}\right\}$ NMR (101 MHz, Chloroform-d) $\delta 163.6(\mathrm{~d}, J=3.2 \mathrm{~Hz}), 156.0(\mathrm{~d}, J=259.3 \mathrm{~Hz}), 145.4$ (d, $J=4.9 \mathrm{~Hz}), 143.6$ (d, $J=18.0 \mathrm{~Hz}$ ), 125.3 (d, $J=3.8 \mathrm{~Hz}), 124.0$ (d, $J=18.5 \mathrm{~Hz}$ ), 47.9, 42.8, 26.3, 25.5, 24.5. ${ }^{19} \mathrm{~F}\left\{{ }^{1} \mathrm{H}\right\}$ NMR (376 MHz, Chloroform-d) $\delta$-124.2. HRMS (ESI) $\mathrm{m} / \mathrm{z}$ calculated for $\left[\mathrm{C}_{11} \mathrm{H}_{13} \mathrm{FN} 2 \mathrm{ONa}\right]\left(\left[\mathrm{M}+\mathrm{Na}^{+}\right]\right) 231.0904$, found 231.0902 .<smiles>O=C(Nc1ccccc1)c1ccncc1F</smiles>

3-Fluoro- $\mathrm{N}$-phenylisonicotinamide: The title compound was prepared according to GP2 from 3-fluoroisonicotinic acid $(423 \mathrm{mg}, 3.0 \mathrm{mmol})$, oxalyl chloride $(0.31 \mathrm{ml}$, $3.6 \mathrm{mmol})$, aniline $(0.30 \mathrm{ml}, 3.3 \mathrm{mmol})$, and NEt $3(0.92 \mathrm{ml}, 6.6 \mathrm{mmol})$. The product was isolated after column chromatography (silica gel, eluent: $n$-pentane/EtOAc 50:50, later $0: 100)$ as a white solid (427 mg, $2.0 \mathrm{mmol}, 66 \%) .{ }^{1} \mathrm{H} \mathrm{NMR}(400 \mathrm{MHz}$, Chloroform- $d) \delta$ $8.67-8.59(\mathrm{~m}, 2 \mathrm{H}), 8.41(\mathrm{~d}, J=13.1 \mathrm{~Hz}, 1 \mathrm{H}), 8.00(\mathrm{dd}, J=6.6,4.9 \mathrm{~Hz}, 1 \mathrm{H}), 7.69-$ $7.61(\mathrm{~m}, 2 \mathrm{H}), 7.39(\mathrm{t}, J=8.0 \mathrm{~Hz}, 2 \mathrm{H}), 7.25-7.16(\mathrm{~m}, 1 \mathrm{H}) .{ }^{13} \mathrm{C}\left\{{ }^{1} \mathrm{H}\right\} \mathrm{NMR}(101 \mathrm{MHz}$, Chloroform- $d$ ) $\delta 159.6(\mathrm{~d}, J=2.8 \mathrm{~Hz}), 156.4(\mathrm{~d}, J=256.3 \mathrm{~Hz}), 147.5(\mathrm{~d}, J=5.2 \mathrm{~Hz})$, $139.8(\mathrm{~d}, J=27.7 \mathrm{~Hz}), 137.4,129.7,128.5(\mathrm{~d}, J=9.7 \mathrm{~Hz}), 125.9,125.1,121.1 .{ }^{19} \mathrm{~F}\left\{{ }^{1} \mathrm{H}\right\}$ NMR (376 MHz, Chloroform- $d$ ) $\delta$-128.6. HRMS (ESI) $\mathrm{m} / \mathrm{z}$ calculated for $\left[\mathrm{C}_{12} \mathrm{H}_{10} \mathrm{FN} \mathrm{N}_{2} \mathrm{O}\right]$ $\left(\left[\mathrm{M}+\mathrm{H}^{+}\right]\right)$217.0772, found 217.0769.<smiles>N#CCc1ccccc1</smiles><smiles>N#CC(c1ccccc1)c1ncccc1F</smiles><smiles>NC(=O)C(c1ccccc1)c1ncccc1F</smiles>

2-(3-Fluoropyridin-2-yl)-2-phenylacetamide: In a $50 \mathrm{ml}$ screw-cap flask equipped with a stir bar, $\mathrm{KOH}(1.2 \mathrm{~g}, 22.0 \mathrm{mmol}, 2.2$ equiv) was suspended in dry DMSO (10 ml). 2-Phenylacetonitrile $(1.0 \mathrm{ml}, 10.0 \mathrm{mmol}, 1.0$ equiv) was added and the mixture was heated to $55{ }^{\circ} \mathrm{C}$. After stirring for $1 \mathrm{~h}$ at $55^{\circ} \mathrm{C}$, 2-bromo-3-fluoropyridine $(1.5 \mathrm{ml}$, 
$13.0 \mathrm{mmol}, 1.2$ equiv) was added and the mixture was stirred for $5 \mathrm{~h}$ at $70^{\circ} \mathrm{C}$. The red suspension was poured into ice-cooled water and stirred for 10 minutes. The precipitate was filtered off and purified by flash column chromatography over silica gel (eluent: $n$-pentane, later $n$-pentane/EtOAc 95:5, 90:10, 85:15) to give a yellowish liquid. The obtained 2-(3-fluoropyridin-2-yl)-2-phenylacetonitrile was added to concentrated $\mathrm{H}_{2} \mathrm{SO}_{4}(2 \mathrm{ml})$ in a $10 \mathrm{ml}$ screw-cap flask equipped with a stir bar and stirred for $12 \mathrm{~h}$ at room temperature. The reaction mixture was poured into water, basified with sat. aq. $\mathrm{Na}_{2} \mathrm{CO}_{3}$ and extracted with EtOAc (3x). The combined organic phases were dried over $\mathrm{MgSO}_{4}$ and concentrated. The product was obtained as a white solid (542 mg, $2.35 \mathrm{mmol}, 24 \%)$ after flash column chromatography using silical gel (eluent: EtOAc). ${ }^{1} \mathrm{H}$ NMR $(400 \mathrm{MHz}$, Chloroform- $d) \delta 8.32(\mathrm{~d}, J=4.6 \mathrm{~Hz}, 1 \mathrm{H}), 7.50(\mathrm{~s}, 1 \mathrm{H}), 7.35(\mathrm{~d}, J=$ $7.3 \mathrm{~Hz}, 2 \mathrm{H}), 7.28(\mathrm{t}, J=8.5 \mathrm{~Hz}, 1 \mathrm{H}), 7.20(\mathrm{t}, J=7.3 \mathrm{~Hz}, 2 \mathrm{H}), 7.18-7.10(\mathrm{~m}, 2 \mathrm{H}), 6.19$ $(\mathrm{s}, 1 \mathrm{H}), 5.26(\mathrm{~s}, 1 \mathrm{H}) \cdot{ }^{13} \mathrm{C}\left\{{ }^{1} \mathrm{H}\right\}$ NMR $(101 \mathrm{MHz}$, Chloroform-d) $\delta$ 172.6, $157.4(\mathrm{~d}, J=$ $258.7 \mathrm{~Hz}$ ), 147.1 (d, $J=13.8 \mathrm{~Hz}), 144.7$ (d, $J=5.5 \mathrm{~Hz}), 137.7,129.0,128.6,127.8$, 123.9, $123.8(\mathrm{~d}, J=15.8 \mathrm{~Hz}), 52.7 .{ }^{19} \mathrm{~F}\left\{{ }^{1} \mathrm{H}\right\}$ NMR $(376 \mathrm{MHz}$, Chloroform- $d) \delta-123.5$. HRMS $(E S I) \mathrm{m} / \mathrm{z}$ calculated for $\left[\mathrm{C}_{13} \mathrm{H}_{11} \mathrm{FN}_{2} \mathrm{ONa}\right]\left(\left[\mathrm{M}+\mathrm{Na}^{+}\right]\right) 253.0748$, found 253.0745 .

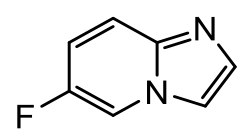

6-Fluoroimidazo[1,2-a]pyridine: The title compound was prepared according to GP3 from 2-amino-5-fluoropyridine (336 mg, $3.0 \mathrm{mmol})$ and chloroacetaldehyde $(1.91 \mathrm{ml}$, $15.0 \mathrm{mmol}, 50 \mathrm{wt} \%$ aq.) in $\mathrm{EtOH}(10 \mathrm{ml})$ at $100{ }^{\circ} \mathrm{C}$. The product was isolated after column chromatography (eluent: 50:50 n-pentane/EtOAc, later 0:100) as a white solid (356 mg, $2.6 \mathrm{mmol}, 87 \%)$. ${ }^{1} \mathrm{H}$ NMR $(400 \mathrm{MHz}$, Chloroform- $d$ ) $\delta 8.08-8.01(\mathrm{~m}, 1 \mathrm{H})$, $7.67-7.62(\mathrm{~m}, 1 \mathrm{H}), 7.60-7.53(\mathrm{~m}, 2 \mathrm{H}), 7.06(\mathrm{ddd}, J=10.2,8.0,2.4 \mathrm{~Hz}, 1 \mathrm{H}) .{ }^{13} \mathrm{C}\left\{{ }^{1} \mathrm{H}\right\}$ NMR (101 MHz, Chloroform- $d) \delta 153.4(\mathrm{~d}, J=236.5 \mathrm{~Hz}), 143.3,135.0(\mathrm{~d}, J=1.9 \mathrm{~Hz})$, $118.4(\mathrm{~d}, J=9.2 \mathrm{~Hz}), 116.5(\mathrm{~d}, J=25.8 \mathrm{~Hz}), 113.8,112.4(\mathrm{~d}, J=40.6 \mathrm{~Hz}) .{ }^{19} \mathrm{~F}\left\{{ }^{1} \mathrm{H}\right\}$ NMR (376 MHz, Chloroform- $d$ ) $\delta-141.2$ HRMS (ESI) m/z calculated for $\left[\mathrm{C}_{7} \mathrm{H}_{6} \mathrm{FN}_{2}\right]$ $\left(\left[\mathrm{M}+\mathrm{H}^{+}\right]\right)$137.0510, found 137.0502 .

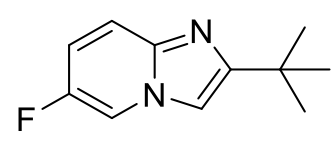

2-(tert-Butyl)-6-fluoroimidazo[1,2-a]pyridine: The title compound was prepared according to GP3 from 2-amino-5-fluoropyridine (336 mg, $3.0 \mathrm{mmol}$ ) and 1-chloro-3,3- 
dimethylbutan-2-one $(0.95 \mathrm{ml}, 7.2 \mathrm{mmol})$ in $\mathrm{EtOH}(10 \mathrm{ml})$ at $100{ }^{\circ} \mathrm{C}$. The product was isolated after column chromatography (eluent: 50:50 n-pentane/EtOAc, later 0:100) as a white solid (177 mg, $0.9 \mathrm{mmol}, 31 \%) .{ }^{1} \mathrm{H}$ NMR $(400 \mathrm{MHz}$, Chloroform- $d) \delta 7.96$ (ddd, $J=3.8,2.4,0.6 \mathrm{~Hz}, 1 \mathrm{H}$ ), 7.52 (dd, $J=9.8,5.1 \mathrm{~Hz}, 1 \mathrm{H}), 7.34(\mathrm{~d}, J=0.7 \mathrm{~Hz}, 1 \mathrm{H}), 7.02$ (ddd, $J=10.3,8.1,2.4 \mathrm{~Hz}, 1 \mathrm{H}), 1.38(\mathrm{~s}, 9 \mathrm{H}) .{ }^{13} \mathrm{C}\left\{{ }^{1} \mathrm{H}\right\}$ NMR (101 MHz, Chloroform- $d$ ) $\delta 158.8(\mathrm{~d}, J=2.0 \mathrm{~Hz}$ ), 153.1 (d, $J=235.2 \mathrm{~Hz}), 142.8,117.7(\mathrm{~d}, J=9.2 \mathrm{~Hz}), 115.8(\mathrm{~d}$, $J=25.4 \mathrm{~Hz}), 112.0(\mathrm{~d}, J=40.2 \mathrm{~Hz}), 108.4,32.5,30.3 .{ }^{19} \mathrm{~F}\left\{{ }^{1} \mathrm{H}\right\}$ NMR $(376 \mathrm{MHz}$, Chloroform- $d) \delta-142.2$ HRMS (ESI) $\mathrm{m} / \mathrm{z}$ calculated for $\left[\mathrm{C}_{11} \mathrm{H}_{14} \mathrm{FN}_{2} \mathrm{O}_{2}\right]\left(\left[\mathrm{M}+\mathrm{H}^{+}\right]\right)$ 193.1136, found 193.1154.

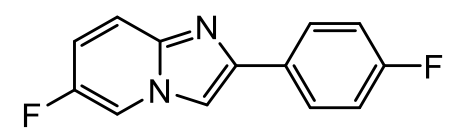

6-Fluoro-2-(4-fluorophenyl)imidazo[1,2-a]pyridine: The title compound was prepared according to GP3 from 2-amino-5-fluoropyridine (280 $\mathrm{mg}, 2.5 \mathrm{mmol}$ ) and 2bromo-1-(4-fluorophenyl)ethan-1-one $(597 \mathrm{mg}, 2.8 \mathrm{mmol})$ in $\mathrm{EtOH}(10 \mathrm{ml})$ at $100{ }^{\circ} \mathrm{C}$. The product was isolated after column chromatography (eluent: 50:50 npentane/EtOAc) as a white solid (174 mg, $0.8 \mathrm{mmol}, 30 \%) .{ }^{1} \mathbf{H}$ NMR $(300 \mathrm{MHz}$, Chloroform- $d$ ) $\delta 8.05$ (ddd, $J=3.5,2.4,0.8 \mathrm{~Hz}, 1 \mathrm{H}), 7.97-7.86(\mathrm{~m}, 2 \mathrm{H}), 7.81(\mathrm{~s}, 1 \mathrm{H})$, $7.58(\mathrm{dd}, J=9.8,5.1 \mathrm{~Hz}, 1 \mathrm{H}), 7.19-7.04(\mathrm{~m}, 3 \mathrm{H}) .{ }^{13} \mathrm{C}\left\{{ }^{1} \mathrm{H}\right\} \mathrm{NMR}(75 \mathrm{MHz}$, Chloroformd) $\delta 162.9(\mathrm{~d}, J=247.3 \mathrm{~Hz}), 153.5(\mathrm{~d}, J=237.0 \mathrm{~Hz}), 146.4(\mathrm{~d}, J=1.9 \mathrm{~Hz}), 143.6,129.8$ (d, $J=2.9 \mathrm{~Hz}), 127.8(\mathrm{~d}, J=8.2 \mathrm{~Hz}), 118.0(\mathrm{~d}, J=9.1 \mathrm{~Hz}), 116.9(\mathrm{~d}, J=25.5 \mathrm{~Hz}$ ), $115.9(\mathrm{~d}, J=21.6 \mathrm{~Hz}), 112.3(\mathrm{~d}, J=40.4 \mathrm{~Hz}), 109.3 .{ }^{19} \mathrm{~F}\left\{{ }^{1} \mathrm{H}\right\}$ NMR $(282 \mathrm{MHz}$, Chloroform- $d$ ) $\delta-113.8,-140.5$ HRMS $(E S I) \mathrm{m} / \mathrm{z}$ calculated for $\left[\mathrm{C}_{13} \mathrm{H}_{9} \mathrm{~F}_{2} \mathrm{~N}_{2}\right]\left(\left[\mathrm{M}+\mathrm{H}^{+}\right]\right)$ 231.0728 , found 231.0746 .

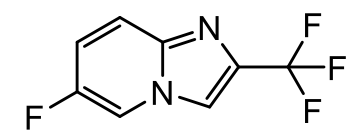

6-Fluoro-2-(trifluoromethyl)imidazo[1,2-a]pyridine: The title compound was prepared according to GP3 from 2-amino-5-fluoropyridine (280 $\mathrm{mg}, 2.5 \mathrm{mmol})$ and 3bromo-1,1,1-trifluoropropan-2-one $(0.79 \mathrm{ml}, 7.5 \mathrm{mmol})$ in $\mathrm{EtOH}(10 \mathrm{ml})$ at $100^{\circ} \mathrm{C}$. The product was isolated after column chromatography (eluent: 50:50 n-pentane/EtOAc) as a white solid (354 mg, $1.7 \mathrm{mmol}, 69 \%)$. ${ }^{1} \mathrm{H}$ NMR (500 MHz, Chloroform- $d$ ) $\delta 8.09$ (ddd, $J=3.4,2.4,0.9 \mathrm{~Hz}, 1 \mathrm{H}$ ), 7.89 (t, $J=0.9 \mathrm{~Hz}, 1 \mathrm{H}$ ), 7.63 (dd, $J=10.0,5.0 \mathrm{~Hz}, 1 \mathrm{H}$ ), 7.22 (ddd, $J=10.1,7.9,2.4 \mathrm{~Hz}, 1 \mathrm{H}) \cdot{ }^{13} \mathrm{C}\left\{{ }^{1} \mathrm{H}\right\}$ NMR $(126 \mathrm{MHz}$, Chloroform- $d$ ) $\delta 154.0$ 
(d, $J=240.2 \mathrm{~Hz}$ ), 143.3, 137.2 (dq, $J=38.9,1.9 \mathrm{~Hz}$ ), 121.5 (q, $J=268.2 \mathrm{~Hz}), 119.5$ (d, $J=9.2 \mathrm{~Hz}), 119.3(\mathrm{~d}, J=26.1 \mathrm{~Hz}), 113.2,112.9 .{ }^{19} \mathrm{~F}\left\{{ }^{1} \mathrm{H}\right\}$ NMR $(282 \mathrm{MHz}$, Chloroform- $d$ ) $\delta-62.9$ (3F), -137.8 (1F) HRMS (ESI) m/z calculated for $\left[\mathrm{C}_{8} \mathrm{H}_{4} \mathrm{~F}_{4} \mathrm{~N}_{2} \mathrm{Na}\right.$ ] $\left(\left[\mathrm{M}+\mathrm{Na}^{+}\right]\right)$227.0203, found 227.0216.

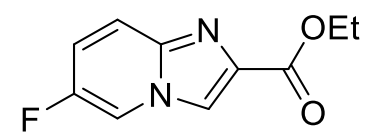

Ethyl 6-fluoroimidazo[1,2-a]pyridine-2-carboxylate: The title compound was prepared according to GP3 from 2-amino-5-fluoropyridine $(336 \mathrm{mg}, 3.0 \mathrm{mmol}$ ) and ethyl 3-bromo-2-oxopropanoate $(0.90 \mathrm{ml}, 7.2 \mathrm{mmol})$ in $\mathrm{EtOH}(10 \mathrm{ml})$ at $100^{\circ} \mathrm{C}$. The product was isolated after column chromatography (eluent: 50:50 n-pentane/EtOAc, later 0:100) as a white solid (435 mg, $2.1 \mathrm{mmol}, 70 \%)$. ${ }^{1} \mathrm{H}$ NMR $(400 \mathrm{MHz}$, Chloroformd) $\delta 8.18(\mathrm{~s}, 1 \mathrm{H}$ ), 8.08 (ddd, $J=3.5,2.4,0.9 \mathrm{~Hz}, 1 \mathrm{H}), 7.65(\mathrm{dd}, J=10.0,5.2 \mathrm{~Hz}, 1 \mathrm{H}$ ), 7.17 (ddd, $J=10.1,7.9,2.4 \mathrm{~Hz}, 1 \mathrm{H}$ ), 4.44 (q, $J=7.1 \mathrm{~Hz}, 2 \mathrm{H}), 1.42(\mathrm{t}, J=7.1 \mathrm{~Hz}, 3 \mathrm{H}$ ). ${ }^{13} \mathrm{C}\left\{{ }^{1} \mathrm{H}\right\}$ NMR $(101 \mathrm{MHz}$, Chloroform-d) $\delta .163 .0,154.0(\mathrm{~d}, J=240.0 \mathrm{~Hz}), 143.1,138.2$ (d, $J=1.9 \mathrm{~Hz}), 119.9$ (d, $J=9.2 \mathrm{~Hz}), 118.9(\mathrm{~d}, J=26.2 \mathrm{~Hz}), 118.2(\mathrm{~d}, J=2.9 \mathrm{~Hz}$ ), $112.8(\mathrm{~d}, J=40.8 \mathrm{~Hz}), 61.4,14.5 .{ }^{19} \mathrm{~F}\left\{{ }^{1} \mathrm{H}\right\}$ NMR $(376 \mathrm{MHz}$, Chloroform- $d) \delta-138.0$ HRMS $(\mathrm{ESI}) \mathrm{m} / \mathrm{z}$ calculated for $\left[\mathrm{C}_{10} \mathrm{H}_{10} \mathrm{FN}_{2} \mathrm{O}_{2}\right]\left(\left[\mathrm{M}+\mathrm{H}^{+}\right]\right)$209.0721, found 209.0729. 


\section{Sensitivity Screen}

Recently, our group has established a reaction-condition based assignment to evaluate the sensitivity of a chemical reaction. ${ }^{2}$ The sensitivity screen conducted in this study is based on a modification of the literature procedure.

\section{Preparation of stock solution A:}

To a $10 \mathrm{ml}$ oven-dried Schlenk flask was added $\mathrm{MeOH}(5.4 \mathrm{ml}, 0.55 \mathrm{M})$, 3fluoropyridine (291 mg, $3.0 \mathrm{mmol}, 1.0$ equiv), and $\mathrm{H}_{2} \mathrm{SO}_{4}$ (353 mg, $3.6 \mathrm{mmol}, 1.2$ equiv).

\section{Standard conditions:}

To a $4 \mathrm{ml}$ glass vial (screw-cap) equipped with a stir bar were added $\mathrm{Pd}(\mathrm{OH})_{2} / \mathrm{C}$ ( $8.8 \mathrm{mg}, 5 \mathrm{~mol} \%)$, stock solution $\mathbf{A}(0.45 \mathrm{ml})$ and additional $\mathrm{MeOH}(0.05 \mathrm{ml})$. The glass vial was placed in a $150 \mathrm{ml}$ stainless steel autoclave under air. The autoclave was pressurized and depressurized four times with hydrogen gas before the final hydrogen pressure was set. The reaction mixture was stirred at the indicated temperature for $24 \mathrm{~h}$. After the autoclave was carefully depressurized, hexafluorobenzene $(5 \mu \mathrm{l})$ was added and the mixture was analyzed by ${ }^{19} \mathrm{~F}$ NMR analysis.

\begin{tabular}{ccccc}
\hline Entry & Modification & Deviation from standard condition & Yield & Deviation \\
\hline $\mathbf{1}$ & High $C$ & without additional $50 \mu \mathrm{l} \mathrm{MeOH}$ & $97 \%$ & $-3 \%$ \\
$\mathbf{2}$ & Low $C$ & $+100 \mu \mathrm{l} \mathrm{MeOH}$ & $101 \%$ & $+1 \%$ \\
$\mathbf{3}$ & $\mathrm{H}_{2} \mathrm{O}$ & $+5 \mu \mathrm{l} \mathrm{H} \mathrm{O}$ & $94 \%$ & $-6 \%$ \\
$\mathbf{4}$ & $\mathrm{Low} \mathrm{O}_{2}$ & degassed solvent, working under $\mathrm{Ar}$ & $101 \%$ & $+1 \%$ \\
$\mathbf{5}$ & $\mathrm{High} \mathrm{O}_{2}$ & $+5 \mathrm{ml}$ air, autoclave not purged & $97 \%$ & $-3 \%$ \\
$\mathbf{6}$ & $\mathrm{High} \mathrm{T}$ & $50{ }^{\circ} \mathrm{C}$ & $100 \%$ & 0 \\
$\mathbf{7}$ & Low $T$ & $30{ }^{\circ} \mathrm{C}$ & $100 \%$ & 0 \\
$\mathbf{8}$ & High $P$ & 60 bar $\mathrm{H}_{2}$ & $97 \%$ & $-3 \%$ \\
$\mathbf{9}$ & Low $P$ & 40 bar $\mathrm{H}_{2}$ & $97 \%$ & $-3 \%$ \\
$\mathbf{1 0}$ & Big scale & Standard scale $\cdot 10$ & $105 \%$ & $+5 \%$ \\
\hline
\end{tabular}




\section{Analysis:}

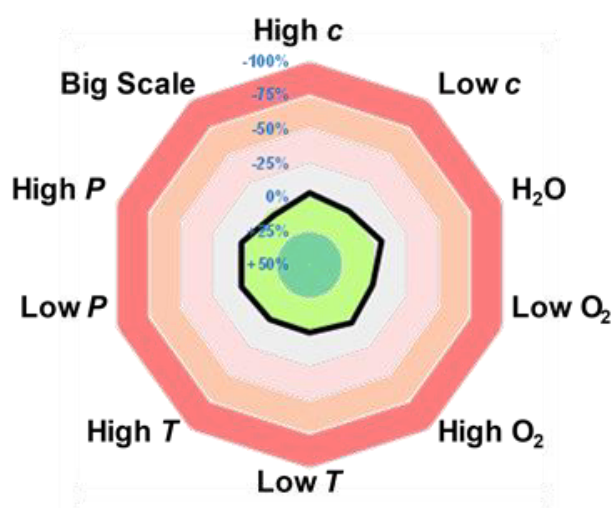

Figure S1: Radar diagram for the reaction-condition based sensitivity assessment. $c=$ concentration. $T=$ temperature. $P=$ pressure.

Small deviations of the reaction concentration did not have a significant impact on the reaction outcome. Many transition metal-catalyzed reactions are sensitive to air and/or moisture, which drives the need for thoroughly dried solvents as well as working under an inert atmosphere. To our delight, the addition of $\mathrm{H}_{2} \mathrm{O}$ or the presence of $\mathrm{O}_{2}$ did not lead to a meaningful reduction of product formation. Further, small deviations in $\mathrm{H}_{2}$ pressure or temperature did not affect the reaction outcome. A slightly increased yield was obtained when the reaction was scaled up. This sensitivity screen underlines the robustness of our protocol, which in addition utilizes simple and inexpensive reagents. 


\section{Hydrogenation Reactions}

\section{General procedure A for the synthesis of fluorinated piperidines (GP-A):}
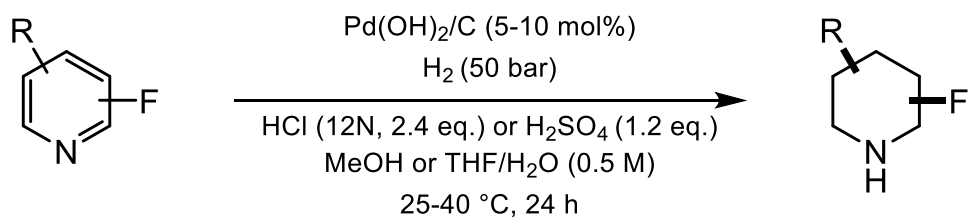

To a $4 \mathrm{ml}$ glass vial (screw-cap) equipped with a stir bar were added $\mathrm{Pd}(\mathrm{OH})_{2} / \mathrm{C}$ (5$10 \mathrm{~mol} \%)$ and solid fluorinated pyridines $(0.5 \mathrm{mmol}, 1.0$ equiv). The indicated solvent was added $\left(0.5 \mathrm{M} \mathrm{MeOH}\right.$ or $\left.0.5 \mathrm{M} \mathrm{THF} / \mathrm{H}_{2} \mathrm{O}, 1: 1\right)$ followed by the addition of liquid fluorinated pyridines ( $0.5 \mathrm{mmol}, 1.0$ equiv) and the indicated acid (100 $\mu \mathrm{l}$ of conc. aq. $\mathrm{HCl}$ or $32 \mu \mathrm{l}$ of conc. $\mathrm{H}_{2} \mathrm{SO}_{4}$ ). The glass vial was placed in a $150 \mathrm{ml}$ stainless steel autoclave under air. The autoclave was pressurized and depressurized four times with hydrogen gas before the final hydrogen pressure was set. If not otherwise stated, the reaction mixture was stirred at $40^{\circ} \mathrm{C}$ for $24 \mathrm{~h}$. After the autoclave was carefully depressurized, the mixture was diluted with $\mathrm{H}_{2} \mathrm{O}(1 \mathrm{ml})$ and $\mathrm{Na}_{2} \mathrm{CO}_{3}(212 \mathrm{mg}$, 4.0 equiv) was added. After the gas evolution has stopped, the mixture was filtered over celite using $\mathrm{CH}_{2} \mathrm{Cl}_{2}$. The solvent was removed in vacuo and the product was purified using column chromatography on silica gel. The diastereoselectivities were determined by GC-MS analysis of the basified reaction mixture.

\section{General procedure B for the synthesis of fluorinated piperidines (GP-B):}<smiles>[R]c1ccncc1F</smiles>

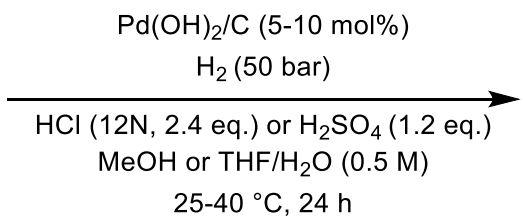<smiles>[R]C1CCNCC1(F)F</smiles>

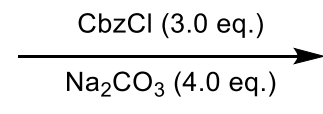

$\mathrm{Na}_{2} \mathrm{CO}_{3}$ (4.0 eq.)<smiles>[R]C1CC2CCC(F)(C1)N(C(=O)OCc1ccccc1)C2</smiles>

To a $4 \mathrm{ml}$ glass vial (screw-cap) equipped with a stir bar were added $\mathrm{Pd}(\mathrm{OH})_{2} / \mathrm{C}$ (5$10 \mathrm{~mol} \%)$ and solid fluorinated pyridines $(0.5 \mathrm{mmol}, 1.0$ equiv). The indicated solvent was added $\left(0.5 \mathrm{M} \mathrm{MeOH}\right.$ or $\left.0.5 \mathrm{M} \mathrm{THF} / \mathrm{H}_{2} \mathrm{O}, 1: 1\right)$ followed by the addition of liquid fluorinated pyridines ( $0.5 \mathrm{mmol}, 1.0$ equiv) and the indicated acid (100 $\mu \mathrm{l}$ of conc. aq. $\mathrm{HCl}$ or $32 \mu \mathrm{l}$ of conc. $\mathrm{H}_{2} \mathrm{SO}_{4}$ ). The glass vial was placed in a $150 \mathrm{ml}$ stainless steel autoclave under air. The autoclave was pressurized and depressurized four times with hydrogen gas before the final hydrogen pressure was set. If not otherwise stated, the reaction mixture was stirred at $40^{\circ} \mathrm{C}$ for $24 \mathrm{~h}$. After the autoclave was carefully depressurized, the mixture was diluted with $\mathrm{H}_{2} \mathrm{O}(1 \mathrm{ml})$ and $\mathrm{Na}_{2} \mathrm{CO}_{3}(212 \mathrm{mg}$, 
4.0 equiv) followed by $\mathrm{CbzCl}$ ( $208 \mu \mathrm{l}, 3.0$ equiv) were added. After stirring for $1-3 \mathrm{~h}$ at room temperature (reaction control by TLC using a ninhydrin staining solution), the mixture was filtered over celite using $\mathrm{CH}_{2} \mathrm{Cl}_{2}$. The solvent was removed in vacuo and the product was purified using column chromatography on silica gel. The diastereoselectivities were determined by GC-MS analysis after the carbamate protection had reached full conversion.

Conformational analysis: The experimental determination of the axial or equatorial orientation of the fluorine atoms on the piperidine ring was done through NMR studies. It has been shown that the vicinal ${ }^{3} \mathrm{~J}\left({ }^{19} \mathrm{~F},{ }^{1} \mathrm{H}\right)$ coupling constants provide useful insights into conformational orientation, as large values of ${ }^{3} \mathrm{~J}\left({ }^{19} \mathrm{~F},{ }^{1} \mathrm{H} \mathrm{Hax}\right)$ indicate axial orientation and small values of ${ }^{3} \mathrm{~J}\left({ }^{19} \mathrm{~F},{ }^{1} \mathrm{Hax}\right)$ indicate equatorial orientation (Karplus equation). This was done using the Pbox provided by Vnmrj of Agilent. For further details regarding conformational analysis of fluorinated piperidines see ref. 3 and ref. 4 .

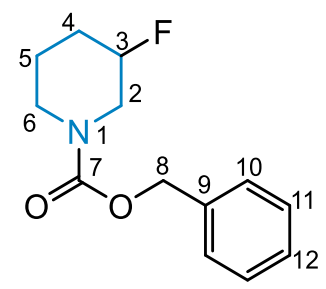

Benzyl 3-fluoropiperidine-1-carboxylate (1): The title compound was synthesized according to GP-B using 5 mol\% Pd-catalyst $(17.6 \mathrm{mg})$ in $\mathrm{MeOH}(0.5 \mathrm{M})$ with aq. $\mathrm{HCl}$ $\left(100 \mu \mathrm{l}, 2.4\right.$ equiv, $12 \mathrm{~N}$ ) at $25^{\circ} \mathrm{C}$. The product was isolated as a colorless liquid (94 mg, $0.40 \mathrm{mmol}, 79 \%$ ) after column chromatography (silical gel, eluent: $n$-pentane, later $n$-pentane/EtOAc 95:5, 90:10). The conformation of the product was not determined, since fast rotation of the carbamate group prohibited determination of $J$ values. However, analogous compounds have been studied in the literature in detail. ${ }^{3}$

${ }^{1} \mathrm{H}$ NMR (599 MHz, Chloroform-d) $\delta 7.36(\mathrm{~m}, 4 \mathrm{H}, \mathrm{HC} 10, H \mathrm{C} 11), 7.34-7.29(\mathrm{~m}, 1 \mathrm{H}$, HC12), 5.14 (s, 2H, $\mathrm{H}_{2} \mathrm{C} 8$ ), 4.62 (d, ${ }^{2} \mathrm{JFH}_{\mathrm{FH}}=46.7 \mathrm{~Hz}, 1 \mathrm{H}, \mathrm{HC} 3$ ), 3.76 (bs, $1 \mathrm{H}, \mathrm{H}_{2} \mathrm{C} 2$ ), $3.71-3.46$ (m, 2H, $\mathrm{H}_{2} \mathrm{C} 6, \mathrm{H}_{2} \mathrm{C} 2$ ), 3.30 (bs, $1 \mathrm{H}, \mathrm{H}_{2} \mathrm{C} 6$ ), $1.93-1.77$ (m, 3H, $\mathrm{H}_{2} \mathrm{C} 4, \mathrm{H}_{2} \mathrm{C} 4$, $\left.\mathrm{H}_{2} \mathrm{C} 5\right), 1.51$ (bs, $\left.1 \mathrm{H}, H_{2} \mathrm{C} 5\right) .{ }^{13} \mathrm{C}\left\{{ }^{1} \mathrm{H}\right\}$ NMR $(151 \mathrm{MHz}$, Chloroform-d) $\delta 155.6(\mathrm{C} 7), 136.8$ (C9), 128.6 (C11), 128.1 (C12), 127.9 (C10), 86.3 (d, $\left.{ }^{1} \mathrm{JFC}_{\mathrm{FC}}=175.0 \mathrm{~Hz}, \mathrm{C} 3\right), 67.3$ (C8), $48.1\left(\mathrm{~d},{ }^{2} \mathrm{JFC}=23.8 \mathrm{~Hz}, \mathrm{C} 2\right), 43.9(\mathrm{C} 6), 29.7\left(\mathrm{~d},{ }^{2} \mathrm{JFC}=20.5 \mathrm{~Hz}, \mathrm{C} 4\right), 23.2-20.3(\mathrm{~m}$, C5). ${ }^{19} \mathrm{~F}$ NMR $\left(564 \mathrm{MHz}\right.$, Chloroform-d) $\delta-184.2--185.0(\mathrm{~m}) .{ }^{19} \mathrm{~F}\left\{{ }^{1} \mathrm{H}\right\}$ NMR $(282 \mathrm{MHz}$, 
Chloroform- $d$ ) $\delta-184.6(\mathrm{~s}$, rotamer A), $-184.8(\mathrm{~s}$, rotamer B). HRMS (ESI) m/z calculated for $\left[\mathrm{C}_{13} \mathrm{H}_{16} \mathrm{FNO}_{2} \mathrm{Na}\right]\left(\left[\mathrm{M}+\mathrm{Na}^{+}\right]\right)$260.1057, found 260.1054.

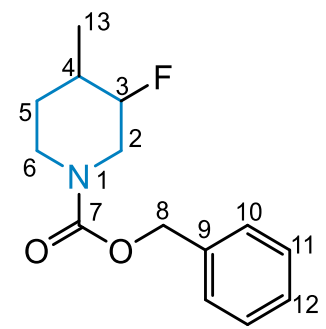

Benzyl 3-fluoro-4-methylpiperidine-1-carboxylate (2): The title compound was synthesized according to GP-B using $5 \mathrm{~mol} \% \mathrm{Pd}$-catalyst $(17.6 \mathrm{mg})$ in $\mathrm{THF} / \mathrm{H}_{2} \mathrm{O}(0.5 \mathrm{M}$, 1:1) with aq. $\mathrm{HCl}(100 \mu \mathrm{l}, 2.4$ equiv, $12 \mathrm{~N})$ at $25^{\circ} \mathrm{C}$. The product was isolated as a colorless liquid (95 mg, $0.38 \mathrm{mmol}, 76 \%,>95: 5$ d.r.) after column chromatography (silical gel, eluent: $n$-pentane, later $n$-pentane/EtOAc 95:5, 90:10). The conformation of the product was not determined, since fast rotation of the carbamate group prohibited determination of $J$ values. However, analogous compounds have been studied in the literature in detail. ${ }^{3}$

Large scale: The title compound was synthesized according to GP-B using $10 \mathrm{~mol} \%$ Pd-catalyst $(702 \mathrm{mg})$ in $\mathrm{MeOH}(0.5 \mathrm{M})$ with $\mathrm{H}_{2} \mathrm{SO}_{4}(1.18 \mathrm{~g}, 1.2$ equiv.). The product was isolated as a colorless liquid $(1.67 \mathrm{~g}, 6.67 \mathrm{mmol}, 67 \%$, >95:5 d.r.) after column chromatography (silical gel, eluent: $n$-pentane, later $n$-pentane/EtOAc 95:5, 90:10).

${ }^{1} \mathrm{H}$ NMR (599 MHz, Chloroform-d) $\delta 7.37-7.33$ (m, 4H, HC10, HC11), $7.33-7.28$ (m, $1 \mathrm{H}, \mathrm{HC} 12), 5.21-5.07$ (m, 2H, $\left.\mathrm{H}_{2} \mathrm{C} 8\right), 4.62-4.36$ (m, 2H, HC3, $\left.\mathrm{H}_{2} \mathrm{C} 2\right), 4.32-4.11$ (m, 1H, $\left.\mathrm{H}_{2} \mathrm{C} 6\right)$, $3.03-2.72$ (m, 2H, $\left.\mathrm{H}_{2} \mathrm{C} 2, \mathrm{H}_{2} \mathrm{C} 6\right), 1.77-1.53$ (m, 2H, HC4, $\left.\mathrm{H}_{2} \mathrm{C} 5\right), 1.51$ - $1.42\left(\mathrm{~m}, 1 \mathrm{H}, \mathrm{H}_{2} \mathrm{C} 5\right), 1.06\left(\mathrm{~d},{ }^{3} \mathrm{HH}=6.7 \mathrm{~Hz}, 3 \mathrm{H}, H_{3} \mathrm{C} 13\right) .{ }^{13} \mathrm{C}\left\{{ }^{1} \mathrm{H}\right\}$ NMR $(151 \mathrm{MHz}$, Chloroform- $d$ ) $\delta 155.8$ (C7), 136.9 (C9), 128.5 (C11), 128.0 (C12), 127.8 (C10), 89.3 $\left(\mathrm{d},{ }^{1} \mathrm{JFC}=178.0 \mathrm{~Hz}, \mathrm{C} 3\right), 67.2(\mathrm{C} 8), 48.4-47.5(\mathrm{~m}, \mathrm{C} 2), 43.7(\mathrm{C} 6), 34.2\left(\mathrm{~d},{ }^{2} \mathrm{JFC}_{\mathrm{FC}} 20.6\right.$ $\mathrm{Hz}, \mathrm{C} 4), 28.2-27.2$ (m, C5), 17.2 (d, $\left.{ }^{3} \mathrm{JFC}=3.6 \mathrm{~Hz}, \mathrm{C} 13\right) .{ }^{19} \mathrm{~F}$ NMR $(564 \mathrm{MHz}$, Chloroform- $d$ ) $\delta-202.6--203.6(\mathrm{~m}) .{ }^{19} \mathrm{~F}\left\{{ }^{1} \mathrm{H}\right\}$ NMR $(282 \mathrm{MHz}$, Chloroform- $d$ ) $\delta-203.0$ (s, rotamer A), -203.3 (s, rotamer B). HRMS (ESI) m/z calculated for [ $\mathrm{C}_{14} \mathrm{H}_{18} \mathrm{FNO} \mathrm{Fa}_{2} \mathrm{Na}$ $\left(\left[\mathrm{M}+\mathrm{Na}^{+}\right]\right)$274.1214, found 274.1209. 


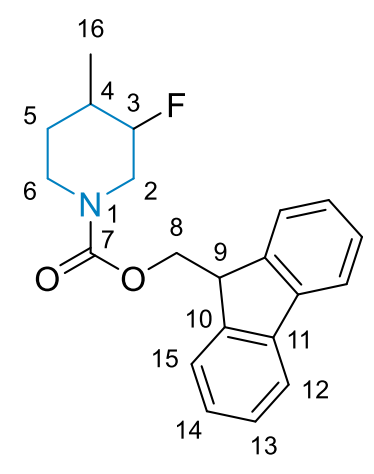

(9H-Fluoren-9-yl)methyl 3-fluoro-4-methylpiperidine-1-carboxylate (3): The title compound was synthesized according to a modification of GP-B using $10 \mathrm{~mol} \% \mathrm{Pd}$ catalyst $(35.1 \mathrm{mg})$ in $\mathrm{THF} / \mathrm{H}_{2} \mathrm{O}(0.5 \mathrm{M}, 1: 1)$ with aq. $\mathrm{HCl}(100 \mu \mathrm{l}, 2.4$ eq., $12 \mathrm{~N})$. The piperidine was trapped with $\mathrm{FmocCl}$ (3.0 equiv). The product was isolated as a white solid (100 mg, $0.30 \mathrm{mmol}, 59 \%$, >95:5 d.r.) after column chromatography (silical gel, eluent: $n$-pentane, later $n$-pentane/EtOAc 90:10, 80:20). The conformation of the product was not determined, since fast rotation of the carbamate group prohibited determination of $J$ values. However, analogous compounds have been studied in the literature in detail. ${ }^{3}$

${ }^{1} \mathbf{H}$ NMR $(500 \mathrm{MHz}$, Chloroform- $d) \delta 7.77\left(\mathrm{~d},{ }^{3}{ }_{\mathrm{HH}}=7.5 \mathrm{~Hz}, 2 \mathrm{H}\right), 7.68-7.56(\mathrm{~m}, 2 \mathrm{H})$, $7.40\left(\mathrm{t},{ }^{3} \mathrm{JHH}_{\mathrm{HH}}=7.5 \mathrm{~Hz}, 2 \mathrm{H}\right), 7.32\left(\mathrm{td},{ }^{3} \mathrm{JHH}_{\mathrm{HH}}=7.4 \mathrm{~Hz},{ }^{3} \mathrm{JHH}_{\mathrm{HH}}=1.2 \mathrm{~Hz}, 2 \mathrm{H}\right), 4.63-4.08(\mathrm{~m}$, $\left.6 \mathrm{H}, \mathrm{HC} 3, \mathrm{H}_{2} \mathrm{C} 8, \mathrm{HC} 9, \mathrm{H}_{2} \mathrm{C} 2, \mathrm{H}_{2} \mathrm{C} 6\right), 3.14-2.72\left(\mathrm{~m}, 2 \mathrm{H}, \mathrm{H}_{2} \mathrm{C} 2, \mathrm{H}_{2} \mathrm{C} 6\right), 1.82-1.42$ (m, $\left.\left.3 \mathrm{H}, \mathrm{HC} 4, \mathrm{H}_{2} \mathrm{C} 5\right), 1.09\left(\mathrm{~d},{ }^{3} \mathrm{JHH}_{\mathrm{HH}}=6.5 \mathrm{~Hz}, 3 \mathrm{H}, \mathrm{H}_{3} \mathrm{C} 16\right).\right) .{ }^{13} \mathrm{C}\left\{{ }^{1} \mathrm{H}\right\} \mathrm{NMR}(126 \mathrm{MHz}$, Chloroform- $d$ ) $\delta 155.8,143.8,141.5,127.8(\mathrm{~m}), 127.2(\mathrm{~m}), 125.2,120.1,89.3\left(\mathrm{~d},{ }^{1} \mathrm{JFC}\right.$ $=178.4 \mathrm{~Hz}, \mathrm{C} 3), 67.9-67.5$ (m, C8), $48.2-47.7$ (m, C2), 47.4 (C9), 43.8 (C6), 34.3 $\left(\mathrm{d},{ }^{2} \mathrm{JFC}=20.0 \mathrm{~Hz}, \mathrm{C} 4\right), 27.7$ (C5), $17.2(\mathrm{C} 16) .{ }^{19} \mathrm{~F}$ NMR $(470 \mathrm{MHz}$, Chloroform- $d$ ) $\delta-$ $202.4--202.9\left(\mathrm{~m}\right.$, rotamer A), $-203.0--203.5\left(\mathrm{~m}\right.$, rotamer B). ${ }^{19} \mathbf{F}\left\{{ }^{1} \mathrm{H}\right\} \mathbf{N M R}(470 \mathrm{MHz}$, Chloroform- $d$ ) $\delta$-202.7 (rotamer A), -203.3 (rotamer B). HRMS (ESI) m/z calculated for $\left[\mathrm{C}_{21} \mathrm{H}_{22} \mathrm{NO}_{2} \mathrm{FNa}\right]\left(\left[\mathrm{M}+\mathrm{Na}^{+}\right]\right) 362.1527$, found 362.1526 . 


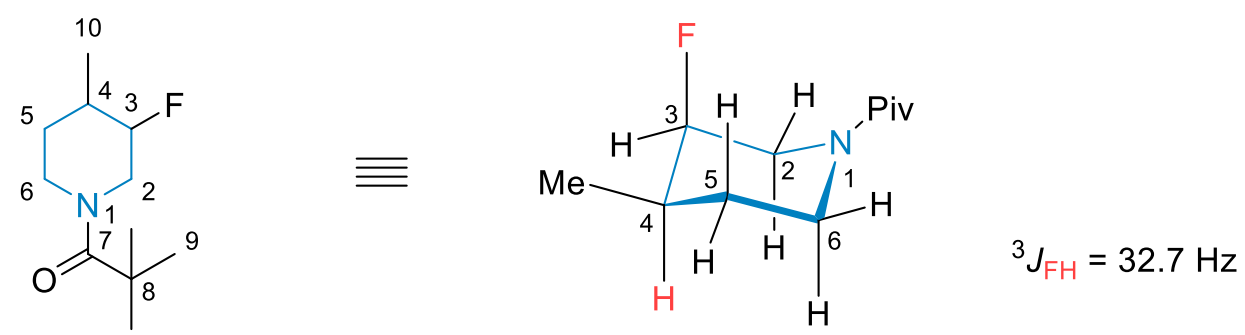

1-(3-Fluoro-4-methylpiperidin-1-yl)-2,2-dimethylpropan-1-one (4): The title compound was synthesized according to a modification of GP-B using $10 \mathrm{~mol} \% \mathrm{Pd}$ catalyst $(35.1 \mathrm{mg})$ in $\mathrm{THF} / \mathrm{H}_{2} \mathrm{O}(0.5 \mathrm{M}, 1: 1)$ with aq. $\mathrm{HCl}(100 \mu \mathrm{l}, 2.4$ equiv, $12 \mathrm{~N})$. The piperidine was trapped with PivCl (1.5 equiv). The product was isolated as a yellow oil (63 mg, $0.31 \mathrm{mmol}, 62 \%$ ) after column chromatography (silical gel, eluent: $n$-pentane, later $n$-pentane/EtOAc 80:20, 70:30). The axial position of the fluorine substituent was determined based on the ${ }^{3} J_{F H}$ coupling between $\mathrm{FC}$ and $H_{\mathrm{ax}} \mathrm{C} 4$.

${ }^{1} \mathrm{H}$ NMR $(500 \mathrm{MHz}$, Chloroform- $d) \delta 4.74-4.66\left(\mathrm{~m}, 1 \mathrm{H}, H_{\mathrm{eq}} \mathrm{C} 2\right), 4.53\left(\mathrm{dm},{ }^{2} \mathrm{JFH}_{\mathrm{FH}}=\right.$ $47.4 \mathrm{~Hz}, 1 \mathrm{H}, \mathrm{HC} 3), 4.43-4.36\left(\mathrm{~m}, 1 \mathrm{H}, \mathrm{H}_{2} \mathrm{C} 6\right), 2.92\left(\mathrm{dm},{ }^{3} \mathrm{JFH}=37.6 \mathrm{~Hz}, 1 \mathrm{H}, \mathrm{Hax}_{\mathrm{C}}\right)$, $2.86-2.78\left(\mathrm{~m}, 1 \mathrm{H}, \mathrm{H}_{2} \mathrm{C} 6\right), 1.75\left(\mathrm{dm},{ }^{3} \mathrm{JFH}_{\mathrm{FH}}=32.7 \mathrm{~Hz}, 1 \mathrm{H}, \mathrm{H}_{\mathrm{ax}} \mathrm{C} 4\right), 1.64-1.54(\mathrm{~m}, 1 \mathrm{H}$, $\mathrm{H}_{2} \mathrm{C} 5$ ), $1.53-1.48\left(\mathrm{~m}, 1 \mathrm{H}, \mathrm{H}_{2} \mathrm{C} 5\right), 1.28\left(\mathrm{~s}, 9 \mathrm{H}, H_{3} \mathrm{C} 9\right), 1.06$ (d, ${ }^{3} \mathrm{HH}=6.2 \mathrm{~Hz}, 2 \mathrm{H}$, $\left.H_{3} \mathrm{C} 10\right) .{ }^{1} \mathrm{H}\left\{{ }^{19} \mathrm{~F}\right\}$ NMR $(500 \mathrm{MHz}$, Chloroform- $d) \delta=4.70\left(\mathrm{dm},{ }^{2} \mathrm{HH}=14.5 \mathrm{~Hz}, 1 \mathrm{H}\right.$, $H_{\text {eq }}$ ), $4.53(\mathrm{~m}, 1 \mathrm{H}, H \mathrm{C} 3), 4.39\left(\mathrm{dm},{ }^{2}{ }_{\mathrm{HH}}=13.9 \mathrm{~Hz}, 1 \mathrm{H}, \mathrm{H}_{2} \mathrm{C} 6\right), 2.92\left(\mathrm{dm},{ }^{2} \mathrm{JHH}_{\mathrm{HH}}=14.5\right.$ $\left.\mathrm{Hz}, 1 \mathrm{H}, H_{\mathrm{ax}} \mathrm{C} 2\right), 2.86-2.77\left(\mathrm{~m}, 1 \mathrm{H}, \mathrm{H}_{2} \mathrm{C} 6\right), 1.75\left(\mathrm{~m}, 1 \mathrm{H}, \mathrm{Hax}_{\mathrm{ax}}\right), 1.65-1.54(\mathrm{~m}, 1 \mathrm{H}$, $\left.\mathrm{H}_{2} \mathrm{C} 5\right), 1.51\left(\mathrm{~m}, 1 \mathrm{H}, \mathrm{H}_{2} \mathrm{C} 5\right), 1.28\left(\mathrm{~s}, 9 \mathrm{H}, \mathrm{H}_{3} \mathrm{C} 9\right), 1.06\left(\mathrm{~d},{ }^{3} \mathrm{JH}=6.9 \mathrm{~Hz}, 3 \mathrm{H}, \mathrm{H}_{3} \mathrm{C} 10\right)$. ${ }^{13} \mathrm{C}\left\{{ }^{1} \mathrm{H}\right\}$ NMR $(126 \mathrm{MHz}$, Chloroform- $d) \delta=177.3(\mathrm{C} 7), 89.7\left(\mathrm{~d},{ }^{1} \mathrm{JFC}=178.3 \mathrm{~Hz}, \mathrm{C} 3\right)$, $48.5\left(\mathrm{~d},{ }^{2} \mathrm{JFC}=20.5 \mathrm{~Hz}, \mathrm{C} 2\right), 45.0$ (C6), $38.9(\mathrm{C} 8), 34.6$ (d, $\left.{ }^{2} \mathrm{JFC}=20.7 \mathrm{~Hz}, \mathrm{C} 4\right), 28.7$ (C9), $28.2\left(\mathrm{~d},{ }^{3} \mathrm{JFC}_{\mathrm{FC}}=1.4 \mathrm{~Hz}, \mathrm{C} 5\right), 16.9\left(\mathrm{~d},{ }^{3} \mathrm{JFC}=3.9 \mathrm{~Hz}, \mathrm{C} 10\right) \cdot{ }^{13} \mathrm{C}\left\{{ }^{1} \mathrm{H},{ }^{19} \mathrm{~F}\right\}$ NMR $(126$ $\mathrm{MHz}$, Chloroform- $d$ ) $\delta=177.3$ (C7), 89.7 (C3), 48.5 (C2), 45.0 (C6), 38.9 (C8), 34.6 (C4), 28.7 (C9), 28.2 (C5), 16.9 (C13). ${ }^{19} \mathrm{~F}$ NMR (470 MHz, Chloroform- $d$ ) $\delta=-202.7$ - $-203.0(\mathrm{~m}) .{ }^{19} \mathrm{~F}\left\{{ }^{1} \mathrm{H}\right\}$ NMR $(470 \mathrm{MHz}$, Chloroform- $d) \delta=-202.9$ (s). HRMS (ESI) $\mathrm{m} / \mathrm{z}$ calculated for $\left[\mathrm{C}_{11} \mathrm{H}_{20} \mathrm{NO}_{2} \mathrm{FNa}\right]\left(\left[\mathrm{M}+\mathrm{Na}^{+}\right]\right)$224.1421, found 224.1415. 


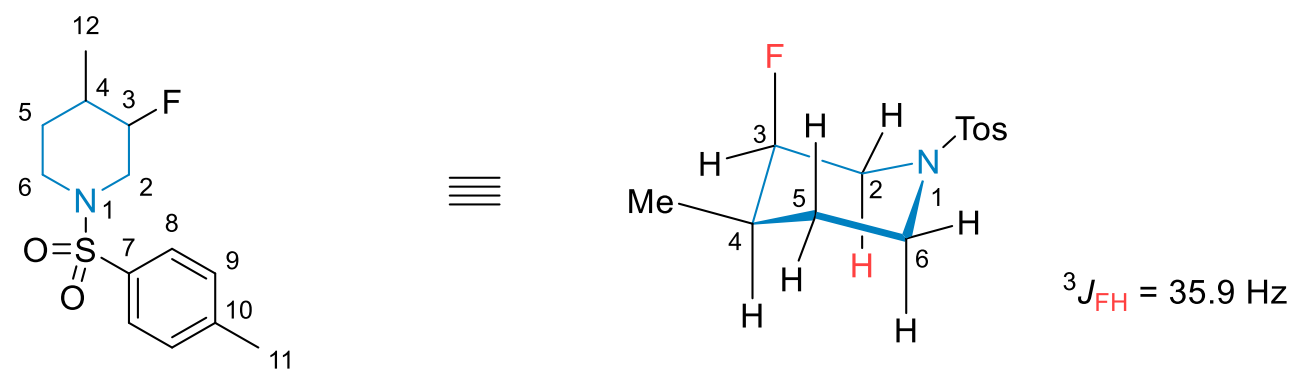

3-Fluoro-4-methyl-1-tosylpiperidine (5): The title compound was synthesized according to a modification of GP-B using $10 \mathrm{~mol} \% \mathrm{Pd}$-catalyst $(35.1 \mathrm{mg})$ in $\mathrm{THF} / \mathrm{H}_{2} \mathrm{O}$ $(0.5 \mathrm{M}, 1: 1)$ with aq. $\mathrm{HCl}(100 \mu \mathrm{l}, 2.4$ equiv, $12 \mathrm{~N})$. The piperidine was trapped with $\mathrm{Tos} \mathrm{Cl}$ (3.0 equiv). The product was isolated as a white solid $(78 \mathrm{mg}, 0.29 \mathrm{mmol}, 58 \%$, $>95: 5$ d.r.) after column chromatography (silical gel, eluent: $n$-pentane, later $n$ pentane/EtOAc 80:20, 70:30). The axial position of the fluorine substituent was determined based on the ${ }^{3} \mathrm{JFH}$ coupling between $\mathrm{FC} 3$ and $\mathrm{HaxC}_{\mathrm{a}}$.

${ }^{1}$ H NMR $(500 \mathrm{MHz}$, Chloroform-d) $\delta 7.70-7.62$ (m, 2H, HC8), $7.36-7.28(\mathrm{~m}, 2 \mathrm{H}$, HC9), $4.59-4.43\left(\mathrm{dm},{ }^{2} \mathrm{JFH}_{\mathrm{FH}}=47.7 \mathrm{~Hz}, 1 \mathrm{H}, H \mathrm{C} 3\right), 3.98\left(\mathrm{~m}, 1 \mathrm{H}, H_{\mathrm{eq}} \mathrm{C} 2\right), 3.78-3.67(\mathrm{~m}$, $1 \mathrm{H}, \mathrm{H}_{2} \mathrm{C} 6$ ), 2.57 (ddd, ${ }^{3} \mathrm{JFH}_{\mathrm{FH}}=35.9 \mathrm{~Hz},{ }^{2} \mathrm{~J}_{\mathrm{HH}}=13.1 \mathrm{~Hz},{ }^{3} \mathrm{JHH}_{\mathrm{HH}}=1.6 \mathrm{~Hz}, 1 \mathrm{H}, H_{\mathrm{ax}} \mathrm{C} 2$ ), 2.49 - $2.42\left(\mathrm{~m}, 1 \mathrm{H}, \mathrm{H}_{2} \mathrm{C} 6\right), 2.43$ (s, 3H, $\left.\mathrm{H}_{3} \mathrm{C} 11\right), 1.68\left(\mathrm{~m}, 1 \mathrm{H}, \mathrm{H}_{2} \mathrm{C} 5\right), 1.60-1.45(\mathrm{~m}, 2 \mathrm{H}$, $\left.\mathrm{H}_{2} \mathrm{C} 5, \mathrm{HC} 4\right), 1.02\left(\mathrm{~d},{ }^{3} \mathrm{JHH}=6.6 \mathrm{~Hz}, 3 \mathrm{H}, H_{3} \mathrm{C} 12\right) .{ }^{1} \mathrm{H}\left\{{ }^{19} \mathrm{~F}\right\} \mathrm{NMR}(500 \mathrm{MHz}$, Chloroformd) $\delta 7.70-7.63(\mathrm{~m}, 2 \mathrm{H}, \mathrm{HC} 8), 7.36-7.28$ (m, 2H, HC9), $4.53-4.49(\mathrm{~m}, 1 \mathrm{H}, H \mathrm{C} 3)$, $3.98\left(\mathrm{dm},{ }^{2} \mathrm{JHH}=13.1 \mathrm{~Hz}, 1 \mathrm{H}, H_{\mathrm{eq}} \mathrm{C}\right), 3.78-3.69\left(\mathrm{~m}, 1 \mathrm{H}, \mathrm{H}_{2} \mathrm{C} 6\right), 2.57\left(\mathrm{dd},{ }^{2} \mathrm{JHH}=\right.$ $\left.13.1 \mathrm{~Hz},{ }^{3} \mathrm{HH}=1.6 \mathrm{~Hz}, 1 \mathrm{H}, \mathrm{HaxC}_{\mathrm{H}}\right), 2.48-2.42\left(\mathrm{dm},{ }^{2} \mathrm{~J} \mathrm{HH}=12.1 \mathrm{~Hz}, 1 \mathrm{H}, \mathrm{H}_{2} \mathrm{C} 6\right), 2.43$ (s, 3H, $\left.H_{3} \mathrm{C} 11\right), 1.76-1.61$ (m, $\left.1 \mathrm{H}, H_{2} \mathrm{C} 5\right), 1.58-1.47$ (m, 2H, $\left.H_{2} \mathrm{C} 5, H_{\mathrm{ax}} \mathrm{C} 4\right), 1.02$ (d, $\left.{ }^{3} \mathrm{JHH}_{\mathrm{HH}}=6.6 \mathrm{~Hz}, 3 \mathrm{H}, \mathrm{H}_{3} \mathrm{C} 12\right) .{ }^{13} \mathrm{C}\left\{{ }^{1} \mathrm{H}\right\}$ NMR $(126 \mathrm{MHz}$, Chloroform-d) $\delta 143.6$ (C7), 134.1 (C10), 129.8 (C9), 127.8 (C8), 88.5 (d, ${ }^{1} \mathrm{JFC}_{\mathrm{FC}}=181.2 \mathrm{~Hz}, \mathrm{C} 3$ ), 49.3 (d, ${ }^{2} \mathrm{JFC}=21.9 \mathrm{~Hz}$, C2), 45.5 (C6), 33.5 (d, $\left.{ }^{2} \mathrm{JFC}=20.5 \mathrm{~Hz}, \mathrm{C} 4\right), 27.4\left(\mathrm{~d},{ }^{3} \mathrm{JFC}=1.7 \mathrm{~Hz}, \mathrm{C} 5\right), 21.7$ (C11), $16.6\left(\mathrm{~d},{ }^{3} \mathrm{JFC}=3.7 \mathrm{~Hz}, \mathrm{C} 12\right) .{ }^{13} \mathrm{C}\left\{{ }^{1} \mathrm{H},{ }^{19} \mathrm{~F}\right\}$ NMR $(126 \mathrm{MHz}$, Chloroform- $d) \delta 143.6(\mathrm{C} 7)$, 134.1 (C10), 129.8 (C9), 127.8 (C8), 88.5 (C3), 49.3 (C2), 45.5 (C6), 33.5 (C4), 27.4 (C5), 21.7 (C11), 16.6 (C12). ${ }^{19} \mathrm{~F}$ NMR (470 MHz, Chloroform- $\left.d\right) \delta-200.6--200.9(\mathrm{~m})$. ${ }^{19} \mathrm{~F}\left\{{ }^{1} \mathrm{H}\right\}$ NMR $(282 \mathrm{MHz}$, Chloroform- $d) \delta=-200.7$ (s). HRMS (ESI) $\mathrm{m} / \mathrm{z}$ calculated for $\left[\mathrm{C}_{13} \mathrm{H}_{18} \mathrm{NO}_{2} \mathrm{SFNa}\right]\left(\left[\mathrm{M}+\mathrm{Na}^{+}\right]\right)$294.0935, found 294.0929. 


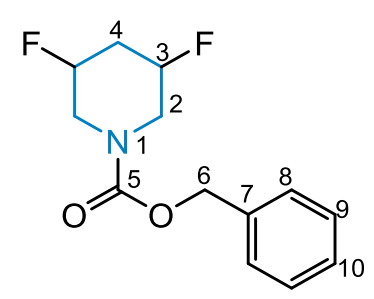

Benzyl 3,5-difluoropiperidine-1-carboxylate (6): The title compound was synthesized according to GP-B using $10 \mathrm{~mol} \%$ Pd-catalyst $(35.1 \mathrm{mg})$ in $\mathrm{THF} / \mathrm{H}_{2} \mathrm{O}$ (1.0 M, 1:1) with $\mathrm{H}_{2} \mathrm{SO}_{4}$ (35 $\mu \mathrm{l}, 1.2$ equiv). The product was isolated as a white solid (38 mg, $0.15 \mathrm{mmol}, 30 \%,>95: 5 \mathrm{~d}$.r.) after column chromatography (silical gel, eluent: $n$-pentane, later $n$-pentane/EtOAc 95:5, 90:10, 80:20). The conformation of the product was not determined, since fast rotation of the carbamate group prohibited determination of $J$ values. However, analogous compounds have been studied in the literature in detail. ${ }^{3}$

${ }^{1} \mathrm{H}$ NMR $(599 \mathrm{MHz}$, Chloroform-d) $\delta 7.38-7.30$ (m, 5H, HC8, HC9, HC10), 5.16 (s, $\left.2 \mathrm{H}, \mathrm{H}_{2} \mathrm{C} 6\right), 4.67\left(\mathrm{dm},{ }^{2} \mathrm{JFH}=43.6,2 \mathrm{H}, \mathrm{HC} 3\right), 3.91$ (bs, $\left.2 \mathrm{H}, \mathrm{H}_{2} \mathrm{C} 2\right), 3.52$ (ddd, ${ }^{3} \mathrm{FFH}=$ $\left.25.9 \mathrm{~Hz},{ }^{2} \mathrm{JHH}=14.0 \mathrm{~Hz},{ }^{3} \mathrm{JHH}=3.0 \mathrm{~Hz}, 2 \mathrm{H}, \mathrm{H}_{2} \mathrm{C} 2\right), 2.29-2.06\left(\mathrm{~m}, 2 \mathrm{H}, \mathrm{H}_{2} \mathrm{C} 4\right) .{ }^{1} \mathrm{H}\left\{{ }^{19} \mathrm{~F}\right\}$ NMR (599 MHz, Chloroform-d) $\delta 7.39-7.30$ (m, 5H, HC8, HC9, HC10), 5.16 (s, 2H, $\mathrm{H}_{2} \mathrm{C} 6$ ), $4.73-4.61$ (m, 2H, $\mathrm{H}_{2} \mathrm{C} 3$ ), 3.91 (bs, 2H, $\mathrm{H}_{2} \mathrm{C} 2$ ), 3.52 (dd, ${ }^{2} \mathrm{JHH}_{\mathrm{HH}}=14.1 \mathrm{~Hz},{ }^{3} \mathrm{JHH}_{\mathrm{HH}}$ $\left.=3.0 \mathrm{~Hz}, 2 \mathrm{H}, \mathrm{H}_{2} \mathrm{C} 2\right), 2.28-2.21\left(\mathrm{~m}, 1 \mathrm{H}, \mathrm{H}_{2} \mathrm{C} 4\right), 2.13\left(\mathrm{dt},{ }^{2} \mathrm{~J}_{\mathrm{HH}}=14.6 \mathrm{~Hz},{ }^{3} \mathrm{~J}_{\mathrm{HH}}=3.9\right.$ $\left.\mathrm{Hz}, 1 \mathrm{H}, \mathrm{H}_{2} \mathrm{C} 4\right) .{ }^{13} \mathrm{C}\left\{{ }^{1} \mathrm{H}\right\}$ NMR (151 MHz, Chloroform- $d$ ) $\delta 155.8$ (C5), $136.4(\mathrm{C} 7), 128.6$ (C8), 128.2 (C10), 128.0 (C9), 84.2 (dd, ${ }^{1} \mathrm{JFC}=174.7 \mathrm{~Hz},{ }^{3} \mathrm{JFC}=4.5 \mathrm{~Hz}, \mathrm{C} 3$ ), 67.7 (C6), $47.6-46.9(\mathrm{~m}, \mathrm{C} 2), 34.8\left(\mathrm{t},{ }^{2} \mathrm{JFC}=20.8 \mathrm{~Hz}, \mathrm{C} 4\right) .{ }^{13} \mathrm{C}\left\{{ }^{1} \mathrm{H},{ }^{19} \mathrm{~F}\right\}$ NMR $(151 \mathrm{MHz}$, Chloroform- $d$ ) $\delta 155.8$ (C5), 136.4 (C7), 128.6 (C8), 128.2 (C10), 128.0 (C9), 84.2 (C3), 67.7 (C6), 47.5 - 46.9 (m, C2), 34.8 (C4). ${ }^{19} \mathrm{~F}$ NMR (564 MHz, Chloroform- $d$ ) $\delta$ $182.9--183.5(\mathrm{~m}) .{ }^{19} \mathrm{~F}\left\{{ }^{1} \mathrm{H}\right\}$ NMR $(564 \mathrm{MHz}$, Chloroform- $d) \delta-183.0$ (bs, rotamer A), 183.3 (bs, rotamer B). HRMS (ESI) $\mathrm{m} / \mathrm{z}$ calculated for $\left[\mathrm{C}_{13} \mathrm{H}_{15} \mathrm{NO}_{2} \mathrm{~F}_{2} \mathrm{Na}\right]\left(\left[\mathrm{M}+\mathrm{Na}^{+}\right]\right)$ 278.0963 , found 278.0959 .

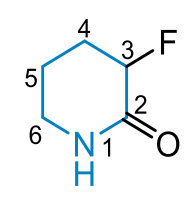

3-Fluoropiperidin-2-one (7): The title compound was synthesized according to GP-A using $5 \mathrm{~mol} \% \mathrm{Pd}$-catalyst $(17.6 \mathrm{mg})$ in $\mathrm{MeOH}(0.5 \mathrm{M})$ with aq. $\mathrm{HCl}(100 \mu \mathrm{l}, 2.4$ equiv, 
$12 \mathrm{~N}$ ). The product was isolated as a white solid (28 $\mathrm{mg}, 0.24 \mathrm{mmol}, 48 \%$ ) after column chromatography (silical gel, eluent: $\mathrm{CH}_{2} \mathrm{Cl}_{2}$ :acetone 90:10, later 80:20).

${ }^{1} \mathrm{H}$ NMR (599 MHz, Chloroform-d) $\delta 7.34$ (s, 1H, HN1), 4.80 (ddd, ${ }^{2} J_{\mathrm{FH}}=47.2 \mathrm{~Hz},{ }^{3} \mathrm{JHH}_{\mathrm{HH}}$ $\left.=8.4 \mathrm{~Hz},{ }^{3} \mathrm{JHH}_{\mathrm{HH}}=5.6 \mathrm{~Hz}, 1 \mathrm{H}, \mathrm{HC} 3\right), 3.38-3.30\left(\mathrm{~m}, 1 \mathrm{H}, \mathrm{H}_{2} \mathrm{C} 6\right), 3.31-3.24(\mathrm{~m}, 1 \mathrm{H}$, $\left.\mathrm{H}_{2} \mathrm{C} 6\right), 2.27-2.15$ (m, $\left.1 \mathrm{H}, \mathrm{H}_{2} \mathrm{C} 4\right), 2.11-2.00$ (m, 1H, $\left.H_{2} \mathrm{C} 4\right), 1.97$ (m, $\left.1 \mathrm{H}, H_{2} \mathrm{C} 5\right), 1.86$ $-1.75\left(\mathrm{~m}, 1 \mathrm{H}, \mathrm{H}_{2} \mathrm{C} 5\right) .{ }^{1} \mathrm{H}\left\{{ }^{19} \mathrm{~F}\right\}$ NMR $(599 \mathrm{MHz}$, Chloroform-d) $\delta 7.34(\mathrm{~s}, 1 \mathrm{H}, H \mathrm{~N} 1), 4.80$ $\left(\mathrm{dd},{ }^{3} \mathrm{~J}_{\mathrm{HH}}=8.4 \mathrm{~Hz},{ }^{3} \mathrm{JHH}_{\mathrm{HH}}=5.6 \mathrm{~Hz}, 1 \mathrm{H}, \mathrm{HC} 3\right), 3.36-3.31\left(\mathrm{~m}, 1 \mathrm{H}, \mathrm{H}_{2} \mathrm{C} 6\right), 3.30-3.24(\mathrm{~m}$, $\left.1 \mathrm{H}, \mathrm{H}_{2} \mathrm{C} 6\right), 2.26-2.17\left(\mathrm{~m}, 1 \mathrm{H}, \mathrm{H}_{2} \mathrm{C} 4\right), 2.11-2.02\left(\mathrm{~m}, 1 \mathrm{H}, \mathrm{H}_{2} \mathrm{C} 4\right), 2.00-1.92(\mathrm{~m}, 1 \mathrm{H}$, $\left.\mathrm{H}_{2} \mathrm{C} 5\right), 1.86-1.75\left(\mathrm{~m}, 1 \mathrm{H}, \mathrm{H}_{2} \mathrm{C} 5\right) .{ }^{13} \mathrm{C}\left\{{ }^{1} \mathrm{H}\right\}$ NMR (151 MHz, Chloroform-d) $\delta 169.0(\mathrm{~d}$, $\left.{ }^{2} J_{F C}=20.0 \mathrm{~Hz}, \mathrm{C} 2\right), 86.0\left(\mathrm{~d},{ }^{1} \mathrm{~J}_{\mathrm{FC}}=178.0 \mathrm{~Hz}, \mathrm{C} 3\right), 42.0(\mathrm{C} 6), 28.0$ (d, ${ }^{2} \mathrm{JFC}_{\mathrm{FC}}=20.2 \mathrm{~Hz}$, C4), 19.2 (d, $\left.{ }^{3} \mathrm{FFC}_{\mathrm{FC}} 7.5 \mathrm{~Hz}, \mathrm{C} 5\right) \cdot{ }^{13} \mathrm{C}\left\{{ }^{1} \mathbf{H},{ }^{19} \mathrm{~F}\right\}$ NMR $(151 \mathrm{MHz}$, Chloroform-d) $\delta 169.0$ (C2), 86.0 (C3), 42.0 (C6), 28.0 (C4), 19.2 (C5). ${ }^{19} \mathrm{~F}$ NMR (564 MHz, Chloroform- $d$ ) $\delta$ $187.0--187.1\left(\mathrm{ddd},{ }^{2} \mathrm{JHF}_{\mathrm{HF}}=47.2 \mathrm{~Hz},{ }^{3} \mathrm{~J}_{\mathrm{HF}}=16.7 \mathrm{~Hz},{ }^{3} J_{\mathrm{HF}}=14.1 \mathrm{~Hz}\right) .{ }^{19} \mathrm{~F}\left\{{ }^{1} \mathrm{H}\right\} \mathbf{N M R}(376$ $\mathrm{MHz}$, Chloroform- $d$ ) $\delta-187.0$ (s). HRMS (ESI) m/z calculated for [ $\mathrm{C}_{5} \mathrm{H}_{8} \mathrm{FNONa}$ ] $\left(\left[\mathrm{M}+\mathrm{Na}^{+}\right]\right)$140.0482, found 140.0475 .

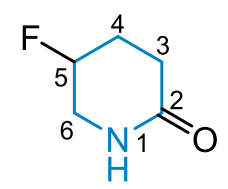

5-Fluoropiperidin-2-one (8): The title compound was synthesized according to GP-A using $5 \mathrm{~mol} \% \mathrm{Pd}$-catalyst (17.6 mg) in $\mathrm{MeOH}(0.5 \mathrm{M})$ with aq. $\mathrm{HCl}(100 \mu \mathrm{l}, 2.4$ equiv, $12 \mathrm{~N}$ ). The product was isolated as a white solid (37 mg, $0.32 \mathrm{mmol}, 64 \%$ ) after column chromatography (silical gel, eluent: $\mathrm{CH}_{2} \mathrm{Cl}_{2}$, later 99:1, 98:2, 95:5, 90:10 $\left.\mathrm{CH}_{2} \mathrm{Cl}_{2} / \mathrm{MeOH}\right)$.

${ }^{1} \mathrm{H}$ NMR (500 MHz, Chloroform-d) $\delta 6.39(\mathrm{~s}, 1 \mathrm{H}, H \mathrm{~N} 1), 4.99\left(\mathrm{dm},{ }^{2} \mathrm{JFH}=48.1 \mathrm{~Hz}, 1 \mathrm{H}\right.$, HC5), $3.65-3.45\left(\mathrm{~m}, 2 \mathrm{H}, \mathrm{H}_{2} \mathrm{C} 6\right), 2.59\left(\mathrm{ddd},{ }^{2} \mathrm{JHH}_{\mathrm{HH}}=18.0 \mathrm{~Hz},{ }^{3} \mathrm{JHH}_{\mathrm{HH}}=11.7 \mathrm{~Hz},{ }^{3} \mathrm{JHH}_{\mathrm{HH}}=6.5\right.$ $\mathrm{Hz}, 1 \mathrm{H}, \mathrm{H}_{2} \mathrm{C} 3$ ), 2.39 (ddd, ${ }^{2} \mathrm{JHH}_{\mathrm{HH}}=17.7 \mathrm{~Hz},{ }^{3} \mathrm{~J}_{\mathrm{HH}}=6.5 \mathrm{~Hz},{ }^{3} \mathrm{JHH}_{\mathrm{HH}}=3.2 \mathrm{~Hz}, 1 \mathrm{H}, \mathrm{H}_{2} \mathrm{C} 3$ ), 2.31 $-2.21\left(\mathrm{~m}, 1 \mathrm{H}, \mathrm{H}_{2} \mathrm{C} 4\right), 1.97\left(\mathrm{dm}, 1 \mathrm{H},{ }^{3} \mathrm{JFH}_{\mathrm{FH}}=38.3 \mathrm{~Hz}, \mathrm{H}_{2} \mathrm{C} 4\right) .{ }^{1} \mathrm{H}\left\{{ }^{19} \mathrm{~F}\right\} \mathbf{N M R}(500 \mathrm{MHz}$, Chloroform-d) $\delta 6.39$ (s, $1 \mathrm{H}, H \mathrm{~N} 1), 4.99$ (m, 1H, HC5), $3.61\left(\mathrm{dm},{ }^{2} \mathrm{JHH}_{\mathrm{HH}}=13.7 \mathrm{~Hz}, 1 \mathrm{H}\right.$, $\mathrm{H}_{2} \mathrm{C} 6$ ), 3.52 (dd, ${ }^{2} \mathrm{JHH}_{\mathrm{HH}}=13.9 \mathrm{~Hz},{ }^{3} \mathrm{~J}_{\mathrm{HH}}=3.6 \mathrm{~Hz}, 1 \mathrm{H}, \mathrm{H}_{2} \mathrm{C} 6$ ), 2.59 (ddd, ${ }^{2} \mathrm{JHH}_{\mathrm{HH}}=18.0 \mathrm{~Hz}$, $\left.{ }^{3} \mathrm{~J}_{\mathrm{HH}}=11.7 \mathrm{~Hz},{ }^{3} \mathrm{JHH}_{\mathrm{HH}}=6.5 \mathrm{~Hz}, 1 \mathrm{H}, \mathrm{H}_{2} \mathrm{C} 3\right), 2.39$ (ddd, ${ }^{2} \mathrm{JHH}_{\mathrm{HH}}=17.8 \mathrm{~Hz},{ }^{3} \mathrm{JHH}_{\mathrm{HH}}=6.5 \mathrm{~Hz},{ }^{3} \mathrm{JHH}_{\mathrm{HH}}$ $=3.3 \mathrm{~Hz}, 1 \mathrm{H}, \mathrm{H}_{2} \mathrm{C} 3$ ), $2.30-2.22\left(\mathrm{~m}, 1 \mathrm{H}, \mathrm{H}_{2} \mathrm{C} 4\right), 1.97$ (dddd, ${ }^{2} \mathrm{JHH}_{\mathrm{HH}}=14.2 \mathrm{~Hz},{ }^{3} \mathrm{JHH}_{\mathrm{HH}}=$ $\left.11.8 \mathrm{~Hz},{ }^{3} \mathrm{JH}=6.5 \mathrm{~Hz},{ }^{3} \mathrm{JHH}_{\mathrm{HH}}=2.1 \mathrm{~Hz}, 1 \mathrm{H}, \mathrm{H}_{2} \mathrm{C} 4\right) .{ }^{13} \mathrm{C}\left\{{ }^{1} \mathrm{H}\right\} \mathrm{NMR}(126 \mathrm{MHz}$, Chloroformd) $\delta 171.2(\mathrm{C} 2), 84.1\left(\mathrm{~d},{ }^{1} \mathrm{JFC}_{\mathrm{FC}} 174.0 \mathrm{~Hz}, \mathrm{C} 5\right), 46.9$ (d, $\left.{ }^{2} \mathrm{JFC}_{\mathrm{FC}}=23.4 \mathrm{~Hz}, \mathrm{C6}\right), 26.3$ (d, 
$\left.{ }^{3} \mathrm{JFC}_{\mathrm{FC}} 5.7 \mathrm{~Hz}, \mathrm{C} 3\right), 25.8\left(\mathrm{~d},{ }^{2} \mathrm{JFC}=21.3 \mathrm{~Hz}, \mathrm{C} 4\right) .{ }^{13} \mathrm{C}\left\{{ }^{1} \mathrm{H},{ }^{19} \mathrm{~F}\right\} \mathrm{NMR}(126 \mathrm{MHz}$, Chloroform- $d$ ) $\delta 171.2$ (C2), 84.1 (C5), 46.9 (C6), 26.3 (C3), 25.8 (C4). ${ }^{19}$ F NMR (470 $\mathrm{MHz}$, Chloroform- $d) \delta-186.8--187.2(\mathrm{~m}) .{ }^{19} \mathrm{~F}\left\{{ }^{1} \mathrm{H}\right\}$ NMR $(470 \mathrm{MHz}$, Chloroform- $d) \delta-$ 187.0. HRMS (ESI) $\mathrm{m} / \mathrm{z}$ calculated for $\left[\mathrm{C}_{5} \mathrm{H}_{8} \mathrm{FNONa}\right]\left(\left[\mathrm{M}+\mathrm{Na}^{+}\right]\right)$140.0482, found 140.0481.
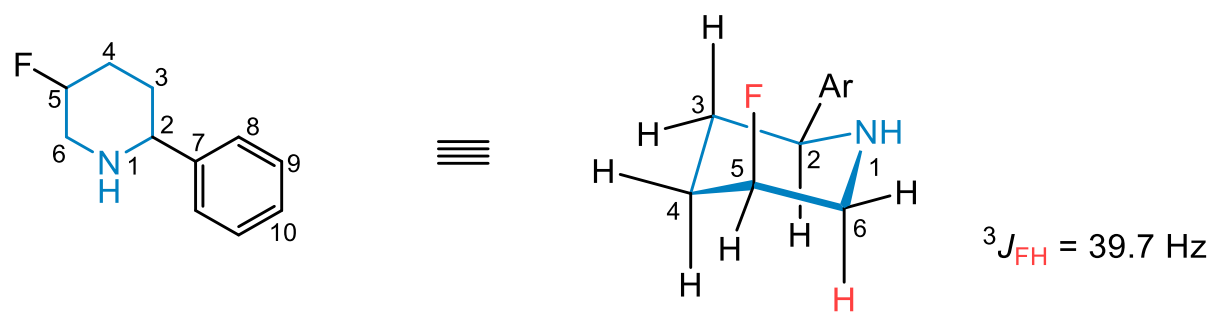

5-Fluoro-2-phenylpiperidine (9): The title compound was synthesized according to GP-A using $10 \mathrm{~mol} \% \mathrm{Pd}$-catalyst $(35.1 \mathrm{mg})$ in $\mathrm{THF} / \mathrm{H}_{2} \mathrm{O}(0.5 \mathrm{M}, 1: 1)$ with aq. $\mathrm{HCl}$ $(100 \mu \mathrm{l}, 2.4$ equiv, $12 \mathrm{~N})$. The product was isolated as a white solid $(51 \mathrm{mg}, 0.29 \mathrm{mmol}$, $52 \%, 80: 20$ d.r.) after column chromatography (silical gel, eluent: $n$-pentane/EtOAc $80: 20$, later 50:50). Only the analytical data of the major diastereomer are listed. The axial position of the fluorine substituent was determined based on the ${ }^{3} \mathrm{JFH}_{\mathrm{FH}}$ coupling between $\mathrm{FC} 5$ and $\mathrm{H}_{\mathrm{ax}} \mathrm{C} 6$ and is in accordance with previous literature results.

${ }^{1} \mathrm{H}$ NMR $(500 \mathrm{MHz}$, Chloroform-d) $\delta 7.40-7.36$ (m, 2H, HC8), $7.36-7.30(\mathrm{~m}, 2 \mathrm{H}$, HC9), $7.28-7.23(\mathrm{~m}, 1 \mathrm{H}, \mathrm{HC} 10), 4.71\left(\mathrm{dm},{ }^{2} \mathrm{JFH}=48.3 \mathrm{~Hz}, 1 \mathrm{H}, H_{\mathrm{eq}} \mathrm{C} 5\right), 3.68\left(\mathrm{~d},{ }^{3} \mathrm{JHH}_{\mathrm{HH}}\right.$ $=10.9 \mathrm{~Hz}, 1 \mathrm{H}, H_{\mathrm{ax} C 2}$ ), 3.42 (dddd, ${ }^{2} \mathrm{JHH}=14.2 \mathrm{~Hz},{ }^{3} \mathrm{JFH}=11.8 \mathrm{~Hz},{ }^{3} \mathrm{JHH}_{\mathrm{HH}}=2.5 \mathrm{~Hz},{ }^{4} \mathrm{JHH}$ $\left.=2.5 \mathrm{~Hz}, 1 \mathrm{H}, H_{\mathrm{eq}} \mathrm{C} 6\right), 2.98\left(\mathrm{dd},{ }^{3} \mathrm{JFH}_{\mathrm{FH}}=39.7 \mathrm{~Hz},{ }^{2} \mathrm{JHH}_{\mathrm{HH}}=14.1 \mathrm{~Hz}, 1 \mathrm{H}, H_{\mathrm{ax}} \mathrm{C} 6\right), 2.30-2.19$ (m, 1H, $\left.H_{2} \mathrm{C} 4\right), 2.06(\mathrm{~s}, 1 \mathrm{H}, H \mathrm{~N} 1), 1.98-1.69\left(\mathrm{~m}, 3 \mathrm{H}, \mathrm{H}_{2} \mathrm{C} 3, \mathrm{H}_{2} \mathrm{C} 4\right) .{ }^{1} \mathrm{H}\left\{{ }^{19} \mathrm{~F}\right\}$ NMR (500 $\mathrm{MHz}$, Chloroform- $d$ ) $\delta 7.40-7.36$ (m, 2H, HC8), $7.36-7.31$ (m, 2H, HC9), $7.29-7.23$ $(\mathrm{m}, 1 \mathrm{H}, H \mathrm{C} 10), 4.71\left(\mathrm{~m}, 1 \mathrm{H}, H_{\mathrm{eq}} \mathrm{C} 5\right), 3.68\left(\mathrm{dd},{ }^{3} \mathrm{~J}_{\mathrm{HH}}=11.4 \mathrm{~Hz},{ }^{3} \mathrm{JHH}_{\mathrm{HH}}=2.3 \mathrm{~Hz}, 1 \mathrm{H}\right.$, $H_{\mathrm{ax}} \mathrm{C}$ ), 3.42 (ddd, $\left.{ }^{2} \mathrm{JHH}_{\mathrm{HH}}=14.0 \mathrm{~Hz},{ }^{3} \mathrm{JHH}_{\mathrm{HH}}=2.5 \mathrm{~Hz},{ }^{3} \mathrm{HHH}_{\mathrm{HH}}=2.5 \mathrm{~Hz}, 1 \mathrm{H}, H_{\mathrm{eq}} \mathrm{C} 6\right), 2.98$ (d, $\left.{ }^{2} \mathrm{HHH}_{\mathrm{H}}=14.1 \mathrm{~Hz}, 1 \mathrm{H}, H_{\mathrm{ax}} \mathrm{C} 6\right), 2.29-2.19\left(\mathrm{~m}, 1 \mathrm{H}, H_{2} \mathrm{C} 4\right), 2.06(\mathrm{~s}, 1 \mathrm{H}, \mathrm{HN} 1), 1.98-1.88$ (m, $\left.1 \mathrm{H}, \mathrm{H}_{2} \mathrm{C} 3\right), 1.84-1.71\left(\mathrm{~m}, 2 \mathrm{H}, \mathrm{H}_{2} \mathrm{C} 3, \mathrm{H}_{2} \mathrm{C} 4\right) .{ }^{13} \mathrm{C}\left\{{ }^{1} \mathrm{H}\right\}$ NMR $(126 \mathrm{MHz}$, Chloroformd) $\delta 144.2$ (C7), 128.6 (C9), 127.4 (C10), 126.6 (C8), 86.0 (d, ' ${ }^{\mathrm{JFC}}=170.3 \mathrm{~Hz}, \mathrm{C}$ ), $60.6(\mathrm{C} 2), 50.7\left(\mathrm{~d},{ }^{2} \mathrm{JFC}=20.4 \mathrm{~Hz}, \mathrm{C} 6\right), 29.8\left(\mathrm{~d},{ }^{2} \mathrm{JFC}_{\mathrm{FC}}=21.6 \mathrm{~Hz}, \mathrm{C} 4\right), 28.7$ (C3). ${ }^{13} \mathrm{C}\left\{{ }^{1} \mathrm{H},{ }^{19} \mathrm{~F}\right\}$ NMR (126 MHz, Chloroform-d) $\delta 144.2$ (C7), 128.6 (C9), 127.4 (C10), 126.6 (C8), 86.0 (C5), 60.6 (C2), 50.7 (C6), 29.8 (C4), 28.7 (C3). ${ }^{19} \mathrm{~F}$ NMR (470 MHz, Chloroform- $d) \delta-188.1--188.5(\mathrm{~m}) .{ }^{19} \mathbf{F}\left\{{ }^{1} \mathbf{H}\right\}$ NMR $(470 \mathrm{MHz}$, Chloroform- $d) \delta-188.3$. HRMS $(E S I) \mathrm{m} / \mathrm{z}$ calculated for $\left[\mathrm{C}_{11} \mathrm{H}_{15} \mathrm{FN}\right]\left(\left[\mathrm{M}+\mathrm{H}^{+}\right]\right) 180.1192$, found 180.1180 . 

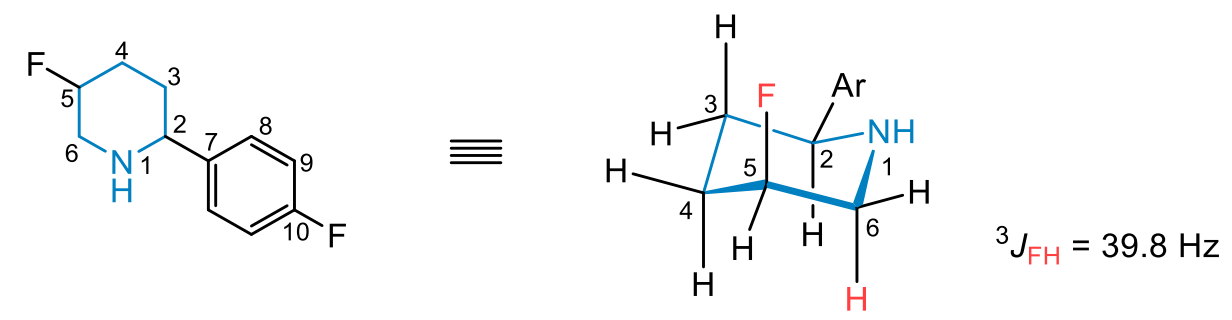

5-Fluoro-2-(4-fluorophenyl)piperidine (10): The title compound was synthesized according to GP-A using $10 \mathrm{~mol} \%$ Pd-catalyst $(35.1 \mathrm{mg})$ in $\mathrm{THF} / \mathrm{H}_{2} \mathrm{O}(0.5 \mathrm{M}, 1: 1)$ with aq. $\mathrm{HCl}(100 \mu \mathrm{l}, 2.4$ equiv, $12 \mathrm{~N}$ ). The product was isolated as a colorless liquid (59 mg, $0.30 \mathrm{mmol}, 60 \%, 88: 12$ d.r.) after column chromatography (silical gel, eluent: $\mathrm{CH}_{2} \mathrm{Cl}_{2} / \mathrm{MeOH} 100: 0$, later 99:1, 98:2). The product was isolated as a mixture of diastereomers. Only the analytical data of the main diastereomer are given. The axial position of the fluorine substituent was determined based on the ${ }^{3} \mathrm{JFH}_{\mathrm{FH}}$ coupling between FC5 and $H_{\mathrm{ax}} \mathrm{C} 6$ and is in accordance with previous literature results. ${ }^{3}$

${ }^{1} \mathrm{H}$ NMR $(500 \mathrm{MHz}$, Chloroform-d) $\delta 7.36-7.31$ (m, 2H, HC8), $7.01-6.97(\mathrm{~m}, 2 \mathrm{H}$, HC9), $4.69\left(\mathrm{dm},{ }^{2} \mathrm{JFH}_{\mathrm{FH}} 49.3 \mathrm{~Hz}, 1 \mathrm{H}, H_{\mathrm{eq}} \mathrm{C}\right), 3.64\left(\mathrm{~d},{ }^{3} \mathrm{JHH}=11.7 \mathrm{~Hz}, 1 \mathrm{H}, H_{\mathrm{ax}} \mathrm{C} 2\right), 3.40$ (dd, $\left.{ }^{2} \mathrm{JHH}_{\mathrm{HH}}=14.1 \mathrm{~Hz},{ }^{3} \mathrm{JFH}_{\mathrm{FH}}=11.6 \mathrm{~Hz}, 1 \mathrm{H}, H_{\mathrm{eq}} \mathrm{C} 6\right), 2.96$ (ddd, ${ }^{3} \mathrm{JFH}_{\mathrm{FH}}=39.8 \mathrm{~Hz},{ }^{2} \mathrm{~J}_{\mathrm{HH}}=14.0$ $\left.\mathrm{Hz},{ }^{3} \mathrm{HH}_{\mathrm{H}}=1.6 \mathrm{~Hz}, 1 \mathrm{H}, H_{\mathrm{ax}} \mathrm{C} 6\right), 2.28-2.17\left(\mathrm{~m}, 1 \mathrm{H}, \mathrm{H}_{2} \mathrm{C} 4\right), 1.93-1.68\left(\mathrm{~m}, 4 \mathrm{H}, \mathrm{H}_{2} \mathrm{C} 4\right.$, $\left.\left.\mathrm{H}_{2} \mathrm{C} 3, \mathrm{HN} 1\right) .{ }^{1} \mathrm{H}_{\{}{ }^{19} \mathrm{~F}\right\}$ NMR $(500 \mathrm{MHz}$, Chloroform- $d) \delta 7.36-7.30(\mathrm{~m}, 2 \mathrm{H}, \mathrm{HC} 8), 7.03$ $-6.96\left(\mathrm{~m}, 2 \mathrm{H}, \mathrm{HC}\right.$ ), $4.69\left(\mathrm{~m}, 1 \mathrm{H}, \mathrm{Heq}_{\mathrm{CC}}\right), 3.64\left(\mathrm{dd},{ }^{3} \mathrm{~J}_{\mathrm{HH}}=11.2 \mathrm{~Hz},{ }^{3} \mathrm{JHH}_{\mathrm{HH}}=2.5 \mathrm{~Hz}, 1 \mathrm{H}\right.$, $\left.H_{\mathrm{ax}} 2\right), 3.40\left(\mathrm{dm},{ }^{2} \mathrm{JHH}=14.1 \mathrm{~Hz}, 1 \mathrm{H}, H_{\mathrm{eq}} \mathrm{C}\right), 2.96\left(\mathrm{dd},{ }^{2} \mathrm{JHH}=14.0 \mathrm{~Hz},{ }^{3} \mathrm{JHH}_{\mathrm{HH}}=1.6 \mathrm{~Hz}\right.$, $\left.1 \mathrm{H}, H_{a x} 6\right)$, $2.28-2.17\left(\mathrm{~m}, 1 \mathrm{H}, \mathrm{H}_{2} \mathrm{C} 4\right), 1.92-1.81\left(\mathrm{~m}, 1 \mathrm{H}, \mathrm{H}_{2} \mathrm{C} 3\right), 1.81-1.67(\mathrm{~m}, 3 \mathrm{H}$, $\left.\mathrm{H}_{2} \mathrm{C} 3, \mathrm{H}_{2} \mathrm{C} 4, H \mathrm{~N} 1\right) .{ }^{13} \mathrm{C}\left\{{ }^{1} \mathrm{H}\right\} \mathrm{NMR}\left(126 \mathrm{MHz}\right.$, Chloroform-d) $\delta 162.0\left(\mathrm{~d},{ }^{1} \mathrm{JFC}=244.9 \mathrm{~Hz}\right.$, C10), $140.1\left(\mathrm{~d},{ }^{4} \mathrm{JFC}=3.1 \mathrm{~Hz}, \mathrm{C} 7\right), 128.1\left(\mathrm{~d},{ }^{3} \mathrm{JFC}=7.9 \mathrm{~Hz}, \mathrm{C} 8\right), 115.3\left(\mathrm{~d},{ }^{2} \mathrm{JFC}=21.1\right.$ $\mathrm{Hz}, \mathrm{C}$ ), 85.9 (d, $\left.{ }^{1} \mathrm{JFC}=170.5 \mathrm{~Hz}, \mathrm{C} 5\right), 59.8(\mathrm{C} 2), 50.6\left(\mathrm{~d},{ }^{2} \mathrm{JFC}=20.4 \mathrm{~Hz}, \mathrm{C} 6\right), 29.7$ (d, $\left.{ }^{2} \mathrm{JFC}=21.7 \mathrm{~Hz}, \mathrm{C} 4\right), 28.8$ (C3). ${ }^{13} \mathrm{C}\left\{{ }^{1} \mathrm{H}, \mathbf{s e l}-{ }^{19} \mathrm{~F}\right.$ at $\left.-188 \mathrm{ppm}\right\} \mathrm{NMR}(126 \mathrm{MHz}$, Chloroform- $d$ ) $\delta 162.0\left(\mathrm{~d},{ }^{1} \mathrm{JFC}=243.5 \mathrm{~Hz}, \mathrm{C} 10\right), 140.1\left(\mathrm{~d},{ }^{4} \mathrm{JFC}=3.1 \mathrm{~Hz}, \mathrm{C} 7\right), 128.1$ $\left(d,{ }^{3} \mathrm{JFC}=7.9 \mathrm{~Hz}, \mathrm{C} 8\right), 115.3\left(\mathrm{~d},{ }^{2} \mathrm{JFC}=21.1 \mathrm{~Hz}, \mathrm{C9}\right), 85.9$ (C5), 59.8 (C2), 50.6 (C6), 29.7 (C4), 28.8 (C3). ${ }^{19} \mathrm{~F}$ NMR (470 MHz, Chloroform- $d$ ) $\delta-115.6--115.8(\mathrm{~m}, 1 \mathrm{~F}$, $F C 10),-188.2--188.6(\mathrm{~m}, 1 \mathrm{~F}, F C 5) .{ }^{19} \mathrm{~F}\left\{{ }^{1} \mathrm{H}\right\}$ NMR $(470 \mathrm{MHz}$, Chloroform- $d) \delta-115.7$ (1F, FC10), -188.4 (1F, FC5). HRMS (ESI) m/z calculated for $\left[\mathrm{C}_{11} \mathrm{H}_{14} \mathrm{~F}_{2} \mathrm{~N}\right]\left(\left[\mathrm{M}+\mathrm{H}^{+}\right]\right)$ 198.1089, found 198.1086 . 

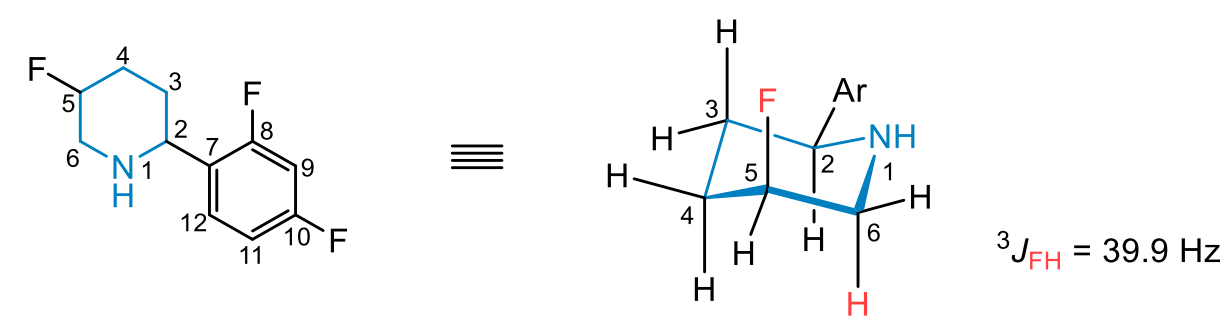

2-(2,4-Difluorophenyl)-5-fluoropiperidine (11): The title compound was synthesized according to GP-A using $10 \mathrm{~mol} \%$ Pd-catalyst $(35.1 \mathrm{mg})$ in $\mathrm{THF} / \mathrm{H}_{2} \mathrm{O}(0.5 \mathrm{M}, 1: 1)$ with aq. $\mathrm{HCl}(100 \mu \mathrm{l}, 2.4$ equiv, $12 \mathrm{~N}$ ). The product was isolated as a colorless liquid (53 mg, $0.25 \mathrm{mmol}, 49 \%, 93: 7$ d.r.) after column chromatography (silical gel, eluent: $\mathrm{CH}_{2} \mathrm{Cl}_{2} / \mathrm{MeOH} 100: 0$, later 99:1, 98:2). The product was isolated as a mixture of diastereomers. Only the analytical data of the main diastereomer are given. The axial position of the fluorine substituent was determined based on the ${ }^{3} \mathrm{JFH}_{\mathrm{FH}}$ coupling between FC5 and $\mathrm{HaxC}_{\mathrm{a}}$ and is in accordance with previous literature results. ${ }^{3}$ Only the analytical of the main diastereomer are listed.

${ }^{1} \mathrm{H}$ NMR $(500 \mathrm{MHz}$, Chloroform-d) $\delta 7.42-7.35$ (m, 1H, HC9), $6.86-6.73(\mathrm{~m}, 2 \mathrm{H}$, $H C 11, H C 12), 4.69\left(\mathrm{dm},{ }^{2} J_{\mathrm{FH}}=46.0 \mathrm{~Hz}, 1 \mathrm{H}, H_{\mathrm{eq}} \mathrm{C} 5\right), 3.94\left(\mathrm{dd},{ }^{3} \mathrm{~J}_{\mathrm{HH}}=10.4 \mathrm{~Hz},{ }^{3} \mathrm{JHH}_{\mathrm{HH}}=\right.$ $2.5 \mathrm{~Hz}, 1 \mathrm{H}, \mathrm{Hax}_{\mathrm{ax}}$ ), 3.40 (dddd, ${ }^{2} \mathrm{JHH}=14.4 \mathrm{~Hz},{ }^{3} \mathrm{JFH}_{\mathrm{FH}}=11.6 \mathrm{~Hz},{ }^{3} \mathrm{~J}_{\mathrm{HH}}=2.8 \mathrm{~Hz},{ }^{4} \mathrm{~J}_{\mathrm{HH}}=$ $2.8 \mathrm{~Hz}, 1 \mathrm{H}, H_{\mathrm{eq}} \mathrm{C} 6$ ), 2.97 (dd, ${ }^{3} \mathrm{JFH}_{\mathrm{FH}}=39.9 \mathrm{~Hz},{ }^{2} \mathrm{JHH}=14.1 \mathrm{~Hz}, 1 \mathrm{H}, H_{\mathrm{ax}} \mathrm{C} 6$ ), $2.25-2.15$ $\left(\mathrm{m}, 1 \mathrm{H}, \mathrm{H}_{2} \mathrm{C} 4\right), 1.93-1.66\left(\mathrm{~m}, 4 \mathrm{H}, \mathrm{H}_{2} \mathrm{C} 3, \mathrm{H}_{2} \mathrm{C} 4, H \mathrm{~N} 1\right) .{ }^{1} \mathrm{H}\left\{{ }^{19} \mathrm{~F}\right\} \mathrm{NMR}(500 \mathrm{MHz}$, Chloroform-d) $\delta 7.41-7.35$ (m, 1H, HC9), $6.88-6.73$ (m, 2H, HC11, HC12), $4.71-$ $4.67\left(\mathrm{~m}, 1 \mathrm{H}\right.$. $\mathrm{Heq}_{\mathrm{CC}}$ ), $3.93\left(\mathrm{dd},{ }^{3} \mathrm{HHH}=11.3 \mathrm{~Hz},{ }^{3} \mathrm{HH}=2.6 \mathrm{~Hz}, 1 \mathrm{H}, \mathrm{HaxC}_{\mathrm{H}}\right), 3.40$ (ddd, $\left.{ }^{2} \mathrm{JHH}_{\mathrm{HH}}=14.1,{ }^{3} \mathrm{JHH}_{\mathrm{HH}}=2.8 \mathrm{~Hz},{ }^{4} \mathrm{JHH}_{\mathrm{HH}}=2.8 \mathrm{~Hz}, 1 \mathrm{H}, \mathrm{Heq}_{\mathrm{eq}} \mathrm{C}\right), 2.97\left(\mathrm{~d},{ }^{2} \mathrm{JHH}_{\mathrm{HH}}=14.1 \mathrm{~Hz}, 1 \mathrm{H}\right.$, $\left.H_{a x} \mathrm{C} 6\right), 2.27-2.17\left(\mathrm{~m}, 1 \mathrm{H}, H_{2} \mathrm{C} 4\right), 1.93-1.67\left(\mathrm{~m}, 4 \mathrm{H}, \mathrm{H}_{2} \mathrm{C} 3, \mathrm{H}_{2} \mathrm{C} 4, \mathrm{HN} 1\right) .{ }^{13} \mathrm{C}\left\{{ }^{1} \mathrm{H}\right\}$ NMR (126 MHz, Chloroform- $d) \delta 162.0$ (dd, ${ }^{1} \mathrm{JFC}=247.7 \mathrm{~Hz},{ }^{3} \mathrm{JFC}_{\mathrm{FC}}=12.4 \mathrm{~Hz}, \mathrm{C} 10$ ), $160.2\left(\mathrm{dd},{ }^{1} \mathrm{JFC}=248.1 \mathrm{~Hz},{ }^{3} \mathrm{JFC}=11.7 \mathrm{~Hz}, \mathrm{C} 8\right), 128.4\left(\mathrm{dd},{ }^{3} \mathrm{JFC}=9.6 \mathrm{~Hz},{ }^{3} \mathrm{JFC}_{\mathrm{FC}}=6.4 \mathrm{~Hz}\right.$, C12), $127.2\left(\mathrm{dd},{ }^{2} \mathrm{JFC}=13.9,{ }^{4} \mathrm{JFC}=3.7 \mathrm{~Hz}, \mathrm{C} 7\right), 111.4\left(\mathrm{dd},{ }^{2} \mathrm{JFC}_{\mathrm{FC}}=20.9 \mathrm{~Hz},{ }^{4} \mathrm{JFC}=3.6\right.$ $\mathrm{Hz}, \mathrm{C} 11), 103.8\left(\mathrm{dd},{ }^{2} \mathrm{JFC}=26.5 \mathrm{~Hz},{ }^{2} \mathrm{JFC}_{\mathrm{FC}} 25.2 \mathrm{~Hz}, \mathrm{C} 9\right), 85.8\left(\mathrm{~d},{ }^{1} \mathrm{JFC}=170.4 \mathrm{~Hz}, \mathrm{C} 5\right)$, $53.8\left(\mathrm{~d},{ }^{3} \mathrm{JFC}=2.0 \mathrm{~Hz}, \mathrm{C} 2\right), 50.7\left(\mathrm{~d},{ }^{2} \mathrm{JFC}=20.4 \mathrm{~Hz}, \mathrm{C} 6\right), 29.7\left(\mathrm{~d},{ }^{2} \mathrm{JFC}=21.7 \mathrm{~Hz}, \mathrm{C} 4\right)$, 27.8 (C3). ${ }^{13} \mathrm{C}\left\{{ }^{1} \mathrm{H}\right.$, sel- ${ }^{19} \mathrm{~F}$ at -188$\}$ NMR $(126 \mathrm{MHz}$, Chloroform- $d) \delta 162.0$ (dd, ${ }^{1} \mathrm{JFC}=$ $\left.246.3 \mathrm{~Hz},{ }^{3} \mathrm{JFC}=12.4 \mathrm{~Hz}, \mathrm{C} 10\right), 160.2\left(\mathrm{dd},{ }^{1} \mathrm{JFC}=246.5 \mathrm{~Hz},{ }^{3} \mathrm{JFC}=11.7 \mathrm{~Hz}, \mathrm{C} 8\right), 128.4$ (dd, $\left.{ }^{3} \mathrm{JFC}=9.5 \mathrm{~Hz},{ }^{3} \mathrm{JFC}=6.3 \mathrm{~Hz}, \mathrm{C} 12\right), 127.2\left(\mathrm{dd},{ }^{2} \mathrm{JFC}=13.9 \mathrm{~Hz},{ }^{4} \mathrm{JFC}=3.7 \mathrm{~Hz}, \mathrm{C} 7\right)$, $111.4\left(\mathrm{dd},{ }^{2} \mathrm{JFC}_{\mathrm{FC}} 20.8,{ }^{4} \mathrm{JFC}=3.6 \mathrm{~Hz}, \mathrm{C} 11\right), 103.8\left(\mathrm{dd},{ }^{3} \mathrm{JFC}_{\mathrm{FC}}=26.3 \mathrm{~Hz},{ }^{3} \mathrm{JFC}=25.1 \mathrm{~Hz}\right.$, C9), 85.8 (C5), 53.8 (d, $\left.{ }^{4} \mathrm{FFC}=1.9 \mathrm{~Hz}, \mathrm{C} 2\right), 50.7$ (C6), 29.7 (C4), 27.8 (C3). ${ }^{19} \mathrm{~F}$ NMR $(470 \mathrm{MHz}$, Chloroform- $d) \delta-112.3--112.4(\mathrm{~m}, 1 \mathrm{~F}, \mathrm{FC} 10),-115.5--115.6(\mathrm{~m}, 1 \mathrm{~F}$, 
FC8), -188.3 - -188.7 (m, 1F, FC5). ${ }^{19} \mathrm{~F}\left\{{ }^{1} \mathrm{H}\right\}$ NMR $(470 \mathrm{MHz}$, Chloroform- $d) \delta-112.3$ (d, $\left.{ }^{3} \mathrm{JFF}=7.1 \mathrm{~Hz}, 1 \mathrm{~F}, F C 10\right),-115.5$ (d, $\left.{ }^{3} \mathrm{JFF}=7.1 \mathrm{~Hz}, 1 \mathrm{~F}, F C 8\right),-188.5(1 \mathrm{~F}, F C 5)$. HRMS (ESI) $\mathrm{m} / \mathrm{z}$ calculated for $\left[\mathrm{C}_{11} \mathrm{H}_{13} \mathrm{~F}_{4} \mathrm{~N}\right]\left(\left[\mathrm{M}+\mathrm{H}^{+}\right]\right) 216.0995$, found 216.0994 .
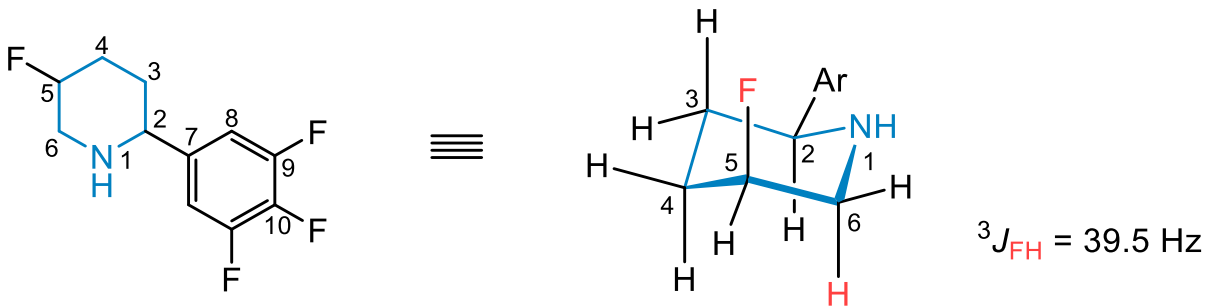

5-Fluoro-2-(3,4,5-trifluorophenyl)piperidine (12): The title compound was synthesized according to GP-A using $10 \mathrm{~mol} \% \mathrm{Pd}$-catalyst $(35.1 \mathrm{mg})$ in $\mathrm{THF} / \mathrm{H}_{2} \mathrm{O}$ $(0.5 \mathrm{M}, 1: 1)$ with aq. $\mathrm{HCl}(100 \mu \mathrm{l}, 2.4$ equiv, $12 \mathrm{~N})$. The product was isolated as a white solid ( $66 \mathrm{mg}, 0.28 \mathrm{mmol}, 57 \%, 95: 5 \mathrm{~d}$.r.) after column chromatography (silical gel, eluent: $n$-pentane/EtOAc 80:20, later 50:50). Only the major diastereomer was isolated. The axial position of the fluorine substituent was determined based on the ${ }^{3} \mathrm{FH}$ coupling between $\mathrm{FC} 5$ and $\mathrm{Hax}_{\mathrm{ax}} 6$ and is in accordance with previous literature results. $^{3}$

${ }^{1} \mathrm{H}$ NMR $(500 \mathrm{MHz}$, Chloroform- $d) \delta 7.07-6.97(\mathrm{~m}, 2 \mathrm{H}, \mathrm{HC} 8), 4.69\left(\mathrm{dm},{ }^{2} \mathrm{JFH}_{\mathrm{FH}}=47.0\right.$ $\mathrm{Hz}, 1 \mathrm{H}, H_{\mathrm{eq}} \mathrm{C}$ ), 3.60 (dd, ${ }^{3} \mathrm{JHH}_{\mathrm{HH}}=10.1 \mathrm{~Hz},{ }^{3} \mathrm{JHH}_{\mathrm{HH}}=2.9 \mathrm{~Hz}, 1 \mathrm{H}, H_{\mathrm{ax}} \mathrm{C} 2$ ), 3.40 (dddd, ${ }^{2} \mathrm{~J}_{\mathrm{HH}}$ $=14.2 \mathrm{~Hz},{ }^{3} \mathrm{JFH}_{\mathrm{FH}}=11.6 \mathrm{~Hz},{ }^{3} \mathrm{JHH}=2.6 \mathrm{~Hz},{ }^{4} \mathrm{JHH}_{\mathrm{HH}}=2.6 \mathrm{~Hz}, 1 \mathrm{H}, H_{\mathrm{eq}} \mathrm{C} 6$ ), 2.94 (ddd, ${ }^{3} \mathrm{JFH}_{\mathrm{FH}}=$ $\left.39.5 \mathrm{~Hz},{ }^{2} \mathrm{JHH}_{\mathrm{HH}}=14.1 \mathrm{~Hz},{ }^{3} \mathrm{JHH}_{\mathrm{HH}}=1.4 \mathrm{~Hz}, 1 \mathrm{H}, H_{\mathrm{ax}} \mathrm{C} 6\right), 2.27-2.17\left(\mathrm{~m}, 1 \mathrm{H}, \mathrm{H}_{2} \mathrm{C} 4\right), 1.86-$ $1.66\left(\mathrm{~m}, 3 \mathrm{H}, \mathrm{H}_{2} \mathrm{C} 4, \mathrm{H}_{2} \mathrm{C} 3, \mathrm{H}_{2} \mathrm{C} 3\right), 1.59(\mathrm{~s}, 1 \mathrm{H}, H \mathrm{~N} 1) .{ }^{1} \mathrm{H}\left\{{ }^{19} \mathrm{~F}\right\} \mathrm{NMR}(500 \mathrm{MHz}$, Chloroform-d) $\delta 7.02$ (d, J=0.7 Hz, 2H, HC8), $4.75-4.62$ (m, 1H, Heq 5 ), $3.64-3.57$ (m, $\left.1 \mathrm{H}, H_{a x} \mathrm{C} 2\right), 3.40$ (ddd, $\left.{ }^{2} J_{\mathrm{HH}}=14.0 \mathrm{~Hz},{ }^{3} \mathrm{JHH}_{\mathrm{HH}}=2.6 \mathrm{~Hz},{ }^{4} \mathrm{JHH}_{\mathrm{HH}}=2.6 \mathrm{~Hz}, 1 \mathrm{H}, H_{e q} \mathrm{C} 6\right)$, $3.01-2.87\left(\mathrm{~m}, 1 \mathrm{H}, \mathrm{H}_{a x} \mathrm{C} 6\right), 2.27-2.16\left(\mathrm{~m}, 1 \mathrm{H}, \mathrm{H}_{2} \mathrm{C} 4\right), 1.86-1.69\left(\mathrm{~m}, 3 \mathrm{H}, \mathrm{H}_{2} \mathrm{C} 4\right.$, $\left.\mathrm{H}_{2} \mathrm{C} 3, \mathrm{H}_{2} \mathrm{C} 3\right), 1.59(\mathrm{~s}, 1 \mathrm{H}, H \mathrm{~N} 1) .{ }^{13} \mathrm{C}\left\{{ }^{1} \mathrm{H}\right\}$ NMR $(126 \mathrm{MHz}$, Chloroform-d) $\delta 151.3$ (ddd, $\left.{ }^{1} \mathrm{JFC}_{2}=249.4,{ }^{2} \mathrm{JFC}_{\mathrm{FC}}=10.0 \mathrm{~Hz},{ }^{3} \mathrm{JFC}=4.1 \mathrm{~Hz}, \mathrm{C} 9\right), 140.7\left(\mathrm{td},{ }^{3} \mathrm{JFC}=6.7 \mathrm{~Hz},{ }^{4} \mathrm{JFC}=4.3 \mathrm{~Hz}\right.$, C7), $138.8\left(\mathrm{dt},{ }^{1} \mathrm{JFC}=250.3 \mathrm{~Hz},{ }^{2} \mathrm{JFC}=15.3 \mathrm{~Hz}, \mathrm{C} 10\right), 110.6\left(\mathrm{dd},{ }^{2} \mathrm{JFC}=16.4 \mathrm{~Hz},{ }^{3} \mathrm{JFC}=\right.$ $4.9 \mathrm{~Hz}, \mathrm{C} 8$ ), 85.6 (d, ${ }^{1} \mathrm{JFC}=171.1 \mathrm{~Hz}, \mathrm{C}$ ), $59.3(\mathrm{C} 2), 50.4$ (d, ${ }^{2} \mathrm{JFC}=20.4 \mathrm{~Hz}, \mathrm{C6}$ ), 29.5 (d, $\left.{ }^{2} J_{F C}=21.6 \mathrm{~Hz}, \mathrm{C} 4\right), 28.7$ (C3). ${ }^{13} \mathrm{C}\left\{{ }^{1} \mathrm{H}\right.$,broadband-19 $\left.\mathrm{F}\right\}$ NMR $(126 \mathrm{MHz}$, Chloroform-d) $\delta 151.3$ (C9), 140.7 (7), 138.8 (10), 110.6 (8), 85.6 (C5), 59.2 (C2), 50.4 (C6), 29.5 (C4), 28.7 (C3). ${ }^{19} \mathrm{~F}$ NMR (282 MHz, Chloroform- $\left.d\right) \delta-134.4--134.7(\mathrm{~m}$, $2 \mathrm{~F}, F C 9),-162.5\left(\mathrm{tt},{ }^{3} \mathrm{JFF}_{\mathrm{FF}}=20.6,{ }^{4} \mathrm{JHF}_{\mathrm{HF}}=6.5 \mathrm{~Hz}, 1 \mathrm{~F}, \mathrm{FC} 10\right),-188.3--189.1(\mathrm{~m}, 1 \mathrm{~F}, F \mathrm{C} 5)$. ${ }^{19} \mathrm{~F}\left\{{ }^{1} \mathrm{H}\right\}$ NMR $(282 \mathrm{MHz}$, Chloroform- $d) \delta-134.5\left(\mathrm{~d},{ }^{3} \mathrm{JFF}=20.6 \mathrm{~Hz}, 2 \mathrm{~F}, F C 9\right),-162.6(\mathrm{t}$, 
$\left.{ }^{3} J_{F F}=20.6 \mathrm{~Hz}, 1 \mathrm{~F}, F C 10\right),-188.7(1 \mathrm{~F}, F C 5)$. HRMS (ESI) $\mathrm{m} / \mathrm{z}$ calculated for $\left[\mathrm{C}_{11} \mathrm{H}_{12} \mathrm{~F}_{4} \mathrm{~N}\right]\left(\left[\mathrm{M}+\mathrm{H}^{+}\right]\right) 234.0900$, found 234.0918 .
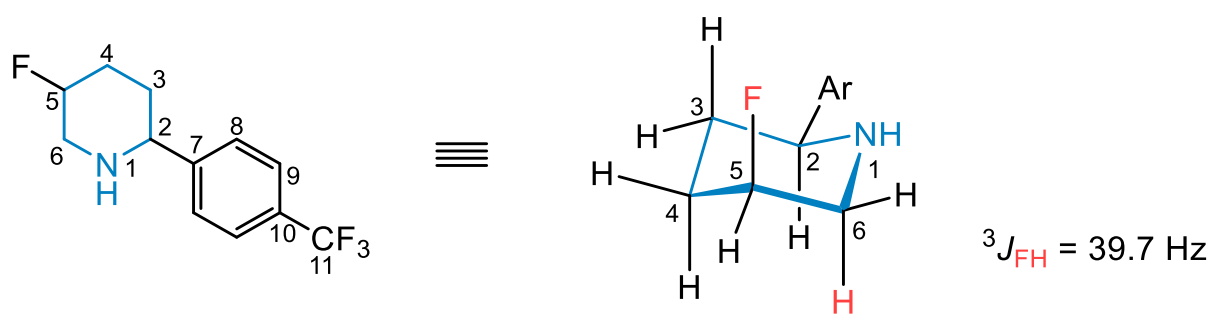

5-Fluoro-2-(4-(trifluoromethyl)phenyl)piperidine (13): The title compound was synthesized according to GP-A using $10 \mathrm{~mol} \%$ Pd-catalyst $(35.1 \mathrm{mg})$ in $\mathrm{THF} / \mathrm{H}_{2} \mathrm{O}$ $(0.5 \mathrm{M}, 1: 1)$ with aq. $\mathrm{HCl}(100 \mu \mathrm{l}, 2.4$ equiv, $12 \mathrm{~N})$. The product was isolated as a white solid (62 mg, $0.28 \mathrm{mmol}, 50 \%, 89: 11$ d.r.) after column chromatography (silical gel, eluent: $n$-pentane/EtOAc 80:20, later 50:50). Only the major diastereomer was isolated. The axial position of the fluorine substituent was determined based on the ${ }^{3} J_{\mathrm{FH}}$ coupling between $\mathrm{FC} 5$ and $\mathrm{Hax}_{\mathrm{ax}} \mathrm{C}$ and is in accordance with previous literature results. ${ }^{3}$

${ }^{1} \mathrm{H}$ NMR $(599 \mathrm{MHz}$, Chloroform- $d) \delta 7.57\left(\mathrm{~d},{ }^{3} \mathrm{HH}=8.1 \mathrm{~Hz}, 2 \mathrm{H}, \mathrm{HC9}\right), 7.49\left(\mathrm{~d},{ }^{3} \mathrm{JHH}_{\mathrm{HH}}=\right.$ $8.2 \mathrm{~Hz}, 2 \mathrm{H}, H \mathrm{C} 8), 4.71\left(\mathrm{dm},{ }^{2} \mathrm{JFH}=47.0 \mathrm{~Hz}, 1 \mathrm{H}, H_{\mathrm{eq}} \mathrm{C} 5\right), 3.72\left(\mathrm{bd},{ }^{3} \mathrm{JHH}=10.9 \mathrm{~Hz}, 1 \mathrm{H}\right.$, $H_{\mathrm{ax}} \mathrm{C}$ ), 3.43 (dddd, ${ }^{2} \mathrm{JHH}_{\mathrm{HH}}=14.2 \mathrm{~Hz},{ }^{3} \mathrm{JFH}_{\mathrm{FH}}=11.6 \mathrm{~Hz},{ }^{3} \mathrm{JHH}_{\mathrm{HH}}=2.6 \mathrm{~Hz},{ }^{4} J_{\mathrm{HH}}=2.6 \mathrm{~Hz}, 1 \mathrm{H}$, $H_{\mathrm{eq}} \mathrm{C}$ ), 2.97 (ddd, ${ }^{3} \mathrm{JFH}_{\mathrm{FH}}=39.7,{ }^{2} \mathrm{~J}_{\mathrm{HH}}=14.1 \mathrm{~Hz},{ }^{3} \mathrm{JHH}_{\mathrm{HH}}=1.4 \mathrm{~Hz}, 1 \mathrm{H}, H_{\mathrm{ax}} \mathrm{C} 6$ ), $2.27-2.19$ (m, $\left.1 \mathrm{H}, \mathrm{H}_{2} \mathrm{C} 4\right), 1.92-1.69\left(\mathrm{~m}, 3 \mathrm{H}, \mathrm{H}_{2} \mathrm{C} 3, \mathrm{H}_{2} \mathrm{C} 3, \mathrm{H}_{2} \mathrm{C} 4\right), 1.62(\mathrm{~s}, 1 \mathrm{H}, H \mathrm{~N} 1) .{ }^{1} \mathrm{H}\left\{{ }^{19} \mathrm{~F}\right\}$ NMR (500 MHz, Chloroform-d) $\delta 7.58\left(\mathrm{~d},{ }^{3} \mathrm{JHH}=8.2 \mathrm{~Hz}, 2 \mathrm{H}, H \mathrm{C} 9\right), 7.49\left(\mathrm{~d},{ }^{3}{ }_{\mathrm{HH}}=8.2\right.$ $\mathrm{Hz}, 2 \mathrm{H}, H \mathrm{C} 8), 4.74-4.69\left(\mathrm{~m}, 1 \mathrm{H}, \mathrm{Heq}_{\mathrm{CO}}\right), 3.73\left(\mathrm{dd},{ }^{3} \mathrm{JHH}_{\mathrm{HH}}=11.1 \mathrm{~Hz},{ }^{3} \mathrm{HH}=2.1 \mathrm{~Hz}, 1 \mathrm{H}\right.$, $H_{\mathrm{ax}} \mathrm{C} 2$ ), $3.43\left(\mathrm{ddd},{ }^{2} \mathrm{~J}_{\mathrm{HH}}=14.0 \mathrm{~Hz},{ }^{3} \mathrm{JHH}_{\mathrm{HH}}=2.6 \mathrm{~Hz},{ }^{4} \mathrm{JHH}_{\mathrm{HH}}=2.6 \mathrm{~Hz}, 1 \mathrm{H}, H_{\mathrm{eq}} \mathrm{C} 6\right), 2.98$ (dd, $\left.{ }^{2} \mathrm{JHH}_{\mathrm{HH}}=14.1 \mathrm{~Hz},{ }^{3} \mathrm{JHH}_{\mathrm{HH}}=1.2 \mathrm{~Hz}, 1 \mathrm{H}, \mathrm{Hax}_{\mathrm{ax}} \mathrm{C}\right), 2.27-2.20\left(\mathrm{~m}, 1 \mathrm{H}, \mathrm{H}_{2} \mathrm{C} 4\right), 1.92-1.72(\mathrm{~m}$, $\left.4 \mathrm{H}, \mathrm{H}_{2} \mathrm{C} 3, \mathrm{H}_{2} \mathrm{C} 4, H \mathrm{~N} 1\right) .{ }^{13} \mathrm{C}\left\{{ }^{1} \mathrm{H}\right\} \mathrm{NMR}(151 \mathrm{MHz}$, Chloroform- $d) \delta 148.3$ (q, ${ }^{5} \mathrm{JFC}=1.3$ $\mathrm{Hz}, \mathrm{C} 7), 129.6$ (q, $\left.{ }^{2} \mathrm{FFC}=32.3 \mathrm{~Hz}, \mathrm{C} 10\right), 126.9(\mathrm{C} 8), 125.5$ (q, $\left.{ }^{3} \mathrm{JFC}=3.8 \mathrm{~Hz}, \mathrm{C} 9\right), 124.3$ (q, $\left.{ }^{1} \mathrm{JFC}=271.9 \mathrm{~Hz}, \mathrm{C} 11\right), 85.8\left(\mathrm{~d},{ }^{1} \mathrm{JFC}=170.7 \mathrm{~Hz}, \mathrm{C} 5\right), 60.2(\mathrm{C} 2), 50.6\left(\mathrm{~d},{ }^{2} \mathrm{JFC}=20.3\right.$ $\mathrm{Hz}, \mathrm{C} 6), 29.7$ (d, $\left.{ }^{2} \mathrm{JFC}=21.7 \mathrm{~Hz}, \mathrm{C} 4\right), 28.9$ (C3). ${ }^{13} \mathrm{C}\left\{{ }^{1} \mathrm{H}\right.$,broadband- $\left.{ }^{19} \mathrm{~F}\right\}$ NMR (151 $\mathrm{MHz}$, Chloroform-d) $\delta 148.3$ (C7), 129.6 (C10), 126.9 (C8), 125.5 (C9), 124.3 (C11), 85.8 (C5), 60.2 (C2), 50.6 (C6), 29.6 (C4), 28.8 (C3). ${ }^{19} \mathrm{~F} \mathrm{NMR}$ (564 MHz, Chloroformd) $\delta-62.5\left(\mathrm{~s}, 3 \mathrm{~F}, F_{3} \mathrm{C} 11\right),-188.4--188.8(\mathrm{~m}, 1 \mathrm{~F}, F C 5) .{ }^{19} \mathrm{~F}\left\{{ }^{1} \mathrm{H}\right\} \mathrm{NMR}(564 \mathrm{MHz}$, Chloroform- $d$ ) $\delta-62.5$ (3F, FC11), -188.6 (1F, FC5). HRMS (ESI) m/z calculated for $\left[\mathrm{C}_{12} \mathrm{H}_{14} \mathrm{~F}_{4} \mathrm{~N}\right]\left(\left[\mathrm{M}+\mathrm{H}^{+}\right]\right)$248.1057, found 248.1077. 


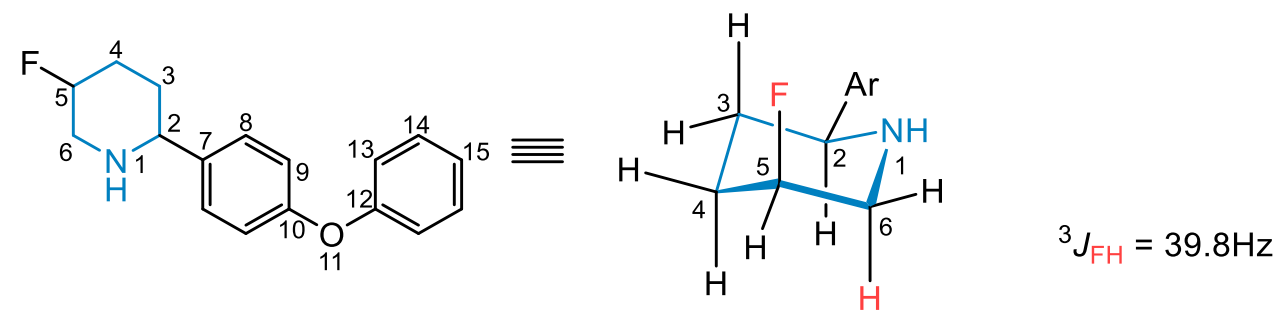

5-Fluoro-2-(4-phenoxyphenyl)piperidine (14): The title compound was synthesized according to GP-A using $10 \mathrm{~mol} \%$ Pd-catalyst $(35.1 \mathrm{mg})$ in $\mathrm{THF} / \mathrm{H}_{2} \mathrm{O}(0.5 \mathrm{M}, 1: 1)$ with aq. $\mathrm{HCl}(100 \mu \mathrm{l}, 2.4$ equiv, $12 \mathrm{~N})$. The product was isolated as a white solid (78 $\mathrm{mg}$, $0.29 \mathrm{mmol}, 57 \%, 91: 9$ d.r.) after column chromatography (silical gel, eluent: $n$ pentane/EtOAc 80:20, later 50:50). Only the major diastereomer was isolated. The axial position of the fluorine substituent was determined based on the ${ }^{3} J_{F H}$ coupling between $\mathrm{FC} 5$ and $\mathrm{H}_{\mathrm{ax}} \mathrm{C} 6$ and is in accordance with previous literature results. ${ }^{3}$

${ }^{1} \mathrm{H}$ NMR (599 MHz, Chloroform-d) $\delta 7.38$ - 7.29 (m, 4H, HC8, HC13), 7.14 - 7.06 (m, $1 \mathrm{H}, \mathrm{HC} 15), 7.04-6.95$ (m, 4H, HC9, HC14), 4.71 (dm, ${ }^{2} \mathrm{JFH}_{\mathrm{FH}}=47.8 \mathrm{~Hz}, 1 \mathrm{H}, H_{\mathrm{eq}} \mathrm{C}$ ), $3.67\left(\mathrm{dm},{ }^{3} \mathrm{JHH}_{\mathrm{HH}}=10.6 \mathrm{~Hz}, 1 \mathrm{H}, \mathrm{HaxC2}_{2}, 3.42\right.$ (dddd, ${ }^{2} \mathrm{JHH}_{\mathrm{HH}}=14.4 \mathrm{~Hz},{ }^{3} \mathrm{JFH}_{\mathrm{FH}}=11.5 \mathrm{~Hz},{ }^{3} \mathrm{JHH}=$ $\left.2.7 \mathrm{~Hz},{ }^{4} \mathrm{JHH}_{\mathrm{HH}}=2.7 \mathrm{~Hz}, 1 \mathrm{H}, H_{\mathrm{eq}} \mathrm{C} 6\right), 2.99\left(\mathrm{dd},{ }^{3} \mathrm{JFH}_{\mathrm{FH}}=39.8 \mathrm{~Hz},{ }^{2} J_{\mathrm{HH}}=14.1 \mathrm{~Hz}, 1 \mathrm{H}, H_{\mathrm{ax}} \mathrm{C} 6\right)$, $2.29-2.20\left(\mathrm{~m}, 1 \mathrm{H}, \mathrm{H}_{2} \mathrm{C} 4\right), 2.10(\mathrm{~s}, 1 \mathrm{H}, \mathrm{HN} 1), 1.98-1.88\left(\mathrm{~m}, 1 \mathrm{H}, \mathrm{H}_{2} \mathrm{CH} 3\right), 1.86-1.71$ (m, $\left.2 \mathrm{H}, \mathrm{H}_{2} \mathrm{CH} 3, \mathrm{H}_{2} \mathrm{CH} 4\right) .{ }^{1} \mathrm{H}\left\{{ }^{19} \mathrm{~F}\right\}$ NMR $(599 \mathrm{MHz}$, Chloroform-d) $\delta 7.37-7.28(\mathrm{~m}, 4 \mathrm{H}$, HC8, HC13), 7.09 (tt, $\left.{ }^{3} \mathrm{JHH}_{\mathrm{HH}}=7.3,{ }^{4} \mathrm{~J}_{\mathrm{HH}}=1.1 \mathrm{~Hz}, 1 \mathrm{H}, \mathrm{HC} 15\right), 7.02-6.95(\mathrm{~m}, 4 \mathrm{H}, \mathrm{HC}$, HC14), 4.71 (bs, $1 \mathrm{H}, H_{\text {eqC }}$ ), 3.68 (dd, $\left.{ }^{3} J_{H H}=11.5 \mathrm{~Hz},{ }^{3} \mathrm{JHH}_{\mathrm{HH}}=2.4 \mathrm{~Hz}, 1 \mathrm{H}, H_{\mathrm{ax}} \mathrm{C} 2\right), 3.42$ (ddd, ${ }^{2} \mathrm{JHH}=14.2 \mathrm{~Hz},{ }^{3} \mathrm{JHH}_{\mathrm{HH}}=2.6 \mathrm{~Hz},{ }^{4} \mathrm{JHH}_{\mathrm{HH}}=2.6 \mathrm{~Hz}, 1 \mathrm{H}, \mathrm{Heq}_{\mathrm{C}}$ ), $2.99\left(\mathrm{~d},{ }^{2} \mathrm{JHH}_{\mathrm{HH}}=14.0 \mathrm{~Hz}\right.$, $\left.1 \mathrm{H}, H_{\mathrm{ax}} \mathrm{C} 6\right), 2.24\left(\mathrm{dm},{ }^{2} \mathrm{~J}_{\mathrm{HH}}=14.1 \mathrm{~Hz}, 1 \mathrm{H}, H_{2} \mathrm{C} 4\right), 2.10(\mathrm{~s}, 1 \mathrm{H}, H \mathrm{~N} 1), 1.98-1.88(\mathrm{~m}$, $\left.1 \mathrm{H}, \mathrm{H}_{2} \mathrm{CH} 3\right), 1.83-1.74\left(\mathrm{~m}, 2 \mathrm{H}, \mathrm{H}_{2} \mathrm{CH} 3, \mathrm{H}_{2} \mathrm{CH} 4\right) .{ }^{13} \mathrm{C}\left\{{ }^{1} \mathrm{H}\right\}$ NMR $(151 \mathrm{MHz}$, Chloroformd) $\delta 157.5$ (C12), 156.4 (C10), 139.1 (C7), 129.8 (C13), 128.0 (C8), 123.3 (C15), 119.0 (C9), 118.9 (C14), 85.9 (d, $\left.{ }^{1} J_{F C}=170.4 \mathrm{~Hz}, \mathrm{C} 5\right), 60.0$ (C2), 50.7 (d, ${ }^{2} \mathrm{JFC}_{\mathrm{FC}}=20.4 \mathrm{~Hz}$, C6), $29.7\left(\mathrm{~d},{ }^{2} \mathrm{JFC}_{\mathrm{FC}}=21.7 \mathrm{~Hz}, \mathrm{C} 4\right), 28.6(\mathrm{C} 3) .{ }^{13} \mathrm{C}\left\{{ }^{1} \mathrm{H},{ }^{19} \mathrm{~F}\right\}$ NMR (151 MHz, Chloroformd) $\delta 157.5$ (C12), 156.4 (C10), 139.1 (C7), 129.8 (C13), 128.0 (C8), 123.3 (C15), 119.1 (C9), 118.9 (C14), 85.9 (C5), 60.0 (C2), 50.7 (C6), 29.7 (C4), 28.6 (C3). ${ }^{19}$ F NMR (564 $\mathrm{MHz}$, Chloroform- $d$ ) $\delta-188.2--188.5(\mathrm{~m}) .{ }^{19} \mathrm{~F}\left\{{ }^{1} \mathrm{H}\right\}$ NMR $(376 \mathrm{MHz}$, Chloroform- $d$ ) $\delta$ 188.4. HRMS (ESI) $\mathrm{m} / \mathrm{z}$ calculated for $\left[\mathrm{C}_{17} \mathrm{H}_{19} \mathrm{FNO}\right]\left(\left[\mathrm{M}+\mathrm{H}^{+}\right]\right) 272.1445$, found 272.1457 . 


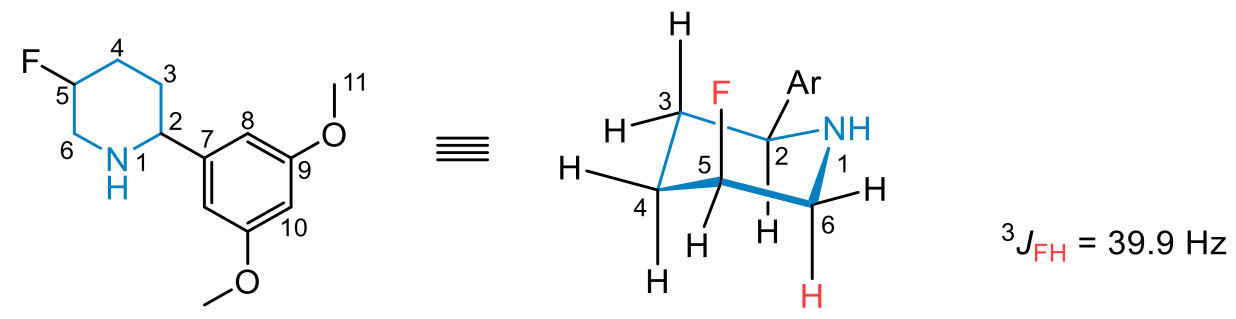

2-(3,5-Dimethoxyphenyl)-5-fluoropiperidine (15): The title compound was synthesized according to GP-A using $10 \mathrm{~mol} \% \mathrm{Pd}$-catalyst $(35.1 \mathrm{mg})$ in $\mathrm{THF} / \mathrm{H}_{2} \mathrm{O}$ $(0.5 \mathrm{M}, 1: 1)$ with aq. $\mathrm{HCl}(100 \mu \mathrm{l}, 2.4$ equiv, $12 \mathrm{~N})$. The product was isolated as a white solid (55 mg, $0.23 \mathrm{mmol}, 46 \%, 93: 7$ d.r.) after column chromatography (silical gel, eluent: $n$-pentane/EtOAc 80:20, later 50:50, 0:100). Only the major diastereomer was isolated. The axial position of the fluorine substituent was determined based on the ${ }^{3} \mathrm{JFH}_{\mathrm{FH}}$ coupling between $\mathrm{FC} 5$ and $\mathrm{Hax}_{\mathrm{ax}} \mathrm{C}$ and is in accordance with previous literature results. ${ }^{3}$

${ }^{1} \mathrm{H}$ NMR $(599 \mathrm{MHz}$, Chloroform- $d) \delta 6.53\left(\mathrm{~d},{ }^{4} \mathrm{JHH}=2.4 \mathrm{~Hz}, 2 \mathrm{H}, H \mathrm{C} 8\right), 6.35\left(\mathrm{t},{ }^{4} \mathrm{JHH}_{\mathrm{HH}}=\right.$ $2.3 \mathrm{~Hz}, 1 \mathrm{H}, H \mathrm{C} 10), 4.69\left(\mathrm{dm},{ }^{2} \mathrm{JFH}=47.3 \mathrm{~Hz}, 1 \mathrm{H}, H_{\mathrm{eq}} \mathrm{C} 5\right), 3.78\left(\mathrm{~s}, 6 \mathrm{H}, \mathrm{H}_{3} \mathrm{C} 11\right), 3.60$ $\left(\mathrm{dd},{ }^{3} \mathrm{JHH}_{\mathrm{HH}}=11.0 \mathrm{~Hz},{ }^{3} \mathrm{~J}_{\mathrm{HH}}=2.3 \mathrm{~Hz}, 1 \mathrm{H}, \mathrm{Hax}_{\mathrm{ax}}\right.$ ), 3.41 (dddd, ${ }^{2} \mathrm{JHH}_{\mathrm{HH}}=14.0 \mathrm{~Hz},{ }^{3} J_{\mathrm{FH}}=$ $\left.11.4 \mathrm{~Hz},{ }^{3} \mathrm{JHH}_{\mathrm{HH}}=2.6 \mathrm{~Hz},{ }^{4} \mathrm{JHH}_{\mathrm{HH}}=2.6 \mathrm{~Hz}, 1 \mathrm{H}, H_{\mathrm{eq}} \mathrm{C} 6\right), 2.95\left(\mathrm{dd},{ }^{3} \mathrm{~J}_{\mathrm{FH}}=39.9 \mathrm{~Hz},{ }^{2} \mathrm{JHH}_{\mathrm{HH}}=14.1\right.$ $\left.\mathrm{Hz}, 1 \mathrm{H}, \mathrm{H}_{\mathrm{ax}} \mathrm{C} 6\right)$ ), 2.26 - 2.17 (m, $\left.1 \mathrm{H}, \mathrm{H}_{2} \mathrm{C} 4\right), 1.96-1.68$ (m, 4H, $\mathrm{H}_{2} \mathrm{C} 4, \mathrm{H}_{2} \mathrm{C} 3, \mathrm{H}_{2} \mathrm{C} 3$, HN1). ${ }^{1} \mathbf{H}\left\{{ }^{19} \mathrm{~F}\right\}$ NMR $(599 \mathrm{MHz}$, Chloroform- $d) \delta 6.53\left(\mathrm{~d},{ }^{4} \mathrm{JHH}=2.2 \mathrm{~Hz}, 2 \mathrm{H}, H \mathrm{C} 8\right), 6.35$ $\left(\mathrm{t},{ }^{4} \mathrm{JHH}_{\mathrm{HH}}=2.3 \mathrm{~Hz}, 1 \mathrm{H}, \mathrm{HC} 10\right), 4.76-4.62\left(\mathrm{~m}, 1 \mathrm{H}, H_{\mathrm{eq}} \mathrm{C} 5\right), 3.78\left(\mathrm{~s}, 6 \mathrm{H}, \mathrm{H}_{3} \mathrm{C} 11\right), 3.60$ (dd, $\left.{ }^{3}{ } \mathrm{HH}=11.0 \mathrm{~Hz},{ }^{3} \mathrm{HHH}=2.3 \mathrm{~Hz}, 1 \mathrm{H}, H_{\mathrm{ax}} \mathrm{C} 2\right), 3.41$ (ddd, ${ }^{2} \mathrm{~J}_{\mathrm{HH}}=14.1 \mathrm{~Hz},{ }^{3} \mathrm{~J}_{\mathrm{HH}}=2.6$

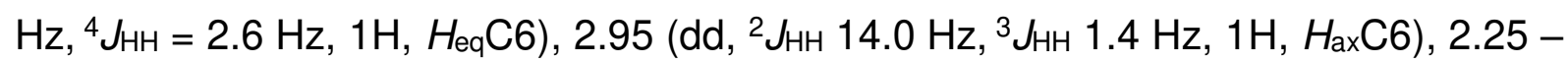
$2.18\left(\mathrm{~m}, 1 \mathrm{H}, \mathrm{H}_{2} \mathrm{C} 4\right), 1.96-1.70\left(\mathrm{~m}, 4 \mathrm{H}, \mathrm{H}_{2} \mathrm{C} 4, \mathrm{H}_{2} \mathrm{C} 3, \mathrm{H}_{2} \mathrm{C} 3, H \mathrm{~N} 1\right) .{ }^{13} \mathrm{C}\left\{{ }^{1} \mathrm{H}\right\}$ NMR $(151$ $\mathrm{MHz}$, Chloroform-d) $\delta 161.0$ (C9), 146.8 (C7), 104.5 (C8), 99.4 (C10), 86.0 (d, ${ }^{1} \mathrm{JFC}=$ $170.4 \mathrm{~Hz}, \mathrm{C}$ ), 60.8 (C2), $55.4(\mathrm{C} 11), 50.7\left(\mathrm{~d},{ }^{2} \mathrm{~J}_{\mathrm{FC}}=20.3 \mathrm{~Hz}, \mathrm{C} 6\right), 29.8\left(\mathrm{~d},{ }^{3} \mathrm{JFC}_{\mathrm{FC}}=21.6\right.$ $\mathrm{Hz}, \mathrm{C} 4), 28.7$ (C3). ${ }^{13} \mathbf{C}\left\{{ }^{1} \mathbf{H},{ }^{19} \mathrm{~F}\right\}$ NMR (151 MHz, Chloroform-d) $\delta 161.0$ (C9), 146.8 (C7), 104.5 (C8), 99.4 (C10), 86.0 (C5), 60.8 (C2), 55.4 (C11), 50.7 (C6), 29.8 (C4), 28.7 (C3). ${ }^{19} \mathrm{~F}$ NMR (564 MHz, Chloroform- $\left.d\right) \delta-188.2(\mathrm{~m}) .{ }^{19} \mathrm{~F}\left\{{ }^{1} \mathrm{H}\right\}$ NMR $(282 \mathrm{MHz}$, Chloroform- $d$ ) $\delta$-188.2. HRMS (ESI) $\mathrm{m} / \mathrm{z}$ calculated for $\left[\mathrm{C}_{13} \mathrm{H}_{19} \mathrm{FNO}_{2}\right]\left(\left[\mathrm{M}+\mathrm{H}^{+}\right]\right)$ 240.1394 , found 240.1415 . 


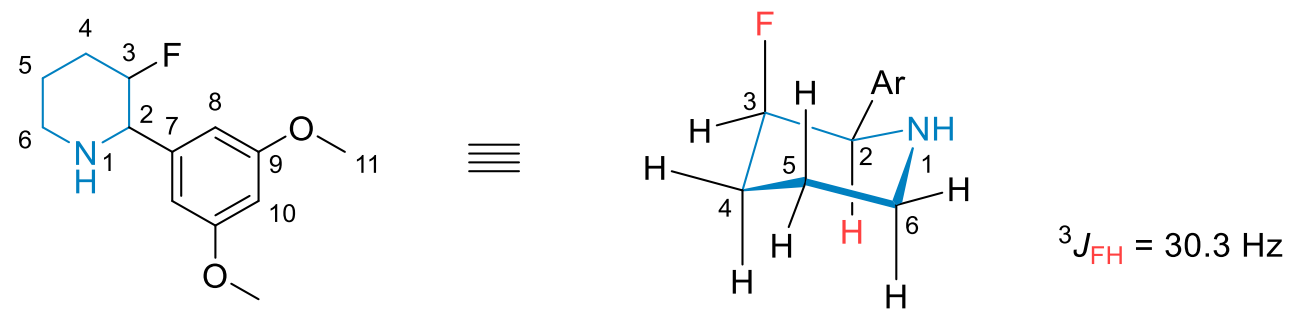

2-(3,5-Dimethoxyphenyl)-3-fluoropiperidine (16): The title compound was synthesized according to GP-A using $10 \mathrm{~mol} \%$ Pd-catalyst $(35.1 \mathrm{mg})$ in $\mathrm{THF} / \mathrm{H}_{2} \mathrm{O}$ $(0.5 \mathrm{M}, 1: 1)$ with aq. $\mathrm{HCl}(100 \mu \mathrm{l}, 2.4$ equiv, $12 \mathrm{~N})$. The product was isolated as a yellow oil (59 mg, $0.25 \mathrm{mmol}, 50 \%,>95: 5$ d.r.) after column chromatography (silical gel, eluent: $\mathrm{CH}_{2} \mathrm{Cl}_{2} / \mathrm{MeOH}$ 100:0, later 99:1, 97:3). The axial position of the fluorine substituent was determined based on the ${ }^{3} \mathrm{JFH}_{\mathrm{F}}$ coupling between $\mathrm{FC} 3$ and $\mathrm{HaxC}_{\mathrm{a}}$ and is in accordance with previous literature results. ${ }^{3}$

${ }^{1} \mathrm{H}$ NMR $(500 \mathrm{MHz}$, Chloroform- $d) \delta 6.57\left(\mathrm{~d},{ }^{4} \mathrm{JHH}=2.3 \mathrm{~Hz}, 2 \mathrm{H}, H C 8\right), 6.38\left(\mathrm{t},{ }^{4} \mathrm{HHH}=\right.$ $2.3 \mathrm{~Hz}, 1 \mathrm{H}, H \mathrm{C} 10), 4.73\left(\mathrm{dm},{ }^{2} \mathrm{JFH}_{\mathrm{FH}}=48.0 \mathrm{~Hz}, 1 \mathrm{H}, H_{\mathrm{eq}} \mathrm{C} 3\right), 3.78\left(\mathrm{~s}, 6 \mathrm{H}, H_{3} \mathrm{C} 11\right), 3.74-$ $3.63\left(\mathrm{dm},{ }^{3} \mathrm{JFH}_{\mathrm{FH}}=30.3 \mathrm{~Hz}, 1 \mathrm{H}, H_{\mathrm{axC}}\right), 3.25\left(\mathrm{dm},{ }^{2} \mathrm{JHH}_{\mathrm{HH}}=12.4 \mathrm{~Hz}, 1 \mathrm{H}, \mathrm{H}_{2} \mathrm{C} 6\right), 2.82(\mathrm{dm}$, $\left.{ }^{2} \mathrm{HHH}=12.6 \mathrm{~Hz}, 1 \mathrm{H}, \mathrm{H}_{2} \mathrm{C} 6\right), 2.28-2.17(\mathrm{~m}, 1 \mathrm{H}, H \mathrm{C} 4), 2.09(\mathrm{~s}, 1 \mathrm{H}, \mathrm{HN} 1), 1.89(\mathrm{~m}, 1 \mathrm{H}$, $\left.\mathrm{H}_{2} \mathrm{C} 5\right), 1.73(\mathrm{~m}, 1 \mathrm{H}, \mathrm{HC} 4), 1.53\left(\mathrm{dm},{ }^{2} \mathrm{JHH}_{\mathrm{HH}} 12.9 \mathrm{~Hz}, 1 \mathrm{H}, \mathrm{H}_{2} \mathrm{C} 5\right) .{ }^{1} \mathrm{H}\left\{{ }^{19} \mathrm{~F}\right\}$ NMR $(500$ $\mathrm{MHz}$, Chloroform-d) $\delta 6.57\left(\mathrm{~d},{ }^{4} \mathrm{JHH}=2.3 \mathrm{~Hz}, 2 \mathrm{H}, H C 8\right), 6.38\left(\mathrm{t},{ }^{4} \mathrm{JHH}_{\mathrm{HH}}=2.3 \mathrm{~Hz}, 1 \mathrm{H}\right.$, HC10), $4.73\left(\mathrm{~m}, 1 \mathrm{H}, H_{\mathrm{eq}} \mathrm{C} 3\right), 3.78\left(\mathrm{~s}, 6 \mathrm{H}, \mathrm{H}_{3} \mathrm{C} 11\right), 3.72-3.67\left(\mathrm{~m}, 1 \mathrm{H}, H_{\mathrm{ax}} \mathrm{C} 2\right), 3.25$ $\left(\mathrm{dm},{ }^{2} \mathrm{~J}_{\mathrm{HH}}=12.4 \mathrm{~Hz}, 1 \mathrm{H}, \mathrm{H}_{2} \mathrm{C} 6\right), 2.82\left(\mathrm{ddd},{ }^{2} \mathrm{JHH}_{\mathrm{HH}}=12.5 \mathrm{~Hz},{ }^{3} \mathrm{JHH}_{\mathrm{HH}}=2.9 \mathrm{~Hz},{ }^{3} \mathrm{JHH}_{\mathrm{HH}}=2.9\right.$ $\left.\mathrm{Hz}, 1 \mathrm{H}, \mathrm{H}_{2} \mathrm{C} 6\right), 2.22\left(\mathrm{dm},{ }^{2} \mathrm{JHH}_{\mathrm{HH}} 14.4 \mathrm{~Hz}, 1 \mathrm{H}, \mathrm{HC} 4\right), 2.09(\mathrm{~s}, 1 \mathrm{H}, H \mathrm{~N} 1), 1.89(\mathrm{~m}, 1 \mathrm{H}$, $\left.\mathrm{H}_{2} \mathrm{C} 5\right), 1.73(\mathrm{~m}, 1 \mathrm{H}, \mathrm{HC} 4), 1.53\left(\mathrm{dm},{ }^{2} \mathrm{JHH}_{\mathrm{H}}=13.4 \mathrm{~Hz}, 1 \mathrm{H}, \mathrm{H}_{2} \mathrm{C} 5\right) .{ }^{13} \mathrm{C}\left\{{ }^{1} \mathrm{H}\right\}$ NMR $(126$ $\mathrm{MHz}$, Chloroform-d) $\delta 160.9$ (C9), 143.3 (C7), 105.4 (C8), 99.8 (C10), 89.3 (d, ${ }^{1} \mathrm{JFC}=$ $178.1 \mathrm{~Hz}, \mathrm{C} 3), 63.8\left(\mathrm{~d},{ }^{2} \mathrm{JFC}=18.2 \mathrm{~Hz}, \mathrm{C} 2\right), 55.5(\mathrm{C} 11), 46.9$ (C6), $30.2\left(\mathrm{~d},{ }^{2} \mathrm{JFC}=21.9\right.$ $\mathrm{Hz}, \mathrm{C} 4), 20.4$ (C5). ${ }^{13} \mathrm{C}\left\{{ }^{1} \mathbf{H},{ }^{19} \mathrm{~F}\right\}$ NMR (126 MHz, Chloroform-d) $\delta 160.9$ (C9), 143.3 (C7), 105.4 (C8), 99.8 (C10), 89.3 (C3), 63.8 (C2), 55.5 (C11), 46.9 (C6), 30.2 (C4), 20.4 (C5). ${ }^{19} \mathrm{~F}$ NMR $\left(470 \mathrm{MHz}\right.$, Chloroform- $d$ ) $\delta-200.0$ (dddd, ${ }^{2} \mathrm{JFH}_{\mathrm{FH}}=48.2 \mathrm{~Hz},{ }^{3} \mathrm{JFH}_{\mathrm{FH}}=$ $\left.44.0 \mathrm{~Hz},{ }^{3} \mathrm{JFH}_{\mathrm{FH}}=30.1 \mathrm{~Hz},{ }^{3} \mathrm{JFH}_{\mathrm{FH}}=9.3 \mathrm{~Hz}\right) .{ }^{19} \mathrm{~F}\left\{{ }^{1} \mathrm{H}\right\} \mathbf{N M R}(470 \mathrm{MHz}$, Chloroform- $d) \delta=-$ 200.0. HRMS (ESI) $\mathrm{m} / \mathrm{z}$ calculated for $\left[\mathrm{C}_{13} \mathrm{H}_{19} \mathrm{NO}_{2} \mathrm{~F}\right]\left(\left[\mathrm{M}+\mathrm{H}^{+}\right]\right) 240.1394$, found 240.1410 . 

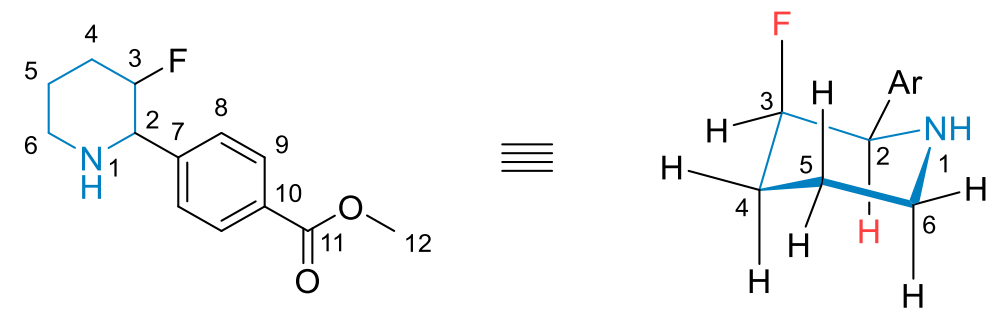

$$
{ }^{3} J_{\mathrm{FH}}=30.1 \mathrm{~Hz}
$$

Methyl 4-(3-fluoropiperidin-2-yl)benzoate (17): The title compound was synthesized according to GP-A using 10 mol\% Pd-catalyst $(35.1 \mathrm{mg})$ in $\mathrm{THF} / \mathrm{H}_{2} \mathrm{O}(0.5 \mathrm{M}, 1: 1)$ with conc. $\mathrm{H}_{2} \mathrm{SO}_{4}(32 \mu \mathrm{l}, 1.2$ equiv). The product was isolated as an yellow oil (31 mg, $0.13 \mathrm{mmol}, 26 \%$, >95:5 d.r.) after column chromatography (silical gel, eluent: $\mathrm{CH}_{2} \mathrm{Cl}_{2} / \mathrm{MeOH}$ 100:0, later 99:1, 98:2). Only the major diastereomer was isolated. The axial position of the fluorine substituent was determined based on the ${ }^{3} J_{F H}$ coupling between $\mathrm{FC} 3$ and $\mathrm{Hax}_{\mathrm{ax}} \mathrm{C}$ and is in accordance with previous literature results. ${ }^{3}$

${ }^{1} \mathrm{H}$ NMR (599 MHz, Chloroform- $\left.d\right) \delta=8.03-7.99$ (m, 2H, HC9), 7.47 (m, 2H, HC8), $4.75\left(\mathrm{dm},{ }^{2} \mathrm{~J}_{\mathrm{FH}}=48.3 \mathrm{~Hz}, 1 \mathrm{H}, H_{\mathrm{eqC}} \mathrm{C}\right), 3.90\left(\mathrm{~s}, 3 \mathrm{H}, H_{3} \mathrm{C} 12\right), 3.83\left(\mathrm{~d},{ }^{3} \mathrm{JFH}_{\mathrm{FH}}=30.1 \mathrm{~Hz}, 1 \mathrm{H}\right.$, $\left.H_{\mathrm{ax}} \mathrm{C}\right), 3.28\left(\mathrm{dm},{ }^{2} \mathrm{JHH}_{\mathrm{HH}} 12.6 \mathrm{~Hz}, 1 \mathrm{H}, \mathrm{H}_{2} \mathrm{C} 6\right), 2.85\left(\mathrm{dm},{ }^{2} \mathrm{JHH}_{\mathrm{HH}}=12.7 \mathrm{~Hz}, 1 \mathrm{H}, \mathrm{H}_{2} \mathrm{C} 6\right)$, $2.29-2.20\left(\mathrm{~m}, 1 \mathrm{H}, \mathrm{H}_{2} \mathrm{C} 4\right), 2.14-2.00(\mathrm{~m}, 1 \mathrm{H}, H \mathrm{~N} 1), 1.89\left(\mathrm{dm},{ }^{2} \mathrm{~J}_{\mathrm{HH}}=12.3 \mathrm{~Hz}, 1 \mathrm{H}\right.$, $\left.H_{2} \mathrm{C} 5\right), 1.77\left(\mathrm{ddm},{ }^{3} J_{\mathrm{FH}}=44.5 \mathrm{~Hz},{ }^{2} J_{\mathrm{HH}}=13.9 \mathrm{~Hz}, \mathrm{H}_{2} \mathrm{C} 4\right), 1.56\left(\mathrm{dm},{ }^{2} J_{\mathrm{HH}}=13.1 \mathrm{~Hz}\right.$, $\left.1 \mathrm{H}, \mathrm{H}_{2} \mathrm{C} 5\right) .{ }^{1} \mathrm{H}\left\{{ }^{19} \mathrm{~F}\right\}$ NMR (599 MHz, Chloroform- $\left.d\right) \delta=8.02-7.99(\mathrm{~m}, 2 \mathrm{H}, \mathrm{HC} 9), 7.49$ - $7.46(\mathrm{~m}, 2 \mathrm{H}, \mathrm{HC} 8), 4.78-4.73\left(\mathrm{~m}, 1 \mathrm{H}, \mathrm{H}_{\mathrm{eq}} \mathrm{C} 3\right), 3.90\left(\mathrm{~s}, 3 \mathrm{H}, \mathrm{H}_{3} \mathrm{C} 12\right), 3.83(\mathrm{~s}, 1 \mathrm{H}$, $\left.H_{\mathrm{ax}} \mathrm{C} 2\right), 3.28\left(\mathrm{dm},{ }^{2} \mathrm{JHH}_{\mathrm{HH}}=12.6 \mathrm{~Hz}, 1 \mathrm{H}, \mathrm{H}_{2} \mathrm{C} 6\right), 2.85\left(\mathrm{dm},{ }^{2} \mathrm{JHH}_{\mathrm{HH}}=12.6 \mathrm{~Hz}, 1 \mathrm{H}, H_{2} \mathrm{C} 6\right)$, $2.25\left(\mathrm{dm},{ }^{2} \mathrm{JHH}_{\mathrm{HH}}=14.4 \mathrm{~Hz}, 1 \mathrm{H}, \mathrm{H}_{2} \mathrm{C} 4\right), 2.11-2.00(\mathrm{~m}, 1 \mathrm{H}, H \mathrm{~N} 1), 1.89\left(\mathrm{dm},{ }^{2} \mathrm{JHH}_{\mathrm{HH}}=\right.$ $\left.13.1 \mathrm{~Hz}, 1 \mathrm{H}, \mathrm{H}_{2} \mathrm{C} 5\right), 1.77\left(\mathrm{dm},{ }^{2} J_{\mathrm{HH}}=13.9 \mathrm{~Hz}, 1 \mathrm{H}, \mathrm{H}_{2} \mathrm{C} 4\right), 1.56\left(\mathrm{dm},{ }^{2} J_{\mathrm{HH}}=12.8 \mathrm{~Hz}\right.$, $\left.1 \mathrm{H}, \mathrm{H}_{2} \mathrm{C} 5\right) .{ }^{13} \mathrm{C}\left\{{ }^{1} \mathrm{H}\right\}$ NMR $(151 \mathrm{MHz}$, Chloroform- $d) \delta=167.1$ (C11), 146.0 (C10), 129.8 (C9), 129.4 (C7), 127.4 (C8), 88.9 (d, $\left.{ }^{1} J_{F C}=178.0 \mathrm{~Hz}, \mathrm{C} 3\right), 63.4$ (d, ${ }^{2} \mathrm{~J}_{\mathrm{FC}}=18.5 \mathrm{~Hz}$, C2), 52.2 (C12), 46.8 (C6), 30.1 (d, $\left.{ }^{3} \mathrm{JFC}_{\mathrm{FC}} 21.7 \mathrm{~Hz}, \mathrm{C} 4\right), 20.4(\mathrm{C} 5) .{ }^{13} \mathrm{C}\left\{{ }^{1} \mathbf{H},{ }^{19} \mathrm{~F}\right\}$ NMR (151 MHz, Chloroform- $d$ ) $\delta=167.1$ (C11), 146.0 (C10), 129.8 (C9), 129.4 (C7), 127.4 (C8), 88.9 (C3), 63.4 (C2), 52.2 (C12), 46.8 (C6), 30.1 (C4), 20.4 (C5). ${ }^{19} \mathrm{~F}$ NMR (564 MHz, Chloroform- $d$ ) $\delta=-200.8$ (dddd, $\mathrm{J}=48.3,44.3,30.0,9.4 \mathrm{~Hz}) .{ }^{19} \mathrm{~F}\left\{{ }^{1} \mathrm{H}\right\} \mathbf{N M R}$ $(564 \mathrm{MHz}$, Chloroform- $d$ ) $\delta$ (ppm) $=-200.8$ (s). HRMS (ESI) m/z calculated for $\left[\mathrm{C}_{13} \mathrm{H}_{16} \mathrm{FNO}_{2} \mathrm{Na}\right]\left(\left[\mathrm{M}+\mathrm{Na}^{+}\right]\right) 260.1057$, found 260.1056 . 


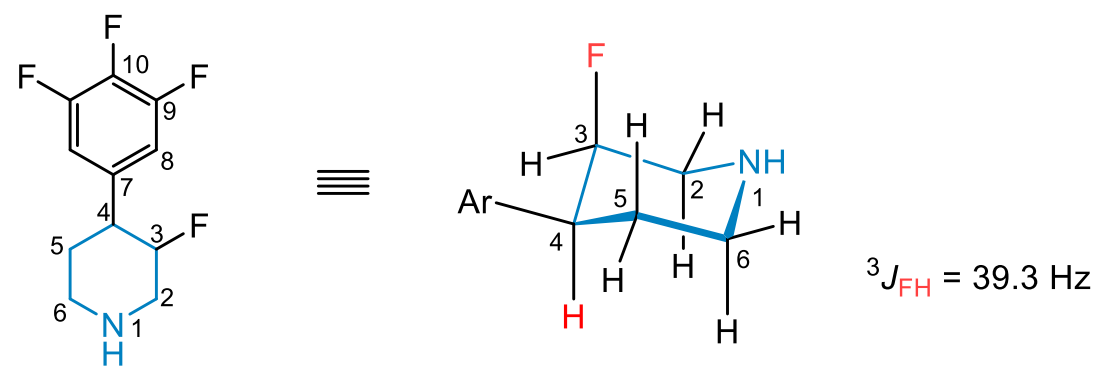

3-Fluoro-4-(3,4,5-trifluorophenyl)piperidine (18): The title compound was synthesized according to GP-A using $10 \mathrm{~mol} \%$ Pd-catalyst $(35.1 \mathrm{mg})$ in $\mathrm{THF} / \mathrm{H}_{2} \mathrm{O}$ ( $0.5 \mathrm{M}, 1: 1)$ with $\mathrm{H}_{2} \mathrm{SO}_{4}$ (32 $\mu \mathrm{l}, 1.2$ equiv). The product was isolated as a white solid (72 mg, $0.31 \mathrm{mmol}, 61 \%,>95: 5$ d.r.) after column chromatography (silical gel, eluent: $\mathrm{CH}_{2} \mathrm{Cl}_{2}$, later 99:1, 98:2, 95:5 $\mathrm{CH}_{2} \mathrm{Cl}_{2} / \mathrm{MeOH}$ ). Only the major diastereomer was isolated. The axial position of the fluorine substituent was determined based on the ${ }^{3} \mathrm{JFH}$ coupling between $\mathrm{FC} 3$ and $\mathrm{Hax}_{\mathrm{ax}} 4$ and is in accordance with previous literature results. ${ }^{3}$

${ }^{1} \mathrm{H}$ NMR $\left(599 \mathrm{MHz}\right.$, Chloroform-d) $\delta 6.95-6.87(\mathrm{~m}, 2 \mathrm{H}, \mathrm{HC} 8), 4.61\left(\mathrm{~d},{ }^{2} \mathrm{JFH}_{\mathrm{FH}}=48.8 \mathrm{~Hz}\right.$, $1 \mathrm{H}, \mathrm{H}_{\mathrm{eqC}}$ ), $3.41-3.33\left(\mathrm{~m}, 1 \mathrm{H}, \mathrm{H}_{2} \mathrm{C} 2\right), 3.24$ (dddd, ${ }^{2} \mathrm{JHH}=13.5 \mathrm{~Hz},{ }^{3} \mathrm{JHH}_{\mathrm{HH}}=3.8 \mathrm{~Hz},{ }^{3} \mathrm{JHH}_{\mathrm{HH}}$ $=1.7 \mathrm{~Hz},{ }^{4} \mathrm{JHH}_{\mathrm{HH}}=1.7 \mathrm{~Hz}, 1 \mathrm{H}, \mathrm{H}_{2} \mathrm{C} 6$ ), 2.87 (ddd, ${ }^{3} \mathrm{FH}=39.3 \mathrm{~Hz},{ }^{2}{ }_{\mathrm{HHH}}=14.6 \mathrm{~Hz},{ }^{3} \mathrm{JHH}_{\mathrm{HH}}=$ $\left.1.1 \mathrm{~Hz}, 1 \mathrm{H}, H_{\mathrm{ax}} \mathrm{C} 4\right), 2.80-2.70$ (m, 2H, $\mathrm{H}_{2} \mathrm{C} 6, H_{2} \mathrm{C} 2$ ), 2.30 (s, 1H, HN1), 1.99 (tdd, $\left.{ }^{3} \mathrm{JHH}_{\mathrm{H}}=12.9 \mathrm{~Hz},{ }^{2} \mathrm{JHH}_{\mathrm{HH}} 12.9 \mathrm{~Hz},{ }^{3} \mathrm{HH}=4.2 \mathrm{~Hz}, 1 \mathrm{H}, \mathrm{H}_{2} \mathrm{C} 5\right), 1.69-1.63\left(\mathrm{dm},{ }^{2} \mathrm{~J}_{\mathrm{HH}}=\right.$ $\left.12.9 \mathrm{~Hz}, 1 \mathrm{H}, \mathrm{H}_{2} \mathrm{C} 5\right) .{ }^{1} \mathrm{H}\left\{{ }^{19} \mathrm{~F}\right\}$ NMR $(599 \mathrm{MHz}$, Chloroform-d) $\delta 6.91$ (s, 2H, HC8), 4.61 (s, $1 \mathrm{H}, H_{\mathrm{eqC}}$ ), $3.37\left(\mathrm{dd},{ }^{2} \mathrm{JHH}_{\mathrm{HH}}=14.4,{ }^{3} \mathrm{JHH}=1.6 \mathrm{~Hz}, 1 \mathrm{H}, \mathrm{H}_{2} \mathrm{C} 2\right), 3.24$ (dddd, ${ }^{2} \mathrm{JHH}=$ $\left.13.5 \mathrm{~Hz},{ }^{3} \mathrm{~J}_{\mathrm{HH}}=3.8 \mathrm{~Hz},{ }^{3} \mathrm{JHH}_{\mathrm{HH}}=1.7 \mathrm{~Hz},{ }^{4} \mathrm{~J}_{\mathrm{HH}}=1.7 \mathrm{~Hz}, 1 \mathrm{H}, \mathrm{H}_{2} \mathrm{C} 6\right), 2.87\left(\mathrm{~d},{ }^{2} \mathrm{JHH}_{\mathrm{HH}}=14.4\right.$ $\left.\mathrm{Hz}, 1 \mathrm{H}, H_{\mathrm{ax}} \mathrm{C} 4\right), 2.80-2.72$ (m, 2H, $\left.H_{2} \mathrm{C} 6, H_{2} \mathrm{C} 2\right), 2.30$ (s, $1 \mathrm{H}, H \mathrm{NN} 1$ ), 1.99 (tdd, ${ }^{3} \mathrm{HHH}_{\mathrm{HH}}=$ $\left.12.9 \mathrm{~Hz},{ }^{2} \mathrm{JHH}=12.9 \mathrm{~Hz},{ }^{3} \mathrm{JHH}_{\mathrm{HH}} 4.2 \mathrm{~Hz}, 1 \mathrm{H}, \mathrm{H}_{2} \mathrm{C} 5\right), 1.69-1.63\left(\mathrm{dm},{ }^{2} \mathrm{JHH}=12.9 \mathrm{~Hz}, 1 \mathrm{H}\right.$, $\left.\mathrm{H}_{2} \mathrm{C} 5\right) \cdot{ }^{13} \mathrm{C}\left\{{ }^{1} \mathrm{H}\right\}$ NMR $\left(151 \mathrm{MHz}\right.$, Chloroform-d) $\delta 151.2\left(\mathrm{ddd},{ }^{1} \mathrm{JFC}_{\mathrm{FC}}=249.3 \mathrm{~Hz},{ }^{2} \mathrm{JFC}=\right.$ $\left.9.8 \mathrm{~Hz},{ }^{3} \mathrm{JFC}_{\mathrm{FC}} 4.1 \mathrm{~Hz}, \mathrm{C} 9\right), 138.8\left(\mathrm{dt},{ }^{1} \mathrm{JFC}_{\mathrm{FC}}=250.3 \mathrm{~Hz},{ }^{2} \mathrm{JFC}=15.3 \mathrm{~Hz}, \mathrm{C} 10\right), 138.5(\mathrm{td}$, $\left.{ }^{3} \mathrm{JFC}=7.0 \mathrm{~Hz},{ }^{4} \mathrm{JFC}_{\mathrm{FC}}=4.5 \mathrm{~Hz}, \mathrm{C} 7\right), 112.0\left(\mathrm{ddd},{ }^{2} \mathrm{JFC}=16.8 \mathrm{~Hz},{ }^{3} \mathrm{JFC}=4.4 \mathrm{~Hz},{ }^{4} \mathrm{JFC}_{\mathrm{FC}}=2.3\right.$ $\mathrm{Hz}, \mathrm{C} 8), 89.0\left(\mathrm{~d},{ }^{1} \mathrm{JFC}=175.8 \mathrm{~Hz}, \mathrm{C} 3\right), 50.4\left(\mathrm{~d},{ }^{2} \mathrm{JFC}=21.2 \mathrm{~Hz}, \mathrm{C} 4\right), 45.8(\mathrm{C} 6), 45.3$ (d, $\left.{ }^{2} \mathrm{JFC}=19.7 \mathrm{~Hz}, \mathrm{C} 2\right), 27.7$ (C5). ${ }^{13} \mathrm{C}\left\{{ }^{1} \mathrm{H}, \mathbf{s e l}-{ }^{19} \mathrm{~F}\right.$ at -201 ppm $\}$ NMR $(151 \mathrm{MHz}$, Chloroform- $d$ ) $\delta 151.2\left(\mathrm{ddd},{ }^{1} \mathrm{JFC}=249.1 \mathrm{~Hz},{ }^{2} \mathrm{JFC}=9.8 \mathrm{~Hz},{ }^{3} \mathrm{JFC}=4.1 \mathrm{~Hz}, \mathrm{C9}\right), 138.8$ $\left(\mathrm{dt},{ }^{1} \mathrm{JFC}=249.7 \mathrm{~Hz},{ }^{2} \mathrm{JFC}=15.3 \mathrm{~Hz}, \mathrm{C} 10\right), 138.5\left(\mathrm{td},{ }^{3} \mathrm{JFC}=7.0 \mathrm{~Hz},{ }^{4} \mathrm{JFC}=4.5 \mathrm{~Hz}, \mathrm{C} 7\right)$, $112.0\left(\mathrm{dd},{ }^{2} \mathrm{JFC}=16.8 \mathrm{~Hz},{ }^{3} \mathrm{JFC}=4.3 \mathrm{~Hz}, \mathrm{C} 8\right), 89.0$ (C5), 50.4 (C4), 45.8 (C6)ff, 45.3 (C2), 27.7 (C5). ${ }^{19} \mathrm{~F}$ NMR (564 MHz, Chloroform- $d$ ) $\delta-134.7--134.8$ (m, 2F, FC9), $163.2\left(\mathrm{tt},{ }^{3} \mathrm{JFF}_{\mathrm{FF}}=20.6 \mathrm{~Hz},{ }^{4} \mathrm{JHF}=6.5 \mathrm{~Hz}, 1 \mathrm{~F}, \mathrm{FC} 10\right),-200.7$ (dddd, ${ }^{2} \mathrm{JHF}_{\mathrm{HF}}=49.5 \mathrm{~Hz},{ }^{3} \mathrm{JHF}_{\mathrm{HF}}$ 
$\left.39.1 \mathrm{~Hz},{ }^{3} \mathrm{JHF}_{\mathrm{HF}}=35.1 \mathrm{~Hz},{ }^{3} \mathrm{JHF}_{\mathrm{HF}}=10.8 \mathrm{~Hz}, 1 \mathrm{~F}, F C 3\right) .{ }^{19} \mathrm{~F}\left\{{ }^{1} \mathrm{H}\right\} \mathrm{NMR}(564 \mathrm{MHz}$, Chloroformd) $\delta-134.7\left(\mathrm{~d},{ }^{3} \mathrm{JFF}=20.6 \mathrm{~Hz}, 2 \mathrm{~F}, F C 9\right),-163.2\left(\mathrm{t},{ }^{3} \mathrm{JFF}=20.5 \mathrm{~Hz}, 1 \mathrm{~F}, F C 10\right),-200.7(\mathrm{~s}$, $1 \mathrm{~F}, \mathrm{FC} 3)$. HRMS $(\mathrm{ESI}) \mathrm{m} / \mathrm{z}$ calculated for $\left[\mathrm{C}_{11} \mathrm{H}_{12} \mathrm{~F}_{4} \mathrm{~N}\right]\left(\left[\mathrm{M}+\mathrm{H}^{+}\right]\right) 234.0900$, found 234.0924.<smiles>FC1CNCCC1=C1CCC(C(F)(F)F)CC1</smiles>

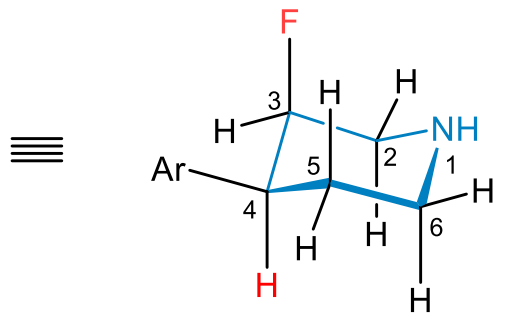

${ }^{3} J_{F H}=37.5 \mathrm{~Hz}$

3-Fluoro-4-(4-(trifluoromethyl)phenyl)piperidine (19): The title compound was synthesized according to GP-A using $10 \mathrm{~mol} \% \mathrm{Pd}$-catalyst $(35.1 \mathrm{mg})$ in $\mathrm{THF} / \mathrm{H}_{2} \mathrm{O}$ ( $0.5 \mathrm{M}, 1: 1)$ with $\mathrm{H}_{2} \mathrm{SO}_{4}$ (32 $\mu \mathrm{l}, 1.2$ equiv). The product was isolated as a white solid (45 mg, $0.18 \mathrm{mmol}, 37 \%,>95: 5 \mathrm{~d} . \mathrm{r}$ )) after column chromatography (silical gel, eluent: $\mathrm{CH}_{2} \mathrm{Cl}_{2}$, later 99:1, 98:2, 95:5 $\mathrm{CH}_{2} \mathrm{Cl}_{2} / \mathrm{MeOH}$ ). Only the major diastereomer was isolated. The axial position of the fluorine substituent was determined based on the ${ }^{3} \mathrm{FH}$ coupling between $\mathrm{FC} 3$ and $\mathrm{HaxC}_{\mathrm{ax}}$ and is in accordance with previous literature results. $^{3}$

${ }^{1} \mathrm{H}$ NMR $(500 \mathrm{MHz}$, Chloroform- $d) \delta 7.58\left(\mathrm{~d},{ }^{3} \mathrm{JHH}_{\mathrm{HH}}=8.0 \mathrm{~Hz}, 2 \mathrm{H}, \mathrm{HC}\right), 7.41\left(\mathrm{~d},{ }^{3} \mathrm{H}_{\mathrm{HH}}=\right.$ $8.0 \mathrm{~Hz}, 2 \mathrm{H}, H \mathrm{C} 8), 4.69\left(\mathrm{dm},{ }^{2} \mathrm{JFH}_{\mathrm{F}}=48.5 \mathrm{~Hz}, 1 \mathrm{H}, H_{\mathrm{eqC}}\right.$ ), $3.43\left(\mathrm{dd},{ }^{2} \mathrm{JHH}_{\mathrm{HH}}=12.7 \mathrm{~Hz},{ }^{3} \mathrm{JFH}_{\mathrm{FH}}\right.$ $\left.=12.7 \mathrm{~Hz}, 1 \mathrm{H}, \mathrm{H}_{2} \mathrm{C} 2\right), 3.35-3.15\left(\mathrm{~m}, 2 \mathrm{H}, H_{2} \mathrm{C} 6, H \mathrm{~N} 1\right), 3.05-2.79$ (m, 3H, $\mathrm{H}_{2} \mathrm{C} 2, H \mathrm{C} 4$, $\left.\mathrm{H}_{2} \mathrm{C} 6\right), 2.16\left(\mathrm{dtd},{ }^{2} \mathrm{JHH}_{\mathrm{HH}}=13.0 \mathrm{~Hz},{ }^{3} \mathrm{JHH}_{\mathrm{HH}}=13.0 \mathrm{~Hz},{ }^{3} \mathrm{JHH}=4.0 \mathrm{~Hz}, 1 \mathrm{H}, \mathrm{H}_{2} \mathrm{C} 5\right), 1.73(\mathrm{dm}$, $\left.{ }^{2} \mathrm{JHH}_{\mathrm{HH}}=13.2 \mathrm{~Hz}, 1 \mathrm{H}, \mathrm{H}_{2} \mathrm{C} 5\right) .{ }^{1} \mathrm{H}\left\{{ }^{19} \mathrm{~F}\right\}$ NMR $(500 \mathrm{MHz}$, Chloroform- $d) \delta 7.58\left(\mathrm{~d},{ }^{3} \mathrm{JHH}=8.1\right.$ $\mathrm{Hz}, 2 \mathrm{H}, H C 9), 7.41\left(\mathrm{~d},{ }^{3} \mathrm{JH}=8.1 \mathrm{~Hz}, 2 \mathrm{H}, H \mathrm{C} 8\right), 4.69\left(\mathrm{~m}, 1 \mathrm{H}, H_{\mathrm{eq}} \mathrm{C} 3\right), 3.43\left(\mathrm{~d},{ }^{2}{ }_{\mathrm{HH}}=\right.$ $\left.13.0 \mathrm{~Hz}, 1 \mathrm{H}, \mathrm{H}_{2} \mathrm{C} 2\right)$, $3.35-3.16\left(\mathrm{~m}, 2 \mathrm{H}, \mathrm{H}_{2} \mathrm{C} 6, H \mathrm{~N} 1\right)$, $3.03-2.87$ (m, 2H, $\left.\mathrm{H}_{2} \mathrm{C} 2, \mathrm{HC} 4\right)$, $2.84\left(\mathrm{dd},{ }^{2} \mathrm{JHH}=13.1 \mathrm{~Hz},{ }^{3} \mathrm{JHH}_{\mathrm{HH}}=13.1 \mathrm{~Hz}, 1 \mathrm{H}, \mathrm{H}_{2} \mathrm{C} 6\right), 2.16\left(\mathrm{dtd},{ }^{2} \mathrm{JHH}_{\mathrm{HH}}=13.0 \mathrm{~Hz},{ }^{3} \mathrm{JHH}_{\mathrm{HH}}\right.$ $\left.=13.0 \mathrm{~Hz},{ }^{3} \mathrm{JHH}_{\mathrm{HH}}=4.1 \mathrm{~Hz}, 1 \mathrm{H}, \mathrm{H}_{2} \mathrm{C} 5\right), 1.73\left(\mathrm{dm},{ }^{2} \mathrm{~J}_{\mathrm{HH}}=13.3 \mathrm{~Hz}, 1 \mathrm{H}, \mathrm{H}_{2} \mathrm{C} 5\right) .{ }^{13} \mathrm{C}\left\{{ }^{1} \mathrm{H}\right\} \mathbf{N M R}$ (126 MHz, Chloroform- $d$ ) $\delta 146.0(\mathrm{C} 7), 129.3\left(\mathrm{q},{ }^{2} \mathrm{JFC}=32.4 \mathrm{~Hz}, \mathrm{C} 10\right), 128.4$ (d, ${ }^{4} \mathrm{JFC}$ $=1.9 \mathrm{~Hz}, \mathrm{C} 8), 125.5\left(\mathrm{q},{ }^{3} \mathrm{JFC}=3.8 \mathrm{~Hz}, \mathrm{C} 9\right), 124.3\left(\mathrm{q},{ }^{1} \mathrm{JFC}=272.7 \mathrm{~Hz}, \mathrm{C} 11\right), 89.1$ (d, $\left.{ }^{1} \mathrm{JFC}_{\mathrm{FC}} 176.4 \mathrm{~Hz}, \mathrm{C} 3\right), 50.3\left(\mathrm{~d},{ }^{2} \mathrm{JFC}_{\mathrm{FC}}=21.8 \mathrm{~Hz}, \mathrm{C} 2\right), 45.9-45.8(\mathrm{~m}, \mathrm{C} 6), 45.7\left(\mathrm{~d},{ }^{2} \mathrm{JFC}=\right.$ $19.6 \mathrm{~Hz}, \mathrm{C} 4), 27.2(\mathrm{C} 5) .{ }^{13} \mathrm{C}\left\{{ }^{1} \mathbf{H}, \mathbf{s e l}-{ }^{19} \mathrm{~F}\right.$ at -200 ppm $\}$ NMR $(126 \mathrm{MHz}$, Chloroform- $d) \delta$ 146.0 (C7), 129.3 (q, ${ }^{2} \mathrm{JFC}=32.4 \mathrm{~Hz}, \mathrm{C} 10$ ), 128.4 (C8), 125.5 (q, ${ }^{3} \mathrm{FFC}=3.8 \mathrm{~Hz}, \mathrm{C} 9$ ), 124.3 (q, ${ }^{1} \mathrm{JFC}=271.7 \mathrm{~Hz}, \mathrm{C} 11$ ), 89.1 (C3), 50.3 (C2), 45.8 (C6), 45.7 (C4), 27.2 (C5). 
${ }^{19} \mathrm{~F}$ NMR $(470 \mathrm{MHz}$, Chloroform- $d) \delta-62.5\left(\mathrm{~s}, 3 \mathrm{~F}, F_{3} \mathrm{C} 11\right),-200.3\left(\mathrm{dtd},{ }^{2} \mathrm{JHF}_{\mathrm{H}}=48.3 \mathrm{~Hz}\right.$, $\left.{ }^{3} \mathrm{JHF}=37.5 \mathrm{~Hz},{ }^{3} \mathrm{JHF}=10.7 \mathrm{~Hz}, 1 \mathrm{~F}, F C 3\right) .{ }^{19} \mathrm{~F}\left\{{ }^{1} \mathrm{H}\right\}$ NMR $(470 \mathrm{MHz}$, Chloroform- $d) \delta-$ 62.5 (3F, $\left.F_{3} \mathrm{C} 11\right)$, -200.3 (1F, FC11).

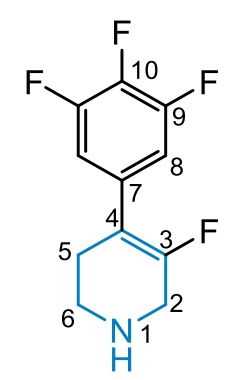

5-Fluoro-4-(3,4,5-trifluorophenyl)-1,2,3,6-tetrahydropyridine (20): The title compound was synthesized according to GP-A using $10 \mathrm{~mol} \%$ Pd-catalyst (35.1 mg) $\mathrm{MeOH}(0.5 \mathrm{M})$ with $\mathrm{H}_{2} \mathrm{SO}_{4}(32 \mu \mathrm{l}, 1.2$ equiv). The product was isolated as a white solid (40 mg, $0.17 \mathrm{mmol}, 34 \%$ ) after column chromatography (silical gel, eluent: $\mathrm{CH}_{2} \mathrm{Cl}_{2}$, later 99:1, 98:2, 95:5 $\left.\mathrm{CH}_{2} \mathrm{Cl}_{2} / \mathrm{MeOH}\right)$.

${ }^{1} \mathrm{H}$ NMR $(500 \mathrm{MHz}$, Chloroform-d) $\delta 7.10-7.03(\mathrm{~m}, 2 \mathrm{H}, \mathrm{HC}), 3.55-3.51(\mathrm{~m}, 2 \mathrm{H}$, $\left.\mathrm{H}_{2} \mathrm{C} 2\right), 3.07-3.01$ (m, 2H, $\left.\mathrm{H}_{2} \mathrm{C} 6\right), 2.43-2.37$ (m, 2H, $\left.\mathrm{H}_{2} \mathrm{C} 5\right), 1.96$ (s, 1H, HN1). ${ }^{1} \mathrm{H}\left\{{ }^{19} \mathrm{~F}\right\}$ NMR $(500 \mathrm{MHz}$, Chloroform- $d) \delta 7.07(\mathrm{~s}, 2 \mathrm{H}, \mathrm{HC} 8), 3.56-3.50\left(\mathrm{~m}, 2 \mathrm{H}, \mathrm{H}_{2} \mathrm{C} 2\right)$, $3.04\left(\mathrm{t},{ }^{3} \mathrm{JHH}=5.6 \mathrm{~Hz}, 2 \mathrm{H}, \mathrm{H}_{2} \mathrm{C} 6\right), 2.42-2.37\left(\mathrm{~m}, 2 \mathrm{H}, \mathrm{H}_{2} \mathrm{C} 5\right), 1.95(\mathrm{~s}, 1 \mathrm{H}, H \mathrm{~N} 1) .{ }^{13} \mathrm{C}\left\{{ }^{1} \mathrm{H}\right\}$ NMR $(126 \mathrm{MHz}$, Chloroform- $d) \delta 156.1$ (d, $\left.{ }^{1} \mathrm{JFC}_{=}=267.9 \mathrm{~Hz}, \mathrm{C} 3\right), 151.1$ (ddd, ${ }^{1} \mathrm{JFC}=$ $\left.248.3 \mathrm{~Hz},{ }^{2} \mathrm{JFC}=9.9 \mathrm{~Hz},{ }^{3} \mathrm{JFC}=4.5 \mathrm{~Hz}, \mathrm{C} 9\right), 138.7\left(\mathrm{dm},{ }^{1} \mathrm{JFC}=251.6 \mathrm{~Hz}, \mathrm{C} 10\right), 132.7-$ $131.8(\mathrm{~m}, \mathrm{C} 7), 111.7\left(\mathrm{dt},{ }^{2} \mathrm{JFC}=17.1 \mathrm{~Hz},{ }^{3} \mathrm{JFC}=5.9 \mathrm{~Hz}, \mathrm{C} 8\right), 110.1-109.8(\mathrm{~m}, \mathrm{C} 4)$, $44.9\left(\mathrm{~d},{ }^{2} \mathrm{JFC}_{\mathrm{FC}}=31.4 \mathrm{~Hz}, \mathrm{C} 2\right), 43.1(\mathrm{C} 6), 28.4\left(\mathrm{~d},{ }^{3} \mathrm{JFC}=2.4 \mathrm{~Hz}, \mathrm{C} 5\right) .{ }^{13} \mathrm{C}\left\{{ }^{1} \mathrm{H}, \mathbf{s e l}-{ }^{19} \mathrm{~F}\right.$ at 111 ppm\} NMR (126 MHz, Chloroform- $d$ ) $\delta 156.1$ (C3), 152.1 - 149.9 (m, C9), 138.6 (dt, $\left.{ }^{1} \mathrm{JFC}=250.3 \mathrm{~Hz},{ }^{2} \mathrm{JFC}_{\mathrm{FC}}=13.4 \mathrm{~Hz}, \mathrm{C} 10\right), 132.3-132.0(\mathrm{~m}, \mathrm{C} 7), 111.8-111.5(\mathrm{~m}$, C8), 110.0 - 109.9 (m, C4), 44.9 (C2), 43.1 (C6), 28.3 (C5). ${ }^{19} \mathrm{~F}$ NMR (470 MHz, Chloroform- $d$ ) $\delta-110.5\left(\mathrm{t},{ }^{3} \mathrm{JHF}=6.0 \mathrm{~Hz}, 1 \mathrm{~F}, F C 3\right),-135.1--135.3(\mathrm{~m}, 2 \mathrm{~F}, F C 9),-162.3$ $\left(\mathrm{tt},{ }^{3} J_{\mathrm{FF}}=20.7 \mathrm{~Hz},{ }^{3} \mathrm{JFF}_{\mathrm{H}}=6.6 \mathrm{~Hz}, 1 \mathrm{~F}, F \mathrm{C} 10\right) .{ }^{19} \mathrm{~F}\left\{{ }^{1} \mathrm{H}\right\} \mathrm{NMR}(470 \mathrm{MHz}$, Chloroform- $d) \delta$ $-110.5\left(\mathrm{~s}, 1 \mathrm{~F}, \mathrm{FC} 3,-135.2\left(\mathrm{~d},{ }^{3} \mathrm{JFF}=20.8 \mathrm{~Hz}, 2 \mathrm{~F}, F C 9\right),-162.3\left(\mathrm{t},{ }^{3} \mathrm{JFF}=20.8 \mathrm{~Hz}, 1 \mathrm{~F}\right.\right.$, FC10). HRMS (ESI) $\mathrm{m} / \mathrm{z}$ calculated for $\left[\mathrm{C}_{11} \mathrm{H}_{10} \mathrm{~F}_{4} \mathrm{~N}\right]\left(\left[\mathrm{M}+\mathrm{H}^{+}\right]\right) 232.0744$, found 232.0763 . 

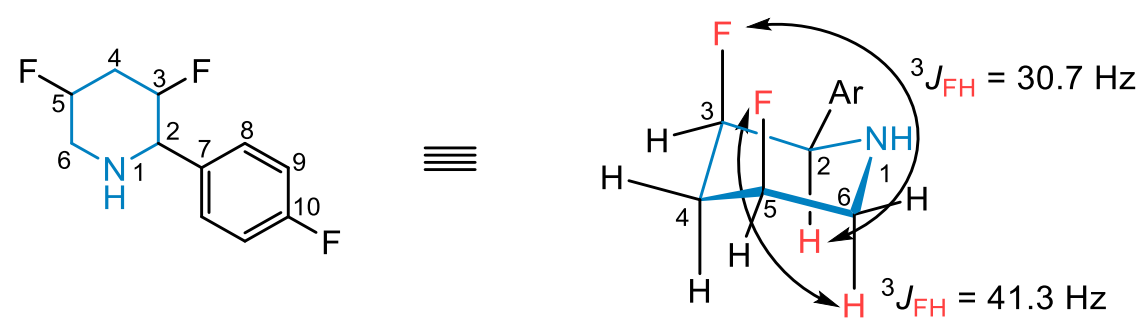

3,5-Difluoro-2-(4-fluorophenyl)piperidine (21): The title compound was synthesized according to GP-A using $10 \mathrm{~mol} \%$ Pd-catalyst $(35.1 \mathrm{mg})$ in $\mathrm{THF} / \mathrm{H}_{2} \mathrm{O}(0.5 \mathrm{M}, 1: 1)$ with $\mathrm{H}_{2} \mathrm{SO}_{4}$ (32 $\mu \mathrm{l}, 1.2$ equiv). The reaction time was increased to $72 \mathrm{~h}$. The product was isolated as a white solid ( $55 \mathrm{mg}, 0.25 \mathrm{mmol}, 51 \%, 95: 5:<5$ d.r.) after column chromatography (silical gel, eluent: $\mathrm{CH}_{2} \mathrm{Cl}_{2}$, later 99:1, 98:2, 95:5 $\mathrm{CH}_{2} \mathrm{Cl}_{2} / \mathrm{MeOH}$ ). Only two diastereomers were observed and the product was isolated as a mixture of diastereomers. The main diastereomer is the all-cis product with both fluorine atoms occupying the axial position on the piperidine. The axial position of the fluorine substituents was determined based on the ${ }^{3} \mathrm{~J}_{\mathrm{FH}}$ coupling between $\mathrm{FC} 5$ and $\mathrm{H}_{\mathrm{ax}} \mathrm{C} 6$ and FC3 and $H_{a x C 2}$, respectively.

${ }^{1} \mathrm{H}$ NMR $(599 \mathrm{MHz}$, Chloroform-d) $\delta 7.39-7.34$ (m, 2H, HC8), $7.07-7.01$ (m, 2H, HC9), $4.79\left(\mathrm{dm},{ }^{2} \mathrm{JFH}_{\mathrm{FH}}=47.5 \mathrm{~Hz}, 1 \mathrm{H}, H_{\mathrm{eq}} \mathrm{C} 3\right), 4.68\left(\mathrm{dm},{ }^{2} \mathrm{JFH}_{\mathrm{FH}}=47.2 \mathrm{~Hz}, 1 \mathrm{H}, H_{\mathrm{eq}} \mathrm{C} 5\right)$, $3.77\left(\mathrm{~d},{ }^{3} \mathrm{JFH}_{\mathrm{FH}}=30.6 \mathrm{~Hz}, 1 \mathrm{H}, \mathrm{Hax}_{\mathrm{ax}}\right.$ ), 3.51 (dddd, ${ }^{2} \mathrm{~J}_{\mathrm{HH}}=14.5 \mathrm{~Hz},{ }^{3} \mathrm{JFH}_{\mathrm{FH}}=14.5 \mathrm{~Hz},{ }^{3} \mathrm{JHH}_{\mathrm{HH}}=$ $2.6 \mathrm{~Hz},{ }^{4} \mathrm{~J}_{\mathrm{FH}}=2.6 \mathrm{~Hz}, 1 \mathrm{H}, \mathrm{Heq}_{\mathrm{eq}}$ ), 3.01 (ddd, ${ }^{3} \mathrm{~J}_{\mathrm{FH}}=40.9 \mathrm{~Hz},{ }^{2} \mathrm{JHH}_{\mathrm{HH}}=14.9 \mathrm{~Hz},{ }^{3} \mathrm{~J}_{\mathrm{HH}}=1.6$ $\left.\mathrm{Hz}, 1 \mathrm{H}, H_{\mathrm{ax}} \mathrm{C} 6\right), 2.69-2.59$ (m, 1H, HeqC4), 2.06 (s, 1H, HN1), 1.99 (tdt, ${ }^{3} \mathrm{JFH}_{\mathrm{FH}}=44.4 \mathrm{~Hz}$, $\left.{ }^{2} \mathrm{JHH}_{\mathrm{HH}}=16.1,{ }^{3} \mathrm{JHH}=3.1 \mathrm{~Hz}, 1 \mathrm{H}, H_{\mathrm{ax}} \mathrm{C} 4\right) .{ }^{1} \mathrm{H}\left\{{ }^{19} \mathrm{~F}\right\} \mathrm{NMR}(599 \mathrm{MHz}$, Chloroform-d) $\delta 7.38$ - 7.35 (m, 2H, HC8), 7.05 - 7.02 (m, 2H, HC9), $4.80-4.77$ (m, 1H, HeqC3), 4.69 $4.66\left(\mathrm{~m}, 1 \mathrm{H}, H_{\mathrm{eqC}}\right.$ ), 3.77 (s, $1 \mathrm{H}, \mathrm{HaxC}_{2}$ ), 3.51 (ddd, ${ }^{2} \mathrm{JHH}=14.9 \mathrm{~Hz},{ }^{3} \mathrm{JHH}=2.5 \mathrm{~Hz},{ }^{4} \mathrm{JHH}$ $=2.5 \mathrm{~Hz}, 1 \mathrm{H}, H_{\mathrm{eq}} \mathrm{C}$ ), 3.01 (dd, $\left.{ }^{2} \mathrm{JHH}=15.0 \mathrm{~Hz},{ }^{3} \mathrm{JHH}=1.6 \mathrm{~Hz}, 1 \mathrm{H}, H_{\mathrm{ax}} \mathrm{C} 6\right), 2.64$ (dtd, $\left.{ }^{2} J_{\mathrm{HH}}=16.1 \mathrm{~Hz},{ }^{3} \mathrm{JHH}_{\mathrm{HH}}=2.8 \mathrm{~Hz},{ }^{4} \mathrm{JHH}_{\mathrm{HH}}=2.8 \mathrm{~Hz}, 1 \mathrm{H}, H_{\mathrm{eq}} \mathrm{C} 4\right), 2.06(\mathrm{~s}, 1 \mathrm{H}, H \mathrm{~N} 1), 1.99(\mathrm{dt}$, $\left.{ }^{2} \mathrm{HHH}=16.2 \mathrm{~Hz},{ }^{3} \mathrm{JHH}=3.1 \mathrm{~Hz}, 1 \mathrm{H}, H_{\mathrm{ax}} \mathrm{C} 4\right) .{ }^{13} \mathrm{C}\left\{{ }^{1} \mathrm{H}\right\}$ NMR $(151 \mathrm{MHz}$, Chloroform- $d) \delta$ $162.2\left(\mathrm{~d},{ }^{1} \mathrm{JFC}_{\mathrm{FC}}=245.6 \mathrm{~Hz}, \mathrm{C} 10\right), 135.6\left(\mathrm{~d},{ }^{4} \mathrm{JFC}_{\mathrm{FC}}=3.2 \mathrm{~Hz}, \mathrm{C} 7\right), 128.7\left(\mathrm{~d},{ }^{3} \mathrm{~J}_{\mathrm{FC}}=8.0 \mathrm{~Hz}\right.$, C8), $115.3\left(\mathrm{~d},{ }^{2} \mathrm{JFC}=21.1 \mathrm{~Hz}, \mathrm{C9}\right), 87.0\left(\mathrm{~d},{ }^{1} \mathrm{JFC}=179.2 \mathrm{~Hz}, \mathrm{C} 3\right), 84.3\left(\mathrm{~d},{ }^{1} \mathrm{JFC}=172.9\right.$ $\mathrm{Hz}, \mathrm{C} 5), 61.4\left(\mathrm{~d},{ }^{2} \mathrm{JFC}=19.6 \mathrm{~Hz}, \mathrm{C} 2\right), 50.4\left(\mathrm{~d},{ }^{2}{ }^{\mathrm{FFC}}=21.9 \mathrm{~Hz}, \mathrm{C} 6\right), 34.1\left(\mathrm{t},{ }^{3} \mathrm{JFC}=20.9\right.$ $\mathrm{Hz}, \mathrm{C} 4) .{ }^{13} \mathrm{C}\left\{{ }^{1} \mathrm{H}\right.$,broadband- $\left.{ }^{19} \mathrm{~F}\right\}$ NMR $(151 \mathrm{MHz}$, Chloroform- $d) \delta 162.2(\mathrm{C} 10), 135.6$ (C7), 128.7 (C8), 115.3 (C9), 87.0 (C3), 84.3 (C5), 61.4 (C2), 50.4 (C6), 34.1 (C4). ${ }^{19} \mathrm{~F}$ NMR $\left(564 \mathrm{MHz}\right.$, Chloroform-d) $\delta-115.2\left(\mathrm{tt},{ }^{3} \mathrm{JHF}=8.7 \mathrm{~Hz},{ }^{4} J_{\mathrm{HF}}=5.4 \mathrm{~Hz}, 1 \mathrm{~F}, F C 10\right)$, 182.1 - -182.4 (m, 1F, FC5), -196.8 - -197.1 (m, 1F, FC3). ${ }^{19} \mathrm{~F}\left\{{ }^{1} \mathrm{H}\right\}$ NMR $(564 \mathrm{MHz}$, Chloroform- $d$ ) $\delta-115.2(1 \mathrm{~F}, F C 10),-182.2\left(\mathrm{~d},{ }^{4} J_{\mathrm{FF}}=15.0 \mathrm{~Hz}, 1 \mathrm{~F}, \mathrm{FC} 5\right),-197.0\left(\mathrm{~d},{ }^{4}{ }_{\mathrm{FFF}}\right.$ 
$=14.9 \mathrm{~Hz}, 1 \mathrm{~F}, \mathrm{FC} 3)$. HRMS $(E S I) \mathrm{m} / \mathrm{z}$ calculated for $\left[\mathrm{C}_{11} \mathrm{H}_{12} \mathrm{~F}_{3} \mathrm{NNa}\right]([\mathrm{M}+\mathrm{Na}+])$ 238.0814, found 238.0812 .
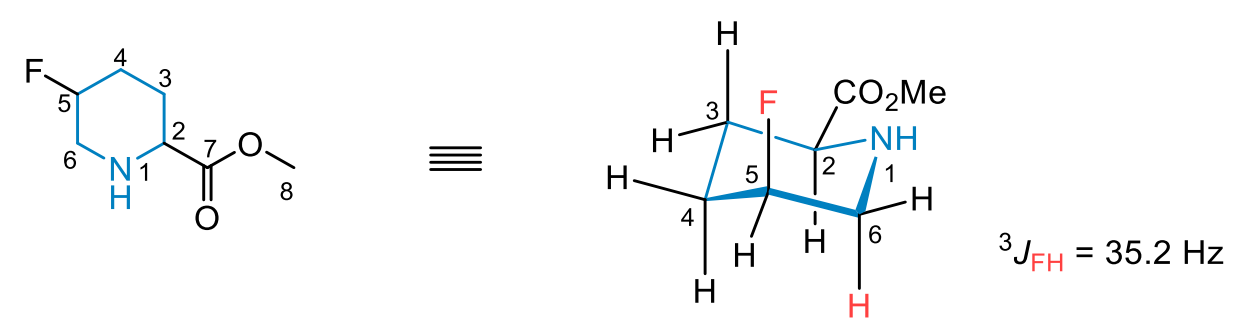

Methyl 5-fluoropiperidine-2-carboxylate (22): The title compound was synthesized according to GP-A using $10 \mathrm{~mol} \% \mathrm{Pd}$-catalyst $(35.1 \mathrm{mg})$ in $\mathrm{MeOH}(0.5 \mathrm{M})$ with aq. $\mathrm{HCl}$ (100 $\mu \mathrm{l}, 2.4$ equiv, $12 \mathrm{~N}$ ). The product was isolated as a white solid (42 $\mathrm{mg}, 0.26 \mathrm{mmol}$, 52\%, 76:24 d.r.) after column chromatography (silical gel, eluent: $\mathrm{CH}_{2} \mathrm{Cl}_{2}$, later 99:1, 98:2, $95: 5 \mathrm{CH}_{2} \mathrm{Cl}_{2} / \mathrm{MeOH}$ ). The product was isolated as a mixture of diastereomers. The axial position of the fluorine substituent was determined based on the ${ }^{3} \mathrm{JFH}$ coupling between $\mathrm{FC} 5$ and $\mathrm{H}_{\mathrm{ax}} \mathrm{C} 6$ and is in accordance with previous literature results. ${ }^{3}$

Analytical data of the main diastereomer: ${ }^{1} \mathbf{H}$ NMR $(599 \mathrm{MHz}$, Chloroform- $d) \delta 4.58$ $\left(\mathrm{dm},{ }^{2} \mathrm{JFH}_{\mathrm{FH}}=47.7 \mathrm{~Hz}, 1 \mathrm{H}, H_{\mathrm{eq}} \mathrm{C} 5\right), 3.69\left(\mathrm{~s}, 3 \mathrm{H}, \mathrm{H}_{3} \mathrm{C} 8\right), 3.45-3.39(\mathrm{~m}, 1 \mathrm{H}, \mathrm{HC} 2), 3.24$ (dddd, ${ }^{2} J_{\mathrm{HH}}=14.4 \mathrm{~Hz},{ }^{3} \mathrm{JFH}=10.9 \mathrm{~Hz},{ }^{3} \mathrm{JHH}_{\mathrm{HH}}=3.6 \mathrm{~Hz},{ }^{4} \mathrm{JHH}_{\mathrm{HH}}=2.2 \mathrm{~Hz}, 1 \mathrm{H}, H_{\mathrm{eqC}}$ ), 2.85 (ddd, $\left.{ }^{3} J_{\mathrm{FH}}=35.2 \mathrm{~Hz},{ }^{2} J_{\mathrm{HH}}=14.2 \mathrm{~Hz},{ }^{3} \mathrm{JHH}_{\mathrm{HH}}=1.9 \mathrm{~Hz}, 1 \mathrm{H}, H_{\mathrm{ax}} \mathrm{C} 6\right), 2.25-2.06(\mathrm{~m}, 2 \mathrm{H}$, $\left.H_{N} 1, H_{2} \mathrm{C} 4\right), 1.86-1.81\left(\mathrm{~m}, 2 \mathrm{H}, H_{2} \mathrm{C} 3\right), 1.82-1.67\left(\mathrm{~m}, 1 \mathrm{H}, \mathrm{H}_{2} \mathrm{C} 4\right) .{ }^{1} \mathrm{H}\left\{{ }^{19} \mathrm{~F}\right\}$ NMR (599 $\mathrm{MHz}$, Chloroform-d) $\delta 4.58\left(\mathrm{tt},{ }^{3} \mathrm{JHH}_{\mathrm{HH}}=4.3 \mathrm{~Hz},{ }^{3} \mathrm{JHH}_{\mathrm{HH}}=2.1 \mathrm{~Hz}, 1 \mathrm{H}, H_{\mathrm{eqC}}\right), 3.72(\mathrm{~s}, 3 \mathrm{H}$, $\mathrm{H}_{3} \mathrm{C} 8$ ), $3.44-3.41(\mathrm{~m}, 1 \mathrm{H}, \mathrm{HC2}), 3.24$ (ddd, ${ }^{2} \mathrm{JHH}_{\mathrm{HH}}=14.2 \mathrm{~Hz},{ }^{3} \mathrm{JHH}_{\mathrm{HH}}=3.5 \mathrm{~Hz},{ }^{3} \mathrm{JHH}_{\mathrm{HH}}=2.2$ $\left.\mathrm{Hz}, 1 \mathrm{H}, H_{\mathrm{eq}} \mathrm{C} 6\right), 2.85\left(\mathrm{dd},{ }^{2} \mathrm{JHH}_{\mathrm{HH}}=14.3 \mathrm{~Hz},{ }^{3} \mathrm{~J}_{\mathrm{HH}}=1.7 \mathrm{~Hz}, 1 \mathrm{H}, H_{\mathrm{ax}} \mathrm{C} 6\right), 2.27-2.05(\mathrm{~m}$, $2 \mathrm{H}, H \mathrm{~N} 1), 1.87-1.79\left(\mathrm{~m}, 2 \mathrm{H}, \mathrm{H}_{2} \mathrm{C} 3\right), 1.78-1.71\left(\mathrm{~m}, 1 \mathrm{H}, \mathrm{H}_{2} \mathrm{C} 4\right) .{ }^{13} \mathrm{C}\left\{{ }^{1} \mathrm{H}\right\}$ NMR $(151$ $\mathrm{MHz}$, Chloroform-d) $\delta 173.4(\mathrm{C} 7), 85.7$ (d, $\left.{ }^{1} \mathrm{JFC}=170.3 \mathrm{~Hz}, \mathrm{C} 5\right), 57.6$ (C2), 52.2 (C8), $48.8\left(\mathrm{~d},{ }^{2} \mathrm{JFC}_{\mathrm{FC}}=21.9 \mathrm{~Hz}, \mathrm{C} 6\right), 28.8\left(\mathrm{~d},{ }^{2} \mathrm{JFC}=21.3 \mathrm{~Hz}, \mathrm{C} 4\right), 24.4\left(\mathrm{~d},{ }^{3} \mathrm{JFC}=1.7 \mathrm{~Hz}, \mathrm{C} 3\right)$. ${ }^{13} \mathrm{C}\left\{{ }^{1} \mathrm{H},{ }^{19} \mathrm{~F}\right\}$ NMR (151 MHz, Chloroform-d) $\delta 173.4$ (C7), 85.7 (C5), 57.6 (C2), 52.2 (C8), 48.8 (C6), 28.8 (C4), 24.4 (C3). ${ }^{19} \mathrm{~F}$ NMR (564 MHz, Chloroform- $d$ ) $\delta-188.3$ (dm, $\left.{ }^{2} \mathrm{JHF}=43.7 \mathrm{~Hz}, 1 \mathrm{~F}\right) .{ }^{19} \mathrm{~F}\left\{{ }^{1} \mathrm{H}\right\}$ NMR $(564 \mathrm{MHz}$, Chloroform- $d) \delta-188.3(\mathrm{~s}, 1 \mathrm{~F})$. HRMS (ESI) $\mathrm{m} / \mathrm{z}$ calculated for $\left[\mathrm{C}_{7} \mathrm{H}_{13} \mathrm{FNO}_{2}\right]\left(\left[\mathrm{M}+\mathrm{H}^{+}\right]\right) 162.0925$, found 162.0928 .

Analytical data of the minor diastereomer: ${ }^{1} \mathrm{H}$ NMR (599 MHz, Chloroform- $d$ ) $\delta 4.52$ (dtt, ${ }^{2} J_{\mathrm{FH}}=48.4 \mathrm{~Hz},{ }^{3} \mathrm{~J}_{\mathrm{HH}}=8.2 \mathrm{~Hz},{ }^{3} \mathrm{~J}_{\mathrm{HH}}=4.2 \mathrm{~Hz}, 1 \mathrm{H}, \mathrm{HC}$ ), $3.72\left(\mathrm{~s}, 3 \mathrm{H}, \mathrm{H}_{3} \mathrm{C} 8\right), 3.39$ (dd, $\left.{ }^{3} \mathrm{JHH}_{\mathrm{HH}}=8.9 \mathrm{~Hz},{ }^{3} \mathrm{JHH}_{\mathrm{HH}}=3.5 \mathrm{~Hz}, 1 \mathrm{H}, H \mathrm{HC} 2\right), 3.31\left(\mathrm{dtd},{ }^{2} \mathrm{~J}_{\mathrm{HH}}=12.3 \mathrm{~Hz},{ }^{3} \mathrm{JFH}=4.1 \mathrm{~Hz}\right.$, $\left.{ }^{3} \mathrm{JHH}_{\mathrm{HH}}=1.7 \mathrm{~Hz}, 1 \mathrm{H}, \mathrm{H}_{2} \mathrm{C} 6\right), 2.74\left(\mathrm{ddd},{ }^{2} \mathrm{JHH}_{\mathrm{HH}}=12.1 \mathrm{~Hz},{ }^{3} \mathrm{JHH}=8.2 \mathrm{~Hz},{ }^{3} \mathrm{JFH}_{\mathrm{FH}}=6.5 \mathrm{~Hz}, 1 \mathrm{H}\right.$, 
$\left.\mathrm{H}_{2} \mathrm{C} 6\right), 2.29-2.05$ (m, 3H, $\left.\mathrm{HN}_{1}, \mathrm{H}_{2} \mathrm{C} 4, \mathrm{H}_{2} \mathrm{C} 5\right), 1.72-1.59$ (m, 2H, $\left.\mathrm{H}_{2} \mathrm{C} 4, \mathrm{H}_{2} \mathrm{C} 5\right)$. ${ }^{1} \mathrm{H}\left\{{ }^{19} \mathrm{~F}\right\}$ NMR $(599 \mathrm{MHz}$, Chloroform- $d) \delta 4.55-4.49(\mathrm{~m}, 1 \mathrm{H}, \mathrm{HC}), 3.72\left(\mathrm{~s}, 3 \mathrm{H}, \mathrm{H}_{3} \mathrm{C} 8\right)$, $3.39\left(\mathrm{dd},{ }^{3} \mathrm{JHH}_{\mathrm{HH}}=8.8 \mathrm{~Hz},{ }^{3} \mathrm{JHH}_{\mathrm{HH}}=3.5 \mathrm{~Hz}, 1 \mathrm{H}, \mathrm{HC} 2\right), 3.31$ (ddd, ${ }^{2} \mathrm{JHH}_{\mathrm{HH}}=12.1 \mathrm{~Hz},{ }^{3} \mathrm{JHH}_{\mathrm{HH}}=$ $\left.4.1 \mathrm{~Hz},{ }^{3} \mathrm{JHH}_{\mathrm{HH}}=1.6 \mathrm{~Hz}, 1 \mathrm{H}, \mathrm{H}_{2} \mathrm{C} 6\right), 2.74\left(\mathrm{dd},{ }^{2} \mathrm{JHH}=12.1,{ }^{3} \mathrm{JHH}=8.2 \mathrm{~Hz}, 1 \mathrm{H}, \mathrm{H}_{2} \mathrm{C} 6\right), 2.28$ - $2.05\left(\mathrm{~m}, 3 \mathrm{H}, H \mathrm{~N} 1, \mathrm{H}_{2} \mathrm{C} 4, \mathrm{H}_{2} \mathrm{C} 5\right), 1.70-1.60\left(\mathrm{~m}, 2 \mathrm{H}, \mathrm{H}_{2} \mathrm{C} 4, \mathrm{H}_{2} \mathrm{C} 5\right) .{ }^{13} \mathrm{C}\left\{{ }^{1} \mathrm{H}\right\}$ NMR $(151$ $\mathrm{MHz}$, Chloroform- $d$ ) $\delta 173.3\left(\mathrm{~d},{ }^{5} \mathrm{JFC}=2.0 \mathrm{~Hz}, \mathrm{C} 7\right), 87.6\left(\mathrm{~d},{ }^{1} \mathrm{JFC}=174.1 \mathrm{~Hz}, \mathrm{C5}\right), 57.0$ (d, $\left.{ }^{4} \mathrm{JFC}=1.3 \mathrm{~Hz}, \mathrm{C} 2\right), 52.2(\mathrm{C} 8), 49.0$ (d, $\left.{ }^{2} \mathrm{JFC}=24.4 \mathrm{~Hz}, \mathrm{C} 6\right), 29.7\left(\mathrm{~d},{ }^{2} \mathrm{JFC}=19.8 \mathrm{~Hz}\right.$, C4), 26.4 (d, $J=8.5 \mathrm{~Hz}, \mathrm{C} 3) .{ }^{13} \mathrm{C}\left\{{ }^{1} \mathrm{H},{ }^{19} \mathrm{~F}\right\}$ NMR $(151 \mathrm{MHz}$, Chloroform-d) $\delta 173.3$ (C7), 87.6 (C5), 57.0 (C2), 52.2 (C8), 49.0 (C6), 29.7 (C4), 26.4 (C3). ${ }^{19} \mathrm{~F} \mathrm{NMR} \mathrm{(564} \mathrm{MHz,}$ Chloroform- $d) \delta-183.0\left(\mathrm{dm},{ }^{2} \mathrm{JHF}=47.9 \mathrm{~Hz}, 1 \mathrm{~F}\right) .{ }^{19} \mathrm{~F}\left\{{ }^{1} \mathrm{H}\right\}$ NMR $(564 \mathrm{MHz}$, Chloroformd) $\delta-183.0(\mathrm{~s}, 1 \mathrm{~F})$.

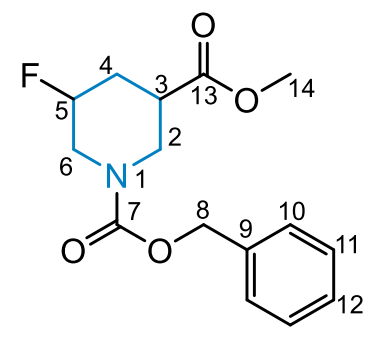

1-Benzyl 3-methyl 5-fluoropiperidine-1,3-dicarboxylate (23): The title compound was synthesized according to GP-B using $5 \mathrm{~mol} \%$ Pd-catalyst (17.6 mg) in $\mathrm{MeOH}$ $(0.5 \mathrm{M})$ with aq. $\mathrm{HCl}(100 \mu \mathrm{l}, 2.4$ equiv, $12 \mathrm{~N})$. The product was isolated as a colorless liquid (45 mg, $0.15 \mathrm{mmol}, 30 \%, 86: 14$ d.r.) after column chromatography (silical gel, eluent: $n$-pentane, later $n$-pentane/EtOAc 95:5, 90:10). Only the analytical data of the main diastereomer are given. The conformation of the product was not determined, since rotation of the carbamate group prohibited determination of $J$ values. However, analogous compounds have been studied in the literature in detail. ${ }^{3}$

${ }^{1} \mathrm{H}$ NMR $(599 \mathrm{MHz}$, Chloroform-d) $\delta 7.38-7.34$ (m, 4H, HC10, HC11), $7.34-7.31$ (m, $1 \mathrm{H}, \mathrm{HC} 12), 5.14$ (bs, 2H, $\left.H_{2} \mathrm{C} 8\right), 4.52\left(\mathrm{~d},{ }^{2} \mathrm{JFH}=47.9 \mathrm{~Hz}, 1 \mathrm{H}, H \mathrm{C} 5\right), 4.35-4.08(\mathrm{~m}, 2 \mathrm{H}$, $\mathrm{H}_{2} \mathrm{C} 6, \mathrm{H}_{2} \mathrm{C} 2$ ), 3.70 (s, $\left.3 \mathrm{H}, \mathrm{H}_{3} \mathrm{C} 14\right), 3.17-2.95$ (m, $\left.1 \mathrm{H}, \mathrm{H}_{2} \mathrm{C} 2\right), 2.94$ (ddd, ${ }^{2} \mathrm{JHH}_{\mathrm{HH}}=$ $\left.12.9 \mathrm{~Hz},{ }^{3} \mathrm{JHH}=9.4 \mathrm{~Hz},{ }^{3} \mathrm{JFH}=5.3 \mathrm{~Hz}, 1 \mathrm{H}, \mathrm{H}_{2} \mathrm{C} 6\right), 2.56$ (bs, $\left.1 \mathrm{H}, H \mathrm{C} 3\right), 2.48-2.40(\mathrm{~m}$, $\left.1 \mathrm{H}, \mathrm{H}_{2} \mathrm{C} 4\right), 1.85\left(\mathrm{ddt},{ }^{2} \mathrm{JHH}=10.9 \mathrm{~Hz},{ }^{3} \mathrm{JFH}=10.9 \mathrm{~Hz},{ }^{3} \mathrm{JHH}=10.9 \mathrm{~Hz}, 1 \mathrm{H}, \mathrm{H}_{2} \mathrm{C} 4\right) .{ }^{1} \mathrm{H}\left\{{ }^{19} \mathrm{~F}\right\}$ NMR (599 MHz, Chloroform-d) $\delta 7.39$ - 7.34 (m, 4H, HC10, HC11), 7.34 - 7.31 (m, $1 \mathrm{H}, H \mathrm{C} 12), 5.14$ (bs, 2H, $\left.\mathrm{H}_{2} \mathrm{C} 8\right), 4.52$ (s, $\left.1 \mathrm{H}, H \mathrm{C} 5\right), 4.37-4.07$ (m, 2H, $\mathrm{H}_{2} \mathrm{C} 6, \mathrm{H}_{2} \mathrm{C} 2$ ), $\left.3.70\left(\mathrm{~s}, 3 \mathrm{H}, H_{3} \mathrm{C} 14\right), 3.13-2.97\left(\mathrm{~m}, 1 \mathrm{H}, \mathrm{H}_{2} \mathrm{C} 2\right)\right), 2.94\left(\mathrm{dd},{ }^{2} \mathrm{JHH}=12.9 \mathrm{~Hz},{ }^{3}{ }_{\mathrm{HH}}=9.4\right.$ $\left.\mathrm{Hz}, 1 \mathrm{H}, H_{2} \mathrm{C} 6\right), 2.56$ (bs, $\left.1 \mathrm{H}, H \mathrm{C} 3\right), 2.49-2.40\left(\mathrm{~m}, 1 \mathrm{H}, \mathrm{H}_{2} \mathrm{C} 4\right), 1.84$ (dt, ${ }^{2} \mathrm{JHH}_{\mathrm{H}}=11.5$ 
$\left.\mathrm{Hz},{ }^{3} \mathrm{JHH}=11.5 \mathrm{~Hz}, 1 \mathrm{H}, \mathrm{H}_{2} \mathrm{C} 4\right) .{ }^{13} \mathrm{C}\left\{{ }^{1} \mathrm{H}\right\}$ NMR $(151 \mathrm{MHz}$, Chloroform- $d) \delta 172.1\left(\mathrm{~d},{ }^{4} \mathrm{JFC}\right.$ $=1.5 \mathrm{~Hz}, \mathrm{C} 13$ ), 136.4 (C9), 128.7 (C11), 128.3 (C12), 128.2 (C10), 85.7 (dm, ${ }^{1} \mathrm{JFC}=$ 178.6 Hz, C5), 67.7 (C8), 52.2 (C14), 47.7 (m, C6), 44.9 (d, $\left.{ }^{4} \mathrm{JFC}=1.3 \mathrm{~Hz}, \mathrm{C} 2\right), 39.5$ (m, C3), $33.3\left(\mathrm{~d},{ }^{2} \mathrm{JFC}=20.8 \mathrm{~Hz}, \mathrm{C} 4\right) .{ }^{13} \mathrm{C}\left\{{ }^{1} \mathrm{H},{ }^{19} \mathrm{~F}\right\}$ NMR $(151 \mathrm{MHz}$, Chloroform- $d) \delta$ 172.0 (C13), 136.4 (C9), 128.7 (C11), 128.3 (C12), 128.2 (C10), 85.7 (bs, C5), 67.7 (C8), 52.2 (C14), 47.7 (bs, C6), 44.9 (C2), 39.5 (bs, C3), 33.3 (C4). ${ }^{19} \mathrm{~F} \mathrm{NMR}(564 \mathrm{MHz}$, Chloroform- $d$ ) $\delta-182.3\left(\mathrm{~d},{ }^{2} \mathrm{JHF}=45.2 \mathrm{~Hz}\right.$, rotamer A), $-182.5\left(\mathrm{~d},{ }^{2} J_{\mathrm{HF}}=44.3 \mathrm{~Hz}\right.$, rotamer B). ${ }^{19} \mathrm{~F}\left\{{ }^{1} \mathrm{H}\right\}$ NMR $(282 \mathrm{MHz}$, Chloroform- $d) \delta-182.3(\mathrm{~s}$, rotamer A), $-182.5(\mathrm{~s}$, rotamer B). HRMS (ESI) $\mathrm{m} / \mathrm{z}$ calculated for $\left[\mathrm{C}_{15} \mathrm{H}_{18} \mathrm{FNO}_{4} \mathrm{Na}\right]\left(\left[\mathrm{M}+\mathrm{Na}^{+}\right]\right) 318.1112$, found 318.1108 .

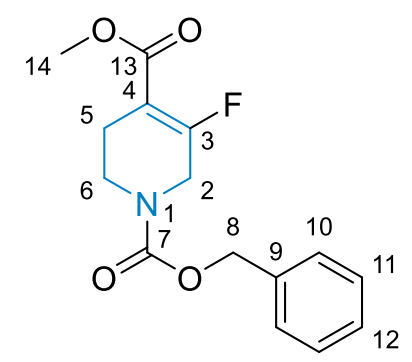

1-Benzyl 4-methyl 3-fluoropiperidine-1,4-dicarboxylate (24): The title compound was synthesized according to GP-B using $5 \mathrm{~mol} \%$ Pd-catalyst $(17.6 \mathrm{mg}$ ) in $\mathrm{MeOH}$ $(0.5 \mathrm{M})$ with aq. $\mathrm{HCl}(100 \mu \mathrm{l}, 2.4$ equiv, $12 \mathrm{~N})$. The product was isolated as a colorless liquid (106 mg, $0.36 \mathrm{mmol}, 72 \%$ ) after column chromatography (silical gel, eluent: $n$ pentane, later $n$-pentane/EtOAc 80:20, 70:30).

${ }^{1} \mathrm{H}$ NMR $(599 \mathrm{MHz}$, Chloroform-d) $\delta 7.38-7.34$ (m, 4H, HC10, HC11), $7.34-7.30$ (m, $1 \mathrm{H}, H \mathrm{C} 12), 5.15$ (s, 2H, $\left.H_{2} \mathrm{C} 8\right), 4.12\left(\mathrm{~s}, 2 \mathrm{H}, H_{2} \mathrm{C} 2\right), 3.77\left(\mathrm{~s}, 3 \mathrm{H}, H_{3} \mathrm{C} 14\right), 3.55$ (t, ${ }^{3} \mathrm{HHH}=$ $\left.5.7 \mathrm{~Hz}, 2 \mathrm{H}, \mathrm{H}_{2} \mathrm{C} 6\right), 2.49-2.40\left(\mathrm{~m}, 2 \mathrm{H}, \mathrm{H}_{2} \mathrm{C} 5\right) .{ }^{13} \mathrm{C}\left\{{ }^{1} \mathrm{H}\right\}$ NMR $(151 \mathrm{MHz}$, Chloroform-d) $\delta 164.8(\mathrm{C} 13), 161.5\left(\mathrm{~d},{ }^{1} \mathrm{JFC}=278.2 \mathrm{~Hz}, \mathrm{C} 3\right.$, rotamer A), $160.9\left(\mathrm{~d},{ }^{1} \mathrm{JFC}=278.3 \mathrm{~Hz}\right.$, C3, rotamer B), 155.5 - 154.1 (m, C7), 136.2 (C9), 128.6 (C11), 128.3 (C12), 128.1 (C10), 107.6 - 107.1 ( $m, C 4$, rotamer A), 107.1 - 106.8 (m, C4, rotamer B), 67.7 (C8), 52.0 (C14), 43.2 (d, $\left.{ }^{2} \mathrm{JFC}=38.6 \mathrm{~Hz}, \mathrm{C} 2\right), 40.6$ (C6, rotamer A), 40.1 (C6, rotamer B), 24.3 (C5, rotamer A), 23.8 (C5, rotamer B). ${ }^{13} \mathbf{C}\left\{{ }^{1} \mathbf{H},{ }^{19} \mathrm{~F}\right\}$ NMR $(151 \mathrm{MHz}$, Chloroformd) $\delta 164.8$ (C13), 161.4 (C3, rotamer A), 160.9 (C3, rotamer B), 155.4 - 154.1 (m, C7), 136.2 (C9), 128.6 (C11), 128.3 (C12), 128.1 (C10), 107.2 (C4, rotamer A), 106.9 (C4, rotamer B), $67.6(\mathrm{C} 8), 51.9(\mathrm{C} 14), 43.2(\mathrm{C} 2), 40.5(\mathrm{C} 6$, rotamer $\mathrm{A}), 40.0$ (C6, rotamer B), 24.3 (C5, rotamer A), 23.8 (C5, rotamer B). ${ }^{19} \mathrm{~F}$ NMR (564 MHz, Chloroform- $\left.d\right) \delta$ 94.7 (s, rotamer A), -96.0 (s, rotamer B). ${ }^{19} \mathrm{~F}\left\{{ }^{1} \mathrm{H}\right\}$ NMR $(282 \mathrm{MHz}$, Chloroform- $d$ ) $\delta$ - 
94.7 (s, rotamer A, -96.0 (s, rotamer B). HRMS (ESI) m/z calculated for $\left[\mathrm{C}_{15} \mathrm{H}_{16} \mathrm{NO}_{4} \mathrm{FNa}\right]\left(\left[\mathrm{M}+\mathrm{Na}^{+}\right]\right)$316.0956, found 316.0792.
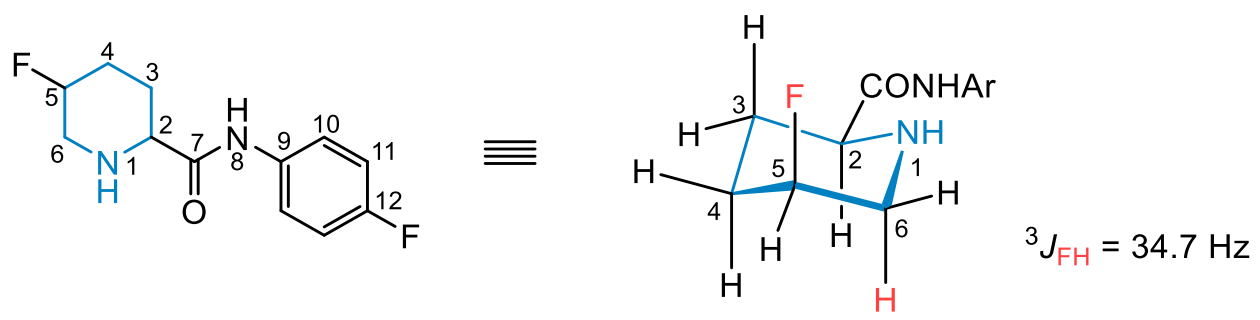

5-Fluoro- $\mathrm{N}$-(4-fluorophenyl)piperidine-2-carboxamide (25): The title compound was synthesized according to GP-A using 10 mol\% Pd-catalyst $(35.1 \mathrm{mg})$ in $\mathrm{THF} / \mathrm{H}_{2} \mathrm{O}$ $(0.5 \mathrm{M}, 1: 1)$ with aq. $\mathrm{HCl}(100 \mu \mathrm{l}, 2.4$ equiv, $12 \mathrm{~N})$. The product was isolated as a white solid (49 mg, $0.20 \mathrm{mmol}$, 41\%, 91:9 d.r.) after column chromatography (silical gel, eluent: $\mathrm{CH}_{2} \mathrm{Cl}_{2}$, later $99: 1,98: 2 \mathrm{CH}_{2} \mathrm{Cl}_{2} / \mathrm{MeOH}$ ). Only the analytical data of the main diastereomer are given. The axial position of the fluorine substituent was determined based on the ${ }^{3} \mathrm{JFH}_{\mathrm{FH}}$ coupling between $\mathrm{FC} 5$ and $\mathrm{Hax}_{\mathrm{ax}} 6$ and is in accordance with previous literature results. ${ }^{3}$

${ }^{1} \mathrm{H}$ NMR (599 MHz, Chloroform-d) $\delta 8.83$ (s, 1H, HN8), $7.55-7.50$ (m, 2H, $\left.\mathrm{H}_{2} \mathrm{C} 10\right)$, $7.05-6.97\left(\mathrm{~m}, 2 \mathrm{H}, \mathrm{H}_{2} \mathrm{C} 11\right), 4.64\left(\mathrm{dm},{ }^{2} \mathrm{JFH}=47.7 \mathrm{~Hz}, 1 \mathrm{H}, \mathrm{Heq}_{\mathrm{C}}\right), 3.31$ (ddd, ${ }^{3} \mathrm{~J}_{\mathrm{HH}}=$ $\left.10.6 \mathrm{~Hz},{ }^{3} \mathrm{~J}_{\mathrm{HH}}=3.3 \mathrm{~Hz},{ }^{5} \mathrm{JFH}_{\mathrm{FH}}=1.8 \mathrm{~Hz}, 1 \mathrm{H}, H_{\mathrm{ax}} \mathrm{C} 2\right), 3.31-3.23\left(\mathrm{~m}, 1 \mathrm{H}, H_{\mathrm{eq}} \mathrm{C} 6\right), 2.90$ $\left(\mathrm{ddd},{ }^{3} \mathrm{JFH}_{\mathrm{FH}}=34.7 \mathrm{~Hz},{ }^{2} \mathrm{JHH}=14.3 \mathrm{~Hz},{ }^{3} \mathrm{JHH}=1.7 \mathrm{~Hz}, 1 \mathrm{H}, \mathrm{HaxC6}\right), 2.20-2.12(\mathrm{~m}, 1 \mathrm{H}$, $\left.\left.\mathrm{H}_{2} \mathrm{C} 4\right), 2.12-2.06\left(\mathrm{~m}, 1 \mathrm{H}, \mathrm{H}_{2} \mathrm{C} 5\right), 1.91-1.68\left(\mathrm{~m}, 3 \mathrm{H}, \mathrm{H}_{2} \mathrm{C} 4, \mathrm{H}_{2} \mathrm{C} 5, H \mathrm{~N} 1\right) .{ }^{1} \mathrm{H}^{19}{ }^{19} \mathrm{~F}\right\}$ NMR (599 MHz, Chloroform-d) $\delta 8.83(\mathrm{~s}, 1 \mathrm{H}, H \mathrm{~N} 8), 7.52\left(\mathrm{~d},{ }^{3} \mathrm{JHH}_{\mathrm{HH}}=9.1 \mathrm{~Hz}, 2 \mathrm{H}, \mathrm{H}_{2} \mathrm{C} 10\right)$, $7.00\left(\mathrm{~d},{ }^{3} \mathrm{JHH}_{\mathrm{HH}}=9.1 \mathrm{~Hz}, 2 \mathrm{H}, \mathrm{H}_{2} \mathrm{C} 11\right), 4.72-4.59\left(\mathrm{~m}, 1 \mathrm{H}, H_{\mathrm{eq}} \mathrm{C} 5\right), 3.31\left(\mathrm{dd},{ }^{3} \mathrm{~J}_{\mathrm{HH}}=\right.$ $10.5 \mathrm{~Hz},{ }^{3} \mathrm{JHH}_{\mathrm{HH}}=3.3 \mathrm{~Hz}, 1 \mathrm{H}, \mathrm{Hax}_{\mathrm{a}}$ ), 3.27 (ddd, ${ }^{2} \mathrm{JHH}_{\mathrm{HH}}=14.3 \mathrm{~Hz},{ }^{3} \mathrm{~J}_{\mathrm{HH}}=3.6 \mathrm{~Hz},{ }^{3} \mathrm{JHH}_{\mathrm{HH}}=2.2$ $\left.\mathrm{Hz}, 1 \mathrm{H}, H_{\mathrm{eq}} \mathrm{C} 6\right), 2.90\left(\mathrm{dd},{ }^{2} J_{\mathrm{HH}}=14.4 \mathrm{~Hz},{ }^{3} J_{\mathrm{HH}}=1.5 \mathrm{~Hz}, 1 \mathrm{H}, H_{\mathrm{ax}} \mathrm{C6}\right), 2.16\left(\mathrm{dm},{ }^{2} J_{\mathrm{HH}}=\right.$ $\left.14.2 \mathrm{~Hz}, 1 \mathrm{H}, \mathrm{H}_{2} \mathrm{C} 4\right), 2.09\left(\mathrm{dm},{ }^{2} \mathrm{~J}_{\mathrm{HH}}=13.5 \mathrm{~Hz}, 1 \mathrm{H}, \mathrm{H}_{2} \mathrm{C} 3\right), 1.90-1.81\left(\mathrm{~m}, 1 \mathrm{H}, \mathrm{H}_{2} \mathrm{C} 3\right)$, $1.80-1.71\left(\mathrm{~m}, 2 \mathrm{H}, \mathrm{H}_{2} \mathrm{C} 4, H \mathrm{~N} 1\right) .{ }^{13} \mathrm{C}\left\{{ }^{1} \mathrm{H}\right\}$ NMR (151 MHz, Chloroform- $\left.d\right) \delta 170.6(\mathrm{C} 7)$, $159.4\left(\mathrm{~d},{ }^{1} \mathrm{JFC}=243.3 \mathrm{~Hz}, \mathrm{C} 12\right), 133.8\left(\mathrm{~d},{ }^{4} \mathrm{JFC}=2.8 \mathrm{~Hz}, \mathrm{C} 9\right), 121.4\left(\mathrm{~d},{ }^{3} \mathrm{JFC}=7.8 \mathrm{~Hz}\right.$, C10), $115.7\left(d,{ }^{2} \mathrm{JFC}=22.4 \mathrm{~Hz}, \mathrm{C} 11\right), 86.1\left(\mathrm{~d},{ }^{1} \mathrm{JFC}=170.8 \mathrm{~Hz}, \mathrm{C} 5\right), 59.2$ (C2), 49.6 (d, $\left.{ }^{2} J_{F C}=21.4 \mathrm{~Hz}, \mathrm{C} 6\right), 28.9\left(\mathrm{~d},{ }^{2} J_{F C}=21.2 \mathrm{~Hz}, \mathrm{C} 4\right), 23.8\left(\mathrm{~d},{ }^{3} J_{F C}=1.2 \mathrm{~Hz}, \mathrm{C} 3\right)$. ${ }^{13} \mathbf{C}\left\{{ }^{1} \mathbf{H}\right.$,broadband- $\left.{ }^{19} \mathrm{~F}\right\}$ NMR (151 MHz, Chloroform- $\left.d\right) \delta 170.6$ (C7), 159.40 (C12), 133.8 (C9), 121.4 (C10), 115.7 (C11), 86.1 (C5), 59.2 (C2), 49.6 (C6), 28.9 (C4), 23.8 (C3). ${ }^{19} \mathrm{~F}$ NMR (564 MHz, Chloroform-d) $\delta-118.3\left(\mathrm{tt},{ }^{3} \mathrm{JHF}_{\mathrm{HF}}=8.3 \mathrm{~Hz},{ }^{4} \mathrm{JHF}_{\mathrm{HF}}=4.8 \mathrm{~Hz}\right.$, FC12), -187.5 (m, FC5). ${ }^{19} \mathrm{~F}\left\{{ }^{1} \mathrm{H}\right\}$ NMR (564 MHz, Chloroform-d) $\delta-118.3$ (FC12), - 
187.5 (FC5). HRMS (ESI) m/z calculated for $\left[\mathrm{C}_{12} \mathrm{H}_{15} \mathrm{~F}_{2} \mathrm{~N}_{2} \mathrm{O}\right]\left(\left[\mathrm{M}+\mathrm{H}^{+}\right]\right) 241.1147$, found 241.1165.
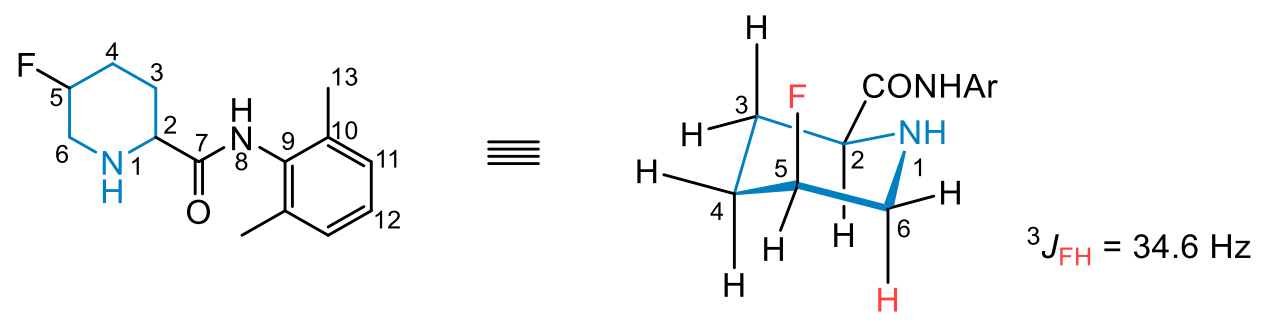

N-(2,6-Dimethylphenyl)-5-fluoropiperidine-2-carboxamide $\quad$ (26): The title compound was synthesized according to GP-A using 10 mol\% Pd-catalyst $(35.1 \mathrm{mg})$ in $\mathrm{THF} / \mathrm{H}_{2} \mathrm{O}(0.5 \mathrm{M}, 1: 1)$ with aq. $\mathrm{HCl}(100 \mu \mathrm{l}, 2.4$ equiv, $12 \mathrm{~N})$. The product was isolated as a white solid (52 $\mathrm{mg}, 0.21 \mathrm{mmol}, 42 \%, 92: 8$ d.r.) after column chromatography (silical gel, eluent: $\mathrm{CH}_{2} \mathrm{Cl}_{2}$, later $99: 1,98: 2 \mathrm{CH}_{2} \mathrm{Cl}_{2} / \mathrm{MeOH}$ ). Only the analytical data of the main diastereomer are given. The axial position of the fluorine substituent was determined based on the ${ }^{3} \mathrm{JFH}_{\mathrm{FH}}$ coupling between FC5 and $\mathrm{H}_{\mathrm{ax}} \mathrm{C} 6$ and is in accordance with previous literature results. ${ }^{3}$

${ }^{1} \mathrm{H}$ NMR (599 MHz, Chloroform-d) $\delta 8.25$ (s, 1H, HN8), $7.12-7.05$ (m, 3H, HC11, HC12), $4.67\left(\mathrm{dm},{ }^{2} \mathrm{JFH}=47.8 \mathrm{~Hz}, 1 \mathrm{H}, H_{\mathrm{eqC}}\right.$ ), 3.43 (ddd, ${ }^{3} \mathrm{JHH}_{\mathrm{HH}}=10.4 \mathrm{~Hz},{ }^{3} \mathrm{JHH}_{\mathrm{HH}}=3.4 \mathrm{~Hz}$, $\left.{ }^{5} J_{\mathrm{FH}}=1.9 \mathrm{~Hz}, 1 \mathrm{H}, H_{\mathrm{ax}} \mathrm{C} 2\right), 3.32$ (dddd, ${ }^{2} \mathrm{~J}_{\mathrm{HH}}=14.5 \mathrm{~Hz},{ }^{3} \mathrm{~J}_{\mathrm{FH}}=11.0 \mathrm{~Hz},{ }^{3} \mathrm{~J}_{\mathrm{HH}}=3.7 \mathrm{~Hz}$, ${ }^{3} \mathrm{JHH}_{\mathrm{HH}}=2.0 \mathrm{~Hz}, 1 \mathrm{H}, \mathrm{Heq}_{\mathrm{C}} 6$ ), 2.96 (ddd, ${ }^{3} \mathrm{JFH}_{\mathrm{FH}}=34.6 \mathrm{~Hz},{ }^{2} \mathrm{JHH}_{\mathrm{HH}}=14.3 \mathrm{~Hz},{ }^{3} \mathrm{JHH}_{\mathrm{HH}}=1.2 \mathrm{~Hz}, 1 \mathrm{H}$, $H_{\mathrm{ax}} \mathrm{C}$ ), 2.22 (s, 6H, $\mathrm{H}_{3} \mathrm{C} 13$ ), $2.20-2.14$ (m, 1H, $\left.\mathrm{H}_{2} \mathrm{C} 4\right), 2.13-2.07$ (m, $1 \mathrm{H}, \mathrm{H}_{2} \mathrm{C} 3$ ), $2.00-1.90\left(\mathrm{~m}, 1 \mathrm{H}, \mathrm{H}_{2} \mathrm{C} 3\right), 1.86-1.72\left(\mathrm{~m}, 2 \mathrm{H}, \mathrm{HN} 1, \mathrm{H}_{2} \mathrm{C} 4\right) .{ }^{1} \mathrm{H}\left\{{ }^{19} \mathrm{~F}\right\}$ NMR $(599 \mathrm{MHz}$, Chloroform- $d$ ) $\delta 8.25$ (s, 1H, HN8), 7.12 - 7.05 (m, 3H, HC11, HC12), 4.76 - 4.60 (m, $1 \mathrm{H}, H_{\text {eq }}$ C5), $3.43\left(\mathrm{dd},{ }^{3} \mathrm{~J}_{\mathrm{HH}}=10.5 \mathrm{~Hz},{ }^{3} \mathrm{~J}_{\mathrm{HH}}=3.3 \mathrm{~Hz}, 1 \mathrm{H}, H_{\mathrm{ax}} \mathrm{C} 2\right), 3.32$ (ddd, ${ }^{2} J_{\mathrm{HH}}=$ $\left.14.1 \mathrm{~Hz},{ }^{3} \mathrm{JHH}_{\mathrm{HH}}=3.7 \mathrm{~Hz},{ }^{3} \mathrm{JHH}_{\mathrm{HH}}=2.1 \mathrm{~Hz}, 1 \mathrm{H}, \mathrm{Heq}_{\mathrm{e}} 6\right), 2.96\left(\mathrm{dd},{ }^{2} \mathrm{JHH}_{\mathrm{HH}}=14.0 \mathrm{~Hz},{ }^{3} \mathrm{JHH}_{\mathrm{HH}}\right.$ $\left.=1.4 \mathrm{~Hz}, 1 \mathrm{H}, H_{\mathrm{ax}} \mathrm{C} 6\right), 2.22\left(\mathrm{~s}, 6 \mathrm{H}, H_{3} \mathrm{C} 13\right), 2.17\left(\mathrm{dm},{ }^{2} \mathrm{JHH}_{\mathrm{H}}=14.4 \mathrm{~Hz}, 1 \mathrm{H}, \mathrm{H}_{2} \mathrm{C} 4\right), 2.10$ $\left(\mathrm{dm},{ }^{2} J_{\mathrm{HH}}=13.7 \mathrm{~Hz}, 1 \mathrm{H}, \mathrm{H}_{2} \mathrm{C} 3\right), 1.99-1.91\left(\mathrm{~m}, 1 \mathrm{H}, \mathrm{H}_{2} \mathrm{C} 3\right), 1.85-1.75(\mathrm{~m}, 2 \mathrm{H}, H \mathrm{~N} 1$, $\left.\mathrm{H}_{2} \mathrm{C} 4\right) .{ }^{13} \mathrm{C}\left\{{ }^{1} \mathrm{H}\right\}$ NMR (151 MHz, Chloroform-d) $\delta 170.9$ (C7), 135.2 (C10), 133.7 (C9), 128.3 (C11), 127.3 (C12), 86.3 (d, $\left.{ }^{1} \mathrm{JFC}=171.1 \mathrm{~Hz}, \mathrm{C} 5\right), 59.3$ (C2), 49.6 (d, ${ }^{2} \mathrm{JFC}=21.4$ $\mathrm{Hz}, \mathrm{C} 6), 29.0\left(\mathrm{~d},{ }^{2} \mathrm{JFC}_{\mathrm{FC}} 21.1 \mathrm{~Hz}, \mathrm{C} 4\right), 24.6\left(\mathrm{~d},{ }^{3} \mathrm{JFC}_{\mathrm{FC}}=1.7 \mathrm{~Hz}, \mathrm{C} 3\right), 18.6(\mathrm{C} 13) .{ }^{13} \mathrm{C}\left\{{ }^{1} \mathrm{H},{ }^{19} \mathrm{~F}\right\}$ NMR (151 MHz, Chloroform-d) $\delta 170.9$ (C7), 135.2 (C10), 133.7 (C9), 128.3 (C11), 127.3 (C12), 86.3 (C5), 59.3 (C2), 49.6 (C6), 29.0 (C4), 24.6 (C3), 18.6 (C13). ${ }^{19}$ F NMR (564 MHz, Chloroform- $d$ ) $\delta-187.1--187.5(\mathrm{~m}) .{ }^{19} \mathrm{~F}\left\{{ }^{1} \mathrm{H}\right\}$ NMR (282 MHz, Chloroform- 
d) $\delta$-187.2. HRMS (ESI) $\mathrm{m} / \mathrm{z}$ calculated for $\left[\mathrm{C}_{14} \mathrm{H}_{20} \mathrm{FN} 2 \mathrm{O}\right]\left(\left[\mathrm{M}+\mathrm{H}^{+}\right]\right) 251.1554$, found 251.1573 .
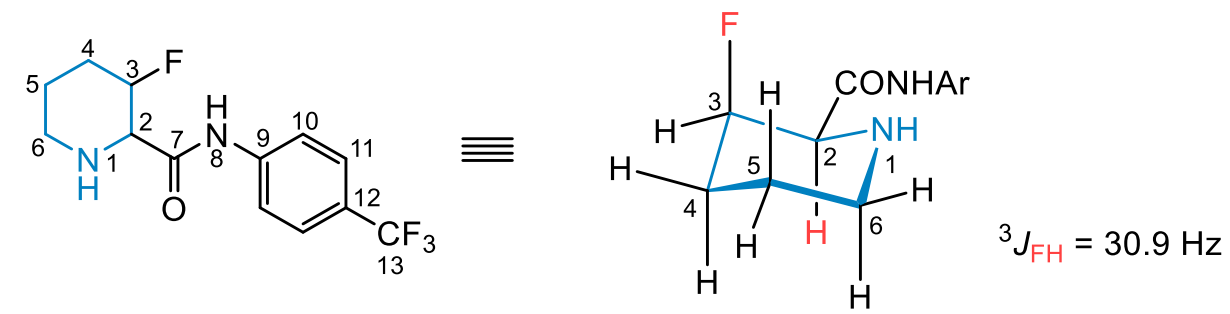

3-Fluoro-N-(4-(trifluoromethyl)phenyl)piperidine-2-carboxamide (27): The title compound was synthesized according to GP-A using 10 mol\% Pd-catalyst $(35.1 \mathrm{mg})$ in $\mathrm{THF} / \mathrm{H}_{2} \mathrm{O}(0.5 \mathrm{M}, 1: 1)$ with aq. $\mathrm{HCl}(100 \mu \mathrm{l}, 2.4$ equiv, $12 \mathrm{~N})$. The product was isolated as a white solid (75 mg, $0.26 \mathrm{mmol}, 52 \%,>95: 5$ d.r.) after column chromatography (silical gel, eluent: $\mathrm{CH}_{2} \mathrm{Cl}_{2}$, later $99: 1,98: 2 \mathrm{CH}_{2} \mathrm{Cl}_{2} / \mathrm{MeOH}$ ). The axial position of the fluorine substituent was determined based on the ${ }^{3} \mathrm{JFH}_{\mathrm{FH}}$ coupling between FC3 and $\mathrm{Hax}_{\mathrm{a} 2}$ and is in accordance with previous literature results. ${ }^{3}$

${ }^{1} \mathbf{H}$ NMR (599 MHz, Chloroform-d) $\delta 8.92(\mathrm{~s}, 1 \mathrm{H}, \mathrm{HN} 8), 7.70\left(\mathrm{~d},{ }^{3} \mathrm{~J}_{\mathrm{HH}}=8.5 \mathrm{~Hz}, 2 \mathrm{H}\right.$, HC10), $7.57\left(\mathrm{~d},{ }^{3} \mathrm{JHH}_{\mathrm{HH}}=8.5 \mathrm{~Hz}, 2 \mathrm{H}, \mathrm{HC} 11\right), 5.27\left(\mathrm{dm},{ }^{2} \mathrm{~J}_{\mathrm{FH}}=47.9 \mathrm{~Hz}, 1 \mathrm{H}, H_{\mathrm{eqC}}\right), 3.36$ $\left(\mathrm{dd},{ }^{3} \mathrm{~J}_{\mathrm{FH}}=30.9 \mathrm{~Hz},{ }^{3} \mathrm{JHH}_{\mathrm{HH}}=1.3 \mathrm{~Hz}, 1 \mathrm{H}, \mathrm{HaxC}_{2}\right), 3.23$ (dddd, ${ }^{2} \mathrm{JHH}_{\mathrm{HH}}=13.1 \mathrm{~Hz},{ }^{3} \mathrm{JHH}_{\mathrm{HH}}=$ $4.2 \mathrm{~Hz},{ }^{3} \mathrm{JHH}_{\mathrm{HH}}=2.0 \mathrm{~Hz},{ }^{4} \mathrm{~J}_{\mathrm{HH}}=2.0 \mathrm{~Hz}, 1 \mathrm{H}, \mathrm{Heq}_{\mathrm{eq}}$ ), 2.73 (dddd, ${ }^{2} J_{\mathrm{HH}}=12.9 \mathrm{~Hz},{ }^{3} \mathrm{JHH}_{\mathrm{HH}}=$ $\left.12.9 \mathrm{~Hz},{ }^{3} \mathrm{JHH}_{\mathrm{HH}}=3.0 \mathrm{~Hz},{ }^{5} \mathrm{JFH}_{\mathrm{FH}} 1.7 \mathrm{~Hz}, 1 \mathrm{H}, \mathrm{H}_{\mathrm{ax}} \mathrm{C} 6\right), 2.32-2.21\left(\mathrm{~m}, 1 \mathrm{H}, \mathrm{H}_{2} \mathrm{C} 4\right), 1.84-$ $1.60\left(\mathrm{~m}, 3 \mathrm{H}, \mathrm{H}_{2} \mathrm{C} 5, \mathrm{HN} 1, \mathrm{H}_{2} \mathrm{C} 4\right), 1.59-1.53\left(\mathrm{~m}, 1 \mathrm{H}, \mathrm{H}_{2} \mathrm{C} 5\right) .{ }^{1} \mathrm{H}\left\{{ }^{19} \mathrm{~F}\right\}$ NMR $(599 \mathrm{MHz}$, Chloroform-d) $\delta 8.92(\mathrm{~s}, 1 \mathrm{H}, \mathrm{HN} 8), 7.70\left(\mathrm{~d},{ }^{3} \mathrm{JHH}_{\mathrm{HH}}=8.4 \mathrm{~Hz}, 2 \mathrm{H}, H \mathrm{C} 10\right), 7.57$ (d, ${ }^{3} \mathrm{~J}_{\mathrm{HH}}=$ $8.5 \mathrm{~Hz}, 2 \mathrm{H}, \mathrm{HC} 11), 5.28-5.26\left(\mathrm{~m}, 1 \mathrm{H}, H_{\mathrm{eq}} \mathrm{C} 3\right), 3.36\left(\mathrm{~d},{ }^{3} \mathrm{~J}_{\mathrm{HH}}=1.3 \mathrm{~Hz}, 1 \mathrm{H}, H_{\mathrm{ax}} \mathrm{C} 2\right)$, 3.22 (dddd, ${ }^{2} J_{H H}=13.1 \mathrm{~Hz},{ }^{3} J_{H H}=4.2 \mathrm{~Hz},{ }^{3} J_{H H}=2.0 \mathrm{~Hz},{ }^{4} J_{H H}=2.0 \mathrm{~Hz}, 1 \mathrm{H}, H_{\text {eqC6) }}$, $2.73\left(\mathrm{ddd},{ }^{2} \mathrm{~J}_{\mathrm{HH}}=12.8 \mathrm{~Hz},{ }^{3} \mathrm{JHH}_{\mathrm{HH}} 12.8 \mathrm{~Hz},{ }^{3} J_{\mathrm{HH}}=3.0 \mathrm{~Hz}, 1 \mathrm{H}, H_{\mathrm{ax}} \mathrm{C6}\right), 2.25\left(\mathrm{dm},{ }^{2} J_{\mathrm{HH}}=\right.$ $\left.14.5 \mathrm{~Hz}, 1 \mathrm{H}, \mathrm{H}_{2} \mathrm{C} 4\right), 1.79\left(\mathrm{dtt},{ }^{2} \mathrm{JHH}_{\mathrm{HH}} 13.0 \mathrm{~Hz},{ }^{3} \mathrm{~J}_{\mathrm{HH}}=13.0 \mathrm{~Hz},{ }^{3} J_{\mathrm{HH}}=4.2 \mathrm{~Hz}, 1 \mathrm{H}, H_{2} \mathrm{C} 5\right)$, $1.73(\mathrm{~s}, 1 \mathrm{H}, \mathrm{HN} 1), 1.71-1.64\left(\mathrm{~m}, 1 \mathrm{H}, \mathrm{H}_{2} \mathrm{C} 4\right), 1.56\left(\mathrm{dm},{ }^{2} \mathrm{JHH}_{\mathrm{HH}}=13.3 \mathrm{~Hz}, 1 \mathrm{H}, \mathrm{H}_{2} \mathrm{C} 5\right)$. ${ }^{13} \mathrm{C}\left\{{ }^{1} \mathrm{H}\right\}$ NMR (151 MHz, Chloroform-d) $\delta 168.6$ (d, $\left.{ }^{3} \mathrm{JFC}=1.4 \mathrm{~Hz}, \mathrm{C} 7\right), 140.7-140.5$ (m, C9), $126.4\left(q,{ }^{3} \mathrm{JFC}=3.8 \mathrm{~Hz}, \mathrm{C} 11\right), 126.2\left(\mathrm{q},{ }^{2} \mathrm{JFC}=32.8 \mathrm{~Hz}, \mathrm{C} 12\right), 124.2\left(\mathrm{q},{ }^{1} \mathrm{JFC}=\right.$ $271.5 \mathrm{~Hz}, \mathrm{C} 13), 119.4(\mathrm{C} 10), 85.8\left(\mathrm{~d},{ }^{1} \mathrm{~J}_{\mathrm{FC}}=173.4 \mathrm{~Hz}, \mathrm{C} 3\right), 63.2\left(\mathrm{~d},{ }^{2} \mathrm{~J}_{\mathrm{FC}}=21.1 \mathrm{~Hz}\right.$, C2), 46.1 (C6), 29.2 (d, $\left.{ }^{2} \mathrm{JFC}_{\mathrm{FC}}=21.3 \mathrm{~Hz}, \mathrm{C} 4\right), 20.6$ (C5). ${ }^{13} \mathrm{C}\left\{{ }^{1} \mathrm{H}\right.$,sel- ${ }^{19} \mathrm{~F}$ at $\left.-62 \mathrm{ppm}\right\}$ NMR (151 MHz, Chloroform-d) $\delta 168.6$ (d, ${ }^{3} J_{F C}=1.4$ Hz, C7), 140.6 (C9), 126.4 (C11), 126.2 (C12) 124.2 (C13), 119.4 (C10), 85.8 (d, $\left.{ }^{1} J_{F C}=173.3 \mathrm{~Hz}, \mathrm{C} 3\right), 63.2\left(\mathrm{~d},{ }^{2} \mathrm{JFC}_{\mathrm{FC}}\right.$ $21.1 \mathrm{~Hz}, \mathrm{C} 2), 46.1$ (C6), 29.2 (d, $\left.{ }^{2} \mathrm{JFC}_{\mathrm{FC}}=21.2 \mathrm{~Hz}, \mathrm{C} 4\right), 20.6$ (C5). ${ }^{19} \mathrm{~F}$ NMR (282 MHz, 
Chloroform- $d$ ) $\delta$-62.1 (3F, $\left.F_{3} \mathrm{C} 13\right),-198.4--199.2(\mathrm{~m}, 1 \mathrm{~F}, \mathrm{FC} 3) .{ }^{19} \mathrm{~F}\left\{{ }^{1} \mathrm{H}\right\}$ NMR $(282$ $\mathrm{MHz}$, Chloroform- $d$ ) $\delta-62.1$ (3F, $\left.F_{3} \mathrm{C} 13\right),-198.8(1 \mathrm{~F}, F C 3)$. HRMS (ESI) $\mathrm{m} / \mathrm{z}$ calculated for $\left[\mathrm{C}_{13} \mathrm{H}_{15} \mathrm{~F}_{4} \mathrm{~N}_{2} \mathrm{O}\right]\left(\left[\mathrm{M}+\mathrm{H}^{+}\right]\right)$291.1115, found 291.1137.
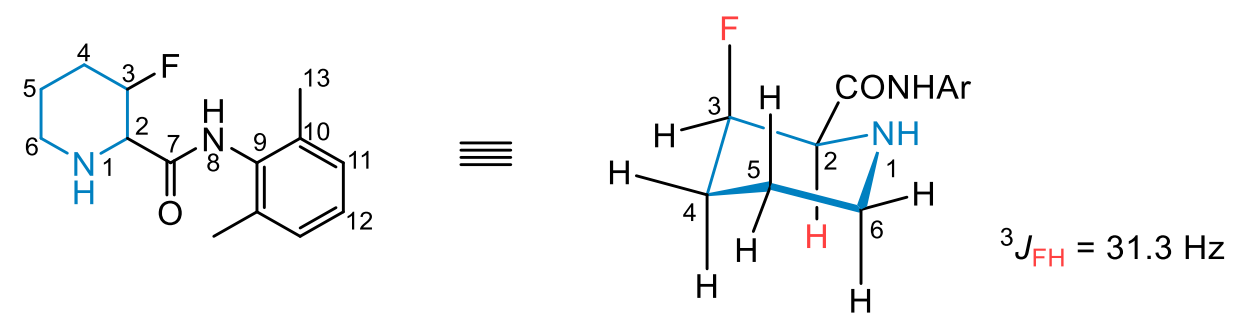

$\mathbf{N}$-(2,6-Dimethylphenyl)-3-fluoropiperidine-2-carboxamide (28): The title compound was synthesized according to GP-A using $10 \mathrm{~mol} \% \mathrm{Pd}$-catalyst $(35.1 \mathrm{mg}$ ) in $\mathrm{THF} / \mathrm{H}_{2} \mathrm{O}(0.5 \mathrm{M}, 1: 1)$ with aq. $\mathrm{HCl}(100 \mu \mathrm{l}, 2.4$ equiv, $12 \mathrm{~N})$. The product was isolated as a white solid (69 mg, $0.28 \mathrm{mmol}, 55 \%,>95: 5$ d.r.) after column chromatography (silical gel, eluent: $\mathrm{CH}_{2} \mathrm{Cl}_{2}$, later $99: 1,98: 2 \mathrm{CH}_{2} \mathrm{Cl}_{2} / \mathrm{MeOH}$ ). The axial position of the fluorine substituent was determined based on the ${ }^{3} \mathrm{FH}$ coupling between $\mathrm{FC} 3$ and $\mathrm{HaxC}_{2}$ and is in accordance with previous literature results. ${ }^{3}$

'H NMR (500 MHz, Chloroform-d) $\delta 8.17$ (s, 1H, HN8), $7.11-7.04(\mathrm{~m}, 3 \mathrm{H}, H \mathrm{C} 11$, $H C 12), 5.21\left(\mathrm{dm},{ }^{2} \mathrm{JFH}_{\mathrm{FH}}=48.0 \mathrm{~Hz}, 1 \mathrm{H}, H_{\mathrm{eq}} \mathrm{C} 3\right), 3.43\left(\mathrm{dd},{ }^{3} \mathrm{JFH}_{\mathrm{FH}}=31.3 \mathrm{~Hz},{ }^{3} \mathrm{JHH}_{\mathrm{HH}}=1.5 \mathrm{~Hz}\right.$, $\left.1 \mathrm{H}, H_{\mathrm{ax}} \mathrm{C} 2\right), 3.19\left(\mathrm{dm},{ }^{2} \mathrm{JHH}_{\mathrm{HH}}=12.8 \mathrm{~Hz}, 1 \mathrm{H}, H_{\mathrm{eq}} \mathrm{C}\right.$ ), 2.74 (dddd, ${ }^{2} \mathrm{JHH}_{\mathrm{HH}}=12.7 \mathrm{~Hz},{ }^{3} \mathrm{JHH}_{\mathrm{HH}}$ $\left.=12.7 \mathrm{~Hz},{ }^{3} \mathrm{JHH}=2.9 \mathrm{~Hz},{ }^{5} \mathrm{JFH}=1.7 \mathrm{~Hz}, 1 \mathrm{H}, H_{\mathrm{ax}} \mathrm{C} 6\right), 2.26-2.17\left(\mathrm{~m}, 1 \mathrm{H}, H_{\mathrm{eq}} \mathrm{C} 4\right), 2.21$ (s, 6H, $\left.H_{3} \mathrm{C} 13\right), 1.92-1.75\left(\mathrm{~m}, 2 \mathrm{H}, H_{\mathrm{N} 1}, H_{2} \mathrm{C} 5\right), 1.74-1.58\left(\mathrm{dm},{ }^{3} \mathrm{JFH}=43.9 \mathrm{~Hz}, 1 \mathrm{H}\right.$, $\left.H_{\mathrm{ax}} \mathrm{C} 4\right), 1.57-1.51\left(\mathrm{~m}, 1 \mathrm{H}, H_{2} \mathrm{C} 5\right) .{ }^{1} \mathrm{H}\left\{{ }^{19} \mathrm{~F}\right\}$ NMR $(500 \mathrm{MHz}$, Chloroform- $d) \delta 8.17(\mathrm{~s}$, $1 \mathrm{H}, H \mathrm{~N} 8), 7.12-7.03(\mathrm{~m}, 3 \mathrm{H}, H \mathrm{C} 11, H \mathrm{C} 12), 5.23-5.19\left(\mathrm{~m}, 1 \mathrm{H}, H_{\mathrm{eq}} \mathrm{C}\right), 3.43\left(\mathrm{~d},{ }^{3} \mathrm{JHH}_{\mathrm{HH}}\right.$ $\left.=1.5 \mathrm{~Hz}, 1 \mathrm{H}, H_{\mathrm{ax}} \mathrm{C} 2\right), 3.19\left(\mathrm{dm},{ }^{2} \mathrm{JHH}_{1}=12.8 \mathrm{~Hz}, 1 \mathrm{H}, H_{\mathrm{eq}} \mathrm{C} 6\right), 2.74$ (ddd, ${ }^{2} \mathrm{JHH}_{\mathrm{H}}=12.7 \mathrm{~Hz}$, $\left.{ }^{3} \mathrm{JHH}_{\mathrm{HH}}=12.7 \mathrm{~Hz},{ }^{3} \mathrm{JHH}_{\mathrm{HH}}=2.9 \mathrm{~Hz}, 1 \mathrm{H}, H_{\mathrm{ax}} \mathrm{C} 6\right), 2.26-2.17\left(\mathrm{~m}, 1 \mathrm{H}, \mathrm{H}_{\mathrm{eq}} \mathrm{C} 4\right), 2.21(\mathrm{~s}, 6 \mathrm{H}$, $\left.\mathrm{H}_{3} \mathrm{C} 13\right), 1.89(\mathrm{~s}, 1 \mathrm{H}, H \mathrm{~N} 1), 1.81\left(\mathrm{dtt},{ }^{2} \mathrm{JHH}=13.2 \mathrm{~Hz},{ }^{3} \mathrm{JHH}_{\mathrm{HH}}=13.2 \mathrm{~Hz},{ }^{3} \mathrm{JHH}=4.2 \mathrm{~Hz}, 1 \mathrm{H}\right.$, $\left.\mathrm{H}_{2} \mathrm{C} 5\right), 1.71-1.62\left(\mathrm{~m}, 1 \mathrm{H}, \mathrm{H}_{\mathrm{ax}} \mathrm{C} 4\right), 1.58-1.51\left(\mathrm{~m}, 1 \mathrm{H}, \mathrm{H}_{2} \mathrm{C} 5\right) .{ }^{13} \mathrm{C}\left\{{ }^{1} \mathrm{H}\right\} \mathrm{NMR}(126 \mathrm{MHz}$, Chloroform-d) $\delta 168.6$ (d, $\left.{ }^{3} J_{F C}=1.4 \mathrm{~Hz}, \mathrm{C} 7\right), 135.3$ (C10), 133.6 (C9), 128.2 (C11), $127.2(\mathrm{C} 12), 86.4\left(\mathrm{~d},{ }^{1} \mathrm{~J}_{F C}=173.6 \mathrm{~Hz}, \mathrm{C} 3\right), 63.1\left(\mathrm{~d},{ }^{2} \mathrm{~J}_{F C}=21.0 \mathrm{~Hz}, \mathrm{C} 2\right), 45.9(\mathrm{C} 6), 29.2$ $\left.\left(\mathrm{d},{ }^{2} J_{F C}=21.5 \mathrm{~Hz}, \mathrm{C} 4\right)\right), 20.5(\mathrm{C} 5), 18.5\left(\mathrm{~d},{ }^{7} J_{F C}=1.0 \mathrm{~Hz}, \mathrm{C} 13\right) .{ }^{13} \mathrm{C}\left\{{ }^{1} \mathrm{H},{ }^{19} \mathrm{~F}\right\}$ NMR $(126$ $\mathrm{MHz}$, Chloroform-d) $\delta 168.6$ (C7), 135.3 (C10), 133.6 (C9), 128.2 (C11), 127.2 (C12), 86.4 (C3), 63.1 (C2), 45.9 (C6), 29.2 (C4), 20.5 (C5), 18.5 (C13). ${ }^{19} \mathrm{~F}$ NMR (470 MHz, Chloroform-d) $\delta-197.8$ (dddm, $\left.{ }^{2} \mathrm{JHF}=47.3 \mathrm{~Hz},{ }^{3} \mathrm{JHF}=42.6 \mathrm{~Hz},{ }^{3}{ }_{\mathrm{HF}}=31.5 \mathrm{~Hz}\right) .{ }^{19} \mathrm{~F}\left\{{ }^{1} \mathrm{H}\right\}$ 
NMR (376 MHz, Chloroform- $d$ ) $\delta$-197.8. HRMS (ESI) m/z calculated for [ $\left.\mathrm{C}_{14} \mathrm{H}_{20} \mathrm{FN}{ }_{2} \mathrm{O}\right]$ $\left(\left[\mathrm{M}+\mathrm{H}^{+}\right]\right) 251.1554$, found 251.1567.
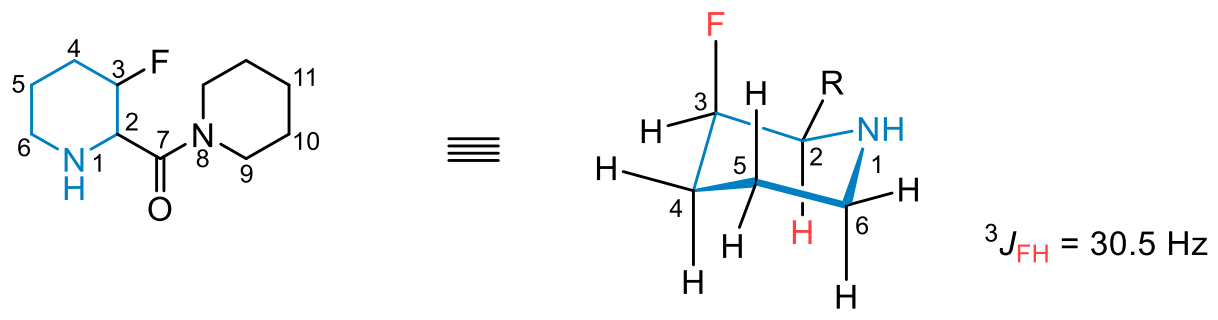

(3-Fluoropiperidin-2-yl)(piperidin-1-yl)methanone (29): The title compound was synthesized according to GP-A using $10 \mathrm{~mol} \%$ Pd-catalyst $(35.1 \mathrm{mg})$ in $\mathrm{THF} / \mathrm{H}_{2} \mathrm{O}$ $(0.5 \mathrm{M}, 1: 1)$ with aq. $\mathrm{HCl}(100 \mu \mathrm{l}, 2.4$ equiv, $12 \mathrm{~N})$. The product was isolated as a white solid (36 mg, $0.17 \mathrm{mmol}, 36 \%,>95: 5$ d.r.) after column chromatography (silical gel, eluent: $\mathrm{CH}_{2} \mathrm{Cl}_{2}$, later 99:1, 98:2 $\mathrm{CH}_{2} \mathrm{Cl}_{2} / \mathrm{MeOH}$ ). The axial position of the fluorine substituent was determined based on the ${ }^{3} \mathrm{JFH}_{\mathrm{FH}}$ coupling between $\mathrm{FC} 3$ and $\mathrm{Hax}_{\mathrm{ax}} \mathrm{C}$ and is in accordance with previous literature results. ${ }^{3}$

${ }^{1} \mathrm{H}$ NMR $(599 \mathrm{MHz}$, Chloroform- $d) \delta 4.70\left(\mathrm{dm},{ }^{2} \mathrm{JFH}_{\mathrm{FH}}=48.1 \mathrm{~Hz}, 1 \mathrm{H}, H_{\mathrm{eq}} \mathrm{C} 3\right), 3.86\left(\mathrm{~d},{ }^{3} \mathrm{JFH}_{\mathrm{FH}}\right.$ $=30.5 \mathrm{~Hz}, 1 \mathrm{H}, H_{\mathrm{ax}} \mathrm{C} 2$ ), 3.66 (bs, $1 \mathrm{H}, H_{2} \mathrm{C} 9$ ), $3.57-3.31$ (m, 3H, $\mathrm{H}_{2} \mathrm{C} 9, \mathrm{H}_{2} \mathrm{C} 9$ ) $), 3.22-$ $3.15\left(\mathrm{~m}, 1 \mathrm{H}, \mathrm{H}_{2} \mathrm{C} 6\right), 2.85-2.68\left(\mathrm{~m}, 2 \mathrm{H}, \mathrm{H}_{2} \mathrm{C} 6, H \mathrm{~N} 1\right), 2.23-2.16\left(\mathrm{~m}, 1 \mathrm{H}, \mathrm{H}_{2} \mathrm{C} 4\right), 1.79$ $-1.49\left(\mathrm{~m}, 8 \mathrm{H}, \mathrm{H}_{2} \mathrm{C} 5, \mathrm{H}_{2} \mathrm{C} 4, \mathrm{H}_{2} \mathrm{C} 10, \mathrm{H}_{2} \mathrm{C} 10\right.$ ', $\left.\mathrm{H}_{2} \mathrm{C} 11\right), 1.46-1.38\left(\mathrm{~m}, 1 \mathrm{H}, \mathrm{H}_{2} \mathrm{C} 5\right) .{ }^{1} \mathrm{H}\left\{{ }^{19} \mathrm{~F}\right\}$ NMR $(599 \mathrm{MHz}$, Chloroform- $d) \delta 4.74-4.66\left(\mathrm{~m}, 1 \mathrm{H}, H_{\mathrm{eq}} \mathrm{C} 3\right), 3.86\left(\mathrm{~m}, 1 \mathrm{H}, \mathrm{Hax}_{\mathrm{ax}}\right), 3.70$ $\left.-3.63\left(\mathrm{~m}, 1 \mathrm{H}, \mathrm{H}_{2} \mathrm{C} 9\right), 3.55-3.30\left(\mathrm{~m}, 3 \mathrm{H}, \mathrm{H}_{2} \mathrm{C} 9, \mathrm{H}_{2} \mathrm{C}\right)^{\prime}\right), 3.18\left(\mathrm{dm},{ }^{2} \mathrm{HH}=14.0 \mathrm{~Hz}, 1 \mathrm{H}\right.$, $\left.\mathrm{H}_{2} \mathrm{C} 6\right), 2.85-2.70$ (m, 2H, $\left.\mathrm{H}_{2} \mathrm{C} 6, H \mathrm{~N} 1\right), 2.26-2.14$ (m, $\left.1 \mathrm{H}, \mathrm{H}_{2} \mathrm{C} 4\right), 1.79-1.51$ (m, $\left.8 \mathrm{H}, \mathrm{H}_{2} \mathrm{C} 5, \mathrm{H}_{2} \mathrm{C} 4, \mathrm{H}_{2} \mathrm{C} 10, \mathrm{H}_{2} \mathrm{C} 10, \mathrm{H}_{2} \mathrm{C} 11\right), 1.46-1.39\left(\mathrm{~m}, 1 \mathrm{H}, \mathrm{H}_{2} \mathrm{C} 5\right) .{ }^{13} \mathrm{C}\left\{{ }^{1} \mathrm{H}\right\}$ NMR (151 MHz, Chloroform- $d$ ) $\delta 167.7(\mathrm{C} 7), 85.9\left(\mathrm{~d},{ }^{1} \mathrm{JFC}=177.2 \mathrm{~Hz}, \mathrm{C} 3\right), 58.1\left(\mathrm{~d},{ }^{2} \mathrm{JFC}=\right.$ $20.2 \mathrm{~Hz}, \mathrm{C} 2$ ), 46.5 (C9'), 44.4 (C6), 43.4 (C9), 29.6 (d, ' $\left.{ }^{\mathrm{FFC}}=21.4 \mathrm{~Hz}, \mathrm{C} 4\right), 26.6$ (C10'), 25.6 (C10), 24.7 (C11), 20.2 (C5). ${ }^{13} \mathbf{C}\left\{{ }^{1} \mathrm{H},{ }^{19} \mathrm{~F}\right\}$ NMR (151 MHz, Chloroform-d) $\delta 167.7$ (C7), 85.9 (C3), 58.1 (C2), 46.5 (C9'), 44.4 (C6), 43.3 (C9), 29.6 (C4), 26.6 (C10'), 25.6 (C10), 24.7 (C11), 20.2 (C5). ${ }^{19} \mathrm{~F}$ NMR (564 MHz, Chloroform- $\left.d\right) \delta-199.5(\mathrm{~m}) .{ }^{19} \mathrm{~F}\left\{{ }^{1} \mathrm{H}\right\}$ NMR (282 MHz, Chloroform- $d) \delta-199.6$. HRMS (ESI) $\mathrm{m} / \mathrm{z}$ calculated for $\left[\mathrm{C}_{11} \mathrm{H}_{20} \mathrm{FN} \mathrm{N}_{2} \mathrm{O}\right]$ $\left(\left[\mathrm{M}+\mathrm{H}^{+}\right]\right)$215.1554, found 215.1577. 


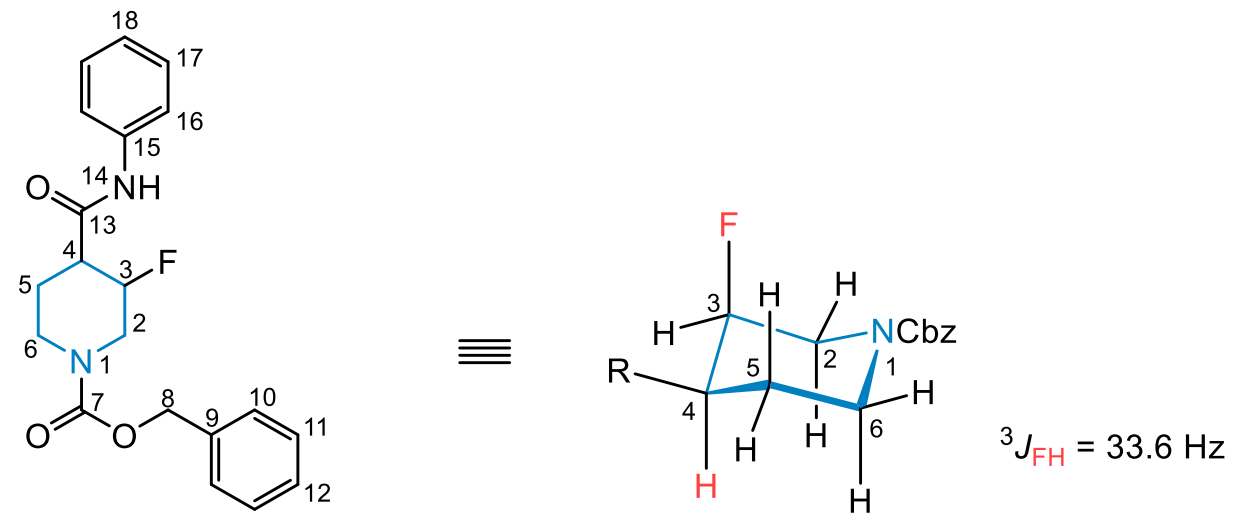

Benzyl 3-fluoro-4-(phenylcarbamoyl)piperidine-1-carboxylate (30): The title compound was synthesized according to GP-B using $5 \mathrm{~mol} \%$ Pd-catalyst $(17.6 \mathrm{mg})$ in $\mathrm{MeOH}(0.5 \mathrm{M})$ with aq. $\mathrm{HCl}(100 \mu \mathrm{l}, 2.4$ equiv, $12 \mathrm{~N})$. The product was isolated as a white solid ( $88 \mathrm{mg}, 0.25 \mathrm{mmol}, 50 \%$, $>95: 5$ d.r.) after column chromatography (silical gel, eluent: $\mathrm{CH}_{2} \mathrm{Cl}_{2}$, later $99: 1,98: 2 \mathrm{CH}_{2} \mathrm{Cl}_{2} / \mathrm{MeOH}$ ). The axial position of the fluorine substituent was determined based on the ${ }^{3} \mathrm{~J}_{\mathrm{FH}}$ coupling between $\mathrm{FC} 3$ and $\mathrm{H}_{\mathrm{ax}} \mathrm{C} 2$ and is in accordance with previous literature results. ${ }^{3}$

${ }^{1} \mathrm{H}$ NMR $\left(500 \mathrm{MHz}\right.$, Chloroform-d) $\delta 8.17-7.96(\mathrm{~m}, 1 \mathrm{H}, H \mathrm{~N} 14), 7.51\left(\mathrm{~d},{ }^{3} \mathrm{JHH}_{\mathrm{HH}}=7.9 \mathrm{~Hz}\right.$, $1 \mathrm{H}, H \mathrm{C} 16), 7.39-7.27(\mathrm{~m}, 7 \mathrm{H}, H \mathrm{C} 10, H \mathrm{C} 11, H \mathrm{C} 12, H \mathrm{C} 17), 7.11$ (tt, ${ }^{3} \mathrm{JHH}_{\mathrm{HH}}=7.3 \mathrm{~Hz}$, $\left.{ }^{4} \mathrm{JHH}_{\mathrm{H}}=1.2 \mathrm{~Hz}, 1 \mathrm{H}, H \mathrm{C} 18\right), 5.23-5.10\left(\mathrm{~m}, 2 \mathrm{H}, \mathrm{H}_{2} \mathrm{C} 8\right), 5.03\left(\mathrm{dm},{ }^{2} \mathrm{JFH}=47.5 \mathrm{~Hz}, 1 \mathrm{H}\right.$, $\left.\mathrm{H}_{\mathrm{eq}} \mathrm{C} 3\right), 4.55-4.38\left(\mathrm{~m}, 1 \mathrm{H}, \mathrm{H}_{2} \mathrm{C} 2\right), 4.36-4.21\left(\mathrm{~m}, 1 \mathrm{H}, \mathrm{H}_{2} \mathrm{C} 6\right), 3.11-2.73(\mathrm{~m}, 2 \mathrm{H}$, $\mathrm{H}_{2} \mathrm{C} 2, \mathrm{H}_{2} \mathrm{C} 6$ ), 2.60 (dddd, ${ }^{3} \mathrm{JFH}_{\mathrm{FH}}=33.6 \mathrm{~Hz},{ }^{3} \mathrm{JHH}_{\mathrm{HH}}=12.4 \mathrm{~Hz},{ }^{3} \mathrm{JHH}_{\mathrm{HH}}=4.3 \mathrm{~Hz},{ }^{3} \mathrm{JHH}_{\mathrm{HH}}=2.1 \mathrm{~Hz}$, $\left.1 \mathrm{H}, H_{\mathrm{ax}} \mathrm{C} 4\right), 2.10\left(\mathrm{dtd},{ }^{2} \mathrm{JHH}_{\mathrm{HH}}=12.4 \mathrm{~Hz},{ }^{3} \mathrm{JHH}_{\mathrm{HH}}=12.4 \mathrm{~Hz},{ }^{3} \mathrm{JHH}_{\mathrm{HH}}=4.3 \mathrm{~Hz}, 1 \mathrm{H}, H_{\mathrm{axC}} \mathrm{C}\right.$ ), 1.82 $\left(\mathrm{dm},{ }^{2} \mathrm{JHH}=12.6 \mathrm{~Hz}, 1 \mathrm{H}, H_{\mathrm{eq}} \mathrm{C} 5\right) .{ }^{1} \mathrm{H}\left\{{ }^{19} \mathrm{~F}\right\}$ NMR $(500 \mathrm{MHz}$, Chloroform-d) $\delta 8.15-7.99$ (m, 1H, HN14), $7.51\left(\mathrm{~d},{ }^{3} \mathrm{JHH}_{\mathrm{HH}}=7.9 \mathrm{~Hz}, 1 \mathrm{H}, H \mathrm{C} 16\right), 7.38-7.27$ (m, 7H, HC10, HC11, HC12, HC17), 7.11 (tt, $\left.{ }^{3} \mathrm{JHH}_{\mathrm{HH}}=7.4 \mathrm{~Hz},{ }^{4} \mathrm{JHH}_{\mathrm{HH}}=1.2 \mathrm{~Hz}, 1 \mathrm{H}, H \mathrm{C} 18\right), 5.21-5.08(\mathrm{~m}, 2 \mathrm{H}$, $\left.\mathrm{H}_{2} \mathrm{C} 8\right), 5.03\left(\mathrm{~m}, 1 \mathrm{H}, \mathrm{H}_{\mathrm{eq}} \mathrm{C} 3\right), 4.53-4.40\left(\mathrm{~m}, 1 \mathrm{H}, \mathrm{H}_{2} \mathrm{C} 2\right), 4.38-4.19\left(\mathrm{~m}, 1 \mathrm{H}, \mathrm{H}_{2} \mathrm{C} 6\right)$, $3.06-2.75\left(\mathrm{~m}, 2 \mathrm{H}, \mathrm{H}_{2} \mathrm{C} 2, \mathrm{H}_{2} \mathrm{C} 6\right), 2.60$ (ddd, ${ }^{3} \mathrm{HHH}_{\mathrm{HH}}=12.3 \mathrm{~Hz},{ }^{3} \mathrm{~J}_{\mathrm{HH}}=4.3 \mathrm{~Hz},{ }^{3} \mathrm{JHH}_{\mathrm{HH}}=2.0$ $\left.\mathrm{Hz}, 1 \mathrm{H}, H_{\mathrm{ax}} \mathrm{C} 4\right), 2.10$ (dtd, ${ }^{2} \mathrm{JHH}_{\mathrm{HH}}=12.4 \mathrm{~Hz},{ }^{3} \mathrm{JHH}_{\mathrm{HH}}=12.4 \mathrm{~Hz},{ }^{3} \mathrm{JHH}_{\mathrm{HH}}=4.3 \mathrm{~Hz}, 1 \mathrm{H}, H_{\mathrm{ax}} \mathrm{C} 5$ ), $1.82\left(\mathrm{dm},{ }^{2}{ }^{\mathrm{HH}}=12.6 \mathrm{~Hz}, 1 \mathrm{H}, H_{\mathrm{eq}} \mathrm{C} 5\right) .{ }^{13} \mathrm{C}\left\{{ }^{1} \mathrm{H}\right\} \mathrm{NMR}(126 \mathrm{MHz}$, Chloroform-d) $\delta 169.4$ (C13), 155.6 (C7), 137.6 (C15), 136.5 (C9), 129.0 (C17), 128.6 (C11), 128.2 (C12), 127.8 (C10), 124.8 (C18), 120.6 (C16), 86.5 (d, $\left.{ }^{1} \mathrm{JFC}=175.6 \mathrm{~Hz}, \mathrm{C} 3\right), 67.5$ (C8), 47.7 - 47.0 (m, C2, C4), 42.8 (C6), $23.2-22.5$ (m, C5). ${ }^{13} \mathrm{C}\left\{{ }^{1} \mathrm{H},{ }^{19} \mathrm{~F}\right\}$ NMR $(126 \mathrm{MHz}$, Chloroform- $d$ ) $\delta 169.4$ (C13), 155.6 (C7), 137.6 (C15), 136.5 (C9), 129.0 (C17), 128.6 (C11), 128.2 (C12), 127.8 (C10), 124.8 (C18), 120.6 (C16), 86.5 (C3), 67.5 (C8), 47.3 (C2), 47.2 (C4), 42.8 (C6), $23.4-22.6$ (m, C5). ${ }^{19} \mathrm{~F}$ NMR (470 MHz, Chloroform- $d$ ) $\delta$ - 
195.2 - -195.7 (m). ${ }^{19} \mathbf{F}\left\{{ }^{1} \mathrm{H}\right\}$ NMR (376 MHz, Chloroform- $d$ ) $\delta$-195.5. HRMS (ESI) $\mathrm{m} / \mathrm{z}$ calculated for $\left[\mathrm{C}_{20} \mathrm{H}_{21} \mathrm{FN}_{2} \mathrm{O}_{3} \mathrm{Na}\right]\left(\left[\mathrm{M}+\mathrm{Na}^{+}\right]\right) 379.1428$, found 379.1423 .
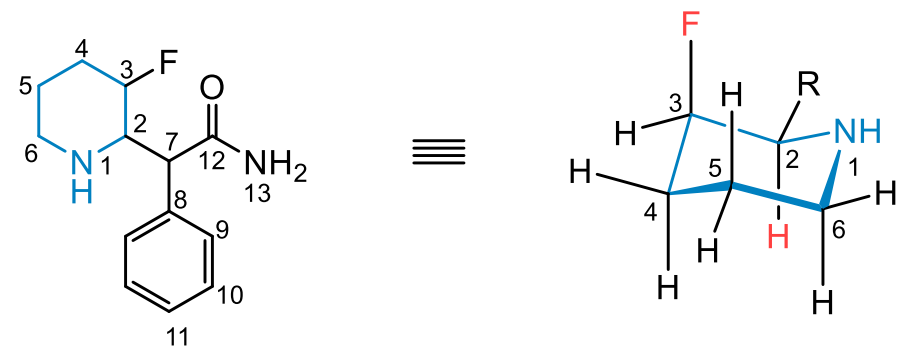

${ }^{3} J_{\mathrm{FH}}=27.9 \mathrm{~Hz}$

2-(3-Fluoropiperidin-2-yl)-2-phenylacetamide (31): The title compound was synthesized on a $1.5 \mathrm{mmol}$ scale according to GP-A using $10 \mathrm{~mol} \%$ Pd-catalyst $(105 \mathrm{mg})$ in $\mathrm{MeOH}(0.5 \mathrm{M})$ with aq. $\mathrm{HCl}(300 \mu \mathrm{l}, 2.4$ equiv, $12 \mathrm{~N})$. The product was isolated as a white solid (185 $\mathrm{mg}, 0.78 \mathrm{mmol}, 52 \%, 71: 29:<5$ d.r.) after column chromatography (silica gel, eluent: $\mathrm{CH}_{2} \mathrm{Cl}_{2}$, later 99:1, 98:2, 95:5 $\mathrm{CH}_{2} \mathrm{Cl}_{2} / \mathrm{MeOH}$ ). Only two diastereomers were observed and isolated. For both diastereomers, the substituents on the piperidine-ring are cis oriented. X-ray analysis revealed the erythro isomer as the main diastereomer. Only the analytical data of the main diastereomer are listed. The axial position of the fluorine substituent was determined based on the ${ }^{3} \mathrm{~J}_{\mathrm{FH}}$ coupling between $\mathrm{FC} 3$ and $\mathrm{H}_{\mathrm{ax}} \mathrm{C} 2$ and is in accordance with previous literature results. ${ }^{3}$

${ }^{1} \mathrm{H}$ NMR (500 MHz, Chloroform- $\left.d\right) \delta 7.46-7.41$ (m, 2H, HC9), $7.37-7.28(\mathrm{~m}, 3 \mathrm{H}$, HC10, HC11), 5.80 (s, 1H, $H_{2} \mathrm{~N} 13$ ), 5.48 (s, $1 \mathrm{H}, H_{2} \mathrm{~N} 13$ ), 4.89 (d, ${ }^{2} \mathrm{JFH}_{\mathrm{FH}}=49.2 \mathrm{~Hz}, 1 \mathrm{H}$, $H_{\mathrm{eq}} \mathrm{C}$ ), 3.60 (d, ${ }^{3} \mathrm{JHH}_{\mathrm{HH}}=10.2 \mathrm{~Hz}, 1 \mathrm{H}, \mathrm{HC}$ ), 3.28 (ddd, ${ }^{3} \mathrm{~J}_{\mathrm{FH}}=27.9 \mathrm{~Hz},{ }^{3} \mathrm{JHH}_{\mathrm{HH}}=10.3 \mathrm{~Hz}$, $\left.{ }^{3} \mathrm{~J}_{\mathrm{HH}}=1.2 \mathrm{~Hz}, 1 \mathrm{H}, \mathrm{Hax}_{\mathrm{a}} 2\right), 3.02-2.91\left(\mathrm{~m}, 1 \mathrm{H}, \mathrm{H}_{2} \mathrm{C} 6\right), 2.60-2.50\left(\mathrm{~m}, 1 \mathrm{H}, \mathrm{H}_{2} \mathrm{C} 6\right), 2.21$ $-2.12\left(\mathrm{~m}, 1 \mathrm{H}, \mathrm{H}_{2} \mathrm{C} 4\right), 1.77\left(\mathrm{dtt},{ }^{2} \mathrm{JHH}_{\mathrm{HH}}=11.9 \mathrm{~Hz},{ }^{3} \mathrm{~J}_{\mathrm{HH}}=11.9 \mathrm{~Hz},{ }^{3} \mathrm{JHH}_{\mathrm{HH}}=3.6 \mathrm{~Hz}, 1 \mathrm{H}\right.$, $\left.H_{\mathrm{ax}} \mathrm{C} 5\right), 1.71-1.54\left(\mathrm{~m}, 1 \mathrm{H}, \mathrm{H}_{2} \mathrm{C} 4\right), 1.52-1.27\left(\mathrm{~m}, 2 \mathrm{H}, \mathrm{H}_{\mathrm{eq}} \mathrm{C} 5, H_{\mathrm{N}} 1\right) .{ }^{1} \mathbf{H}\left\{{ }^{19} \mathrm{~F}\right\} \mathbf{N M R}$ (500 MHz, Chloroform-d) $\delta 7.44-7.41$ (m, 2H, HC9), 7.39 - 7.27 (m, 3H, HC10, HC11), 5.80 (s, 1H, $\left.H_{2} \mathrm{~N} 13\right), 5.48$ (s, $\left.1 \mathrm{H}, H_{2} \mathrm{~N} 13\right), 4.89$ (bs, $1 \mathrm{H}, H_{\mathrm{eq}} \mathrm{C} 3$ ), 3.60 (d, ${ }^{3} \mathrm{JHH}_{\mathrm{H}}=$ $10.2 \mathrm{~Hz}, 1 \mathrm{H}, \mathrm{HC} 7), 3.28$ (dd, $\left.{ }^{3} \mathrm{~J}_{\mathrm{HH}}=10.2 \mathrm{~Hz},{ }^{3} \mathrm{~J}_{\mathrm{HH}}=1.1 \mathrm{~Hz}, 1 \mathrm{H}, H_{\mathrm{ax}} \mathrm{C} 2\right), 3.01-2.93$ $\left(\mathrm{m}, 1 \mathrm{H}, \mathrm{H}_{2} \mathrm{C} 6\right), 2.55\left(\mathrm{ddd},{ }^{2} \mathrm{~J}_{\mathrm{HH}}=12.2 \mathrm{~Hz},{ }^{3} \mathrm{JHH}_{\mathrm{HH}}=12.2 \mathrm{~Hz},{ }^{3} \mathrm{~J}_{\mathrm{HH}}=2.7 \mathrm{~Hz}, 1 \mathrm{H}, \mathrm{H}_{2} \mathrm{C} 6\right)$, $2.21-2.12\left(\mathrm{~m}, 1 \mathrm{H}, \mathrm{H}_{2} \mathrm{C} 4\right), 1.77\left(\mathrm{dtt},{ }^{2} \mathrm{JHH}_{\mathrm{HH}}=11.9 \mathrm{~Hz},{ }^{3} \mathrm{JHH}_{\mathrm{HH}}=11.9 \mathrm{~Hz},{ }^{3} \mathrm{JHH}_{\mathrm{HH}}=3.6 \mathrm{~Hz}, 1 \mathrm{H}\right.$, $\left.H_{\mathrm{ax}} \mathrm{C} 5\right), 1.68-1.57\left(\mathrm{~m}, 1 \mathrm{H}, \mathrm{H}_{2} \mathrm{C} 4\right), 1.51-1.32\left(\mathrm{~m}, 2 \mathrm{H}, \mathrm{Heq}_{\mathrm{C}} 5, H \mathrm{~N} 1\right) .{ }^{13} \mathrm{C}\left\{{ }^{1} \mathrm{H}\right\} \mathbf{N M R}$ (126 MHz, Chloroform-d) $\delta 173.8$ (C12), 136.5 (C8), 129.2 (C10), 128.6 (C9), 128.2 (C11), $87.8\left(\mathrm{~d},{ }^{1} \mathrm{~J}_{\mathrm{FC}}=174.0 \mathrm{~Hz}, \mathrm{C} 3\right), 60.7\left(\mathrm{~d},{ }^{2} \mathrm{~J}_{\mathrm{FC}}=17.5 \mathrm{~Hz}, \mathrm{C} 2\right), 54.3\left(\mathrm{~d},{ }^{3} \mathrm{~J}_{\mathrm{FC}}=3.8\right.$ $\mathrm{Hz}, \mathrm{C} 7), 46.6$ (C6), 29.6 (d, $\left.{ }^{2} \mathrm{JFC}=21.4 \mathrm{~Hz}, \mathrm{C} 4\right), 20.5$ (C5). ${ }^{13} \mathrm{C}\left\{{ }^{1} \mathbf{H},{ }^{19} \mathrm{~F}\right\}$ NMR $(126 \mathrm{MHz}$, 
Chloroform- $d$ ) $\delta 173.8$ (C12), 136.5 (C8), 129.2 (C10), 128.6 (C9), 128.2 (C11), 87.8 (C5), 60.7 (C2), 54.3 (C7), 46.6 (C6), 29.6 (C4), 20.5 (C5). ${ }^{19} \mathrm{~F}$ NMR (470 MHz, Chloroform- $d$ ) $\delta-201.1--201.5(\mathrm{~m}) .{ }^{19} \mathrm{~F}\left\{{ }^{1} \mathrm{H}\right\}$ NMR $(376 \mathrm{MHz}$, Chloroform- $d$ ) $\delta-201.3$ (s). HRMS (ESI) $\mathrm{m} / \mathrm{z}$ calculated for $\left[\mathrm{C}_{13} \mathrm{H}_{18} \mathrm{FN} \mathrm{N}_{2} \mathrm{O}\right]\left(\left[\mathrm{M}+\mathrm{H}^{+}\right]\right) 237.1398$, found 237.1416.

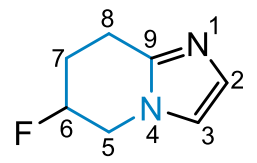

6-Fluoro-5,6,7,8-tetrahydroimidazo[1,2-a]pyridine (32): The title compound was synthesized according to GP-A using $10 \mathrm{~mol} \%$ Pd-catalyst $(35.1 \mathrm{mg})$ in $\mathrm{MeOH}(0.5 \mathrm{M})$ with aq. $\mathrm{HCl}(100 \mu \mathrm{l}, 2.4$ equiv, $12 \mathrm{~N})$. The product was isolated as a white solid (31 mg, $0.22 \mathrm{mmol}, 44 \%$ ) after column chromatography (silical gel, eluent: $\mathrm{CH}_{2} \mathrm{Cl}_{2}$, later $99: 1$, 98:2, $95: 5 \mathrm{CH}_{2} \mathrm{Cl}_{2} / \mathrm{MeOH}$ ).

${ }^{1} \mathrm{H}$ NMR $(599 \mathrm{MHz}$, Chloroform- $d) \delta 7.02\left(\mathrm{~d},{ }^{3} \mathrm{JHH}_{\mathrm{H}}=1.4 \mathrm{~Hz}, 1 \mathrm{H}, \mathrm{HC2}\right), 6.80\left(\mathrm{~d},{ }^{3} \mathrm{HHH}_{\mathrm{HH}}=\right.$ $1.4 \mathrm{~Hz}, 1 \mathrm{H}, H \mathrm{C} 3), 5.20\left(\mathrm{dm},{ }^{2} \mathrm{JFH}_{\mathrm{FH}}=47.4 \mathrm{~Hz}, 1 \mathrm{H}, H \mathrm{C} 6\right), 4.31-4.23\left(\mathrm{~m}, 1 \mathrm{H}, \mathrm{H}_{2} \mathrm{C} 5\right), 4.12$ (ddd, $\left.{ }^{3} \mathrm{JFH}_{\mathrm{FH}}=34.3 \mathrm{~Hz},{ }^{2} \mathrm{JHH}_{\mathrm{HH}}=13.8 \mathrm{~Hz},{ }^{3} \mathrm{JHH}_{\mathrm{HH}}=3.5 \mathrm{~Hz}, 1 \mathrm{H}, \mathrm{H}_{2} \mathrm{C} 5\right), 3.08-2.94(\mathrm{~m}, 2 \mathrm{H}$, $\left.\mathrm{H}_{2} \mathrm{C} 8\right), 2.47-2.38\left(\mathrm{~m}, 1 \mathrm{H}, \mathrm{H}_{2} \mathrm{C} 7\right), 2.02\left(\mathrm{dm},{ }^{3} \mathrm{JFH}=38.9 \mathrm{~Hz}, 1 \mathrm{H}, \mathrm{H}_{2} \mathrm{C} 7\right) .{ }^{1} \mathrm{H}\left\{{ }^{19} \mathrm{~F}\right\}$ NMR (599 MHz, Chloroform-d) $\delta 7.02\left(\mathrm{~d},{ }^{3} \mathrm{HHH}=1.4 \mathrm{~Hz}, 1 \mathrm{H}, H \mathrm{C} 2\right), 6.80\left(\mathrm{~d},{ }^{3} \mathrm{JHH}=1.4 \mathrm{~Hz}\right.$, $1 \mathrm{H}, H \mathrm{C} 3), 5.21-5.18(\mathrm{~m}, 1 \mathrm{H}, \mathrm{HC} 6), 4.26\left(\mathrm{dm},{ }^{2} \mathrm{JHH}_{\mathrm{HH}}=13.8 \mathrm{~Hz}, 1 \mathrm{H}, \mathrm{H}_{2} \mathrm{C} 5\right), 4.12(\mathrm{dd}$, $\left.{ }^{2} \mathrm{JHH}_{\mathrm{HH}}=13.8 \mathrm{~Hz},{ }^{3} \mathrm{JHH}_{\mathrm{HH}}=3.5 \mathrm{~Hz}, 1 \mathrm{H}, \mathrm{H}_{2} \mathrm{C} 5\right), 3.07-2.95\left(\mathrm{~m}, 2 \mathrm{H}, \mathrm{H}_{2} \mathrm{C} 8\right), 2.42\left(\mathrm{dm},{ }^{2} \mathrm{HHH}_{\mathrm{HH}}=\right.$ $14.7 \mathrm{~Hz}, 1 \mathrm{H}, \mathrm{H}_{2} \mathrm{C} 7$ ), 2.02 (dddd, ${ }^{2} \mathrm{JHH}_{\mathrm{HH}}=14.3 \mathrm{~Hz},{ }^{3} \mathrm{JHH}_{\mathrm{HH}}=11.3 \mathrm{~Hz},{ }^{3} \mathrm{~J}_{\mathrm{HH}}=6.5 \mathrm{~Hz},{ }^{3} \mathrm{~J}_{\mathrm{HH}}=$ $\left.1.9 \mathrm{~Hz}, 1 \mathrm{H}, \mathrm{H}_{2} \mathrm{C} 7\right) .{ }^{13} \mathrm{C}\left\{{ }^{1} \mathrm{H}\right\}$ NMR (151 MHz, Chloroform-d) $\delta 143.5$ (C9), 128.7 (C2), 118.0 (C3), $85.3\left(\mathrm{~d},{ }^{1} \mathrm{JFC}=175.1 \mathrm{~Hz}, \mathrm{C} 6\right), 49.2\left(\mathrm{~d},{ }^{2} \mathrm{JFC}=24.3 \mathrm{~Hz}, \mathrm{C} 5\right), 26.2\left(\mathrm{~d},{ }^{2} \mathrm{JFC}=\right.$ $21.1 \mathrm{~Hz}, \mathrm{C} 7), 19.3\left(\mathrm{~d},{ }^{3} \mathrm{JFC}=5.5 \mathrm{~Hz}, \mathrm{C} 8\right) \cdot{ }^{13} \mathrm{C}\left\{{ }^{1} \mathrm{H},{ }^{19} \mathrm{~F}\right\} \mathrm{NMR}(151 \mathrm{MHz}$, Chloroform- $d) \delta$ 143.5 (C9), 128.7 (C2), 118.0 (C3), 85.3 (C6), 49.2 (C5), 26.2 (C7), 19.3 (C8). ${ }^{19} \mathrm{~F}$ NMR (564 MHz, Chloroform- $d$ ) $\delta-187.9--188.2(\mathrm{~m}) .{ }^{19} \mathrm{~F}\left\{{ }^{1} \mathrm{H}\right\}$ NMR $(564 \mathrm{MHz}$, Chloroformd) $\delta-188.1$. HRMS $(\mathrm{ESI}) \mathrm{m} / \mathrm{z}$ calculated for $\left[\mathrm{C}_{7} \mathrm{H}_{10} \mathrm{FN} \mathrm{N}_{2}\right]\left(\left[\mathrm{M}+\mathrm{H}^{+}\right]\right) 141.0823$, found 141.0827 .

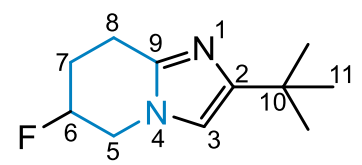

2-(tert-Butyl)-6-fluoro-5,6,7,8-tetrahydroimidazo[1,2-a]pyridine (33): The title compound was synthesized according to GP-A using $10 \mathrm{~mol} \%$ Pd-catalyst (35.1 mg) in $\mathrm{MeOH}(0.5 \mathrm{M})$ with aq. $\mathrm{HCl}(100 \mu \mathrm{l}, 2.4$ equiv, $12 \mathrm{~N})$. The product was isolated as a 
white solid (48 mg, $0.24 \mathrm{mmol}, 49 \%$ ) after column chromatography (silical gel, eluent: n-pentane/EtOAc 50:50, later 25:75, 0:100).

${ }^{1} \mathrm{H}$ NMR $(599 \mathrm{MHz}$, Chloroform-d) $\delta 6.45$ (s, $1 \mathrm{H}, H C 3), 5.13\left(\mathrm{~d},{ }^{2} \mathrm{~J} F \mathrm{H}=47.3 \mathrm{~Hz}, 1 \mathrm{H}\right.$, HC6), $4.15\left(\mathrm{ddm},{ }^{3} \mathrm{JFH}_{\mathrm{FH}}=15.1 \mathrm{~Hz},{ }^{2} \mathrm{JHH}_{\mathrm{HH}}=13.8 \mathrm{~Hz}, 1 \mathrm{H}, \mathrm{H}_{2} \mathrm{C} 5\right), 4.03\left(\mathrm{ddd},{ }^{3} \mathrm{~J}_{\mathrm{FH}}=33.8 \mathrm{~Hz}\right.$, $\left.{ }^{2} \mathrm{JHH}_{\mathrm{HH}}=13.7 \mathrm{~Hz},{ }^{3} \mathrm{JHH}_{\mathrm{HH}}=3.5 \mathrm{~Hz}, 1 \mathrm{H}, \mathrm{H}_{2} \mathrm{C} 5\right), 3.02-2.89\left(\mathrm{~m}, 2 \mathrm{H}, \mathrm{H}_{2} \mathrm{C} 8\right), 2.39-2.31(\mathrm{~m}$, $\left.1 \mathrm{H}, \mathrm{H}_{2} \mathrm{C} 7\right), 1.98\left(\mathrm{dm},{ }^{3} \mathrm{JFH}_{\mathrm{FH}}=38.7 \mathrm{~Hz}, 1 \mathrm{H}, \mathrm{H}_{2} \mathrm{C} 7\right), 1.28-1.19\left(\mathrm{~m}, 9 \mathrm{H}, H_{3} \mathrm{C} 11\right) .{ }^{1} \mathrm{H}\left\{{ }^{19} \mathrm{~F}\right\}$ NMR $(599 \mathrm{MHz}$, Chloroform- $d) \delta 6.45\left(\mathrm{~d},{ }^{5} \mathrm{JHH}=1.2 \mathrm{~Hz}, 1 \mathrm{H}, H \mathrm{C} 3\right), 5.14-5.11(\mathrm{~m}, 1 \mathrm{H}$, HC6), $4.15\left(\mathrm{dm},{ }^{2} \mathrm{JHH}=13.6 \mathrm{~Hz}, 1 \mathrm{H}, H_{2} \mathrm{C} 5\right), 4.03\left(\mathrm{dd},{ }^{2} \mathrm{~J} \mathrm{HH}=13.8,{ }^{3} \mathrm{JHH}=2.7 \mathrm{~Hz}, 1 \mathrm{H}\right.$, $\left.\mathrm{H}_{2} \mathrm{C} 5\right), 3.02-2.89\left(\mathrm{~m}, 2 \mathrm{H}, \mathrm{H}_{2} \mathrm{C} 8\right), 2.38-2.32\left(\mathrm{~m}, 1 \mathrm{H}, \mathrm{H}_{2} \mathrm{C} 7\right), 2.02-1.94(\mathrm{~m}, 1 \mathrm{H}$, $\left.\mathrm{H}_{2} \mathrm{C} 7\right), 1.24\left(\mathrm{~d},{ }^{5} \mathrm{JHH}=1.2 \mathrm{~Hz}, 9 \mathrm{H}, H_{3} \mathrm{C} 11\right) .{ }^{13} \mathrm{C}\left\{{ }^{1} \mathrm{H}\right\} \mathbf{N M R}(126 \mathrm{MHz}$, Chloroform-d $\delta$ 152.1 (C2), 142.4 (C9), 111.5 (C3), 85.3 (d, $\left.{ }^{1} \mathrm{JFC}=175.1 \mathrm{~Hz}, \mathrm{C} 6\right), 48.8\left(\mathrm{~d},{ }^{2} \mathrm{JFC}=24.3\right.$ $\mathrm{Hz}, \mathrm{C5}$ ), 31.7 (C10), 30.2 (C11), $26.2\left(\mathrm{~d},{ }^{2} \mathrm{JFC}=21.1 \mathrm{~Hz}, \mathrm{C} 7\right), 19.4\left(\mathrm{~d},{ }^{3} \mathrm{JFC}=5.8 \mathrm{~Hz}\right.$, C8). ${ }^{13} \mathbf{C}\left\{{ }^{1} \mathbf{H},{ }^{19} \mathrm{~F}\right\}$ NMR (126 MHz, Chloroform- $\left.d\right) \delta 152.1$ (C2), 142.4 (C9), 111.5 (C3), 85.3 (C6), 48.8 (C5), 31.7 (C10), 30.2 (C11), 26.2 (C7), 19.4 (C8). ${ }^{19} \mathrm{~F} \mathrm{NMR} \mathrm{(564} \mathrm{MHz,}$ Chloroform- $d$ ) $\delta-187.6--187.9(\mathrm{~m}) .{ }^{19} \mathrm{~F}\left\{{ }^{1} \mathrm{H}\right\}$ NMR $(564 \mathrm{MHz}$, Chloroform- $d) \delta-187.7$.

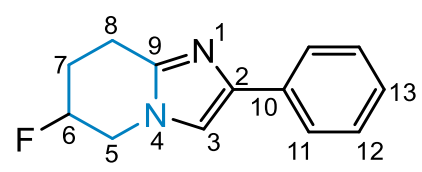

6-Fluoro-2-phenyl-5,6,7,8-tetrahydroimidazo[1,2-a]pyridine (34): The title compound was synthesized according to GP-A using 5 mol\% Pd-catalyst (17.6 mg) in $\mathrm{MeOH}(0.5 \mathrm{M})$ with aq. $\mathrm{HCl}(100 \mu \mathrm{l}, 2.4$ equiv, $12 \mathrm{~N})$. The product was isolated as a white solid (60 mg, $0.28 \mathrm{mmol}, 55 \%$ ) after column chromatography (silical gel, eluent: n-pentane/EtOAc 50:50, later 25:75, 0:100).

${ }^{1} \mathrm{H}$ NMR (599 MHz, Chloroform- $\left.d\right) \delta 7.74-7.71(\mathrm{~m}, 2 \mathrm{H}, \mathrm{HC} 11), 7.37-7.32(\mathrm{~m}, 2 \mathrm{H}$, HC12), $7.24-7.20(\mathrm{~m}, 1 \mathrm{H}, H \mathrm{C} 13), 7.05$ (s, 1H, HC3), $5.18\left(\mathrm{dm},{ }^{2} \mathrm{JFH}_{\mathrm{FH}}=47.1 \mathrm{~Hz}, 1 \mathrm{H}\right.$, HC6), $4.26\left(\mathrm{ddm},{ }^{3} \mathrm{~J}_{\mathrm{FH}}=16.1 \mathrm{~Hz},{ }^{2} J_{\mathrm{HH}}=13.8,1 \mathrm{H}, \mathrm{H}_{2} \mathrm{C} 5\right), 4.10$ (ddd, ${ }^{3} J_{\mathrm{FH}}=34.5 \mathrm{~Hz}$, $\left.{ }^{2} \mathrm{~J}_{\mathrm{HH}}=13.8 \mathrm{~Hz},{ }^{3} \mathrm{~J}_{\mathrm{HH}}=3.5 \mathrm{~Hz}, 1 \mathrm{H}, \mathrm{H}_{2} \mathrm{C} 5\right), 3.12-2.99\left(\mathrm{~m}, 2 \mathrm{H}, \mathrm{H}_{2} \mathrm{C} 8\right), 2.46-2.39(\mathrm{~m}$, $\left.1 \mathrm{H}, \mathrm{H}_{2} \mathrm{C} 7\right), 2.07-1.90\left(\mathrm{~m}, 1 \mathrm{H}, \mathrm{H}_{2} \mathrm{C} 7\right) .{ }^{1} \mathrm{H}\left\{{ }^{19} \mathrm{~F}\right\}$ NMR $(599 \mathrm{MHz}$, Chloroform-d) $\delta 7.74$ - $7.71(\mathrm{~m}, 2 \mathrm{H}, \mathrm{HC} 11), 7.37$ - 7.32 (m, 2H, HC12), $7.24-7.20$ (m, 1H, HC13), 7.05 (s, $1 \mathrm{H}, H \mathrm{C} 3), 5.20-5.16(\mathrm{~m}, 1 \mathrm{H}, \mathrm{HC} 6), 4.25\left(\mathrm{dm},{ }^{2} \mathrm{JHH}_{\mathrm{H}}=13.8 \mathrm{~Hz}, 1 \mathrm{H}, \mathrm{H}_{2} \mathrm{C} 5\right), 4.10$ (dd, $\left.{ }^{2} \mathrm{~J}_{\mathrm{HH}}=13.8 \mathrm{~Hz},{ }^{3} \mathrm{JHH}_{\mathrm{H}}=3.3 \mathrm{~Hz}, 1 \mathrm{H}, \mathrm{H}_{2} \mathrm{C} 5\right), 3.12-2.99\left(\mathrm{~m}, 2 \mathrm{H}, \mathrm{H}_{2} \mathrm{C} 8\right), 2.46-2.39(\mathrm{~m}$, $\left.1 \mathrm{H}, \mathrm{H}_{2} \mathrm{C} 7\right), 2.05-1.90\left(\mathrm{~m}, 1 \mathrm{H}, H_{2} \mathrm{C} 7\right) .{ }^{13} \mathrm{C}\left\{{ }^{1} \mathrm{H}\right\}$ NMR $(151 \mathrm{MHz}$, Chloroform-d) $\delta 143.9$ (C9), 141.4 (C2), 134.3 (C10), 128.7 (C12), 126.8 (C13), 124.9 (C11), 113.8 (C3), 85.1 
(d, $\left.{ }^{1} \mathrm{JFC}=175.4 \mathrm{~Hz}, \mathrm{C6}\right), 49.2\left(\mathrm{~d},{ }^{2} \mathrm{JFC}=24.0 \mathrm{~Hz}, \mathrm{C} 5\right), 26.0\left(\mathrm{~d},{ }^{2} \mathrm{JFC}=21.4 \mathrm{~Hz}, \mathrm{C} 7\right), 19.3$ $\left(d,{ }^{3} \mathrm{JFC}=5.8 \mathrm{~Hz}, \mathrm{C} 8\right) \cdot{ }^{13} \mathrm{C}\left\{{ }^{1} \mathrm{H},{ }^{19} \mathrm{~F}\right\}$ NMR $(151 \mathrm{MHz}$, Chloroform- $d) \delta 143.9(\mathrm{C} 9), 141.4$ (C2), 134.3 (C10), 128.7 (C12), 126.8 (C13), 124.9 (C11), 113.8 (C3), 85.1 (C6), 49.2 (C5), 26.0 (C7), 19.3 (C8). ${ }^{19} \mathrm{~F}$ NMR (564 MHz, Chloroform- $d$ ) $\delta-187.9--188.2(\mathrm{~m})$. ${ }^{19} \mathrm{~F}\left\{{ }^{1} \mathrm{H}\right\}$ NMR (282 MHz, Chloroform- $d$ ) $\delta$-188.0. HRMS (ESI) $\mathrm{m} / \mathrm{z}$ calculated for $\left[\mathrm{C}_{13} \mathrm{H}_{14} \mathrm{FN} 2\right]\left(\left[\mathrm{M}+\mathrm{H}^{+}\right]\right)$217.1136, found 217.1146.

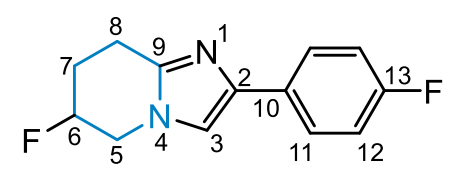

6-Fluoro-2-(4-fluorophenyl)-5,6,7,8-tetrahydroimidazo[1,2-a]pyridine (35): The title compound was synthesized according to GP-A using $10 \mathrm{~mol} \% \mathrm{Pd}$-catalyst $(35.1 \mathrm{mg})$ in $\mathrm{MeOH}(0.5 \mathrm{M})$ with aq. $\mathrm{HCl}(100 \mu \mathrm{l}, 2.4$ equiv, $12 \mathrm{~N})$. The product was isolated as a white solid ( $72 \mathrm{mg}, 0.31 \mathrm{mmol}, 61 \%$ ) after column chromatography (silical gel, eluent: $n$-pentane/EtOAc 50:50, later 25:75, 0:100) accompanied with defluorinated tetrahydroimidazo[1,2-a]pyridine.

${ }^{1} \mathrm{H}$ NMR (500 MHz, Chloroform-d) $\delta 7.74-7.62(\mathrm{~m}, 2 \mathrm{H}, H \mathrm{C} 11), 7.06-6.93(\mathrm{~m}, 2 \mathrm{H}$, $H C 12), 5.18\left(\mathrm{dm},{ }^{2} \mathrm{JFH}_{1}=47.3 \mathrm{~Hz}, 1 \mathrm{H}, H \mathrm{C} 6\right), 4.30-4.20(\mathrm{~m}, 1 \mathrm{H}, H \mathrm{C} 5), 4.10$ (dd, ${ }^{3} \mathrm{FFH}$ $\left.=34.8 \mathrm{~Hz},{ }^{2} \mathrm{HHH}=13.9 \mathrm{~Hz}, 1 \mathrm{H}, H \mathrm{C} 5\right), 3.13-2.95\left(\mathrm{~m}, 2 \mathrm{H}, \mathrm{H}_{2} \mathrm{C} 8\right), 2.48-2.36(\mathrm{~m}, 1 \mathrm{H}$, $\left.\mathrm{H}_{2} \mathrm{C} 7\right), 2.11-1.87\left(\mathrm{~m}, 1 \mathrm{H}, \mathrm{H}_{2} \mathrm{C} 7\right) .{ }^{1} \mathrm{H}\left\{{ }^{19} \mathrm{~F}\right\}$ NMR $(500 \mathrm{MHz}$, Chloroform- $d) \delta 7.68(\mathrm{~d}$, $\left.{ }^{3} \mathrm{JHH}_{\mathrm{H}}=8.6 \mathrm{~Hz}, 2 \mathrm{H}, \mathrm{HC} 11\right), 7.06-6.95(\mathrm{~m}, 2 \mathrm{H}, H \mathrm{C} 12), 5.18(\mathrm{~m}, 1 \mathrm{H}, H \mathrm{C} 6), 4.25$ (dd, $\left.{ }^{2} \mathrm{JHH}_{\mathrm{HH}}=13.9 \mathrm{~Hz},{ }^{3} \mathrm{JHH}_{\mathrm{HH}}=2.8 \mathrm{~Hz}, 1 \mathrm{H}, \mathrm{H}_{2} \mathrm{C} 5\right), 4.10\left(\mathrm{dd},{ }^{2} \mathrm{JHH}_{\mathrm{HH}}=13.8,{ }^{3} \mathrm{JHH}_{\mathrm{HH}}=2.8 \mathrm{~Hz}, 1 \mathrm{H}\right.$, $\left.\mathrm{H}_{2} \mathrm{C} 5\right), 3.13-2.95\left(\mathrm{~m}, 2 \mathrm{H}, \mathrm{H}_{2} \mathrm{C} 8\right), 2.47-2.37\left(\mathrm{~m}, 1 \mathrm{H}, \mathrm{H}_{2} \mathrm{C} 7\right), 2.05-1.88(\mathrm{~m}, 1 \mathrm{H}$, $\left.\mathrm{H}_{2} \mathrm{C} 7\right) .{ }^{13} \mathrm{C}\left\{{ }^{1} \mathrm{H}\right\}$ NMR $(126 \mathrm{MHz}$, Chloroform- $d) \delta 162.0\left(\mathrm{~d},{ }^{1} \mathrm{JFC}=245.1 \mathrm{~Hz}, \mathrm{C} 13\right), 143.9$ (C9), 140.6 (C2), 130.5 (d, $\left.{ }^{4} \mathrm{JFC}=3.1 \mathrm{~Hz}, \mathrm{C} 10\right), 126.4$ (d, $\left.{ }^{3} \mathrm{JFC}=7.9 \mathrm{~Hz}, \mathrm{C} 11\right), 115.4$ $\left(d,{ }^{2} \mathrm{JFC}=21.5 \mathrm{~Hz}, \mathrm{C} 12\right), 113.4\left(\mathrm{~d},{ }^{4} \mathrm{JFC}=1.2 \mathrm{~Hz}, \mathrm{C} 3\right), 85.0\left(\mathrm{~d},{ }^{1} \mathrm{JFC}=175.4 \mathrm{~Hz}, \mathrm{C} 6\right)$, $49.2\left(\mathrm{~d},{ }^{2} \mathrm{JFC}=24.1 \mathrm{~Hz}, \mathrm{C} 5\right), 25.9\left(\mathrm{~d},{ }^{2} \mathrm{JFC}_{\mathrm{FC}}=21.2 \mathrm{~Hz}, \mathrm{C} 7\right), 19.2\left(\mathrm{~d},{ }^{3} \mathrm{JFC}=5.5 \mathrm{~Hz}, \mathrm{C} 8\right.$ ). ${ }^{13} \mathrm{C}\left\{{ }^{1} \mathrm{H}\right.$, sel- ${ }^{19} \mathrm{~F}$ at $\left.-188 \mathrm{ppm}\right\}$ NMR $(126 \mathrm{MHz}$, Chloroform- $d) \delta 162.0\left(\mathrm{~d},{ }^{1} \mathrm{JFC}=243.8\right.$ $\mathrm{Hz}, \mathrm{C} 13), 143.9$ (C9), 140.6 (C2), 130.5 (d, $\left.{ }^{4} \mathrm{JFC}=3.1 \mathrm{~Hz}, \mathrm{C} 10\right), 126.4\left(\mathrm{~d},{ }^{3} \mathrm{JFC}=7.8\right.$ $\mathrm{Hz}, \mathrm{C} 11), 115.4$ (d, ${ }^{2} \mathrm{JFC}=21.4 \mathrm{~Hz}, \mathrm{C} 12$ ), 113.4 (C3), 85.0 (C6), 49.2 (C5), 25.9 (C7), $19.2(\mathrm{C} 8) .{ }^{19} \mathrm{~F}$ NMR $(470 \mathrm{MHz}$, Chloroform- $d) \delta-116.1$ (tt, ${ }^{3} \mathrm{FFH}_{\mathrm{FH}}=8.7 \mathrm{~Hz},{ }^{4} \mathrm{~J}_{\mathrm{FH}}=5.4 \mathrm{~Hz}$, $1 \mathrm{~F}, \mathrm{FC} 13),-187.9--188.3(\mathrm{~m}, 1 \mathrm{~F}, \mathrm{FC} 6) .{ }^{19} \mathrm{~F}\left\{{ }^{1} \mathrm{H}\right\}$ NMR $(470 \mathrm{MHz}$, Chloroform- $d) \delta-$ 116.1 (1F, FC13), -188.1 (1F, FC6). HRMS (ESI) m/z calculated for $\left[\mathrm{C}_{13} \mathrm{H}_{12} \mathrm{~F}_{2} \mathrm{~N}_{2} \mathrm{Na}\right.$ ] $\left(\left[\mathrm{M}+\mathrm{Na}^{+}\right]\right)$257.0861, found 257.0857 . 


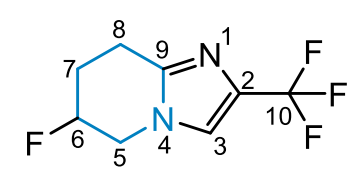

6-Fluoro-2-(trifluoromethyl)-5,6,7,8-tetrahydroimidazo[1,2-a]pyridine (36): The title compound was synthesized according to GP-A using $10 \mathrm{~mol} \% \mathrm{Pd}$-catalyst $(35.1 \mathrm{mg})$ in $\mathrm{MeOH}(0.5 \mathrm{M})$ with aq. $\mathrm{HCl}(100 \mu \mathrm{l}, 2.4$ equiv, $12 \mathrm{~N})$. The product was isolated as a white solid ( $54 \mathrm{mg}, 0.26 \mathrm{mmol}, 52 \%$ ) after column chromatography (silical gel, eluent: $n$-pentane/EtOAc 50:50, later 25:75, 0:100) accompanied with defluorinated tetrahydroimidazo[1,2-a]pyridine.

${ }^{1} \mathrm{H}$ NMR $\left(599 \mathrm{MHz}\right.$, Chloroform-d) $\delta 7.14-7.12(\mathrm{~m}, 1 \mathrm{H}, \mathrm{HC} 3), 5.21\left(\mathrm{dm},{ }^{2} \mathrm{JFH}=47.0\right.$ $\mathrm{Hz}, 1 \mathrm{H}, H \mathrm{C} 6), 4.28\left(\mathrm{ddm},{ }^{2} \mathrm{JHH}_{\mathrm{HH}}=14.1 \mathrm{~Hz},{ }^{3} \mathrm{JFH}_{\mathrm{FH}}=14.1 \mathrm{~Hz}, 1 \mathrm{H}, \mathrm{H}_{2} \mathrm{C} 5\right), 4.11$ (ddd, ${ }^{3} \mathrm{FFH}_{\mathrm{FH}}=$ $\left.35.7 \mathrm{~Hz},{ }^{2} \mathrm{JHH}=14.0 \mathrm{~Hz},{ }^{3} \mathrm{JHH}=3.3 \mathrm{~Hz}, 1 \mathrm{H}, \mathrm{H}_{2} \mathrm{C} 5\right), 3.06-2.94\left(\mathrm{~m}, 2 \mathrm{H}, \mathrm{H}_{2} \mathrm{C} 8\right), 2.48-$ $2.41\left(\mathrm{~m}, 1 \mathrm{H}, \mathrm{H}_{2} \mathrm{C} 7\right), 2.05-1.88\left(\mathrm{~m}, 1 \mathrm{H}, \mathrm{H}_{2} \mathrm{C} 7\right) .{ }^{1} \mathrm{H}\left\{{ }^{19} \mathrm{~F}\right\}$ NMR $(599 \mathrm{MHz}$, Chloroformd) $\delta 7.14-7.12(\mathrm{~m}, 1 \mathrm{H}, H \mathrm{C} 3), 5.23-5.19(\mathrm{~m}, 1 \mathrm{H}, \mathrm{HC} 6), 4.28\left(\mathrm{dm},{ }^{2} \mathrm{JHH}=14.0 \mathrm{~Hz}\right.$, $\left.1 \mathrm{H}, \mathrm{H}_{2} \mathrm{C} 5\right), 4.11\left(\mathrm{dd},{ }^{2} \mathrm{HHH}=14.0 \mathrm{~Hz},{ }^{3} \mathrm{JH}=3.3 \mathrm{~Hz}, 1 \mathrm{H}, \mathrm{H}_{2} \mathrm{C} 5\right), 3.05-2.94(\mathrm{~m}, 2 \mathrm{H}$, $\left.\mathrm{H}_{2} \mathrm{C} 8\right), 2.48-2.41\left(\mathrm{~m}, 1 \mathrm{H}, \mathrm{H}_{2} \mathrm{C} 7\right), 2.02-1.88\left(\mathrm{~m}, 1 \mathrm{H}, \mathrm{H}_{2} \mathrm{C} 7\right) .{ }^{13} \mathrm{C}\left\{{ }^{1} \mathrm{H}\right\} \mathrm{NMR}(151 \mathrm{MHz}$, Chloroform- $d$ ) $\delta 145.0$ (C9), 131.9 (q, $\left.{ }^{2} \mathrm{JFC}=38.6 \mathrm{~Hz}, \mathrm{C} 2\right), 121.9\left(\mathrm{q},{ }^{1} \mathrm{JFC}=266.7 \mathrm{~Hz}\right.$, $\mathrm{C} 10), 118.2-118.0(\mathrm{~m}, \mathrm{C} 3), 84.5\left(\mathrm{~d},{ }^{1} \mathrm{JFC}=176.0 \mathrm{~Hz}, \mathrm{C} 6\right), 49.5\left(\mathrm{~d},{ }^{2} \mathrm{JFC}=24.0 \mathrm{~Hz}\right.$, C5), $25.5\left(\mathrm{~d},{ }^{2} \mathrm{JFC}=21.1 \mathrm{~Hz}, \mathrm{C} 7\right), 19.0\left(\mathrm{~d},{ }^{3} \mathrm{JFC}_{\mathrm{FC}}=5.2 \mathrm{~Hz}, \mathrm{C} 8\right) .{ }^{13} \mathrm{C}\left\{{ }^{1} \mathrm{H}\right.$, broadband $\left.-{ }^{19} \mathrm{~F}\right\}$ NMR (151 MHz, Chloroform- $d$ ) $\delta 145.0$ (C9), 131.9 (C2), 121.9 (C10), 118.1 (C3), 84.5 (C6), 49.5 (C5), 25.5 (C7), 19.0 (C8). ${ }^{19} \mathrm{~F}$ NMR (564 MHz, Chloroform- $d$ ) $\delta-63.0$ (s, 3F, $\left.F_{3} \mathrm{C} 10\right),-188.5--188.9(\mathrm{~m}, 1 \mathrm{~F}, F C 6) .{ }^{19} \mathrm{~F}\left\{{ }^{1} \mathrm{H}\right\}$ NMR $(564 \mathrm{MHz}$, Chloroform- $d) \delta-63.0$ $\left(3 \mathrm{~F}, \mathrm{~F}_{3} \mathrm{C} 10\right),-188.7(1 \mathrm{~F}, \mathrm{FC} 6)$. HRMS (ESI) $\mathrm{m} / \mathrm{z}$ calculated for $\left[\mathrm{C}_{8} \mathrm{H}_{8} \mathrm{~F}_{4} \mathrm{~N}_{2} \mathrm{Na}\right]\left(\left[\mathrm{M}+\mathrm{Na}^{+}\right]\right)$ 231.0516, found 231.0512 .

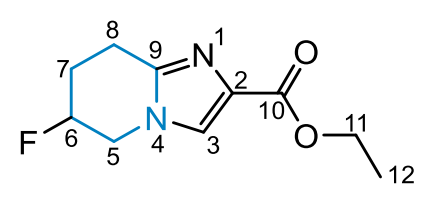

Ethyl 6-fluoro-5,6,7,8-tetrahydroimidazo[1,2-a]pyridine-2-carboxylate (37): The title compound was synthesized according to GP-A using $10 \mathrm{~mol} \%$ Pd-catalyst $(35.1 \mathrm{mg})$ in $\mathrm{MeOH}(0.5 \mathrm{M})$ with aq. $\mathrm{HCl}(100 \mu \mathrm{l}, 2.4$ equiv, $12 \mathrm{~N})$. The product was isolated as a white solid ( $62 \mathrm{mg}, 0.29 \mathrm{mmol}, 58 \%$ ) after column chromatography (silical gel, eluent: $n$-pentane/EtOAc 50:50, later 25:75, 0:100) accompanied with defluorinated tetrahydroimidazo[1,2-a]pyridine. 
${ }^{1} \mathrm{H}$ NMR $(500 \mathrm{MHz}$, Chloroform-d) $\delta 7.41$ (s, $1 \mathrm{H}, H \mathrm{CC} 3), 5.14\left(\mathrm{~d},{ }^{2} \mathrm{JFH}=46.9 \mathrm{~Hz}, 1 \mathrm{H}\right.$, HC6), $4.29-4.19$ (m, 3H, $\mathrm{H}_{2} \mathrm{C} 11, \mathrm{H}_{2} \mathrm{C} 5$ ), 4.08 (ddd, ${ }^{3} \mathrm{JFH}_{\mathrm{FH}}=35.6 \mathrm{~Hz},{ }^{2} \mathrm{JHH}_{\mathrm{HH}}=14.1 \mathrm{~Hz}$, $\left.{ }^{3} \mathrm{HHH}_{\mathrm{H}}=3.2 \mathrm{~Hz}, 1 \mathrm{H}, \mathrm{H}_{2} \mathrm{C} 5\right), 2.97-2.91\left(\mathrm{~m}, 2 \mathrm{H}, \mathrm{H}_{2} \mathrm{C} 8\right), 2.40-2.32\left(\mathrm{~m}, 1 \mathrm{H}, \mathrm{H}_{2} \mathrm{C} 7\right), 2.01$ $-1.81\left(\mathrm{~m}, 1 \mathrm{H}, \mathrm{H}_{2} \mathrm{C} 7\right), 1.28\left(\mathrm{t},{ }^{3} \mathrm{JHH}=7.1 \mathrm{~Hz}, 3 \mathrm{H}, \mathrm{H}_{3} \mathrm{C} 12\right) .{ }^{1} \mathrm{H}\left\{{ }^{19} \mathrm{~F}\right\} \mathbf{N M R}(500 \mathrm{MHz}$, Chloroform- $d$ ) $\delta 7.41$ (s, 1H, HC3), $5.17-5.13(\mathrm{~m}, 1 \mathrm{H}, H \mathrm{C} 6), 4.29-4.21(\mathrm{~m}, 3 \mathrm{H}$, $\left.\mathrm{H}_{2} \mathrm{C} 11, \mathrm{H}_{2} \mathrm{C} 5\right), 4.08\left(\mathrm{dd},{ }^{2} \mathrm{JHH}_{\mathrm{HH}}=14.1 \mathrm{~Hz},{ }^{3} \mathrm{JHH}_{\mathrm{HH}}=3.2 \mathrm{~Hz}, 1 \mathrm{H}, \mathrm{H}_{2} \mathrm{C} 5\right), 2.96-2.92(\mathrm{~m}, 2 \mathrm{H}$, $\left.\mathrm{H}_{2} \mathrm{C} 8\right), 2.40-2.32\left(\mathrm{~m}, 1 \mathrm{H}, \mathrm{H}_{2} \mathrm{C} 7\right), 1.98-1.81\left(\mathrm{~m}, 1 \mathrm{H}, \mathrm{H}_{2} \mathrm{C} 7\right), 1.28\left(\mathrm{t},{ }^{3} \mathrm{HH}=7.1 \mathrm{~Hz}\right.$, $\left.3 \mathrm{H}, \mathrm{H}_{3} \mathrm{C} 12\right) .{ }^{13} \mathrm{C}\left\{{ }^{1} \mathrm{H}\right\}$ NMR (126 MHz, Chloroform-d) $\delta 162.9$ (C10), 144.5 (C9), 133.0 (C2), 124.3 (C3), 84.5 (d, $\left.{ }^{1} \mathrm{JFC}=175.7 \mathrm{~Hz}, \mathrm{C} 6\right), 60.3(\mathrm{C} 11), 49.4\left(\mathrm{~d},{ }^{2} \mathrm{JFC}=23.9 \mathrm{~Hz}\right.$, C5), $25.4\left(\mathrm{~d},{ }^{2} \mathrm{JFC}=21.2 \mathrm{~Hz}, \mathrm{C} 7\right), 19.0\left(\mathrm{~d},{ }^{3} \mathrm{JFC}=5.3 \mathrm{~Hz}, \mathrm{C} 8\right), 14.4(\mathrm{C} 12),{ }^{13} \mathrm{C}\left\{{ }^{1} \mathrm{H},{ }^{19} \mathrm{~F}\right\}$ NMR (126 MHz, Chloroform- $d$ ) $\delta 162.9$ (C10), 144.5 (C9), 133.0 (C2), 124.3 (C3), 84.5 (C6), 60.3 (C11), 49.4 (C5), 25.4 (C7), 19.0 (C8), 14.4 (C12). ${ }^{19} \mathrm{~F}$ NMR (470 MHz, Chloroform- $d$ ) $\delta-188.5--188.8(\mathrm{~m}) .{ }^{19} \mathrm{~F}\left\{{ }^{1} \mathrm{H}\right\}$ NMR $(376 \mathrm{MHz}$, Chloroform- $d) \delta-188.7$. HRMS $(\mathrm{ESI}) \mathrm{m} / \mathrm{z}$ calculated for $\left[\mathrm{C}_{10} \mathrm{H}_{14} \mathrm{FN}_{2} \mathrm{O}_{2}\right]\left(\left[\mathrm{M}+\mathrm{H}^{+}\right]\right)$213.1034, found 213.1035 . 


\section{Application}

\section{Synthesis of fluoromethylphenidat (38):}

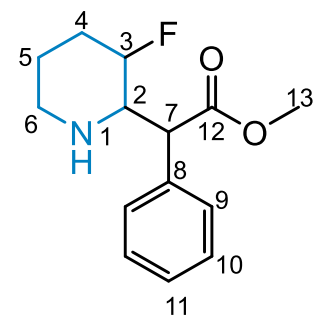

Fluorinated piperidine 31 ( $0.3 \mathrm{mmol}, 70.9 \mathrm{mg}, 1.0$ equiv) was added to a $10 \mathrm{ml} \mathrm{screw}$ cap flask equipped with a stir bar. $\mathrm{MeOH}(0.8 \mathrm{ml})$ and conc. $\mathrm{H}_{2} \mathrm{SO}_{4}(85 \mu \mathrm{l}, 1.6 \mathrm{mmol}$, 5.3 equiv) were added and the mixture was stirred for $72 \mathrm{~h}$ at $60^{\circ} \mathrm{C}$. Afterwards, the mixture was diluted with water, basified with sat. aq. $\mathrm{NaHCO}_{3}$ solution and extracted with EtOAc (3x). The organic phases were combined, dried over $\mathrm{MgSO}_{4}$ and the solvent was evaporated to yield methyl 2-(3-fluoropiperidin-2-yl)-2-phenylacetate (67.3 mg, $0.27 \mathrm{mmol}, 89 \%$ ).

${ }^{1} \mathbf{H}$ NMR $(599 \mathrm{MHz}$, Chloroform-d) $\delta 7.39-7.36(\mathrm{~m}, 2 \mathrm{H}, \mathrm{HC}), 7.36-7.32(\mathrm{~m}, 2 \mathrm{H}$, HC10), $7.31-7.28(\mathrm{~m}, 1 \mathrm{H}, H \mathrm{C} 11), 4.77\left(\mathrm{dm},{ }^{2} \mathrm{JFH}_{\mathrm{FH}}=50.3 \mathrm{~Hz}, 1 \mathrm{H}\right), 3.84\left(\mathrm{~d},{ }^{3} J_{\mathrm{HH}}=10.9\right.$ $\mathrm{Hz}, 1 \mathrm{H}, \mathrm{HC}$ ), 3.64 (s, 3H, $\mathrm{H}_{3} \mathrm{C} 13$ ), 3.27 (ddd, ${ }^{3} \mathrm{JFH}_{\mathrm{FH}}=26.8 \mathrm{~Hz},{ }^{3} \mathrm{~J}_{\mathrm{HH}}=10.8 \mathrm{~Hz},{ }^{3} \mathrm{JHH}_{\mathrm{HH}}=$ $\left.1.3 \mathrm{~Hz}, 1 \mathrm{H}, H_{\mathrm{ax}} \mathrm{C} 2\right), 2.95\left(\mathrm{dm},{ }^{3} \mathrm{JHH}_{\mathrm{H}}=11.9 \mathrm{~Hz}, 1 \mathrm{H}, \mathrm{H}_{2} \mathrm{C} 6\right), 2.56-2.50\left(\mathrm{~m}, 1 \mathrm{H}, \mathrm{H}_{2} \mathrm{C} 6\right)$, $2.21-2.12\left(\mathrm{~m}, 1 \mathrm{H}, \mathrm{H}_{2} \mathrm{C} 4\right), 1.83-1.73\left(\mathrm{~m}, 1 \mathrm{H}, \mathrm{H}_{2} \mathrm{C} 5\right), 1.70-1.54\left(\mathrm{~m}, 1 \mathrm{H}, \mathrm{H}_{2} \mathrm{C} 4\right), 1.49$ - $1.42\left(\mathrm{~m}, 1 \mathrm{H}, \mathrm{H}_{2} \mathrm{C} 5\right), 1.29(\mathrm{~s}, 1 \mathrm{H}, H \mathrm{~N} 1) .{ }^{13} \mathrm{C}\left\{{ }^{1} \mathrm{H}\right\} \mathbf{N M R}(151 \mathrm{MHz}$, Chloroform-d) $\delta$ 172.8 (C12), 135.8 (C8), 129.1 (C10), 128.6 (C9), 128.2 (C11), 87.3 (d, ${ }^{1} \mathrm{JFC}=174.9$ $\mathrm{Hz}, \mathrm{C} 3), 60.7\left(\mathrm{~d},{ }^{2} \mathrm{JFC}=17.7 \mathrm{~Hz}, \mathrm{C} 2\right), 53.0\left(\mathrm{~d},{ }^{3} \mathrm{JFC}_{\mathrm{FC}}=4.0 \mathrm{~Hz}, \mathrm{C} 7\right), 52.2(\mathrm{C} 13), 46.4$ (C6), $29.6\left(\mathrm{~d},{ }^{2} \mathrm{JFC}_{\mathrm{FC}}=21.4 \mathrm{~Hz}, \mathrm{C} 4\right), 20.5(\mathrm{C} 5) .{ }^{19} \mathrm{~F}\left\{{ }^{1} \mathrm{H}\right\} \mathrm{NMR}(376 \mathrm{MHz}$, Chloroform- $d) \delta-201.3$. HRMS (ESI) $\mathrm{m} / \mathrm{z}$ calculated for $\left[\mathrm{C}_{14} \mathrm{H}_{19} \mathrm{FNO}_{2}\right]\left(\left[\mathrm{M}+\mathrm{H}^{+}\right]\right) 252.1394$, found 252.1409 . 


\section{Synthesis of fluorobupivacaine (39):}

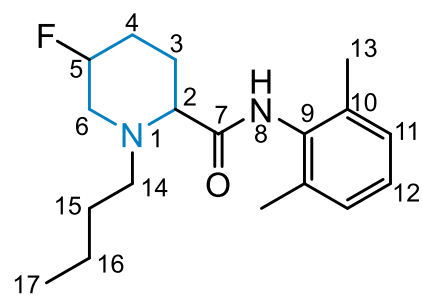

Fluorinated piperidine 26 (25.0 mg, $0.10 \mathrm{mmol}, 1.0$ equiv) and $\mathrm{K}_{2} \mathrm{CO}_{3}(28.0 \mathrm{mg}$, $0.20 \mathrm{mmol}, 2.0$ equiv) were weight into a $5 \mathrm{ml} \mathrm{screw}$-cap flask equipped with a stir bar. The solids were dissolved in DMF $(1 \mathrm{ml})$ and $n$-butyl iodide $(23 \mu \mathrm{l}, 0.20 \mathrm{mmol}$, 2.0 equiv) was added via syringe. The mixture was stirred overnight at $40^{\circ} \mathrm{C}$. After the mixture had reached room temperature, water was added and the aqueous phase was extracted with EtOAc (3x). The combined organic phases were dried over $\mathrm{MgSO}_{4}$ and concentrated. 1-Butyl- $N$-(2,6-dimethylphenyl)-5-fluoropiperidine-2-carboxamide was obtained as a white solid ( $22.1 \mathrm{mg}, 0.07 \mathrm{mmol}, 72 \%)$ after column chromatography on silical gel (eluent: $n$-pentane/EtOAc: 90:10, later 80:20, 50:50).

${ }^{1} \mathrm{H}$ NMR $(599 \mathrm{MHz}$, Chloroform-d) $\delta 8.22$ (s, 1H, HN8), $7.12-7.05$ (m, 3H, HC11, HC12), $4.84(\mathrm{dm}, J=47.8 \mathrm{~Hz}, 1 \mathrm{H}, H \mathrm{C} 5), 3.46-3.36\left(\mathrm{~m}, 1 \mathrm{H}, \mathrm{H}_{2} \mathrm{C} 6\right), 3.04-3.00(\mathrm{~m}$, $1 \mathrm{H}, H C 2$ ), 2.89 (ddd, ${ }^{2} J_{\mathrm{HH}}=12.6 \mathrm{~Hz},{ }^{3} \mathrm{~J}_{\mathrm{HH}}=10.5 \mathrm{~Hz},{ }^{3} \mathrm{~J}_{\mathrm{HH}}=6.0 \mathrm{~Hz}, 1 \mathrm{H}, \mathrm{H}_{2} \mathrm{C} 14$ ), 2.42 - 2.29 (m, $\left.2 \mathrm{H}, \mathrm{H}_{2} \mathrm{C} 6, \mathrm{H}_{2} \mathrm{C} 14\right), 2.25$ (s, 6H, $\left.\mathrm{H}_{3} \mathrm{C} 13\right), 2.22$ - 2.15 (m, 1H, $\mathrm{H}_{2} \mathrm{C} 3$ ), $2.08-$ $1.97\left(\mathrm{~m}, 2 \mathrm{H}, \mathrm{H}_{2} \mathrm{C} 3, \mathrm{H}_{2} \mathrm{C} 4\right), 1.77-1.58\left(\mathrm{~m}, 2 \mathrm{H}, \mathrm{H}_{2} \mathrm{C} 4, \mathrm{H}_{2} \mathrm{C} 15\right), 1.57-1.48(\mathrm{~m}, 1 \mathrm{H}$, $\left.\mathrm{H}_{2} \mathrm{C} 15\right), 1.46-1.29\left(\mathrm{~m}, 2 \mathrm{H}, \mathrm{H}_{2} \mathrm{C} 16\right), 0.93\left(\mathrm{t},{ }^{3} \mathrm{JHH}=7.4 \mathrm{~Hz}, 3 \mathrm{H}, \mathrm{H}_{3} \mathrm{C} 17\right) .{ }^{13} \mathrm{C}\left\{{ }^{1} \mathrm{H}\right\}$ NMR (126 MHz, Chloroform- $d$ ) $\delta 172.1$ (C7), 135.4 (C10), 133.6 (C9), 128.5 (C11), 127.3 (C12), 86.2 (d, $\left.{ }^{1} \mathrm{JFC}=173.9 \mathrm{~Hz}, \mathrm{C} 5\right), 66.8$ (C2), 57.3 (C14), 54.1 (d, ${ }^{2} \mathrm{JFC}=20.0 \mathrm{~Hz}$, C6), 29.6 (C15), 28.0 (d, $\left.{ }^{2} \mathrm{JFC}=20.8 \mathrm{~Hz}, \mathrm{C} 4\right), 24.6\left(\mathrm{~d},{ }^{3} \mathrm{JFC}=3.1 \mathrm{~Hz}, \mathrm{C} 3\right), 20.7$ (C16), $18.9(\mathrm{C} 13), 14.2(\mathrm{C} 17) .{ }^{19} \mathrm{~F} \mathrm{NMR}\left(564 \mathrm{MHz}\right.$, Chloroform-d) $\delta-185.6$ (dtt, ${ }^{2} \mathrm{JFF}=45.7 \mathrm{~Hz}$, $\left.{ }^{3} \mathrm{HFF}=34.9 \mathrm{~Hz},{ }^{3} \mathrm{JHF}=10.2 \mathrm{~Hz}\right) .{ }^{19} \mathrm{~F}\left\{{ }^{1} \mathrm{H}\right\}$ NMR $(282 \mathrm{MHz}$, Chloroform- $d) \delta-185.6$. HRMS (ESI) $\mathrm{m} / \mathrm{z}$ calculated for $\left[\mathrm{C}_{18} \mathrm{H}_{28} \mathrm{FN}_{2} \mathrm{O}\right]\left(\left[\mathrm{M}+\mathrm{H}^{+}\right]\right) 307.2180$, found 307.2199 . 


\section{Synthesis of fluororopivacaine (40):}

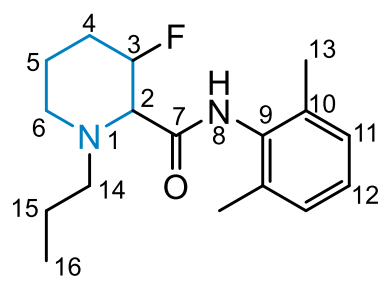

Fluorinated piperidine 28 (25.0 mg, $0.10 \mathrm{mmol}, 1.0$ equiv) and $\mathrm{K}_{2} \mathrm{CO}_{3}(28.0 \mathrm{mg}$, $0.20 \mathrm{mmol}, 2.0$ equiv) were weight into a $5 \mathrm{ml} \mathrm{screw}$-cap flask equipped with a stir bar. The solids were dissolved in DMF $(1 \mathrm{ml})$ and $n$-propyl bromide $(18 \mu \mathrm{l}, 0.20 \mathrm{mmol}$, 2.0 equiv) was added via syringe. The mixture was stirred overnight at $40^{\circ} \mathrm{C}$. After the mixture had reached room temperature, water was added and the aqueous phase was extracted with EtOAc (3x). The combined organic phases were dried over $\mathrm{MgSO}_{4}$ and concentrated. 1-Propyl- $N$-(2,6-dimethylphenyl)-3-fluoropiperidine-2-carboxamide was obtained as a white solid ( $25.4 \mathrm{mg}, 0.09 \mathrm{mmol}, 87 \%)$ after column chromatography on silical gel (eluent: $n$-pentane/EtOAc: 90:10, later 80:20, 50:50).

${ }^{1}$ H NMR $(599 \mathrm{MHz}$, Chloroform-d) $\delta 7.97$ (s, 1H, HN8), $7.12-7.05$ (m, 3H, HC11, HC12), $4.99\left(\mathrm{dm},{ }^{2} \mathrm{JFH}_{\mathrm{FH}}=47.5 \mathrm{~Hz}, 1 \mathrm{H}, H \mathrm{C} 3\right), 3.30-3.25\left(\mathrm{~m}, 1 \mathrm{H}, \mathrm{H}_{2} \mathrm{C} 6\right), 2.96\left(\mathrm{dm},{ }^{3} \mathrm{JFH}_{\mathrm{FH}}\right.$ $=31.2 \mathrm{~Hz}, 1 \mathrm{H}, \mathrm{HaxC}_{\mathrm{ax}}$ ), 2.86 (ddd, ${ }^{2} \mathrm{JHH}_{\mathrm{HH}}=12.5 \mathrm{~Hz},{ }^{3} \mathrm{JHH}_{\mathrm{HH}}=10.6 \mathrm{~Hz},{ }^{3} \mathrm{JHH}_{\mathrm{HH}}=6.3 \mathrm{~Hz}, 1 \mathrm{H}$, $\left.\mathrm{H}_{2} \mathrm{C} 14\right), 2.27$ (s, 6H, $\mathrm{H}_{3} \mathrm{C} 13$ ), $2.22-2.13$ (m, 2H, $\left.\mathrm{H}_{2} \mathrm{C} 4, \mathrm{H}_{2} \mathrm{C} 14\right), 2.08-1.93(\mathrm{~m}, 2 \mathrm{H}$, $\left.\mathrm{H}_{2} \mathrm{C} 5, \mathrm{H}_{2} \mathrm{C} 6\right), 1.76-1.66$ (m, $\left.1 \mathrm{H}, \mathrm{H}_{2} \mathrm{C} 15\right), 1.64-1.44$ (m, 3H, $\left.\mathrm{H}_{2} \mathrm{C} 4, \mathrm{H}_{2} \mathrm{C} 5, \mathrm{H}_{2} \mathrm{C} 15\right)$, $0.91\left(\mathrm{t},{ }^{3} \mathrm{JHH}_{\mathrm{HH}}=7.4 \mathrm{~Hz}, 3 \mathrm{H}, \mathrm{H}_{2} \mathrm{C} 16\right) .{ }^{13} \mathrm{C}\left\{{ }^{1} \mathrm{H}\right\}$ NMR $(151 \mathrm{MHz}$, Chloroform-d) $\delta 169.2$ (C7), 135.5 (C10), 133.9 (C9), 128.5 (C11), 127.3 (C12), 88.4 (d, ${ }^{1} \mathrm{JFC}=176.3 \mathrm{~Hz}, \mathrm{C} 3$ ), $71.6\left(\mathrm{~d},{ }^{2} \mathrm{JFC}=18.4 \mathrm{~Hz}, \mathrm{C} 2\right), 60.0(\mathrm{C} 14), 51.6(\mathrm{C} 6), 28.8\left(\mathrm{~d},{ }^{2} \mathrm{JFC}=21.8 \mathrm{~Hz}, \mathrm{C} 4\right), 20.5$ (C15), $19.3\left(\mathrm{~d},{ }^{3} \mathrm{JFC}=2.5 \mathrm{~Hz}, \mathrm{C} 5\right), 18.9(\mathrm{~d}, \mathrm{JFC}=2.7 \mathrm{~Hz}, \mathrm{C} 13), 11.7$ (C16). ${ }^{19} \mathrm{~F}$ NMR (564 MHz, Chloroform- $d$ ) $\delta-185.7--190.5(\mathrm{~m})$. HRMS (ESI) $\mathrm{m} / \mathrm{z}$ calculated for $\left[\mathrm{C}_{17} \mathrm{H}_{26} \mathrm{FN}_{2} \mathrm{O}\right]\left(\left[\mathrm{M}+\mathrm{H}^{+}\right]\right)$293.2024, found 293.2022. 


\section{Synthesis of enantioenriched 3-fluoropiperidine:}<smiles>CC(C)[C@H]1COC(=O)N1c1ccc(F)cn1</smiles>

(R)-3-(5-Fluoropyridin-2-yl)-4-isopropyloxazolidin-2-one (41): Into a $10 \mathrm{ml}$ screwcap Schlenk vial equipped with a stir bar were added $(R)$-4-isopropyloxazolidin-2-one (5.0 mmol, $646 \mathrm{mg}, 1.0$ equiv), Cul ( $0.25 \mathrm{mmol}, 48 \mathrm{mg}, 5 \mathrm{~mol} \%), 1,10$-phenanthrolin (0.50 mmol, $90 \mathrm{mg}, 10 \mathrm{~mol} \%$ ), and $\mathrm{K}_{2} \mathrm{CO}_{3}(6.5 \mathrm{mmol}, 898 \mathrm{mg}, 1.3$ equiv). The solids were suspended in dry toluene $(5 \mathrm{ml})$ and 2-bromo-5-fluoropyridine $(5.0 \mathrm{mmol}$, $880 \mathrm{mg}, 1.0$ equiv) was added via syringe. The mixture was heated to $140{ }^{\circ} \mathrm{C}$ and stirred overnight at that temperature. After cooling to room temperature, the mixture was filtered over celite using $\mathrm{CH}_{2} \mathrm{Cl}_{2}$ and concentrated. The product was obtained after column chromatography on silica gel (eluent: $n$-pentane/EtOAc 95:5, later 90:10, 80:20) as a white solid. ${ }^{1} \mathbf{H}$ NMR (400 MHz, Chloroform- $\left.d\right) \delta 8.22-8.08(\mathrm{~m}, 2 \mathrm{H}), 7.45$ (ddd, $J=9.3,7.6,3.0 \mathrm{~Hz}, 1 \mathrm{H}$ ), 4.81 (dt, $J=8.8,3.8 \mathrm{~Hz}, 1 \mathrm{H}), 4.38(\mathrm{t}, J=8.9 \mathrm{~Hz}, 1 \mathrm{H}$ ), $4.28(\mathrm{dd}, J=9.0,3.9 \mathrm{~Hz}, 1 \mathrm{H}), 2.52-2.40(\mathrm{~m}, 1 \mathrm{H}), 0.93(\mathrm{~d}, J=7.1 \mathrm{~Hz}, 3 \mathrm{H}), 0.83(\mathrm{~d}, J$ $=6.9 \mathrm{~Hz}, 3 \mathrm{H}) .{ }^{13} \mathrm{C}\left\{{ }^{1} \mathrm{H}\right\}$ NMR $(101 \mathrm{MHz}$, Chloroform- $d) \delta 156.4(\mathrm{~d}, J=251.4 \mathrm{~Hz}), 155.6$, 146.7 (d, $J=1.8 \mathrm{~Hz}), 135.0$ (d, $J=25.4 \mathrm{~Hz}$ ), 125.3 (d, $J=19.8 \mathrm{~Hz}), 115.5$ (d, J = 4.4 $\mathrm{Hz}$ ), 63.0, 59.3, 27.7, 18.0, 14.4. ${ }^{19} \mathrm{~F}\left\{{ }^{1} \mathrm{H}\right\}$ NMR (376 MHz, Chloroform- $d$ ) $\delta-133.5$. HRMS (ESI) m/z calculated for $\left[\mathrm{C}_{11} \mathrm{H}_{13} \mathrm{FN}_{2} \mathrm{O}_{2} \mathrm{Na}\right]\left(\left[\mathrm{M}+\mathrm{Na}^{+}\right]\right) 247.0853$, found 247.0861 .

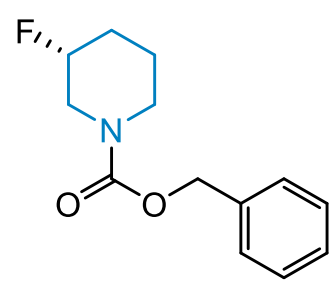

Benzyl (R)-3-fluoropiperidine-1-carboxylate (42): An $8 \mathrm{ml}$ screw-cap glass vial equipped with a stir bar was charged with $(R)$-3-(5-fluoropyridin-2-yl)-4isopropyloxazolidin-2-one (112.0 mg, $0.5 \mathrm{mmol}, 1.0$ equiv) and $\mathrm{Pd}(\mathrm{OH})_{2} / \mathrm{C}(17.6 \mathrm{mg}$, $0.025 \mathrm{~mol}, 5 \mathrm{~mol} \%, 20 \mathrm{wt} \%$ on carbon). Dry $\mathrm{MeOH}(5 \mathrm{ml}, 0.1 \mathrm{M})$ was added followed by conc. $\mathrm{H}_{2} \mathrm{SO}_{4}(35 \mu \mathrm{l}, 0.6 \mathrm{mmol}, 1.2$ equiv). The glass vial was placed in a $150 \mathrm{ml}$ stainless steel autoclave under air. The autoclave was pressurized and depressurized four times with hydrogen gas before the final hydrogen pressure (50 bar) was set. The reaction mixture was stirred at $40^{\circ} \mathrm{C}$ for $24 \mathrm{~h}$. After the autoclave was carefully 
depressurized, the mixture was diluted with $\mathrm{H}_{2} \mathrm{O}(1 \mathrm{ml})$ followed by the addition of $\mathrm{Na}_{2} \mathrm{CO}_{3}$ (212 mg, 4.0 equiv) and $\mathrm{CbzCl} / 208 \mu \mathrm{l}, 3.0$ equiv). After stirring for $1 \mathrm{~h}$ at room temperature, the mixture was filtered over celite using $\mathrm{CH}_{2} \mathrm{Cl}_{2}$. The solvent was removed in vacuo and the product was purified using column chromatography on silica gel (eluent: $n$-pentane, later 95:5, 90:10 n-pentane/EtOAc) to yield a colorless liquid ( $65 \mathrm{mg}, 0.27 \mathrm{mmol}, 55 \%, 95: 5$ e.r.). The enantiomeric ratio of the product was determined after purification using gas chromatography (HYDRODEX B-6TBDM column, $25 \mathrm{~m}, 0.25 \mathrm{~mm}$ ID; carrier gas: $\mathrm{H}_{2}$; injection temperature: $230^{\circ} \mathrm{C}$; oven temperature: $50^{\circ} \mathrm{C}$, heating rate: $0.5^{\circ} \mathrm{C} / \mathrm{min}$ ).

For determination of the absolute configuration, the carboxybenzyl group was removed (10 mol\% Pd/C, $\mathrm{H}_{2}$ (10 bar), $\mathrm{MeOH}, 40{ }^{\circ} \mathrm{C}$, overnight) and the free piperidine was treated with $\mathrm{HCl}$ in dioxane. Recrystallization from $\mathrm{CDCl}_{3} / \mathrm{CD}_{3} \mathrm{OD}$ afforded a crystal suitable for X-Ray analysis.

${ }^{1} \mathrm{H}$ NMR $(300 \mathrm{MHz}$, Chloroform- $d) \delta 7.41-7.25(\mathrm{~m}, 5 \mathrm{H}), 5.14(\mathrm{~s}, 2 \mathrm{H}), 4.62$ (dm, J = $47.2 \mathrm{~Hz}, 1 \mathrm{H}), 3.87-3.40(\mathrm{~m}, 3 \mathrm{H}), 3.38-3.20(\mathrm{~m}, 1 \mathrm{H}), 1.97-1.74(\mathrm{~m}, 3 \mathrm{H}), 1.62-$ $1.41(\mathrm{~m}, 1 \mathrm{H}) .{ }^{13} \mathrm{C}\left\{{ }^{1} \mathrm{H}\right\}$ NMR $(101 \mathrm{MHz}$, Chloroform- $d) \delta 155.6,136.8,128.6,128.1$, 127.9, $86.2(\mathrm{~d}, J=175.0 \mathrm{~Hz}), 67.3,48.0(\mathrm{~d}, J=25.1 \mathrm{~Hz}), 43.9,29.7$ (d, $J=20.5 \mathrm{~Hz})$, $21.5-20.7(\mathrm{~m}) .{ }^{19} \mathrm{~F}\left\{{ }^{1} \mathrm{H}\right\}$ NMR $(376 \mathrm{MHz}$, Chloroform- $d) \delta-184.6,-184.8$.

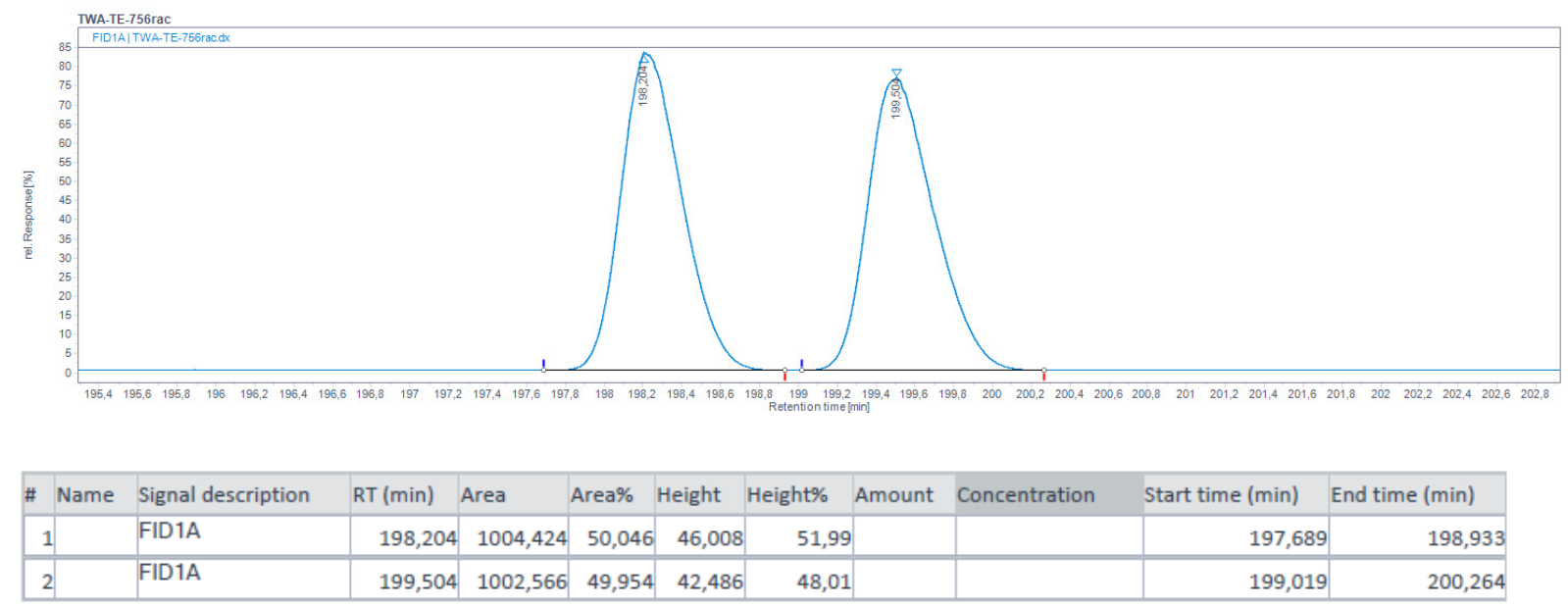




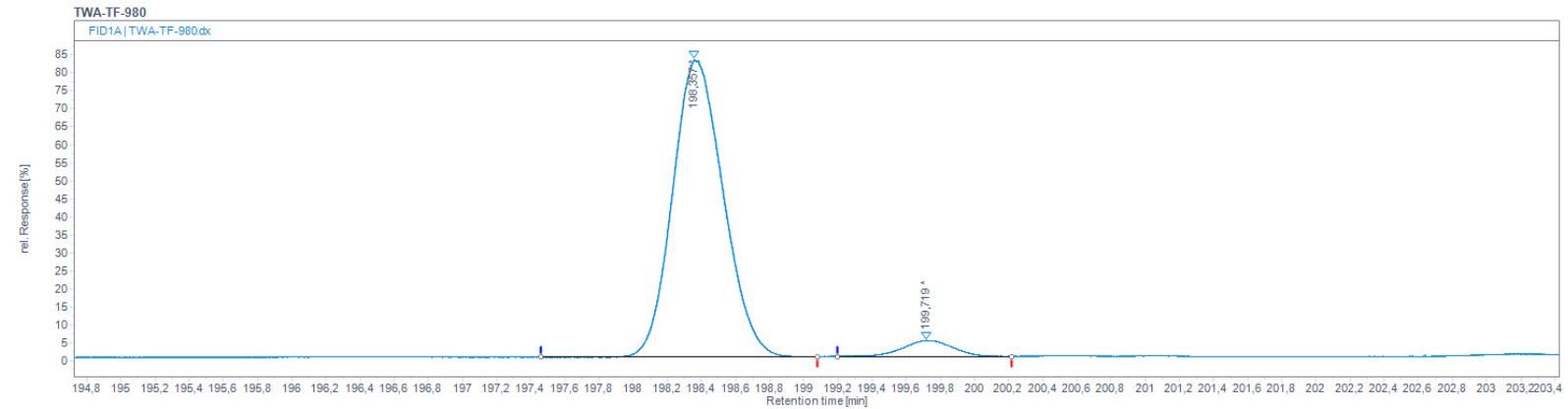

\begin{tabular}{|c|c|c|c|c|c|c|c|c|c|c|}
\hline \# Name & Signal description & $\mathrm{RT}(\mathrm{min})$ & Area & Area\% & Height & Height\% & Amount & Concentration & Start time ( $\mathrm{min}$ ) & End time ( $\mathrm{min})$ \\
\hline 1 & FID1A & 198,357 & 7) 477,681 & 95,401 & 23,267 & 95,01 & & & 197,458 & 199,198 \\
\hline 2 & FID1A & 199,719 & 23,027 & 4,599 & 1,222 & 4,99 & & & 199,198 & 200,495 \\
\hline
\end{tabular}




\section{Mechanistic Considerations}

\section{Detection of defluorinated pyridine:}

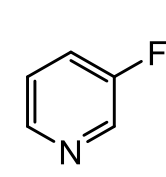

$0.25 \mathrm{mmol}$

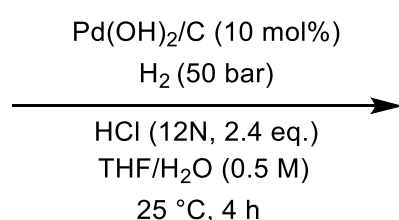

$25^{\circ} \mathrm{C}, 4 \mathrm{~h}$<smiles>Fc1cccnc1</smiles>

detected<smiles>FC1CCCNC1</smiles>

detected<smiles>C1CCNCC1</smiles>

detected

The reaction was carried out following general procedure A using 3-fluoropyridine, $10 \mathrm{~mol} \% \mathrm{Pd}(\mathrm{OH})_{2} / \mathrm{C}$ (20 wt\%), $\mathrm{HCl}\left(2.4\right.$ eq., $12 \mathrm{~N}$ ), THF/ $\mathrm{H}_{2} \mathrm{O}(0.5 \mathrm{M})$ and 50 bar hydrogen pressure. After 4 hours the reaction was stopped, the mixture was basified using $\mathrm{Na}_{2} \mathrm{CO}_{3}$ and filtered over celite. GC-MS analysis of the crude reaction mixture reveled presence of starting material (3-fluoropyridine), 3-fluoropiperidine and defluorinated piperidine. No defluorinated pyridine starting material was detected.

\section{Product decomposition:}<smiles>Cc1cccc(C)c1NC(=O)C1CCC(F)CN1</smiles>

cis-26

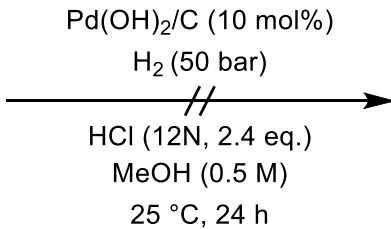

$25^{\circ} \mathrm{C}, 24 \mathrm{~h}$<smiles>Cc1cccc(C)c1NC(=O)C1CCCCN1</smiles>

not detected

The reaction was carried out following general procedure A using cis-26, $10 \mathrm{~mol} \%$ $\mathrm{Pd}(\mathrm{OH})_{2} / \mathrm{C}(20 \mathrm{wt} \%), \mathrm{HCl}(2.4$ eq., $12 \mathrm{~N}), \mathrm{MeOH}(0.5 \mathrm{M})$ and 50 bar hydrogen pressure. After $24 \mathrm{~h}$ reaction time, the mixture was basified using $\mathrm{Na}_{2} \mathrm{CO}_{3}$ and filtered over celite. Only starting material and no further reduced or defluorinated products could be detected by GC-MS analysis.

These preliminary mechanistic experiments indicate that defluorination does neither occur on the fluoropyridine starting material nor does the fluorinated piperidine show any decomposition under the reaction conditions. Thus, hydrodefluorination pathways are likely to occur on dearomatized intermediates upon hydrogenation. 
To confirm the heterogeneous nature of our catalyst, we have carried out the following experiments. The reactions were carried out following general procedure $\mathrm{A}$ using 3fluoropyridine, $10 \mathrm{~mol} \% \mathrm{Pd}(\mathrm{OH})_{2} / \mathrm{C}(20 \mathrm{wt} \%), \mathrm{HCl}(2.4$ eq., $12 \mathrm{~N}), \mathrm{MeOH}(0.5 \mathrm{M})$ and 50 bar hydrogen pressure. The reaction was determined by ${ }^{19} \mathrm{~F}$ NMR analysis using hexafluorobenzene as internal standard.

\section{Filtration test:}<smiles>Fc1cccnc1</smiles><smiles>FC1CCCNC1</smiles>

$\mathrm{Pd}(\mathrm{OH})_{2} / \mathrm{C}: 85 \%$ NMR yield recovered catalyst: $82 \%$ NMR yield

In a filtration test, the residue of a hydrogenation reaction was recovered, washed with organic solvents and reused as catalyst. The recovered catalyst achieved only a slightly worse result compared to unused Pearlman's catalyst.

\section{Poisoning experiment:}

Furthermore, a sub-stoichiometric poisoning experiment using a thiol was performed. Sub-stoichiometric amounts in relation to the catalyst of a catalyst poison can cover the entire surface of a heterogeneous catalyst and thus prevent the conversion of substrate. However, the same amount of catalyst poison cannot poison all homogeneous catalyst species, so conversion should still be possible. With a catalyst to poison ratio of $10: 1$, complete poisoning of the reaction occurred and no conversion of the starting material was observed.

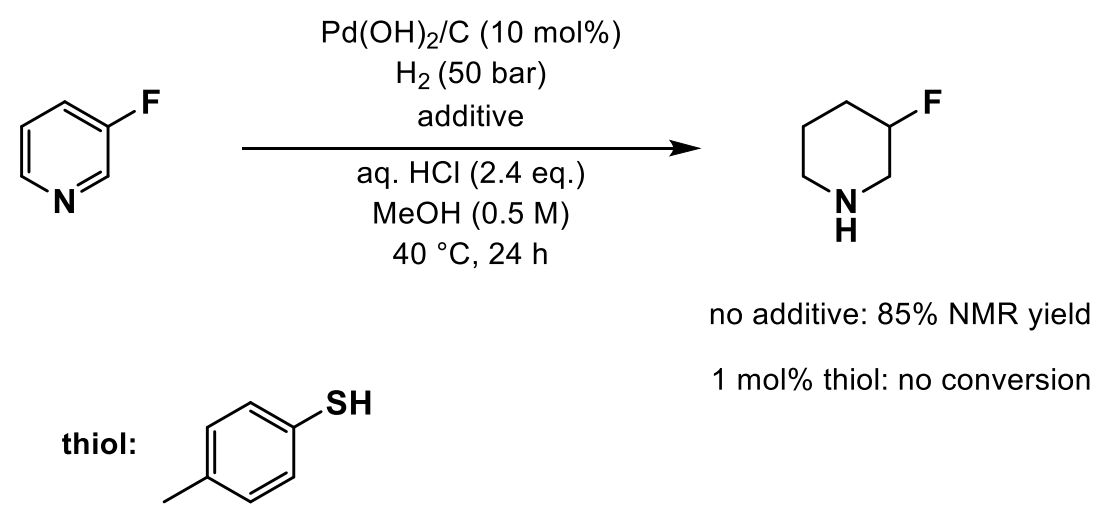




\section{X-Ray Analysis}

X-Ray diffraction: Data sets for compounds 7, 31, 32 and $\mathbf{4 3}$ were collected with a Bruker D8 Venture CMOS diffractometer. For compounds 8, 13 and 28 data sets were collected with a Bruker APEX II CCD diffractometer. Programs used: data collection: APEX3 V2016.1-0; ${ }^{5}$ cell refinement: SAINT V8.37A $; 5$ data reduction: SAINT V8.37A $;{ }^{5}$ absorption correction, SADABS V2014/7;5 structure solution SHELXT-2015;6 structure refinement SHELXL-2015 and graphics, $X P^{8}{ }^{8} R$-values are given for observed reflections, and $w R^{2}$ values are given for all reflections.

Exceptions and special features: For compound 7 the six membered ring and for compound 13 the fluorine atom were found disordered over two positions. Several restraints (SADI, SAME, ISOR and SIMU) were used in order to improve refinement stability.

X-ray crystal structure analysis of 7: A colorless plate-like specimen of $\mathrm{C}_{5} \mathrm{H}_{8} \mathrm{FNO}$, approximate dimensions $0.060 \mathrm{~mm} \times 0.210 \mathrm{~mm} \times 0.558 \mathrm{~mm}$, was used for the X-ray crystallographic analysis. The X-ray intensity data were measured. A total of 905 frames were collected. The total exposure time was 7.54 hours. The frames were integrated with the Bruker SAINT software package using a narrow-frame algorithm. The integration of the data using a monoclinic unit cell yielded a total of 6571 reflections to a maximum $\theta$ angle of $27.49^{\circ}$ ( $0.77 \AA$ resolution), of which 1216 were independent (average redundancy 5.404 , completeness $=99.0 \%, R_{\text {int }}=2.35 \%, R_{\text {sig }}=1.65 \%$ ) and $1163(95.64 \%)$ were greater than $2 \sigma\left(F^{2}\right)$. The final cell constants of $\underline{a}=10.9416(17) \AA$, $\underline{b}=5.8946(9) \AA, \underline{c}=8.9783(13) \AA, \beta=112.366(6)^{\circ}$, volume $=535.51(14) \AA^{3}$, are based upon the refinement of the XYZ-centroids of 3456 reflections above $20 \sigma(\mathrm{I})$ with $8.001^{\circ}$ $<2 \theta<54.96^{\circ}$. Data were corrected for absorption effects using the multi-scan method (SADABS). The ratio of minimum to maximum apparent transmission was 0.941 . The calculated minimum and maximum transmission coefficients (based on crystal size) are 0.9340 and 0.9930 . The structure was solved and refined using the Bruker SHELXTL Software Package, using the space group $P 2_{1} / c$, with $Z=4$ for the formula unit, $\mathrm{C}_{5} \mathrm{H}_{8} \mathrm{FNO}$. The final anisotropic full-matrix least-squares refinement on $\mathrm{F}^{2}$ with 114 variables converged at $R 1=3.83 \%$, for the observed data and $w R 2=9.34 \%$ for all data. The goodness-of-fit was 1.074. The largest peak in the final difference electron density synthesis was $0.411 \mathrm{e}^{-/} \AA^{3}$ and the largest hole was $-0.168 \mathrm{e}^{-/} / \AA^{3}$ with an RMS 
deviation of $0.040 \mathrm{e}^{-/} / \AA^{3}$. On the basis of the final model, the calculated density was $1.453 \mathrm{~g} / \mathrm{cm}^{3}$ and $\mathrm{F}(000), 248 \mathrm{e}$. The hydrogen at $\mathrm{N} 1$ atom was refined freely. CCDC Nr.: 1999050.

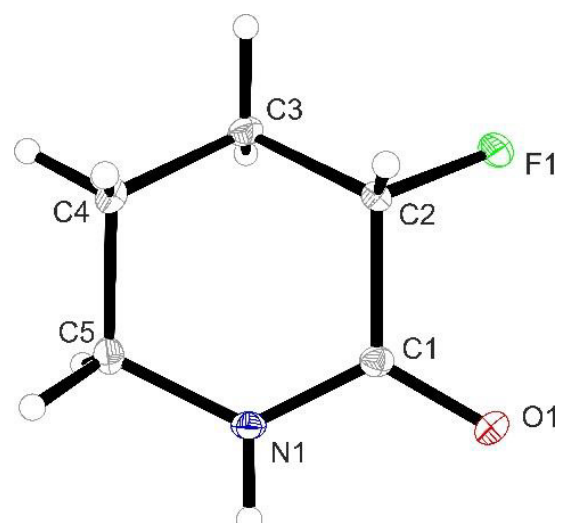

Figure S2: Crystal structure of compound 7. Thermal ellipsoids are shown at $30 \%$ probability.

X-ray crystal structure analysis of 8: A colorless needle-like specimen of $\mathrm{C}_{5} \mathrm{H}_{8} \mathrm{FNO}$, approximate dimensions $0.040 \mathrm{~mm} \times 0.080 \mathrm{~mm} \times 0.120 \mathrm{~mm}$, was used for the $X$-ray crystallographic analysis. The X-ray intensity data were measured. A total of 1267 frames were collected. The total exposure time was 17.63 hours. The frames were integrated with the Bruker SAINT software package using a wide-frame algorithm. The integration of the data using a monoclinic unit cell yielded a total of 7570 reflections to a maximum $\theta$ angle of $66.55^{\circ}$ ( $0.84 \AA$ resolution), of which 956 were independent (average redundancy 7.918 , completeness $=99.9 \%$, $R_{\text {int }}=4.44 \%, R_{\text {sig }}=2.33 \%$ ) and $833(87.13 \%)$ were greater than $2 \sigma\left(F^{2}\right)$. The final cell constants of $\underline{a}=6.8234(8) \AA, \underline{b}$ $=7.0364(7) \AA, \underline{C}=11.2928(13) \AA, \beta=93.296(5)^{\circ}$, volume $=541.29(10) \AA^{3}$, are based upon the refinement of the XYZ-centroids of 2457 reflections above $20 \sigma(I)$ with $12.99^{\circ}$ $<2 \theta<133.0^{\circ}$. Data were corrected for absorption effects using the multi-scan method (SADABS). The ratio of minimum to maximum apparent transmission was 0.872 . The calculated minimum and maximum transmission coefficients (based on crystal size) are 0.8840 and 0.9590 . The structure was solved and refined using the Bruker SHELXTL Software Package, using the space group $P 2_{1} / c$, with $Z=4$ for the formula unit, $\mathrm{C}_{5} \mathrm{H}_{8} \mathrm{FNO}$. The final anisotropic full-matrix least-squares refinement on $\mathrm{F}^{2}$ with 77 variables converged at $\mathrm{R} 1=3.62 \%$, for the observed data and $\mathrm{wR} 2=8.91 \%$ for all data. The goodness-of-fit was 1.121. The largest peak in the final difference electron 
density synthesis was $0.202 \mathrm{e}^{-/} / \AA^{3}$ and the largest hole was $-0.215 \mathrm{e}^{-/} / \AA^{3}$ with an RMS deviation of $0.049 \mathrm{e}^{-} / \AA^{3}$. On the basis of the final model, the calculated density was $1.437 \mathrm{~g} / \mathrm{cm}^{3}$ and $\mathrm{F}(000), 248 \mathrm{e}$. The hydrogen at $\mathrm{N} 1$ atom was refined freely. CCDC Nr.: 1999051.

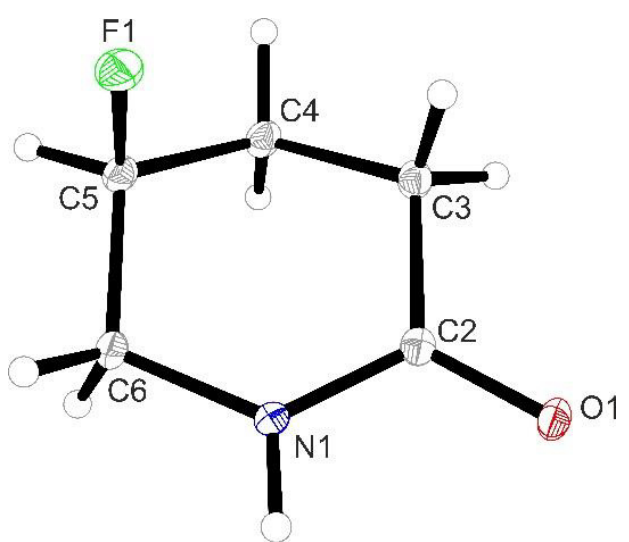

Figure S3: Crystal structure of compound 8. Thermal ellipsoids are shown at $30 \%$ probability.

X-ray crystal structure analysis of 13: A colorless prism-like specimen of $\mathrm{C}_{12} \mathrm{H}_{13} \mathrm{~F}_{4} \mathrm{~N}$, approximate dimensions $0.060 \mathrm{~mm} \times 0.100 \mathrm{~mm} \times 0.180 \mathrm{~mm}$, was used for the X-ray crystallographic analysis. The X-ray intensity data were measured. A total of 1146 frames were collected. The total exposure time was 15.10 hours. The frames were integrated with the Bruker SAINT software package using a wide-frame algorithm. The integration of the data using an orthorhombic unit cell yielded a total of 14196 reflections to a maximum $\theta$ angle of $66.65^{\circ}(0.84 \AA$ resolution), of which 1962 were independent (average redundancy 7.235 , completeness $=99.9 \%$, Rint $=4.43 \%$, Rsig $=$ $2.57 \%)$ and $1671(85.17 \%)$ were greater than $2 \sigma\left(\mathrm{F}^{2}\right)$. The final cell constants of $\underline{a}=$ 16.6845(4) $\AA, \underline{b}=6.2599(2) \AA, \underline{c}=21.2252(5) \AA$, volume $=2216.83(10) \AA^{3}$, are based upon the refinement of the XYZ-centroids of 3690 reflections above $20 \mathrm{\sigma}(\mathrm{I})$ with $8.331^{\circ}$ $<2 \theta<133.3^{\circ}$. Data were corrected for absorption effects using the multi-scan method (SADABS). The ratio of minimum to maximum apparent transmission was 0.831 . The calculated minimum and maximum transmission coefficients (based on crystal size) are 0.8170 and 0.9330 . The structure was solved and refined using the Bruker SHELXTL Software Package, using the space group $P b c n$, with $Z=8$ for the formula unit, $\mathrm{C}_{12} \mathrm{H}_{13} \mathrm{~F}_{4} \mathrm{~N}$. The final anisotropic full-matrix least-squares refinement on $\mathrm{F}^{2}$ with 168 variables converged at $R 1=4.14 \%$, for the observed data and $w R 2=11.57 \%$ for 
all data. The goodness-of-fit was 1.029. The largest peak in the final difference electron density synthesis was $0.257 \mathrm{e}^{-/} \AA^{3}$ and the largest hole was $-0.240 \mathrm{e}^{-/} / \AA^{3}$ with an RMS deviation of $0.047 \mathrm{e}^{-/} / \AA^{3}$. On the basis of the final model, the calculated density was $1.482 \mathrm{~g} / \mathrm{cm}^{3}$ and $\mathrm{F}(000), 1024 \mathrm{e}$. The hydrogen at $\mathrm{N} 1$ atom was refined freely. CCDC Nr.: 1999052.

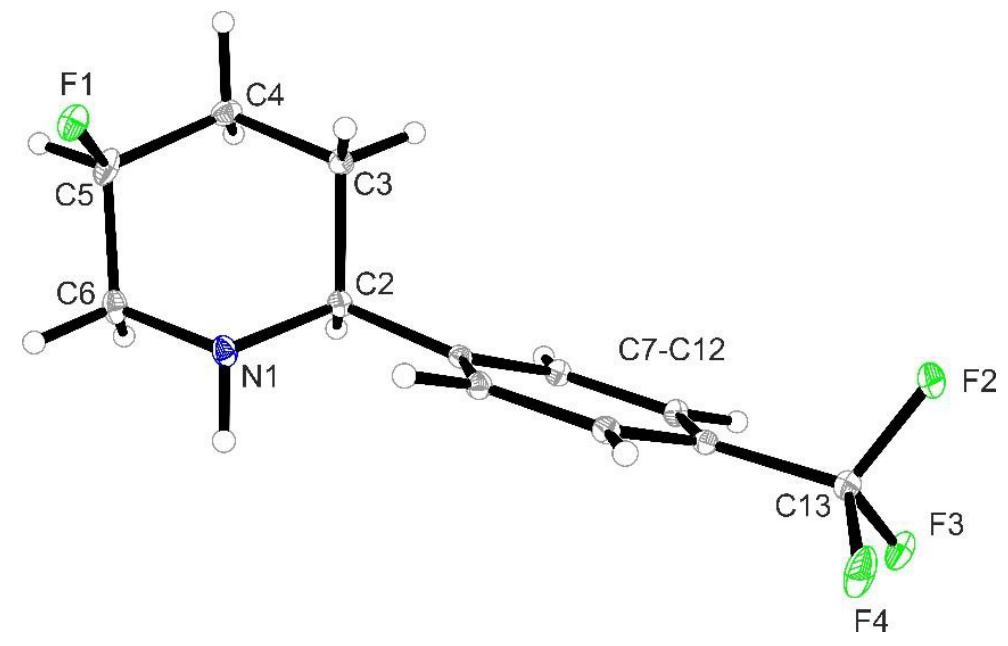

Figure S4: Crystal structure of compound 13. Thermal ellipsoids are shown at $15 \%$ probability.

X-ray crystal structure analysis of 27: A colorless prism-like specimen of $\mathrm{C}_{13} \mathrm{H}_{14} \mathrm{~F}_{4} \mathrm{~N}_{2} \mathrm{O}$, approximate dimensions $0.140 \mathrm{~mm} \times 0.140 \mathrm{~mm} \times 0.240 \mathrm{~mm}$, was used for the $\mathrm{X}$-ray crystallographic analysis. The $\mathrm{X}$-ray intensity data were measured. $\mathrm{A}$ total of 1493 frames were collected. The total exposure time was 21.59 hours. The frames were integrated with the Bruker SAINT software package using a wide-frame algorithm. The integration of the data using a monoclinic unit cell yielded a total of 17836 reflections to a maximum $\theta$ angle of $66.83^{\circ}$ ( $0.84 \AA$ resolution), of which 2273 were independent (average redundancy 7.847 , completeness $=99.3 \%$, Rint $=4.36 \%$, $\left.R_{\text {sig }}=2.35 \%\right)$ and $1989(87.51 \%)$ were greater than $2 \sigma\left(\mathrm{F}^{2}\right)$. The final cell constants of $\underline{\mathrm{a}}=12.8484(3) \AA$, $\underline{\mathrm{b}}=8.4676(2) \AA, \underline{\mathrm{c}}=11.8938(3) \AA, \beta=95.5190(10)^{\circ}$, volume $=$ $1287.99(5) \AA^{3}$, are based upon the refinement of the $X Y Z$-centroids of 7181 reflections above $20 \sigma(\mathrm{I})$ with $6.912^{\circ}<2 \theta<133.2^{\circ}$. Data were corrected for absorption effects using the multi-scan method (SADABS). The ratio of minimum to maximum apparent transmission was 0.843. The calculated minimum and maximum transmission coefficients (based on crystal size) are 0.7640 and 0.8520 . The structure was solved and refined using the Bruker SHELXTL Software Package, using the space group 
$P 21 / c$, with $Z=4$ for the formula unit, $\mathrm{C}_{13} \mathrm{H}_{14} \mathrm{~F}_{4} \mathrm{~N}_{2} \mathrm{O}$. The final anisotropic full-matrix least-squares refinement on $\mathrm{F}^{2}$ with 189 variables converged at $\mathrm{R} 1=3.34 \%$, for the observed data and $w R 2=8.92 \%$ for all data. The goodness-of-fit was 1.042 . The largest peak in the final difference electron density synthesis was $0.211 \mathrm{e}^{\mathrm{e} / \AA^{3}}$ and the largest hole was $-0.232 \mathrm{e}^{-} / \AA^{3}$ with an RMS deviation of $0.043 \mathrm{e}^{-} / \AA^{3}$. On the basis of the final model, the calculated density was $1.497 \mathrm{~g} / \mathrm{cm}^{3}$ and $F(000), 600 \mathrm{e}$. The hydrogen atoms at N1 and N2 atom was refined freely. CCDC Nr.: 1999053.

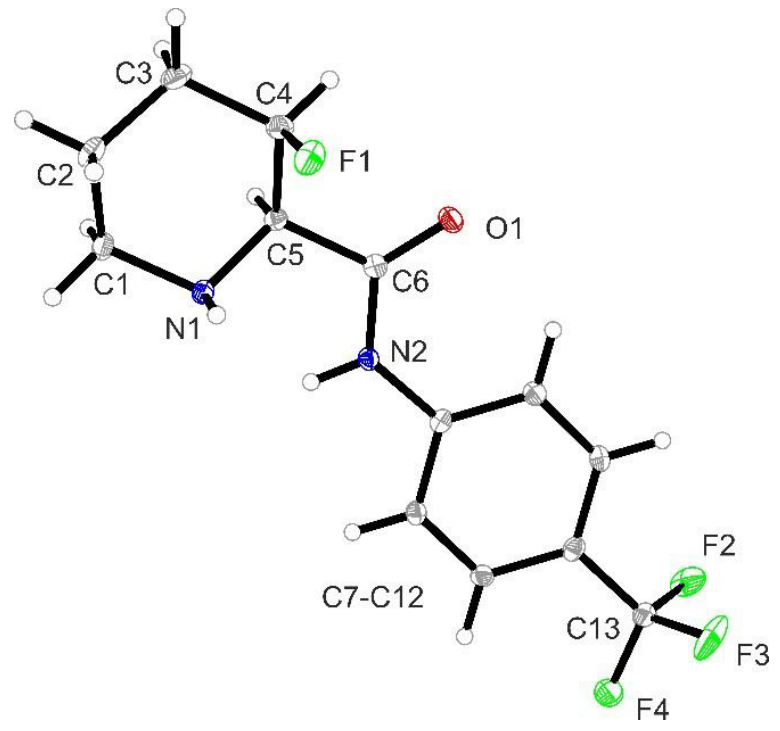

Figure S5: Crystal structure of compound 27 . Thermal ellipsoids are shown at $30 \%$ probability.

X-ray crystal structure analysis of 31: A colorless plate-like specimen of $\mathrm{C}_{13} \mathrm{H}_{19} \mathrm{FN}_{2} \mathrm{O}_{2}$, approximate dimensions $0.038 \mathrm{~mm} \times 0.088 \mathrm{~mm} \times 0.129 \mathrm{~mm}$, was used for the $\mathrm{X}$-ray crystallographic analysis. The $\mathrm{X}$-ray intensity data were measured. A total of 917 frames were collected. The total exposure time was 15.43 hours. The frames were integrated with the Bruker SAINT software package using a wide-frame algorithm. The integration of the data using a monoclinic unit cell yielded a total of 16859 reflections to a maximum $\theta$ angle of $68.28^{\circ}$ ( $0.83 \AA$ resolution), of which 2406 were independent (average redundancy 7.007 , completeness $=99.4 \%, R_{\text {int }}=4.91 \%$, $\left.R_{\text {sig }}=2.97 \%\right)$ and $2015(83.75 \%)$ were greater than $2 \sigma\left(F^{2}\right)$. The final cell constants of $\underline{\mathrm{a}}=11.8985(2) \AA, \underline{\mathrm{b}}=7.4145(2) \AA, \underline{\mathrm{c}}=15.3304(3) \AA \AA$ 1324.92(5) $\AA^{3}$, are based upon the refinement of the $X Y Z$-centroids of 8495 reflections above $20 \sigma(\mathrm{I})$ with $8.618^{\circ}<2 \theta<136.4^{\circ}$. Data were corrected for absorption effects 
using the multi-scan method (SADABS). The ratio of minimum to maximum apparent transmission was 0.871. The calculated minimum and maximum transmission coefficients (based on crystal size) are 0.9040 and 0.9700 . The structure was solved and refined using the Bruker SHELXTL Software Package, using the space group $P 21 / n$, with $\mathrm{Z}=4$ for the formula unit, $\mathrm{C}_{13} \mathrm{H}_{19} \mathrm{FN}_{2} \mathrm{O}_{2}$. The final anisotropic full-matrix least-squares refinement on $\mathrm{F}^{2}$ with 181 variables converged at $\mathrm{R} 1=4.86 \%$, for the observed data and $w R 2=13.38 \%$ for all data. The goodness-of-fit was 1.059 . The largest peak in the final difference electron density synthesis was $0.752 \mathrm{e}^{-} / \AA^{3}$ and the largest hole was $-0.319 \mathrm{e} / \AA^{3}$ with an RMS deviation of $0.048 \mathrm{e}^{-} / \AA^{3}$. On the basis of the final model, the calculated density was $1.275 \mathrm{~g} / \mathrm{cm}^{3}$ and $F(000), 544 \mathrm{e}^{-}$. The hydrogen atoms at $\mathrm{N} 1$ and $\mathrm{N} 2$ were refined freely. The hydrogen atoms from water oxygen were refined freely, but with O-H distance restraints (SADI, DFIX). CCDC Nr.: 1999054.

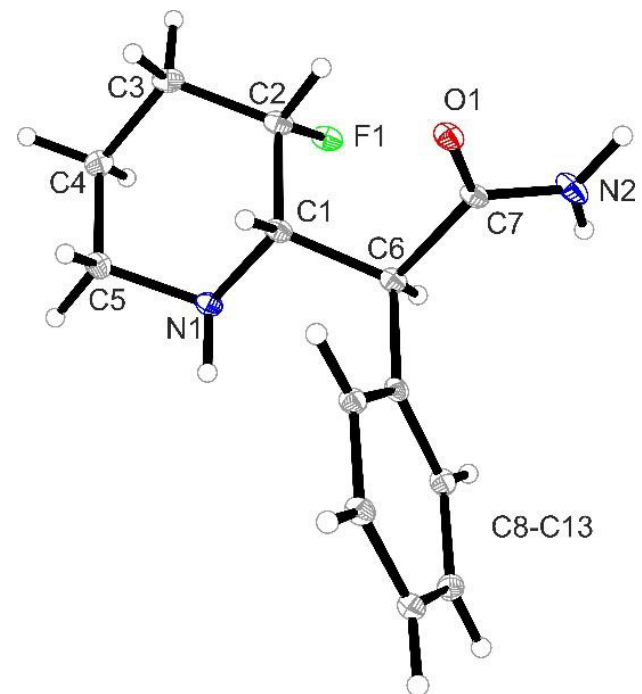

Figure S6: Crystal structure of compound 31. Thermal ellipsoids are shown at $15 \%$ probability.

X-ray crystal structure analysis of 32: A colorless prism-like specimen of $\mathrm{C}_{7} \mathrm{H}_{11} \mathrm{FN}_{2} \mathrm{O}$, approximate dimensions $0.104 \mathrm{~mm} \times 0.147 \mathrm{~mm} \times 0.317 \mathrm{~mm}$, was used for the $\mathrm{X}$-ray crystallographic analysis. The $\mathrm{X}$-ray intensity data were measured. A total of 633 frames were collected. The total exposure time was 5.28 hours. The frames were integrated with the Bruker SAINT software package using a narrow-frame algorithm. The integration of the data using a monoclinic unit cell yielded a total of 19749 reflections to a maximum $\theta$ angle of $27.51^{\circ}$ ( $0.77 \AA$ resolution), of which 1804 were independent (average redundancy 10.947 , completeness $=99.4 \%$, $R_{\text {int }}=4.02 \%$, 
$\left.R_{\text {sig }}=1.67 \%\right)$ and $1662(92.13 \%)$ were greater than $2 \sigma\left(F^{2}\right)$. The final cell constants of $\underline{\mathrm{a}}=4.7931(2) \AA, \underline{\mathrm{b}}=12.9627(5) \AA \stackrel{\mathrm{c}}{\mathrm{c}}=12.8030(5) \AA, \beta=97.4690(10)^{\circ}$, volume $=$ $788.72(5) \AA^{3}$, are based upon the refinement of the XYZ-centroids of 7413 reflections above $20 \sigma(I)$ with $6.286^{\circ}<2 \theta<54.99^{\circ}$. Data were corrected for absorption effects using the multi-scan method (SADABS). The ratio of minimum to maximum apparent transmission was 0.927 . The calculated minimum and maximum transmission coefficients (based on crystal size) are 0.9670 and 0.9890 . The structure was solved and refined using the Bruker SHELXTL Software Package, using the space group $P 2{ }_{1} / c$, with $\mathrm{Z}=4$ for the formula unit, $\mathrm{C}_{7} \mathrm{H}_{11} \mathrm{FN}_{2} \mathrm{O}$. The final anisotropic full-matrix leastsquares refinement on $\mathrm{F}^{2}$ with 113 variables converged at $\mathrm{R} 1=3.74 \%$, for the observed data and $w R 2=9.30 \%$ for all data. The goodness-of-fit was 1.090 . The largest peak in the final difference electron density synthesis was $0.297 \mathrm{e}^{-/} \AA^{3}$ and the largest hole was $-0.186 \mathrm{e}^{-} / \AA^{3}$ with an RMS deviation of $0.039 \mathrm{e} / \AA^{3}$. On the basis of the final model, the calculated density was $1.332 \mathrm{~g} / \mathrm{cm}^{3}$ and $F(000), 336 \mathrm{e}$. One hydrogen atom from the water oxygen was found disordered over two position. The hydrogen atoms were refined freely, but with $\mathrm{O}-\mathrm{H}$ distance restraints (SADI). CCDC Nr.: 1999055.

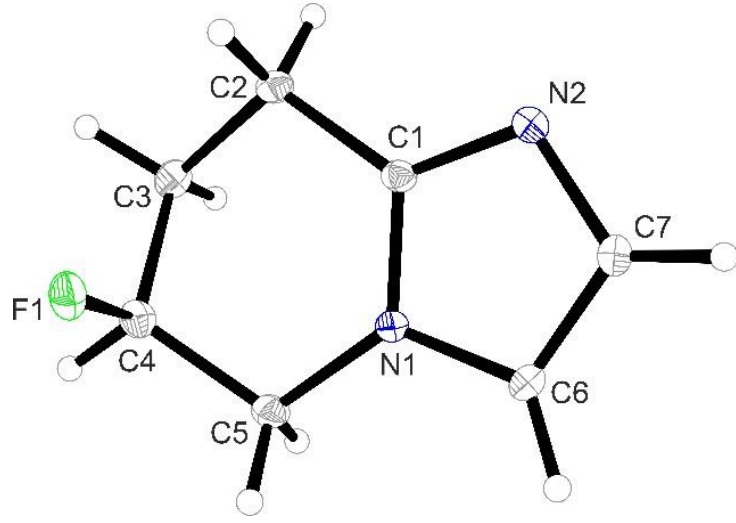

Figure S7: Crystal structure of compound 32. Thermal ellipsoids are shown at $30 \%$ probability.

X-ray crystal structure analysis of 43: A colorless plate-like specimen of $\mathrm{C}_{5} \mathrm{H}_{11} \mathrm{CIFN}$, approximate dimensions $0.057 \mathrm{~mm} \times 0.107 \mathrm{~mm} \times 0.111 \mathrm{~mm}$, was used for the $X$-ray crystallographic analysis. The X-ray intensity data were measured on a Bruker D8 Venture PHOTON III Diffractometer system equipped with a micro focus tube $\mathrm{Cu}$ Ims (CuKa, $\lambda=1.54178 \AA$ ) and a MX mirror monochromator. A total of 1092 frames were 
collected. The total exposure time was 17.69 hours. The frames were integrated with the Bruker SAINT software package using a wide-frame algorithm. The integration of the data using a monoclinic unit cell yielded a total of 5303 reflections to a maximum $\theta$ angle of $68.18^{\circ}$ (0.83 $\AA$ resolution), of which 1204 were independent (average redundancy 4.404 , completeness $\left.=98.7 \%, R_{\text {int }}=4.46 \%, R_{\text {sig }}=4.29 \%\right)$ and 1165 $(96.76 \%)$ were greater than $2 \sigma\left(F^{2}\right)$. The final cell constants of $\underline{a}=6.6025(2) \AA$, $\underline{b}=$ 7.3672(2) $\AA, \underline{c}=7.0360(2) \AA, \beta=90.746(2)^{\circ}$, volume $=342.216(17) \AA^{3}$, are based upon the refinement of the $X Y Z$-centroids of 4796 reflections above $20 \sigma(I)$ with $12.58^{\circ}$ $<2 \theta<136.3^{\circ}$. Data were corrected for absorption effects using the Multi-Scan method (SADABS). The ratio of minimum to maximum apparent transmission was 0.791 . The calculated minimum and maximum transmission coefficients (based on crystal size) are 0.6460 and 0.7910 . The structure was solved and refined using the Bruker SHELXTL Software Package, using the space group $P 2{ }_{1}$, with $Z=2$ for the formula unit, $\mathrm{C}_{5} \mathrm{H}_{11} \mathrm{CIFN}$. The final anisotropic full-matrix least-squares refinement on $\mathrm{F}^{2}$ with 81 variables converged at $\mathrm{R} 1=3.30 \%$, for the observed data and $\mathrm{wR} 2=8.66 \%$ for all data. The goodness-of-fit was 1.172. The largest peak in the final difference electron density synthesis was $0.269 \mathrm{e}^{-/} / \AA^{3}$ and the largest hole was $-0.273 \mathrm{e}^{-/} / \AA^{3}$ with an RMS deviation of $0.062 \mathrm{e}^{-} / \AA^{3}$. On the basis of the final model, the calculated density was $1.355 \mathrm{~g} / \mathrm{cm}^{3}$ and $\mathrm{F}(000), 148 \mathrm{e}$. The hydrogens at $\mathrm{N} 1$ atom were refined freely. Flack parameter was refined to $0.078(14)$. CCDC number: 2026631.

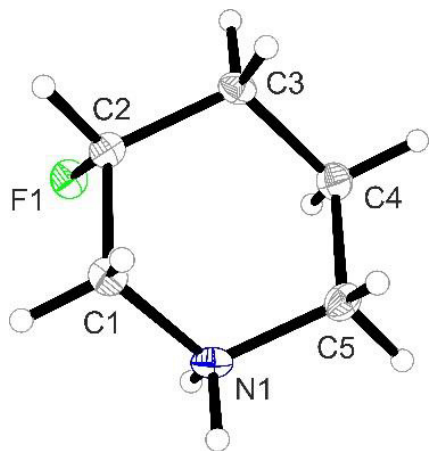

Figure S8: Crystal structure of compound 43. Thermal ellipsoids are shown at $30 \%$ probability. 


\section{Literature}

(1) Suveges, N. S.; de Souza, R. O. M. A.; Gutman, B.; Kappe, C. O. Synthesis of Mepivacaine and Its Analogues by a Continuous-Flow Tandem Hydrogenation/Reductive Amination Strategy. Eur. J. Org. Chem. 2017, 65116517.

(2) Pitzer, L.; Schäfers, F.; Glorius, F. Rapid Assessment of the Reaction-ConditionBased Sensitivity of Chemical Transformations. Angew. Chem., Int. Ed. 2019, 58, 8572-8576.

(3) Nairoukh, Z.; Wollenburg, M.; Schlepphorst, C.; Bergander, K.; Glorius, F. The formation of all-cis-(multi)fluorinated piperidines by a dearomatizationhydrogenation process. Nat. Chem. 2019, 11, 264-270.

(4) Nairoukh, Z.; Strieth-Kalthoff, F.; Bergander, K.; Glorius, F. Understanding the Conformational Behavior of Fluorinated Piperidines: The Origin of the Axial-F Preference. Chem. Eur. J. 2020, 26, 6141-6146.

(5) APEX3 (2016), SAINT (2015) and SADABS (2015), Bruker AXS Inc., Madison, Wisconsin, USA.

(6) G. M. Sheldrick, Acta Cryst., 2015, A71, 3-8.

(7) G. M. Sheldrick, Acta Cryst., 2015, C71 (1), 3-8.

(8) XP - Interactive molecular graphics, Version 5.1, Bruker AXS Inc., Madison, Wisconsin, USA, 1998. 


\section{NMR Spectra}

1H NMR (Chloroforme)

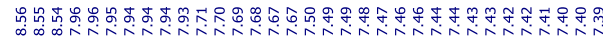<smiles>Fc1ccc(-c2ccccc2)nc1</smiles>

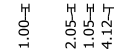

\begin{tabular}{lllllllllllllllllllllllll}
\hline 16 & 15 & 14 & 13 & 12 & 11 & 10 & 9 & 8 & 7 & 6 & 5 & 4 & 3 & 2 & 1 & 0 & -1 & -2 & -3 & -4
\end{tabular}

${ }^{13} \mathrm{C}\{1 \mathrm{H}\}$ NMR (Chloroforme)

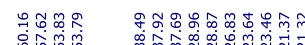

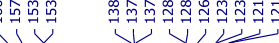<smiles>Fc1ccc(-c2ccccc2)nc1</smiles> 
${ }^{19} \mathrm{~F}\{1 \mathrm{H}\}$ NMR (Chloroforme)

\begin{tabular}{l}
$\stackrel{0}{0}$ \\
\multirow{7}{7}{}
\end{tabular}
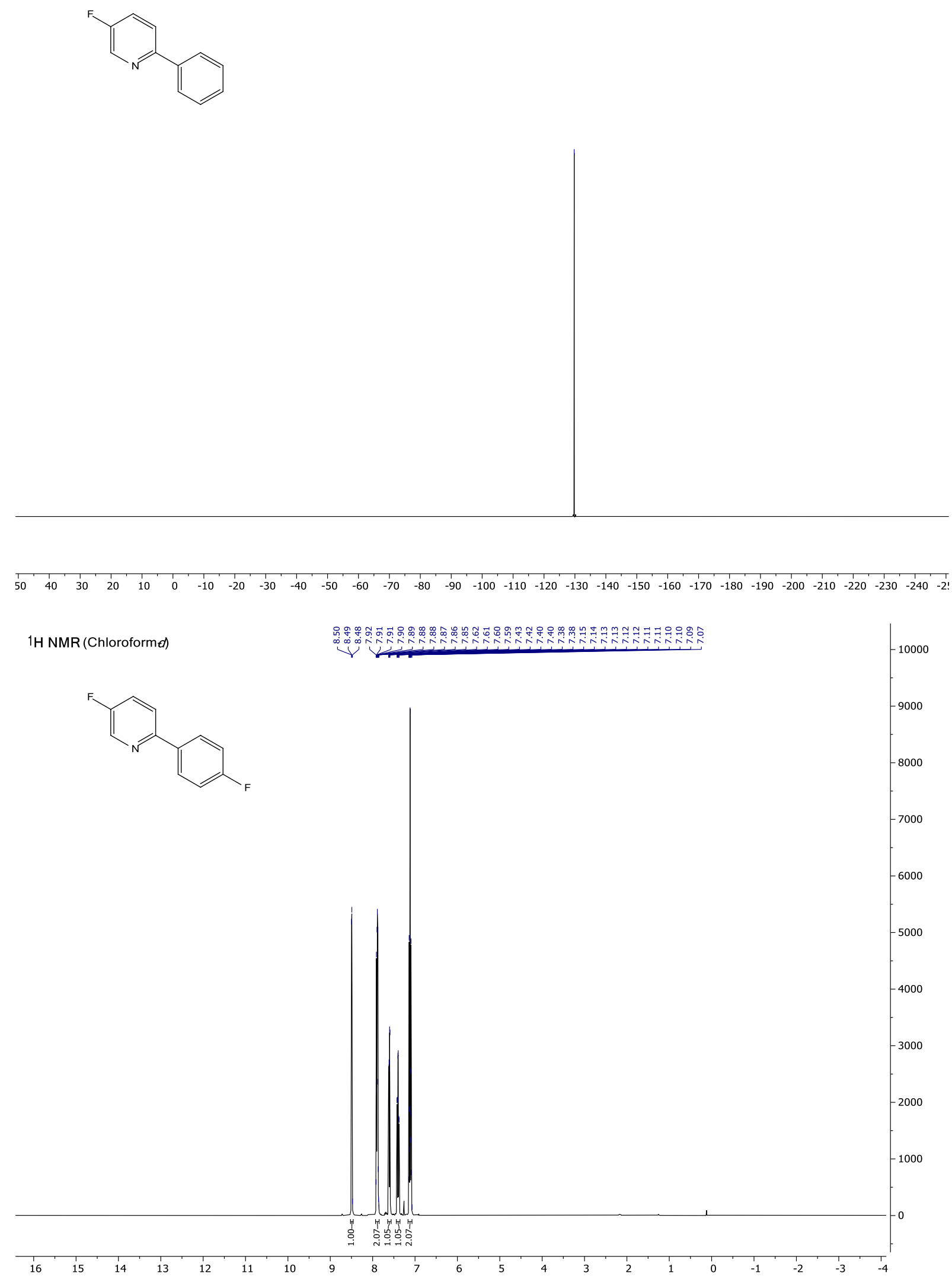

S75 
${ }^{13} \mathrm{C}\{1 \mathrm{H}\}$ NMR (Chloroforme)

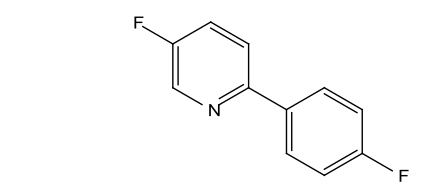

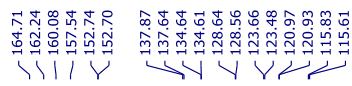

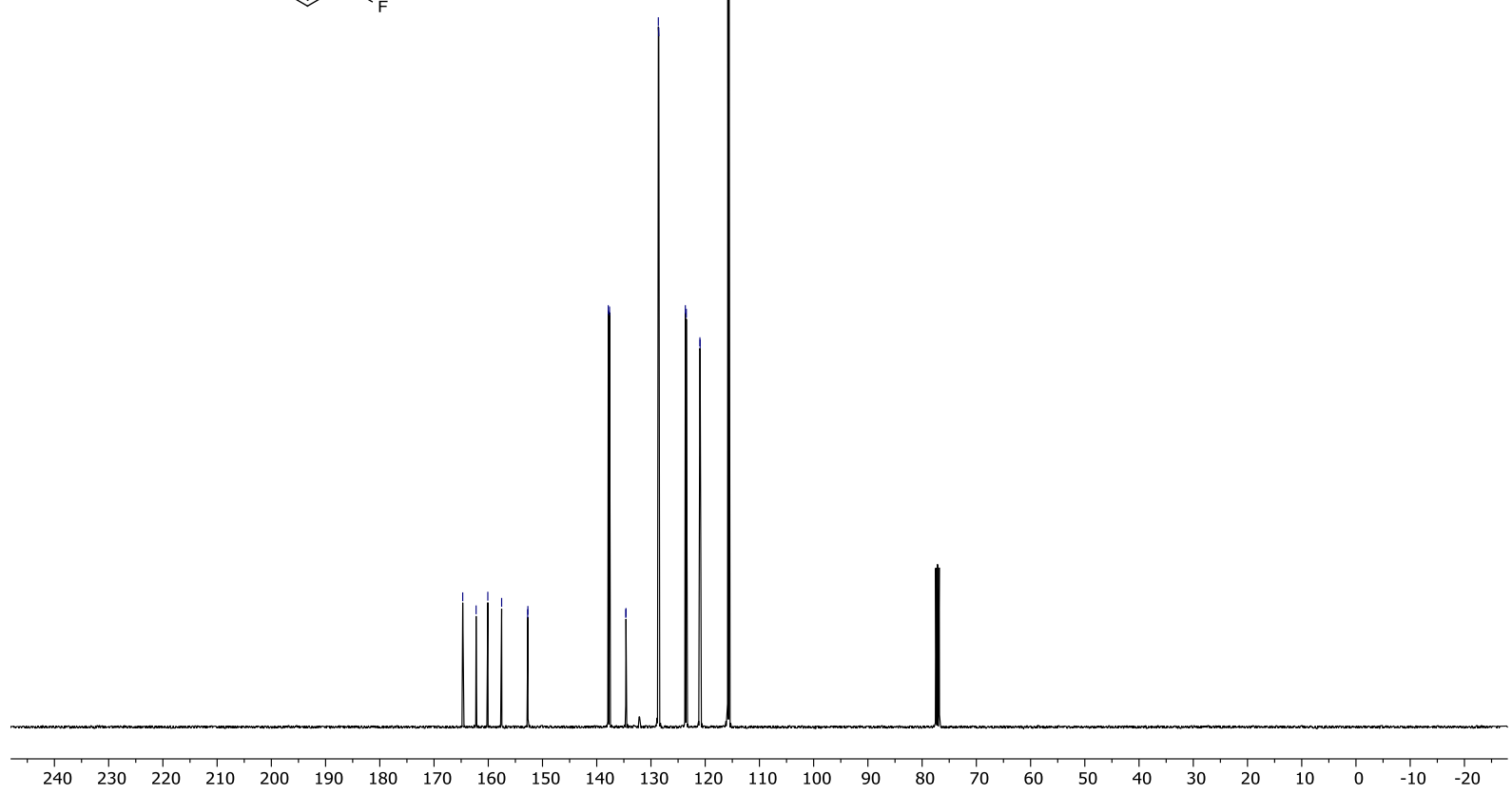

${ }_{19}^{19}\{1 \mathrm{H}\}$ NMR (Chloroformel)
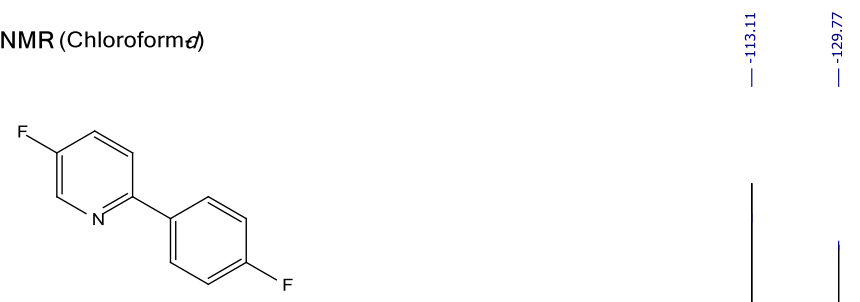

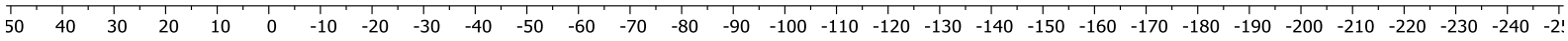

S76 
1H NMR (Chloroformd)

Y
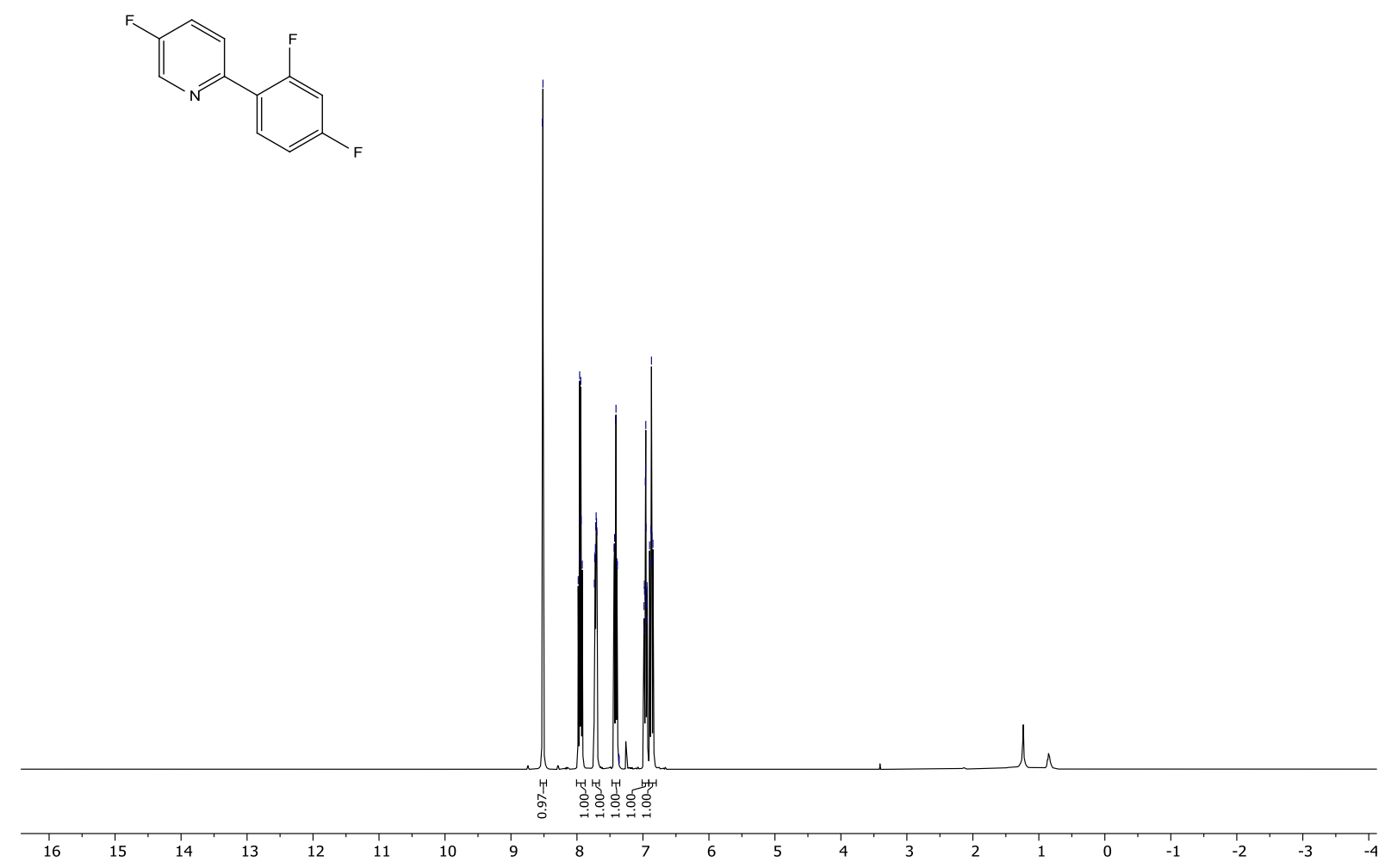

13C\{1H\} NMR (Chloroformed)

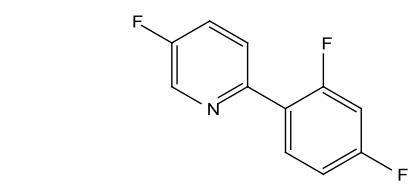

S77 
${ }^{19} \mathrm{~F}\{\mathrm{H} H\}$ NMR (Chloroformed)

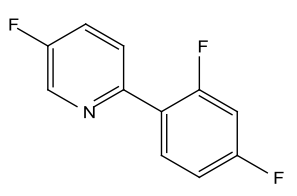

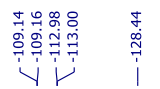

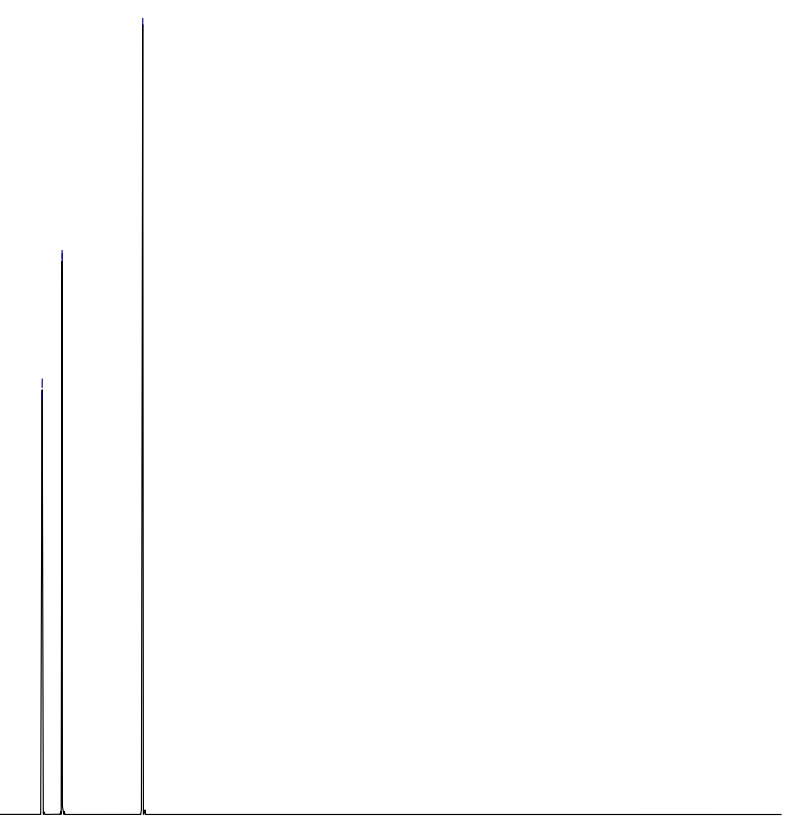

$\begin{array}{llllllllllllllllllllllllllllllllllllllll}50 & 40 & 30 & 20 & 10 & 0 & -10 & -20 & -30 & -40 & -50 & -60 & -70 & -80 & -90 & -100 & -110 & -120 & -130 & -140 & -150 & -160 & -170 & -180 & -190 & -200 & -210 & -220 & -230 & -240 & -2 !\end{array}$

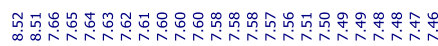
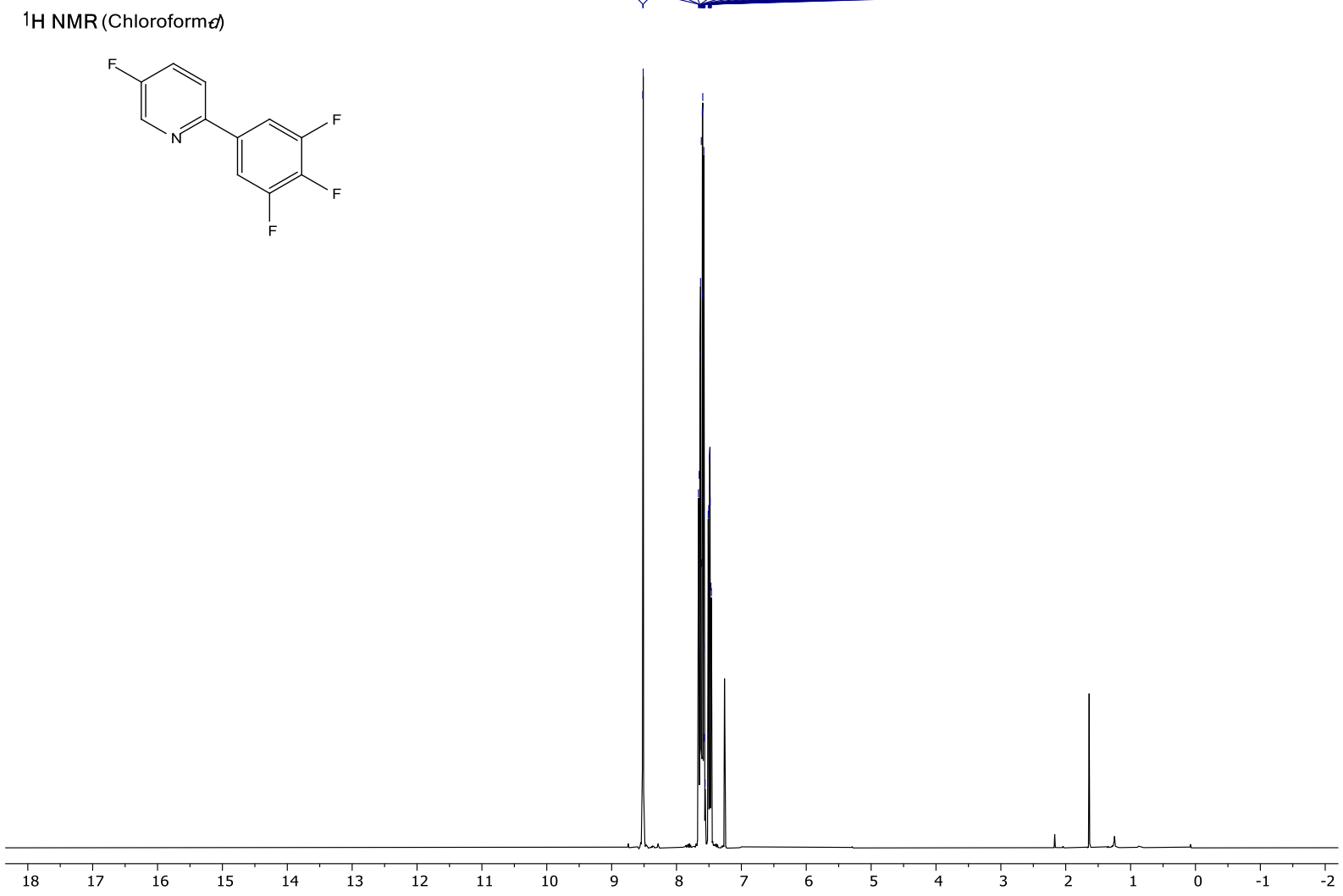

S78 


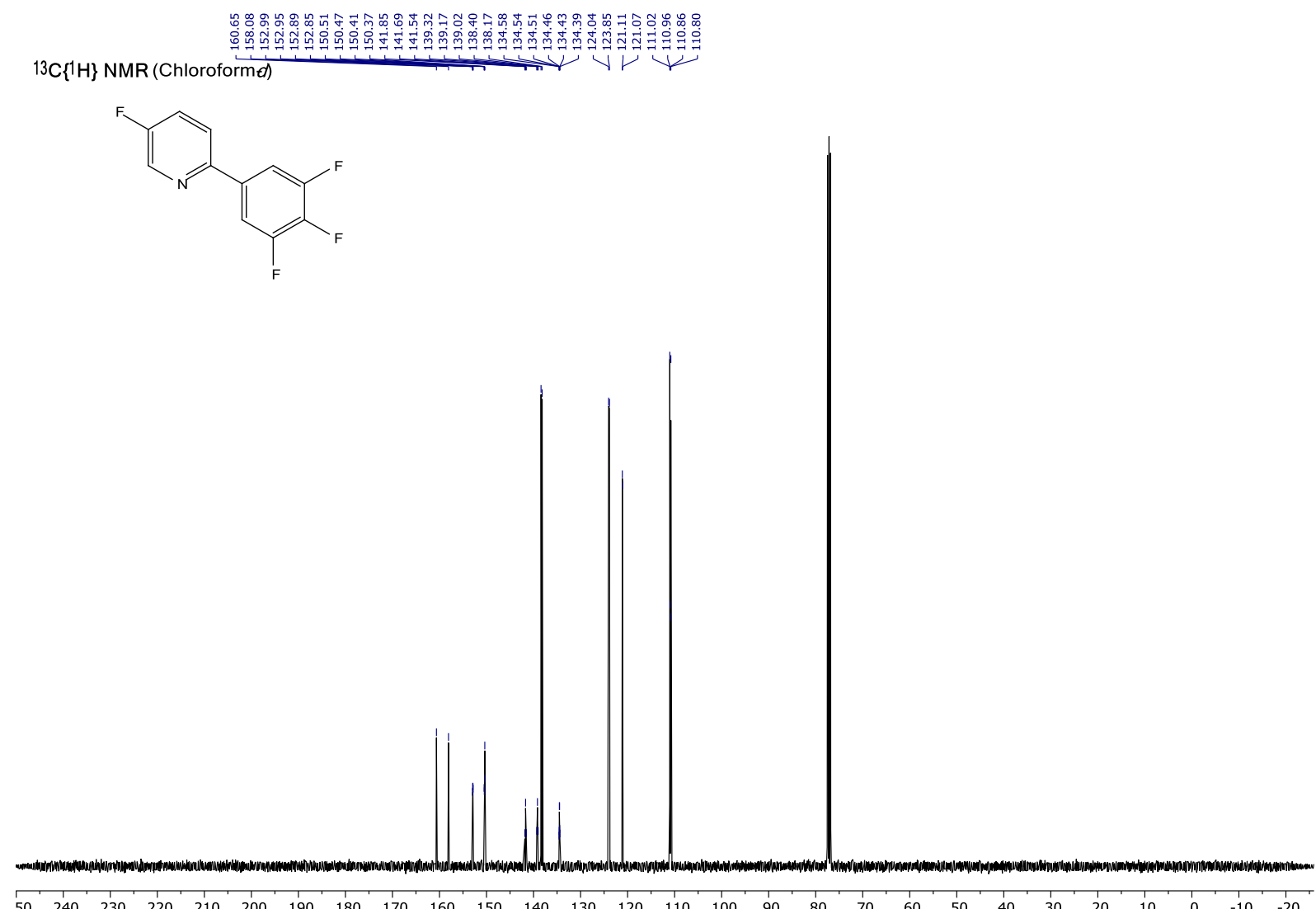

19F $\{1 \mathrm{H}\}$ NMR (Chloroforme)

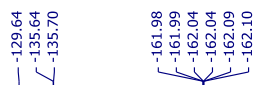<smiles>Fc1ccc(-c2cc(F)c(F)c(F)c2)nc1</smiles>

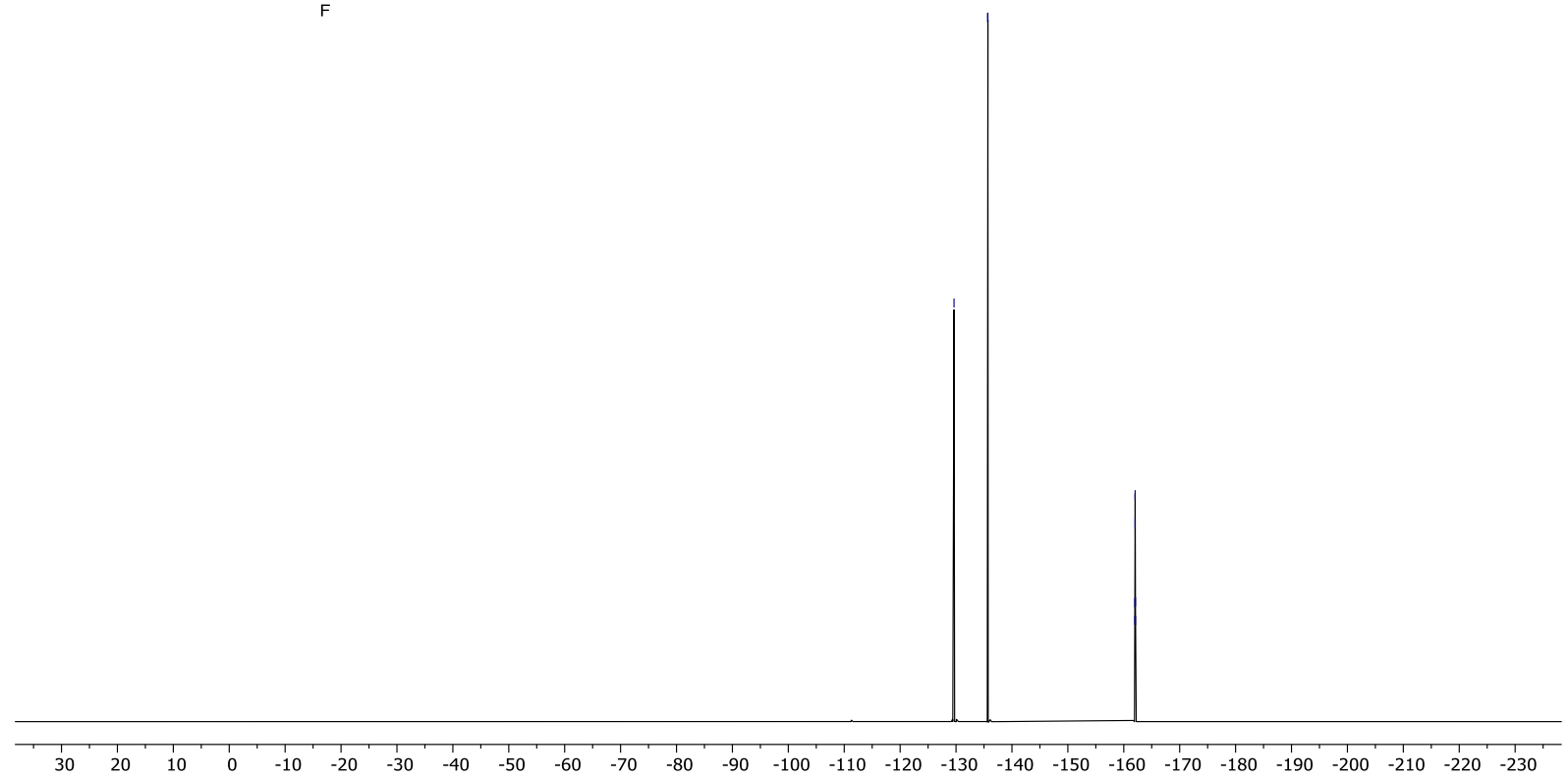




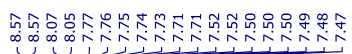

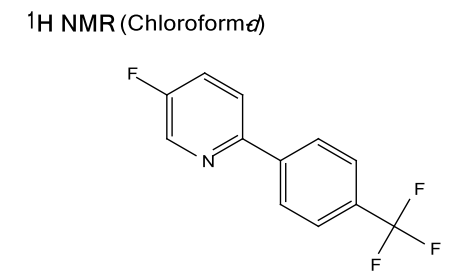

Y V 
${ }^{19} \mathrm{~F}\{\mathrm{H} \mathrm{H}\}$ NMR (Chloroformed)

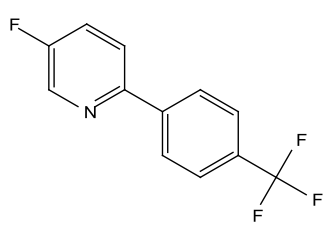

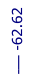

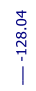
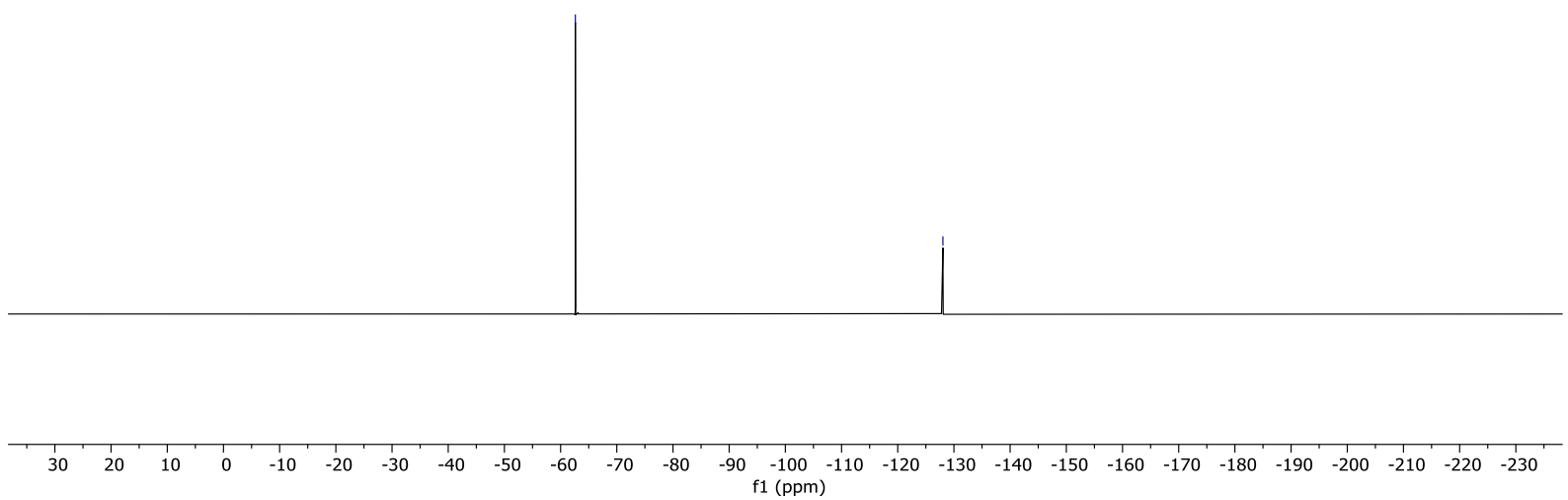

1H NMR (Chlorofor mel)

$\underbrace{\infty}$
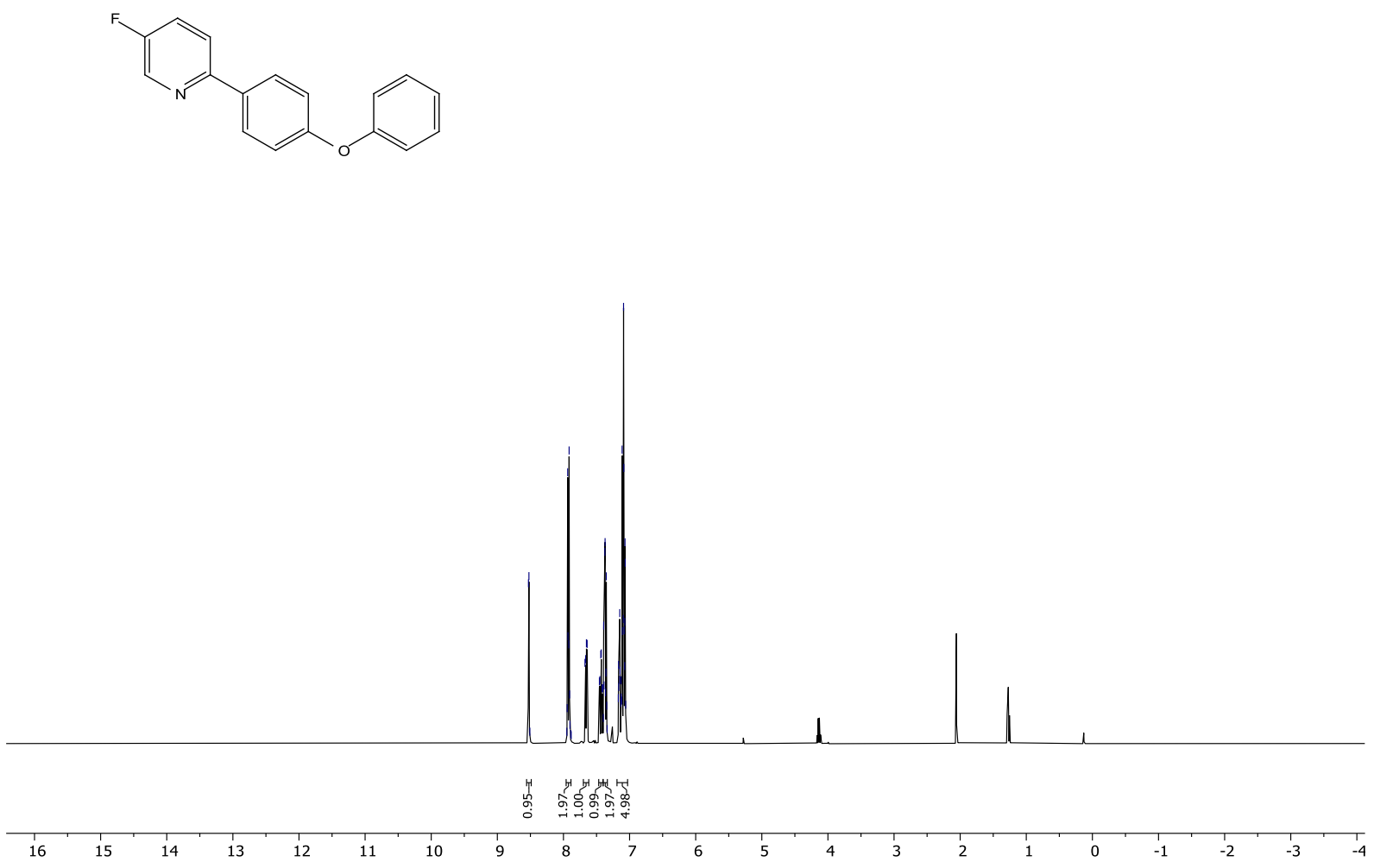

S81 
${ }^{13} \mathrm{C}\{1 \mathrm{H}\}$ NMR (Chlorofor me)

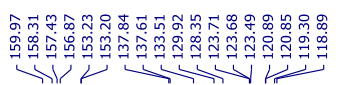
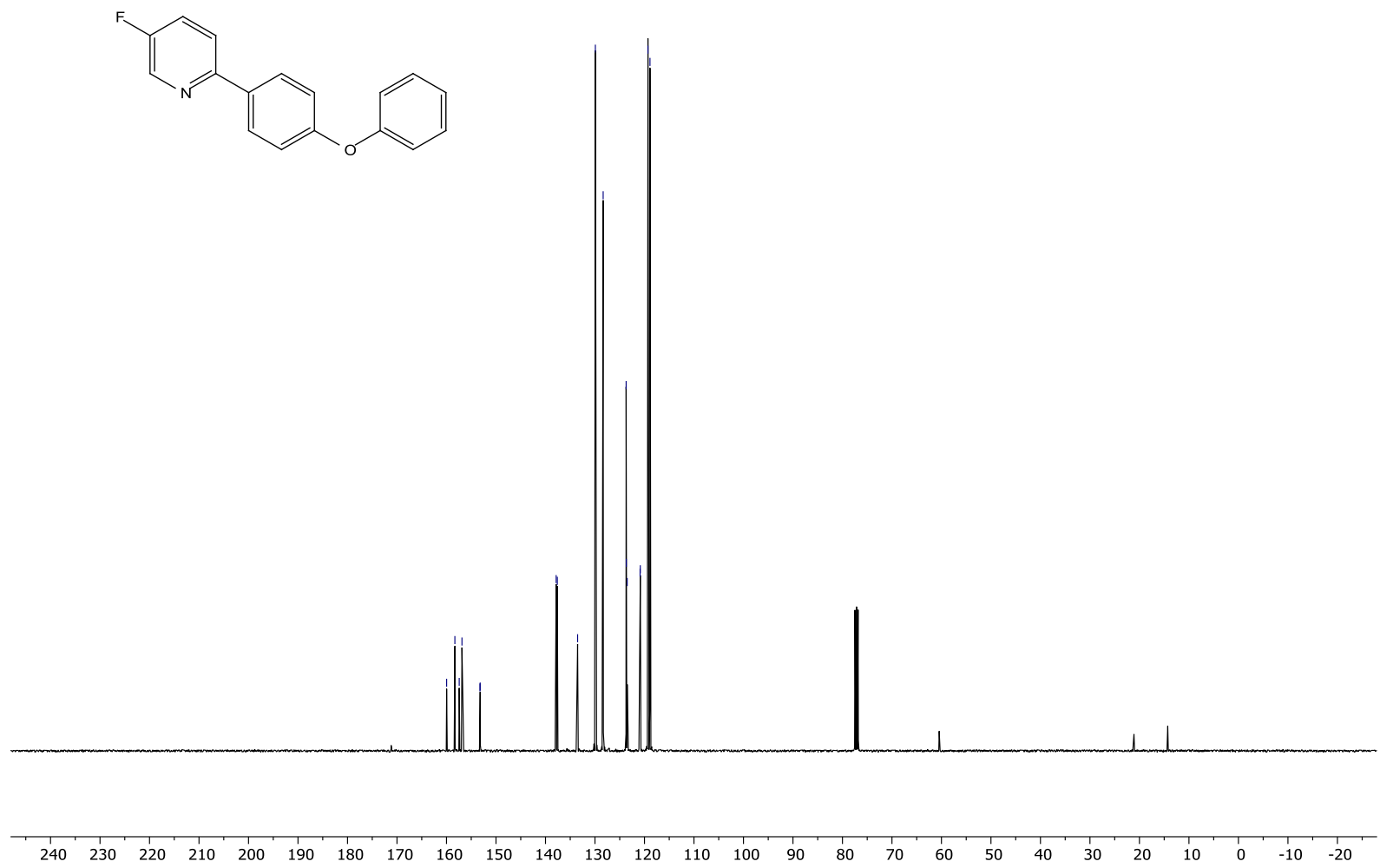

${ }^{19} \mathrm{~F}\left\{{ }^{1} \mathrm{H}\right\}$ NMR (Chloroformed)

in:
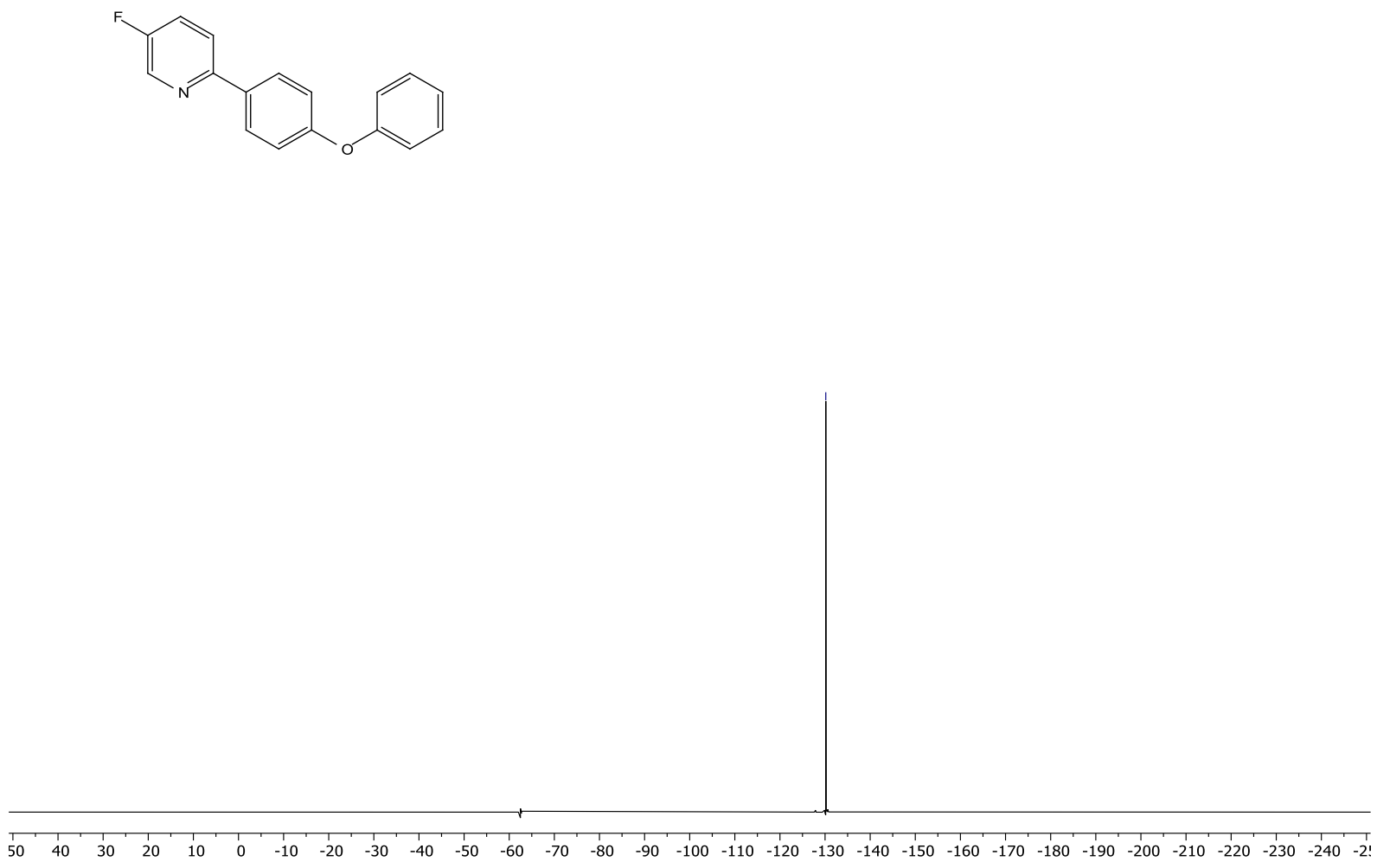

S82 

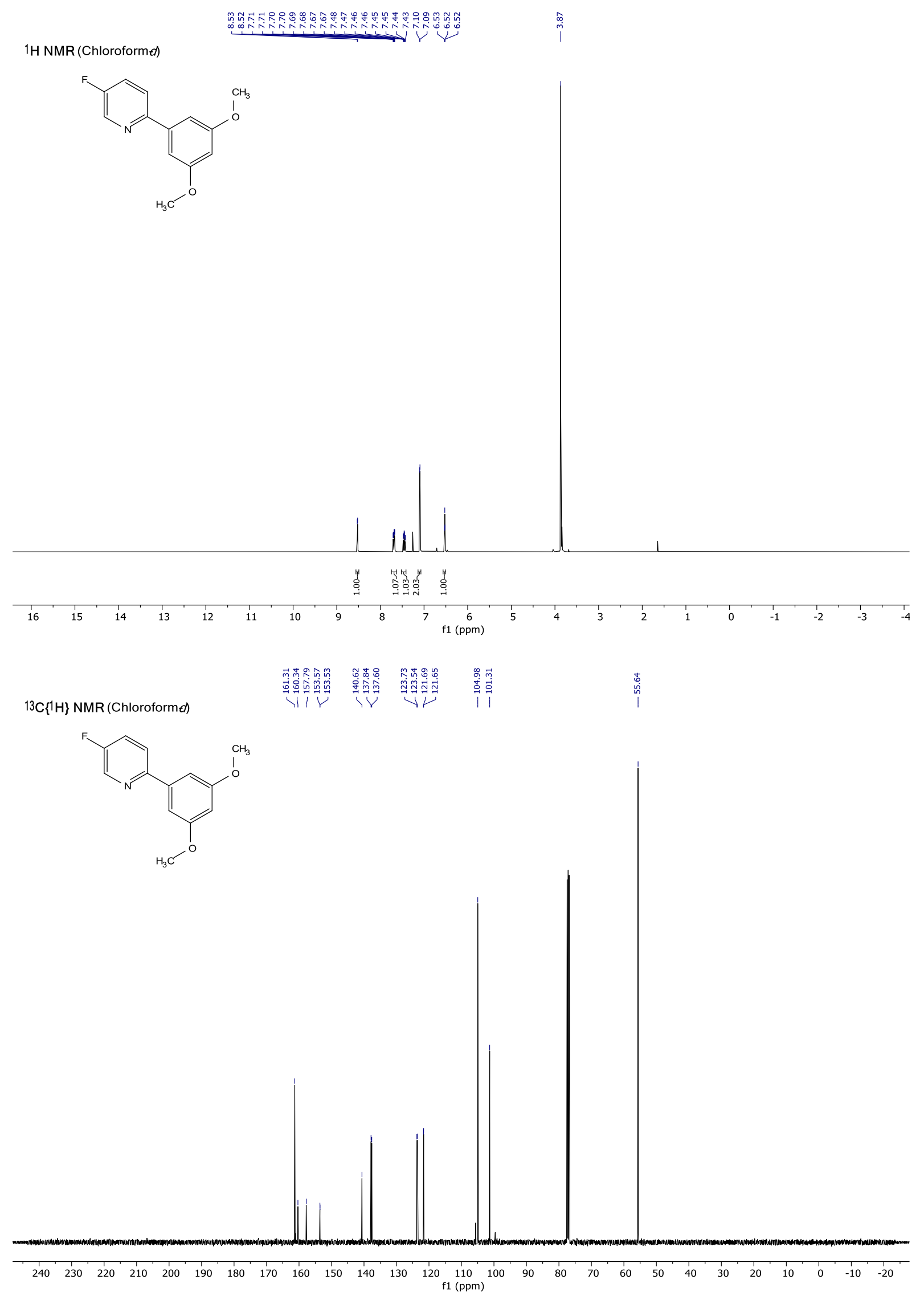
${ }^{19} \mathrm{~F}\{1 \mathrm{H}\}$ NMR (Chloroformed)

ํํำ

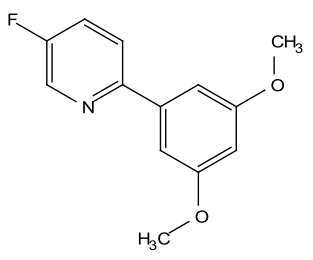

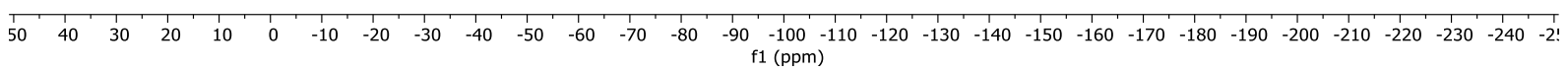

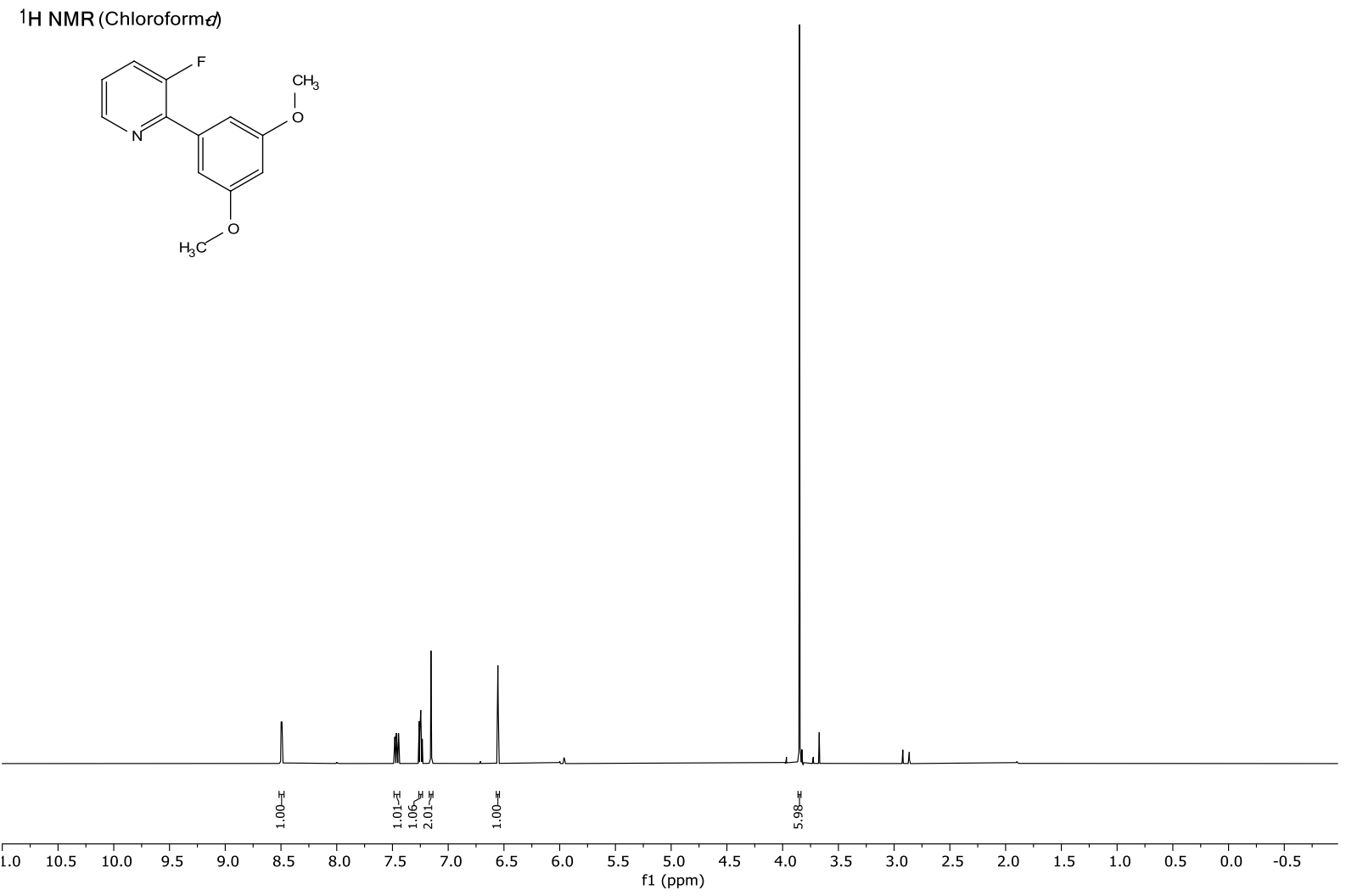

S84 

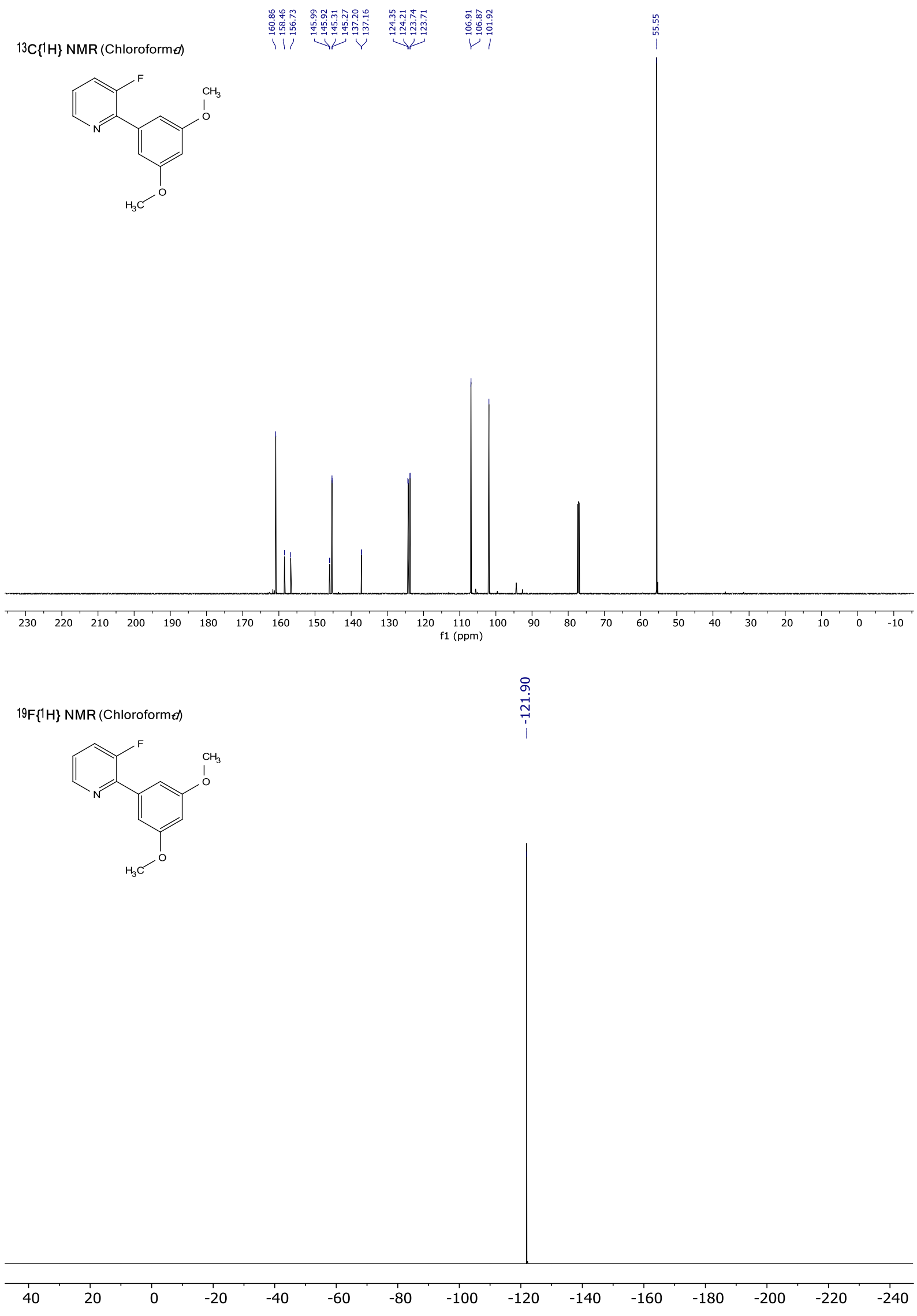

S85 
$1 \mathrm{HNMR}$ (Chloroformd)
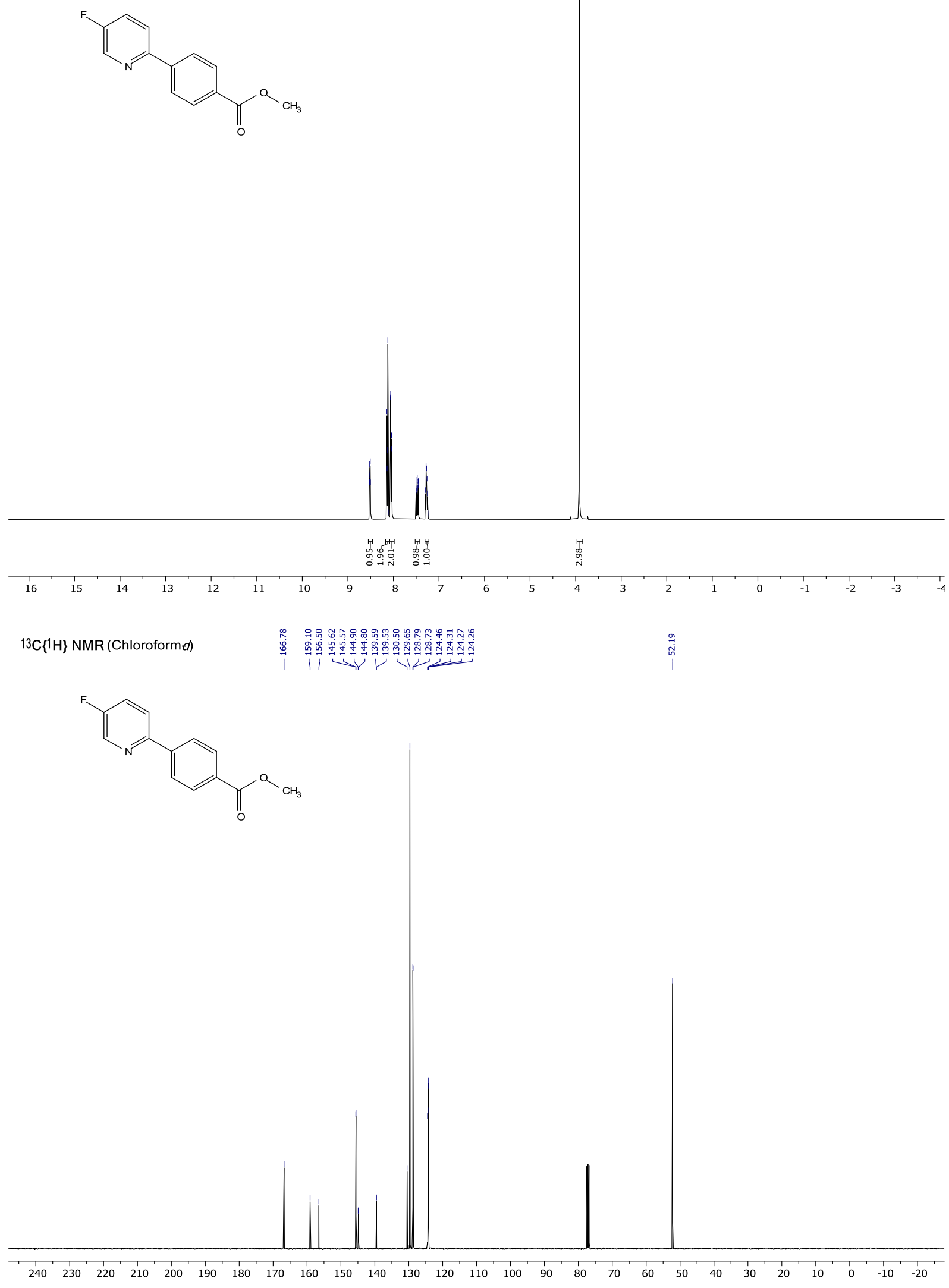

S86 
${ }^{19} \mathrm{~F}\left\{{ }^{1} \mathrm{H}\right\}$ NMR (Chloroform $d$ )

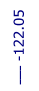
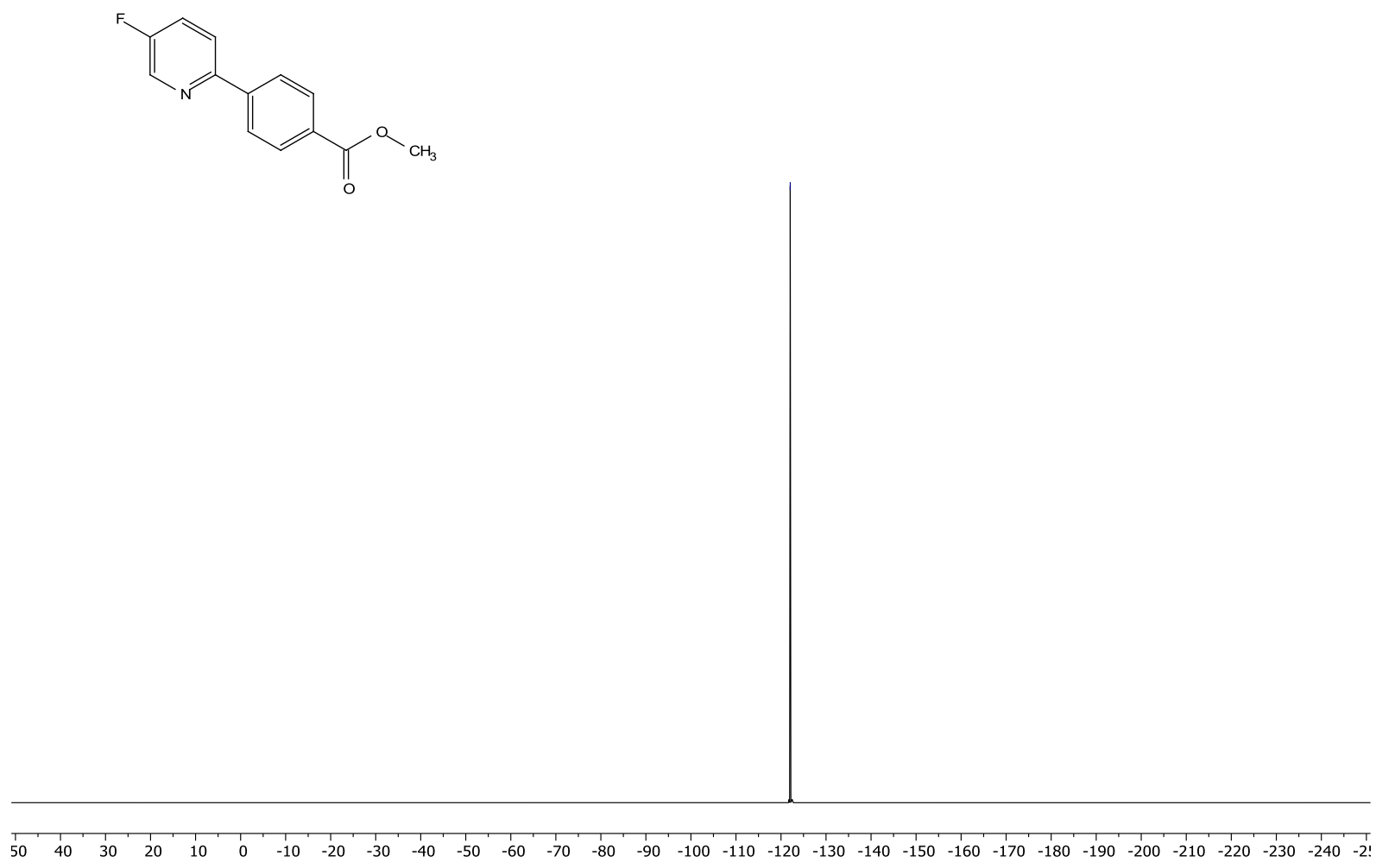

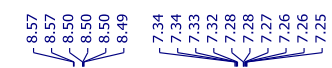

1H NMR (Chlorofor md)
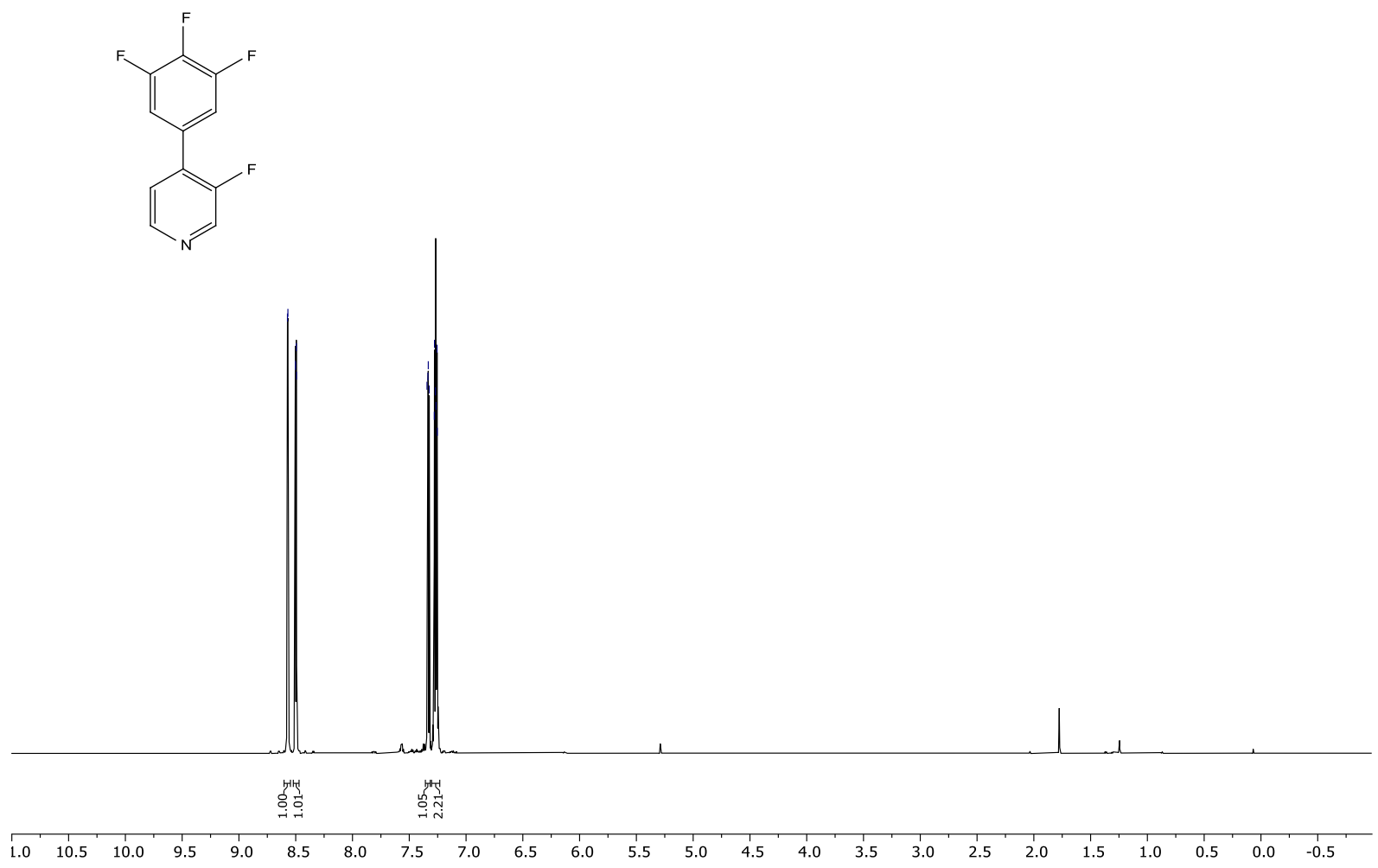

S87 


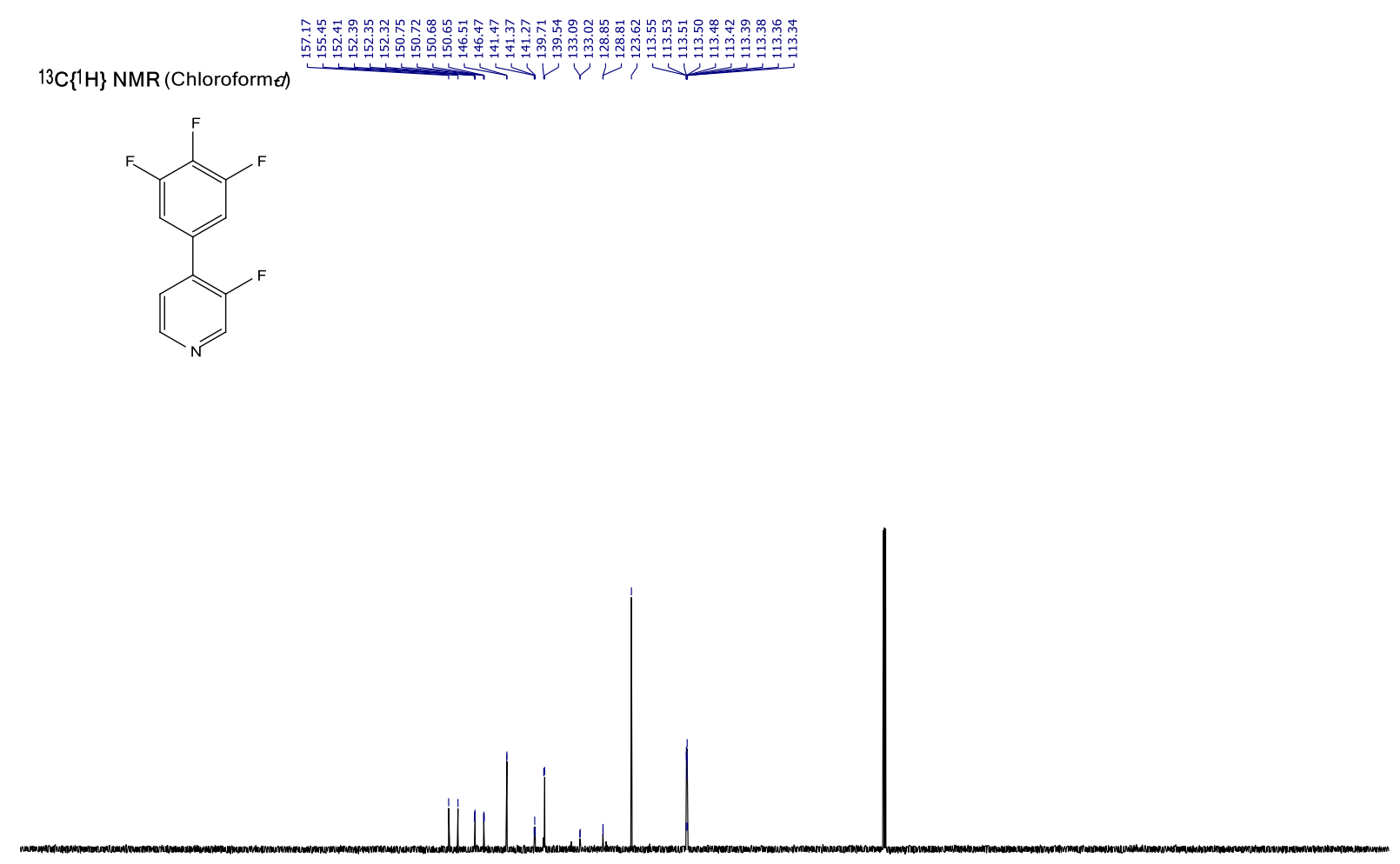

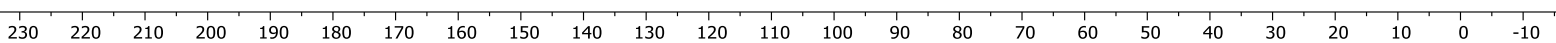

${ }^{19} \mathrm{~F}\{1 \mathrm{H}\}$ NMR (Chloroformed)

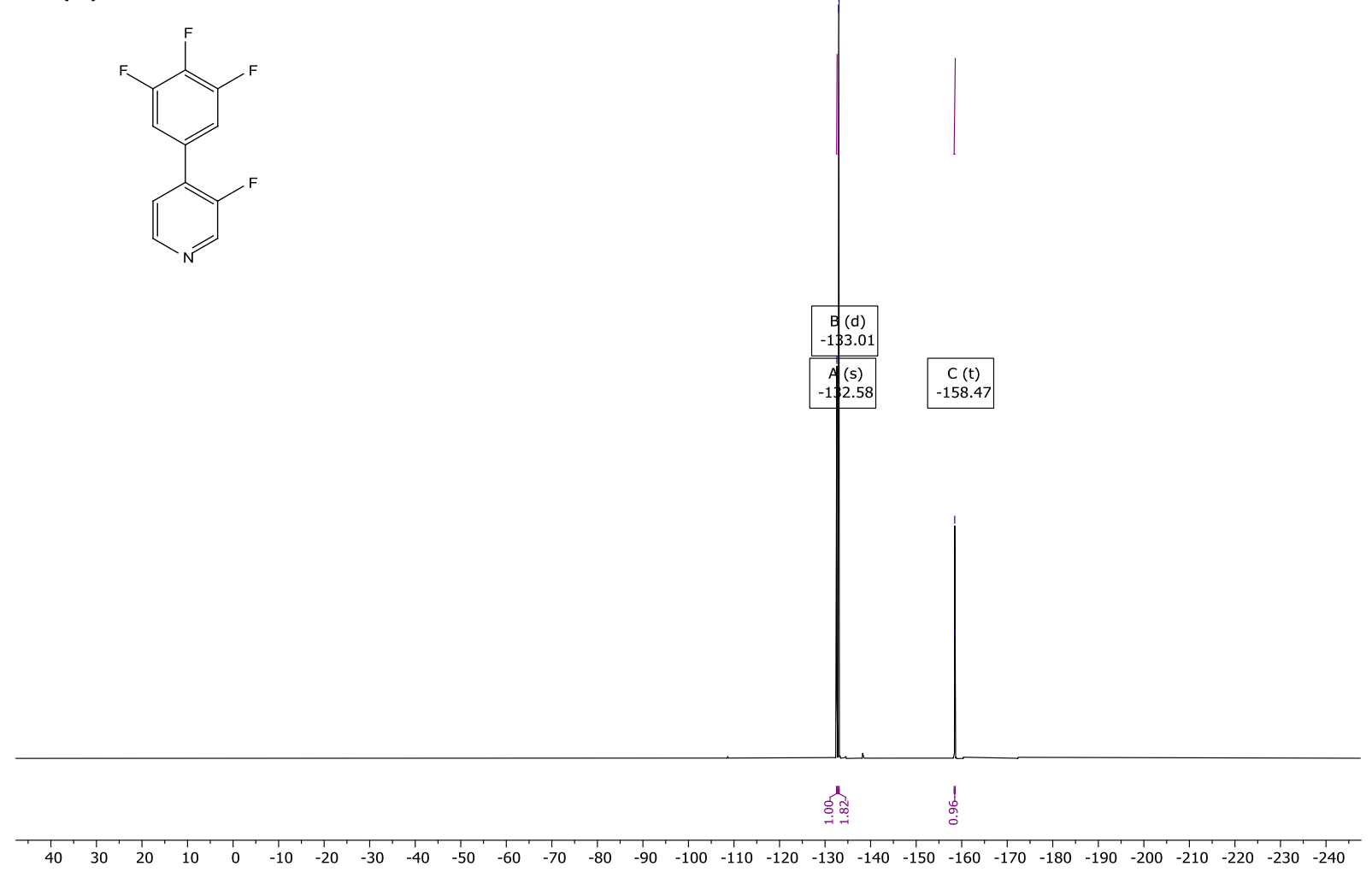




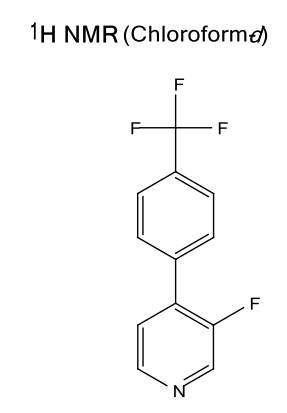

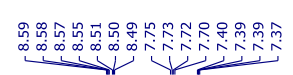
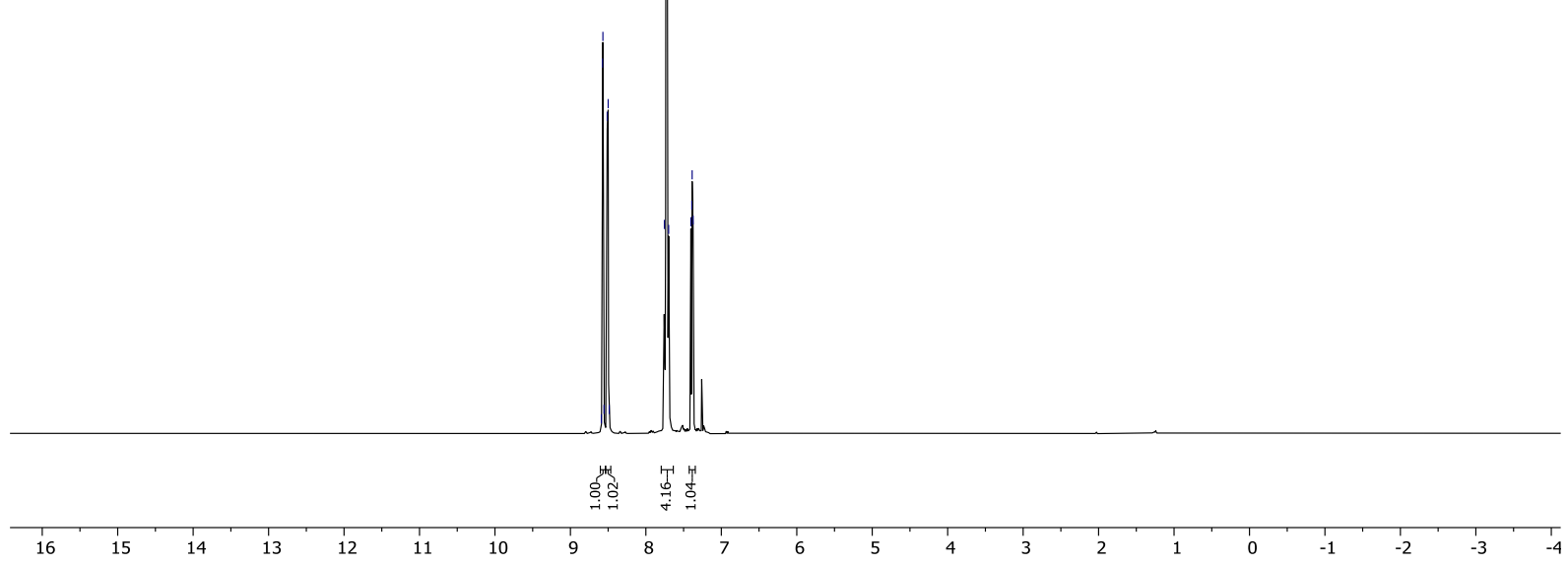

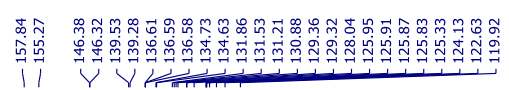

${ }^{13} \mathrm{C}\{1 \mathrm{H}\}$ NMR (Chloroformed)

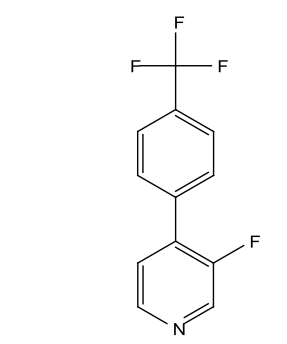

S89 
${ }^{19} \mathrm{~F}\{1 \mathrm{H}\}$ NMR (Chloroformel)

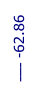

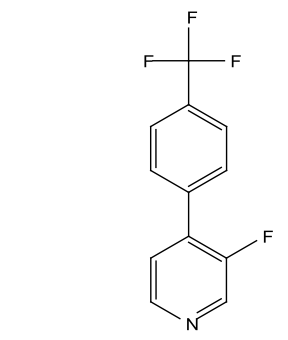

i
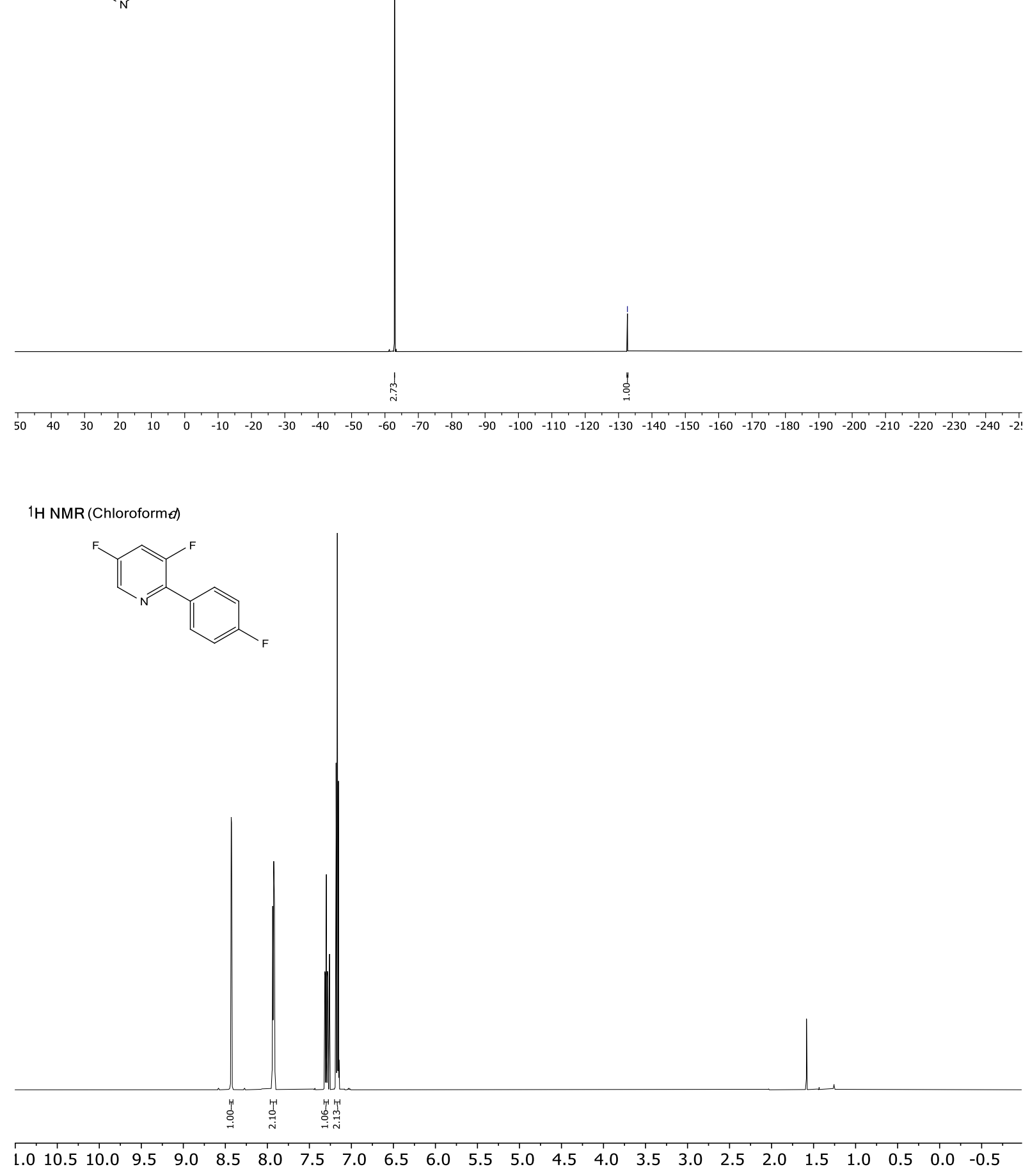

$\mathrm{S} 90$ 
\{ $\{1 \mathrm{H}\}$ NMR (Chloroforme)

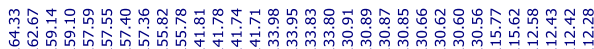

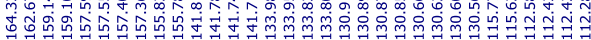<smiles>Fc1ccc(-c2ncc(F)cc2F)cc1</smiles>

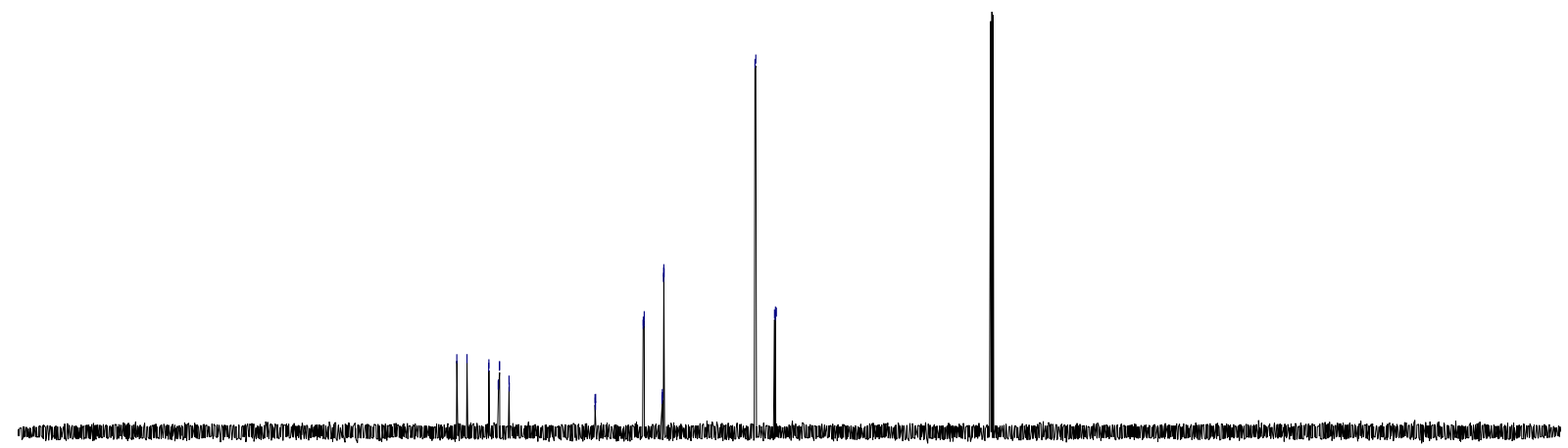

$\begin{array}{lllllllllllllllllllllllll}230 & 220 & 210 & 200 & 190 & 180 & 170 & 160 & 150 & 140 & 130 & 120 & 110 & 100 & 90 & 80 & 70 & 60 & 50 & 40 & 30 & 20 & 10 & 0 & -10\end{array}$

19F $\{1 \mathrm{H}\}$ NMR (Chloroformed)

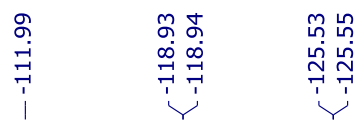<smiles>Fc1ccc(-c2ncc(F)cc2F)cc1</smiles>

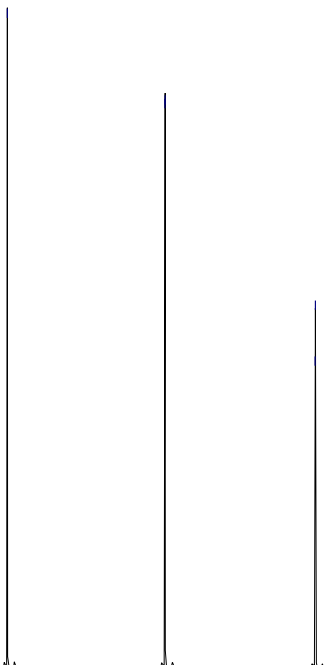

$-75$

$-80$

$-85$

$-90 \quad-95$

$-100$

$-105$

$-110$

$-115$

$-120$

$-130$

$-135$ 
1H NMR (Chloroforme)

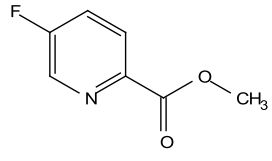

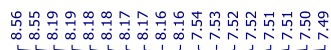

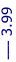

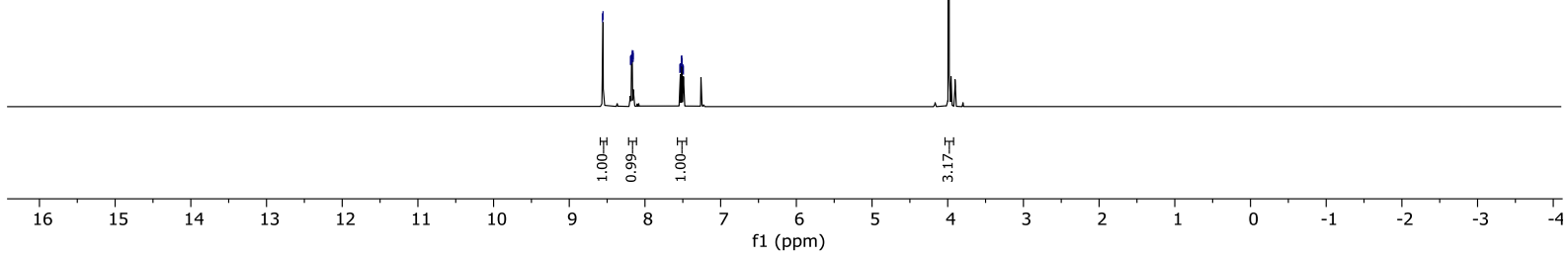

13C\{1H\} NMR (Chloroforme)

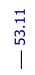
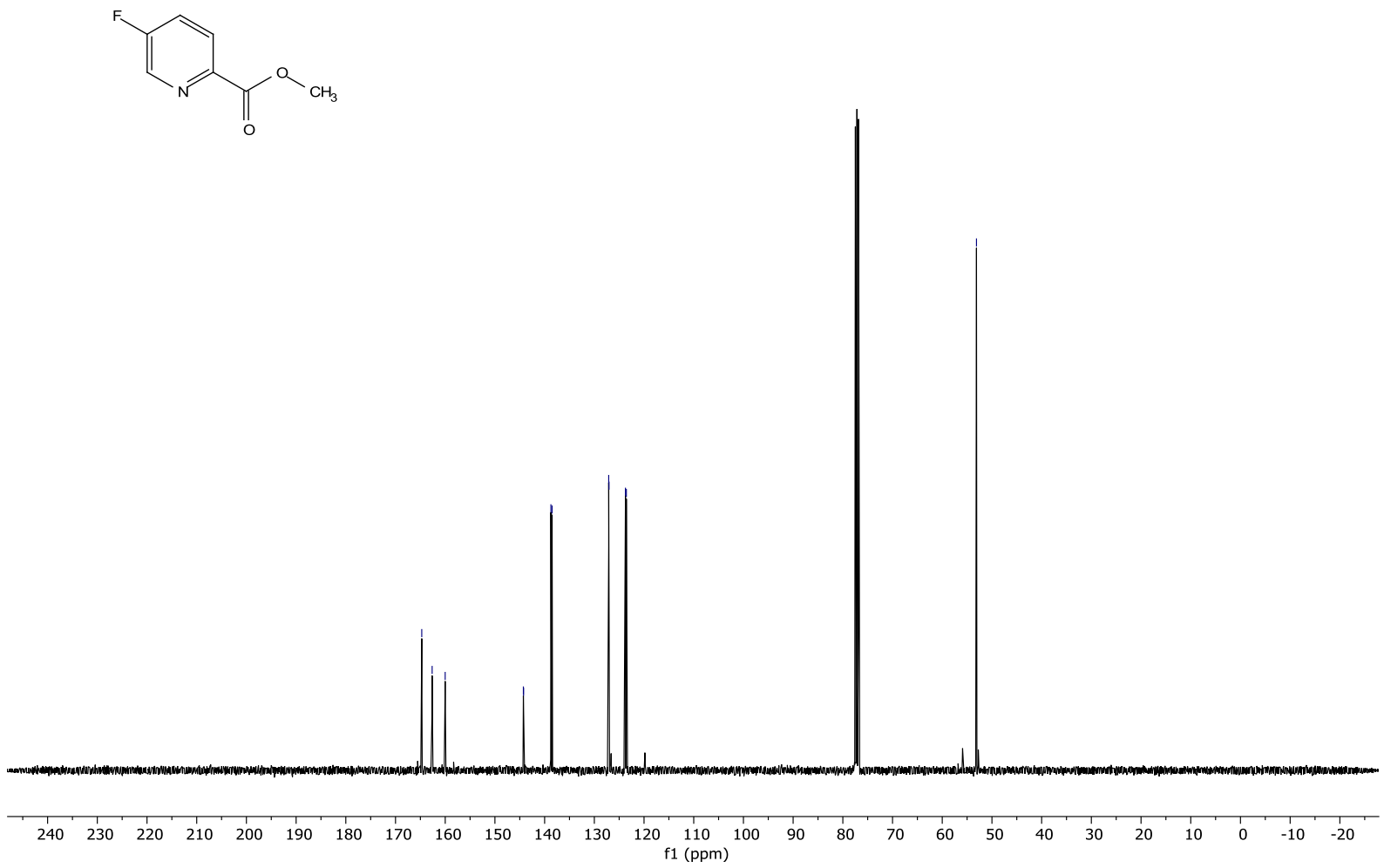

S92 
${ }^{19} \mathrm{~F}\{1 \mathrm{H}\}$ NMR (Chloroform $d$ )

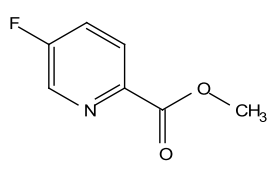

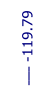

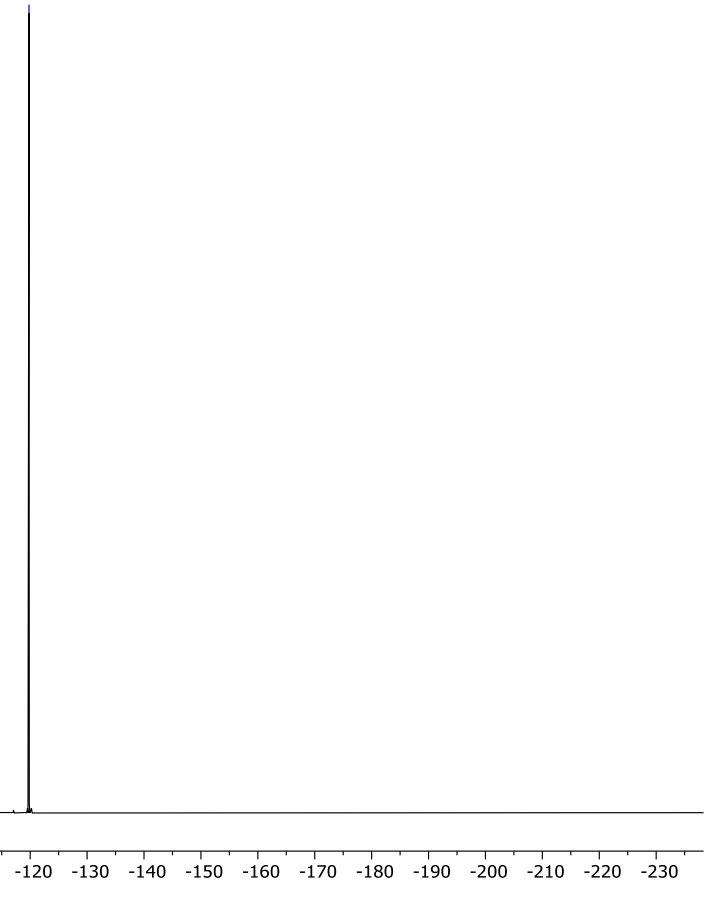

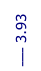
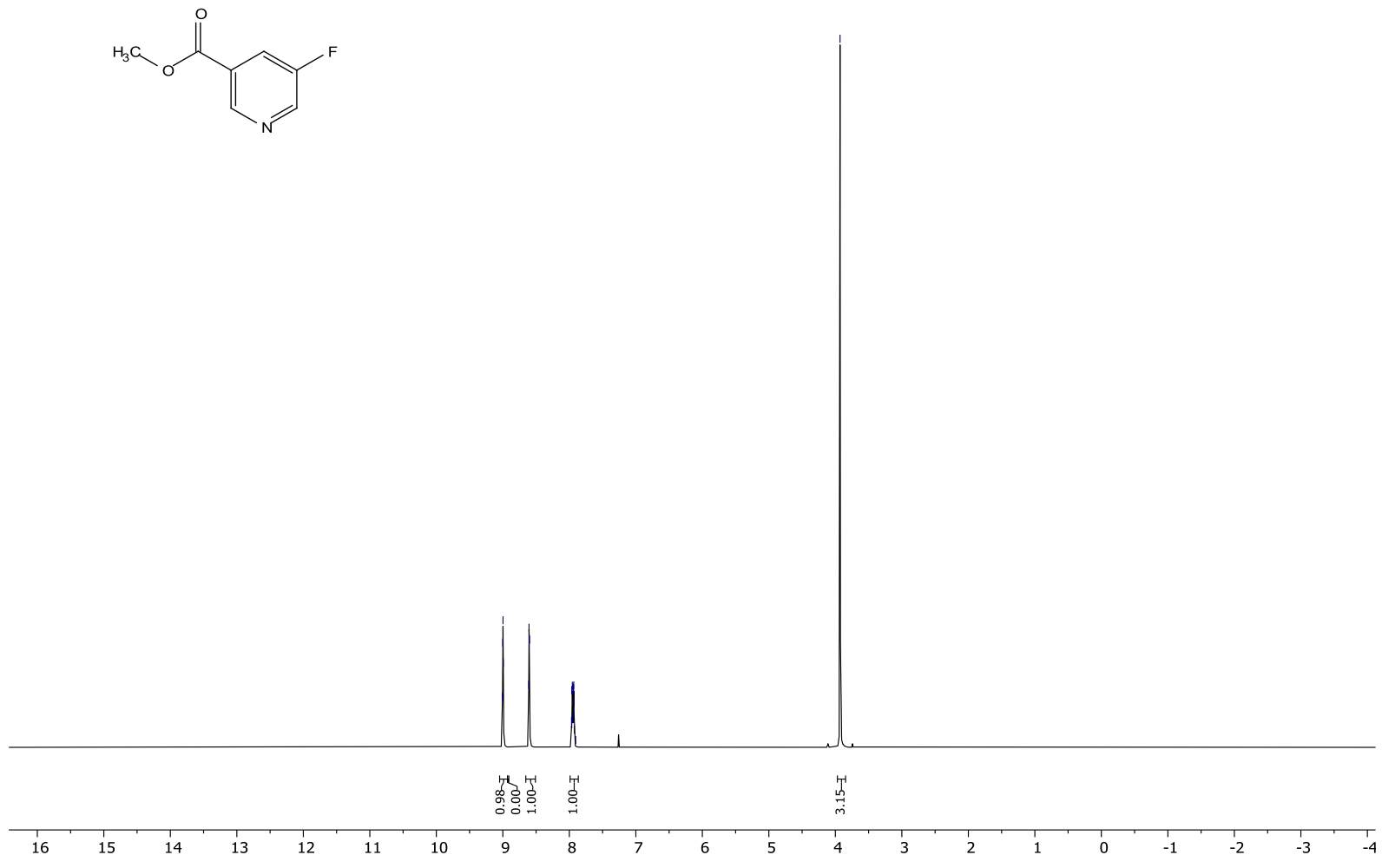

S93 
${ }^{13} \mathrm{C}\{1 \mathrm{H}\} \mathrm{NMR}$ (Chloroforme)

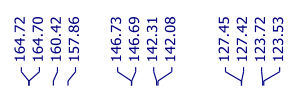

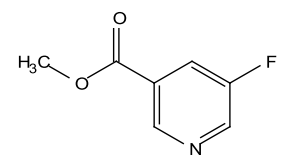

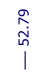
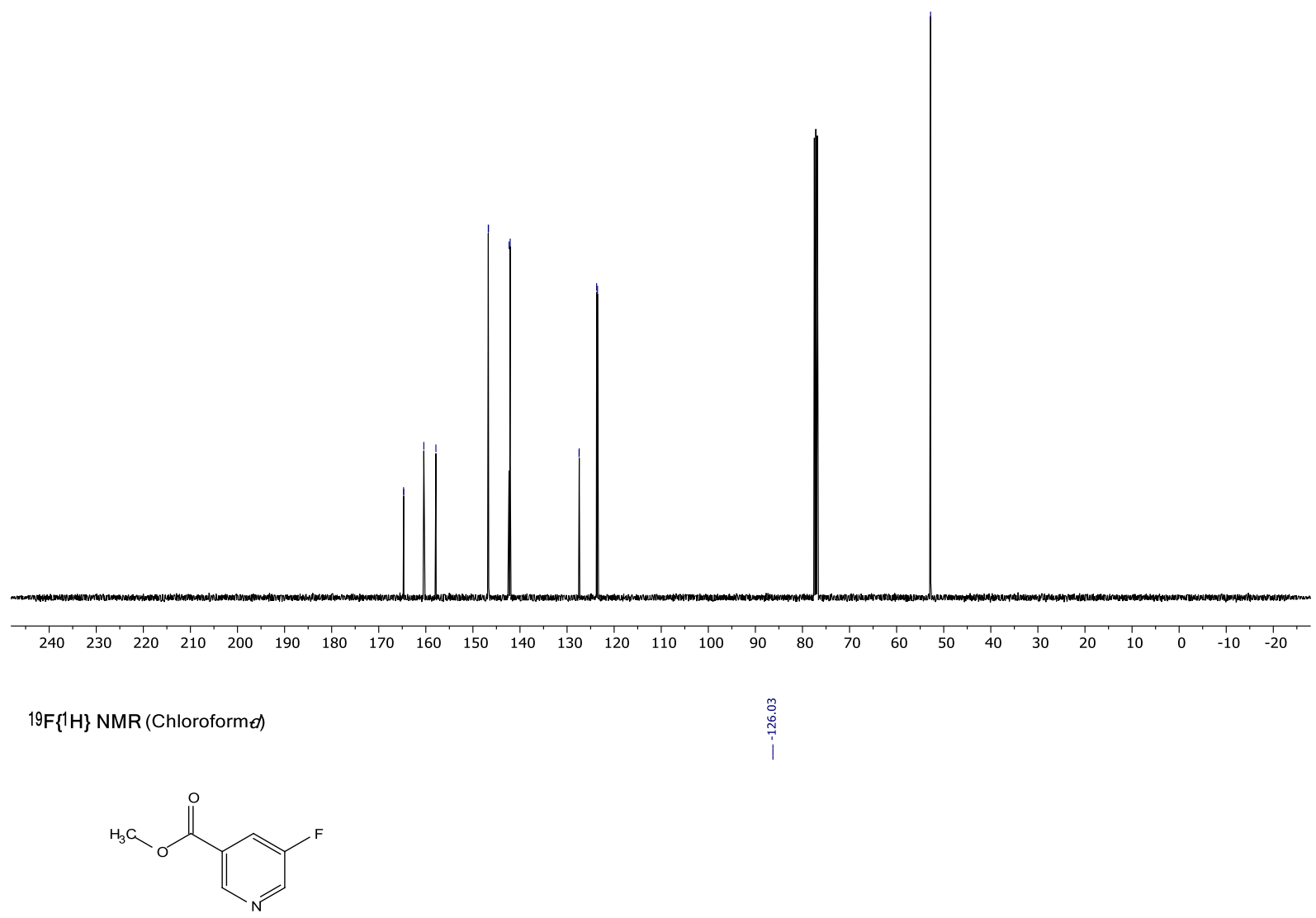

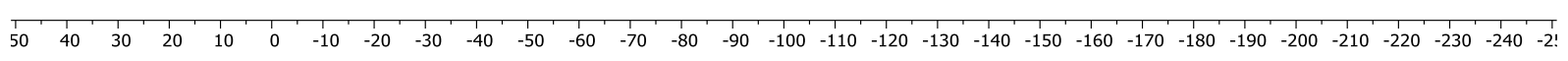

S94 
1H NMR (Chlorofor me)

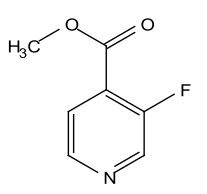

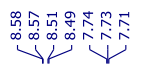

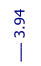

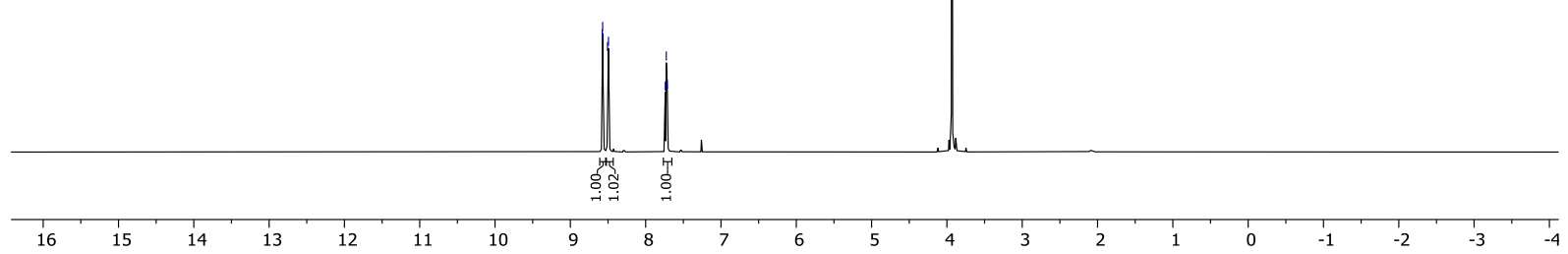

${ }^{13} \mathrm{C}\{1 \mathrm{H}\}$ NMR (Chloroformed)

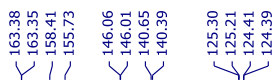

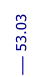
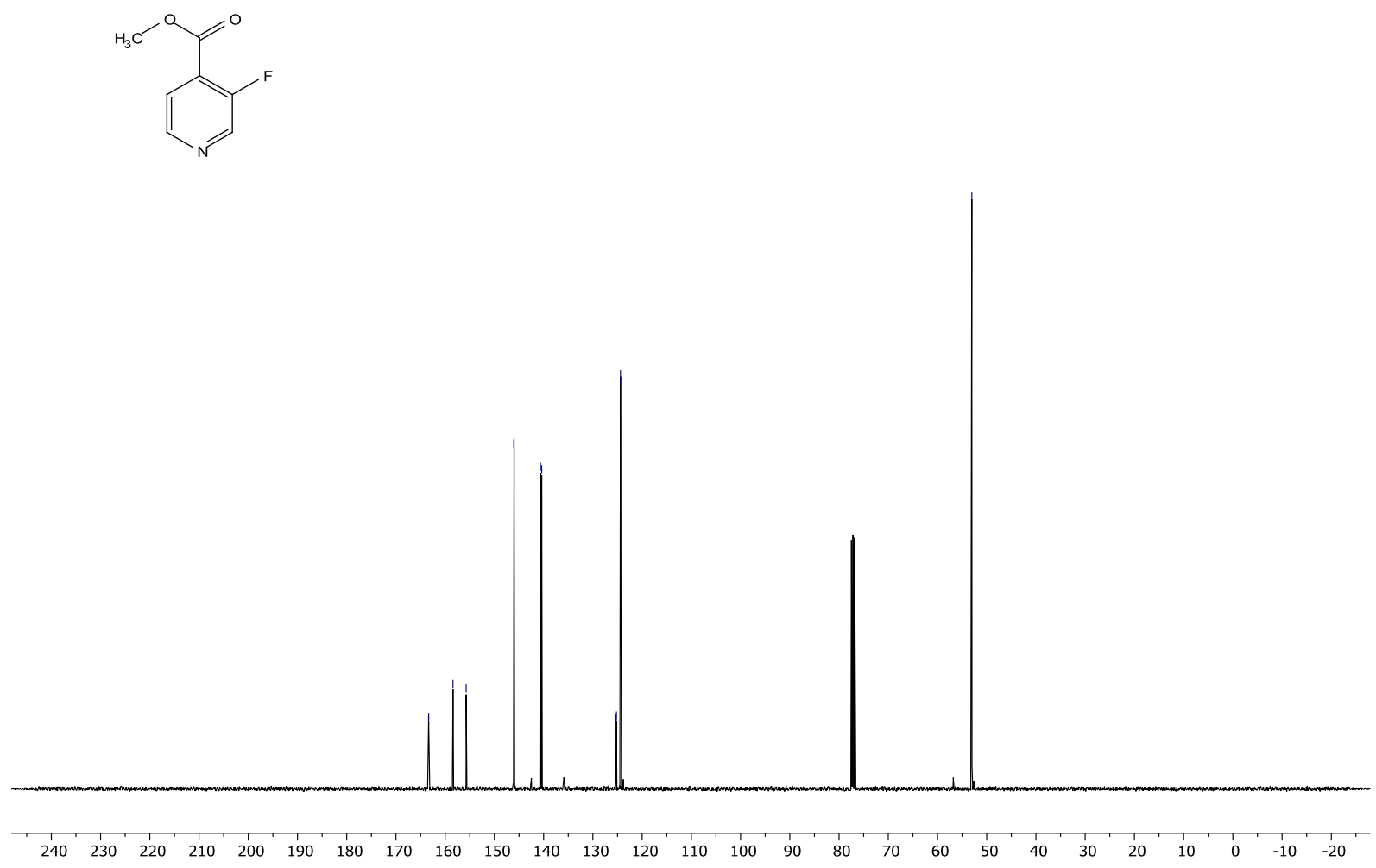

S95 
${ }^{19} \mathrm{~F}\{1 \mathrm{H}\}$ NMR (Chloroformed)

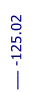

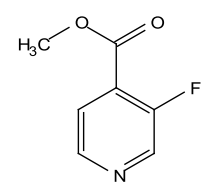

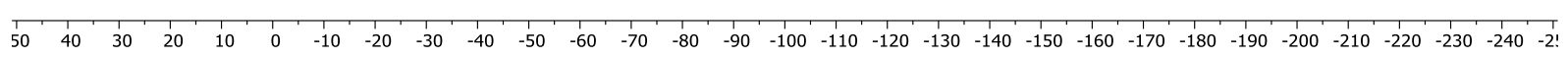

1H NMR (Chlorofor md)

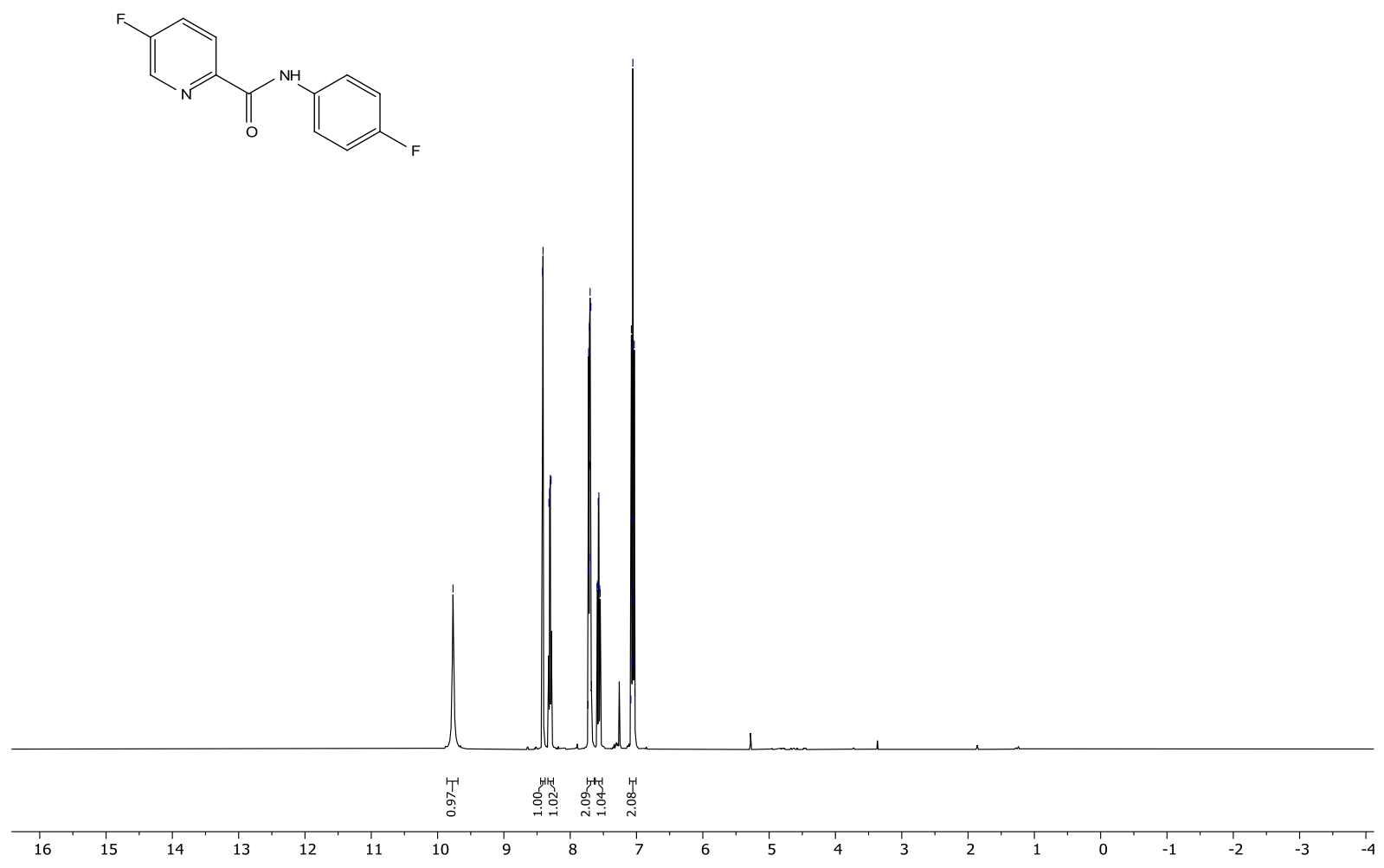

S96 
13C $\{1 \mathrm{H}\}$ NMR (Chloroforme)

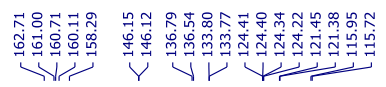
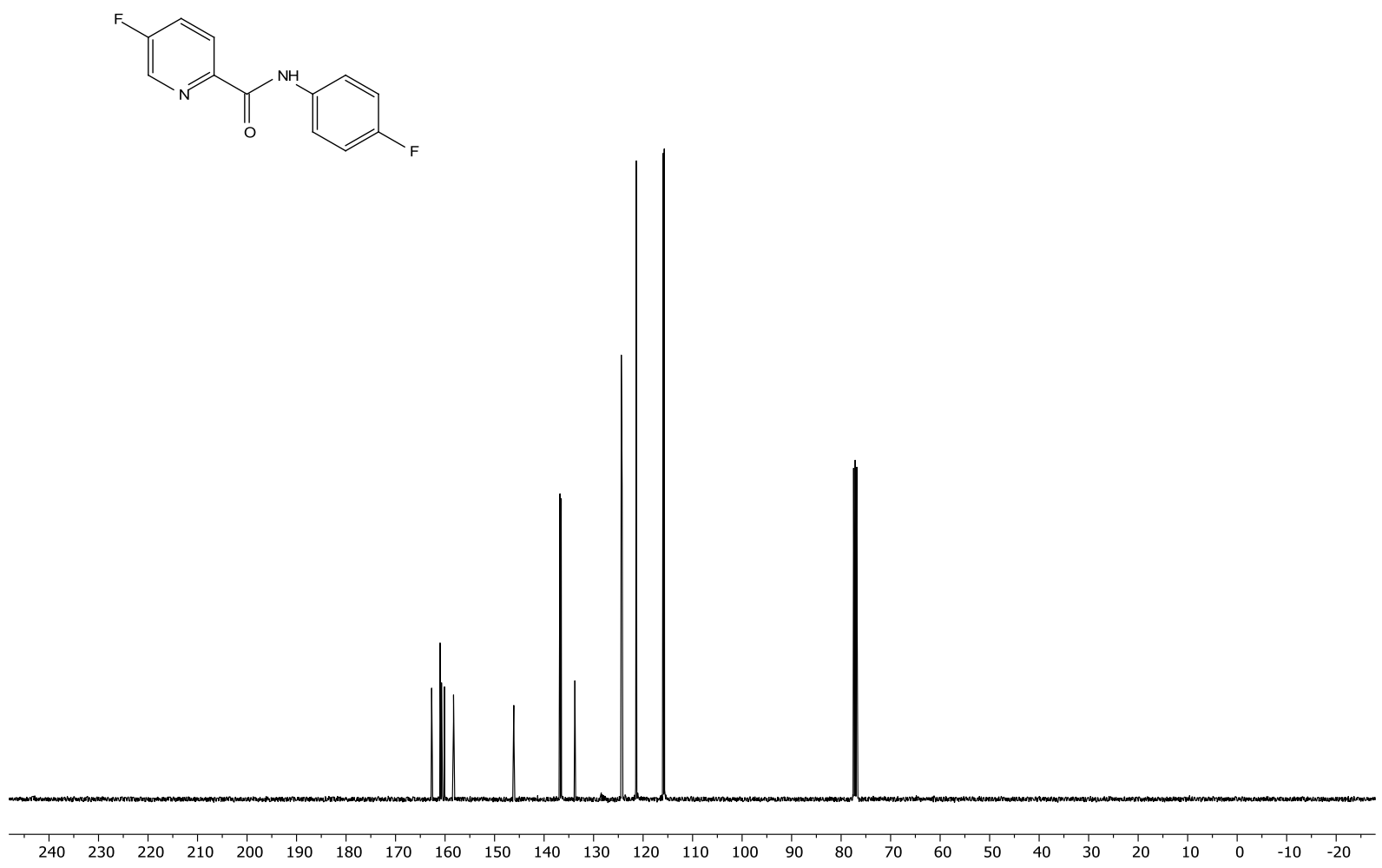

${ }^{19} \mathrm{~F}\{1 \mathrm{H}\} \mathrm{NMR}$ (Chloroformel)



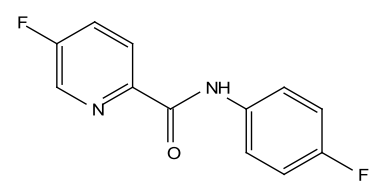

\begin{tabular}{lllllllllllllllllllllllllllllllllll}
\hline 50 & 40 & 30 & 20 & 10 & 0 & -10 & -20 & -30 & -40 & -50 & -60 & -70 & -80 & -90 & -100 & -110 & -120 & -130 & -140 & -150 & -160 & -170 & -180 & -190 & -200 & -210 & -220 & -230 & -240 & $-2 !$
\end{tabular}

S97 

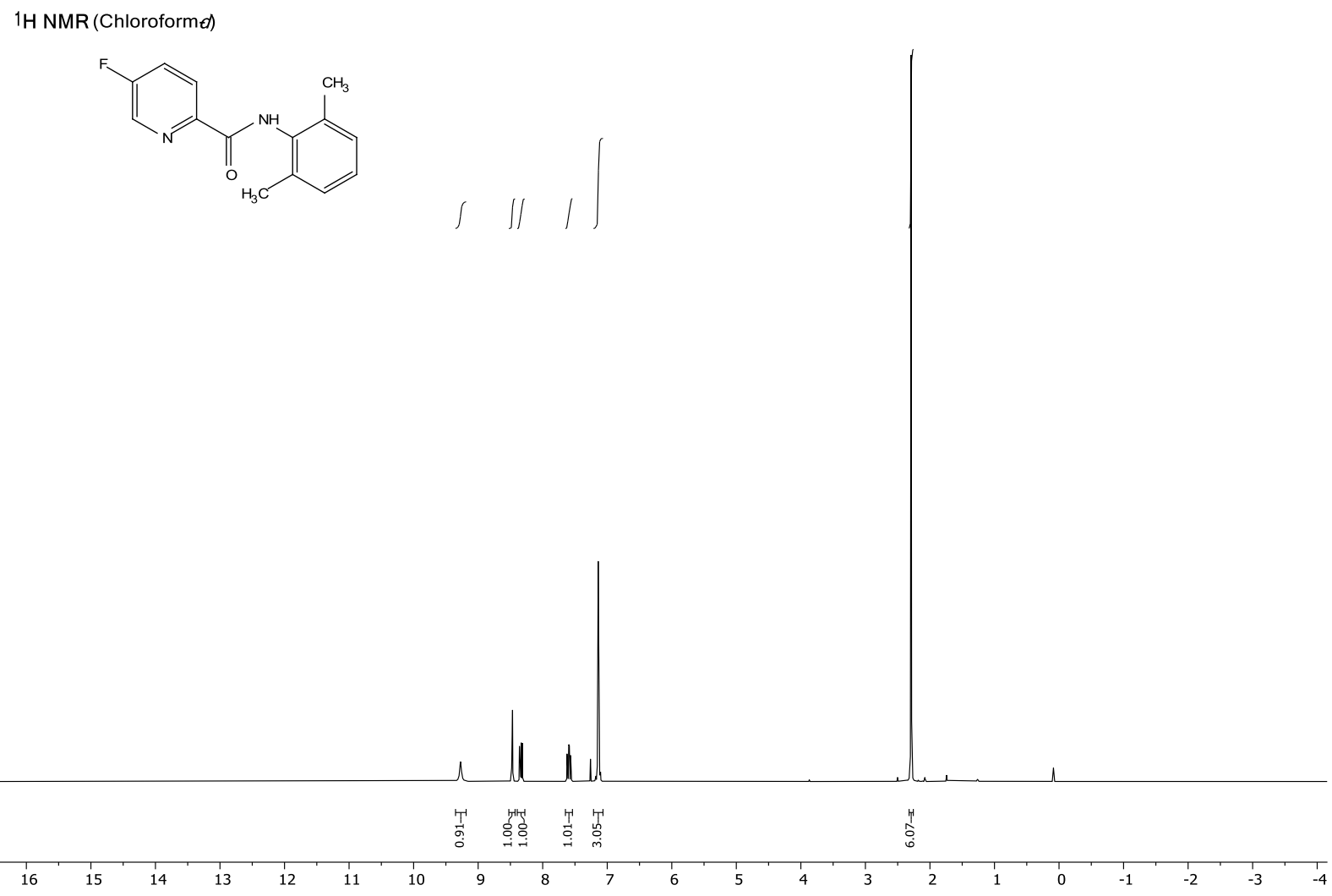

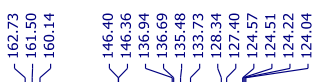

$\stackrel{\substack{\infty \\ \text { I }}}{1}$

13C $\{1 \mathrm{H}\}$ NMR (Chloroformed)
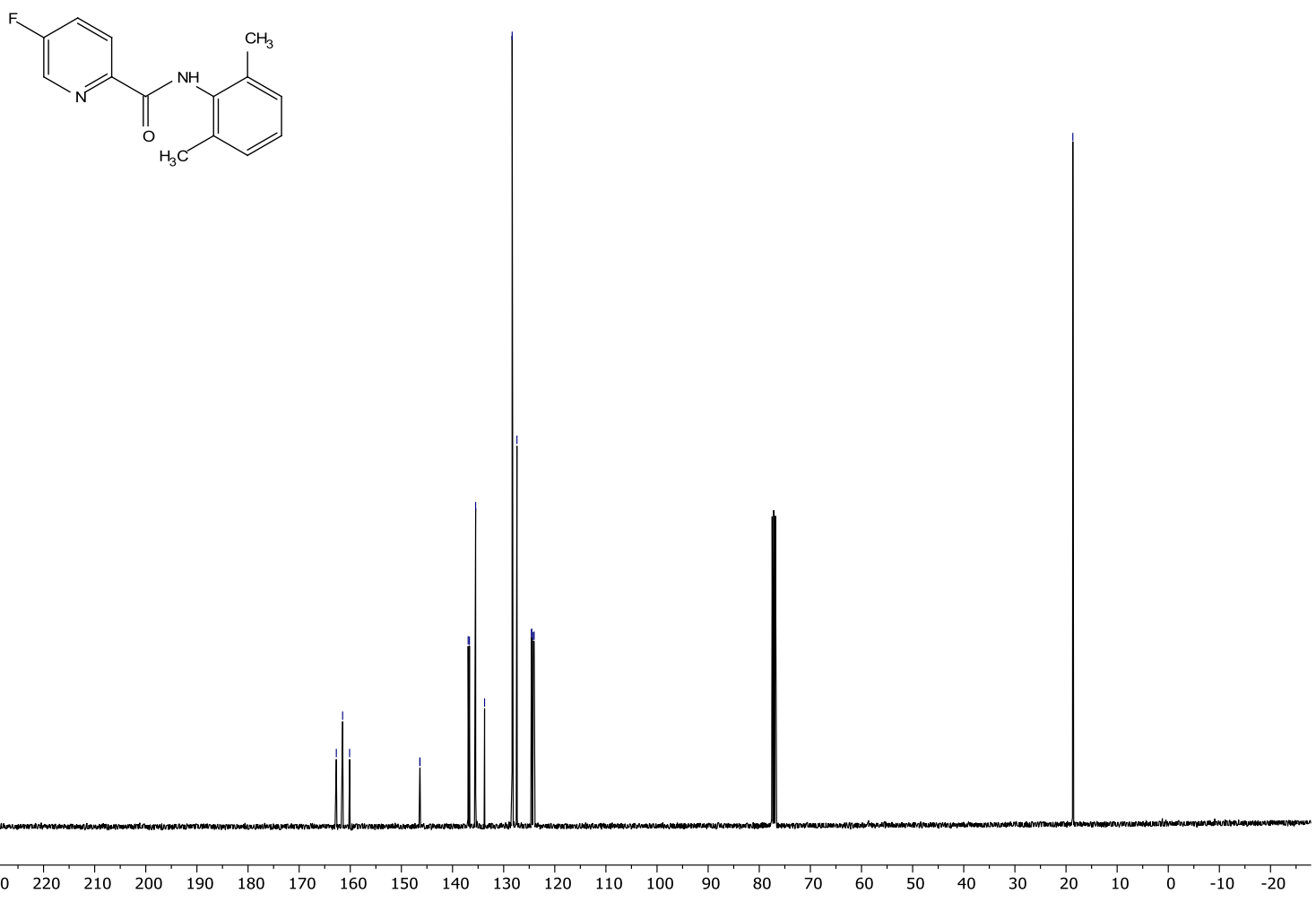

S98 
${ }^{19} \mathrm{~F}\{1 \mathrm{H}\}$ NMR (Chloroformed)

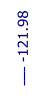
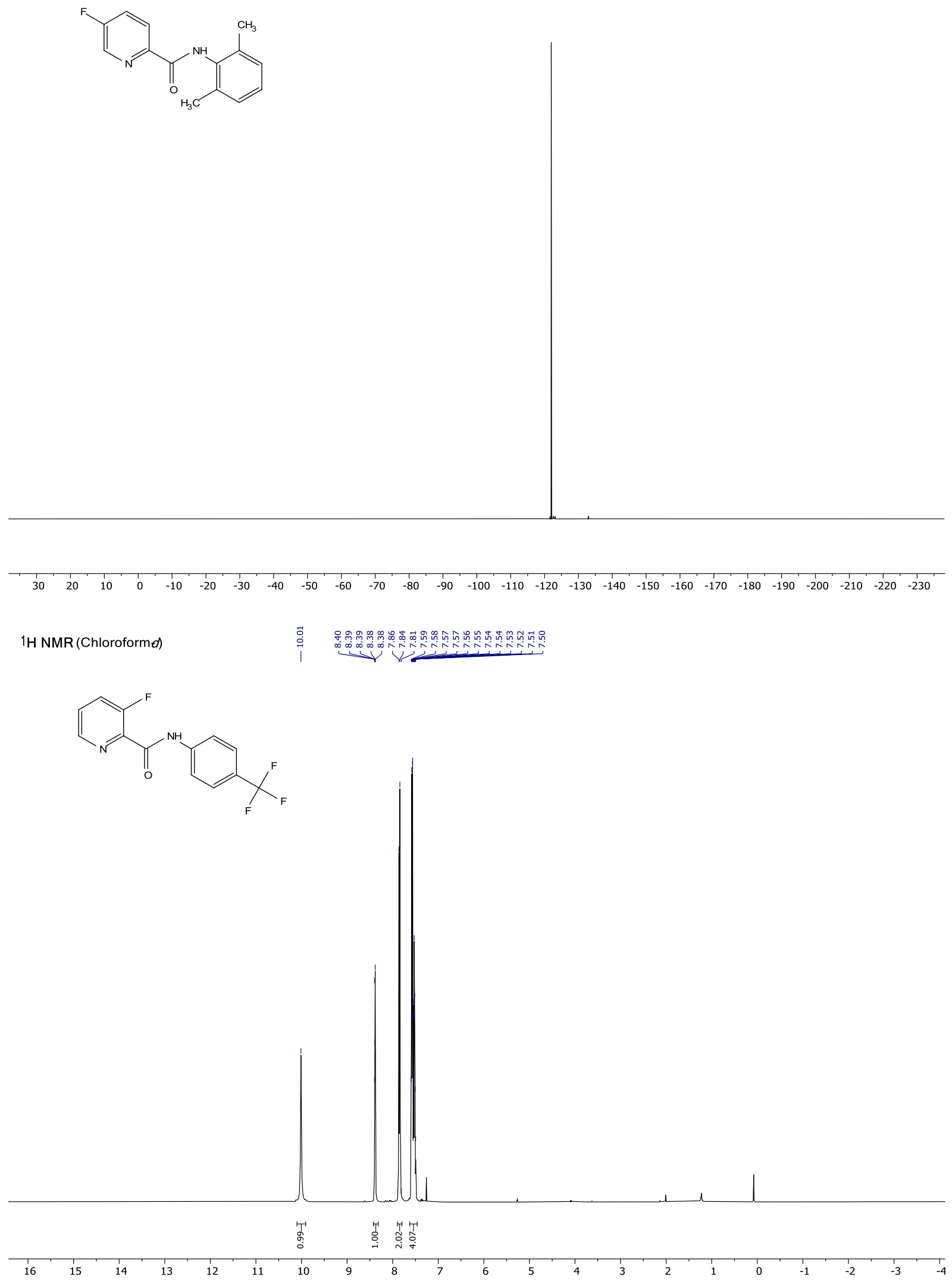

S99 
${ }^{13 C}\{1 \mathrm{H}\}$ NMR (Chloroforme)

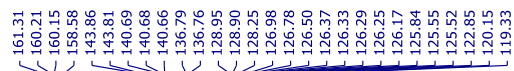
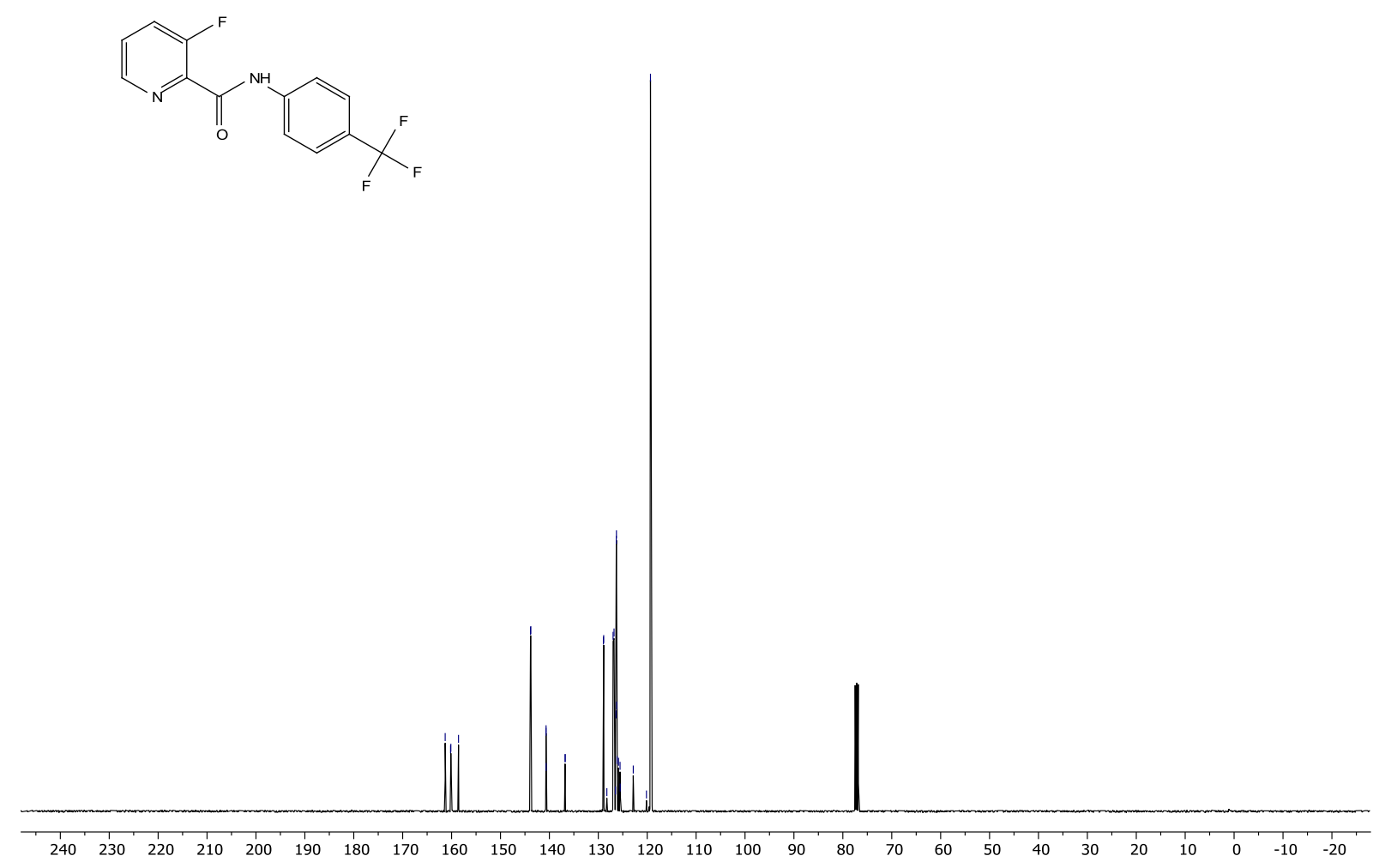

${ }^{19} \mathrm{~F}\{1 \mathrm{H}\} \mathrm{NMR}$ (Chloroforme) i
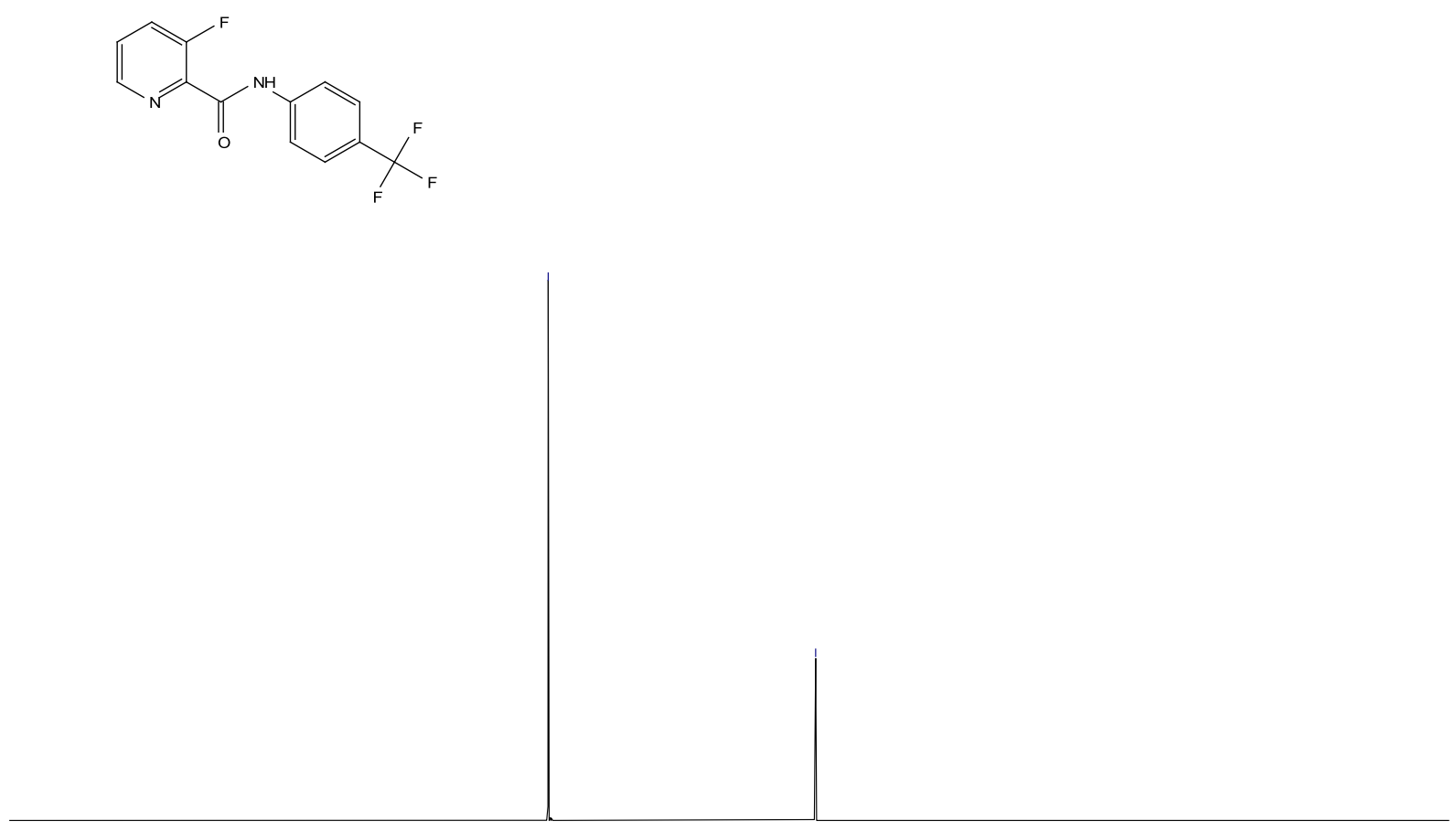

$\begin{array}{llllllllllllllllllllllllllllllllll}50 & 40 & 30 & 20 & 10 & 0 & -10 & -20 & -30 & -40 & -50 & -60 & -70 & -80 & -90 & -100 & -110 & -120 & -130 & -140 & -150 & -160 & -170 & -180 & -190 & -200 & -210 & -220 & -230 & -240 & -2 !\end{array}$

$\mathrm{S} 100$ 
1H NMR (Chloroformed)<smiles>Cc1cccc(C)c1NC(=O)c1ncccc1F</smiles>

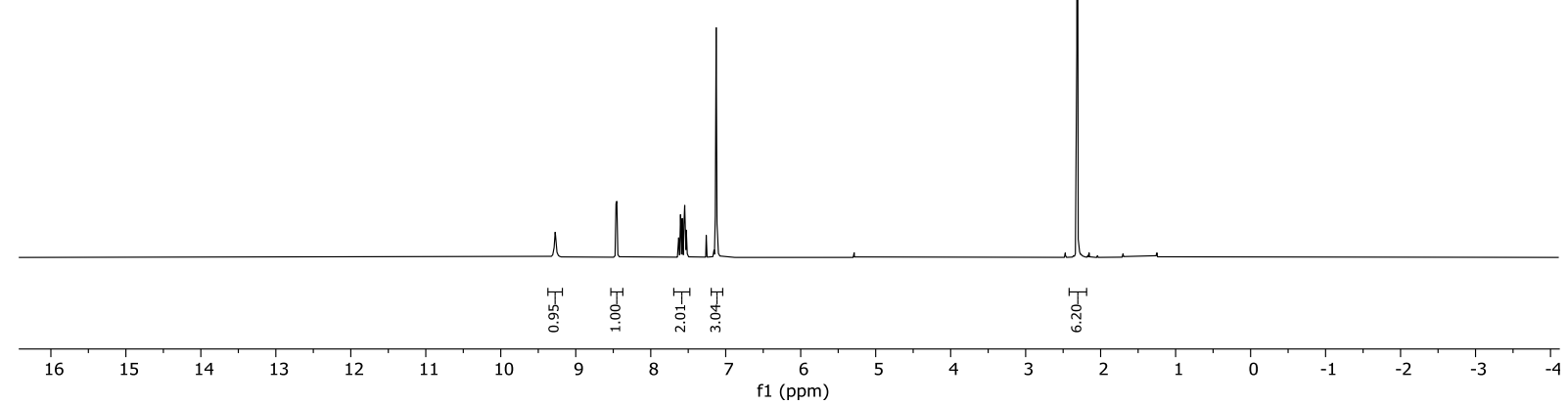

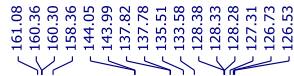

${ }^{13} \mathrm{C}\{1 \mathrm{H}\}$ NMR (Chloroformed)<smiles>Cc1cccc(C)c1NC(=O)c1ncccc1F</smiles>

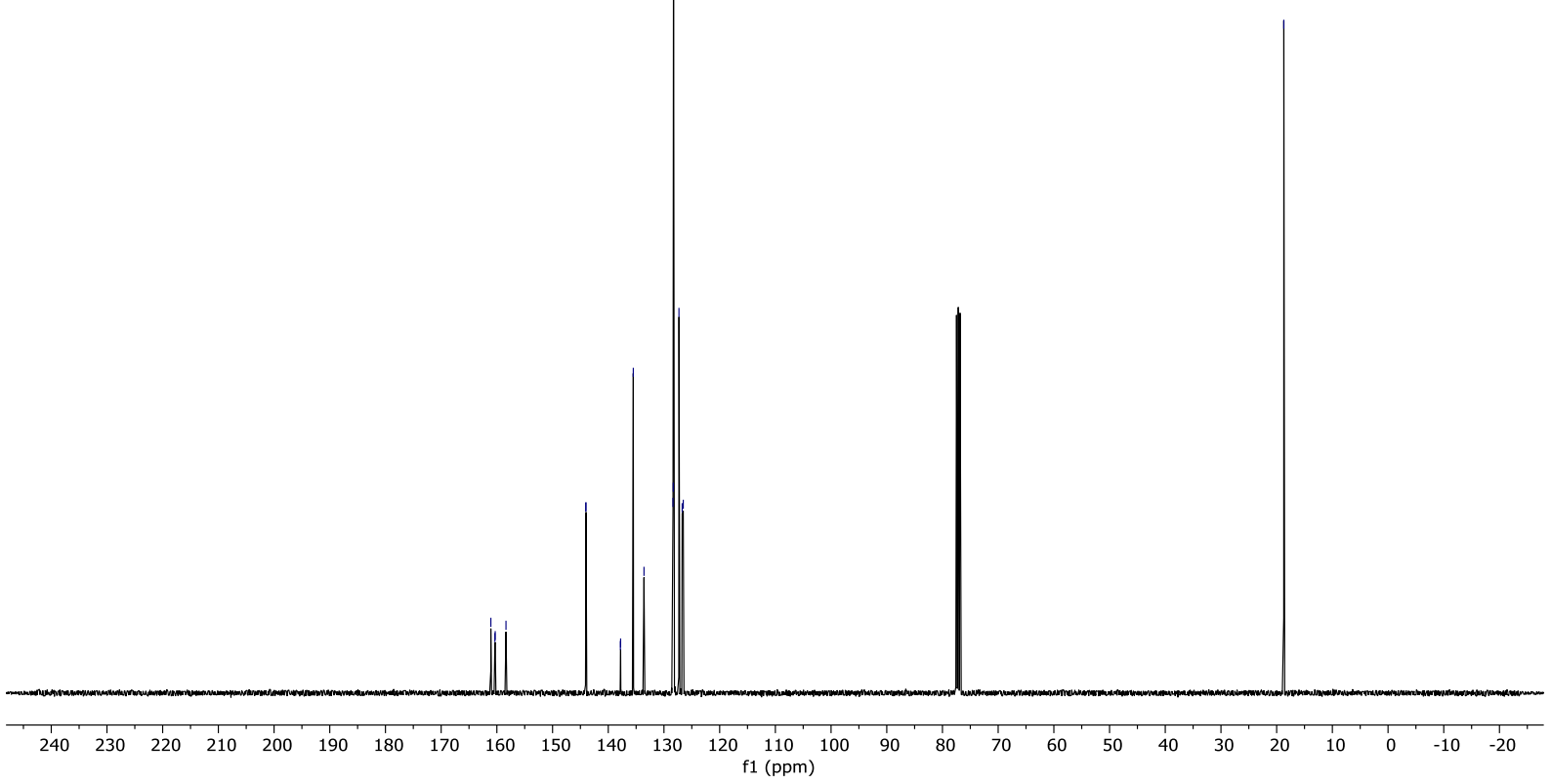


${ }^{19} \mathrm{~F}\{1 \mathrm{H}\}$ NMR (Chloroformel)<smiles>Cc1cccc(C)c1NC(=O)c1ncccc1F</smiles><smiles>O=C(c1ccc(F)cn1)N1CCCCC1</smiles>

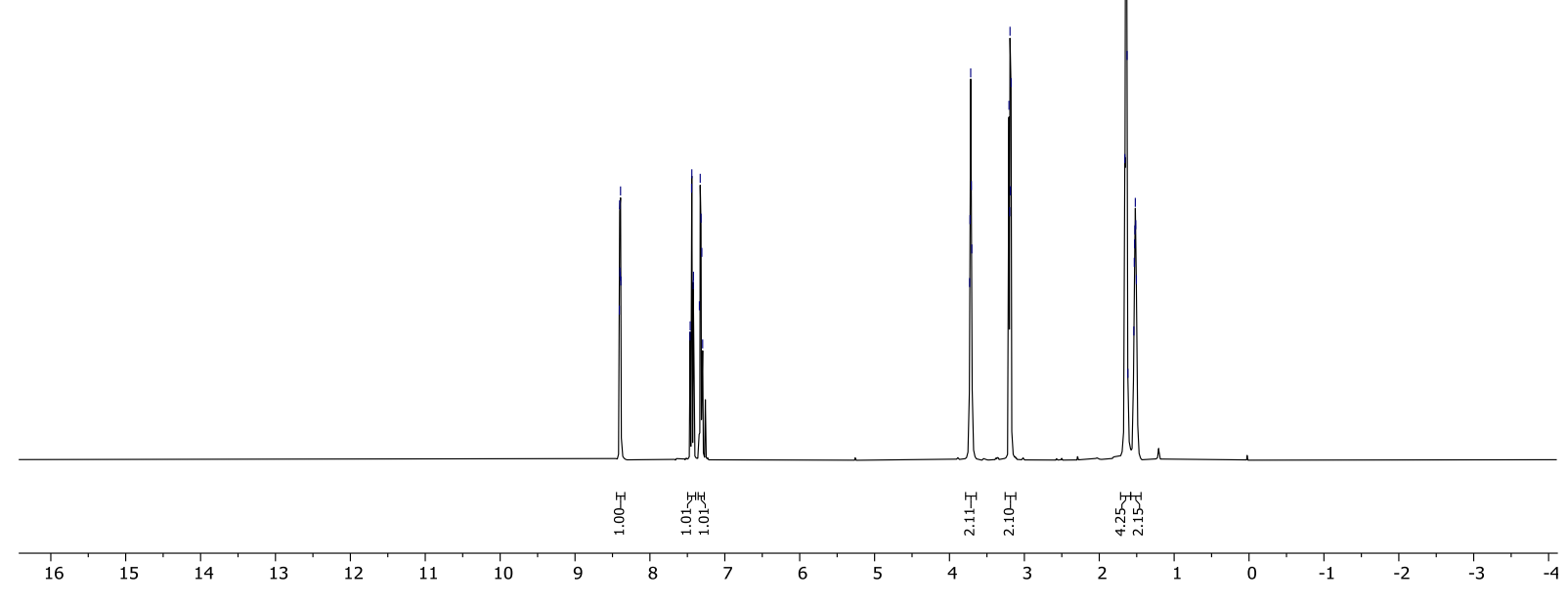


13C $\{1 \mathrm{H}\}$ NMR (Chloroforme)<smiles>O=C(c1ccc(F)cn1)N1CCCCC1</smiles>

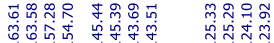

족

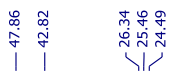

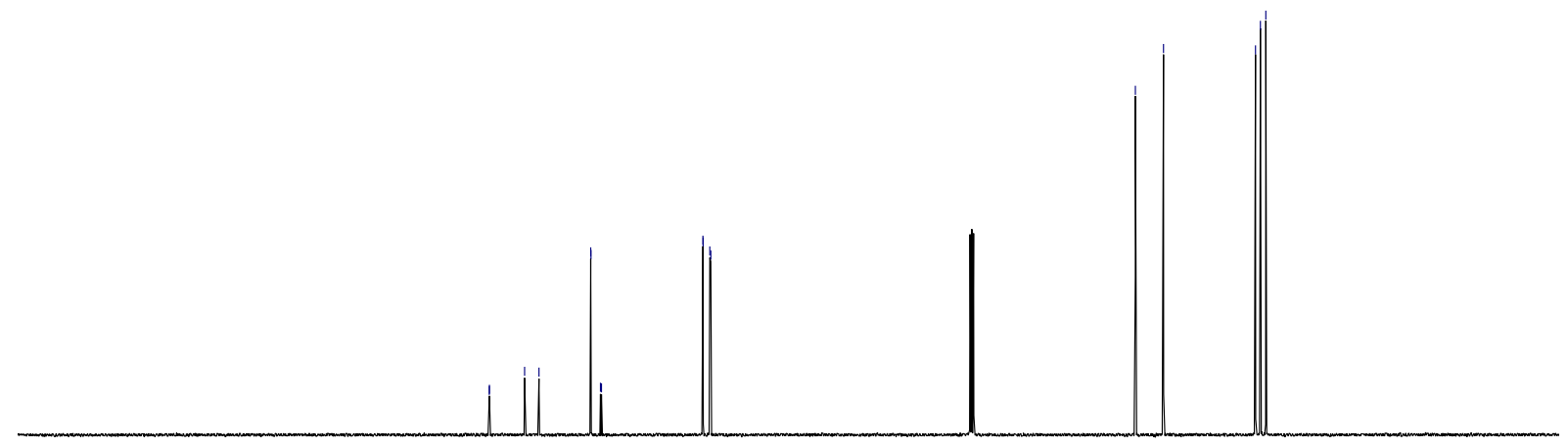

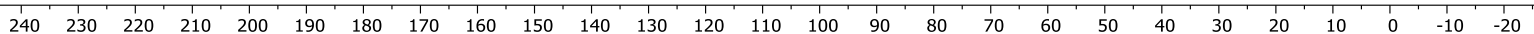

$19 \mathrm{~F}\{1 \mathrm{H}\}$ NMR (Chloroforme $)$<smiles>O=C(c1ccc(F)cn1)N1CCCCC1</smiles>

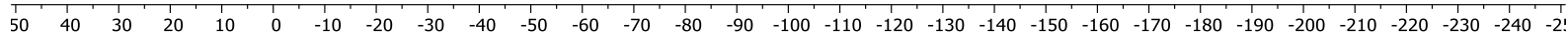




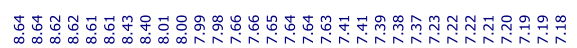

1H NMR (Chloroforme)
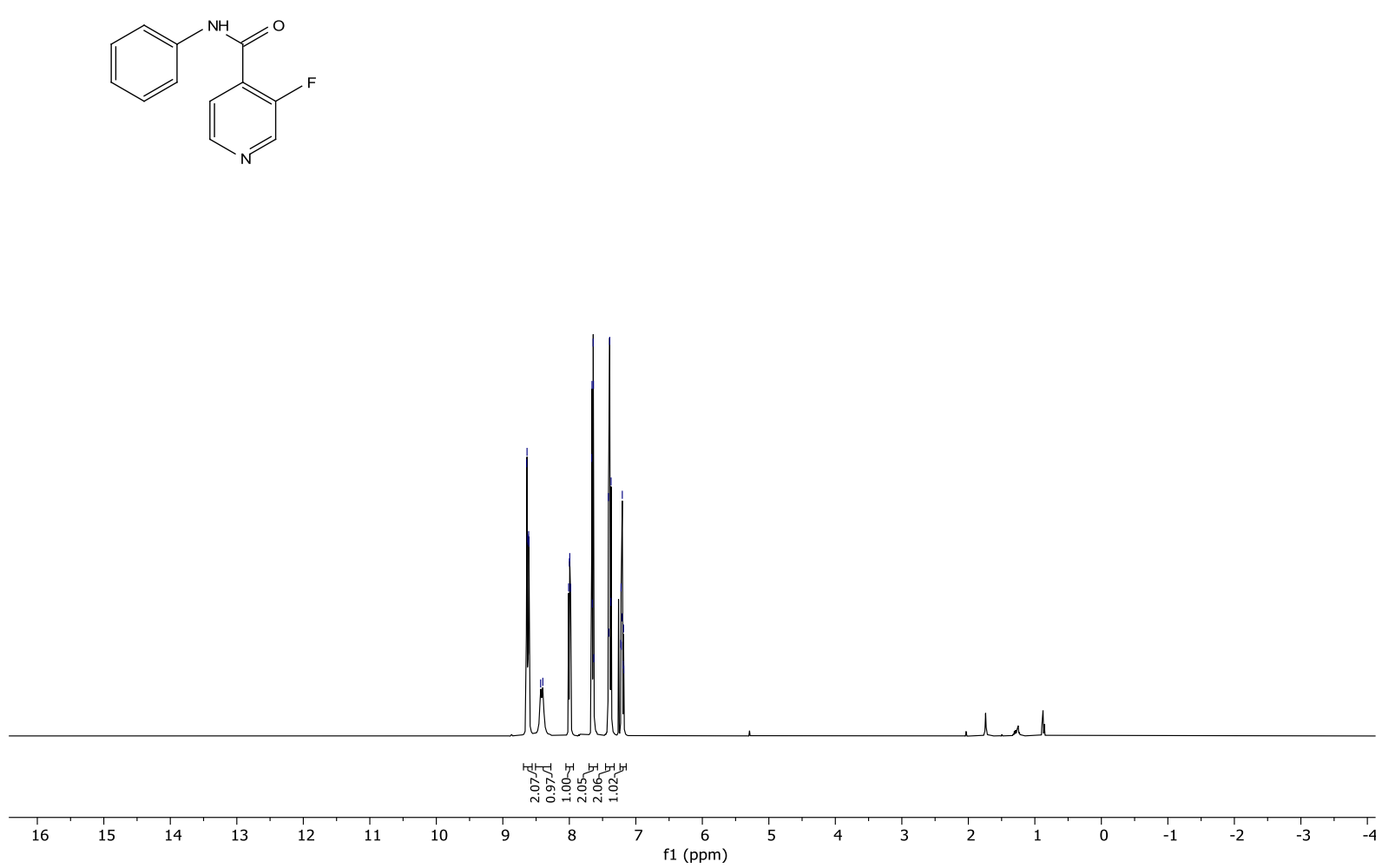

13C $\{1 \mathrm{H}\}$ NMR (Chloroforme)

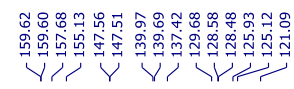
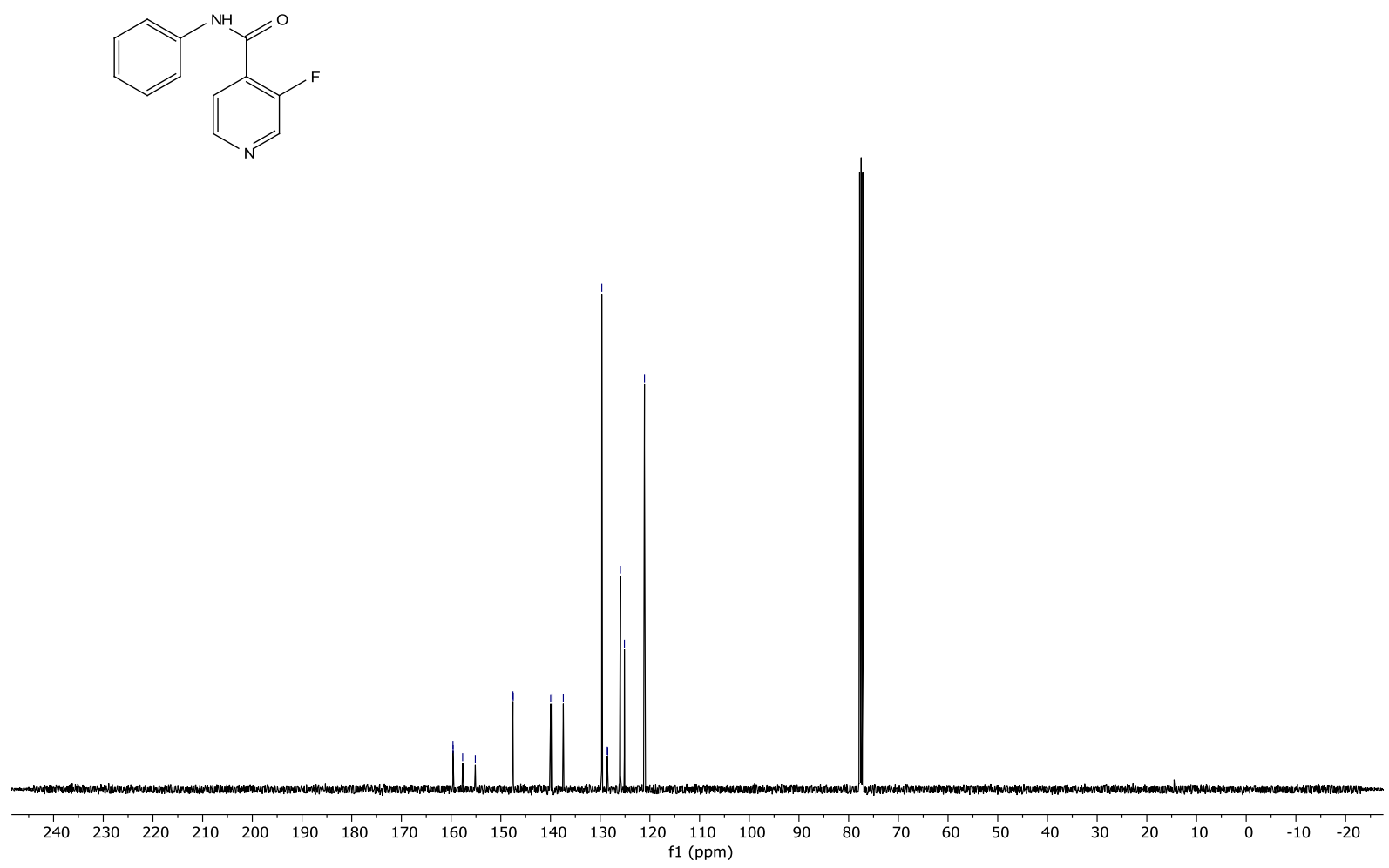

S104 
${ }^{19} \mathrm{~F}\{1 \mathrm{H}\}$ NMR (Chloroforme)<smiles>O=C(Nc1ccccc1)c1ccncc1F</smiles>

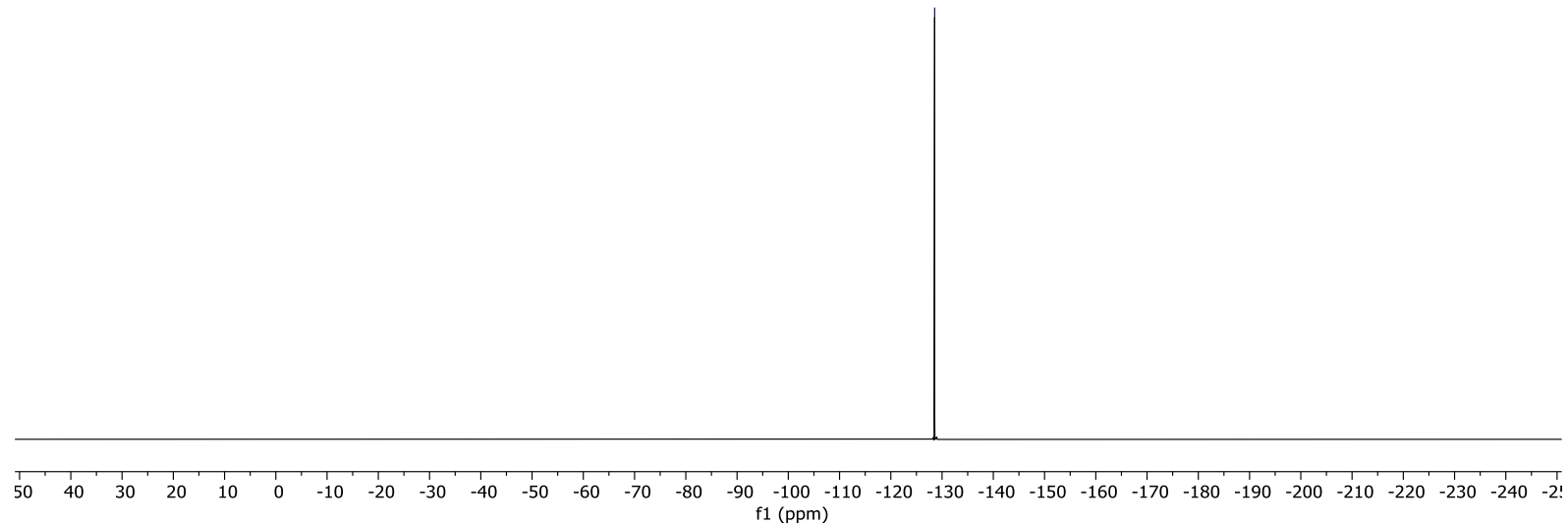

1H NMR (Chloroforme)

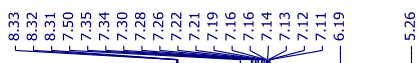

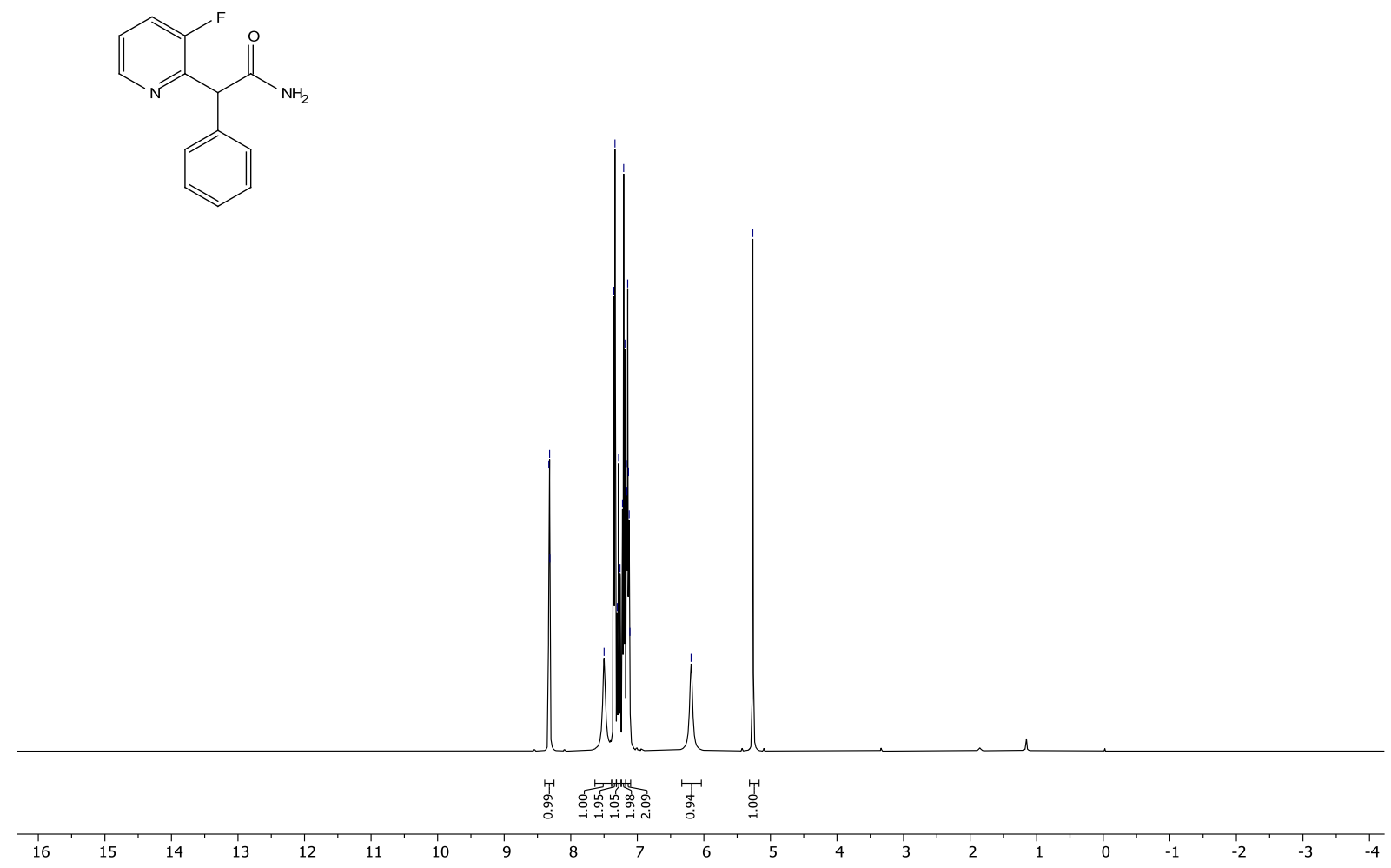




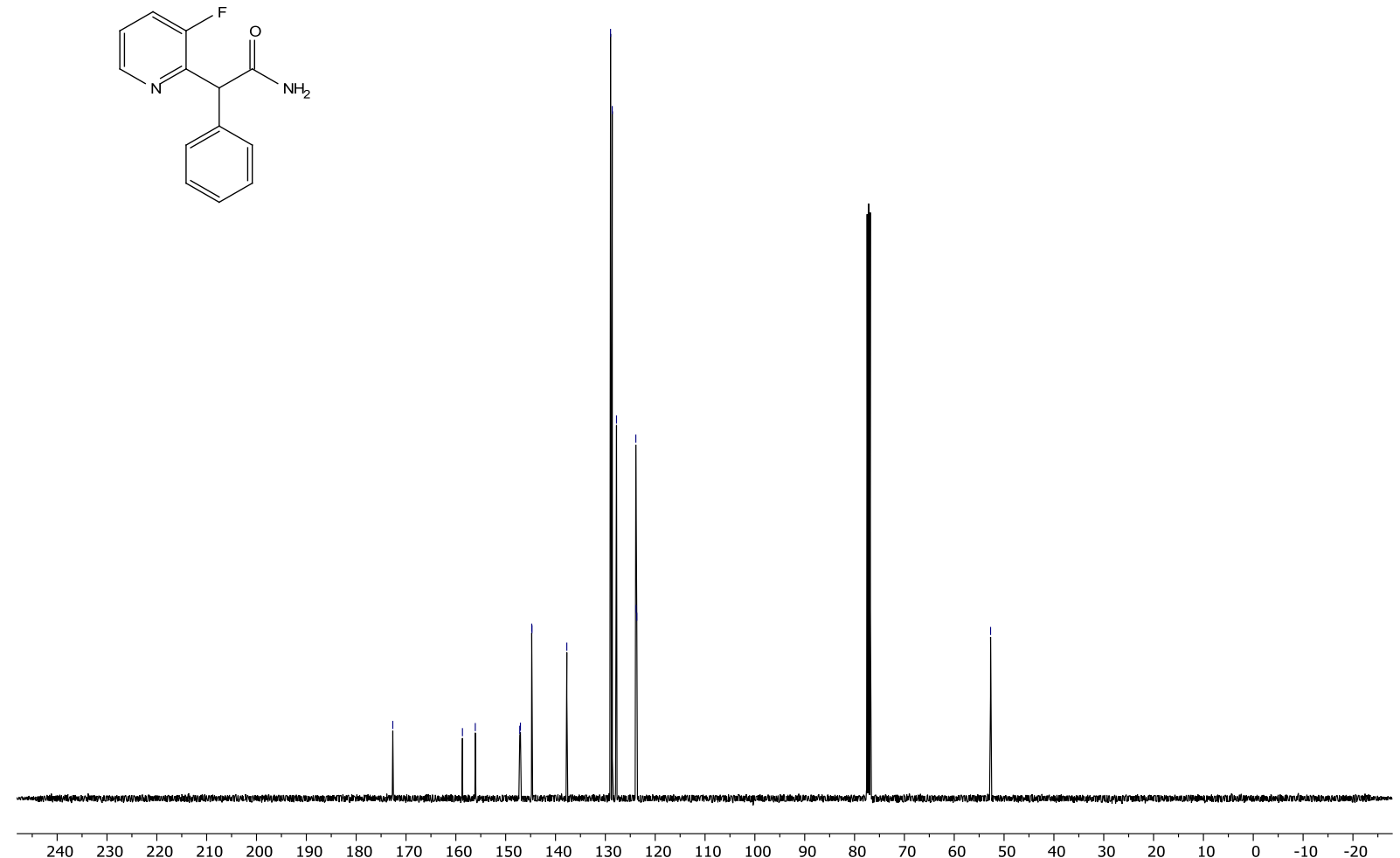

${ }^{19} \mathrm{~F}\{1 \mathrm{H}\}$ NMR (Chloroformel)<smiles>NC(=O)C(c1ccccc1)c1ncccc1F</smiles> 


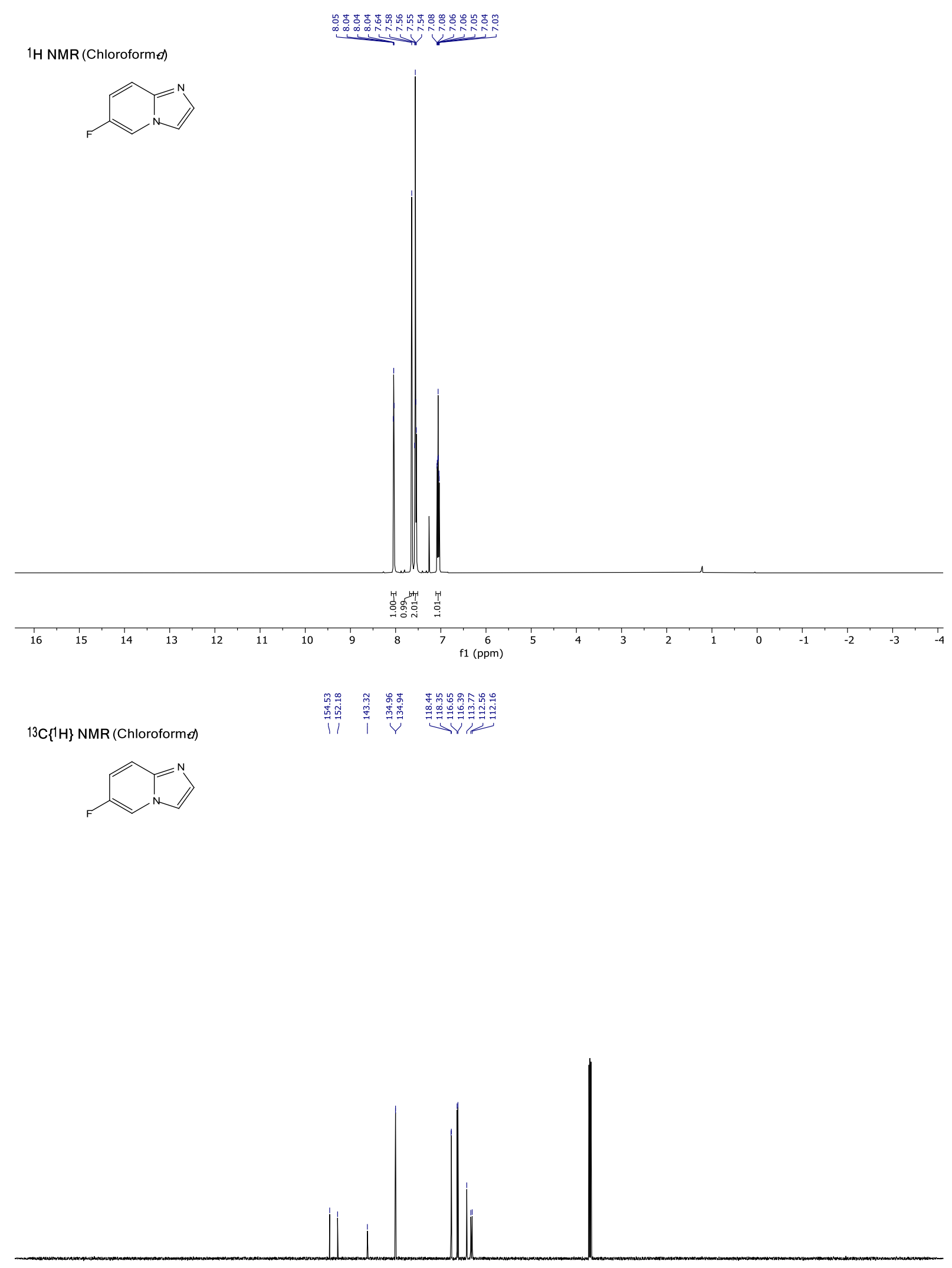

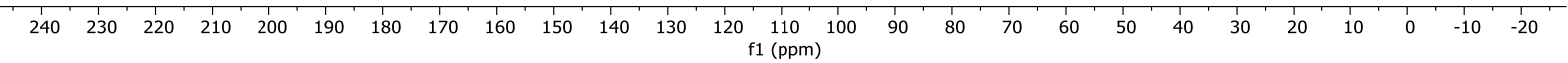


19F $\{1 \mathrm{H}\}$ NMR (Chloroformed)<smiles>Fc1ccc2nccn2c1</smiles>

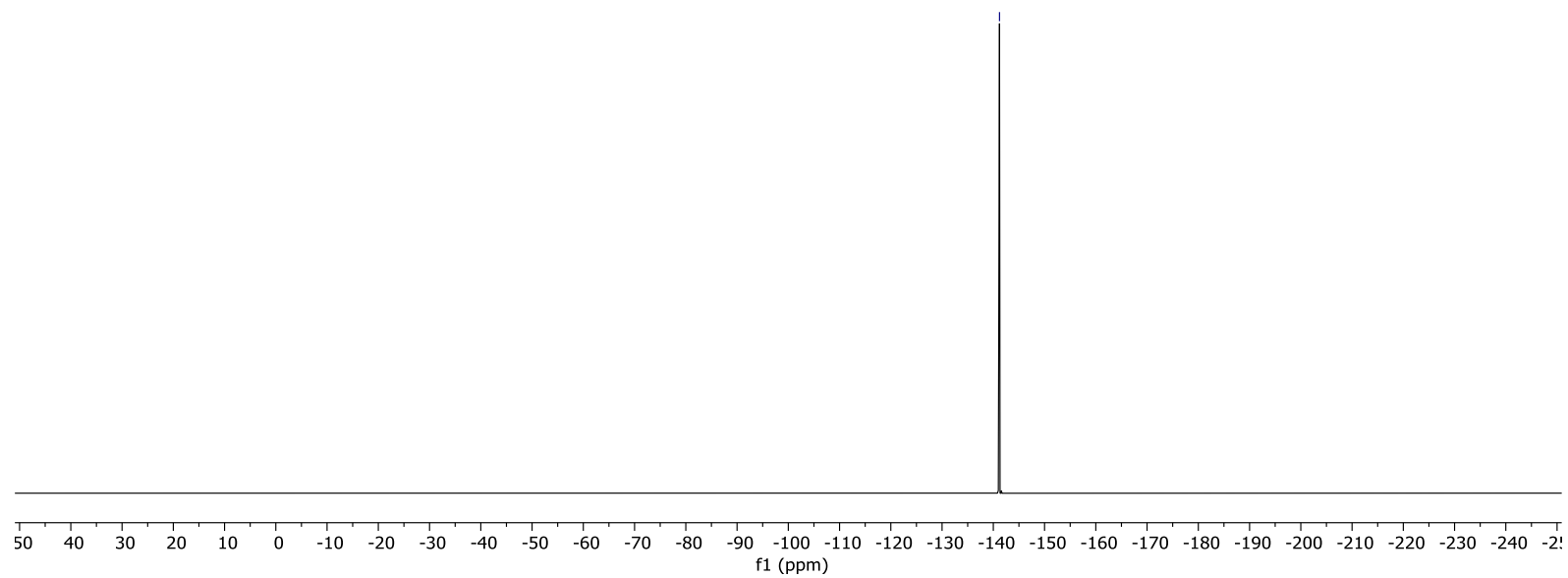

1H NMR (Chloroforme)<smiles>CC(C)(C)c1cn2cc(F)ccc2n1</smiles>

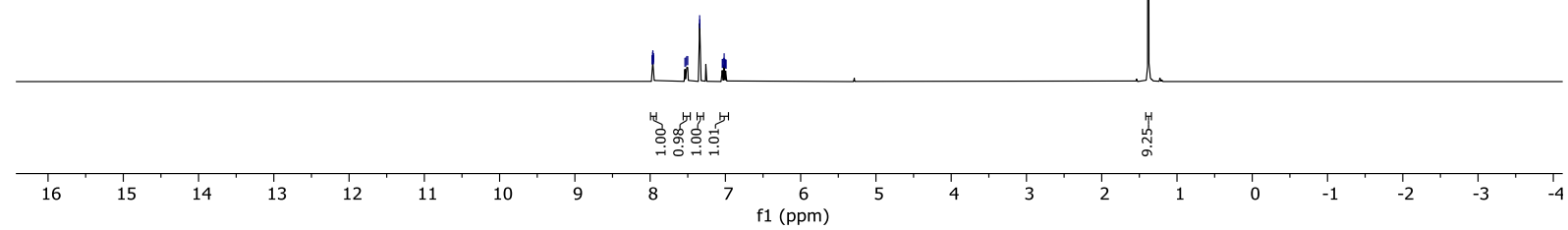


${ }^{13} \mathrm{C}\{1 \mathrm{H}\}$ NMR (Chloroformed)

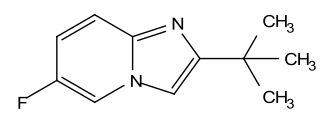

作

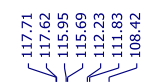

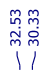



19F $\{1 \mathrm{H}\}$ NMR (Chloroformel)

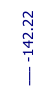

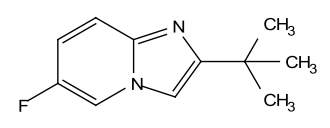

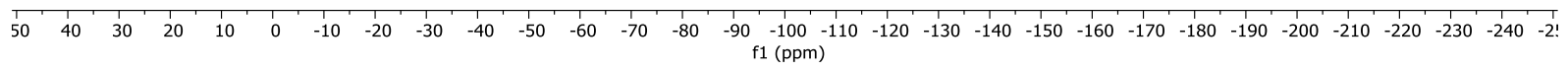

S109 
1H NMR (Chloroforme)<smiles>Fc1ccc(-c2cn3cc(F)ccc3n2)cc1</smiles>

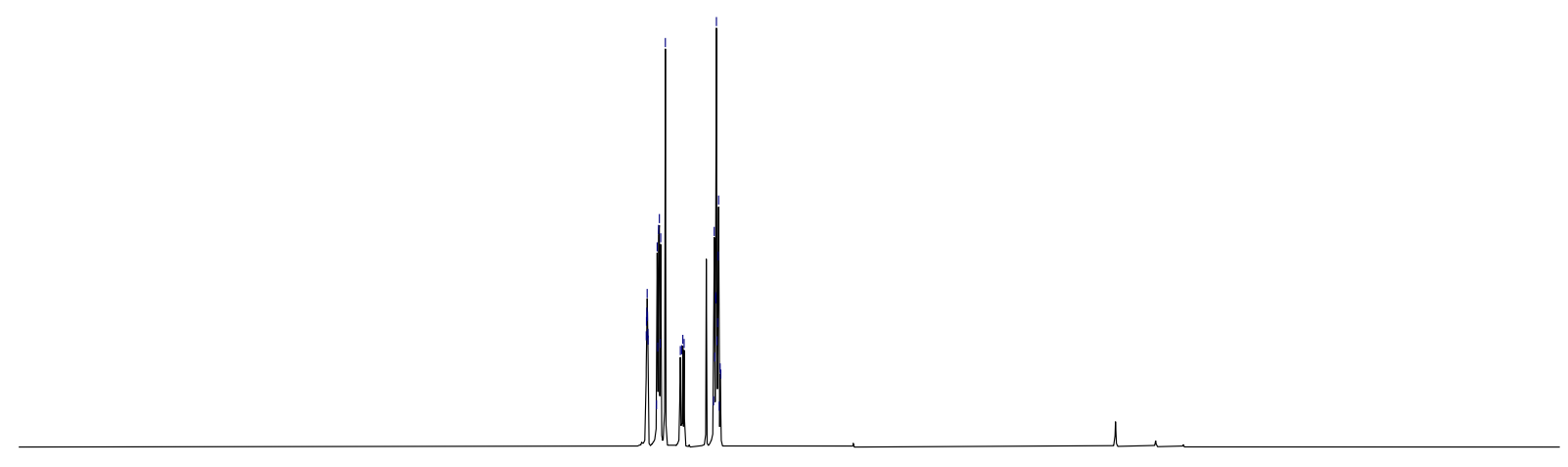

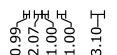

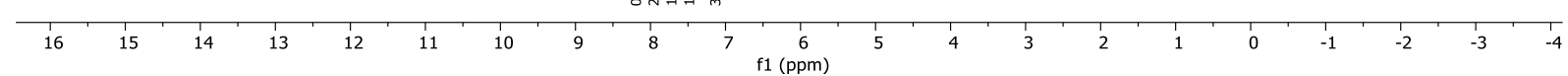

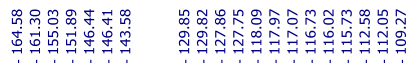

${ }^{13 C}\{1 \mathrm{H}\}$ NMR (Chloroforme) 1人र<smiles>Fc1ccc(-c2cn3cc(F)ccc3n2)cc1</smiles>

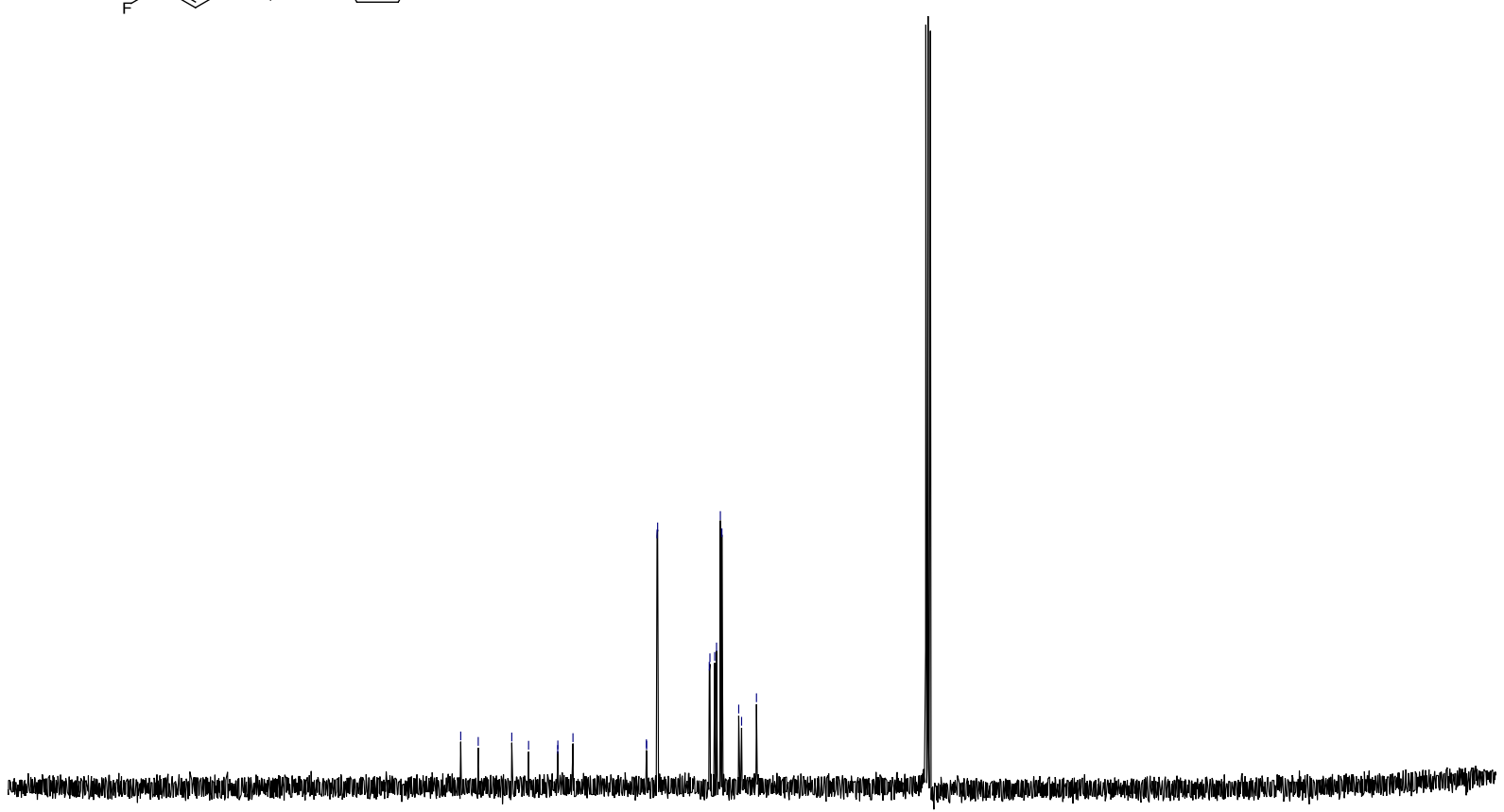

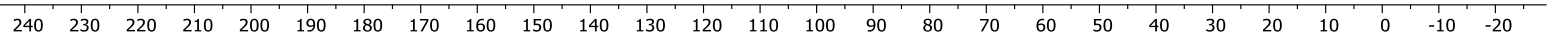


${ }^{19} \mathrm{~F}\{1 \mathrm{H}\}$ NMR (Chloroformed)<smiles>Fc1ccc(-c2cn3cc(F)ccc3n2)cc1</smiles>

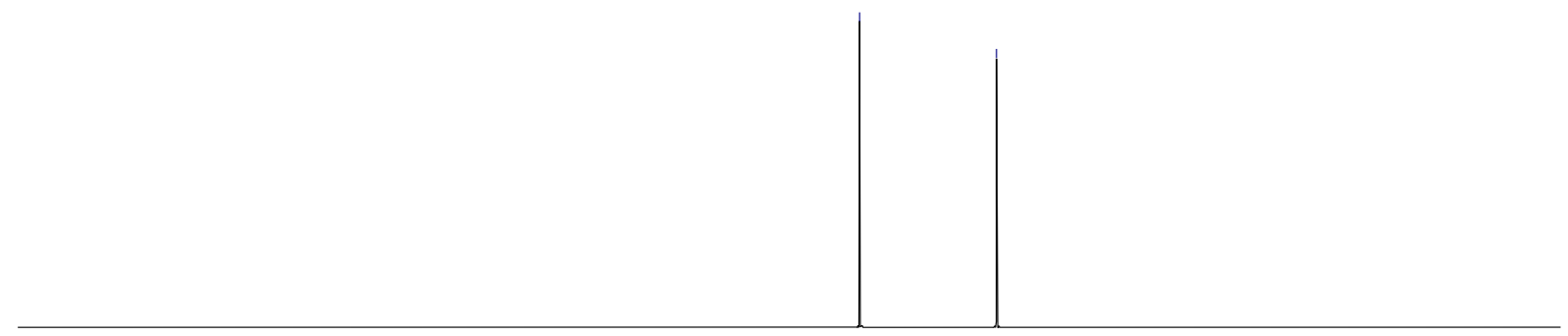
$\begin{array}{lllllllllllllllllllllllllllllllllllllllllll}50 & 40 & 30 & 20 & 10 & 0 & -10 & -20 & -30 & -40 & -50 & -60 & -70 & -80 & -90 & -100 & -110 & -120 & -130 & -140 & -150 & -160 & -170 & -180 & -190 & -200 & -210 & -220 & -230 & -240 & -2 !\end{array}$

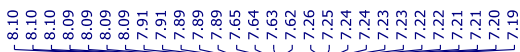

\section{H NMR (Chloroformed)}<smiles>Fc1ccc2nc(C(F)(F)F)cn2c1</smiles>

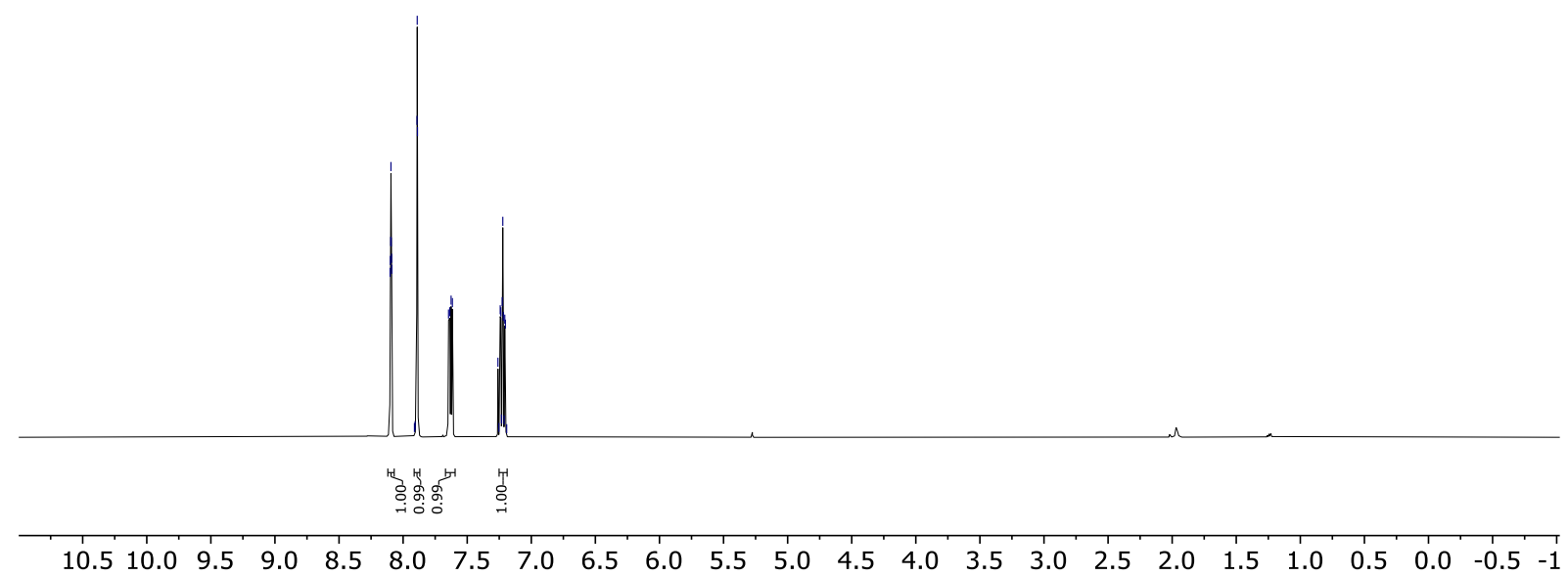




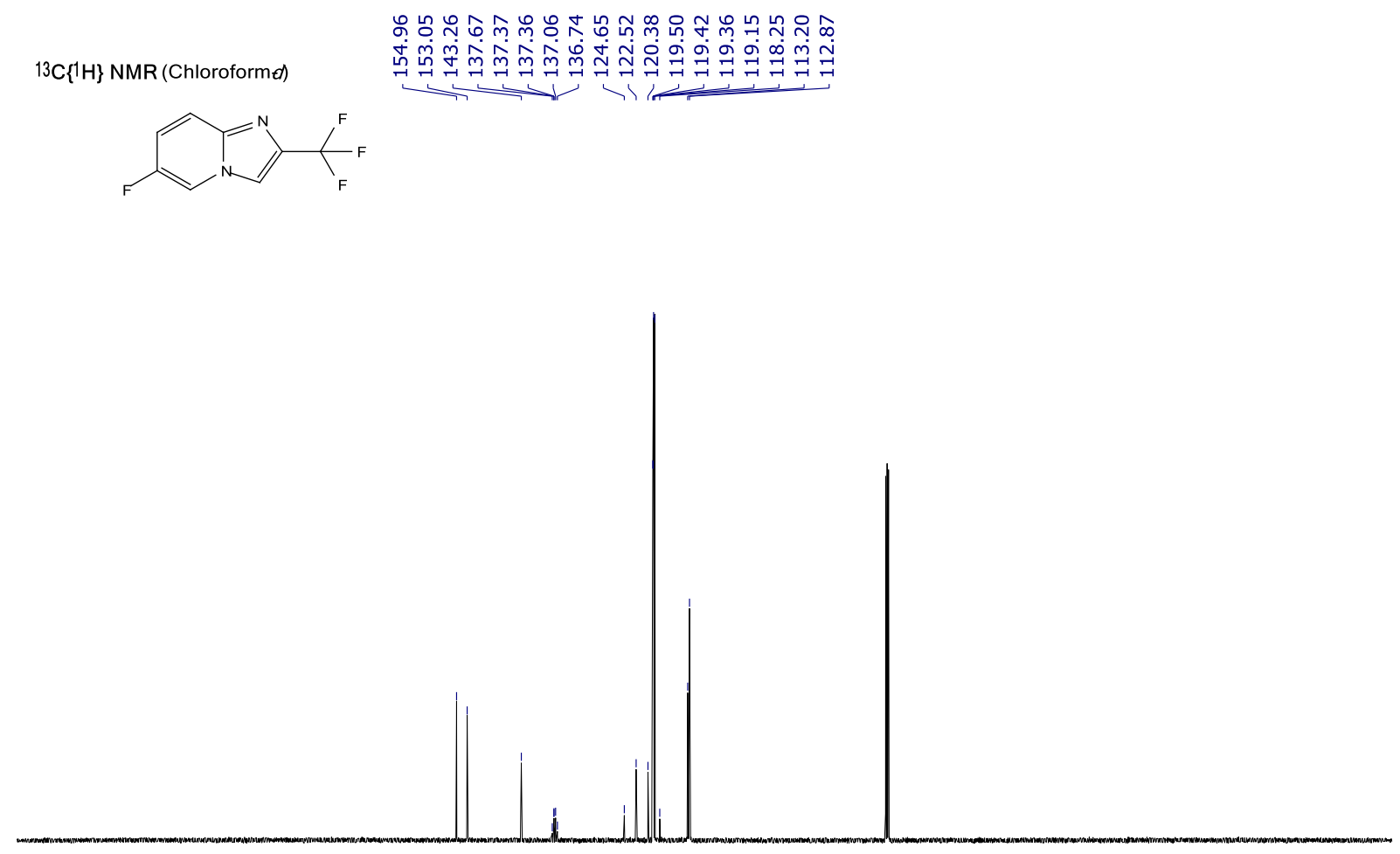

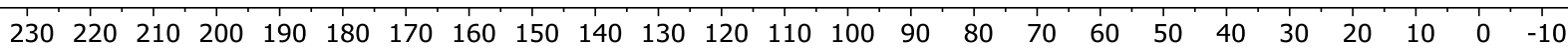

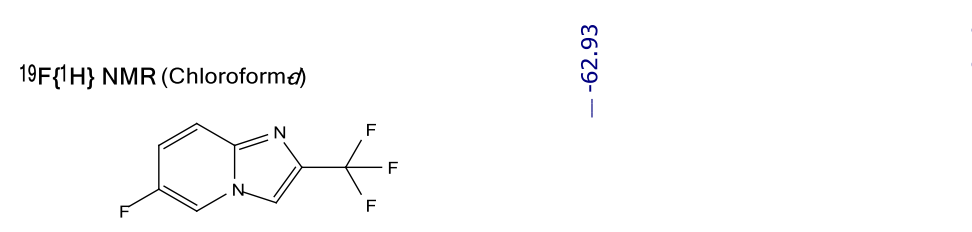

$\underset{\substack{\infty \\ \stackrel{m}{m} \\ i}}{i}$

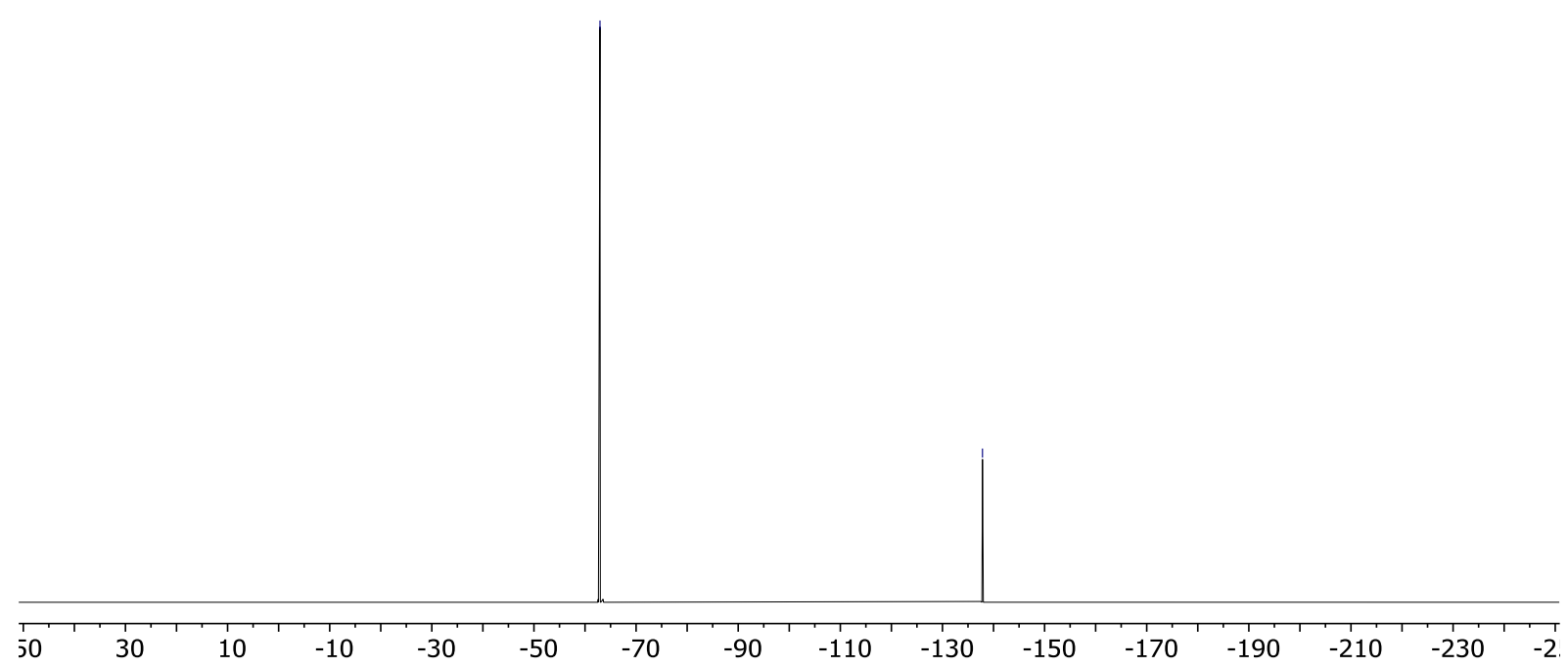


地

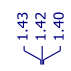

1H NMR (Chloroforme)
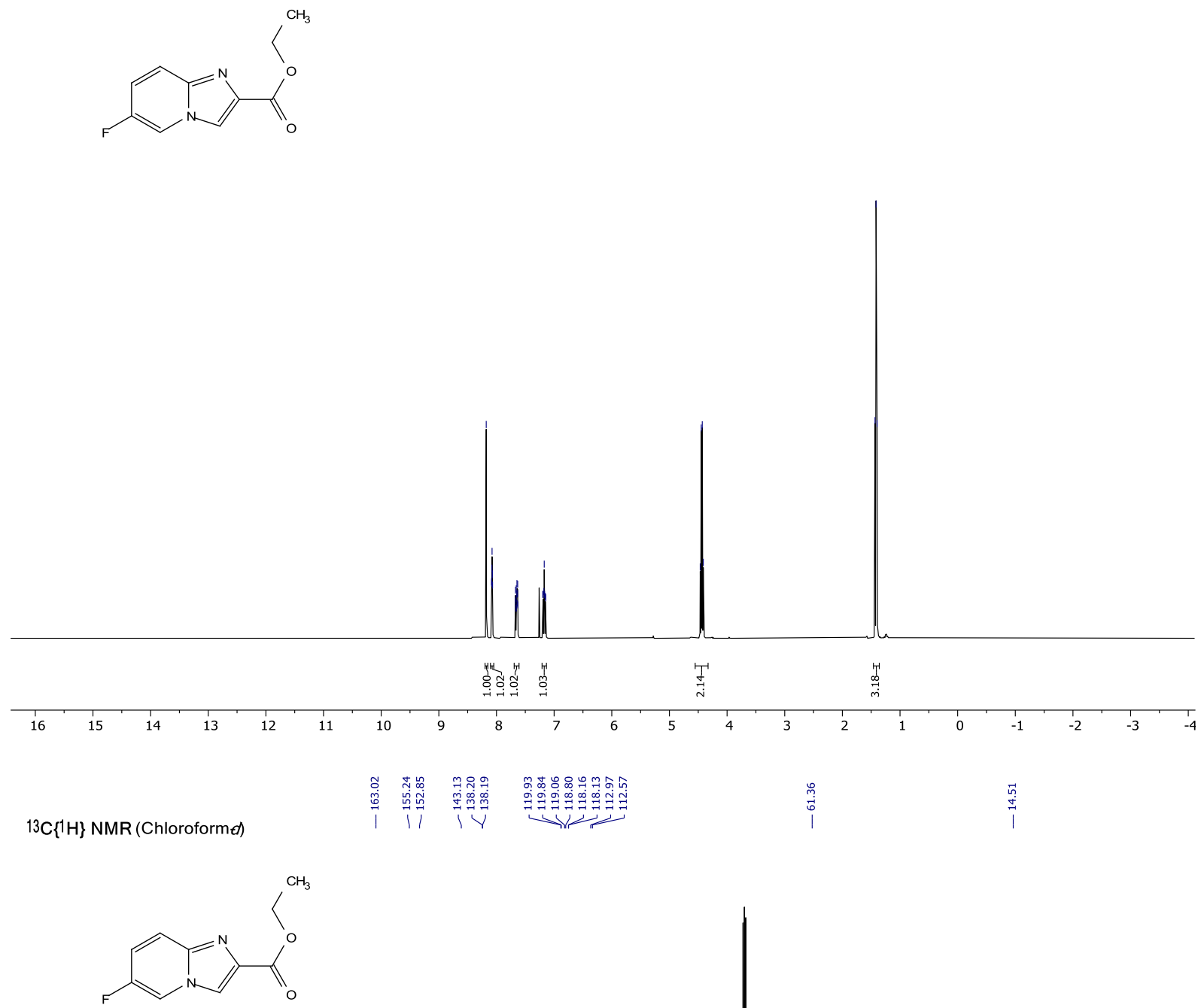

$\mathrm{S} 113$ 
${ }^{19} \mathrm{~F}\{1 \mathrm{H}\}$ NMR (Chloroformed)<smiles>CCOC(=O)c1cn2cc(I)ccc2n1</smiles>

$\begin{array}{lllllllllllllllllllllllllllllllllllll}50 & 40 & 30 & 20 & 10 & 0 & -10 & -20 & -30 & -40 & -50 & -60 & -70 & -80 & -90 & -100 & -110 & -120 & -130 & -140 & -150 & -160 & -170 & -180 & -190 & -200 & -210 & -220 & -230 & -240 & -2 !\end{array}$ 1H NMR (Chloroforme)

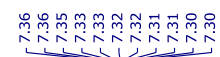

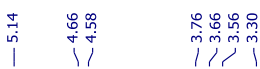
象象品品

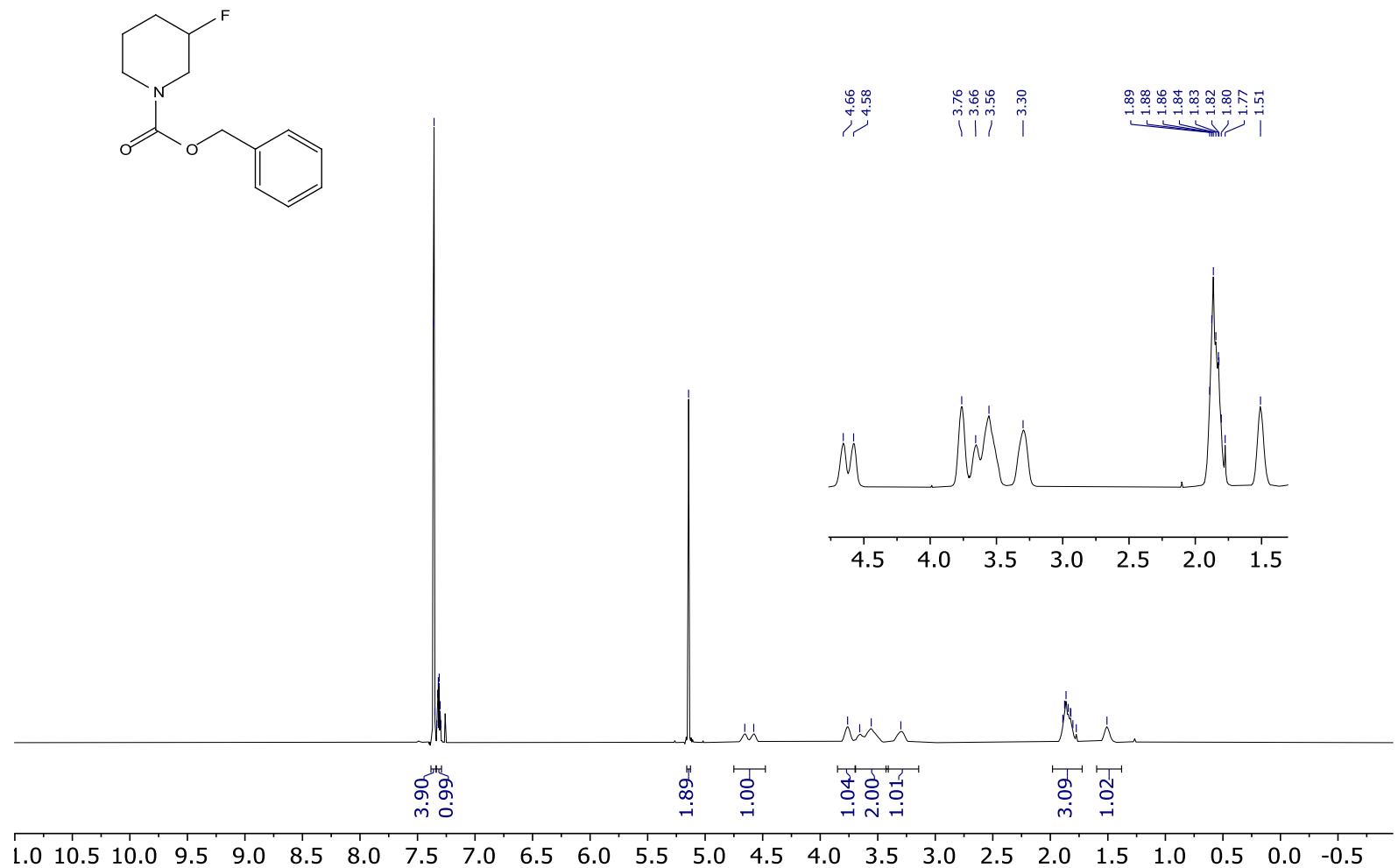


<smiles>O=C(OCc1ccccc1)N1CCCC(F)C1</smiles>

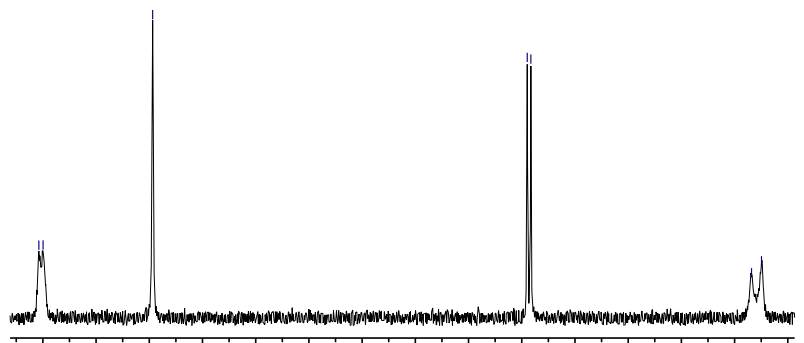

$\begin{array}{lllllllllllllll}48 & 46 & 44 & 42 & 40 & 38 & 36 & 34 & 32 & 30 & 28 & 26 & 24 & 22 & 21\end{array}$

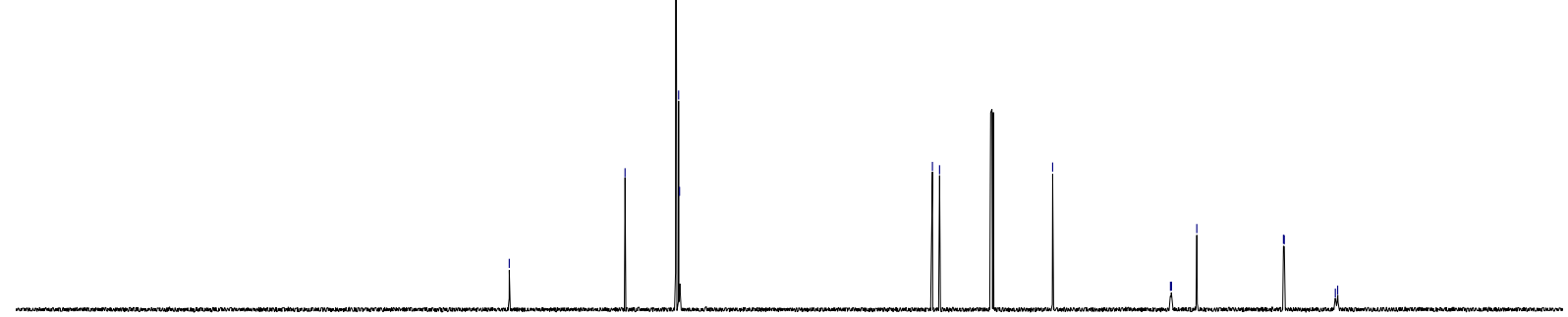

$\begin{array}{lllllllllllllllllllllllllll}230 & 220 & 210 & 200 & 190 & 180 & 170 & 160 & 150 & 140 & 130 & 120 & 110 & 100 & 90 & 80 & 70 & 60 & 50 & 40 & 30 & 20 & 10 & 0 & -10\end{array}$

19F NMR (Chloroforme)
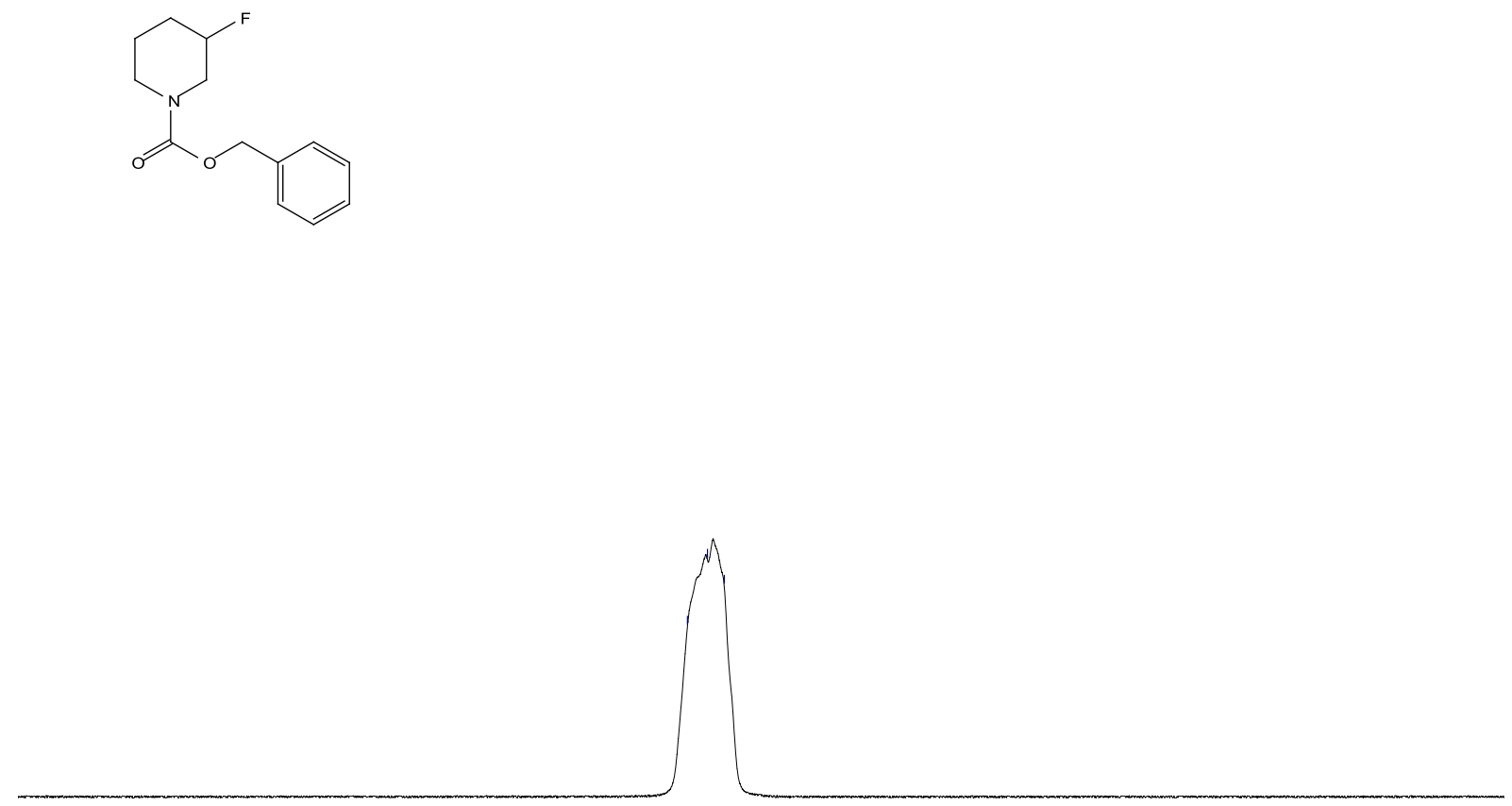
${ }^{19} \mathrm{~F}\{1 \mathrm{H}\}$ NMR (Chloroform $\left.d\right)$<smiles>O=C(OCc1ccccc1)N1CCCC(F)C1</smiles>

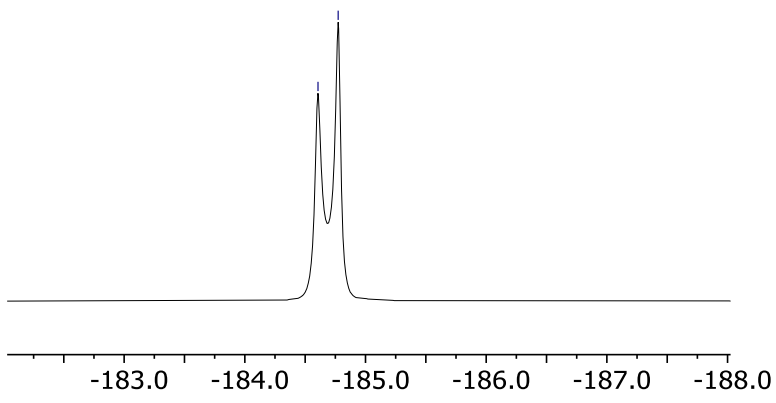

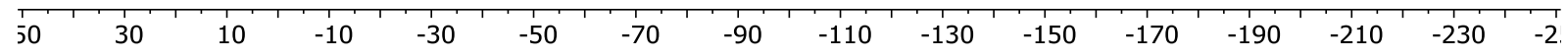

1H NMR (Chloroforme)

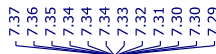

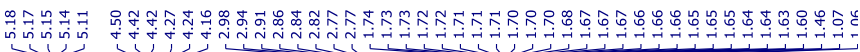

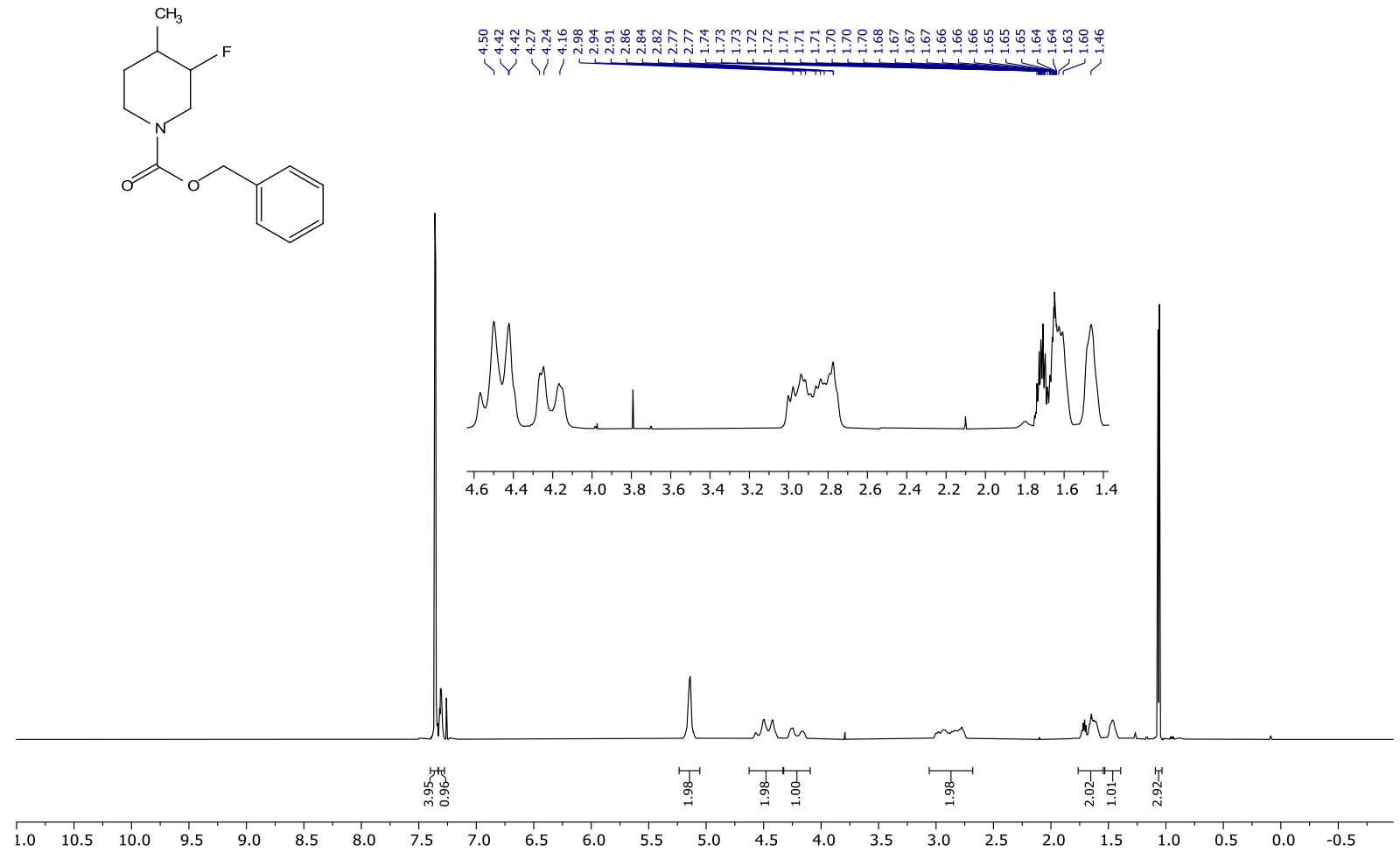


<smiles>CC1CCN(C(=O)OCc2ccccc2)CC1F</smiles>
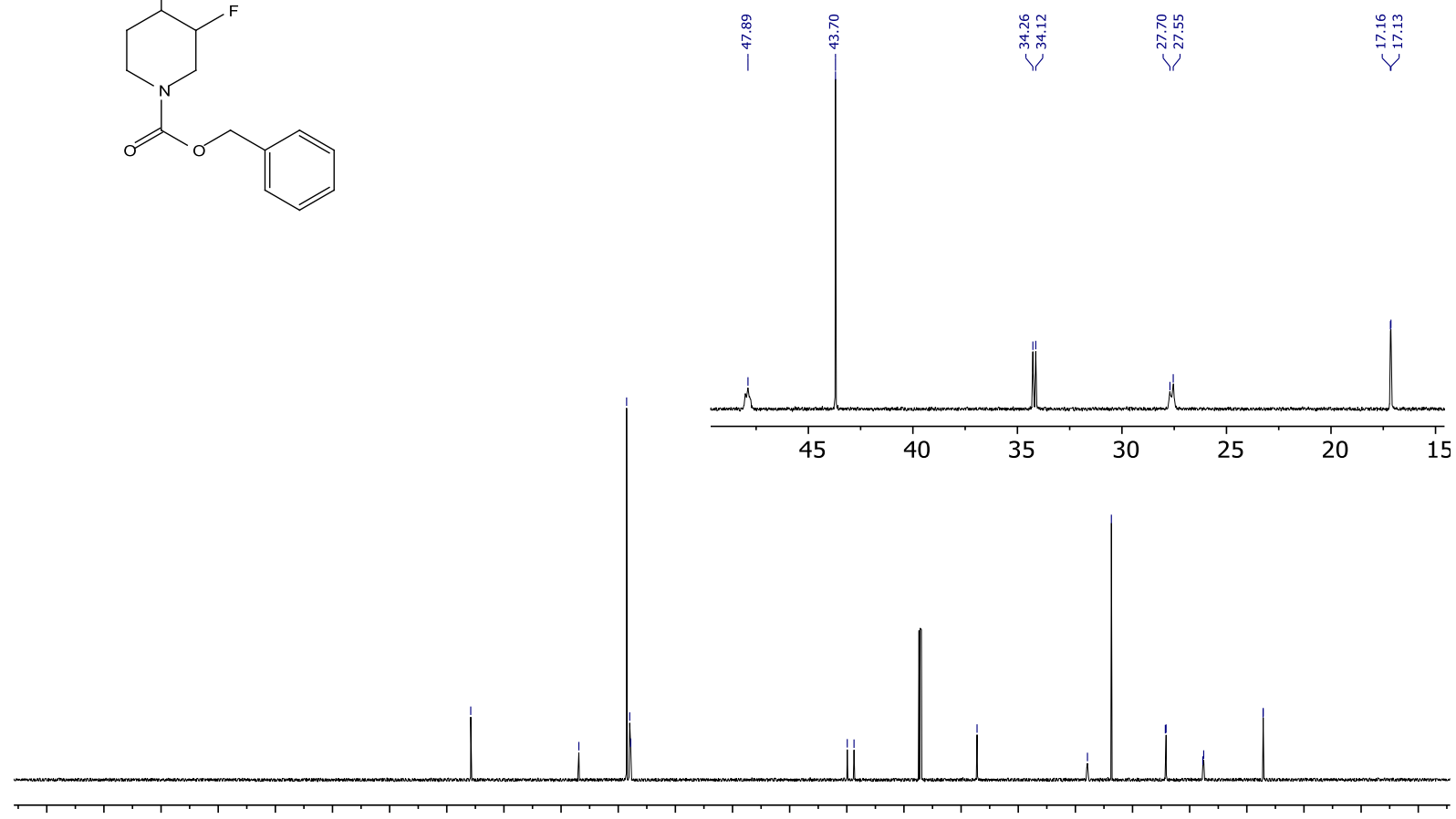

19F NMR (Chloroforme)

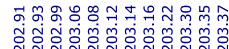

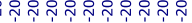<smiles>CC1CCN(C(=O)OCc2ccccc2)CC1F</smiles>

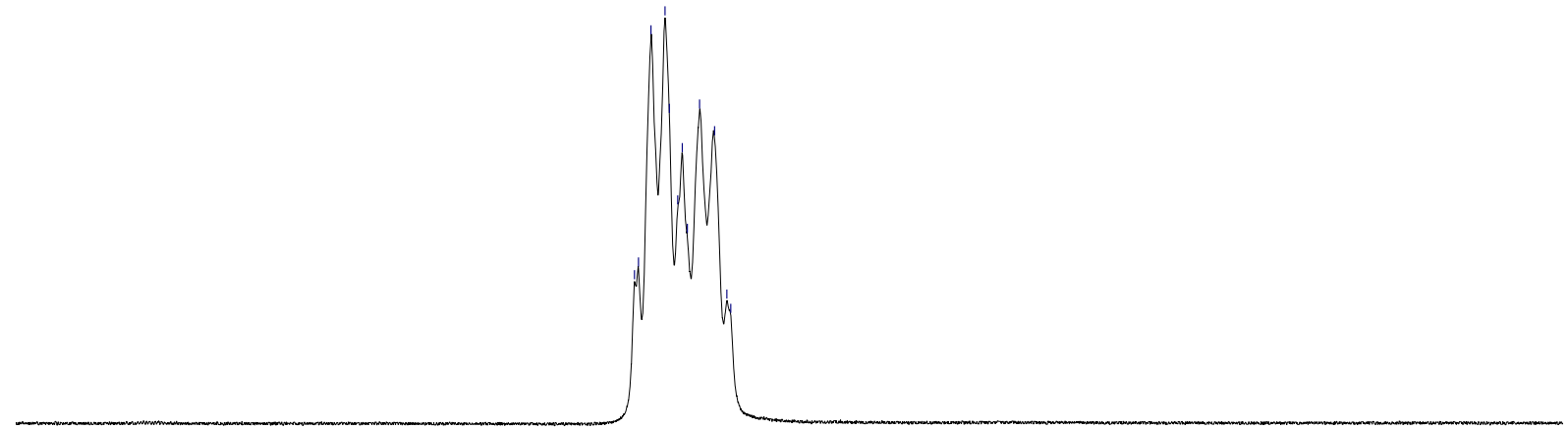

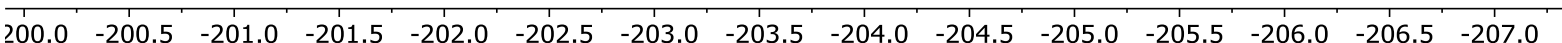


${ }^{19} \mathrm{~F}\{\mathrm{H} \mathrm{H}\}$ NMR (Chloroformed)<smiles>CC1CCN(C(=O)OCc2ccccc2)CC1F</smiles>

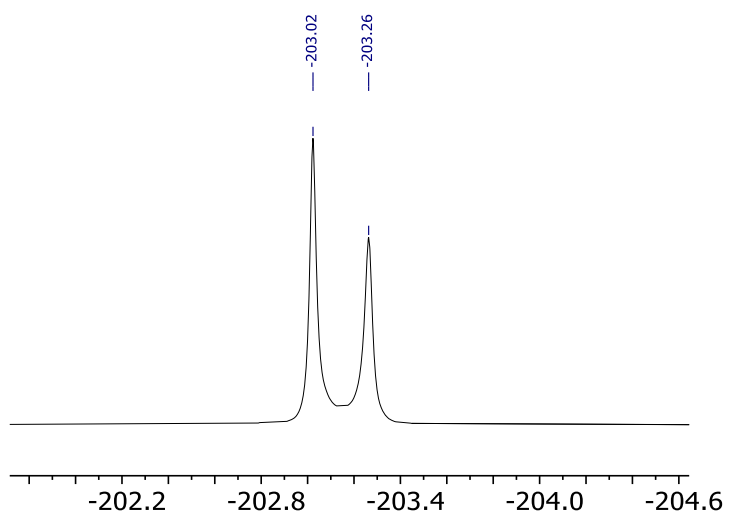

1H NMR (Chloroforme)

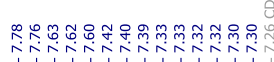

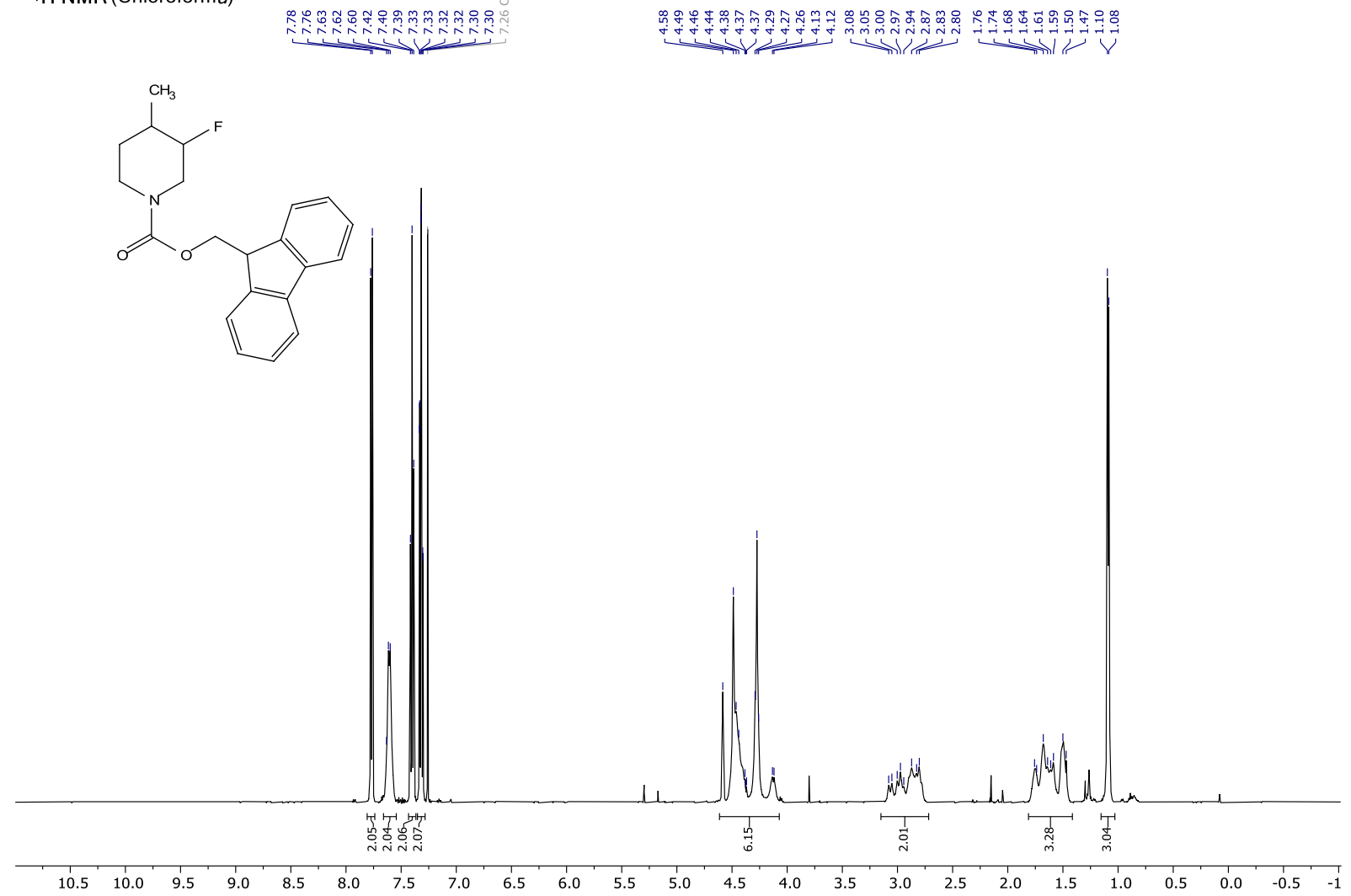

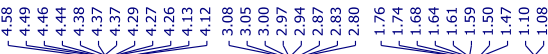

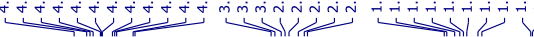


13C $\{1 \mathrm{H}\}$ NMR (Chloroformel)

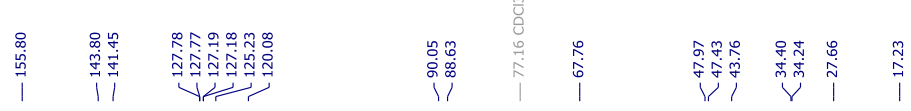
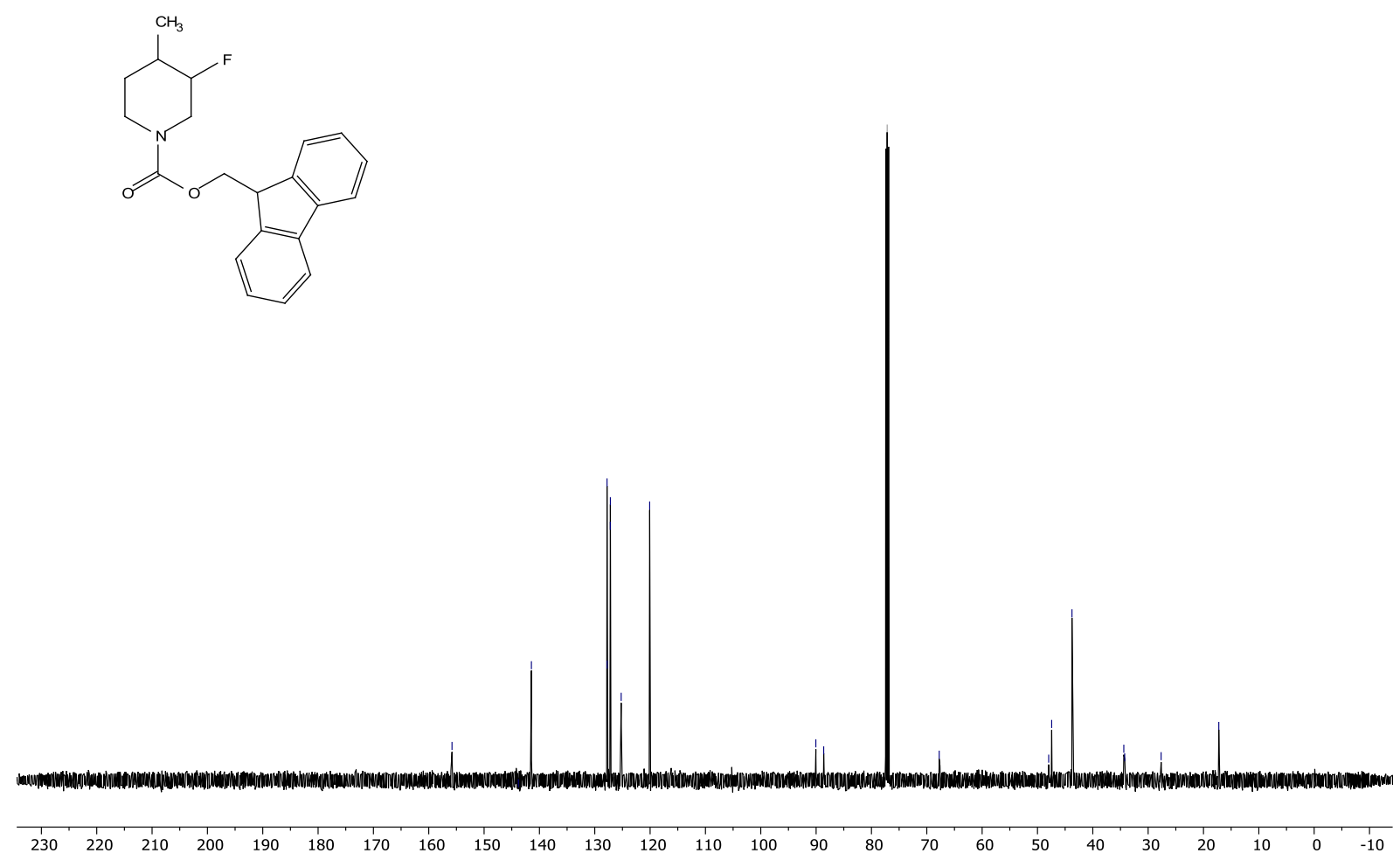

19F NMR (Chloroforme)

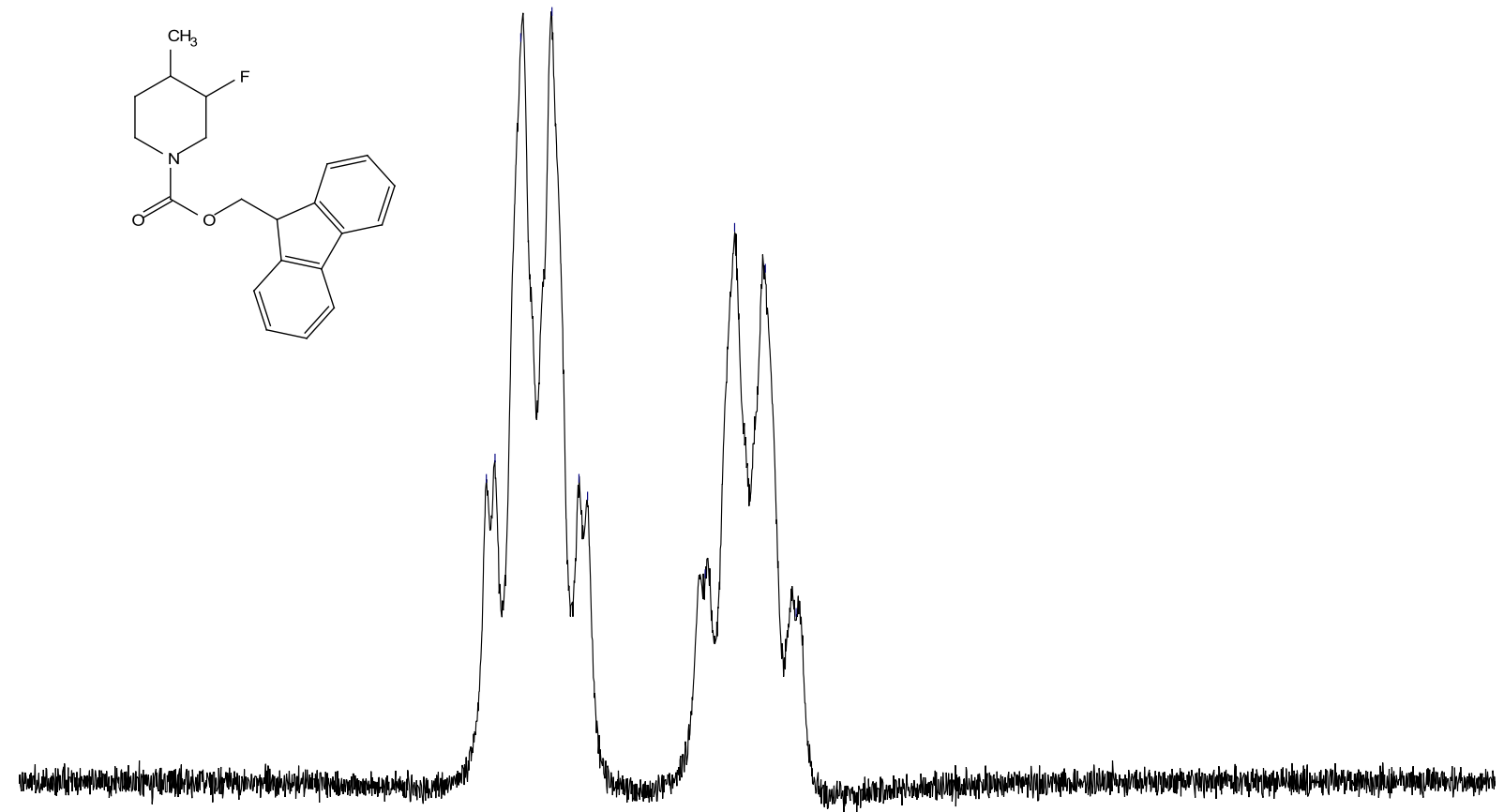

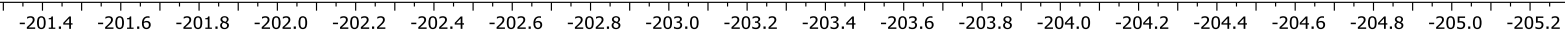


<smiles>CC1CCN(C(=O)OCC2c3ccccc3-c3ccccc32)CC1F</smiles>

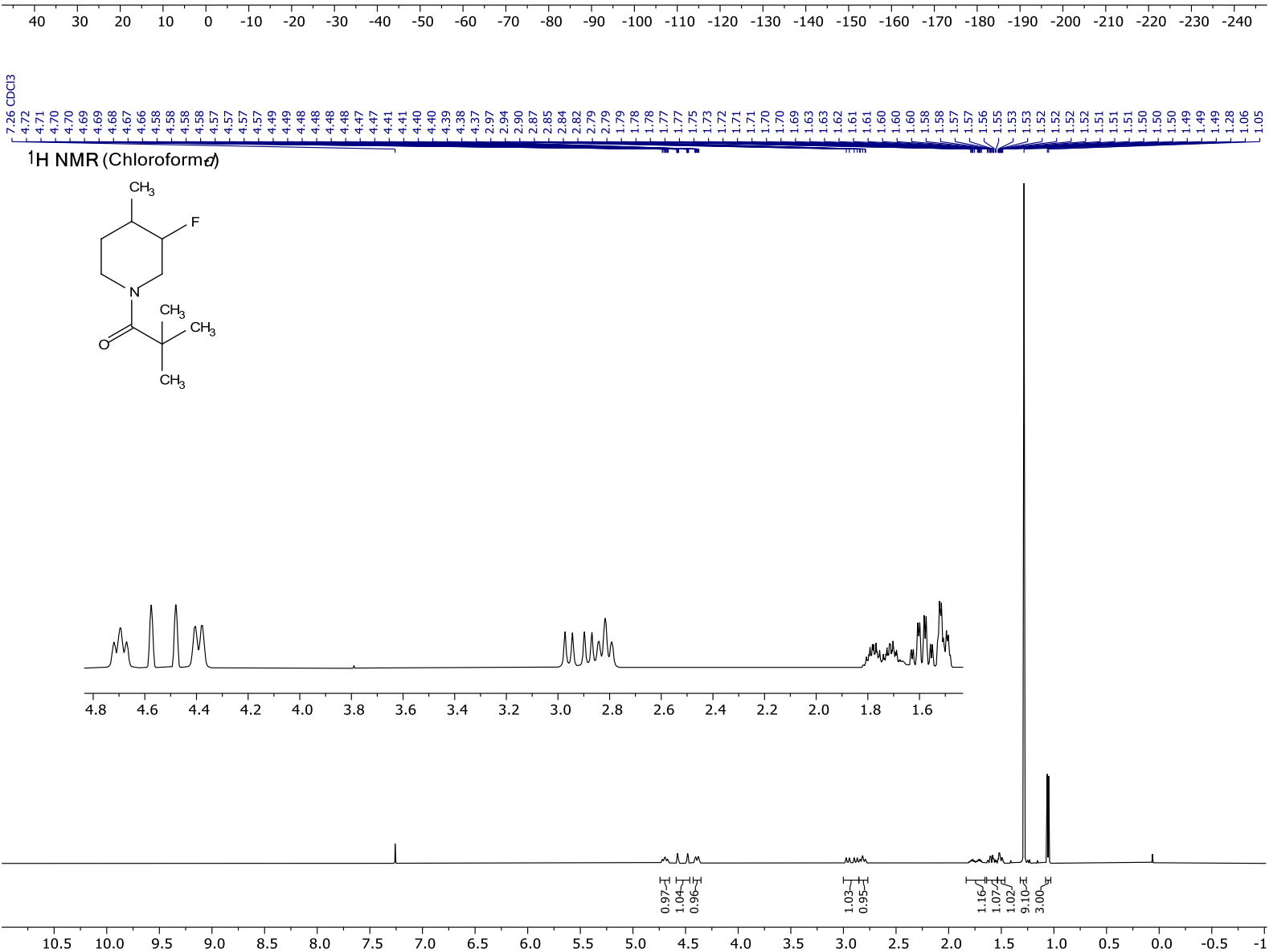


范

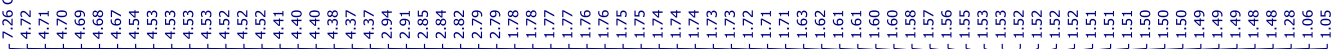
$1 \mathrm{H}\{19 \mathrm{~F}\}$ NMR (Chloroforme)<smiles>CC1CCN(C(=O)C(C)(C)C)CC1F</smiles>
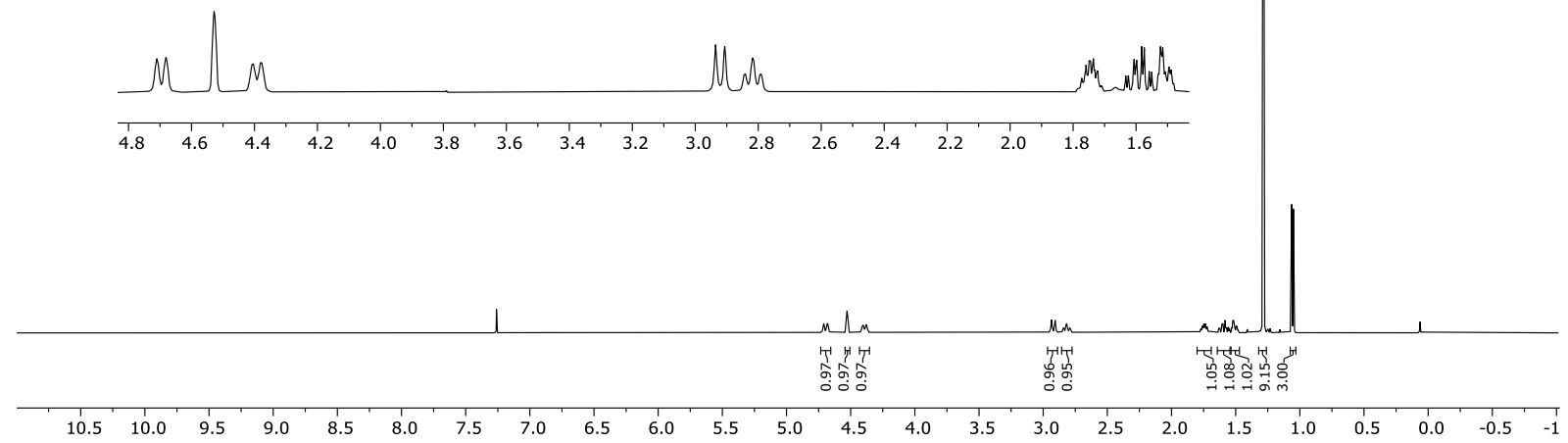

$13 \mathrm{C}\{1 \mathrm{H}\} \mathrm{NMR}($ Chloroforme) $\stackrel{\text { I }}{\stackrel{M}{1}}$

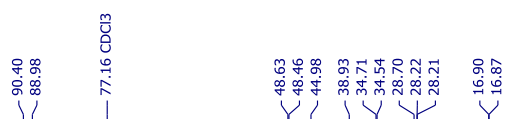<smiles>CC1CCN(C(=O)C(C)(C)C)CC1F</smiles>

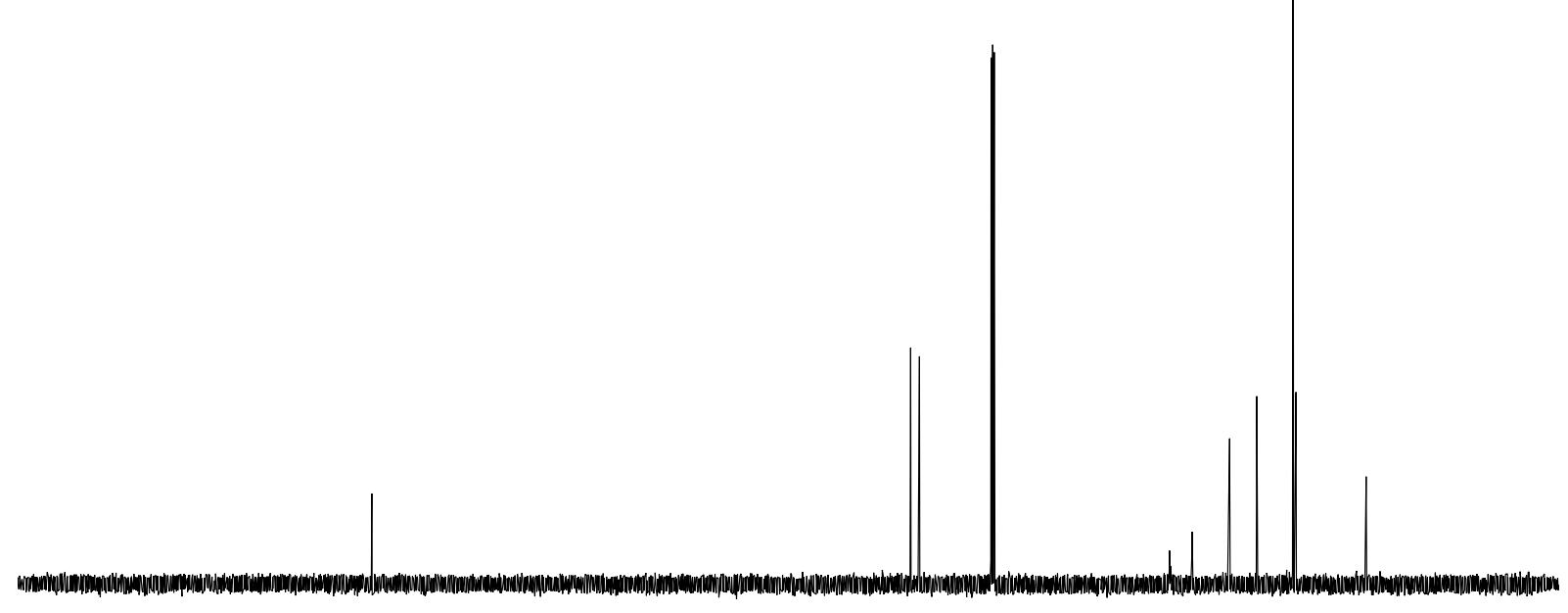
\begin{tabular}{llllllllllllllllllllllllllllllll}
\hline & 130 & 220 & 210 & 200 & 190 & 180 & 170 & 160 & 150 & 140 & 130 & 120 & 110 & 100 & 90 & 80 & 70 & 60 & 50 & 40 & 30 & 20 & 10 & 0 & -10
\end{tabular} 
$13 \mathrm{C}\left\{{ }^{1} \mathrm{H}, 19 \mathrm{~F}\right\}$ NMR (Chloroforme)<smiles>CC1CCN(C(=O)C(C)(C)C)CC1F</smiles>

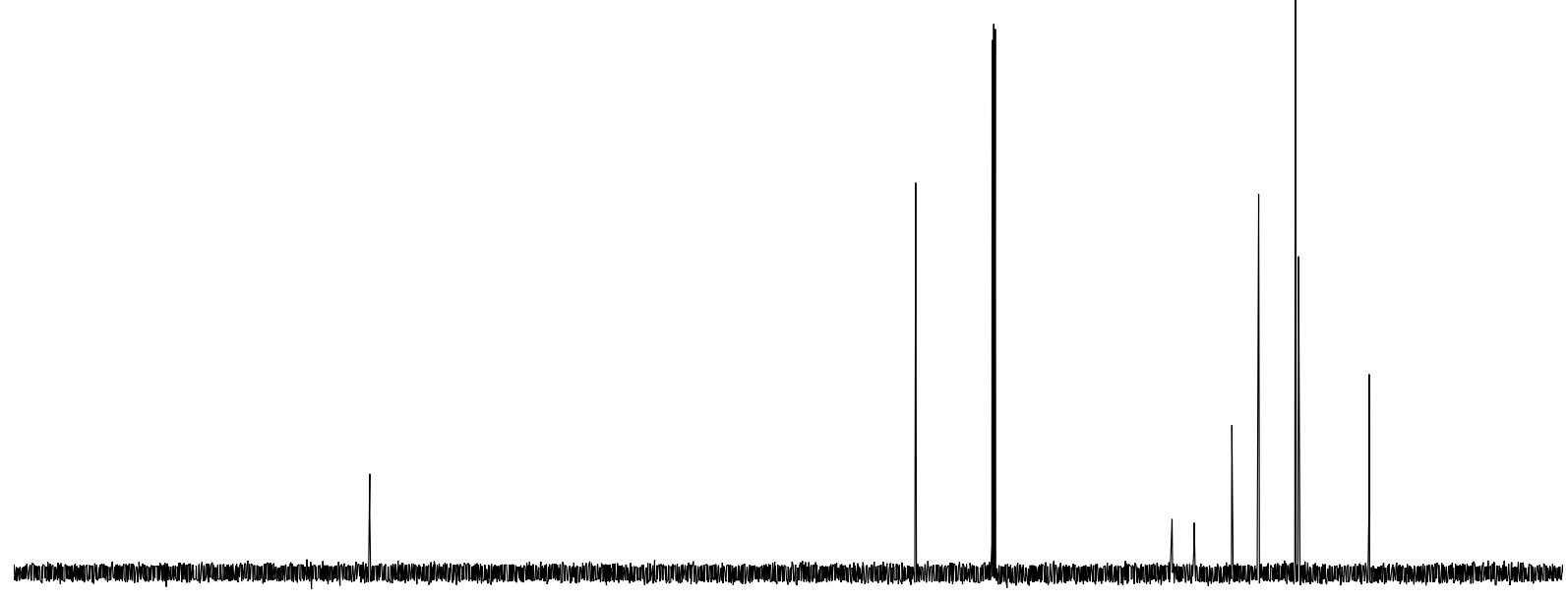
\begin{tabular}{llllllllllllllllllllllllll}
\hline 230 & 220 & 210 & 200 & 190 & 180 & 170 & 160 & 150 & 140 & 130 & 120 & 110 & 100 & 90 & 80 & 70 & 60 & 50 & 40 & 30 & 20 & 10 & 0 & -10
\end{tabular}

19F NMR (Chloroformed)

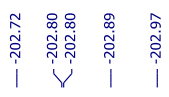<smiles>CC1CCN(C(=O)C(C)(C)C)CC1F</smiles>

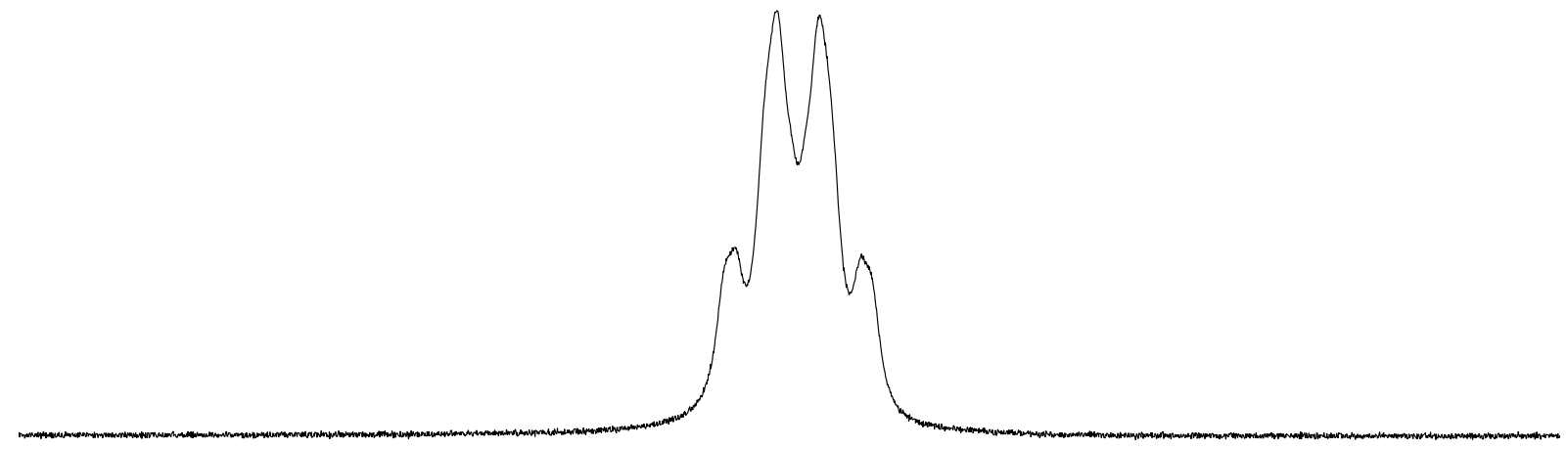


${ }^{19} \mathrm{~F}\{1 \mathrm{H}\}$ NMR (Chloroforme)<smiles>CC1CCN(C(=O)C(C)(C)C)CC1F</smiles>

突

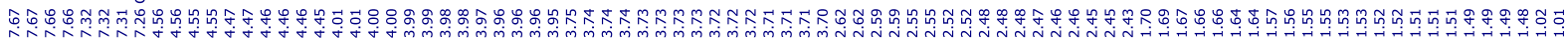
1 H NMR (Chloroforme)<smiles>Cc1ccc(S(=O)(=O)N2CCC(C)C(F)C2)cc1</smiles>

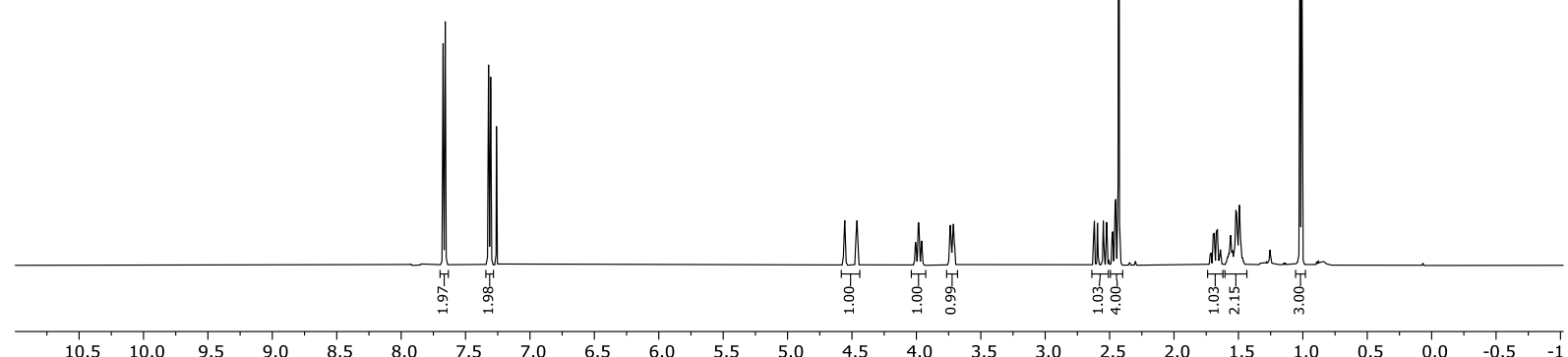


1H\{19F $\}$ NMR (Chloroformed)

位
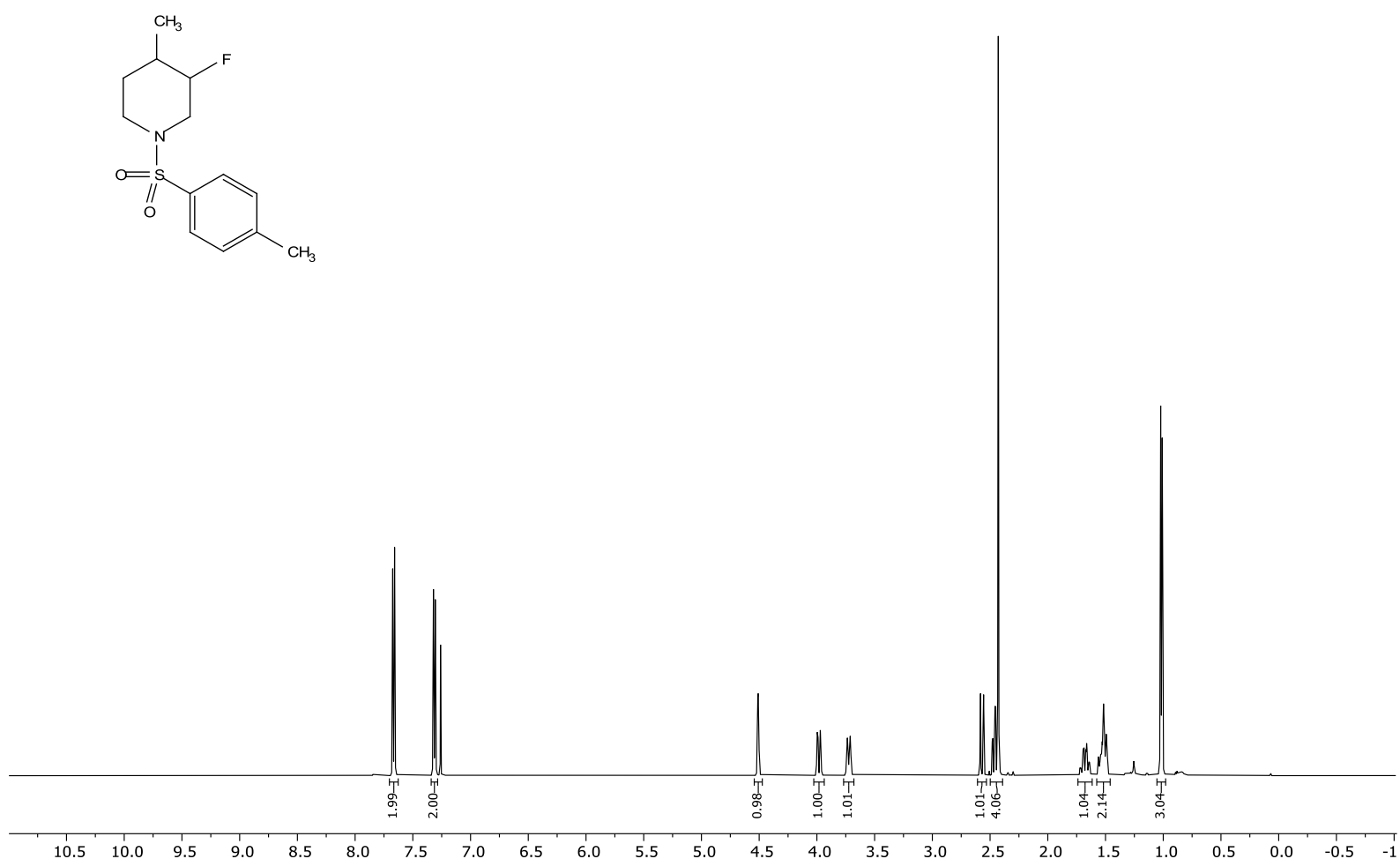

${ }^{13} \mathrm{C}\{1 \mathrm{H}\}$ NMR (Chloroformed)

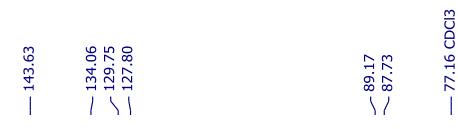

每
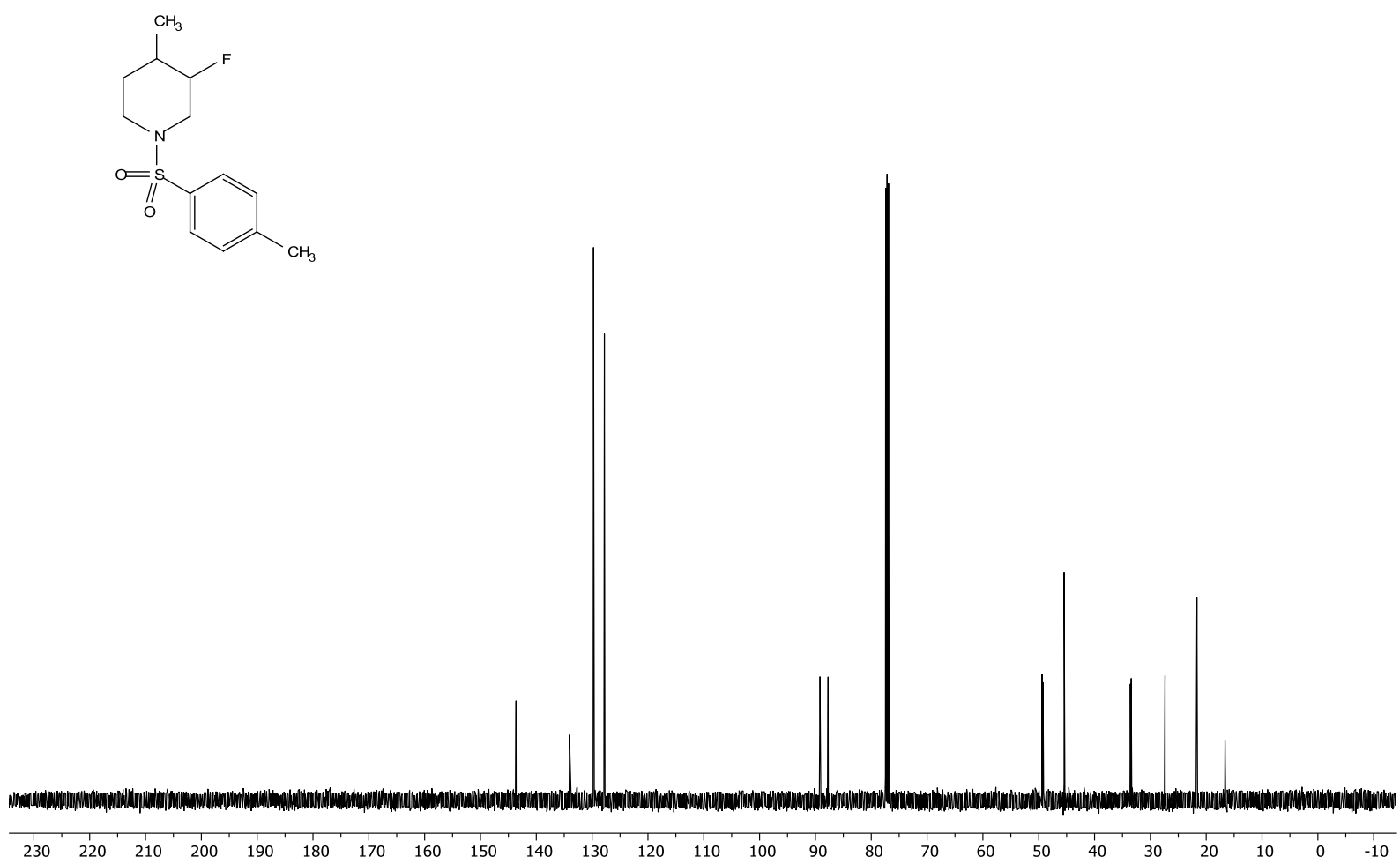

S124 


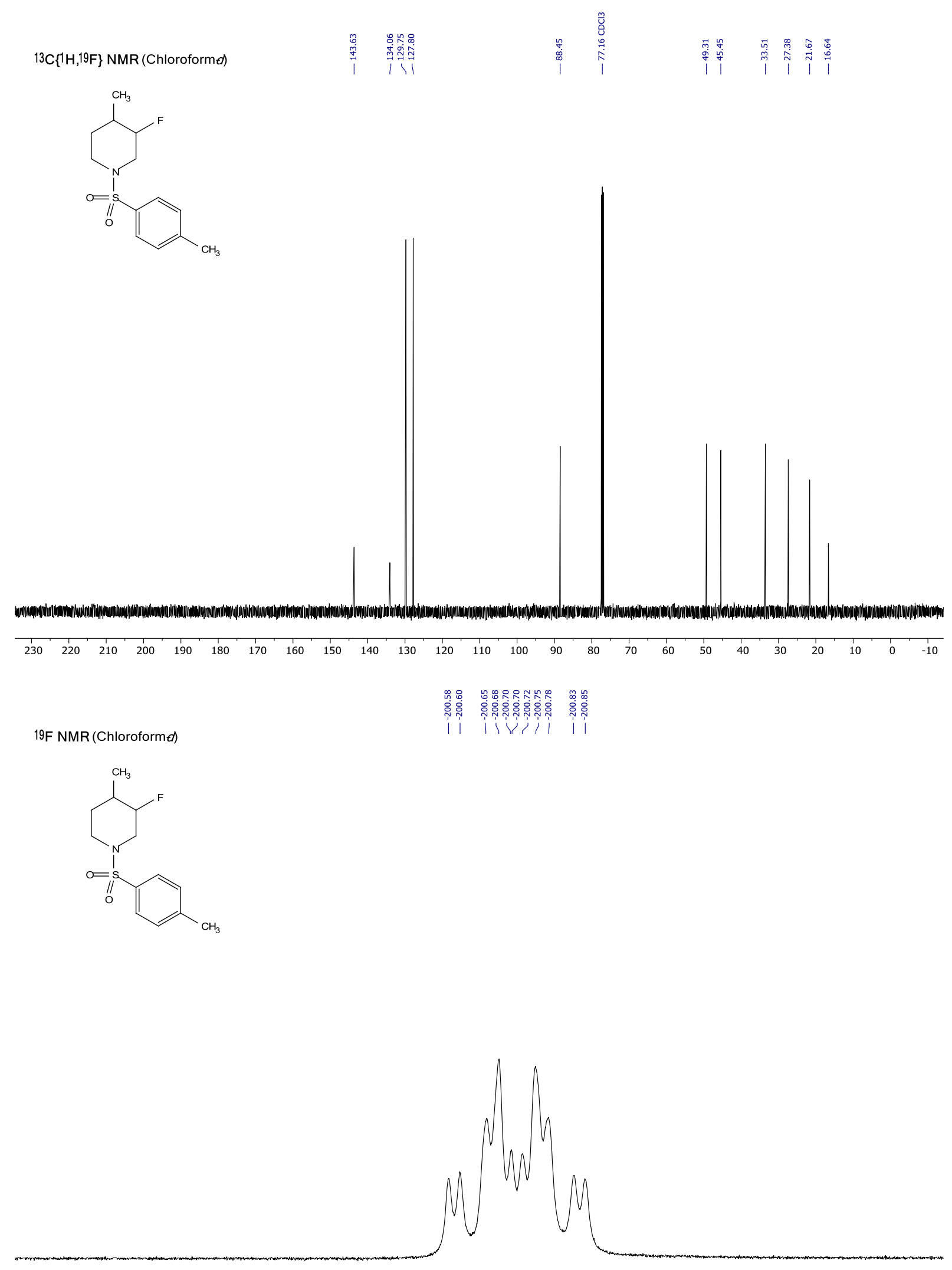

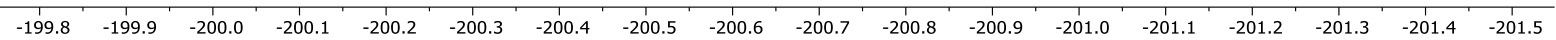


${ }^{19} \mathrm{~F}\{1 \mathrm{H}\}$ NMR (Chloroformed)

in
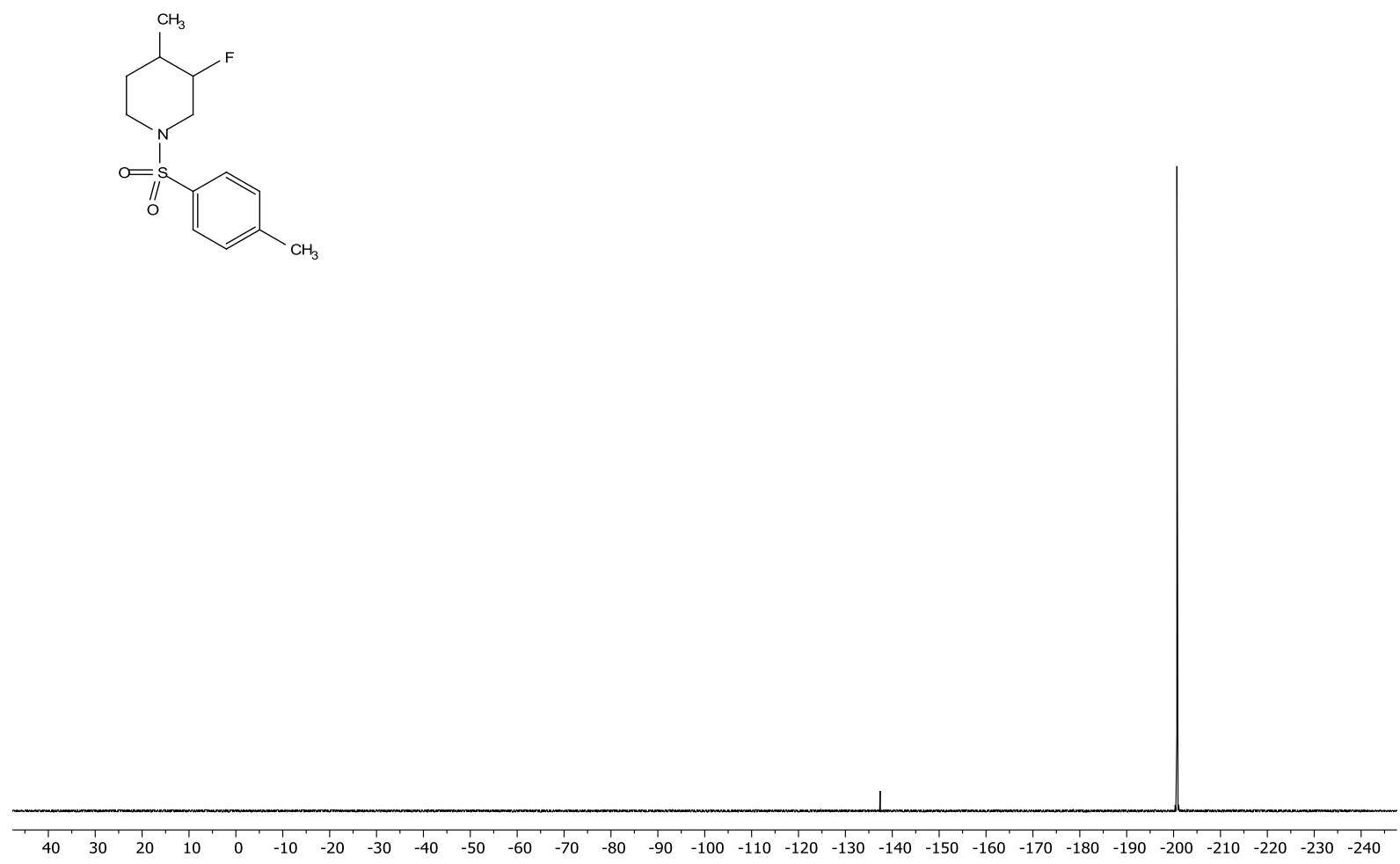

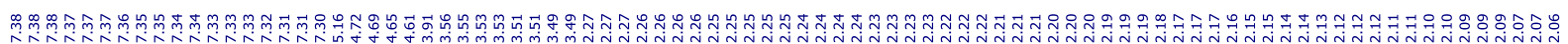

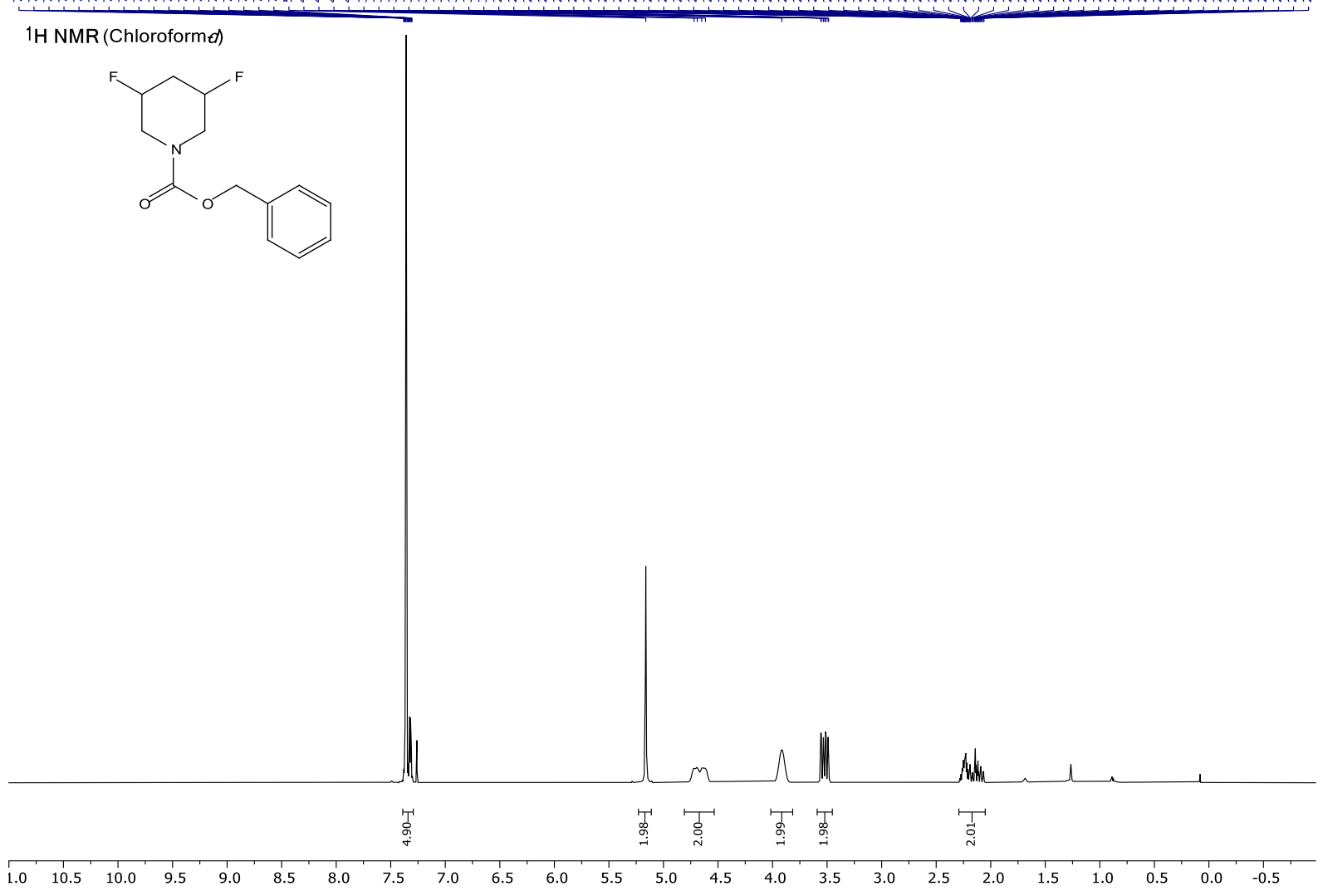

S126 
$1 \mathrm{H}\{19 \mathrm{~F}\}$ NMR (Chloroform $d)$

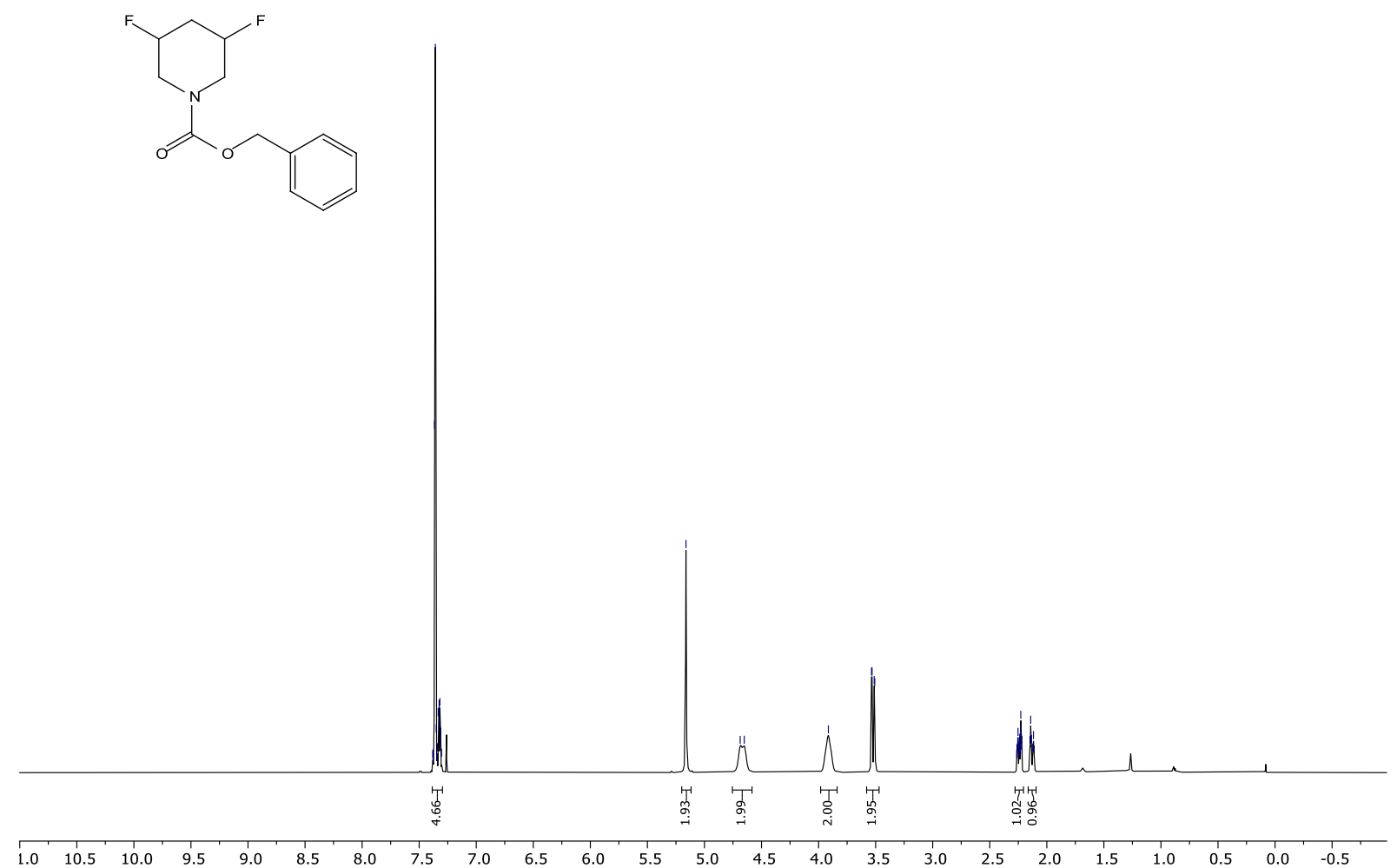

13C\{1H\} NMR (Chloroforme)

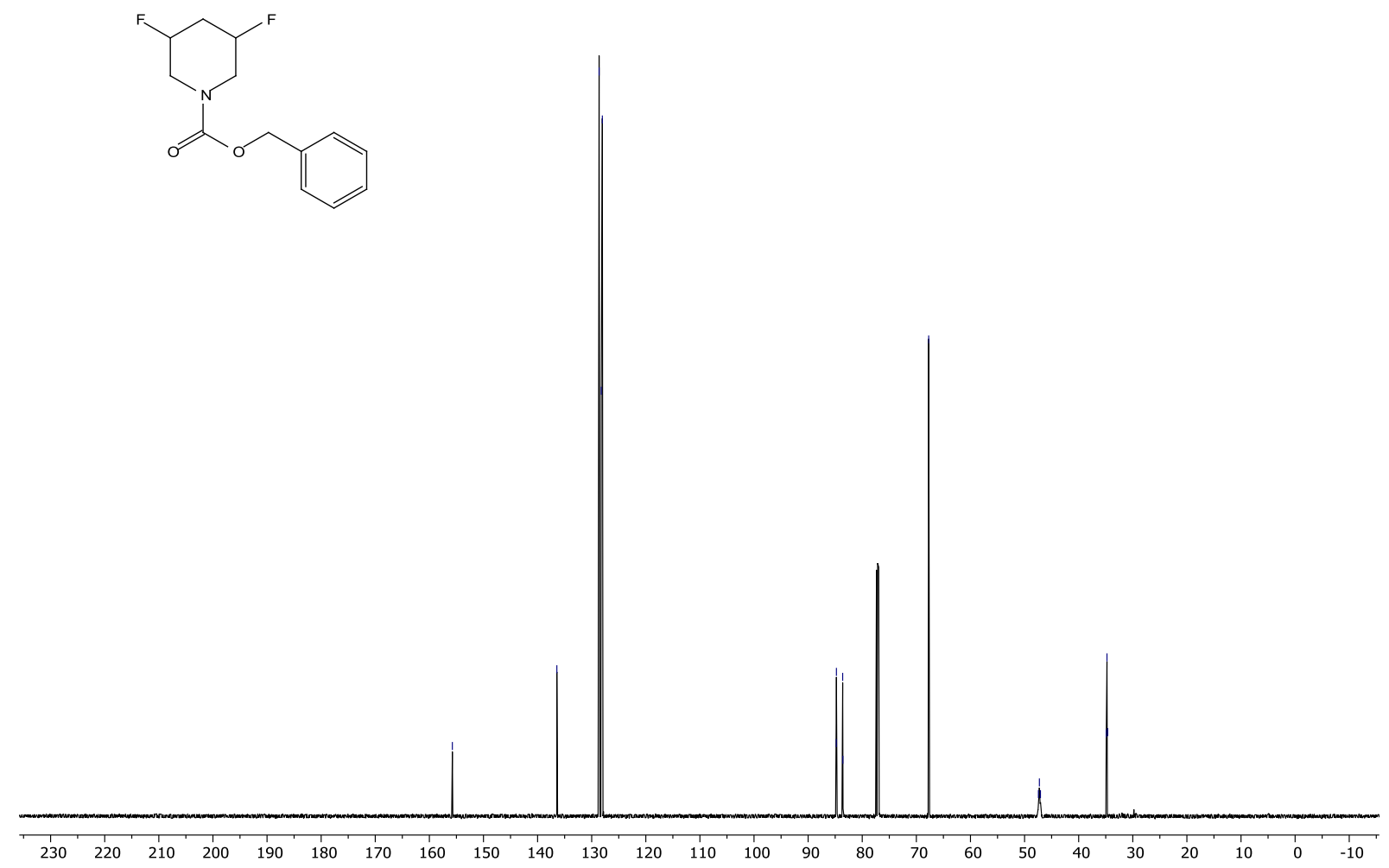


${ }^{13} \mathrm{C}\{1 \mathrm{H}, 19 \mathrm{~F}\}$ NMR (Chloroformed)

لv

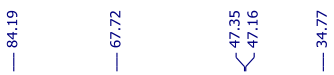<smiles>O=C(OCc1ccccc1)N1CC(F)CC(F)C1</smiles>

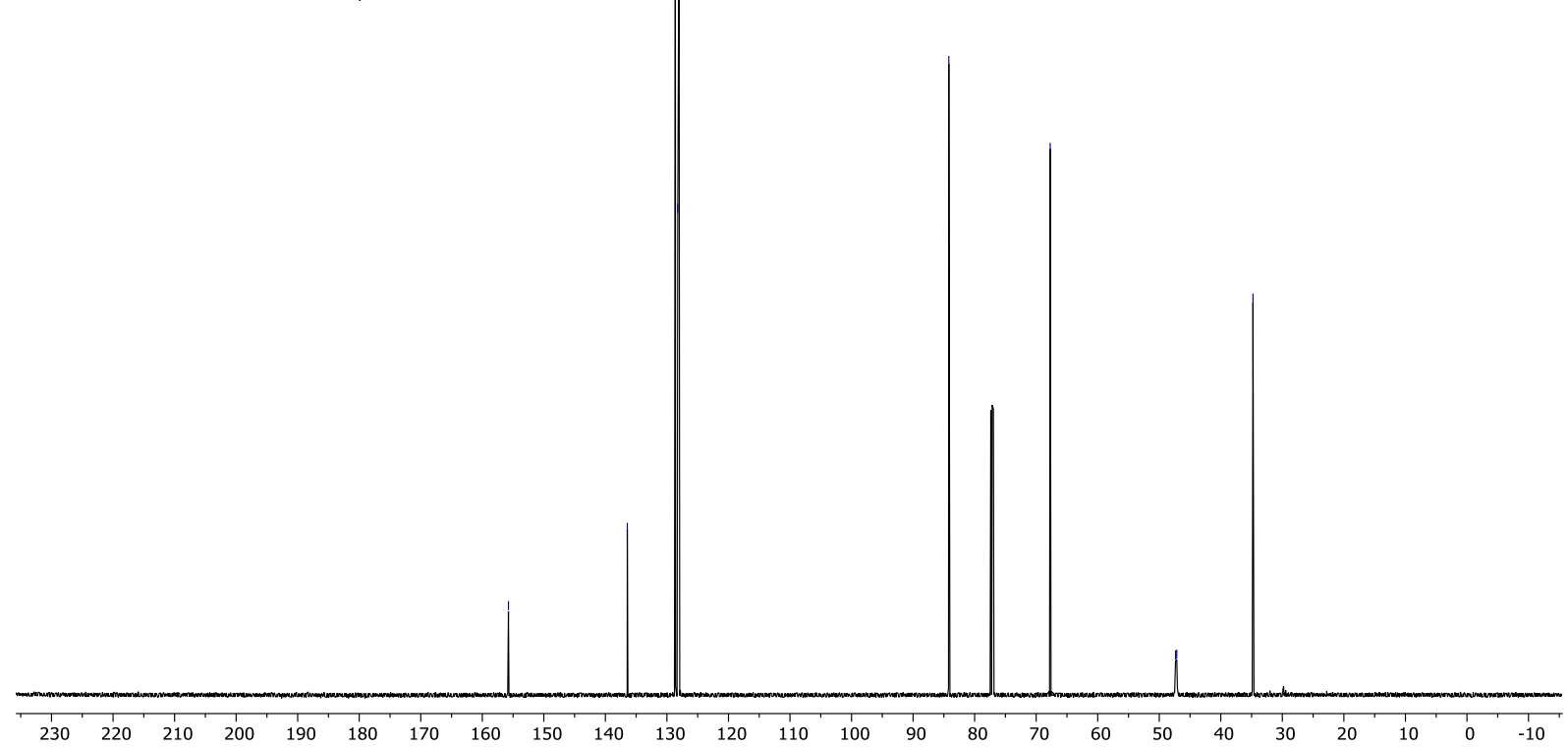

19F NMR (Chloroformed)

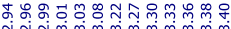

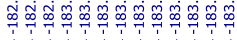<smiles>O=C(OCc1ccccc1)N1CC(F)CC(F)C1</smiles>

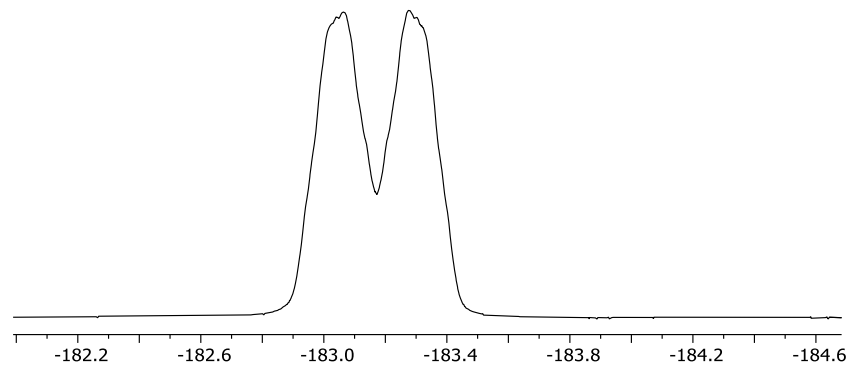

$\begin{array}{lllllllllllllllllllllllllllllllllllll}40 & 30 & 20 & 10 & 0 & -10 & -20 & -30 & -40 & -50 & -60 & -70 & -80 & -90 & -100 & -110 & -120 & -130 & -140 & -150 & -160 & -170 & -180 & -190 & -200 & -210 & -220 & -230 & -240\end{array}$ 
${ }^{19} \mathrm{~F}\{1 \mathrm{H}\}$ NMR (Chloroforme)<smiles>O=C(OCc1ccccc1)N1CC(F)CC(F)C1</smiles>

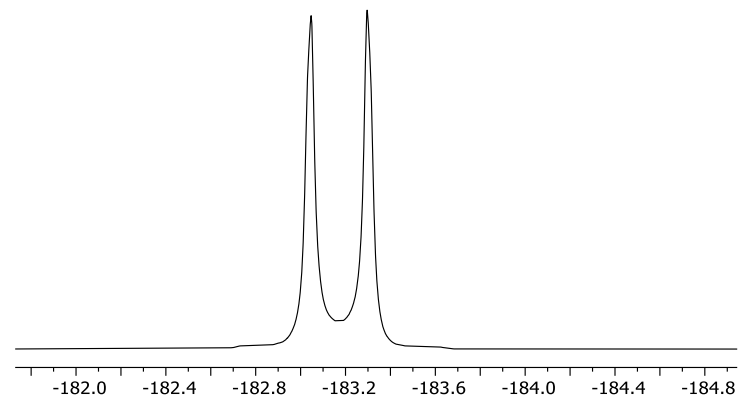

$\begin{array}{lllllllllllllllllllllllllllllllllll}40 & 30 & 20 & 10 & 0 & -10 & -20 & -30 & -40 & -50 & -60 & -70 & -80 & -90 & -100 & -110 & -120 & -130 & -140 & -150 & -160 & -170 & -180 & -190 & -200 & -210 & -220 & -230 & -240 & 1\end{array}$

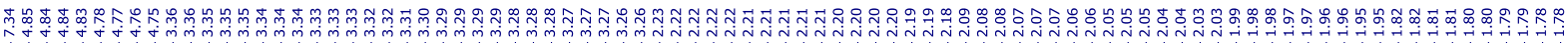
1H NMR (Chloroformed)<smiles>O=C1NCCCC1F</smiles>

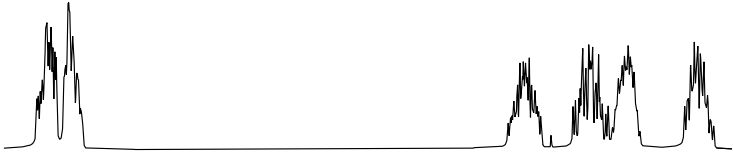

$\begin{array}{lllllllllllllllllllll}3.4 & 3.3 & 3.2 & 3.1 & 3.0 & 2.9 & 2.8 & 2.7 & 2.6 & 2.5 & 2.4 & 2.3 & 2.2 & 2.1 & 2.0 & 1.9 & 1.8\end{array}$

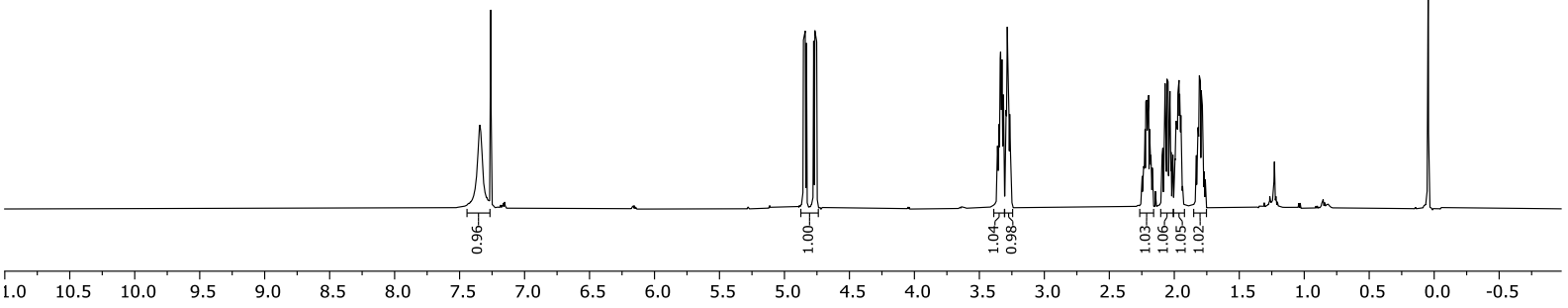




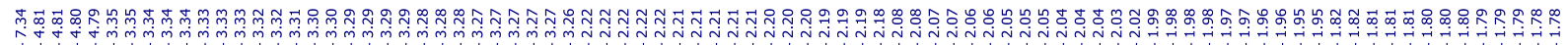
${ }^{1} \mathrm{H}\left\{{ }^{19} \mathrm{~F}\right\} \operatorname{NMR}$ (Chloroforme)<smiles>O=C1NCCCC1F</smiles>

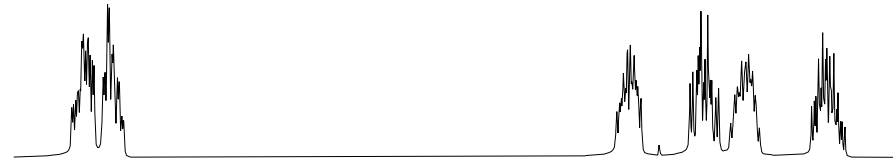

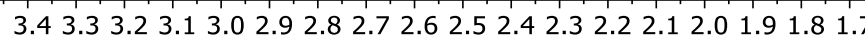

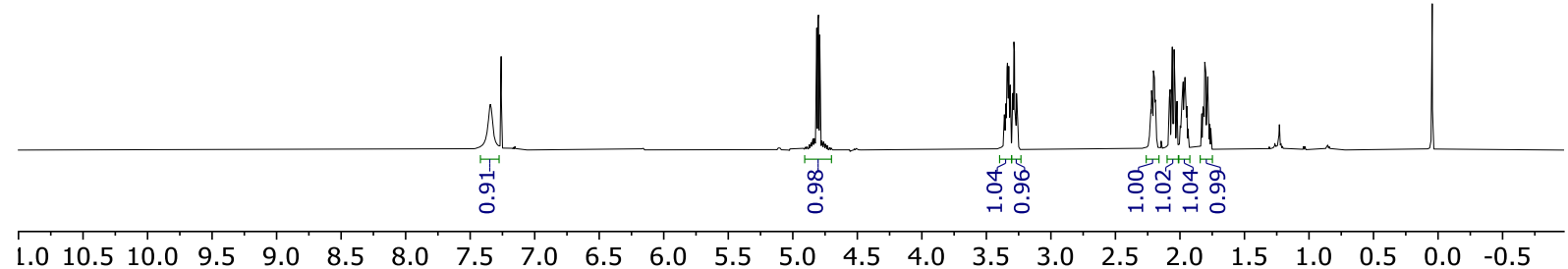<smiles>O=C1NCCCC1F</smiles>

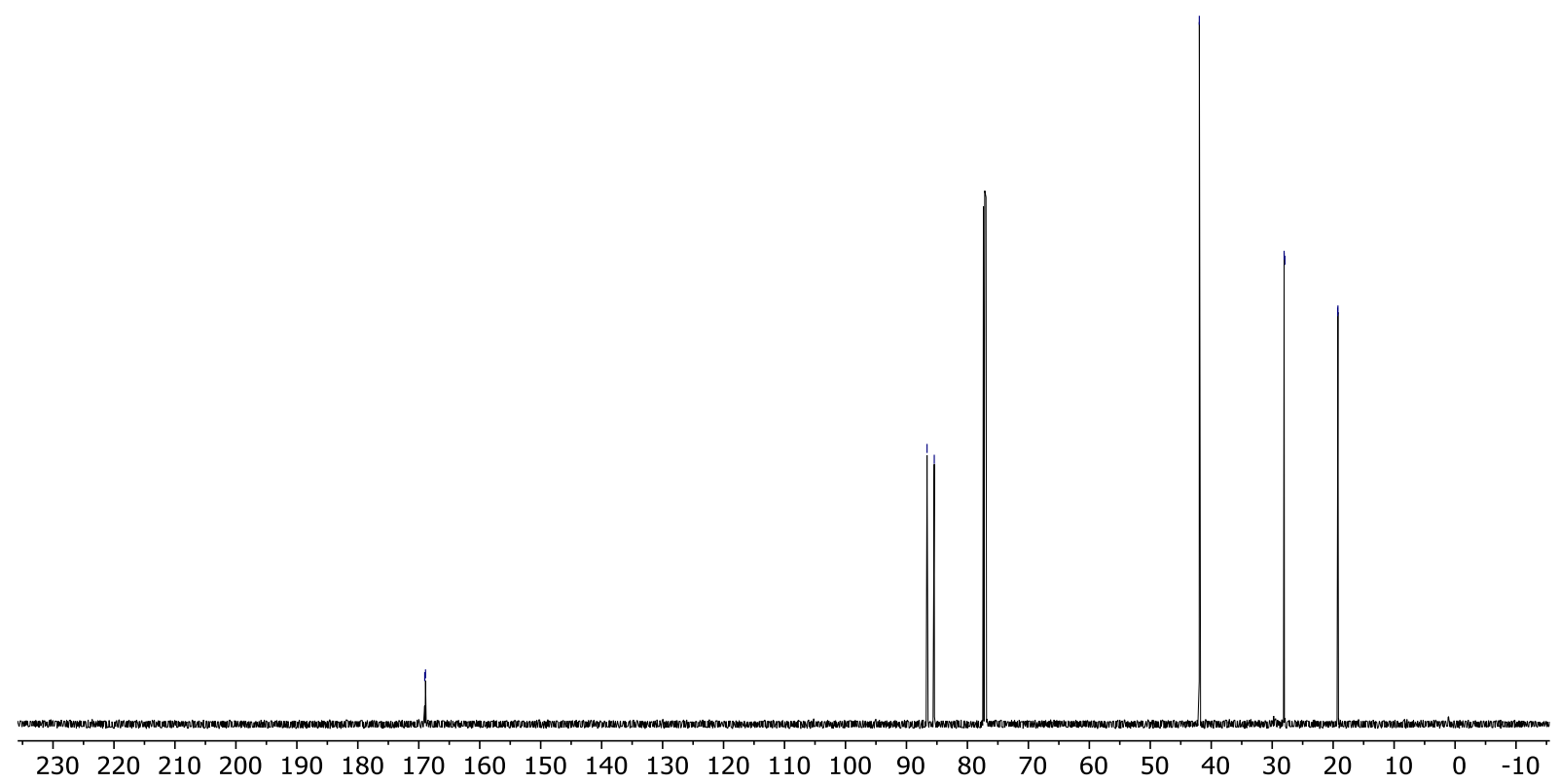


<smiles>O=C1NCCCC1F</smiles>

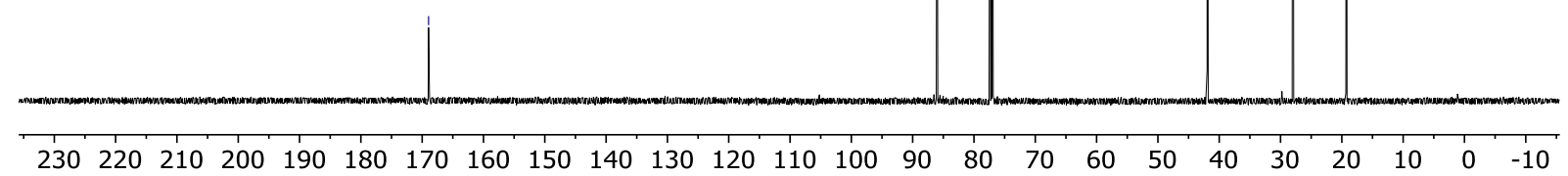

19F NMR (Chloroforme)

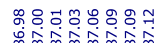

$\stackrel{\infty}{1}$

$\overbrace{H}^{C}$

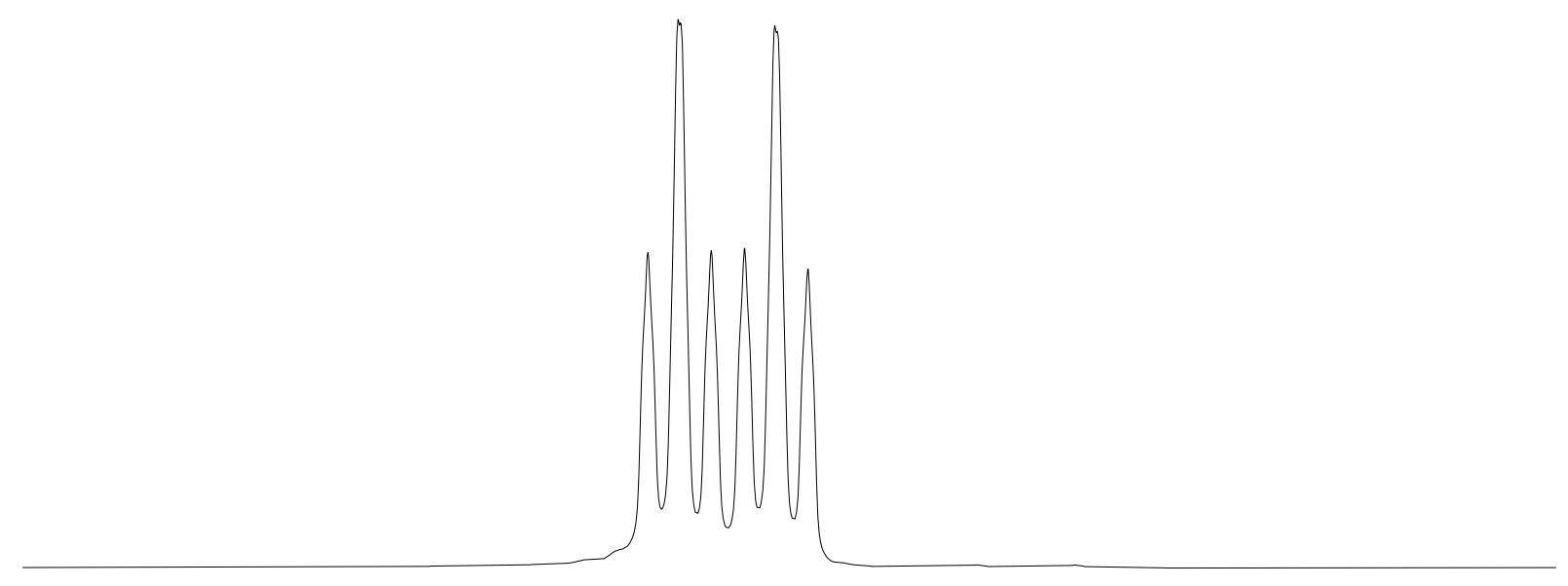

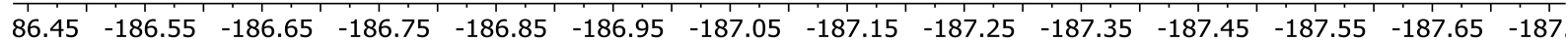


<smiles>O=C1NCCCC1F</smiles>

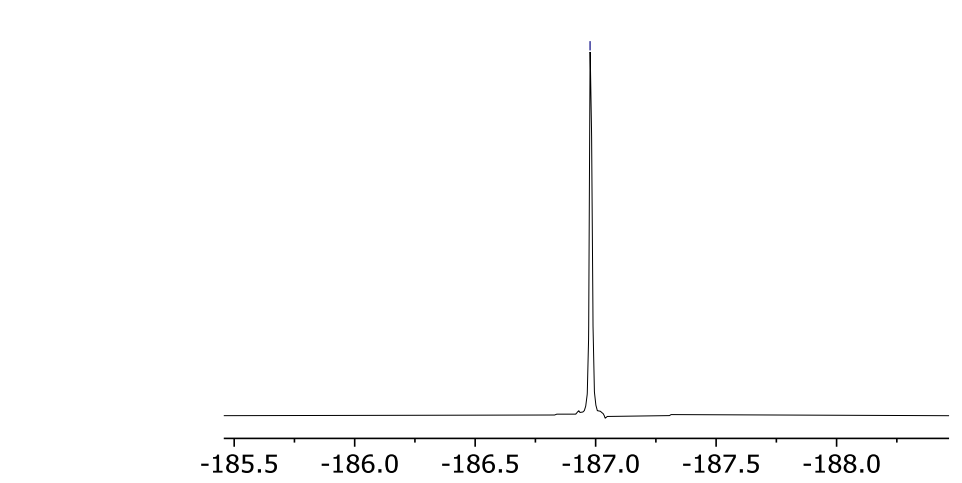

\begin{tabular}{lllllllllllllll}
\hline 30 & 10 & -10 & -30 & -50 & -70 & -90 & -110 & -130 & -150 & -170 & -190 & -210 & -230
\end{tabular}

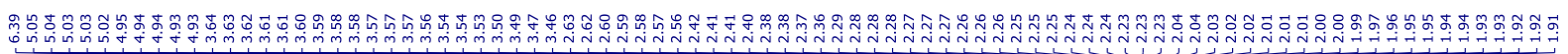
1H NMR (Chloroforme)<smiles>O=C1CCC(F)CN1</smiles>

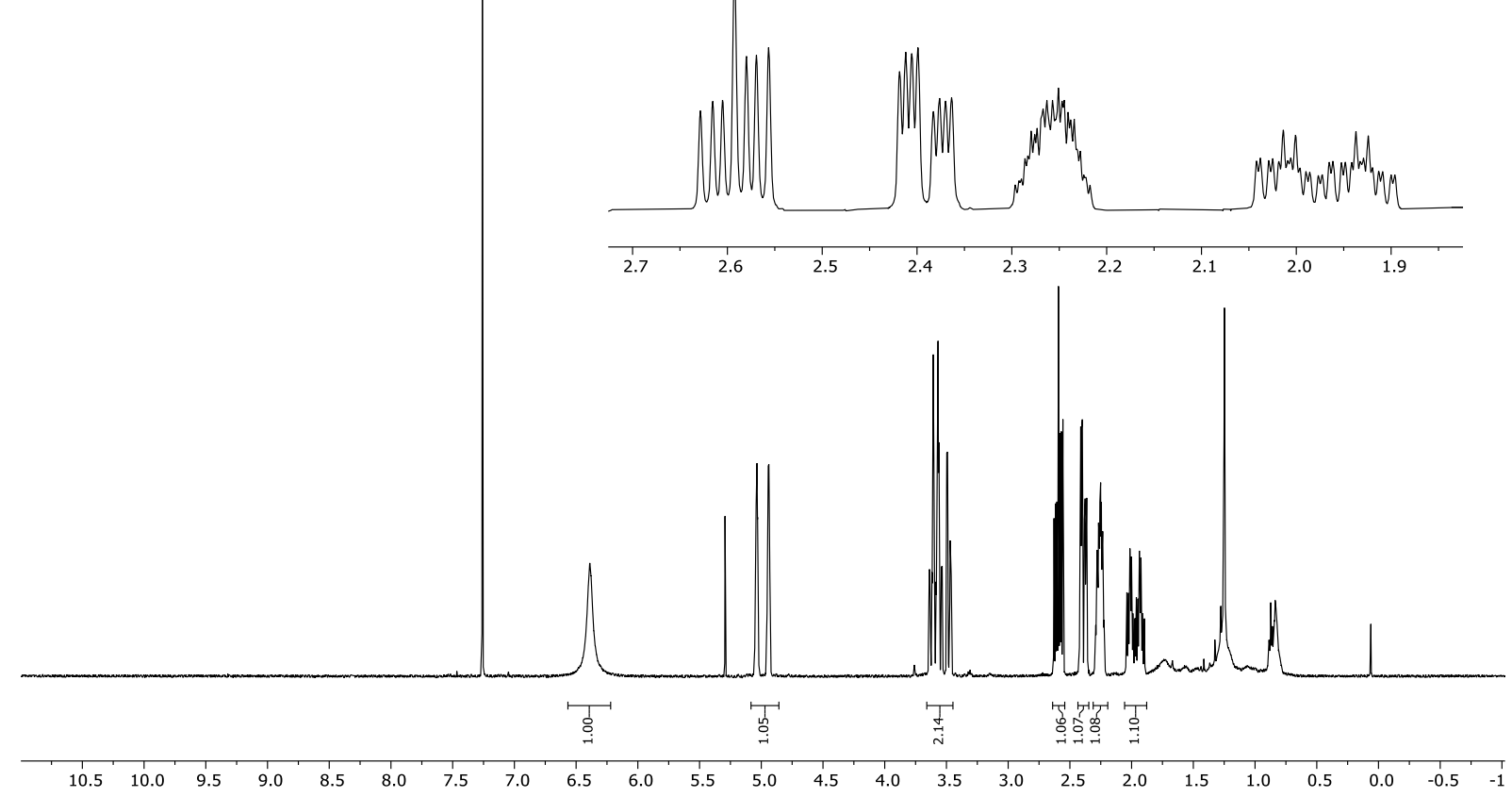


$1 \mathrm{H}\left\{{ }^{19} \mathrm{~F}\right\}$ NMR (Chloroformed)<smiles>O=C1CCC(F)CN1</smiles>

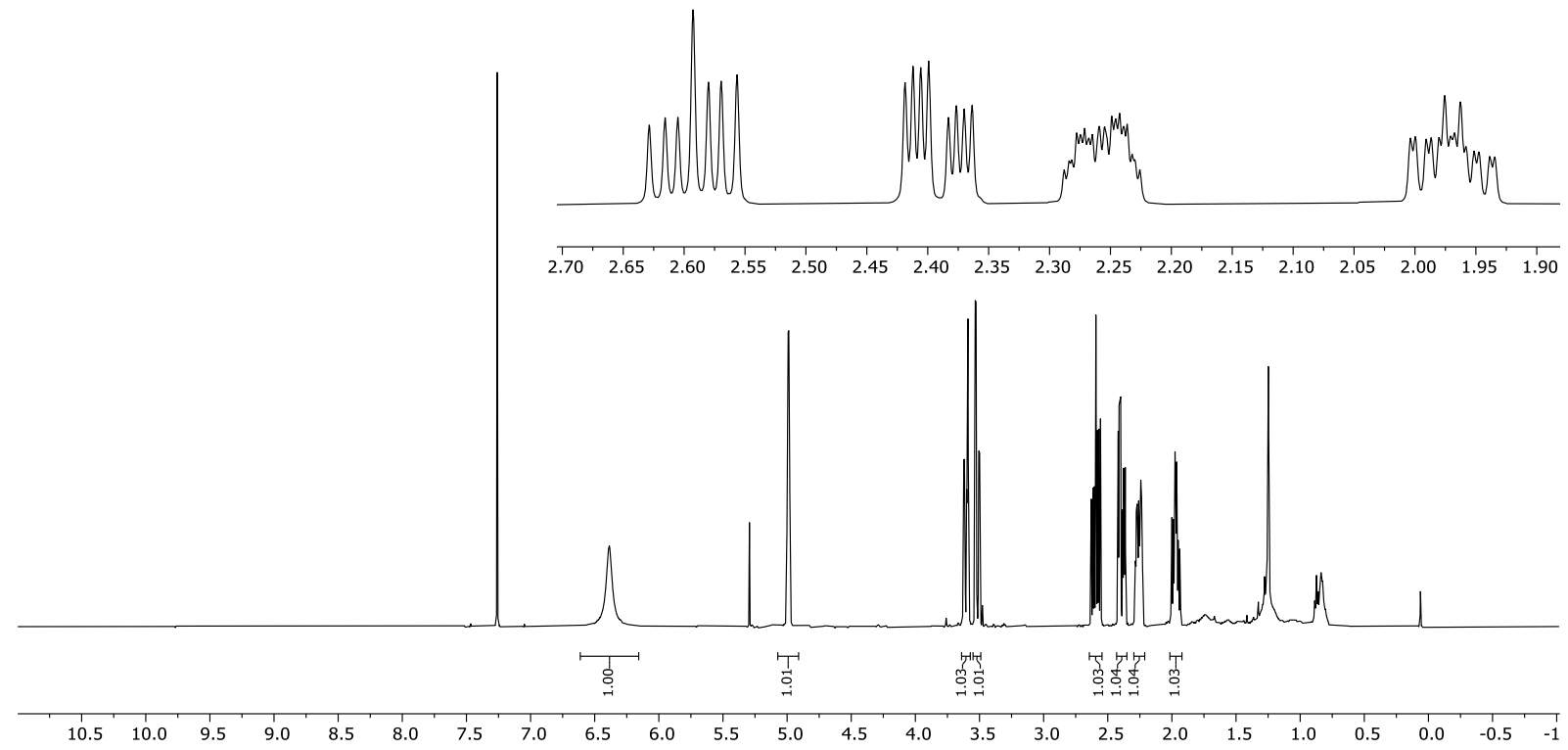

${ }^{13} \mathrm{C}\{1 \mathrm{H}\}$ NMR (Chloroforme)

$\substack{\infty \\ \infty \\ 0 \\ \infty \\ \infty \\ 1}$
1

$\underbrace{\infty}$<smiles>O=C1CCC(F)CN1</smiles>

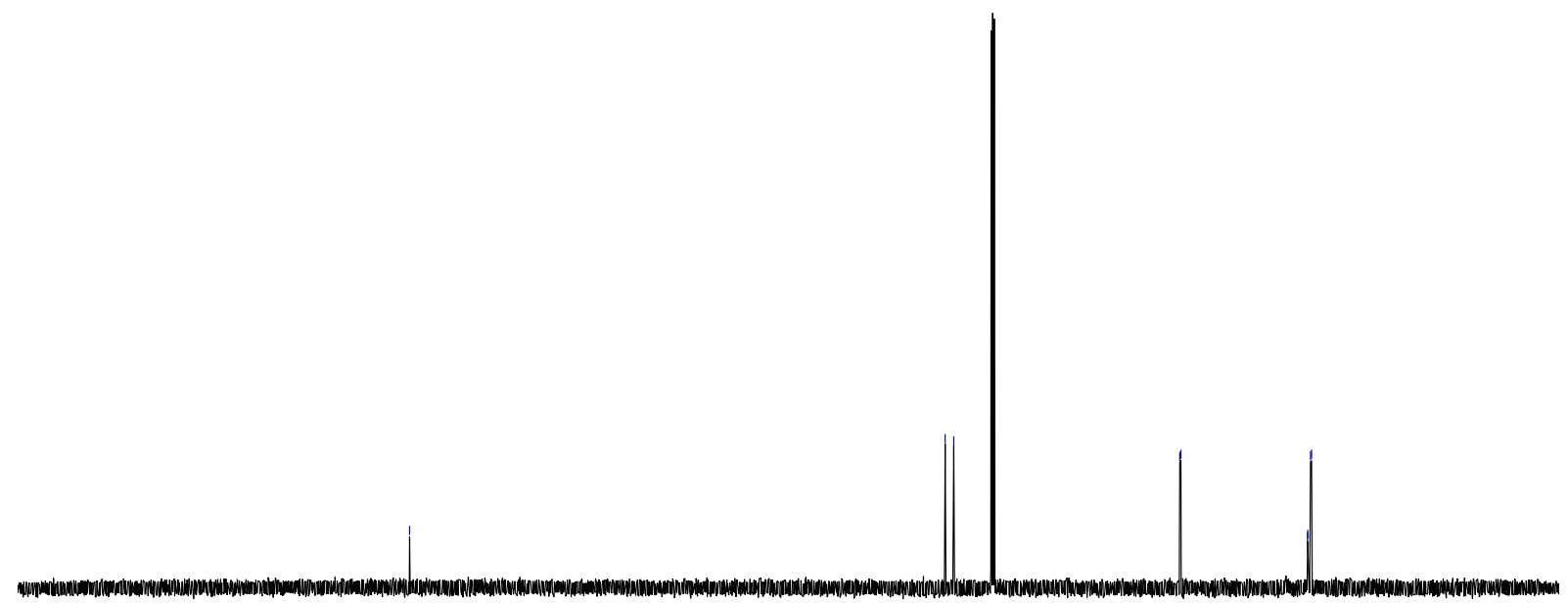
\begin{tabular}{lllllllllllllllllllllllllll}
\hline 230 & 220 & 210 & 200 & 190 & 180 & 170 & 160 & 150 & 140 & 130 & 120 & 110 & 100 & 90 & 80 & 70 & 60 & 50 & 40 & 30 & 20 & 10 & 0 & -10
\end{tabular} 


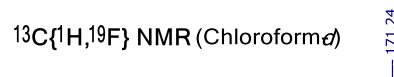

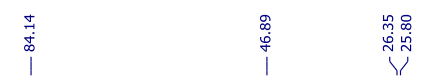<smiles>O=C1CCC(F)CN1</smiles>

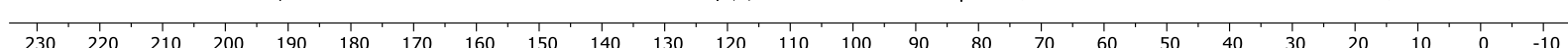

19F NMR (Chloroformed)

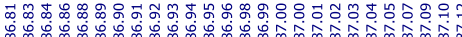

垈<smiles>O=C1CCC(F)CN1</smiles> 
${ }^{19} \mathrm{~F}\{1 \mathrm{H}\}$ NMR (Chloroformed)<smiles>O=C1CCC(F)CN1</smiles>

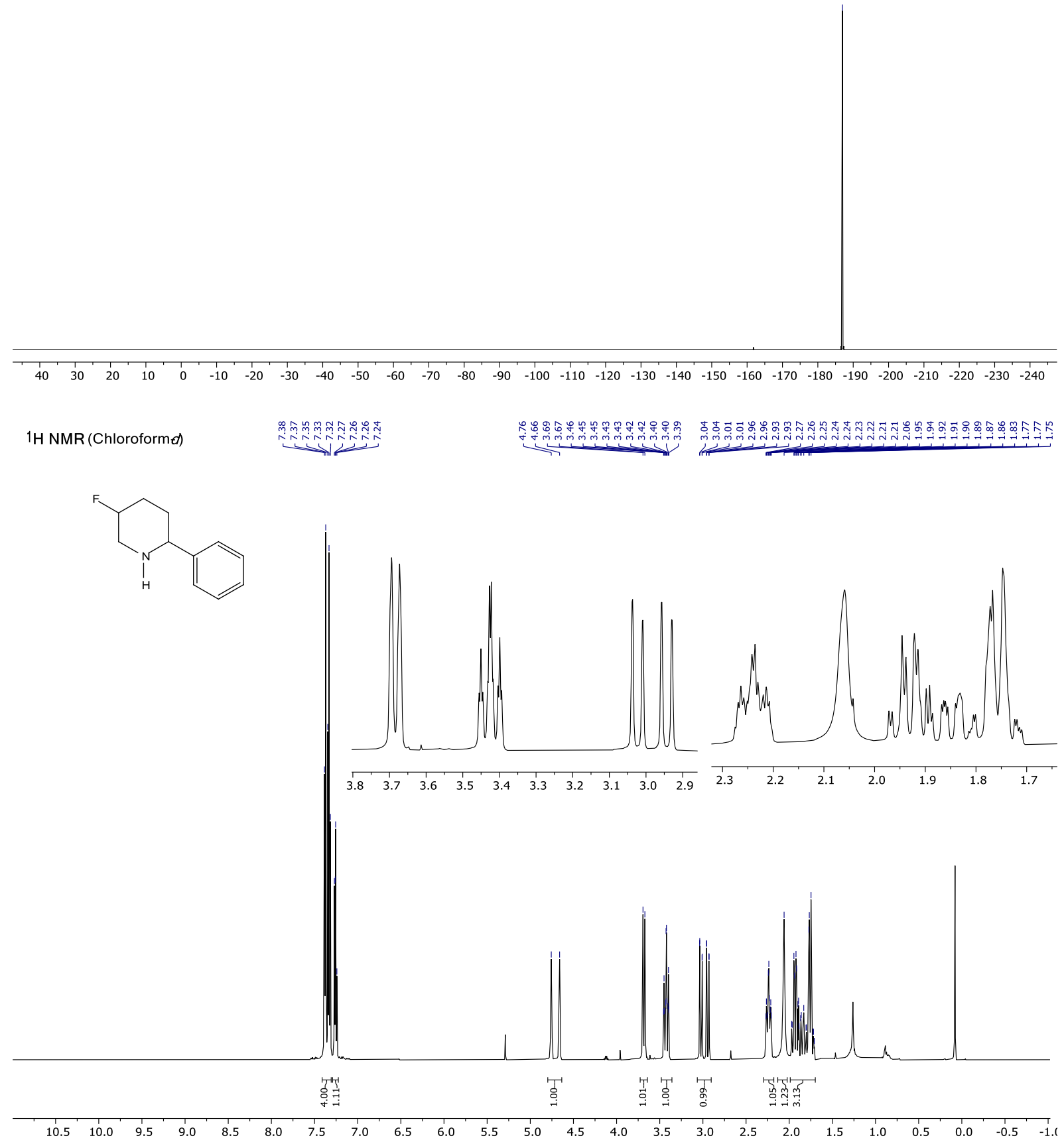


1H\{19F\} NMR (Chloroformel<smiles>FC1CCC(c2ccccc2)NC1</smiles>
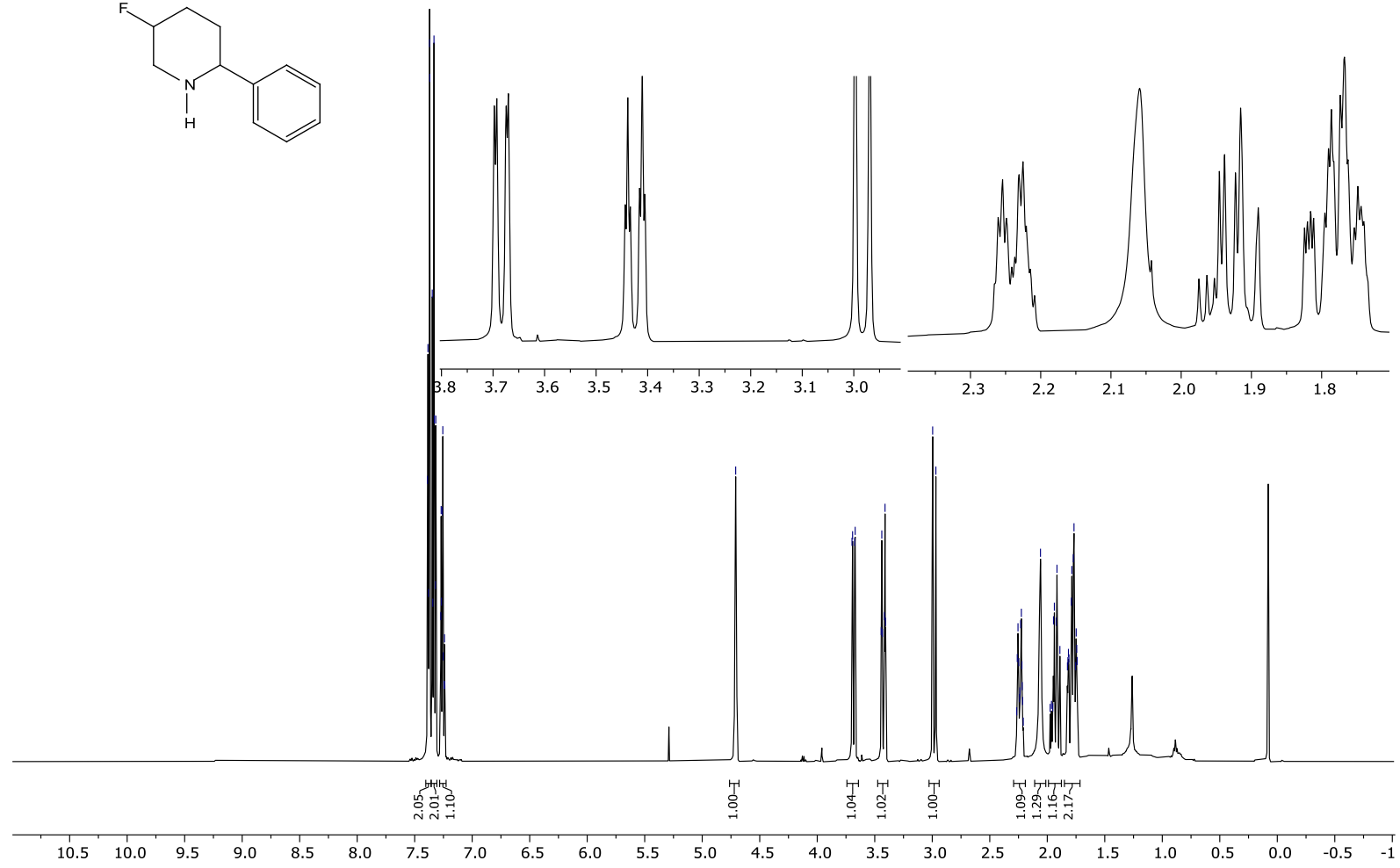

${ }^{13} \mathrm{C}\{1 \mathrm{H}\}$ NMR (Chloroforme)<smiles>FC1CCC(c2ccccc2)NC1</smiles>

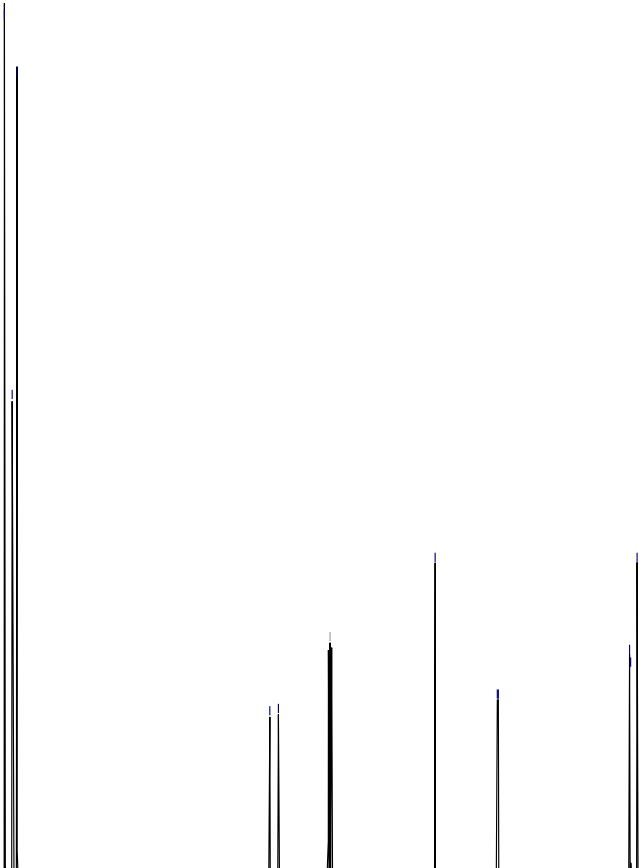




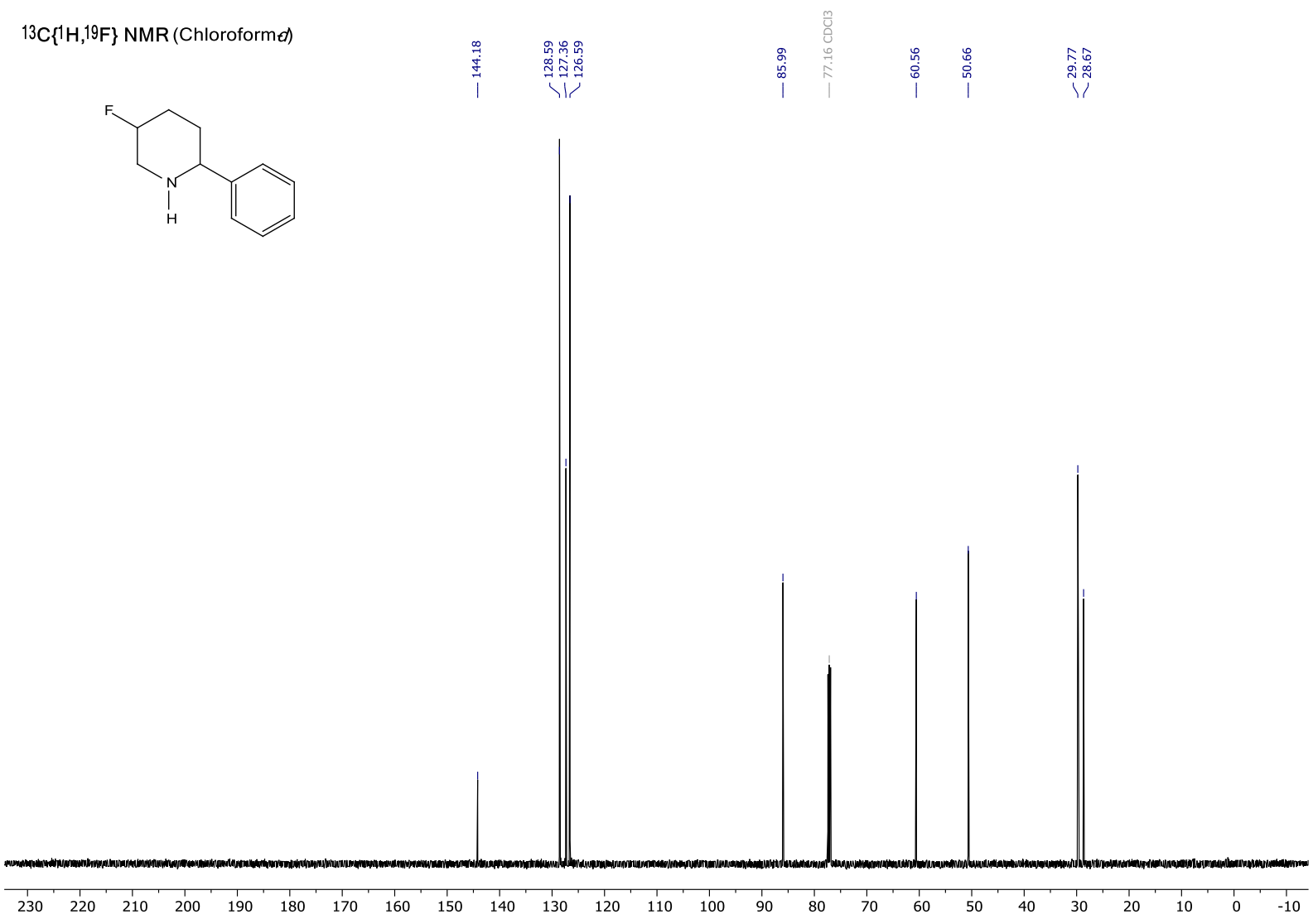

19 F NMR (Chloroforme)

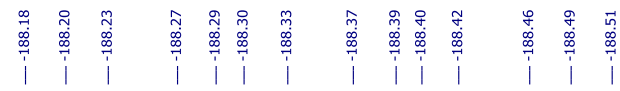<smiles>FC1CCC(c2ccccc2)NC1</smiles>

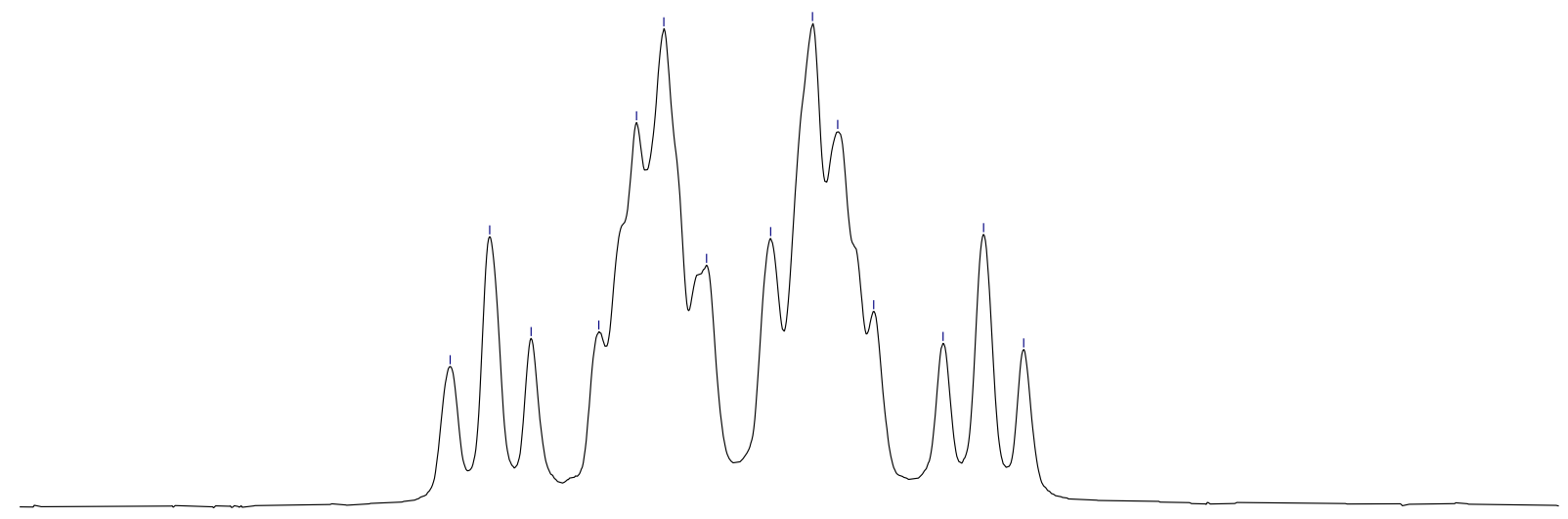


${ }^{19} \mathrm{~F}\{1 \mathrm{H}\}$ NMR (Chloroforme)

$\underset{\substack{m \\ \infty \\ \infty \\ \infty}}{\substack{1 \\ \mid}}$
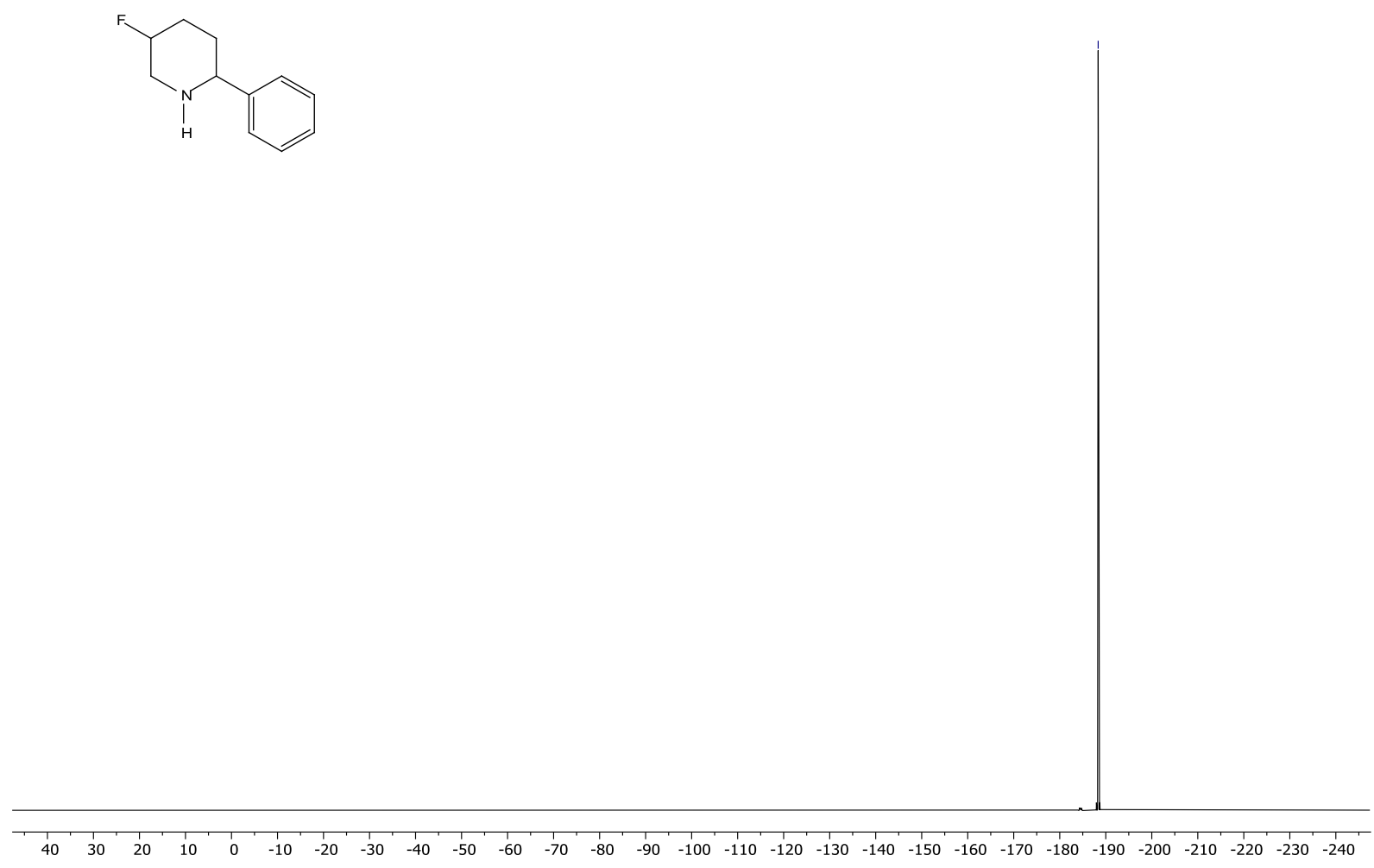

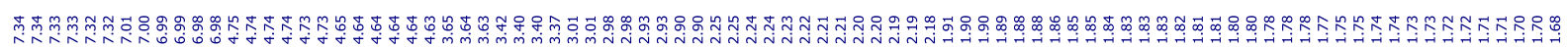

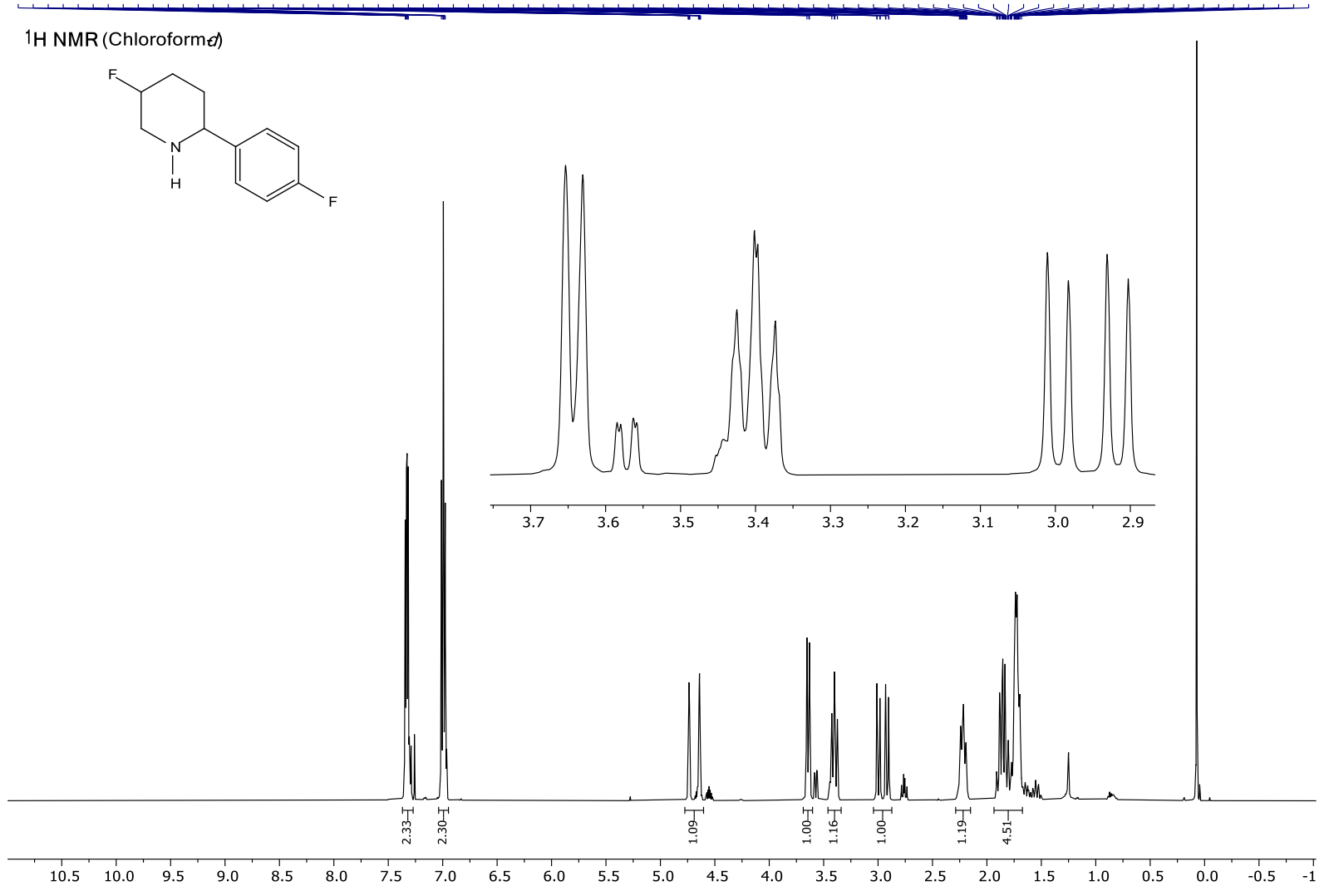

S138 
<smiles>Fc1ccc(C2CCC(F)CN2)cc1</smiles>

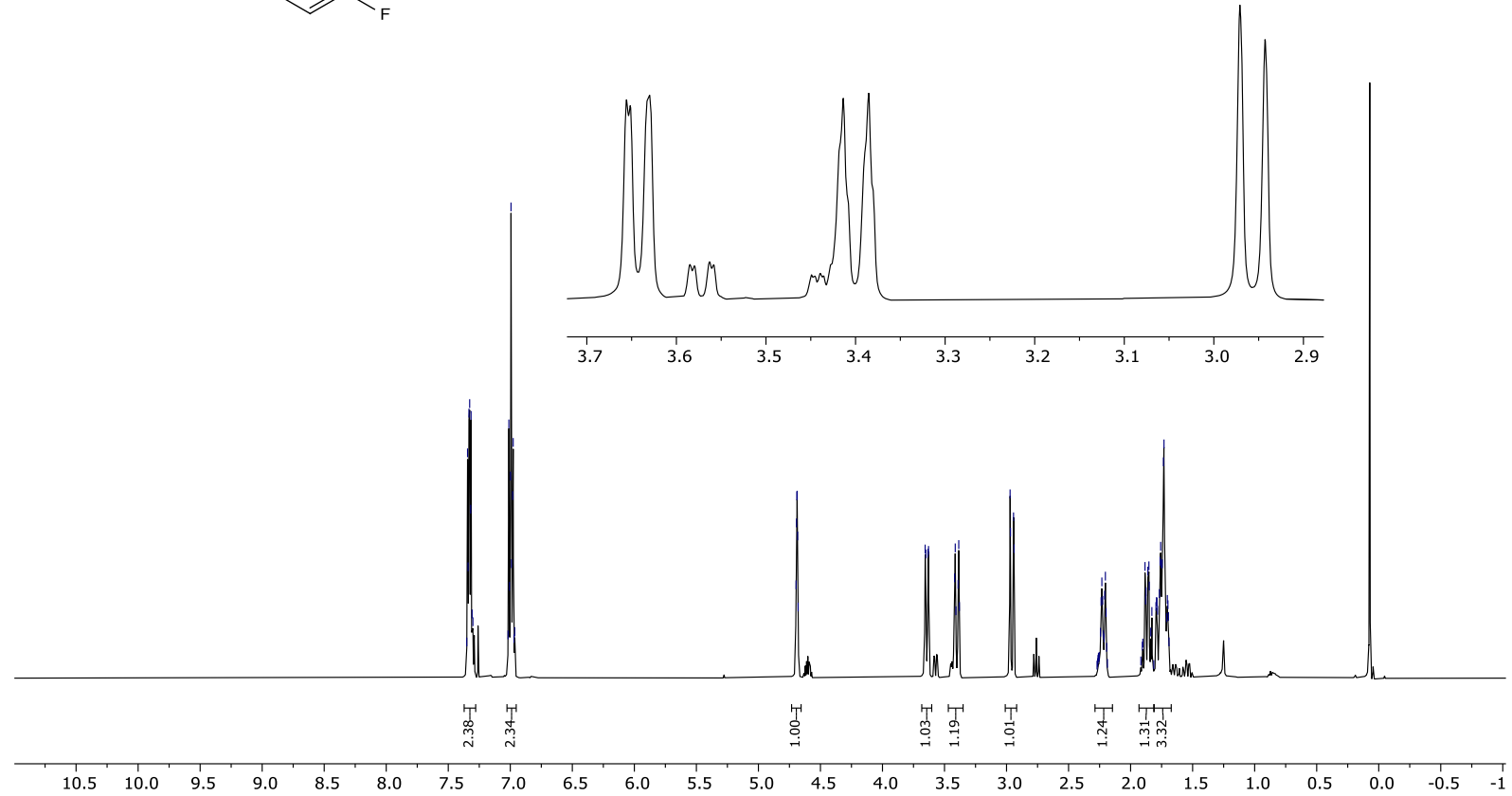

${ }_{13}^{13}\{1 \mathrm{H}\}$ NMR (Chloroformed)

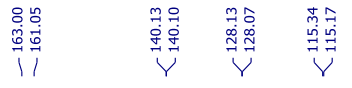

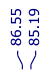

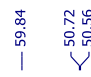

ปั<smiles>Fc1ccc(C2CCC(F)CN2)cc1</smiles>

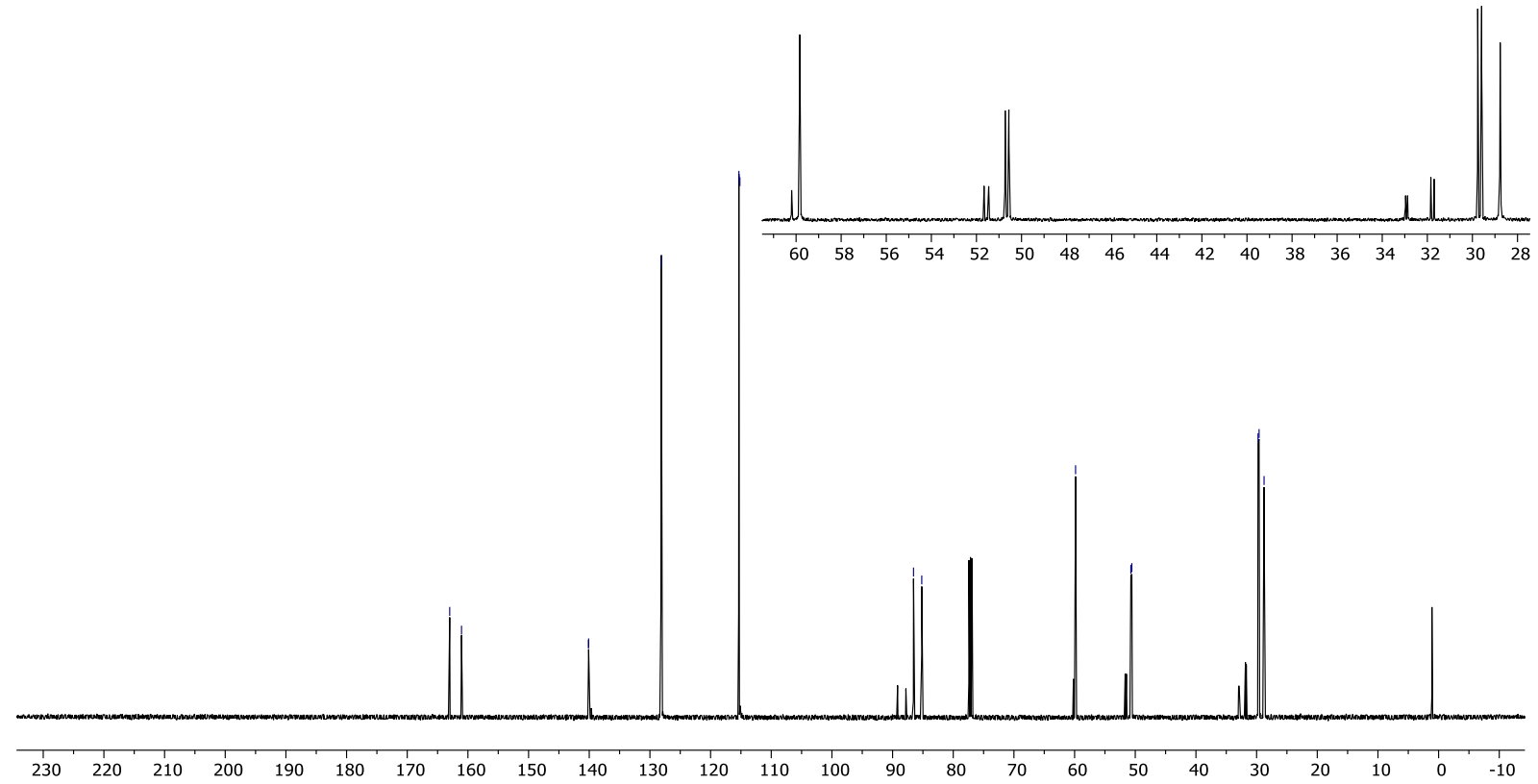


${ }^{13} \mathrm{C}\left\{{ }^{1} \mathrm{H}, 19 \mathrm{~F}\right\}$ NMR (Chloroform d)

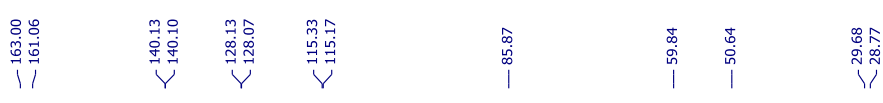<smiles>Fc1ccc(C2CCC(F)CN2)cc1</smiles>

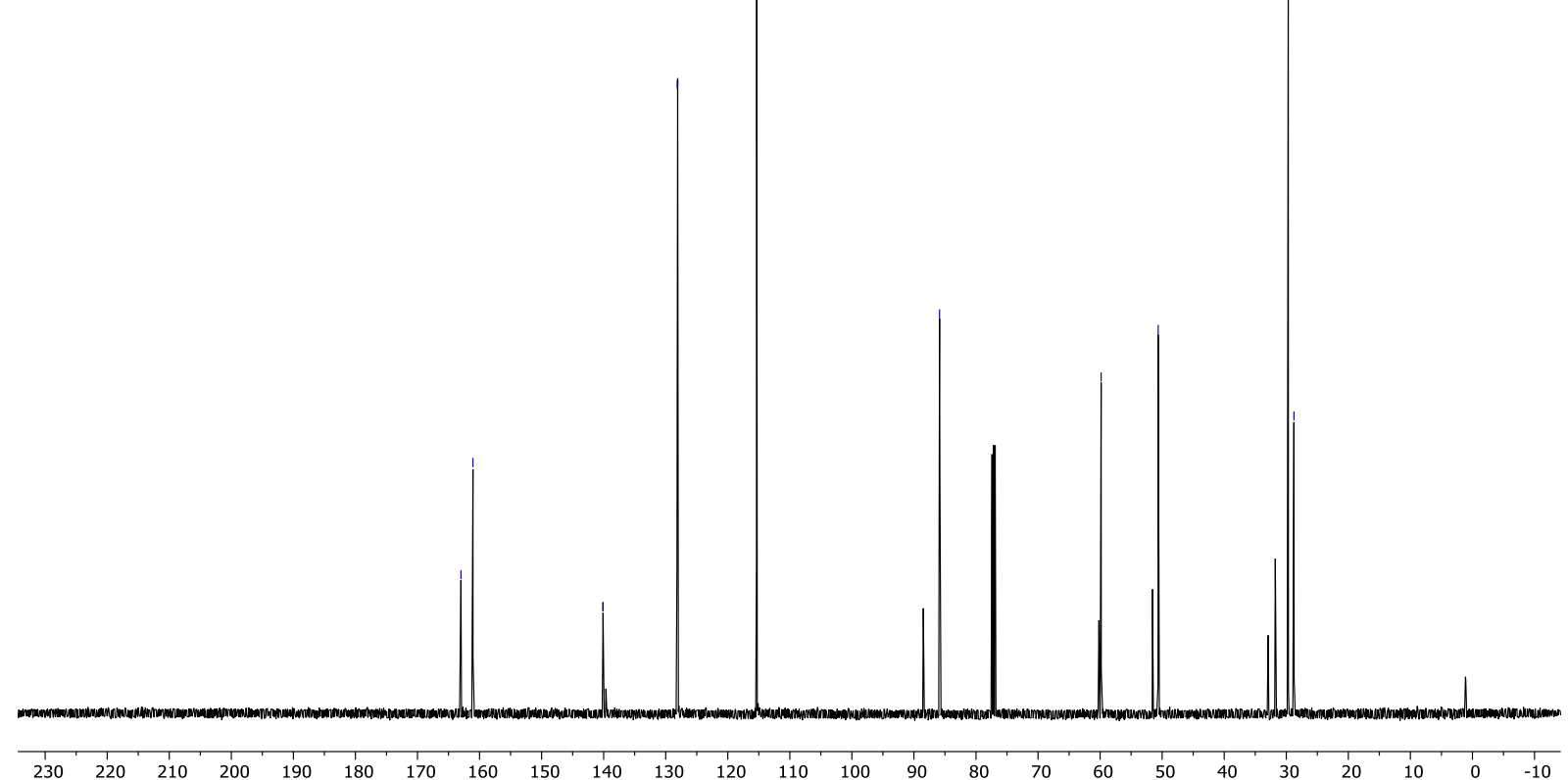

19F NMR (Chloroforme)

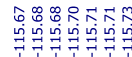

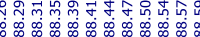<smiles>Fc1ccc(C2CCC(F)CN2)cc1</smiles>

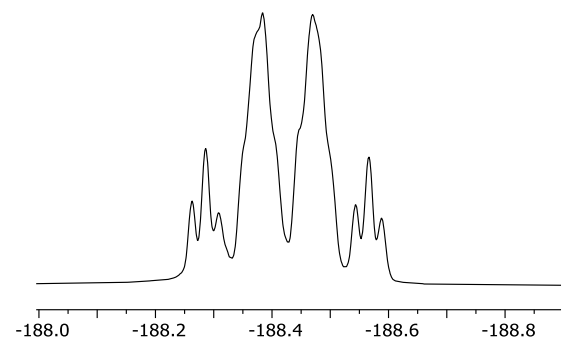

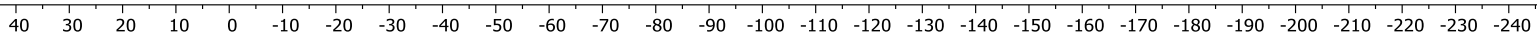


${ }^{19} \mathrm{~F}\left\{{ }^{1} \mathrm{H}\right\}$ NMR (Chloroform $d$ )

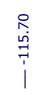

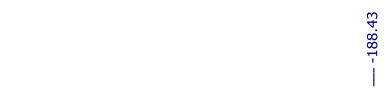
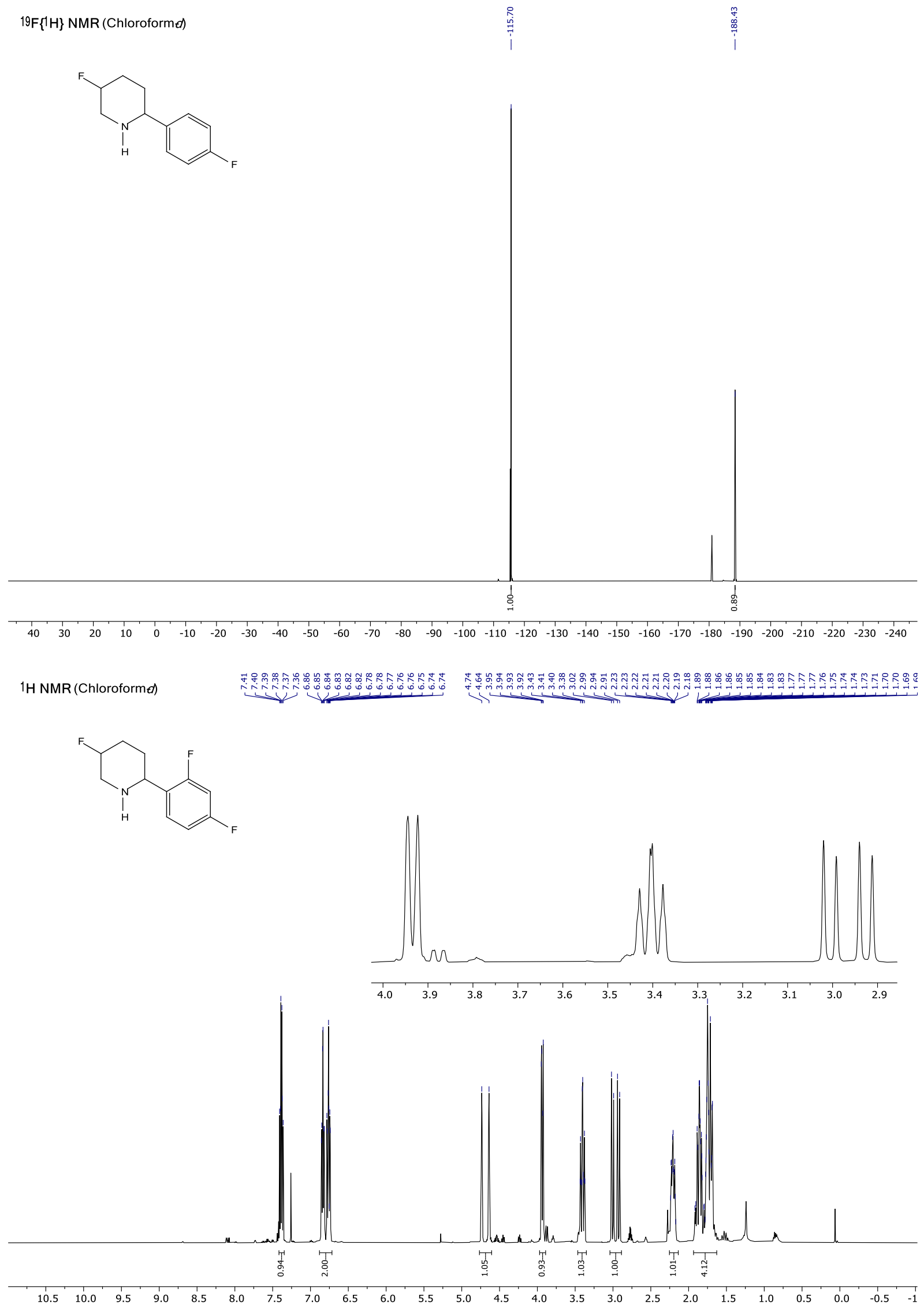

S141 
$1 \mathrm{H}\{19 \mathrm{~F}\}$ NMR (Chloroforme)

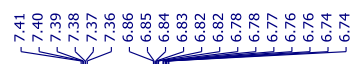

V

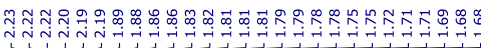
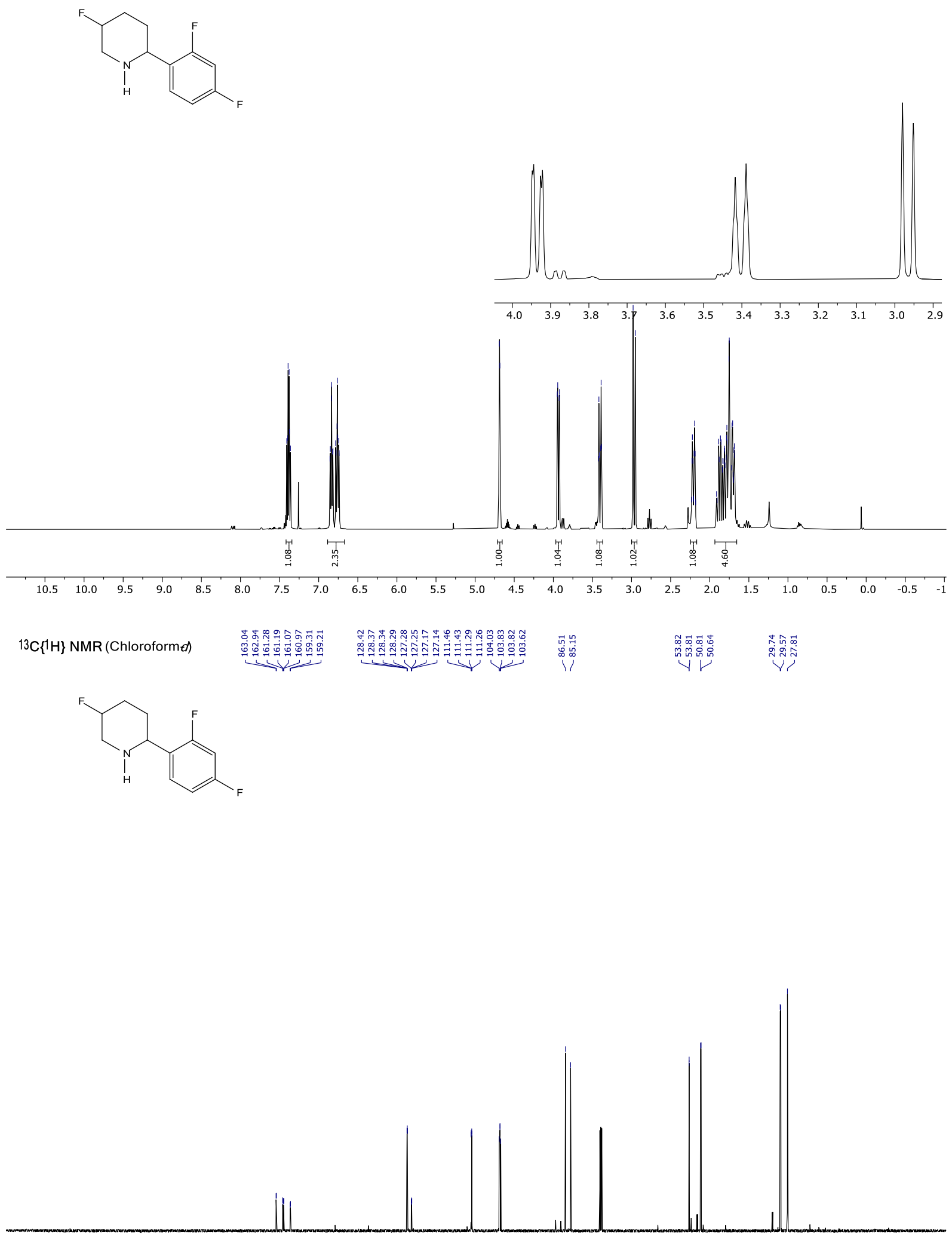

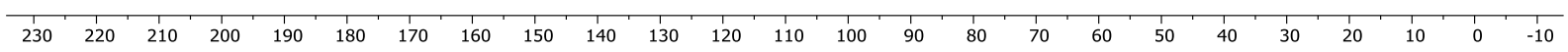

S142 
<smiles>Fc1ccc(C2CCC(F)CN2)c(F)c1</smiles>

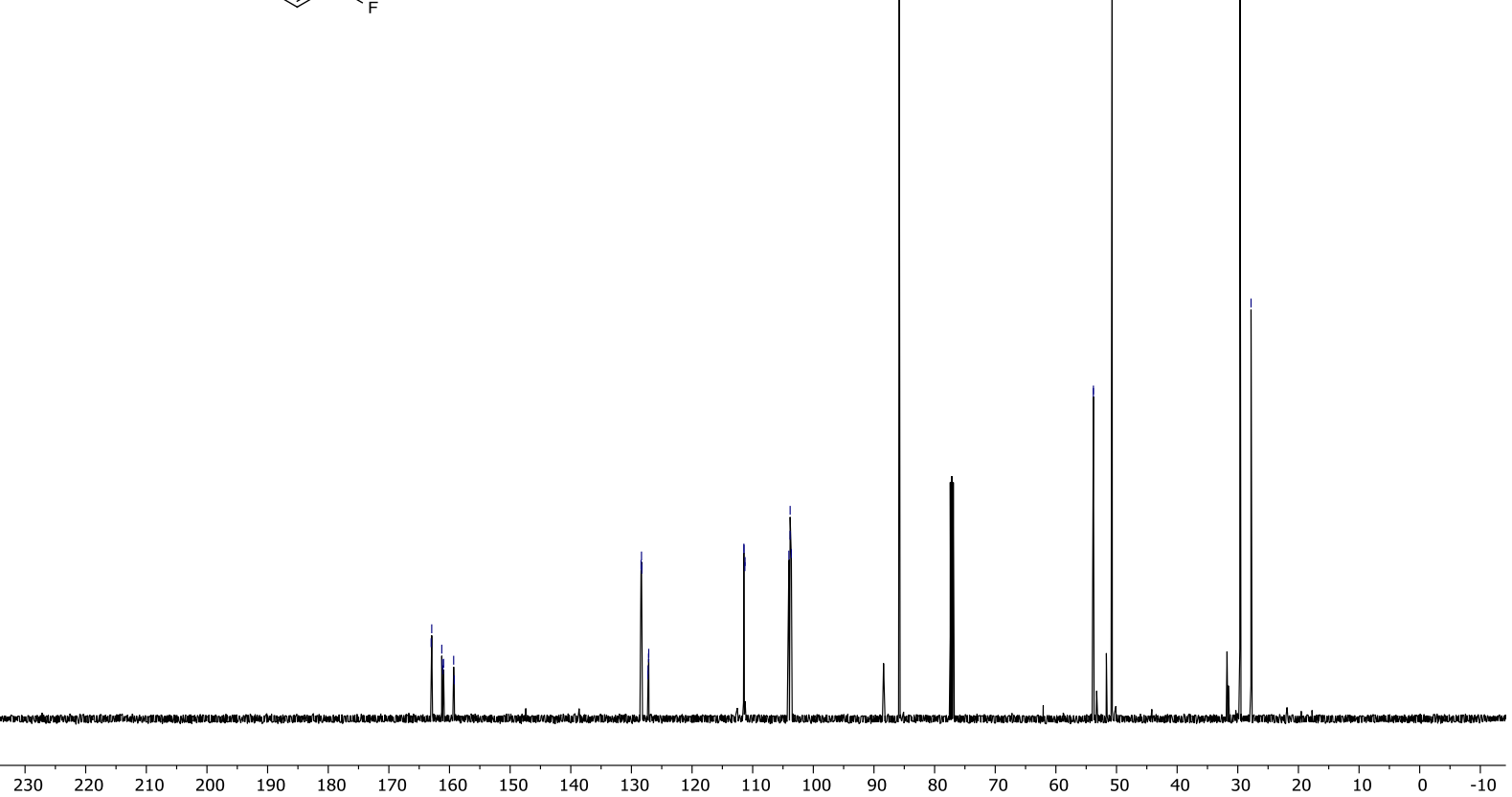

19F NMR (Chloroforme)

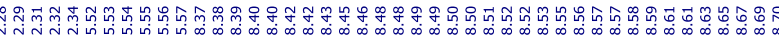

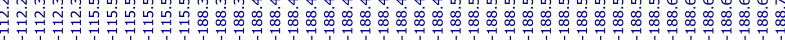<smiles>Fc1ccc(C2CCC(F)CN2)c(F)c1</smiles>

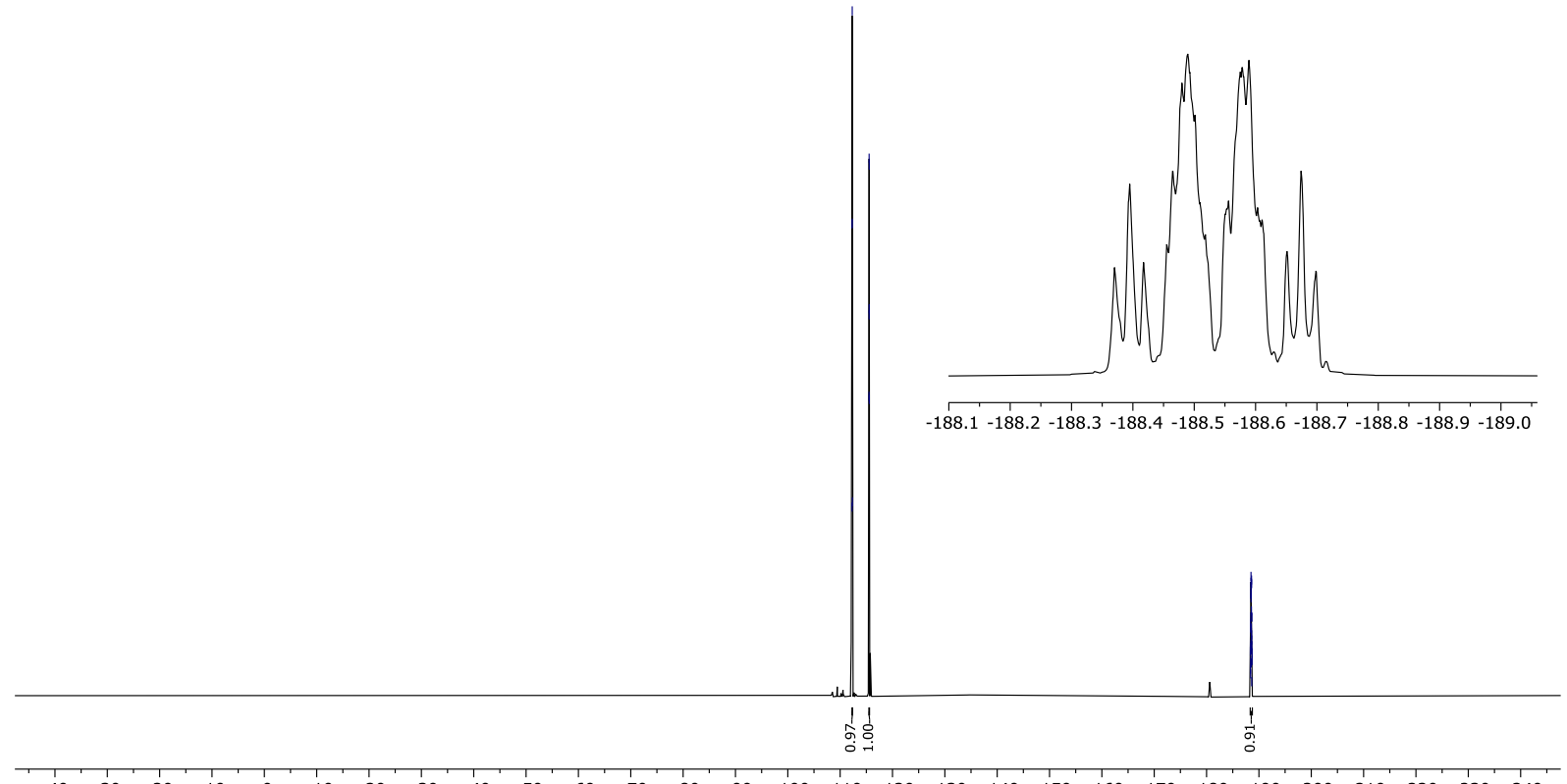


${ }^{19} \mathrm{~F}\{1 \mathrm{H}\}$ NMR (Chloroformed)<smiles>Fc1ccc(C2CCC(F)CN2)c(F)c1</smiles>

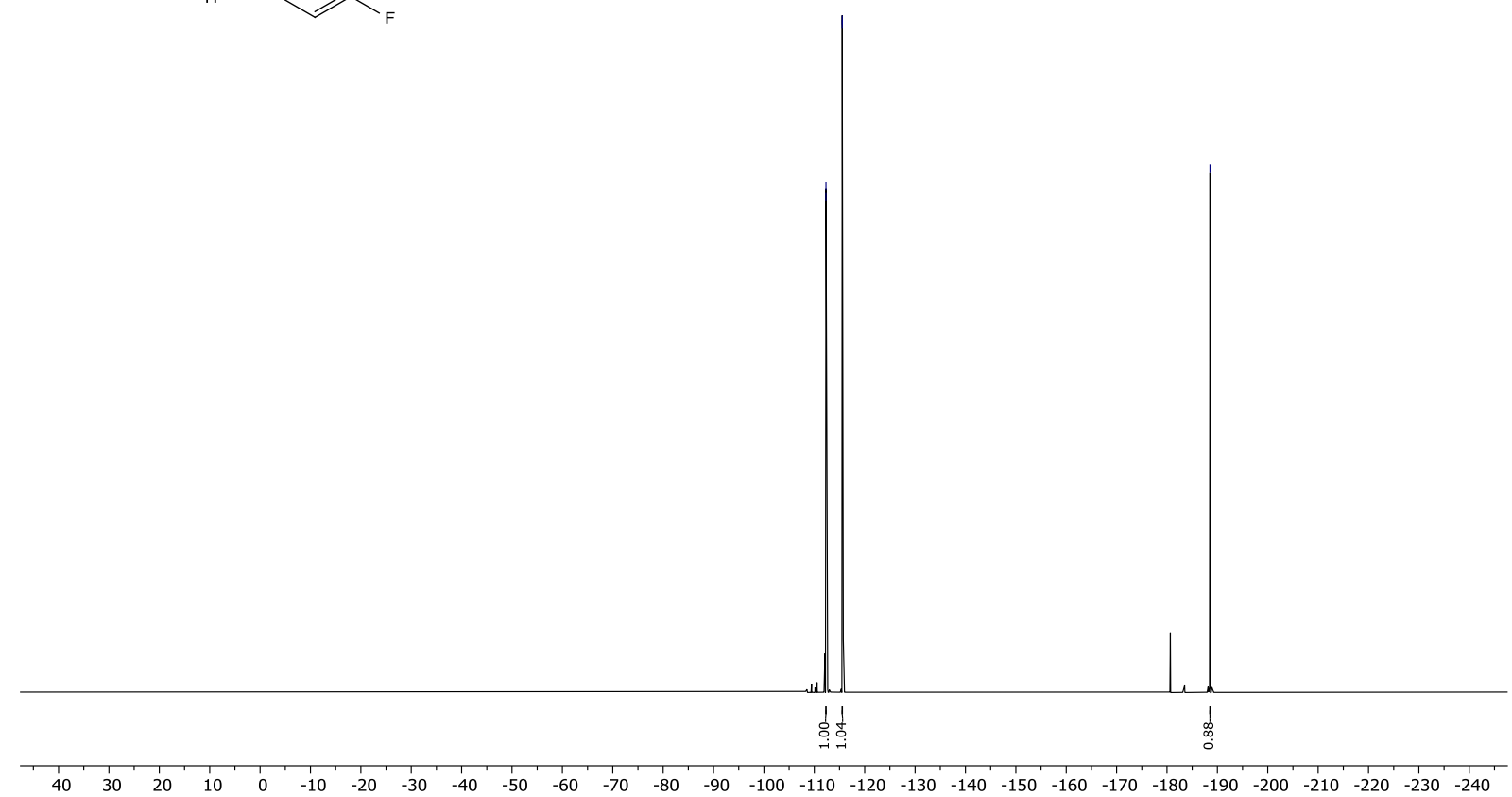

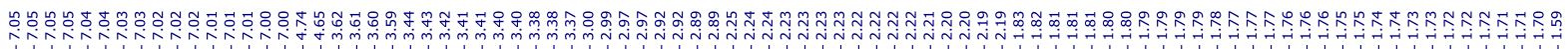
1H NMR (Chloroformel)<smiles>Fc1cc(C2CCC(F)CN2)cc(F)c1F</smiles>

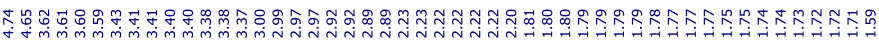
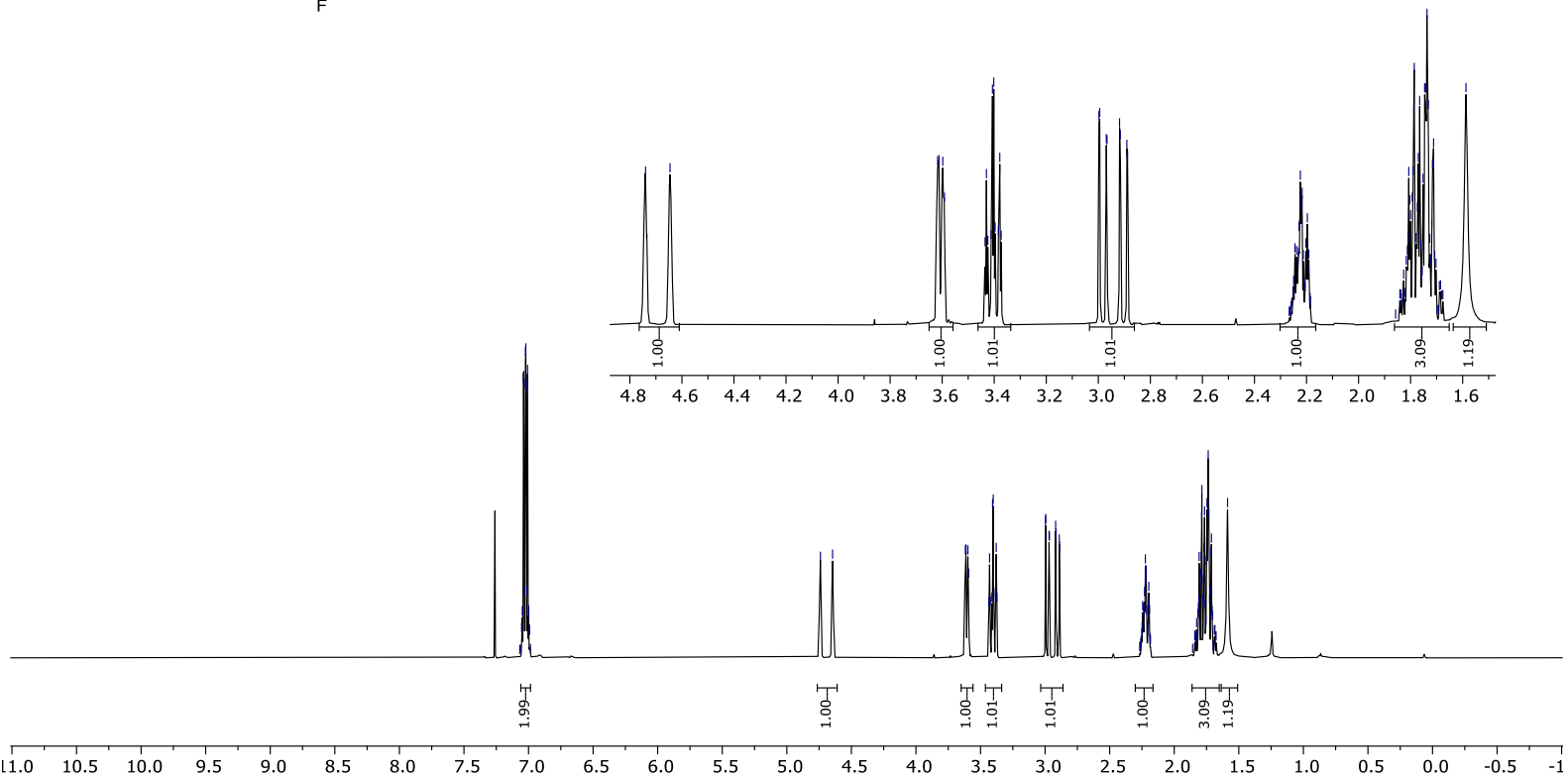


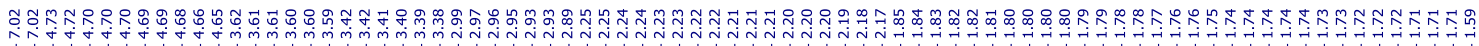
$1 \mathrm{H}\{19 \mathrm{~F}\}$ NMR (Chloroforme)

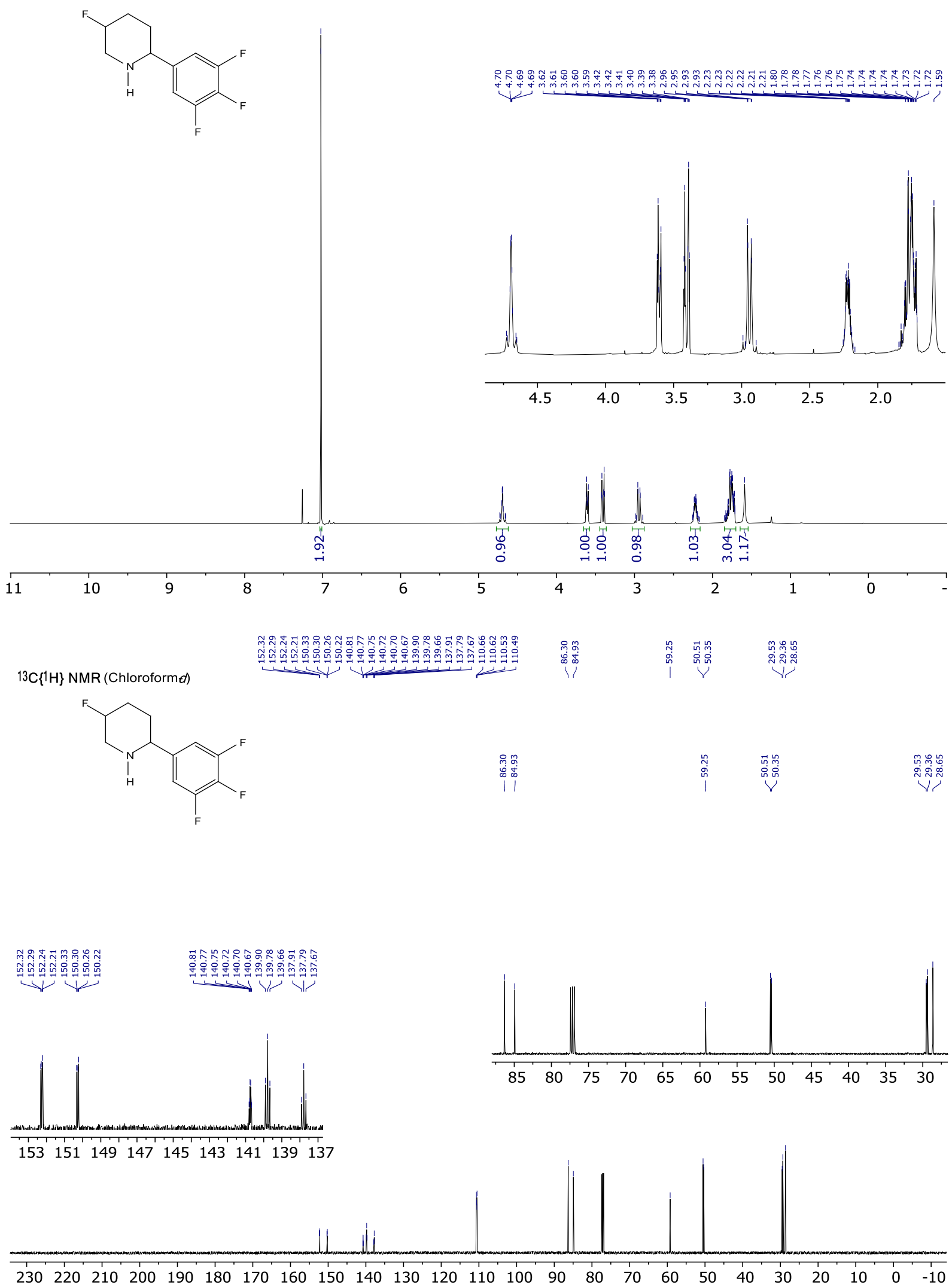


${ }^{13} \mathrm{C}\left\{{ }^{1} \mathrm{H},{ }^{19} \mathrm{~F}\right\}$ NMR (Chloroformed)

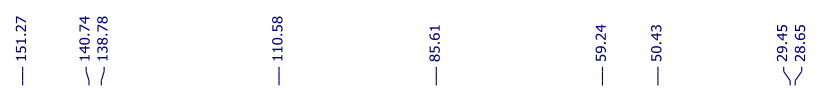<smiles>Fc1cc(C2CCC(F)CN2)cc(F)c1F</smiles>

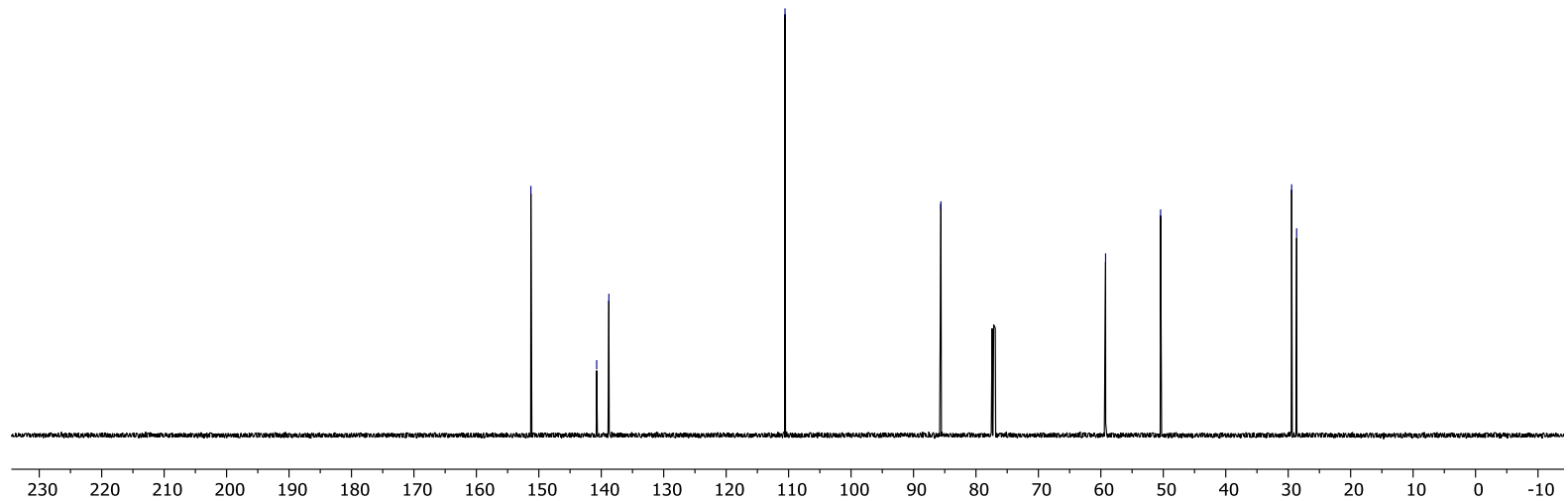

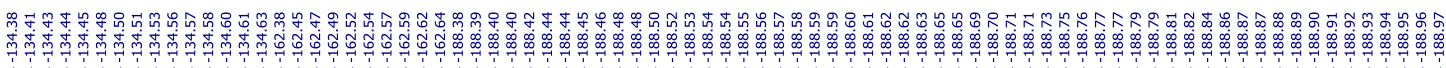

19F NMR (Chloroforme)<smiles>Fc1cc(C2CCC(F)CN2)cc(F)c1F</smiles>
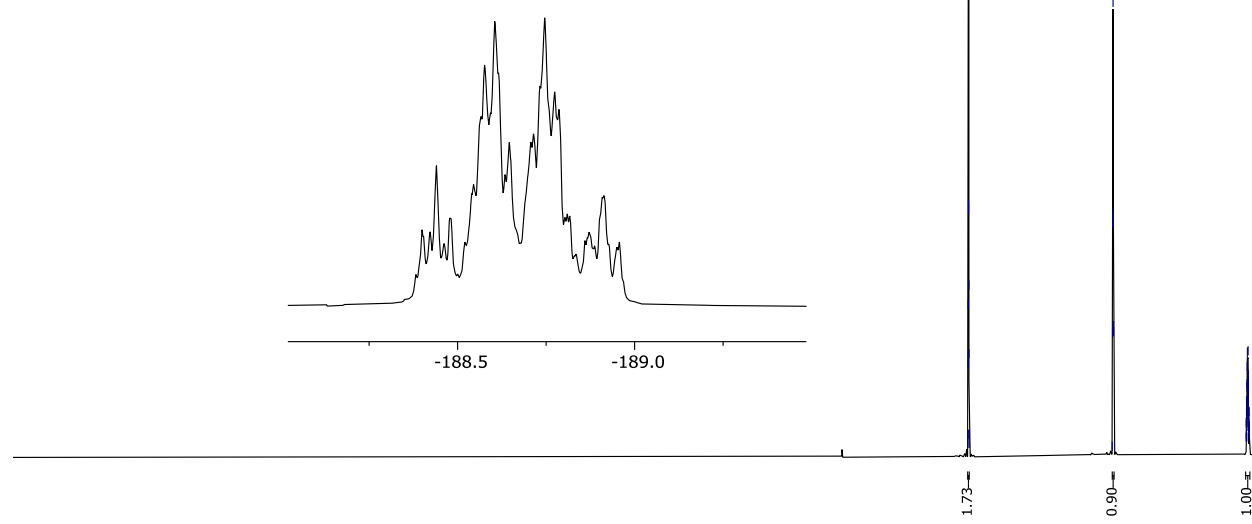

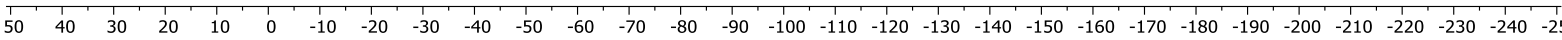


19F $\{1 \mathrm{H}\}$ NMR (Chloroformed)

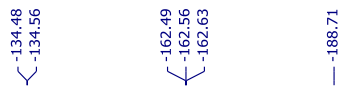<smiles>Fc1cc(C2CCC(F)CN2)cc(F)c1F</smiles>

ㅇำ ํํำ

กู่ ชู่
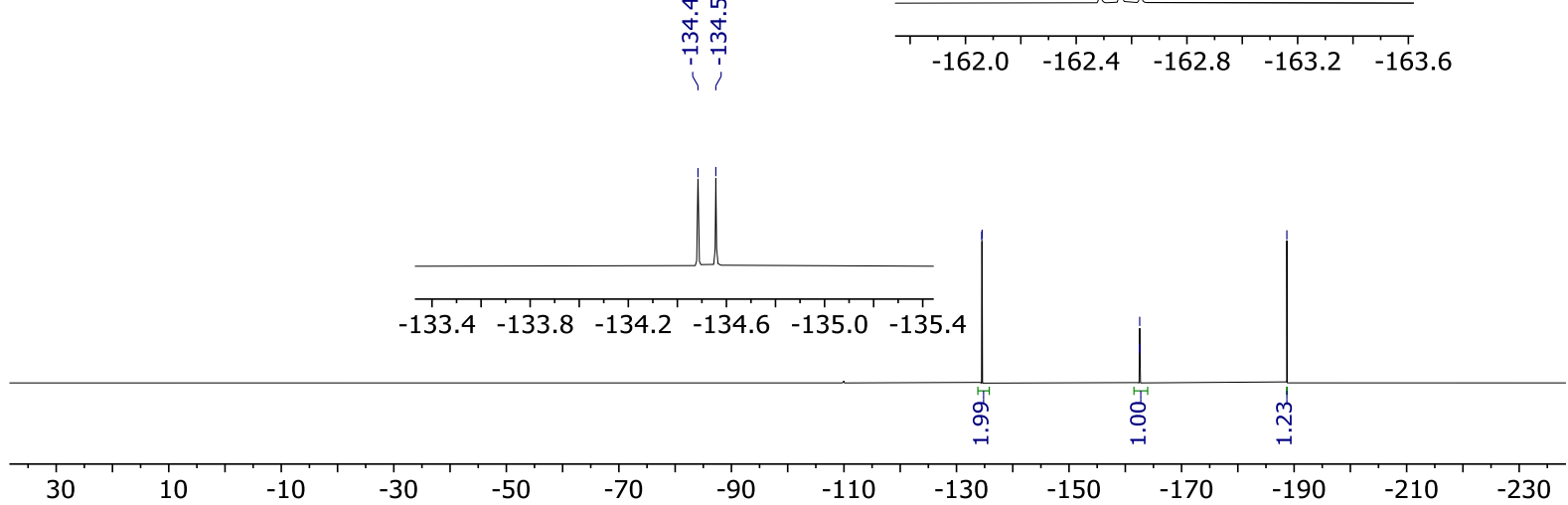

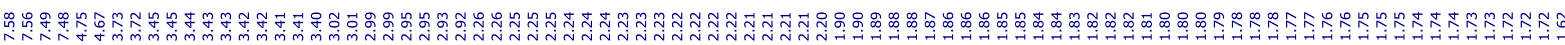
1H NMR (Chloroforme)<smiles>FC1CCC(c2ccc(C(F)(F)F)cc2)NC1</smiles>

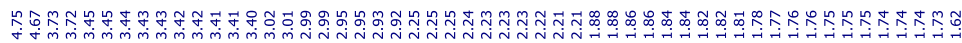

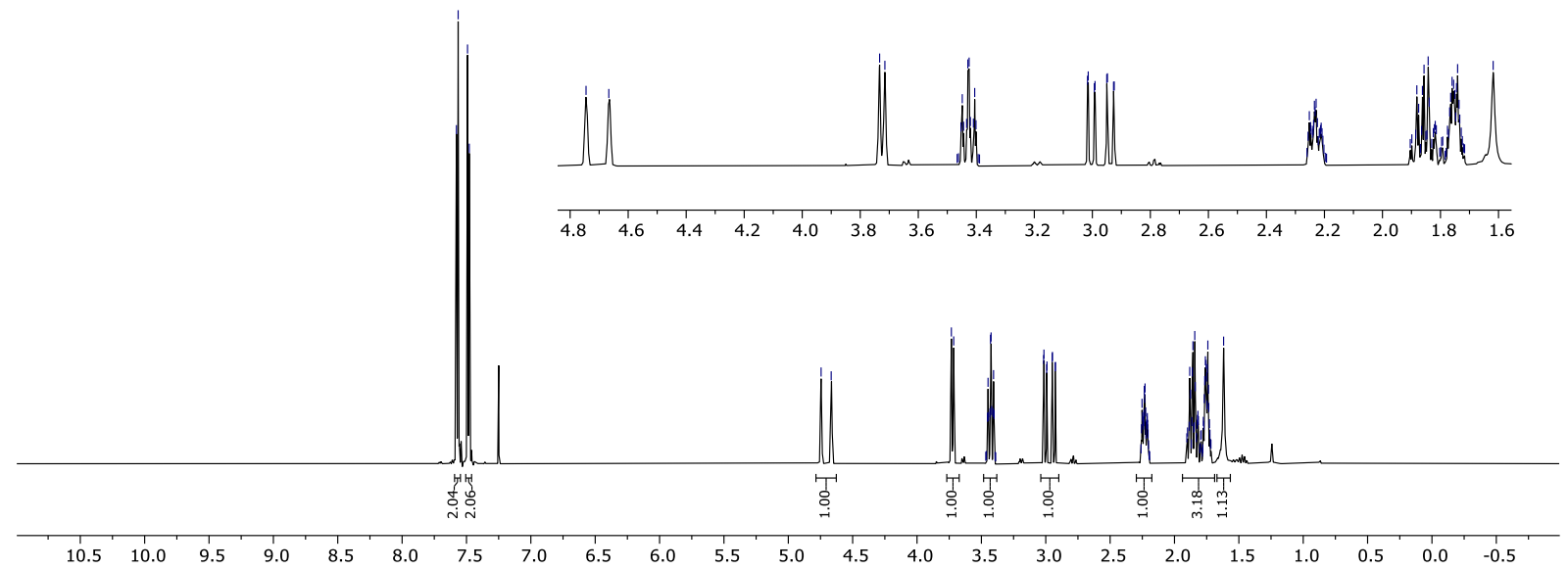



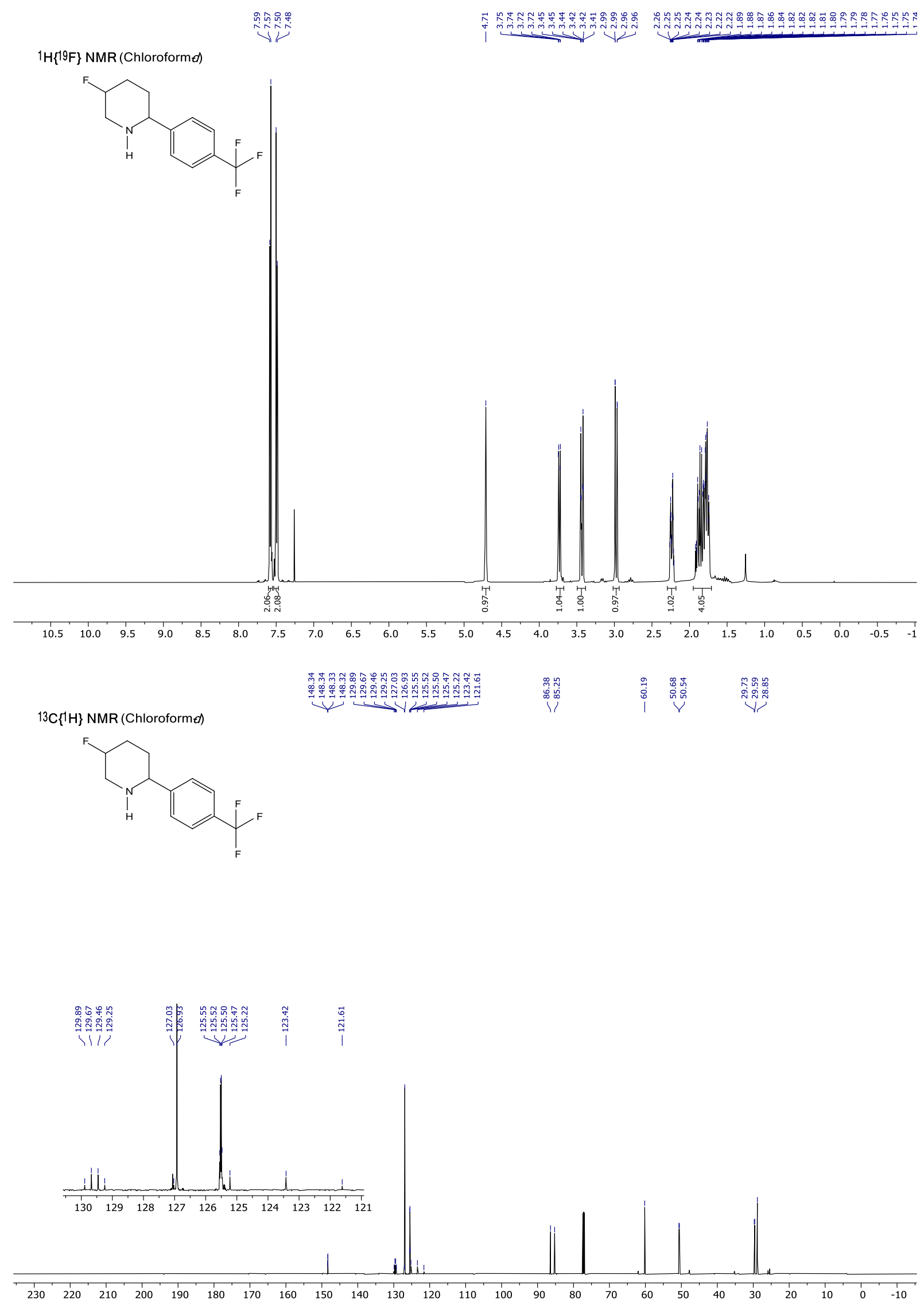
${ }^{13} \mathrm{C}\{1 \mathrm{H}, 19 \mathrm{~F}\}$ NMR (Chloroformen)

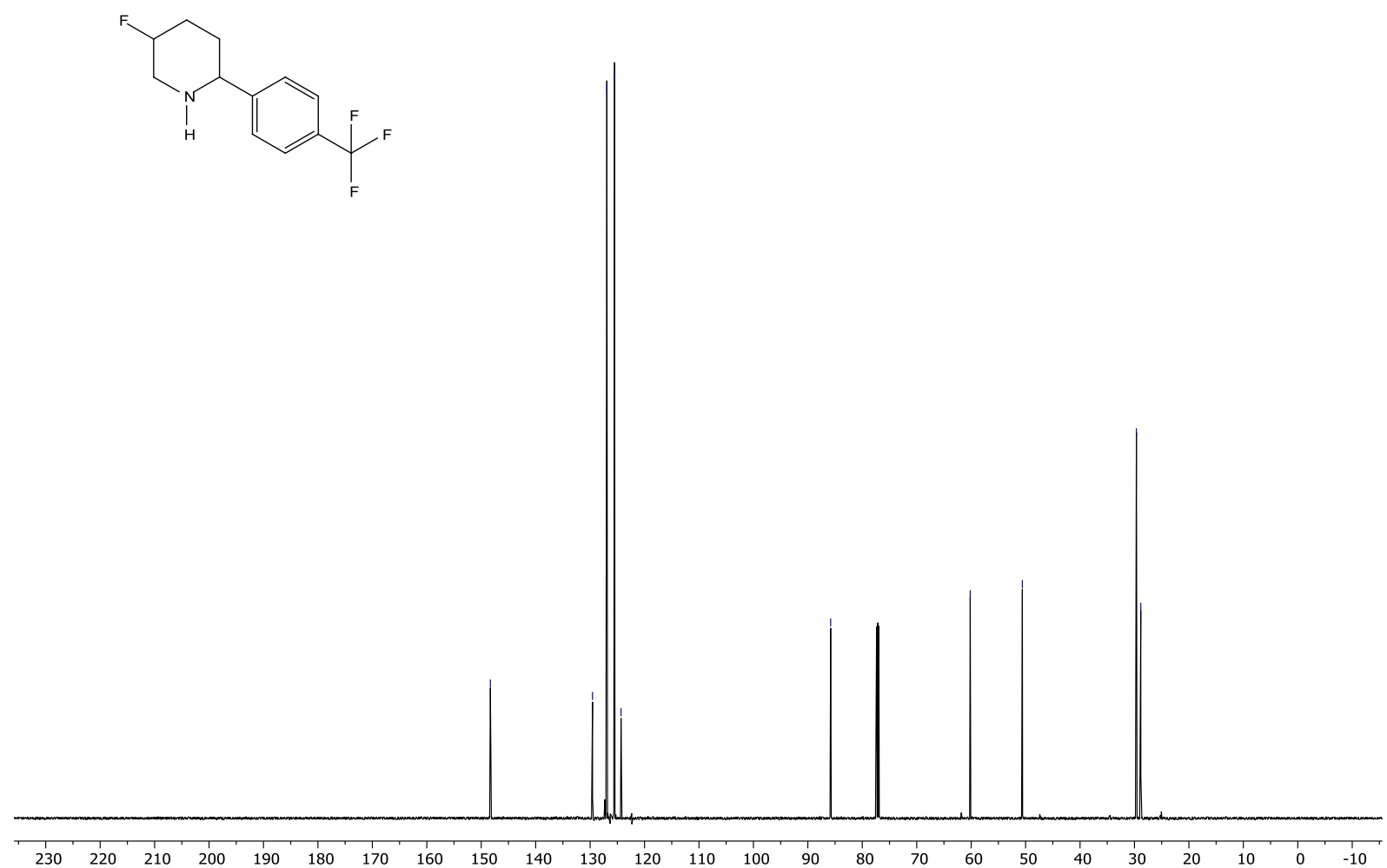

\section{F NMR (Chloroformed)}<smiles>FC1CCC(c2ccc(C(F)(F)F)cc2)NC1</smiles>

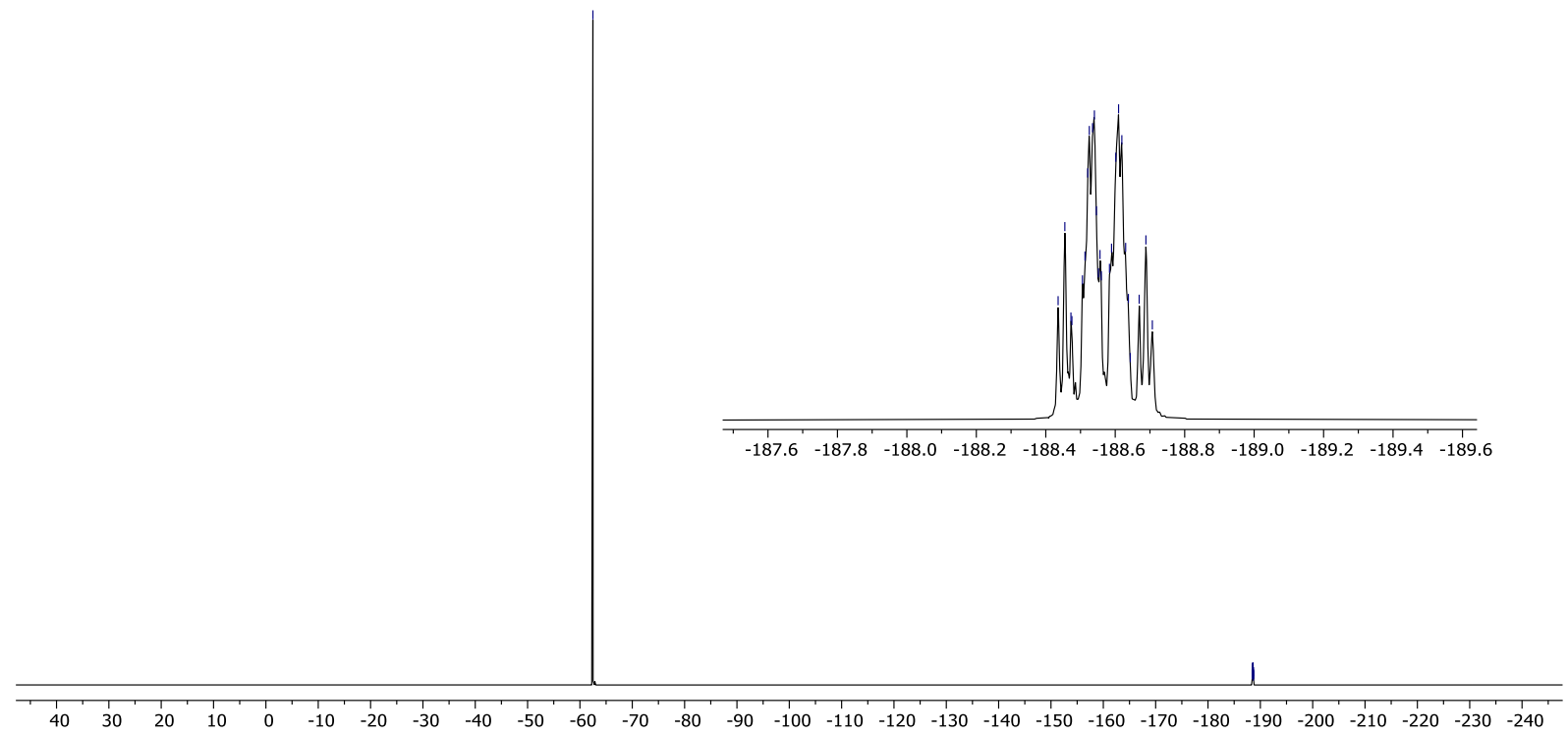


${ }^{19} \mathrm{~F}\{1 \mathrm{H}\}$ NMR (Chloroformed)

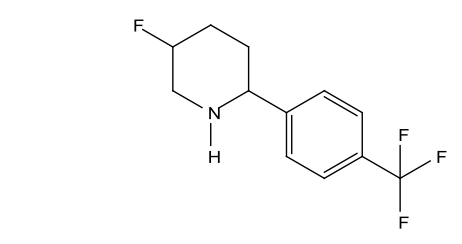

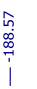
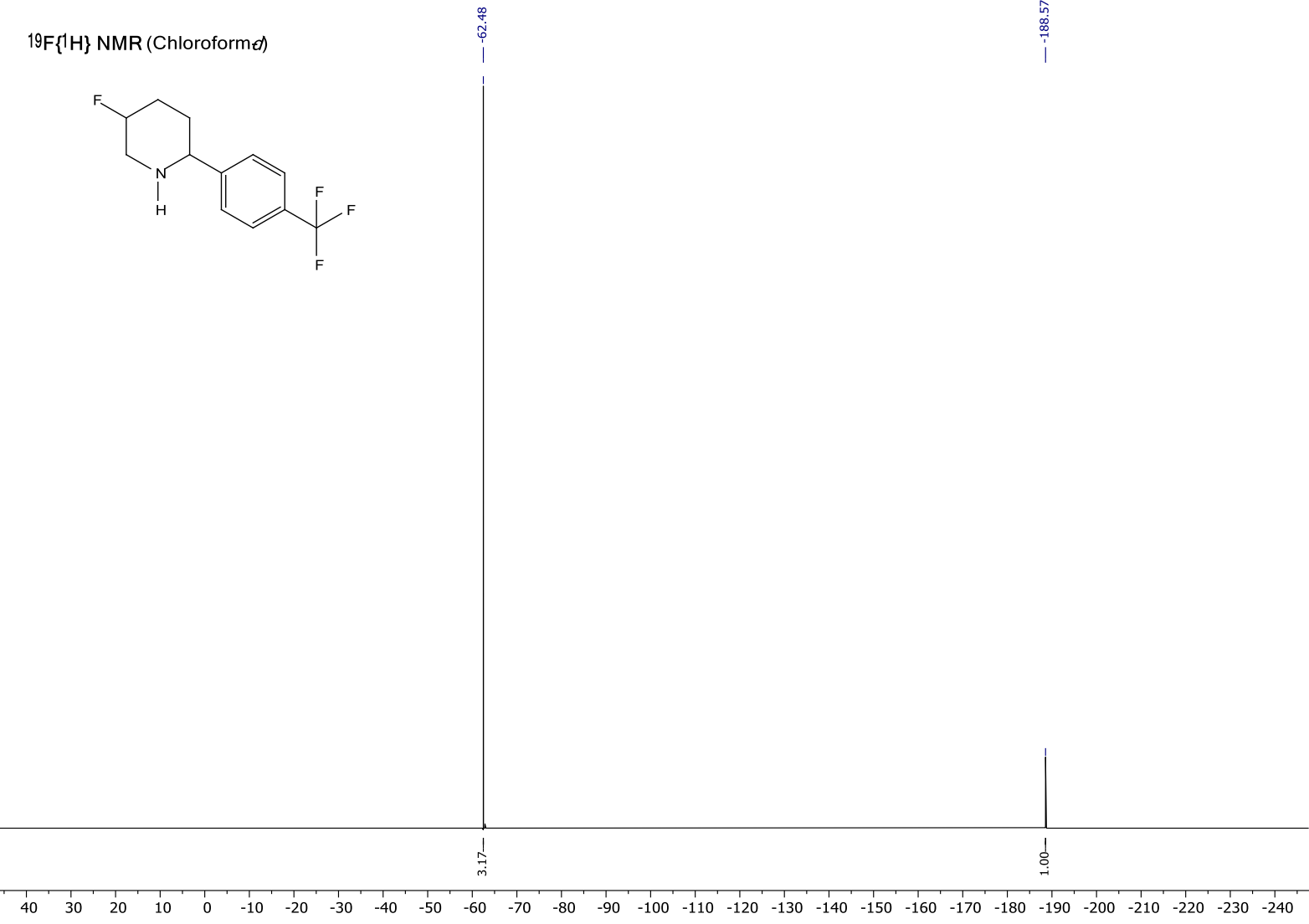

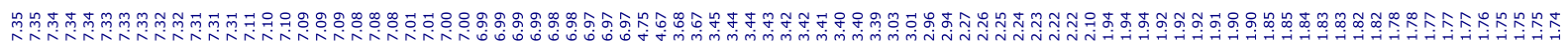
1H NMR (Chloroformed)

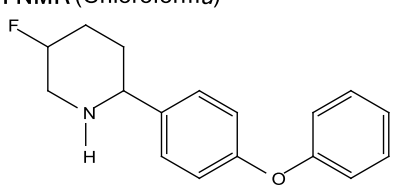

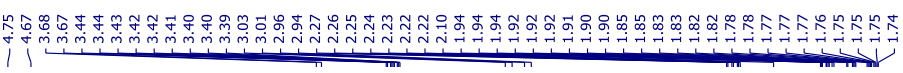
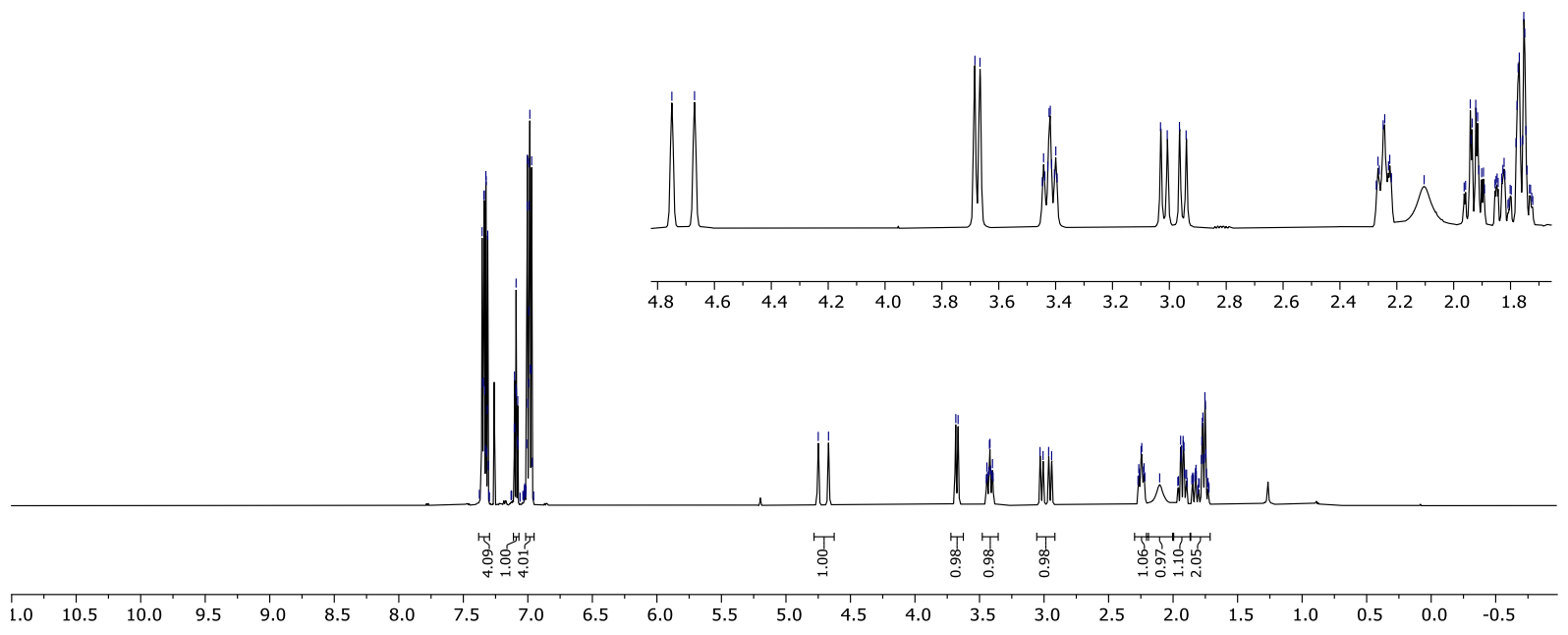

S150 


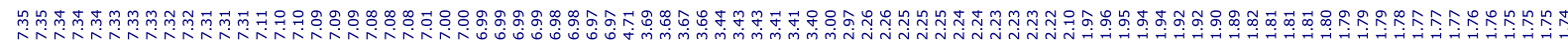
$1 \mathrm{H}\{19 \mathrm{~F}\}$ NMR (Chloroformel)<smiles>FC1CCC(c2ccc(Oc3ccccc3)cc2)NC1</smiles>

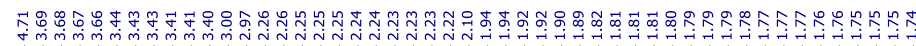
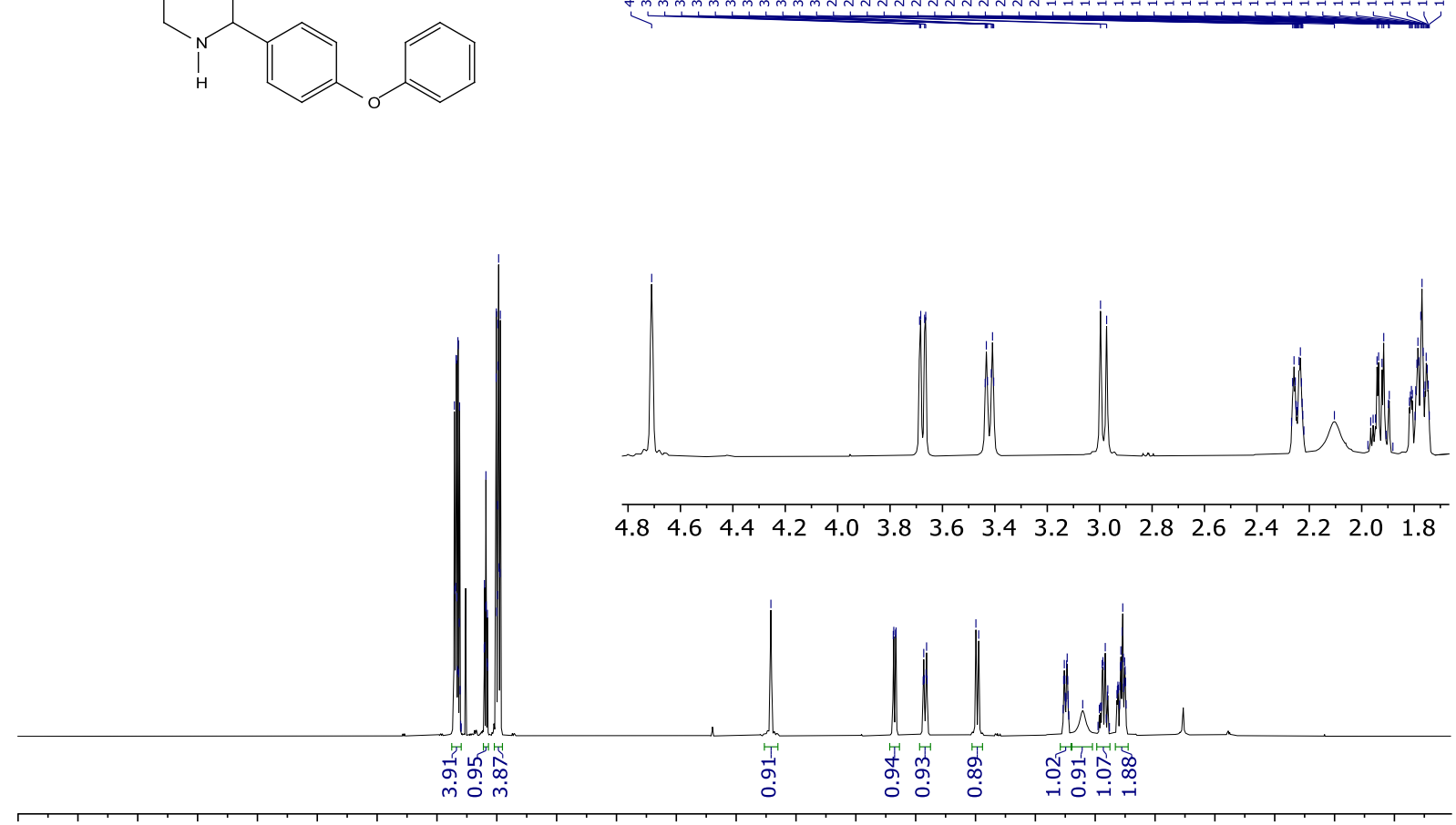

\begin{tabular}{lllllllllllllllllllllllllll}
\hline 1.0 & 10.5 & 10.0 & 9.5 & 9.0 & 8.5 & 8.0 & 7.5 & 7.0 & 6.5 & 6.0 & 5.5 & 5.0 & 4.5 & 4.0 & 3.5 & 3.0 & 2.5 & 2.0 & 1.5 & 1.0 & 0.5 & 0.0 & -0.5
\end{tabular}

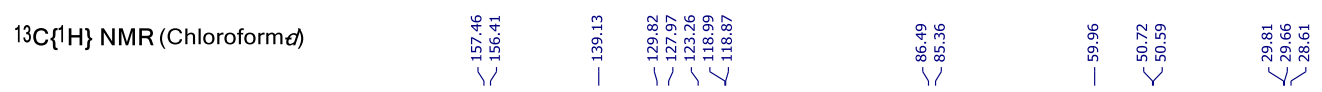<smiles>FC1CCC(c2ccc(Oc3ccccc3)cc2)NC1</smiles>
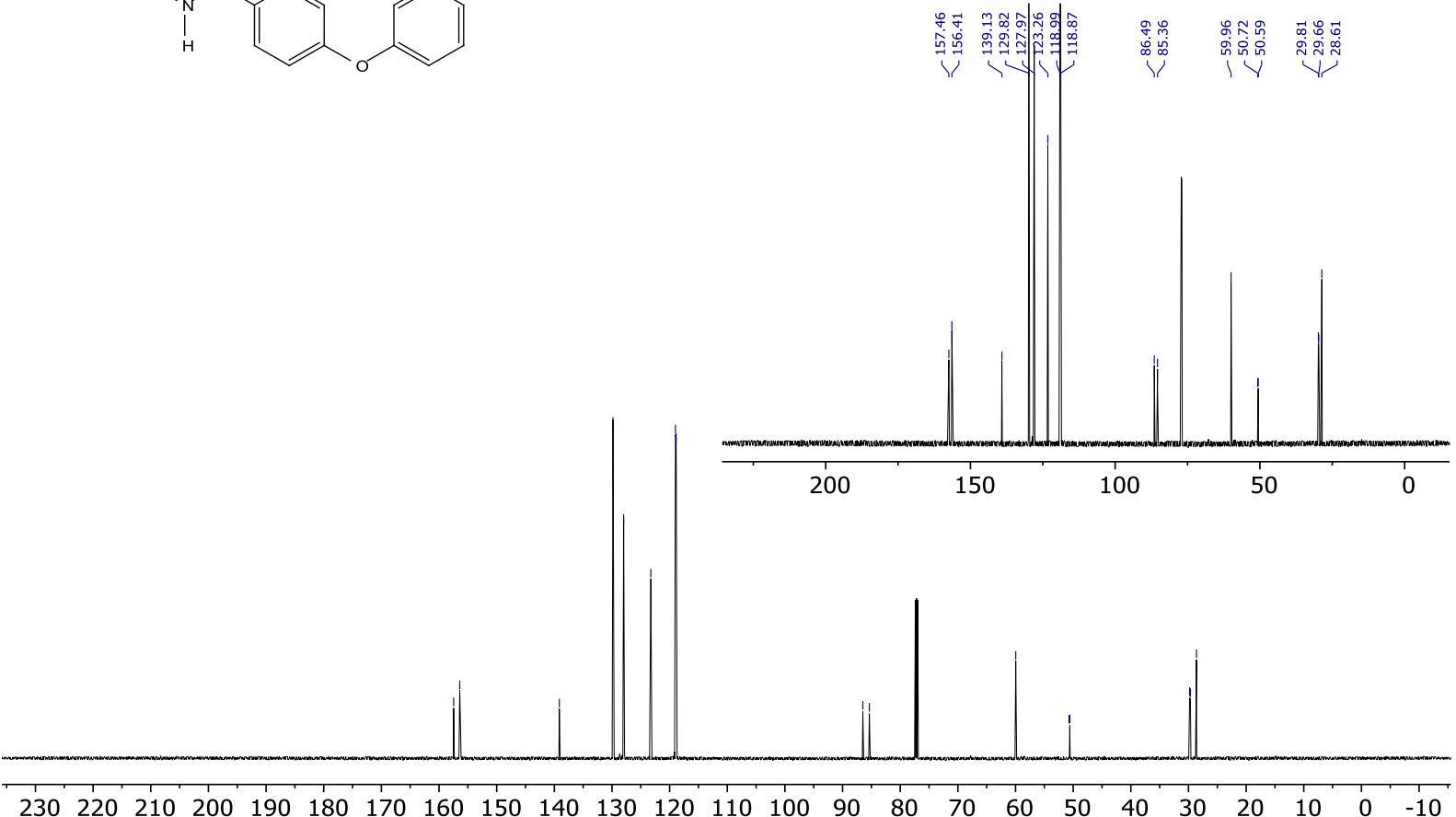


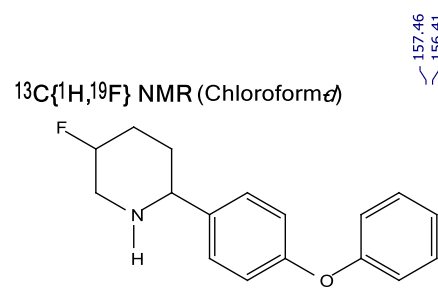

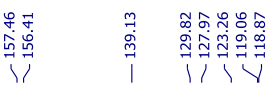

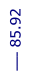

$\underset{\substack{0 \\ 0}}{\substack{0 \\ 0}}$

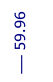

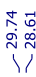

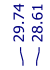

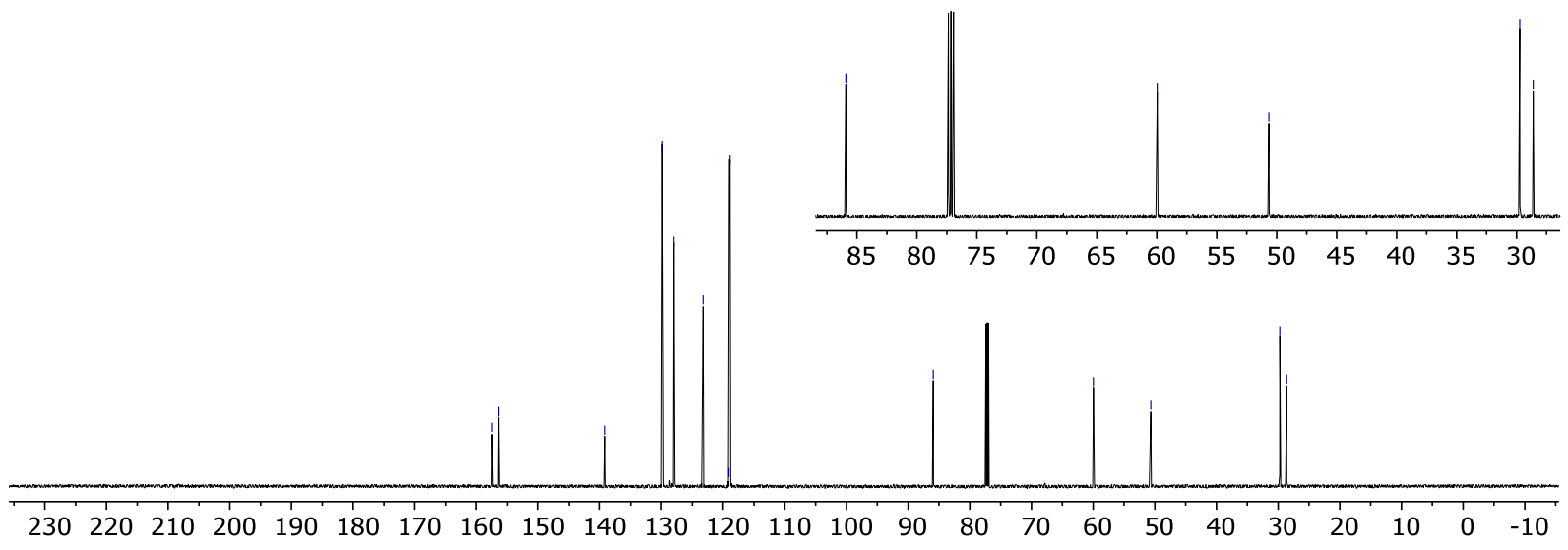

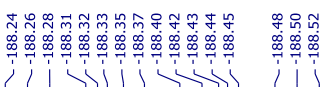

19F NMR (Chloroforme)
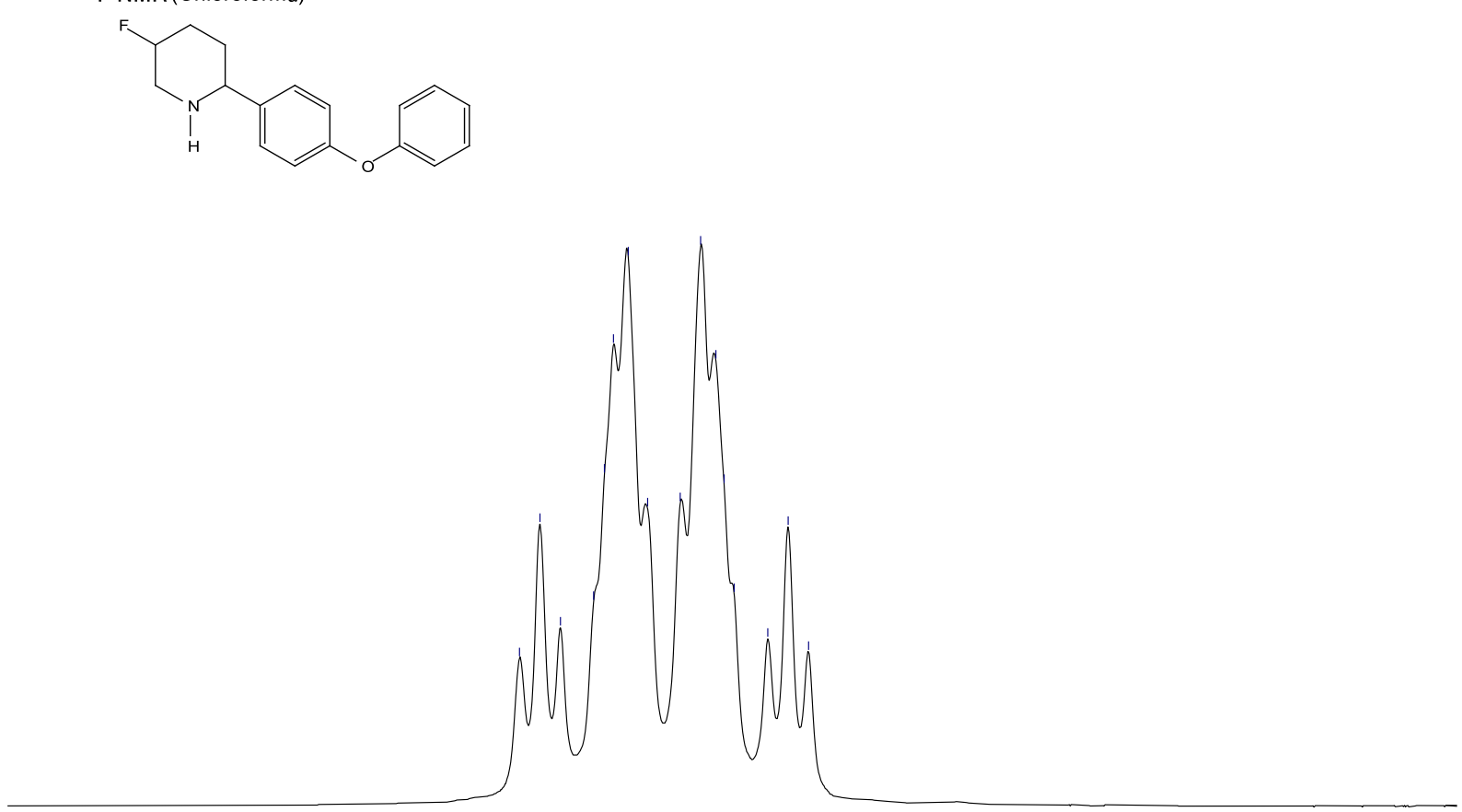

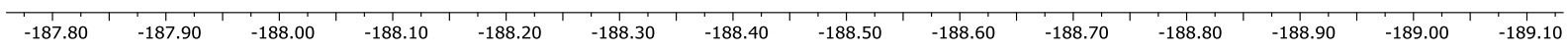

S152 
19F $\{1 \mathrm{H}\}$ NMR (Chloroformed)<smiles>FC1CCC(c2ccc(Oc3ccccc3)cc2)NC1</smiles>

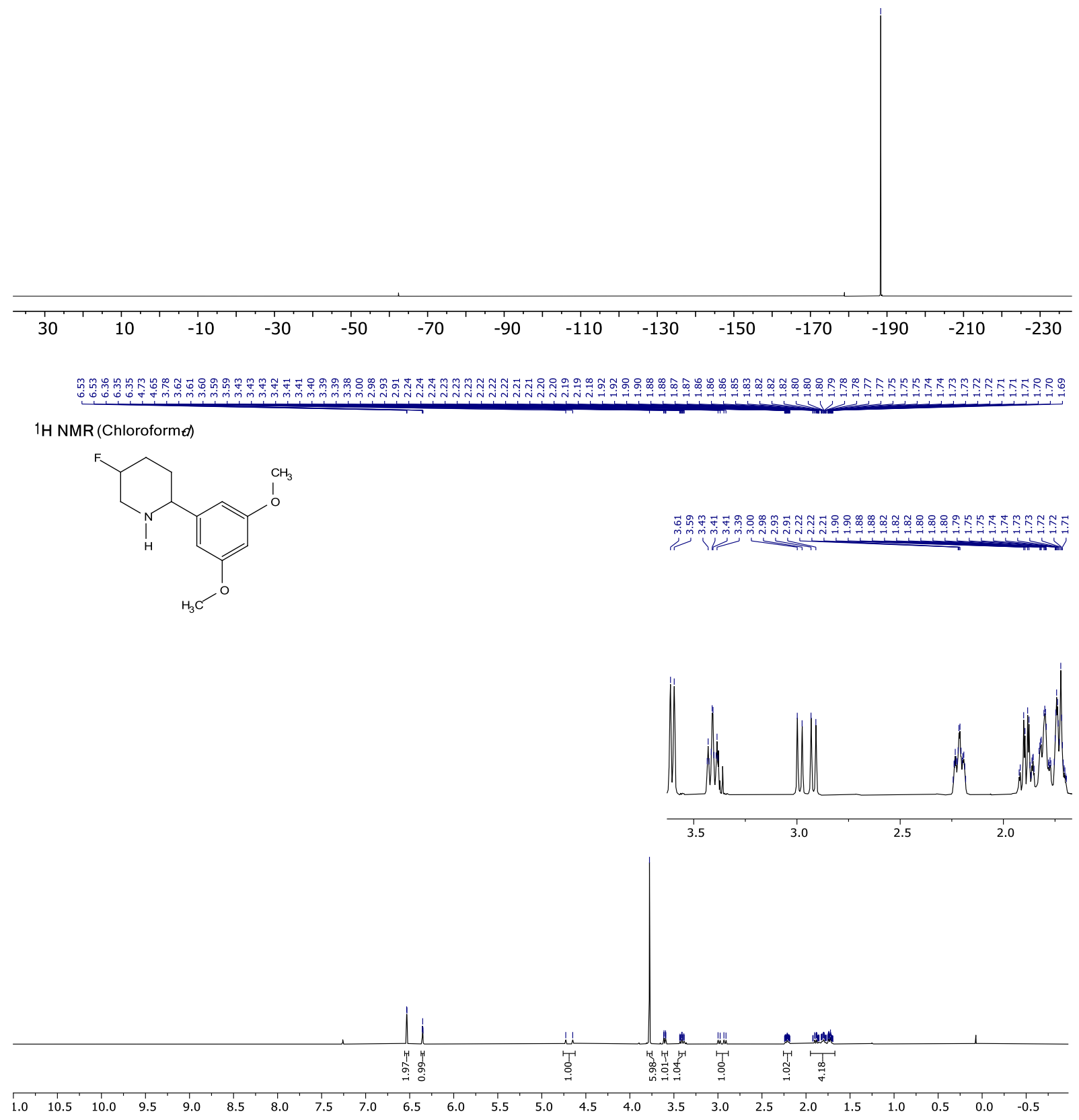




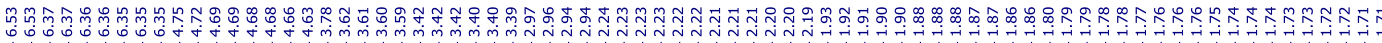
$1 \mathrm{H}\{19 \mathrm{~F}\}$ NMR (Chloroforme)<smiles>COc1cc(OC)cc(C2CCC(F)CN2)c1</smiles>

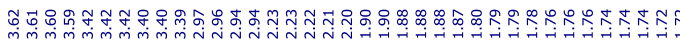

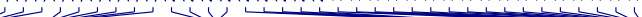
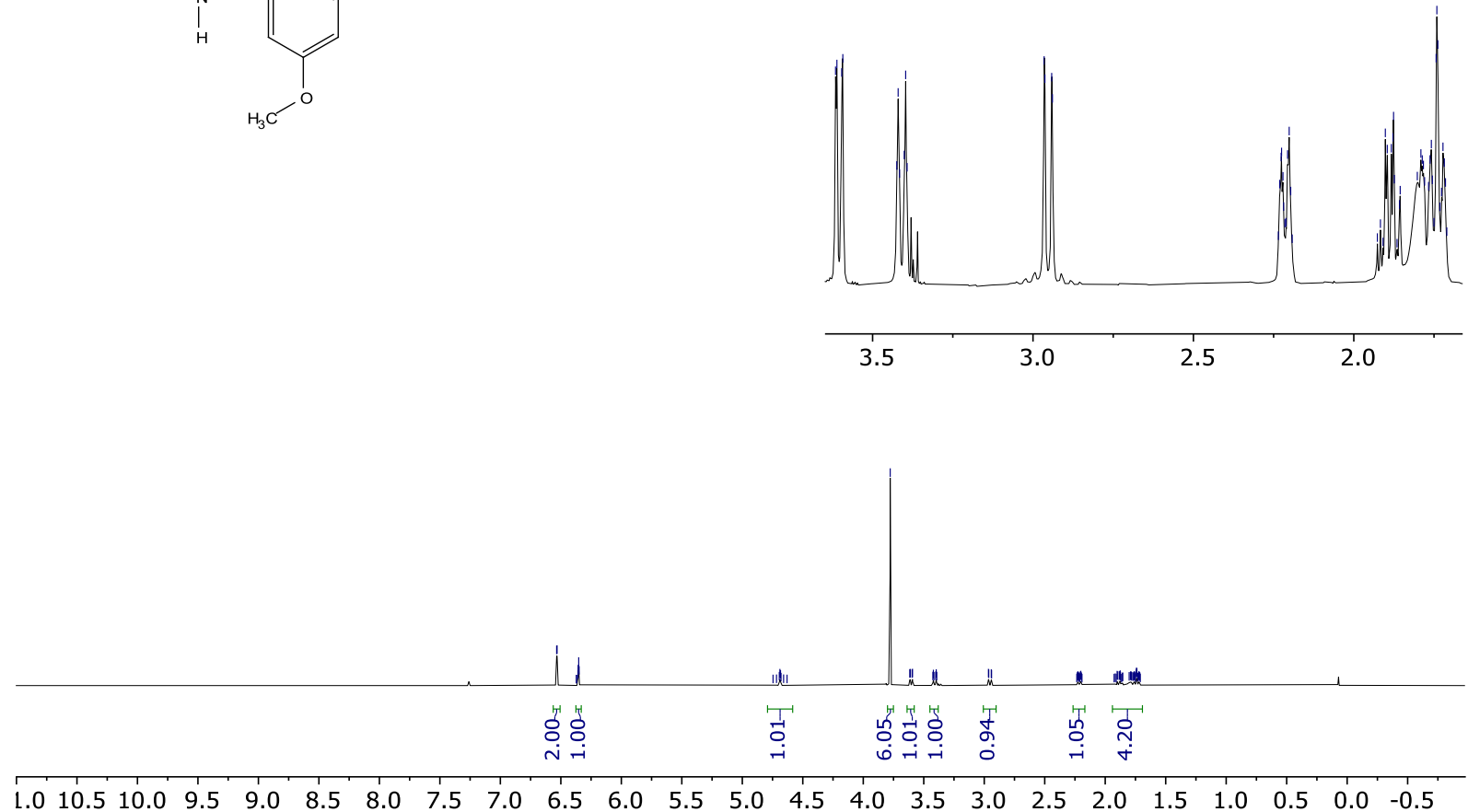

${ }^{13 C}\{1 \mathrm{H}\}$ NMR (Chloroforme)

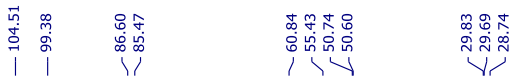<smiles>COc1cc(OC)cc(C2CCC(F)CN2)c1</smiles>

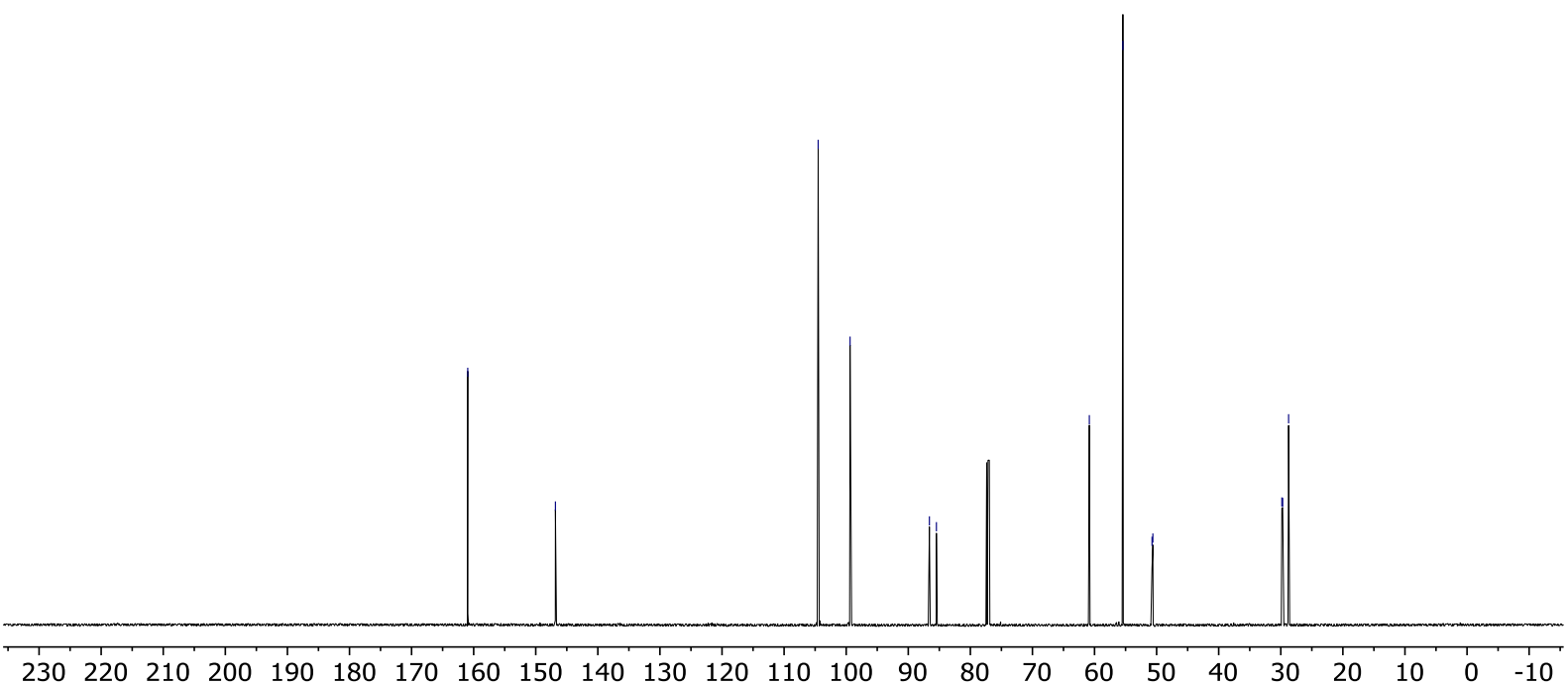


13C\{1H,19F\} NMR (Chloroforme) $\quad$ l<smiles>COc1cc(OC)cc(C2CCC(F)CN2)c1</smiles>

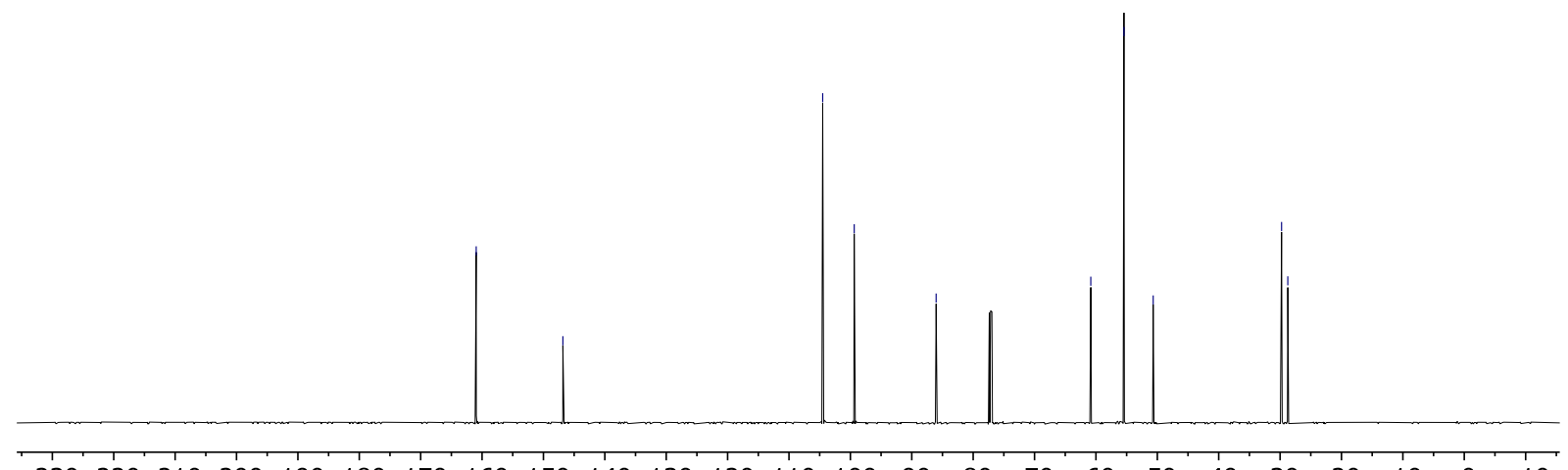

$\begin{array}{lllllllllllllllllllllllll}230 & 220 & 210 & 200 & 190 & 180 & 170 & 160 & 150 & 140 & 130 & 120 & 110 & 100 & 90 & 80 & 70 & 60 & 50 & 40 & 30 & 20 & 10 & 0 & -10\end{array}$

19F NMR (Chloroforme)

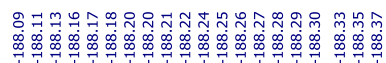<smiles>COc1cc(OC)cc(C2CCC(F)CN2)c1</smiles>

\section{ن}

$\mathrm{H}_{3} \mathrm{C}^{\prime}$

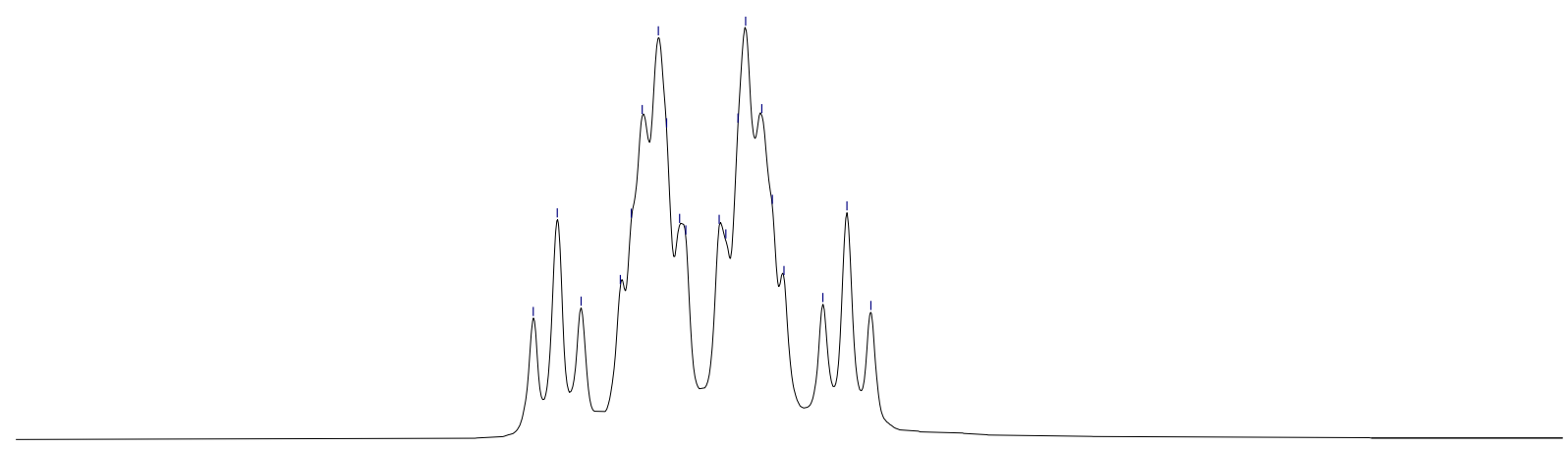

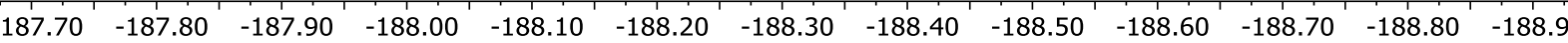


${ }^{19} \mathrm{~F}\left\{{ }^{1} \mathrm{H}\right\}$ NMR (Chloroformed)<smiles>COc1cc(OC)cc(C2CCC(F)CN2)c1</smiles>

$\begin{array}{lllllllllllllllll}50 & 30 & 10 & -10 & -30 & -50 & -70 & -90 & -110 & -130 & -150 & -170 & -190 & -210 & -230 & -2\end{array}$

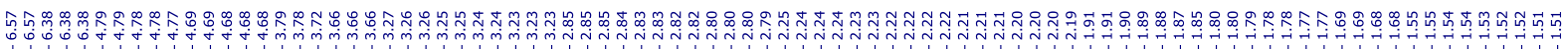
1H NMR (Chloroforme)<smiles>COc1cc(OC)cc(C2NCCCC2F)c1</smiles>
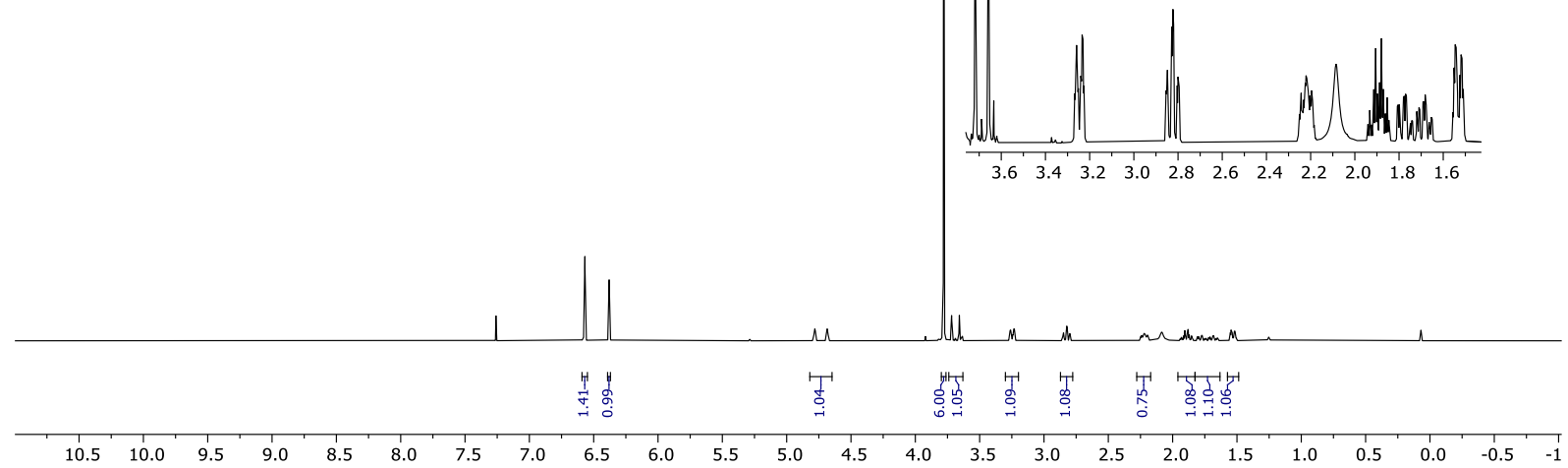


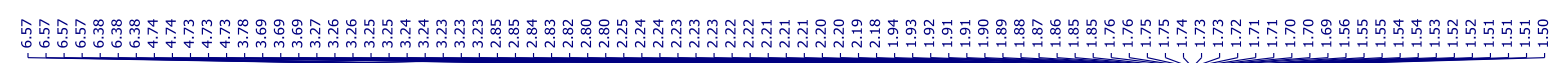

1H\{19F\} NMR (Chloroforme)
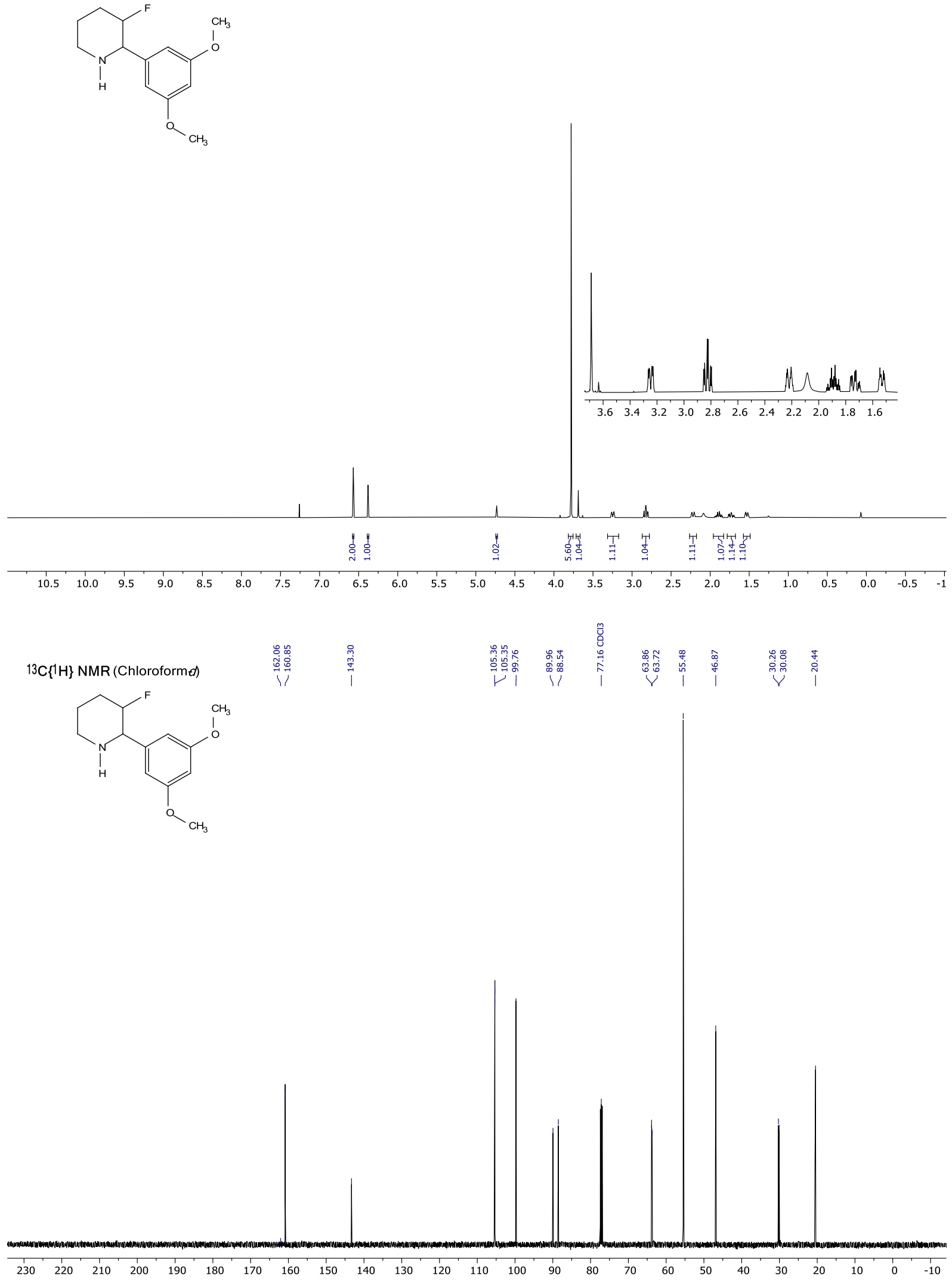

S157 

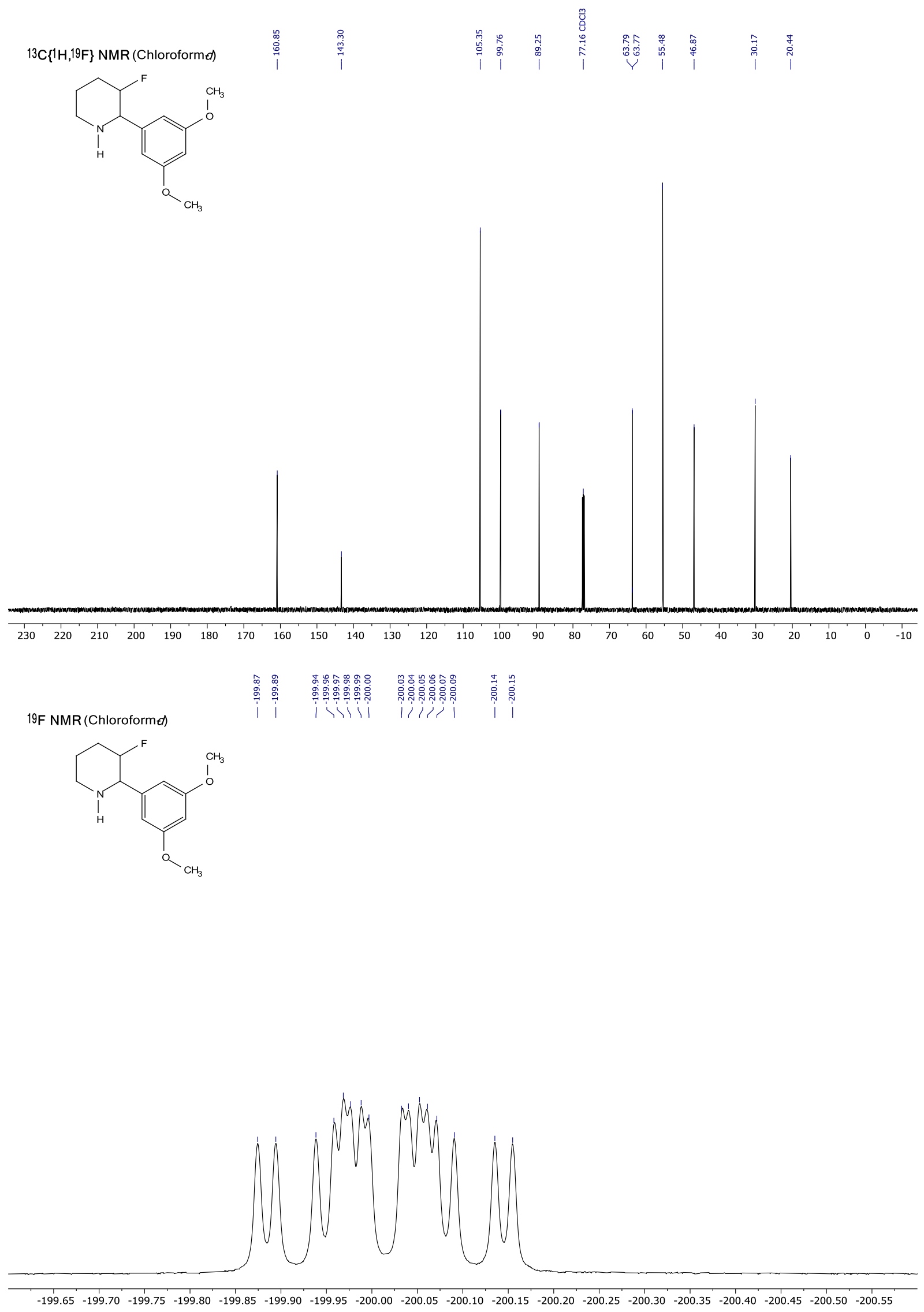

S158 
$19 \mathrm{~F}\{1 \mathrm{H}\}$ NMR (Chloroforme)

iั.

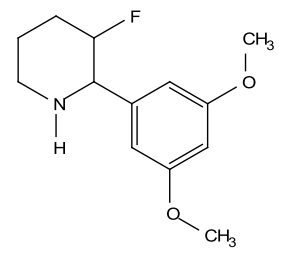

\begin{tabular}{lllllllllllllllllllllllllllllllllll}
\hline 50 & 40 & 30 & 20 & 10 & 0 & -10 & -20 & -30 & -40 & -50 & -60 & -70 & -80 & -90 & -100 & -110 & -120 & -130 & -140 & -150 & -160 & -170 & -180 & -190 & -200 & -210 & -220 & -230 & -240 & $-2 !$
\end{tabular}

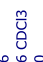

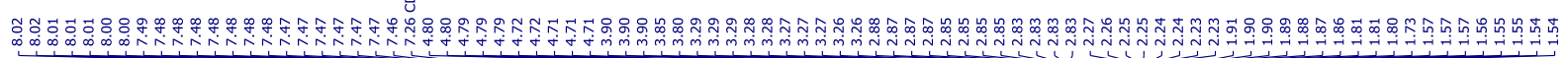

1H NMR (Chloroformed)
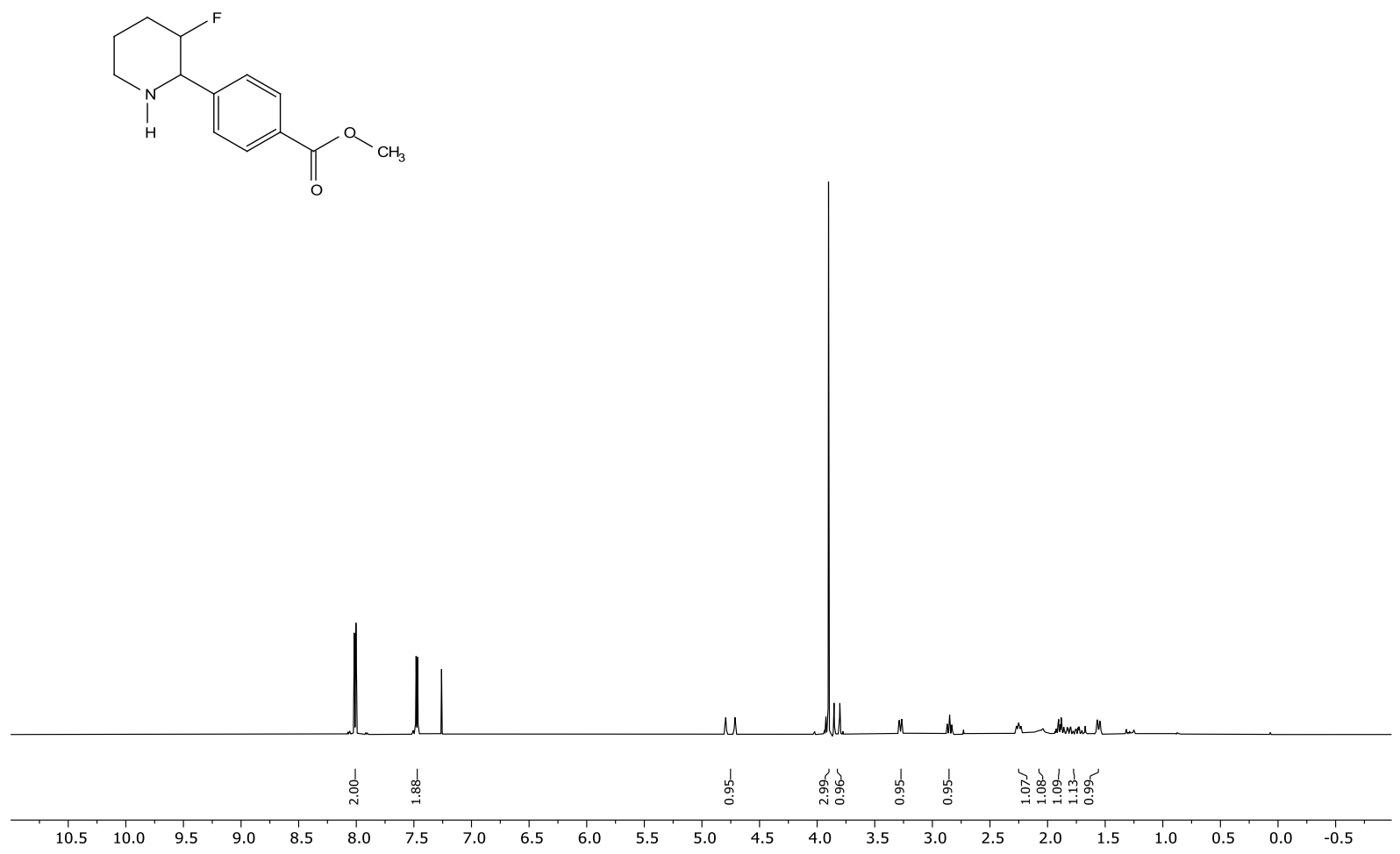

S159 
萮

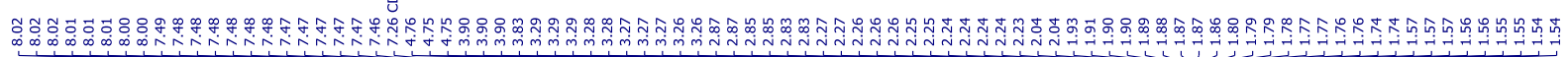

$1 \mathrm{H}\{19 \mathrm{~F}\}$ NMR (Chloroforme)
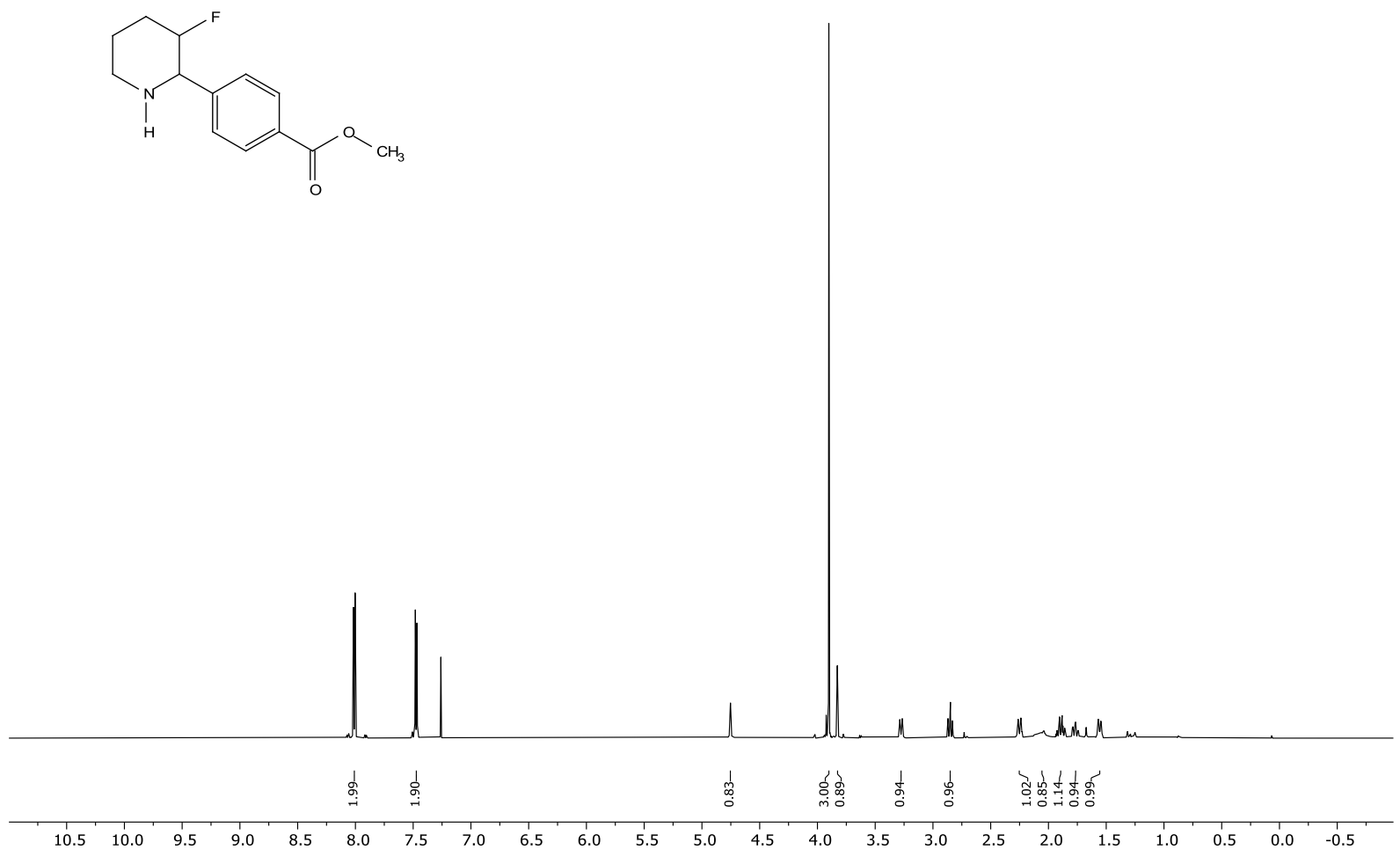

$13 \mathrm{C}\{1 \mathrm{H}\}$ NMR (Chloroformet)

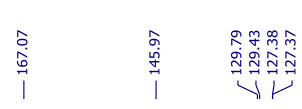

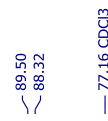

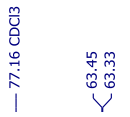

భ.

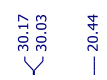
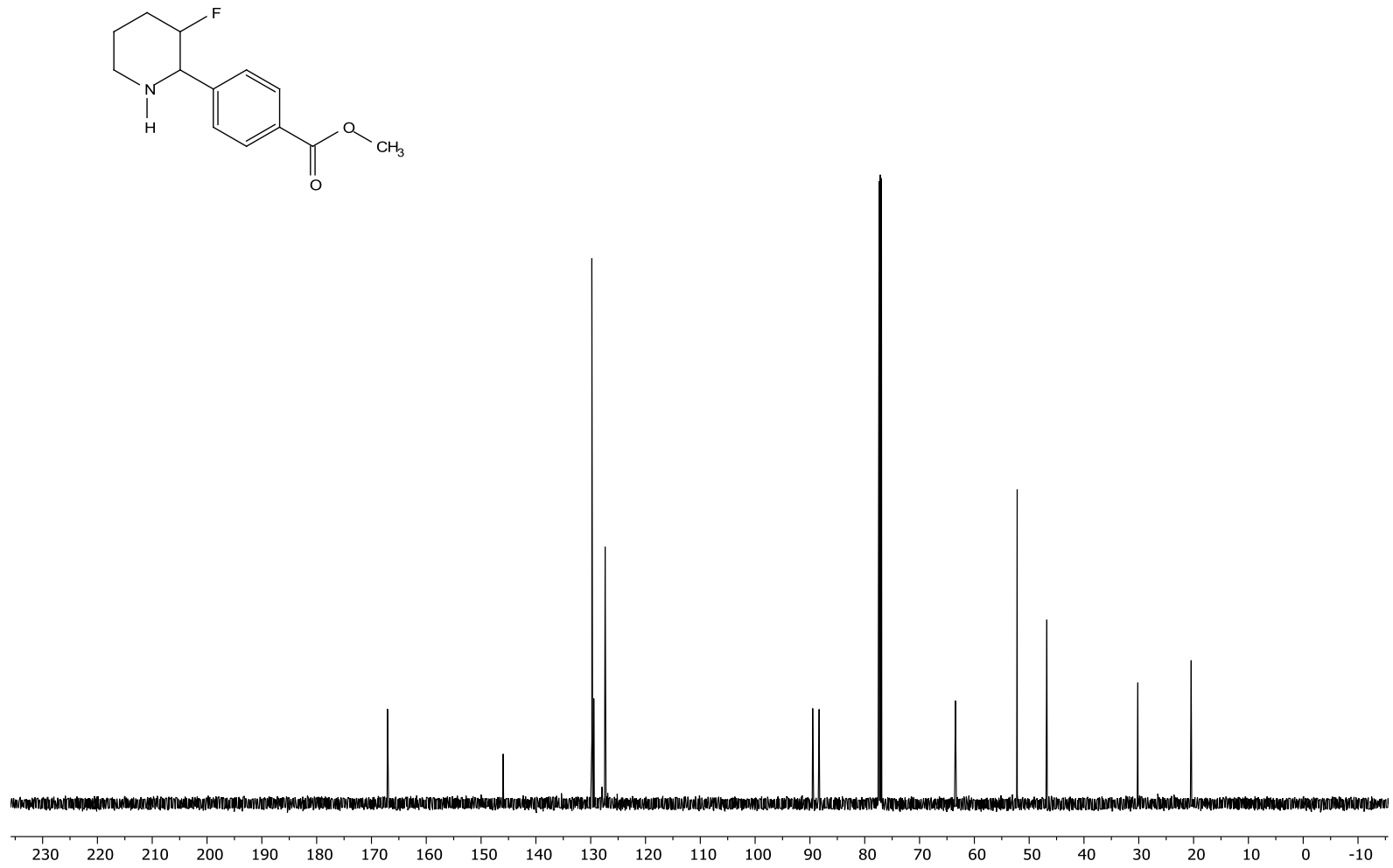

$\mathrm{S} 160$ 

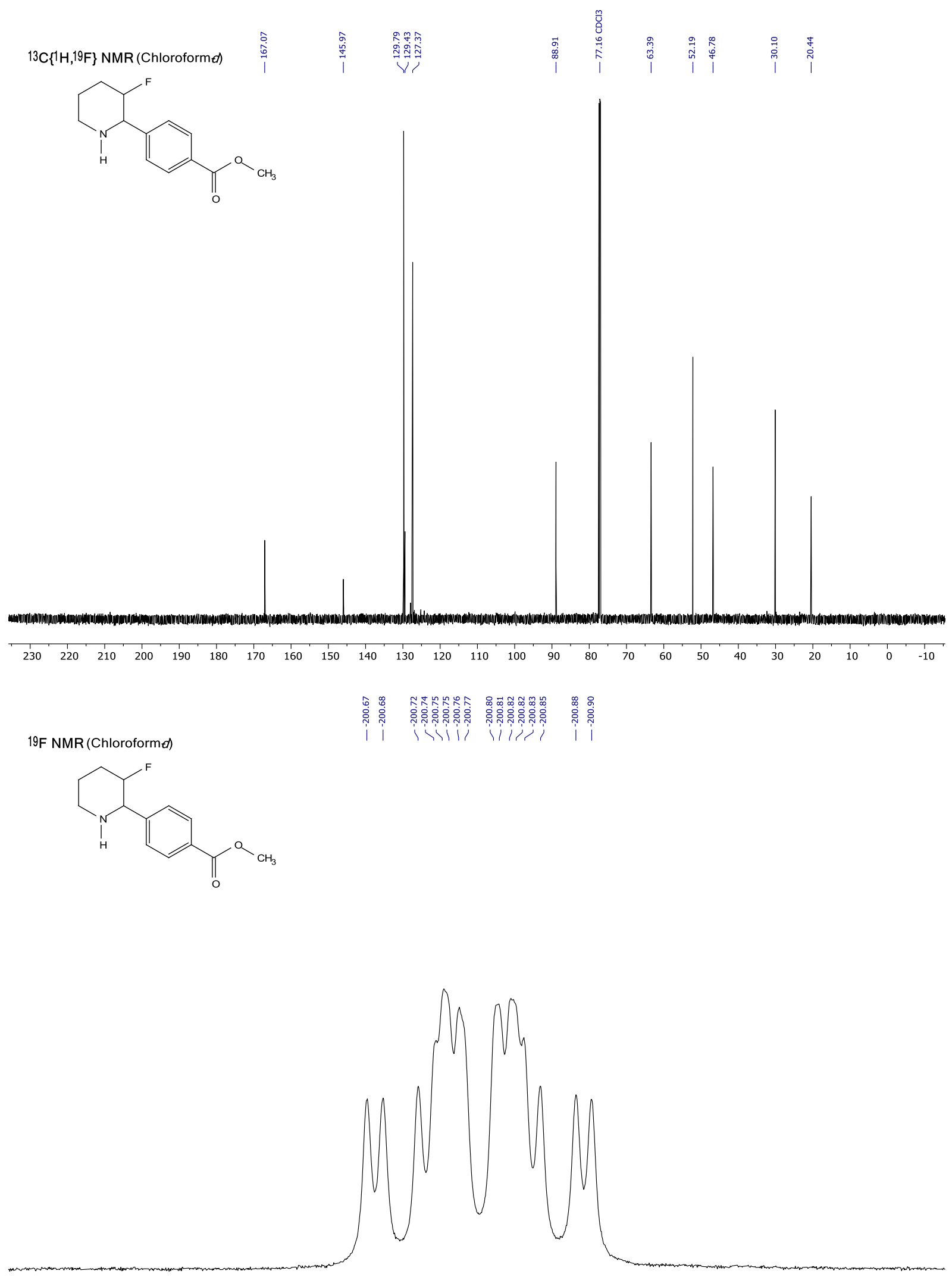

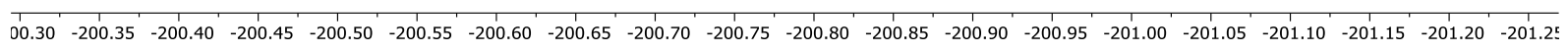

S161 
19F $\{1 \mathrm{H}\}$ NMR (Chloroformed)
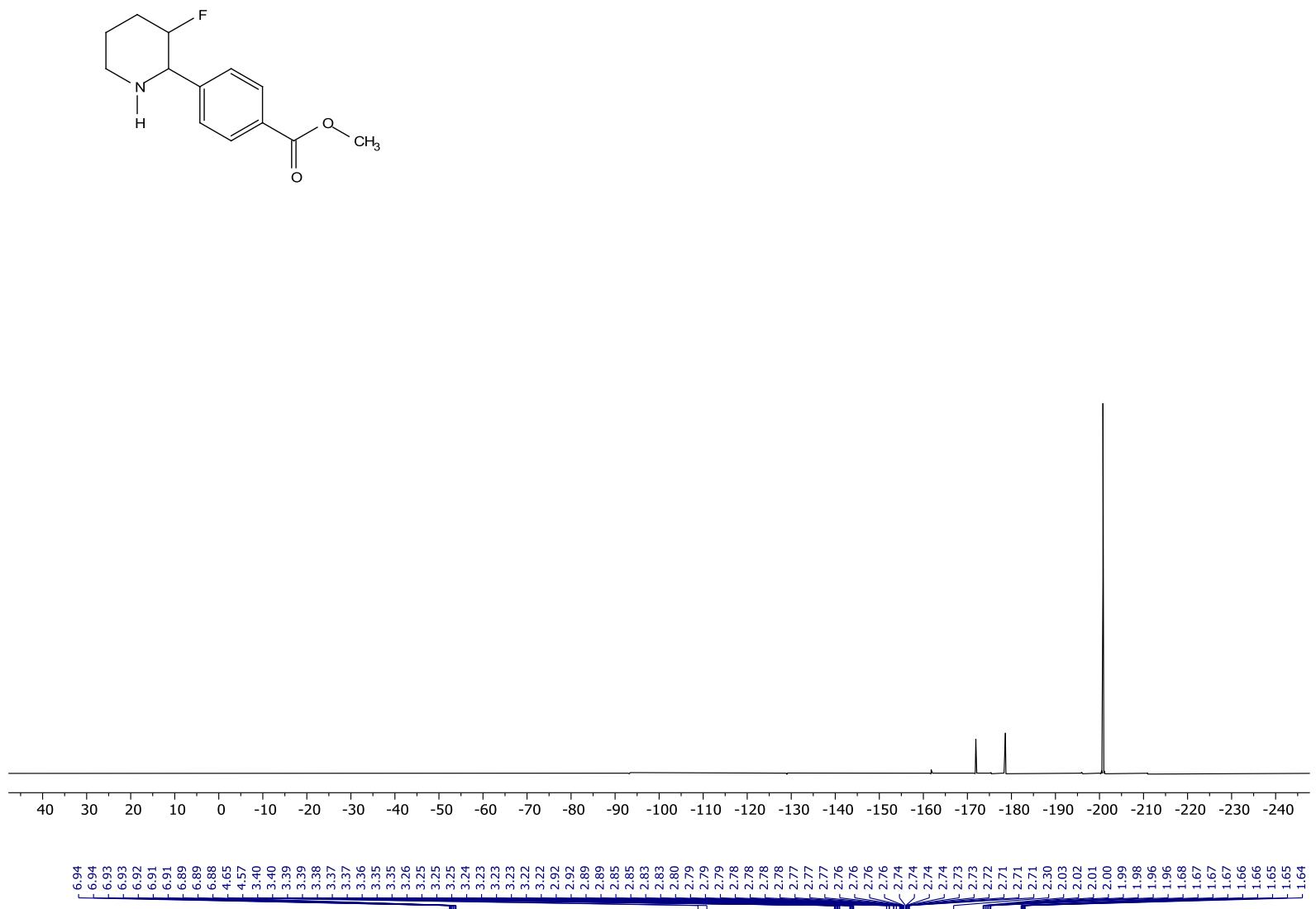

1H NMR (Chloroformed)
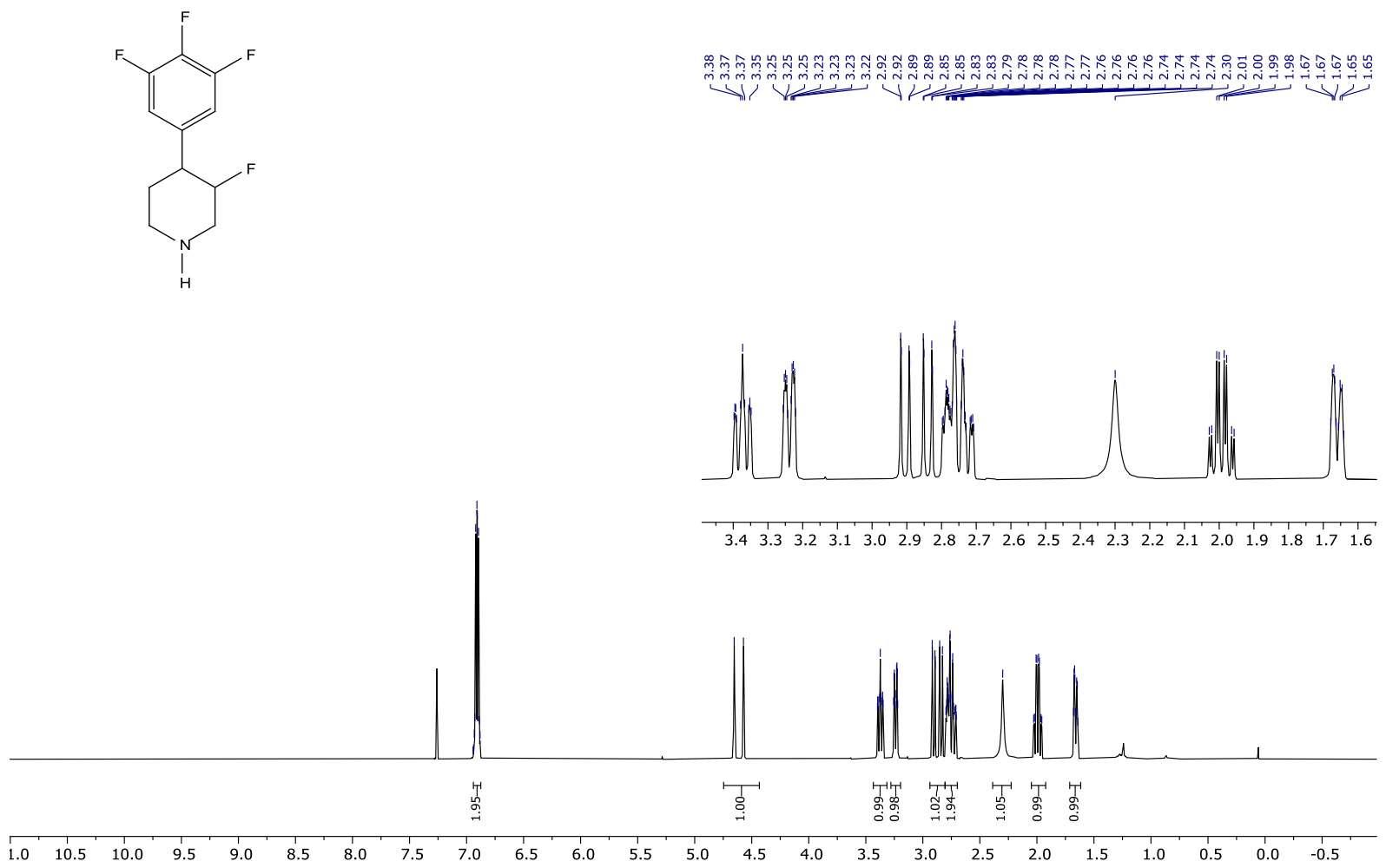

S162 

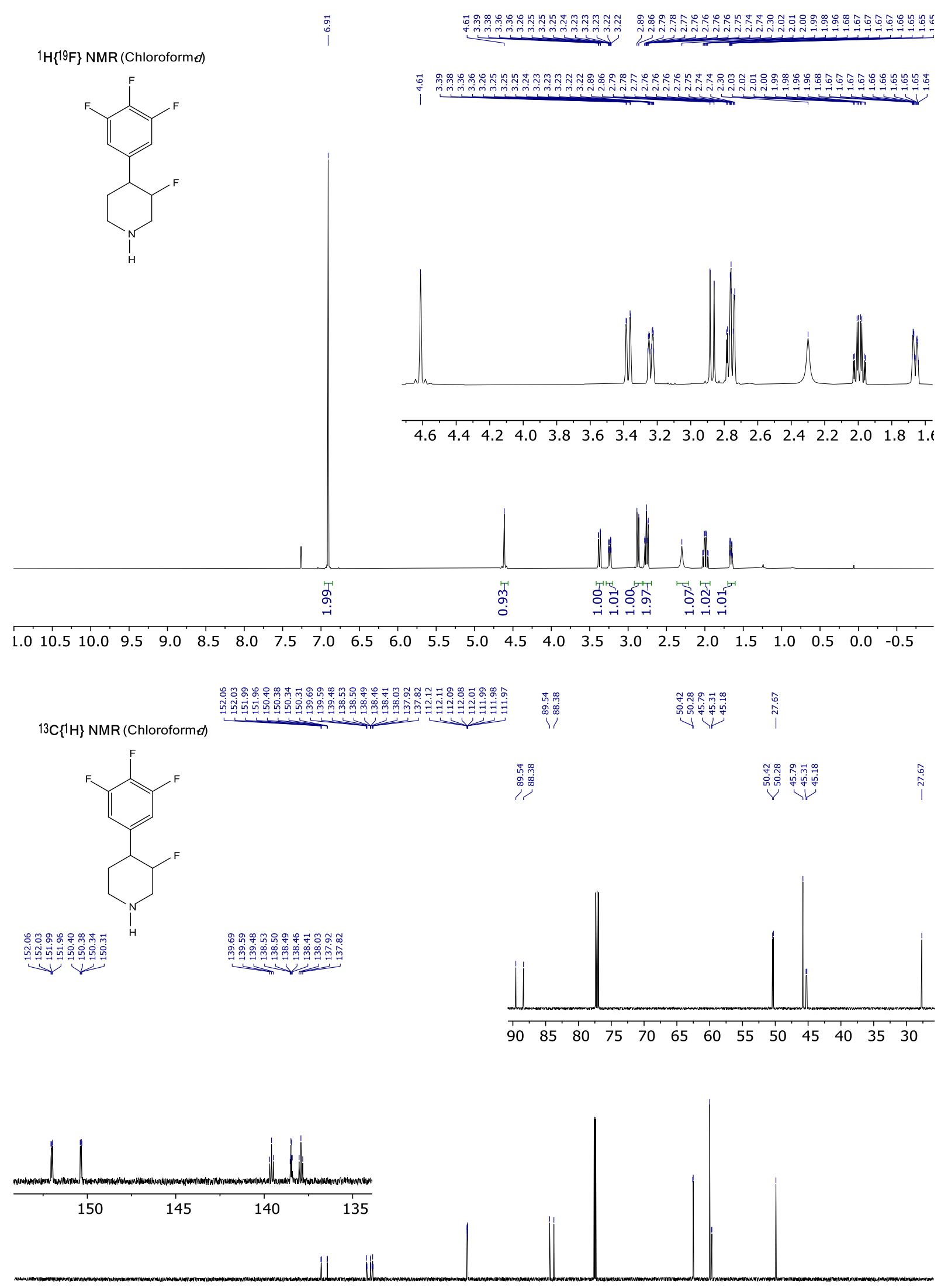

$\begin{array}{llllllllllllllllllllllllll}230 & 220 & 210 & 200 & 190 & 180 & 170 & 160 & 150 & 140 & 130 & 120 & 110 & 100 & 90 & 80 & 70 & 60 & 50 & 40 & 30 & 20 & 10 & 0 & -10\end{array}$ 


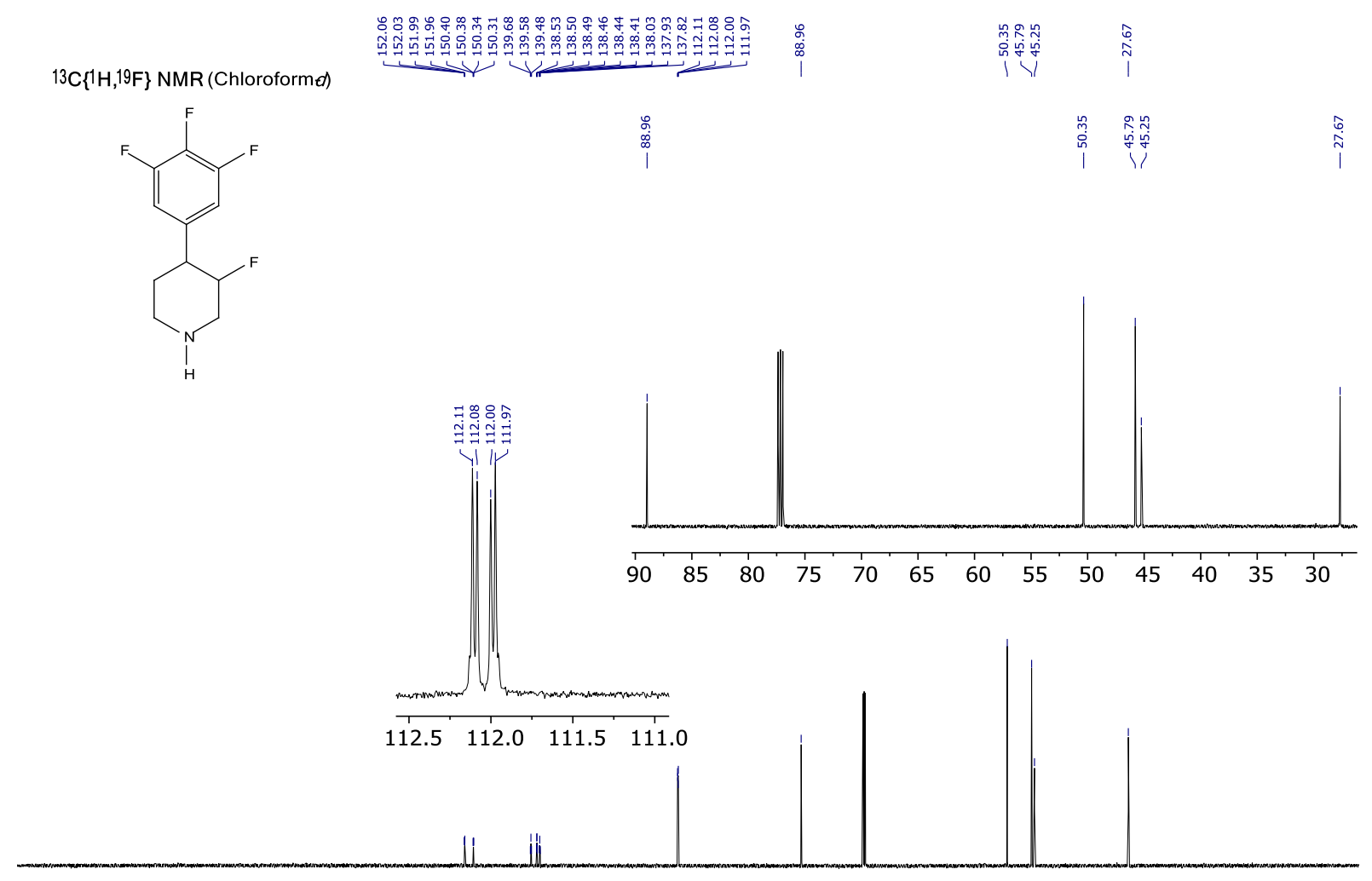

$\begin{array}{lllllllllllllllllllllllll}230 & 220 & 210 & 200 & 190 & 180 & 170 & 160 & 150 & 140 & 130 & 120 & 110 & 100 & 90 & 80 & 70 & 60 & 50 & 40 & 30 & 20 & 10 & 0 & -10\end{array}$ 19F NMR (Chloroforme)

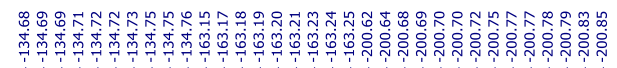<smiles>Fc1cc(C2CCNCC2F)cc(F)c1F</smiles>

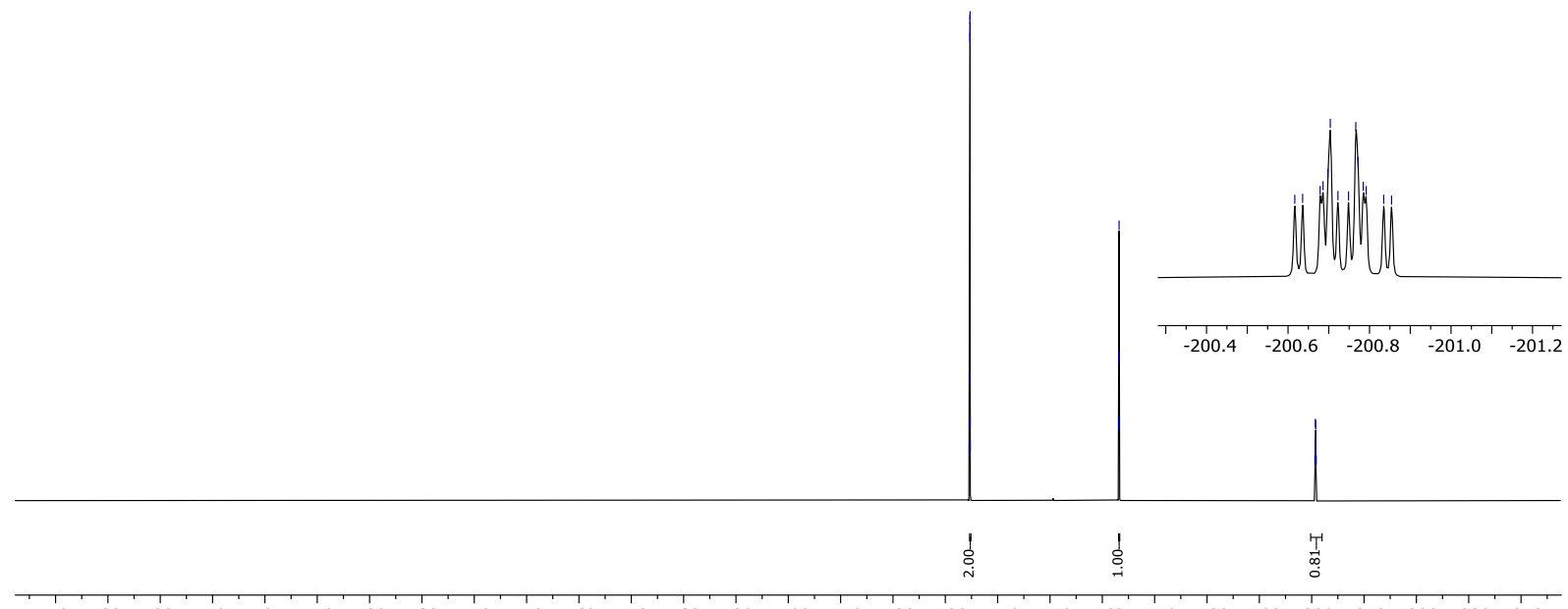


19F $\{1 \mathrm{H}\}$ NMR (Chloroformel)

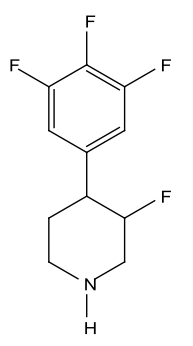

ن

议

ì̃

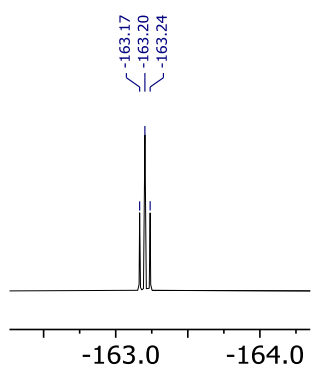

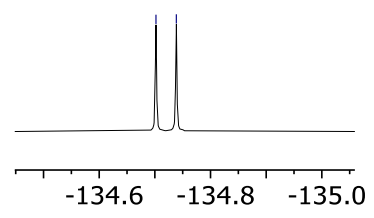
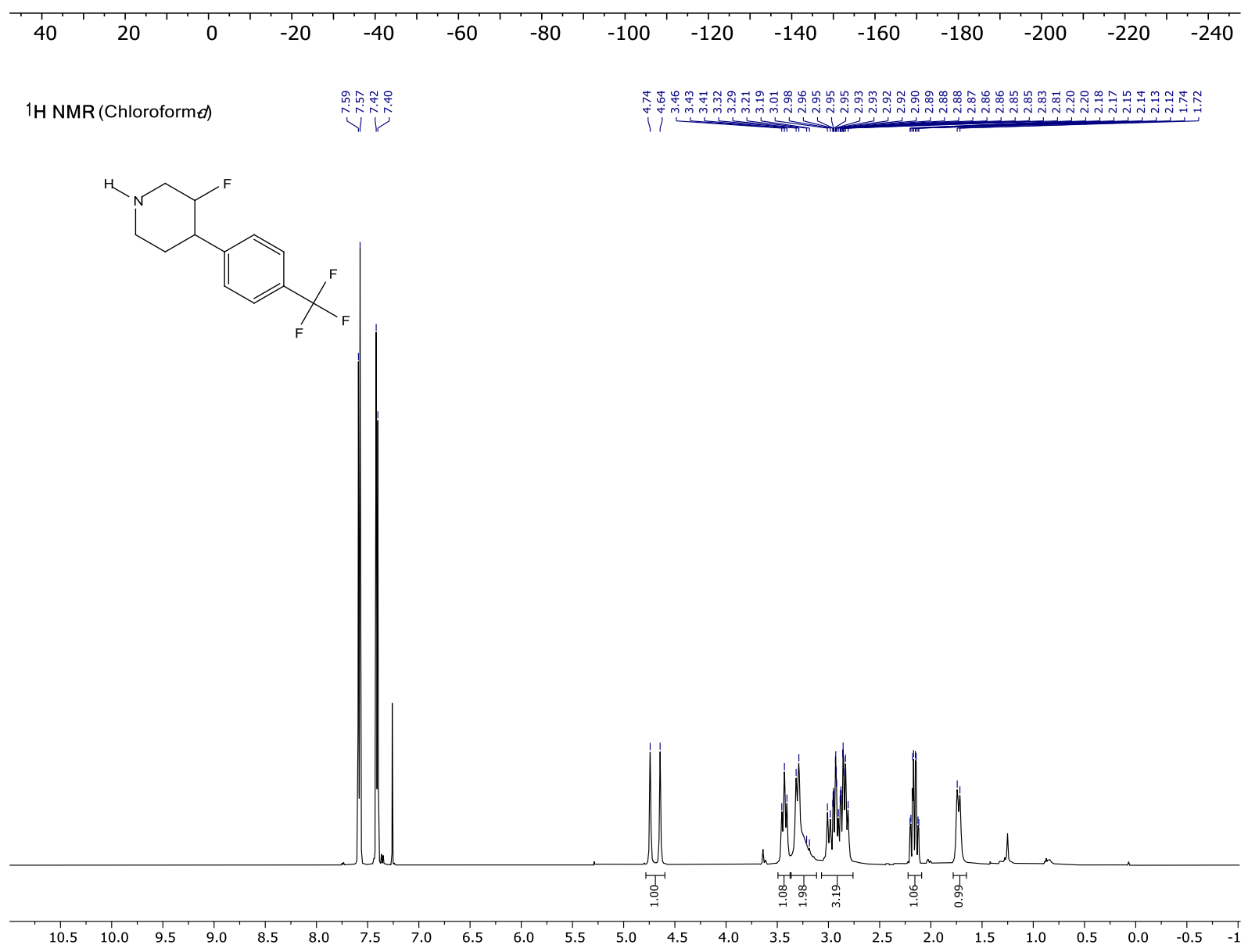

S165 
$1 \mathrm{H}\{19 \mathrm{~F}\}$ NMR (Chloroformed)

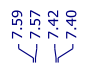

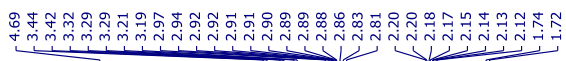

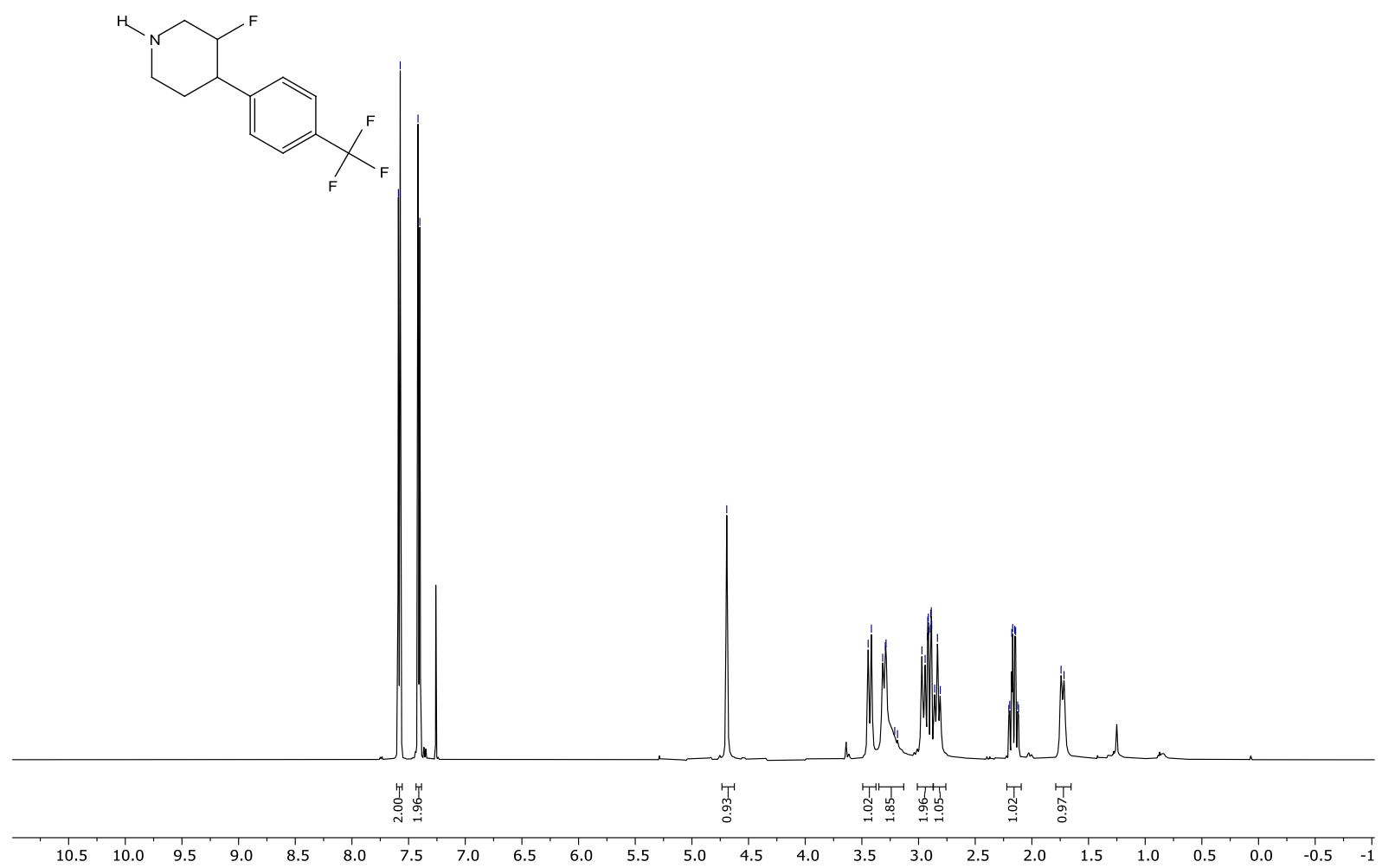

${ }^{13} \mathrm{C}\{1 \mathrm{H}\}$ NMR (Chloroformed)

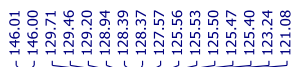

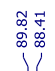

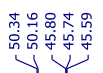

$\stackrel{\stackrel{\sim}{N}}{\stackrel{1}{1}}$
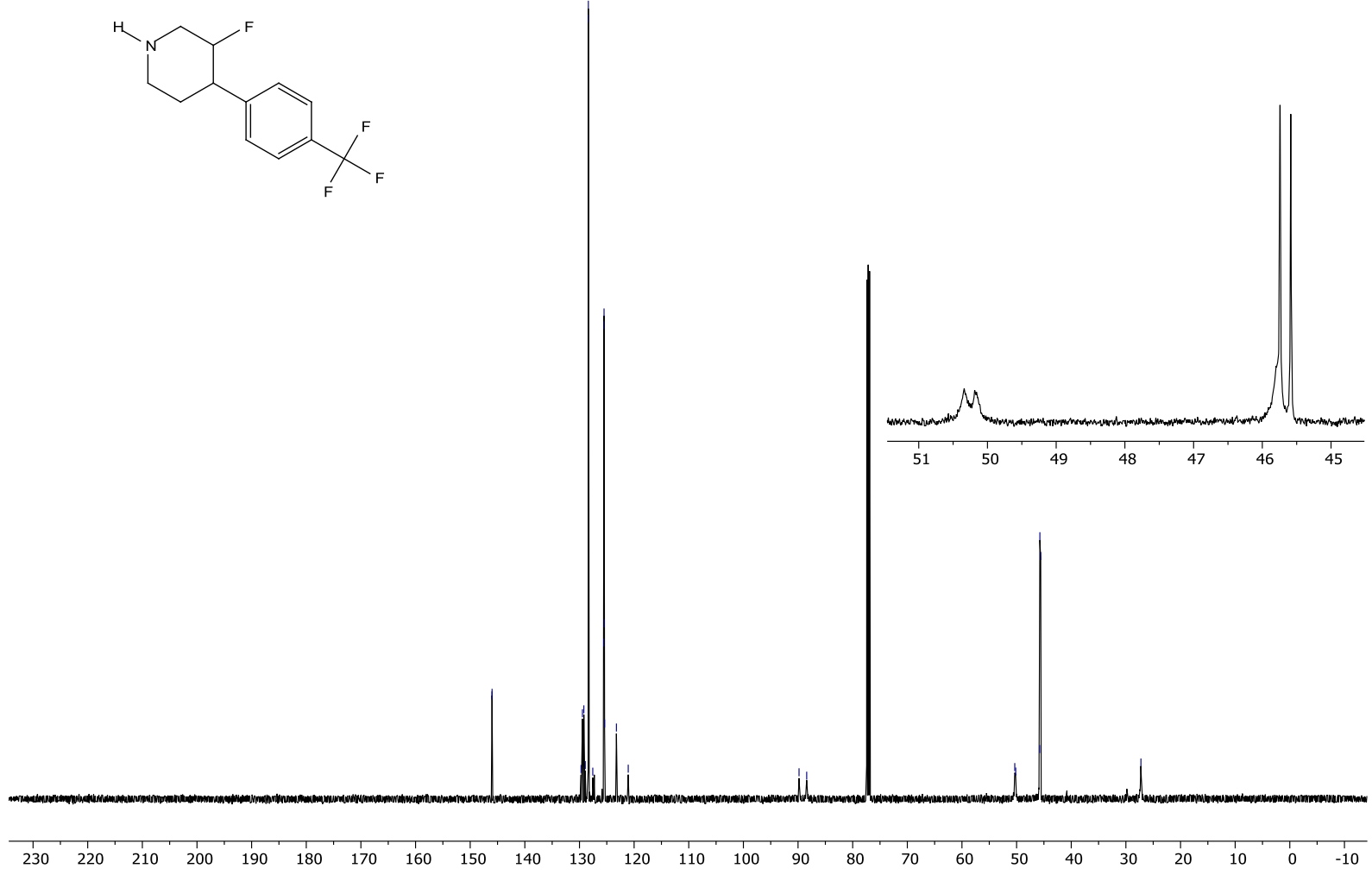

S166 
${ }^{13} \mathrm{C}\left\{{ }^{1} \mathrm{H}, 19 \mathrm{~F}\right\}$ NMR (Chloroforme)

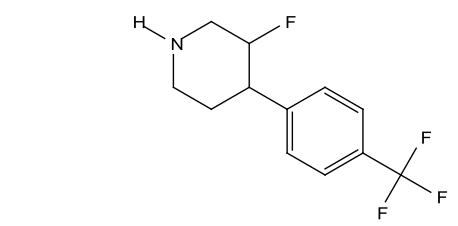

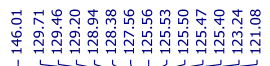

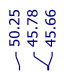

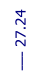

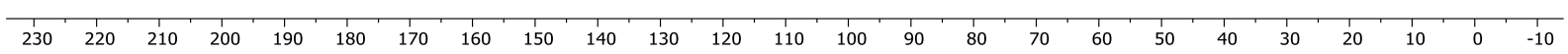

19F NMR (Chloroforme)

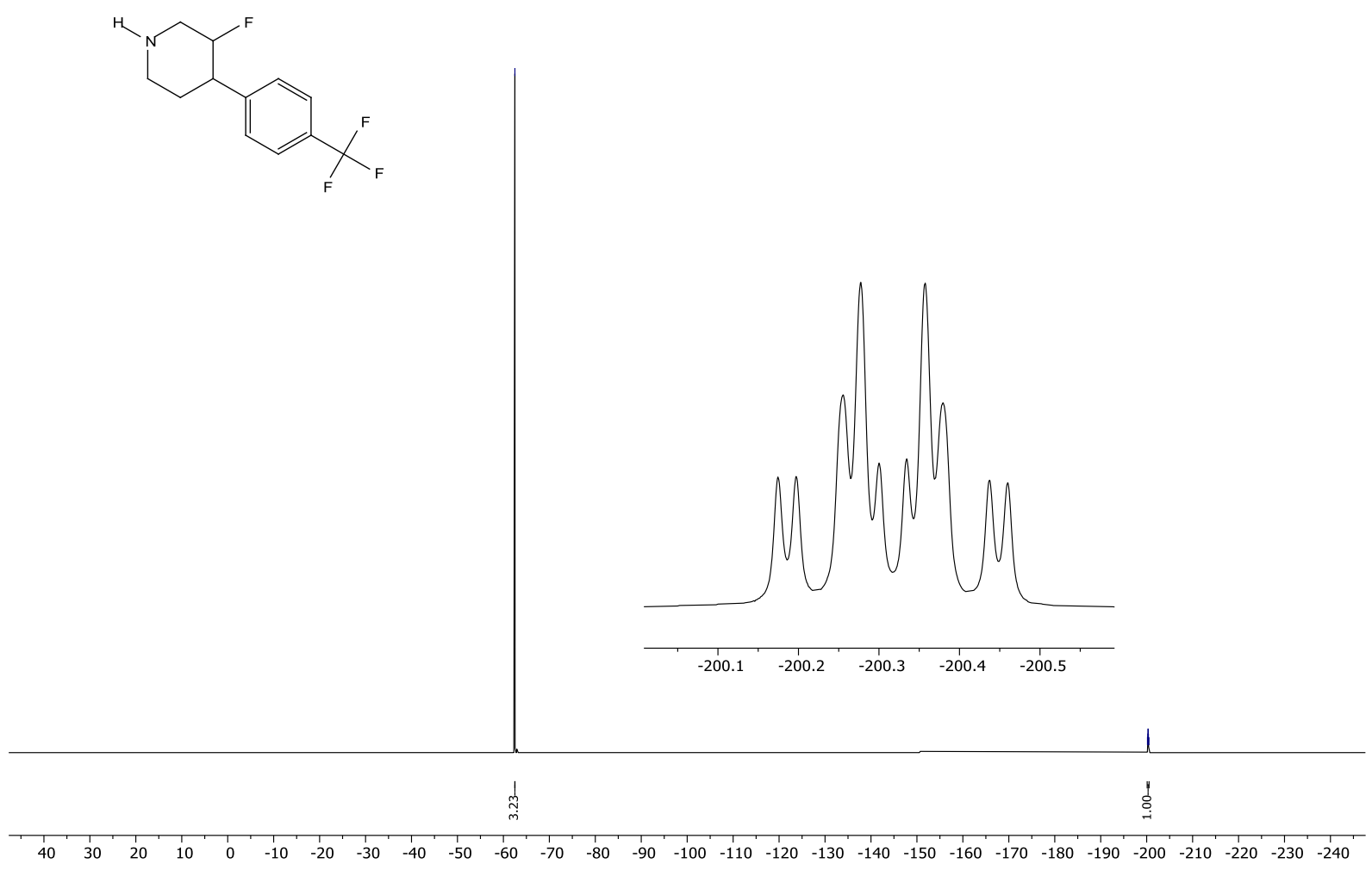

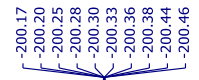

S167 
${ }^{19} \mathrm{~F}\{\mathrm{l} H \mathrm{H}\} \mathrm{NMR}$ (Chloroformed)

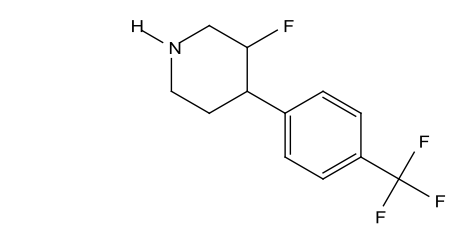

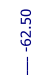

ก̃

TH NMR (Chloroformed)

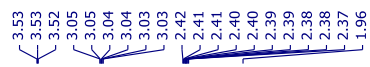
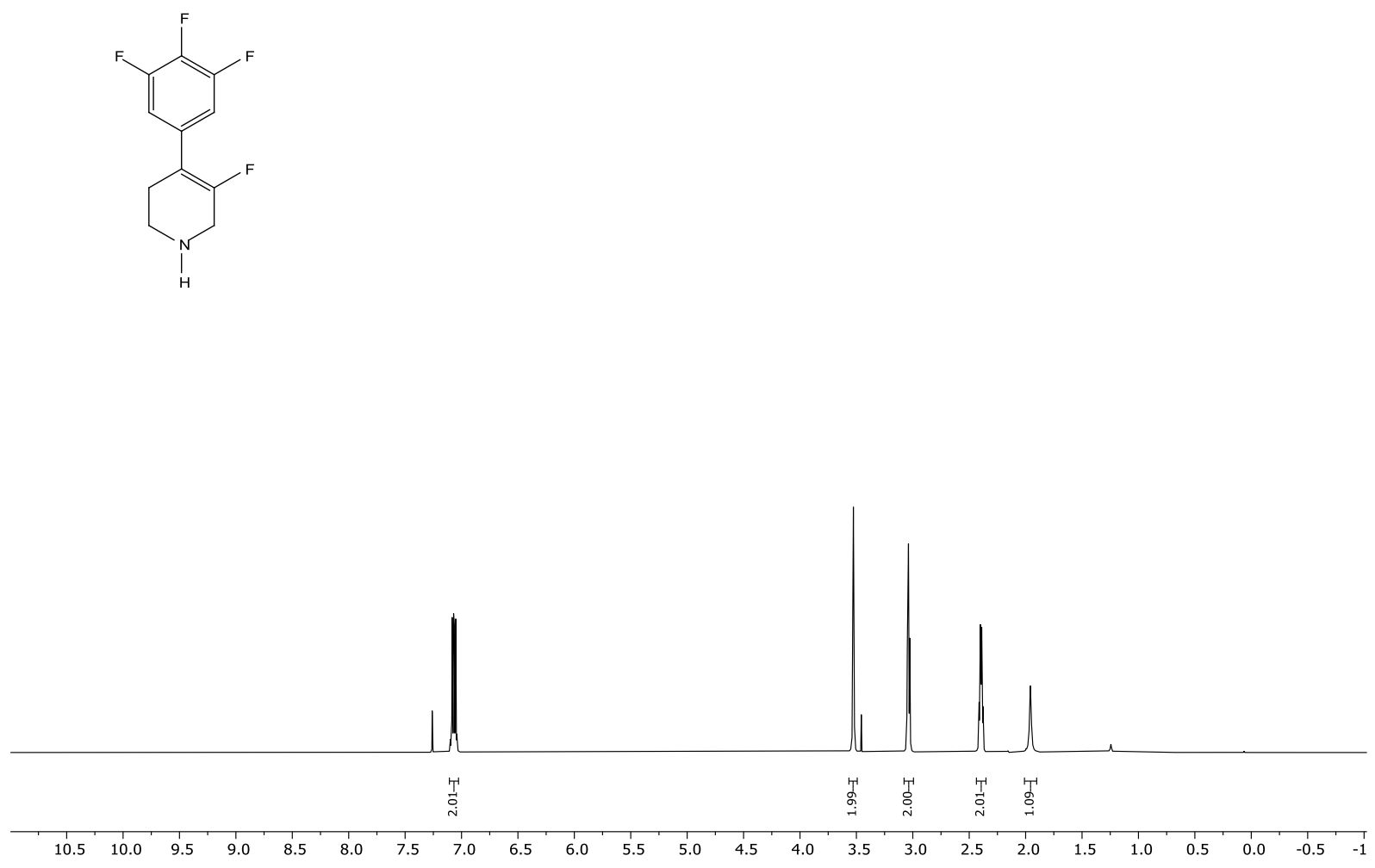

S168 
<smiles>FC1=C(c2cc(F)c(F)c(F)c2)CCNC1</smiles>

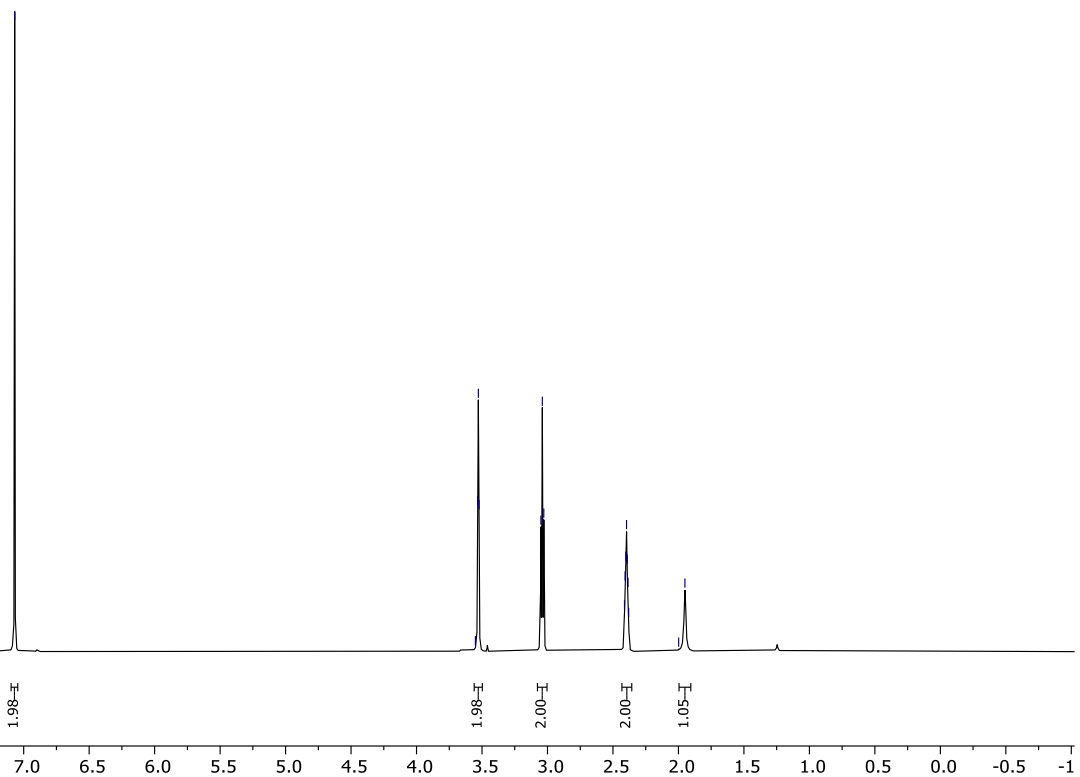

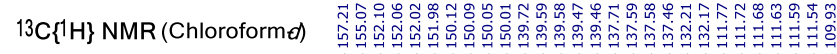

Vִ<smiles>FC1=C(c2cc(F)c(F)c(F)c2)CCNC1</smiles> 

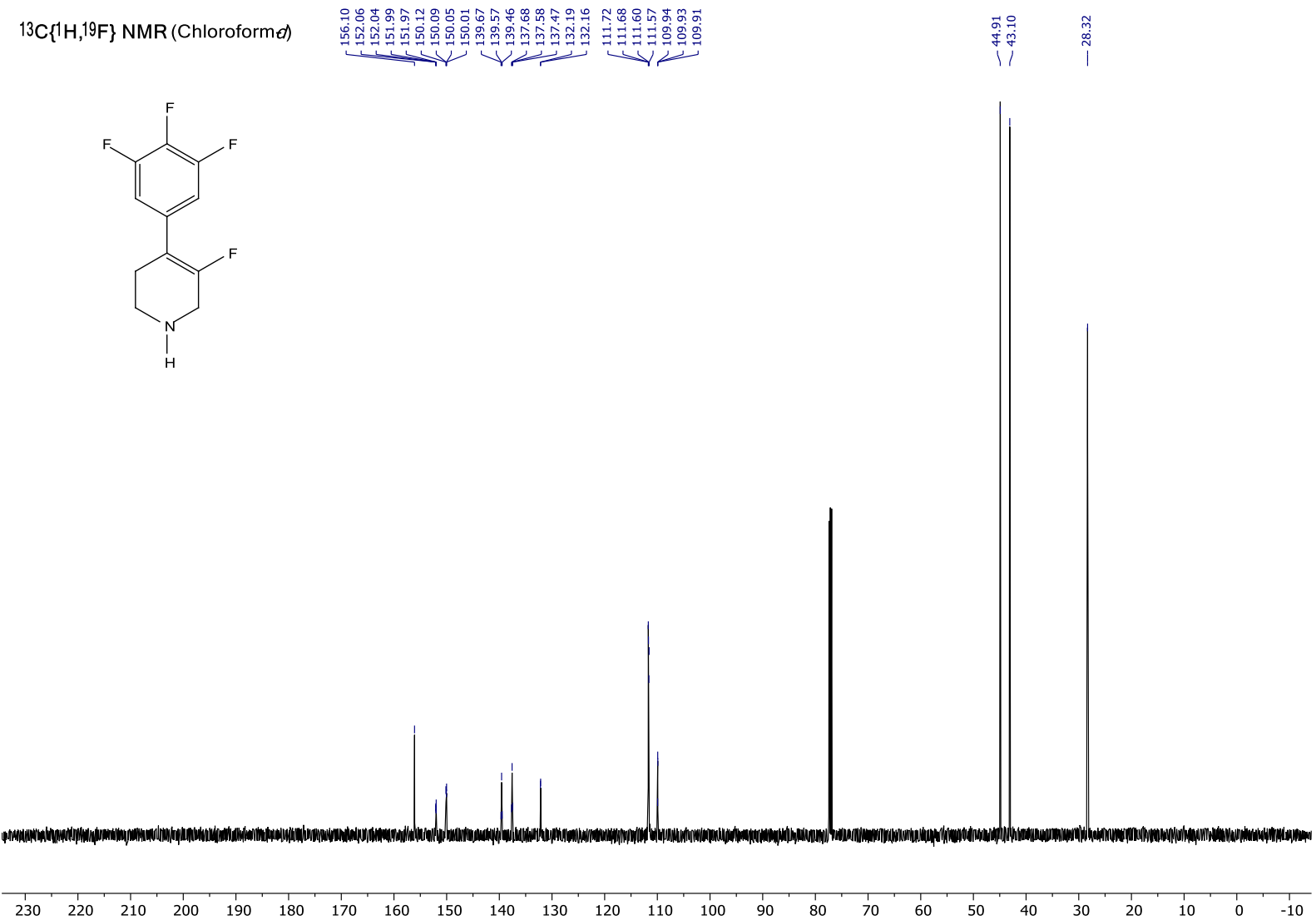

19F NMR (Chloroforme)

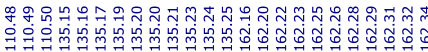

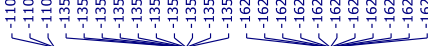

(C)
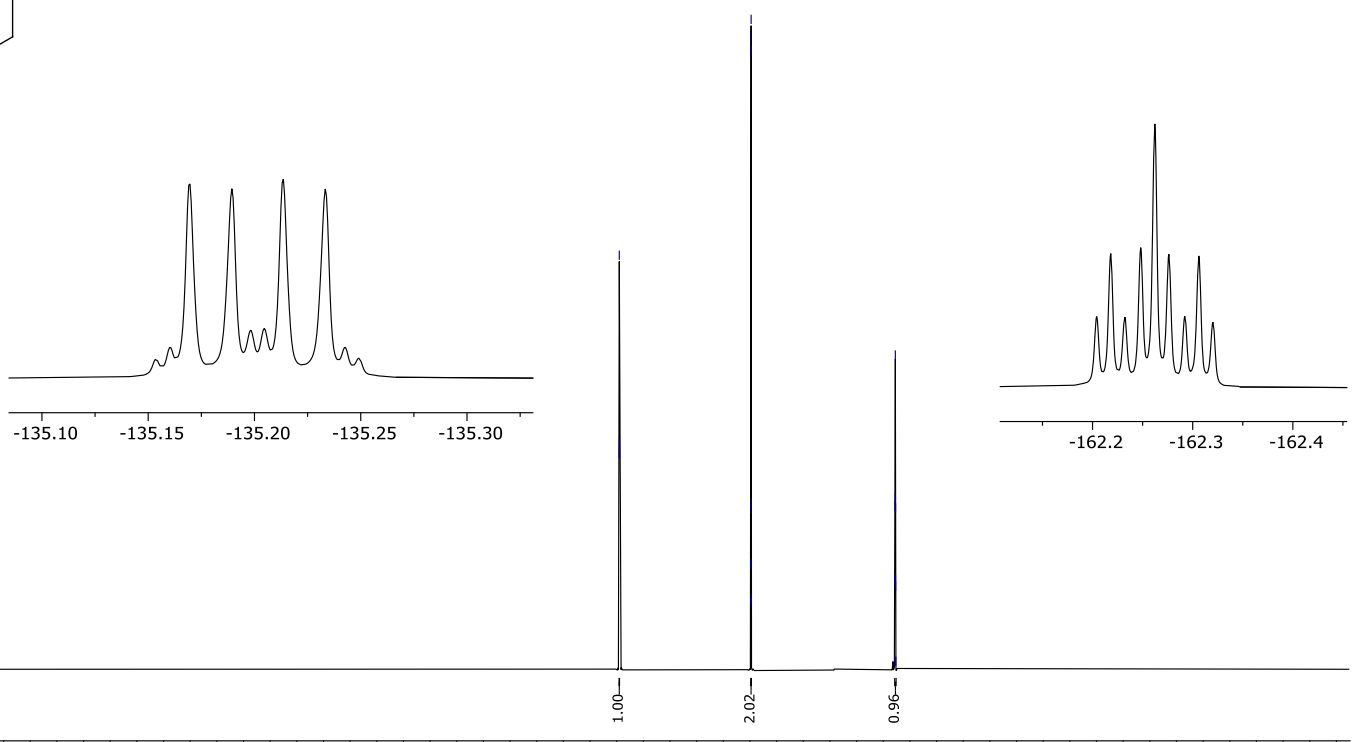

$\begin{array}{llllllllllllllllllllllllllllllllll}40 & 30 & 20 & 10 & 0 & -10 & -20 & -30 & -40 & -50 & -60 & -70 & -80 & -90 & -100 & -110 & -120 & -130 & -140 & -150 & -160 & -170 & -180 & -190 & -200 & -210 & -220 & -230 & -240\end{array}$ 
${ }^{19} \mathrm{~F}\{1 \mathrm{H}\}$ NMR (Chloroformed)

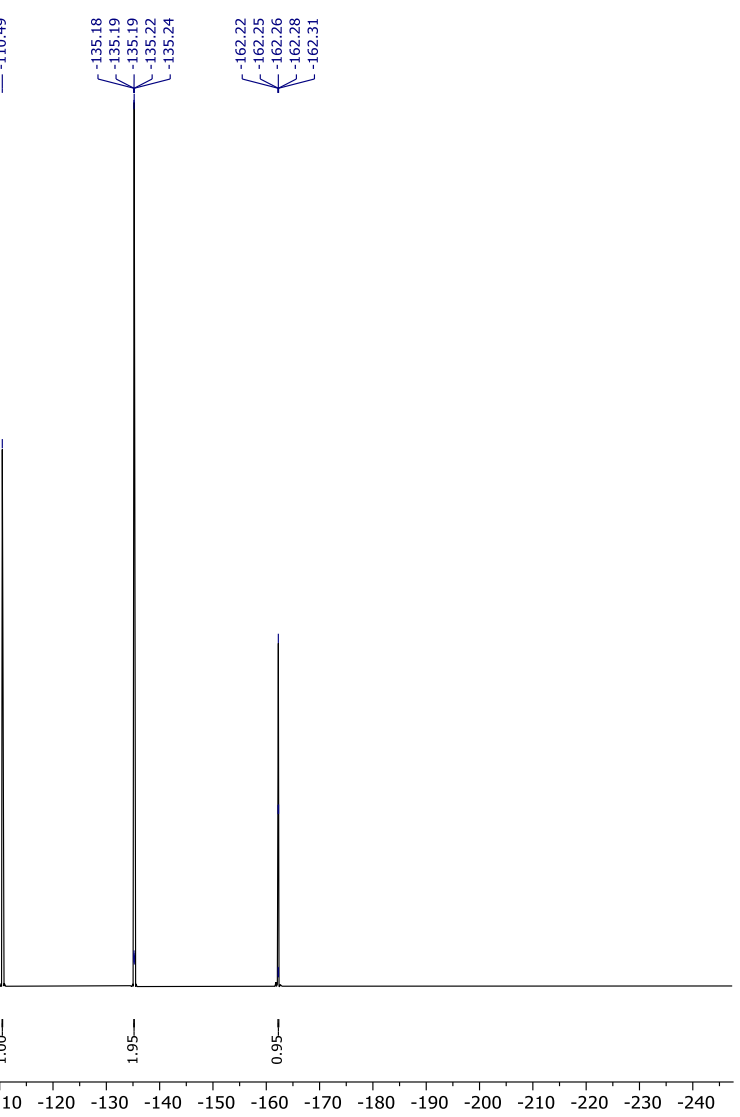

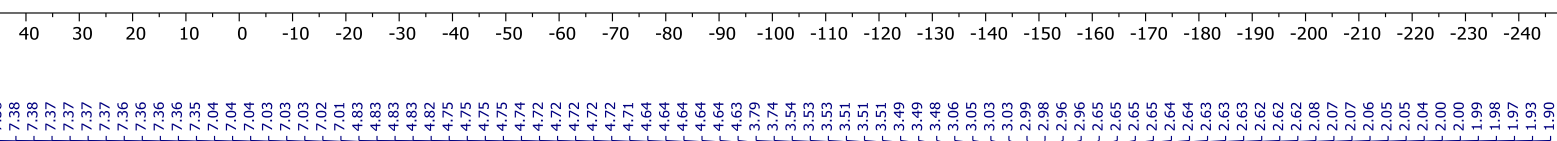

1H NMR (Chlorofor me)
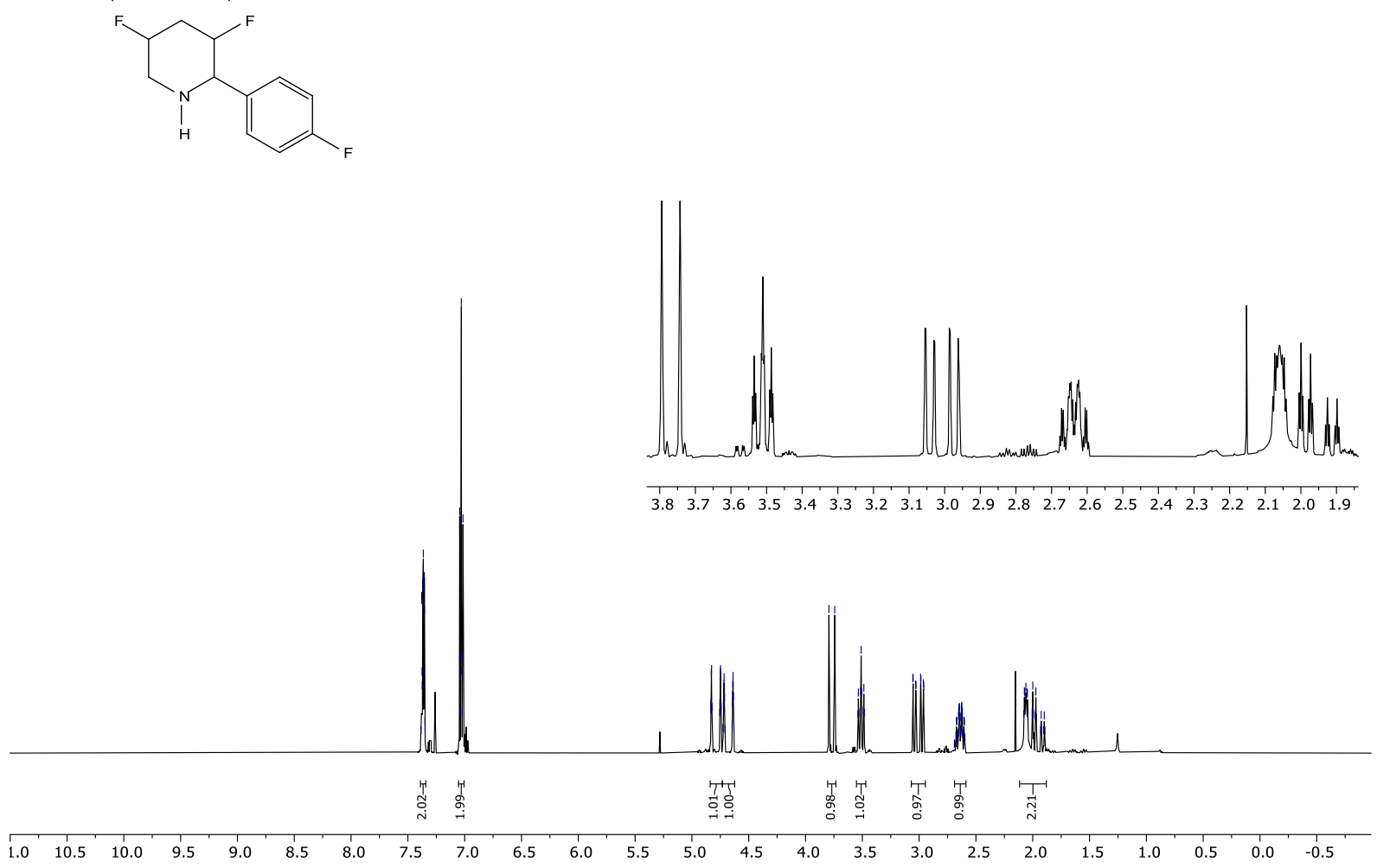

S171 


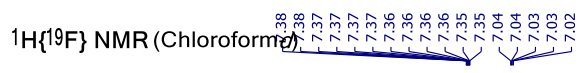

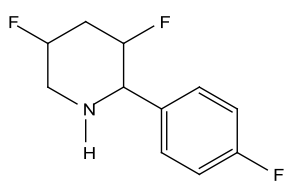

|

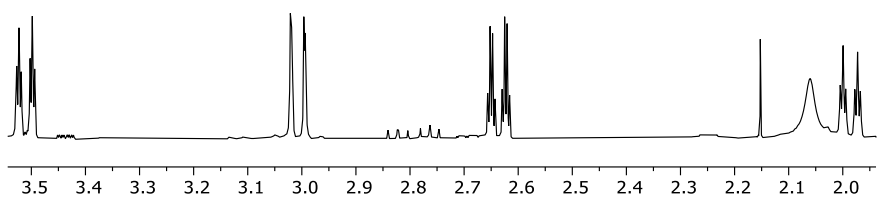

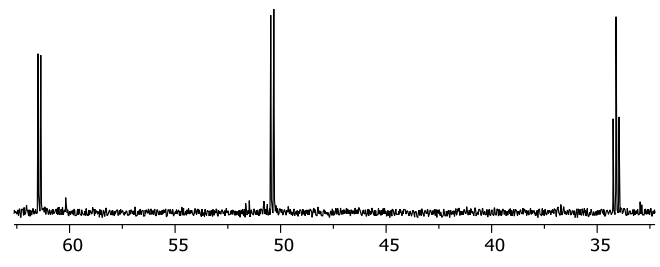

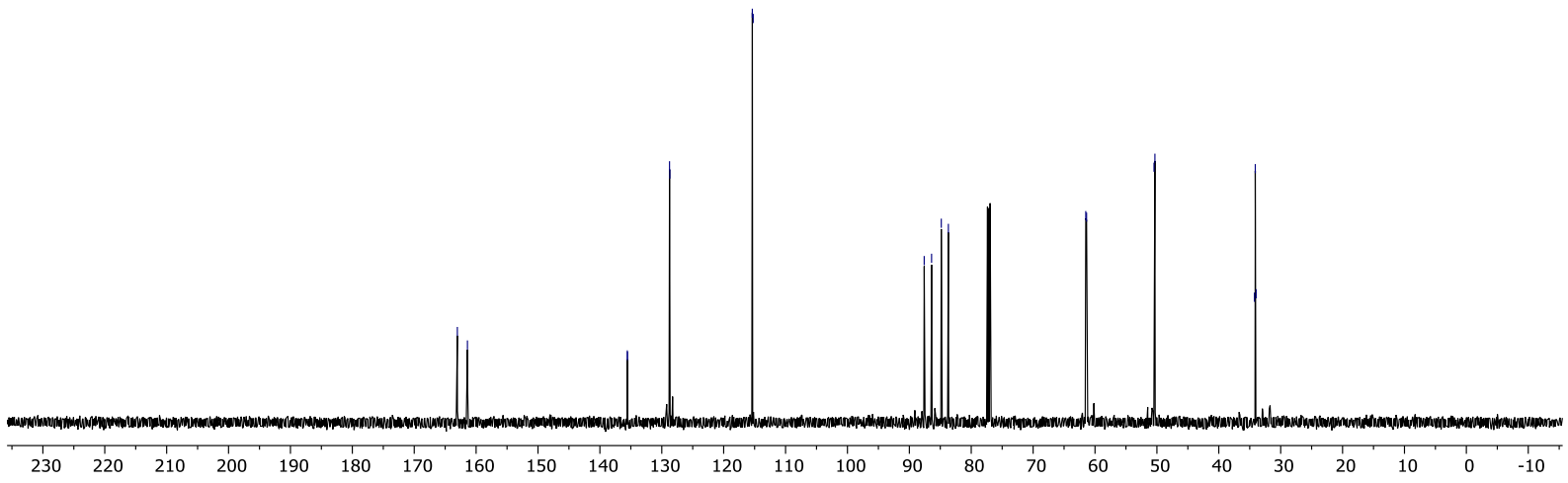

S172 
<smiles>Fc1ccc(C2NCC(F)CC2F)cc1</smiles>

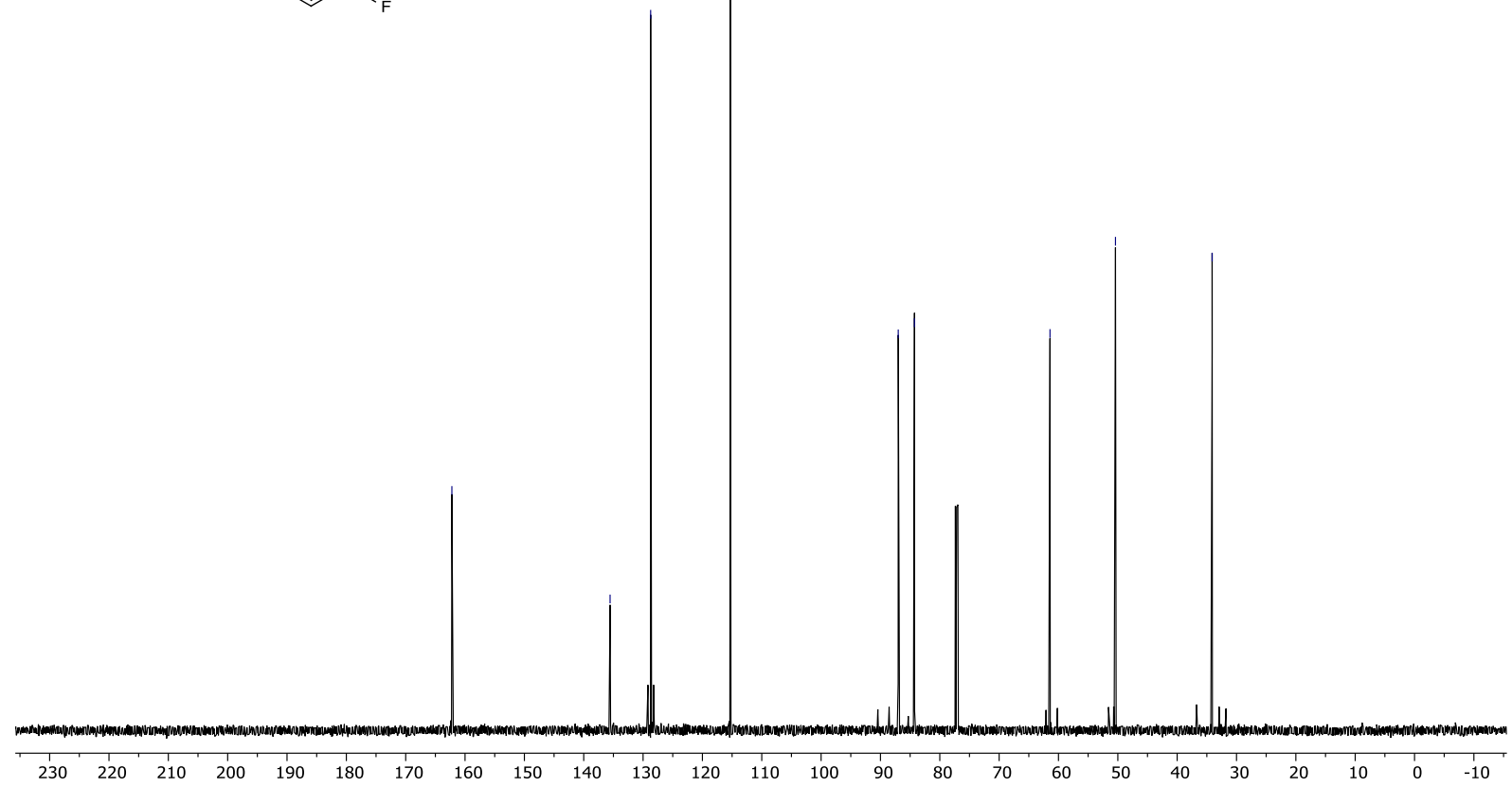

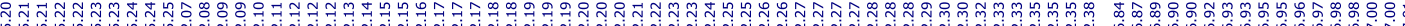

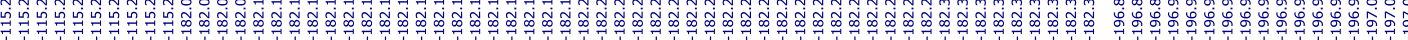
19F NMR (Chloroforme)<smiles>Fc1ccc(C2NCC(F)CC2F)cc1</smiles>
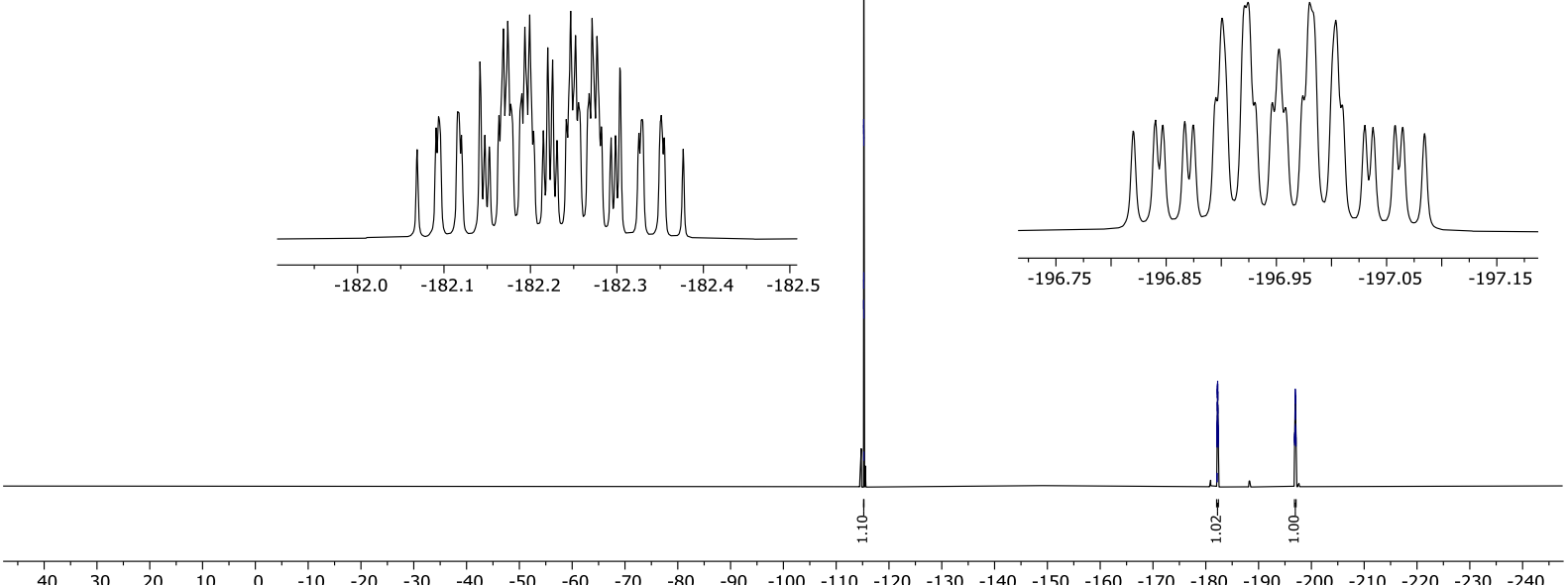
${ }^{19} \mathrm{~F}\{\mathrm{l} H\}$ NMR (Chloroformed)

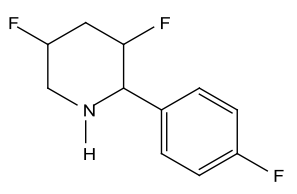

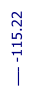

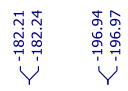

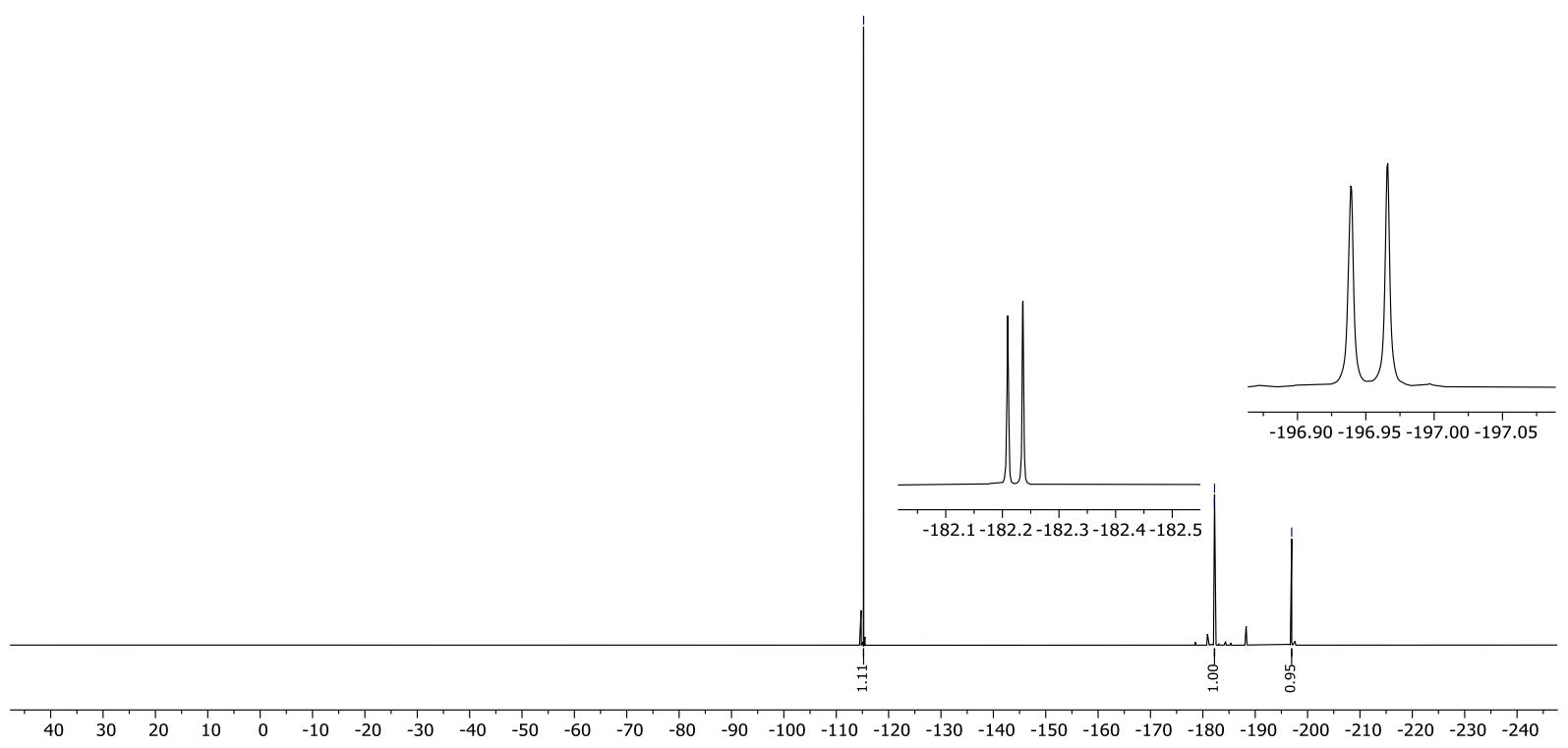

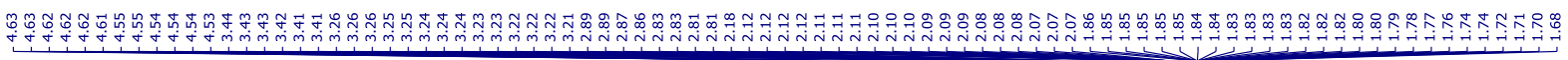

1H NMR (Chlorofor me)
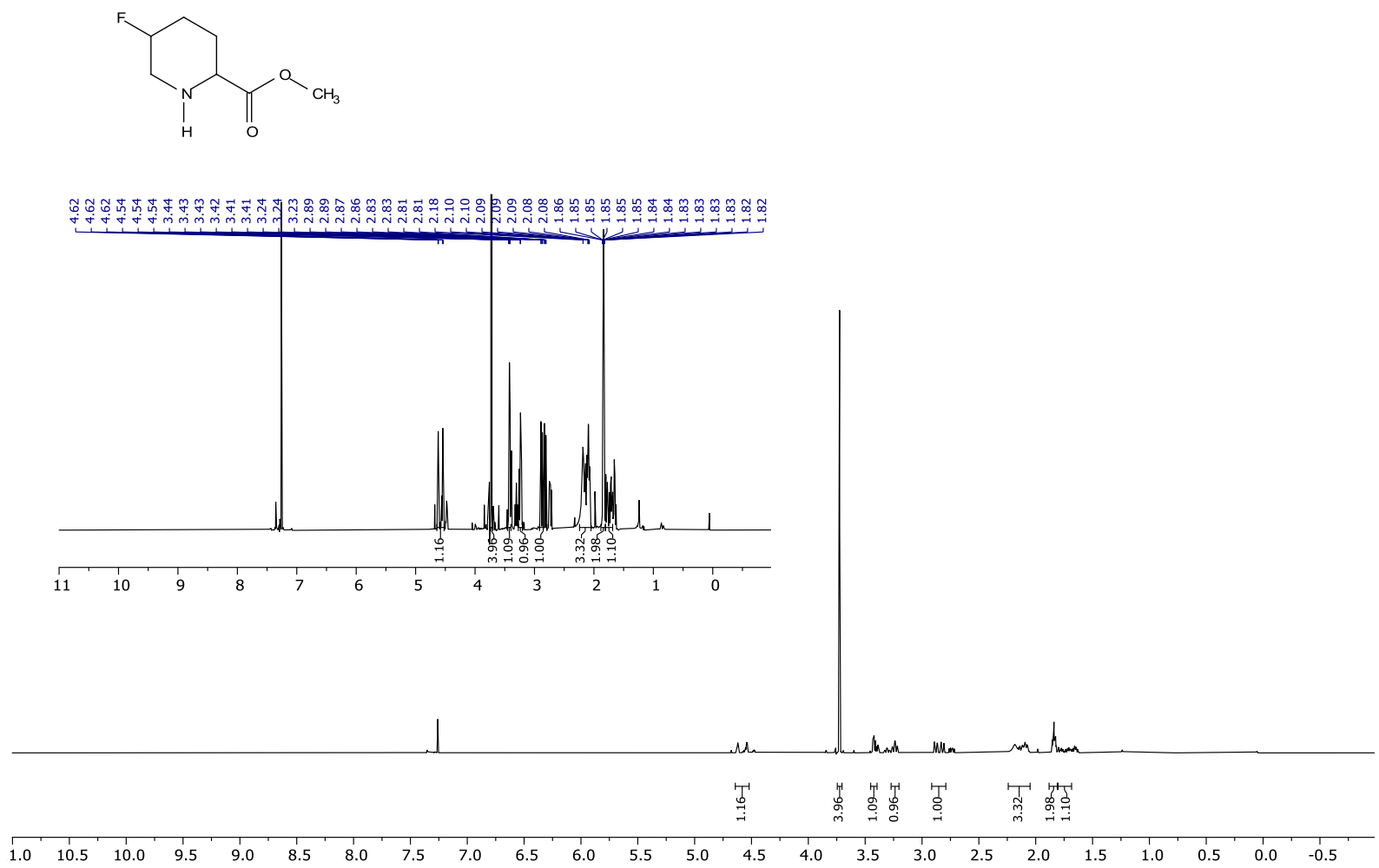

S174 
<smiles>COC(=O)C1CCC(F)CN1</smiles>

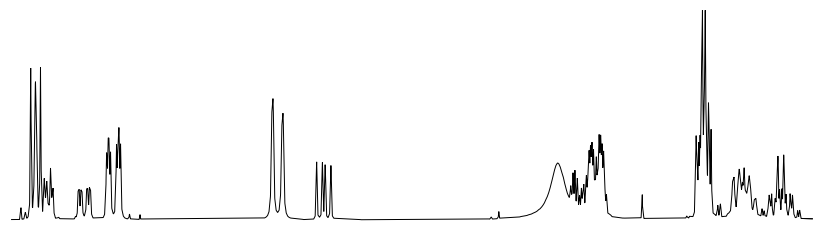

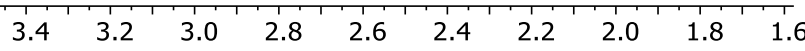

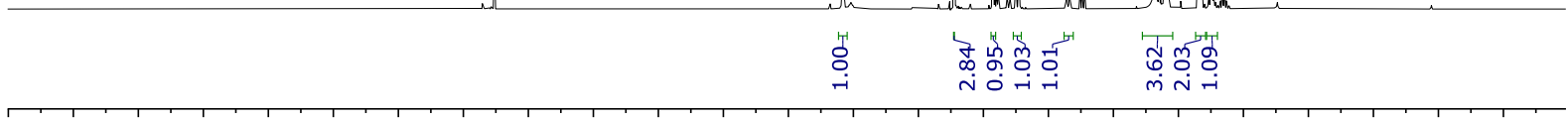
\begin{tabular}{llllllllllllllllllllllllllllll}
\hline 1.0 & 10.5 & 10.0 & 9.5 & 9.0 & 8.5 & 8.0 & 7.5 & 7.0 & 6.5 & 6.0 & 5.5 & 5.0 & 4.5 & 4.0 & 3.5 & 3.0 & 2.5 & 2.0 & 1.5 & 1.0 & 0.5 & 0.0 & -0.5
\end{tabular}
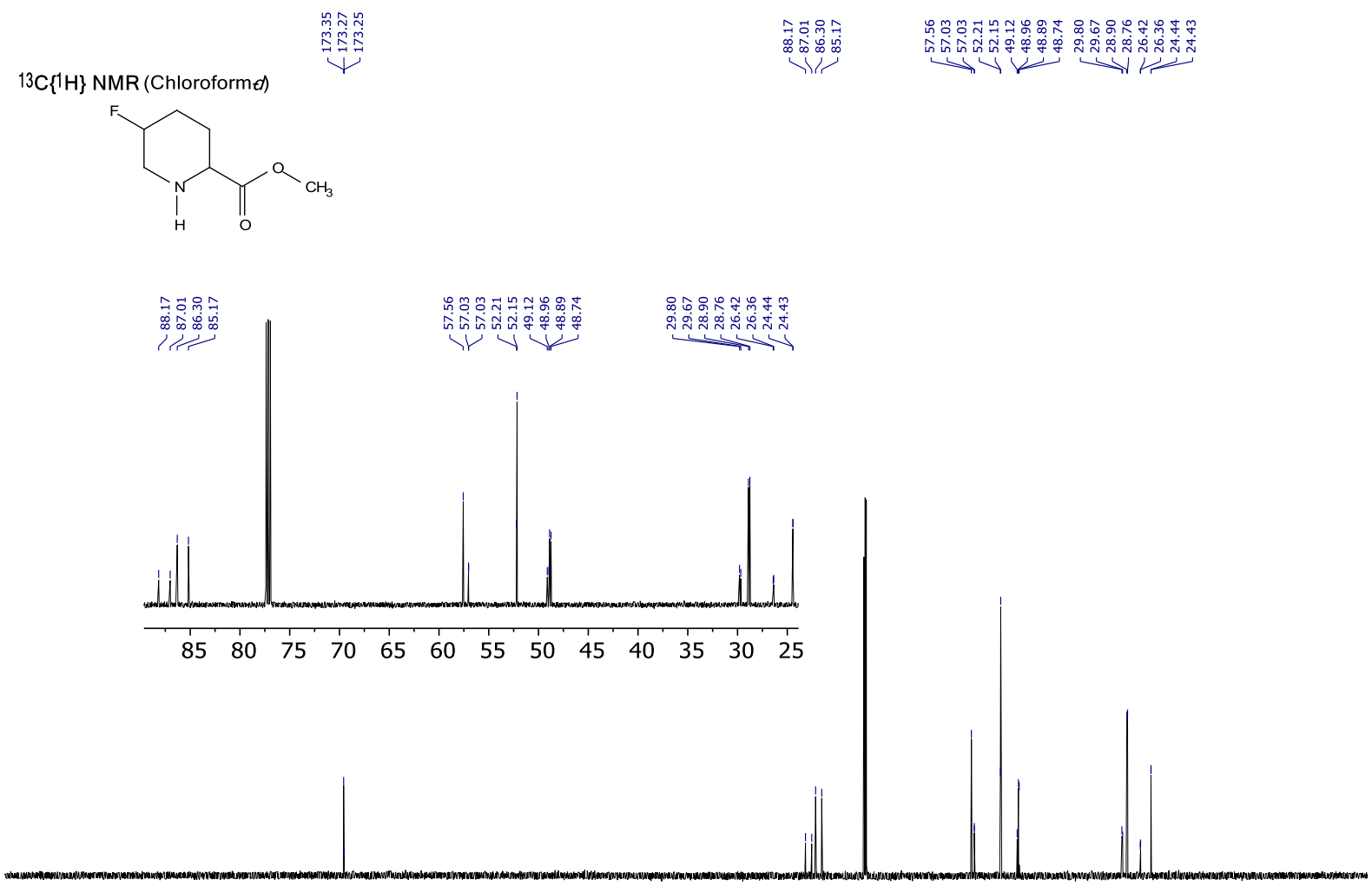

$\begin{array}{lllllllllllllllllllllllllll}230 & 220 & 210 & 200 & 190 & 180 & 170 & 160 & 150 & 140 & 130 & 120 & 110 & 100 & 90 & 80 & 70 & 60 & 50 & 40 & 30 & 20 & 10 & 0 & -10\end{array}$ 


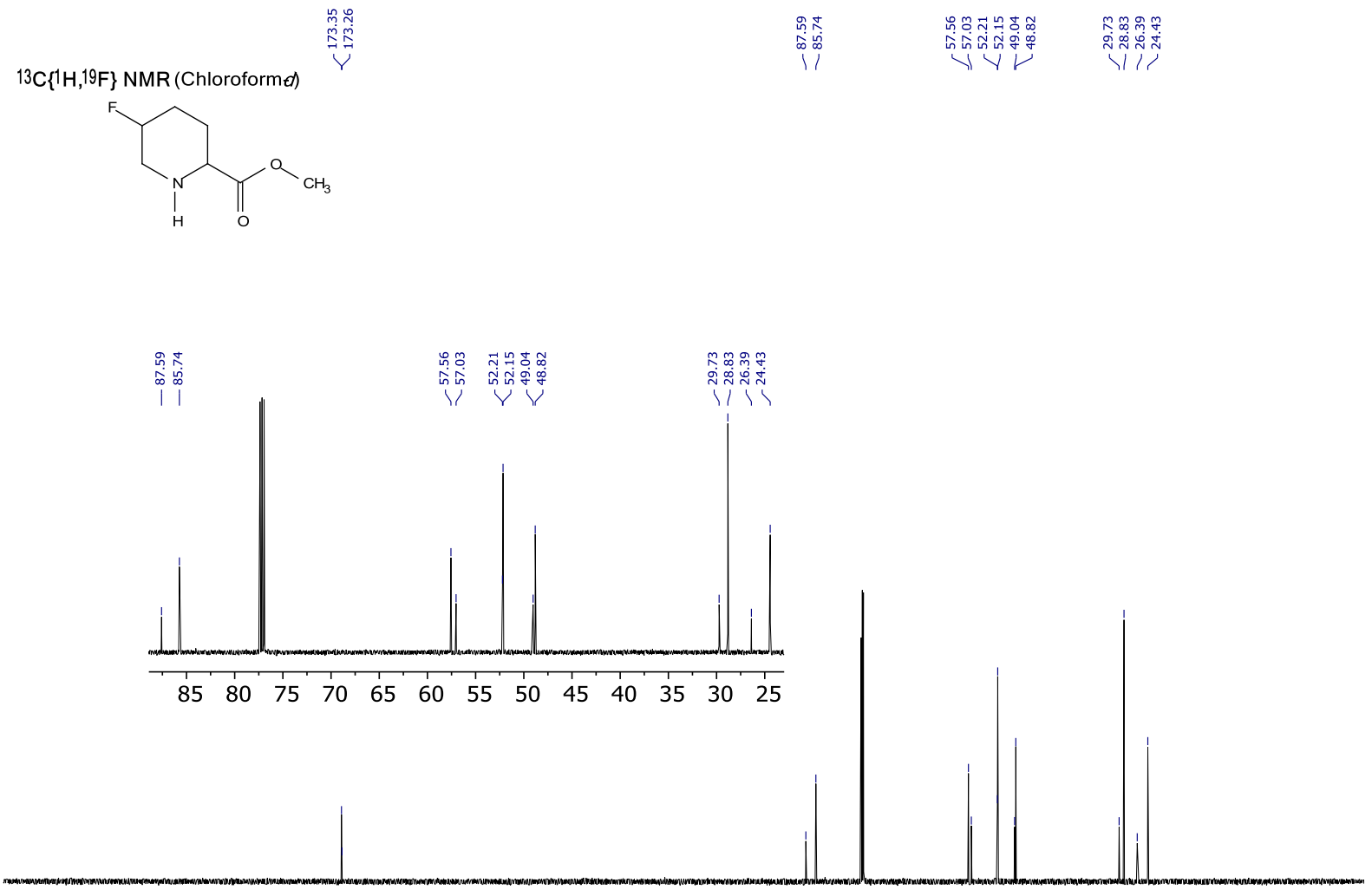

$\begin{array}{lllllllllllllllllllllllll}230 & 220 & 210 & 200 & 190 & 180 & 170 & 160 & 150 & 140 & 130 & 120 & 110 & 100 & 90 & 80 & 70 & 60 & 50 & 40 & 30 & 20 & 10 & 0 & -10\end{array}$ 19F NMR (Chloroforme)

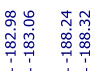<smiles>COC(=O)C1CCC(F)CN1</smiles>

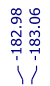

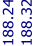

ij

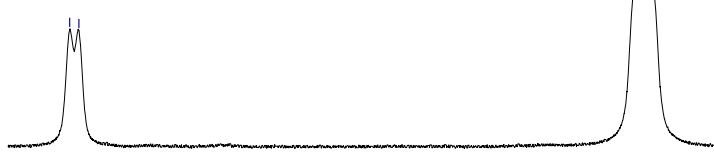

$\begin{array}{lllllll}-182.5 & -183.5 & -184.5 & -185.5 & -186.5 & -187.5 & -188.5\end{array}$

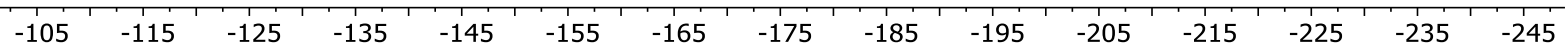


19F $\{1 \mathrm{H}\}$ NMR (Chloroformel)<smiles>COC(=O)C1CCC(F)CN1</smiles>

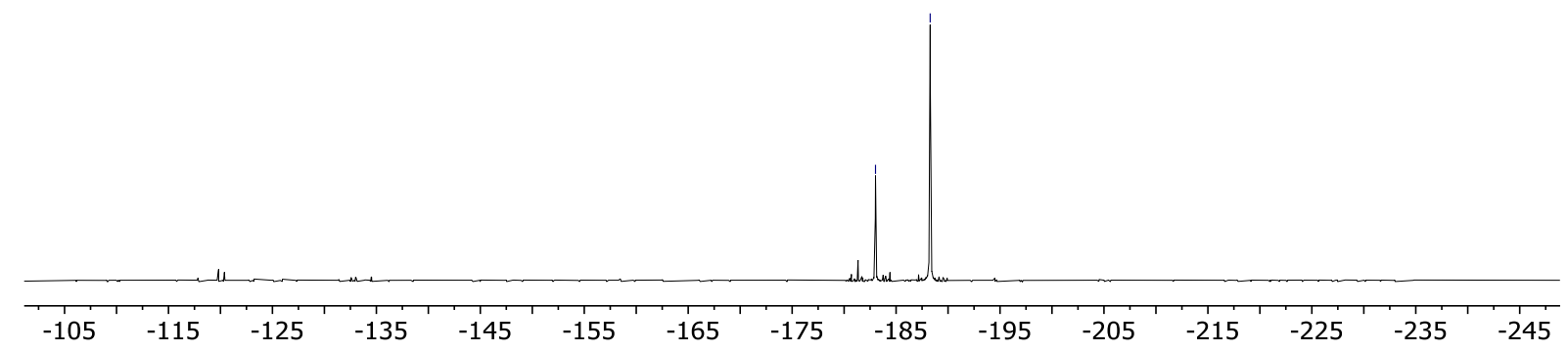

1H NMR (Chloroforme)

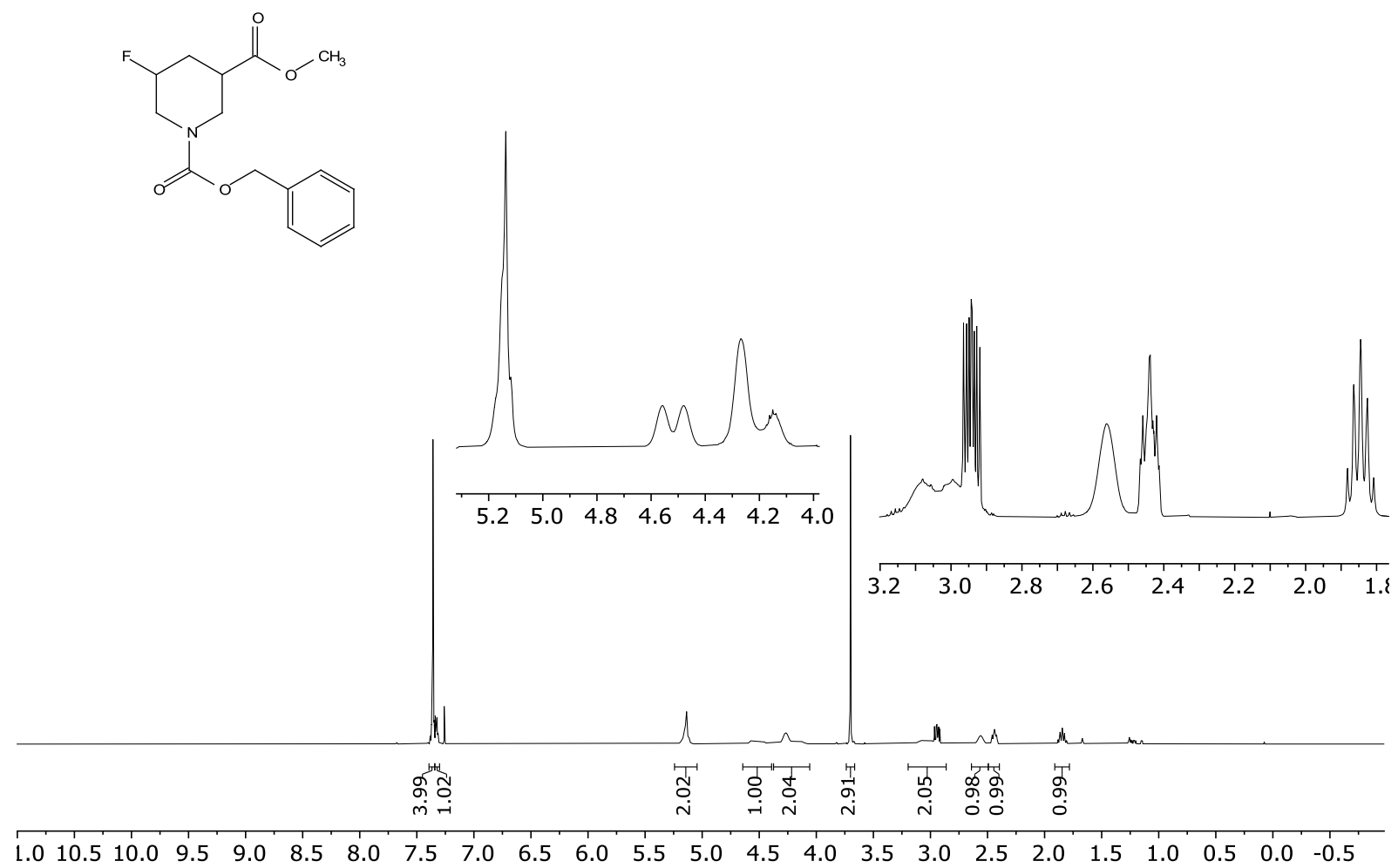


1H\{19F\} NMR (Chloroforme)<smiles>COC(=O)C1CC(F)CN(C(=O)OCc2ccccc2)C1</smiles>

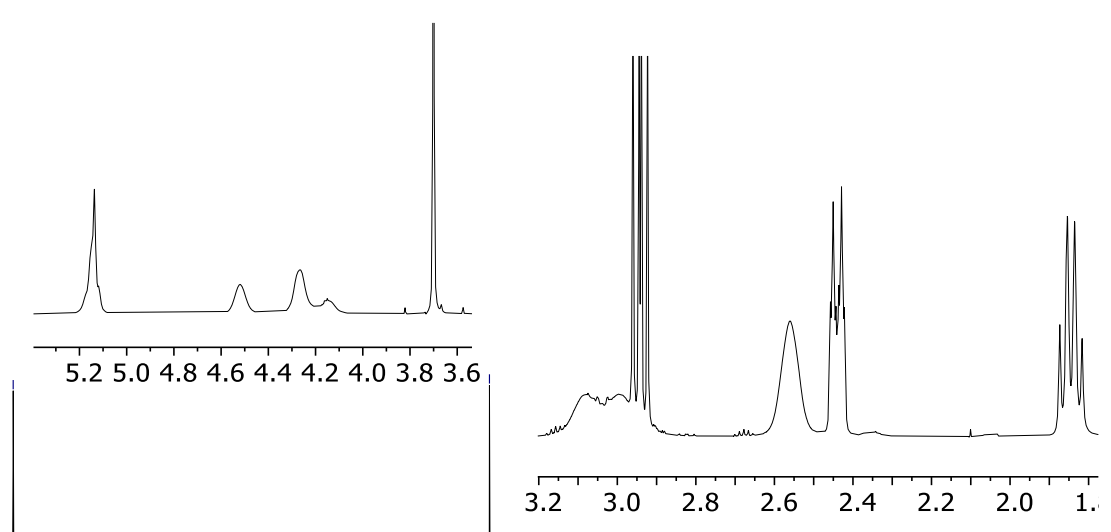

$\begin{array}{lllllllllllllllllllllllllllllll}1 & 1.0 & 10.5 & 10.0 & 9.5 & 9.0 & 8.5 & 8.0 & 7.5 & 7.0 & 6.5 & 6.0 & 5.5 & 5.0 & 4.5 & 4.0 & 3.5 & 3.0 & 2.5 & 2.0 & 1.5 & 1.0 & 0.5 & 0.0 & -0.5\end{array}$

13C\{1H\} NMR (Chloroforme)<smiles>COC(=O)C1CC(F)CN(C(=O)OCc2ccccc2)C1</smiles>

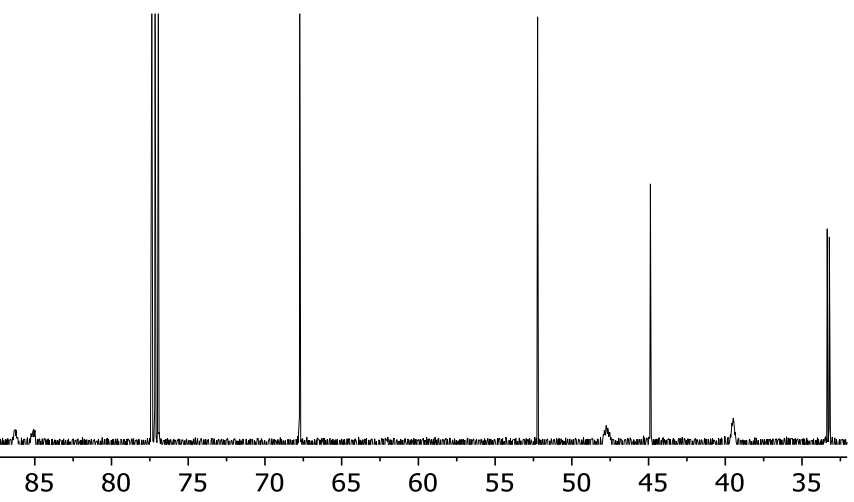

\begin{tabular}{llllllllllllllllllllllllll}
\hline 230 & 220 & 210 & 200 & 190 & 180 & 170 & 160 & 150 & 140 & 130 & 120 & 110 & 100 & 90 & 80 & 70 & 60 & 50 & 40 & 30 & 20 & 10 & 0 & -10
\end{tabular} 
$13 \mathrm{C}\{1 \mathrm{H}, 19 \mathrm{~F}\}$ NMR (Chloroformed)

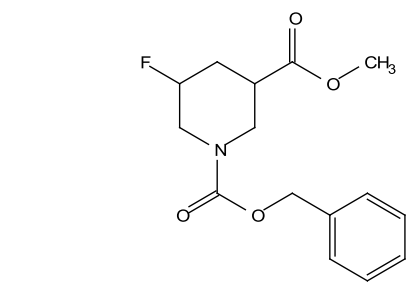

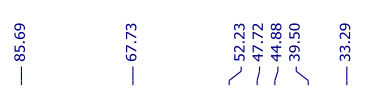

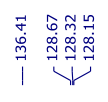

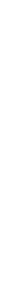

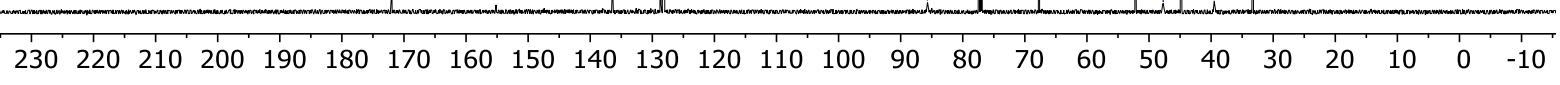

${ }^{19} \mathrm{~F}\{1 \mathrm{H}\}$ NMR (Chloroformed)

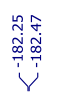

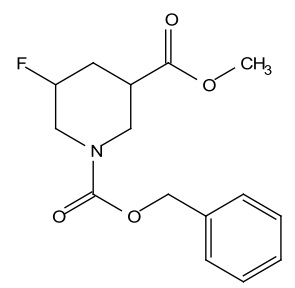

\begin{tabular}{lllllllllllll|l|l|l|}
50 & 30 & 10 & -10 & -30 & -50 & -70 & -90 & -110 & -130 & -150 & -170 & -190 & -210 & -230 & -2
\end{tabular}

S179 
19F NMR (Chloroforme)

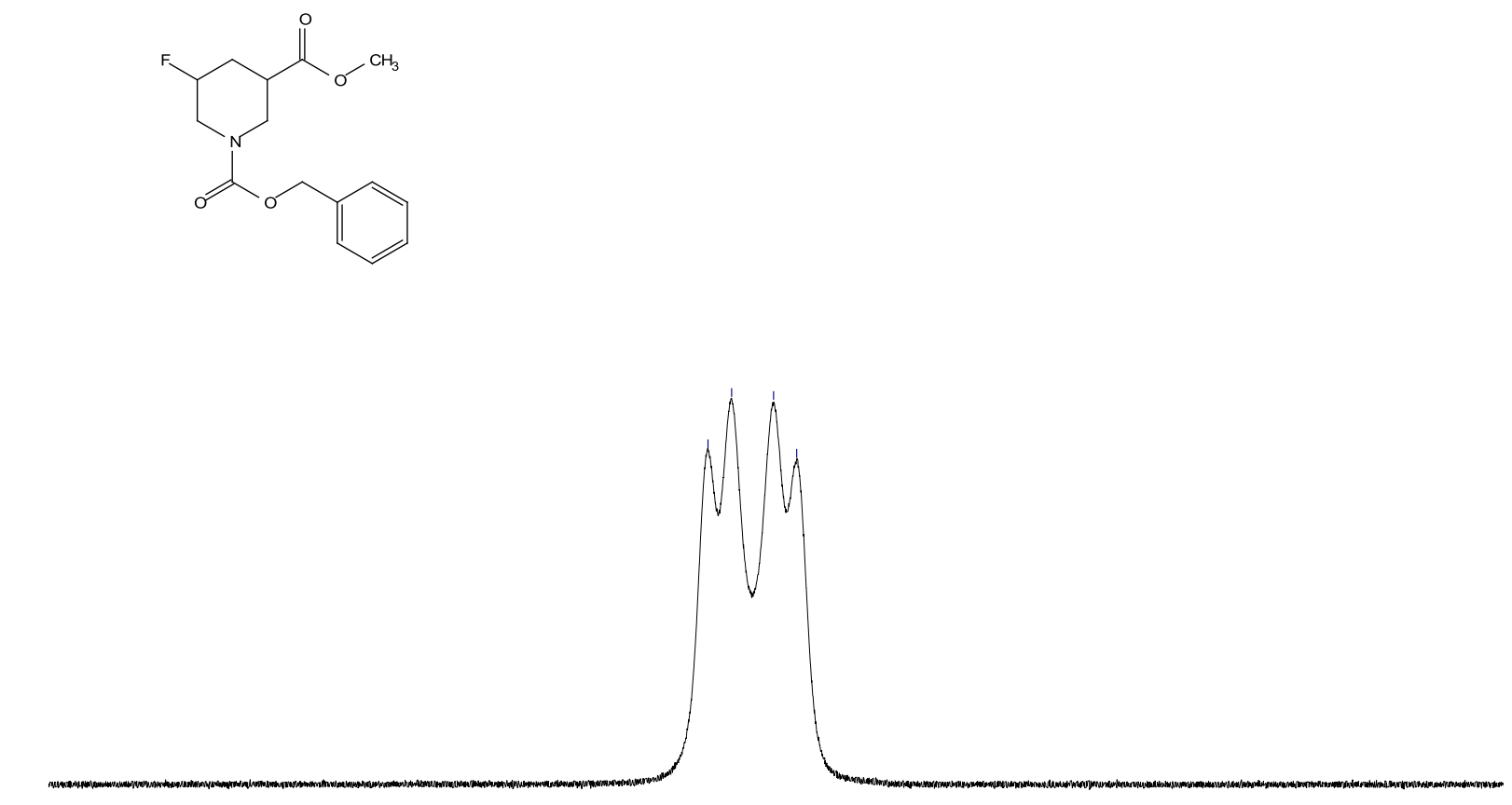

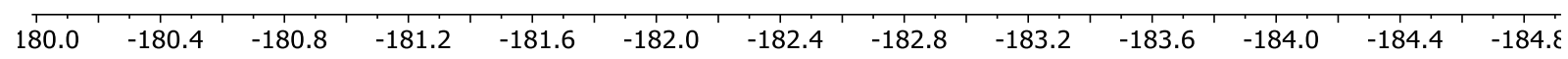

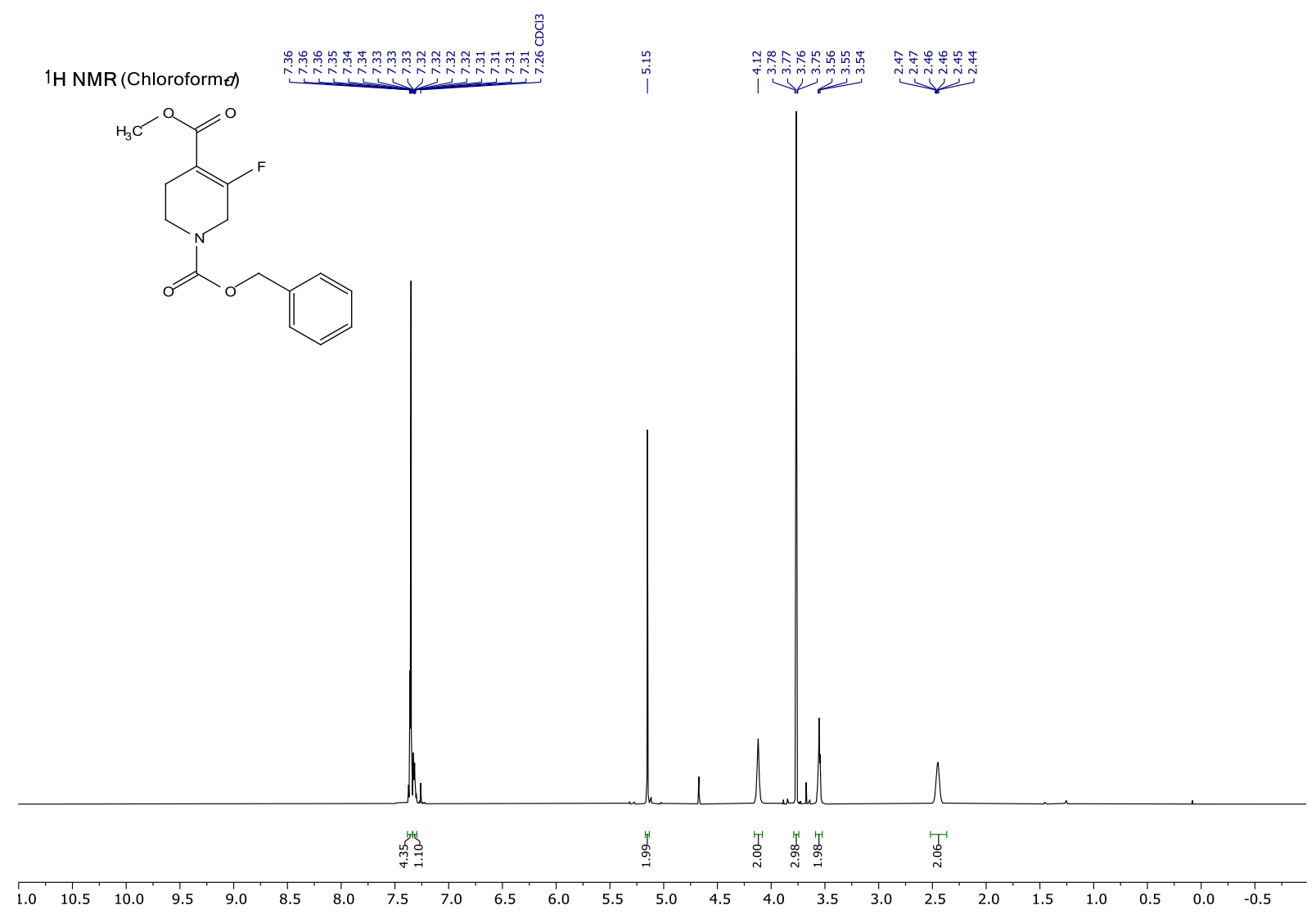




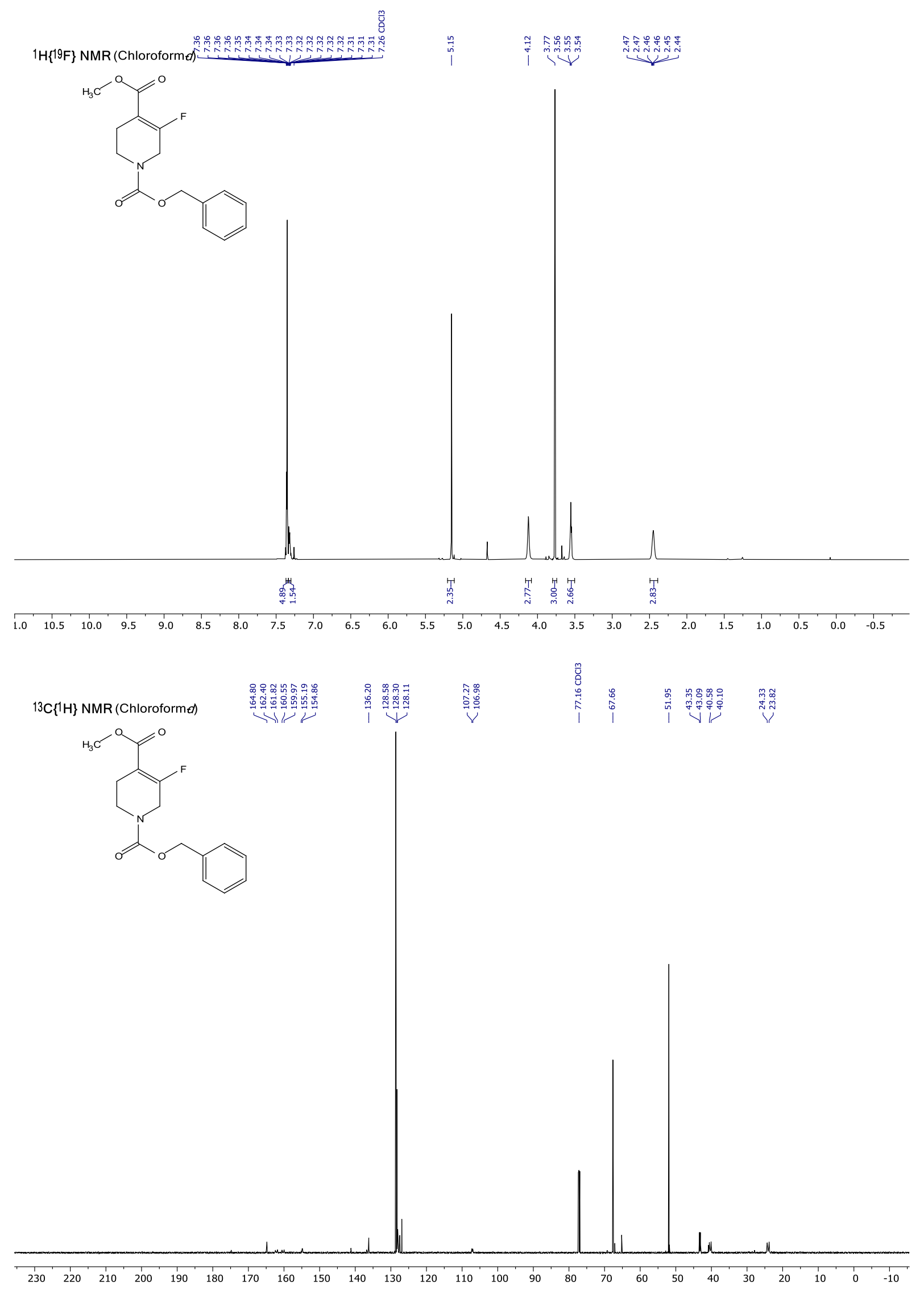



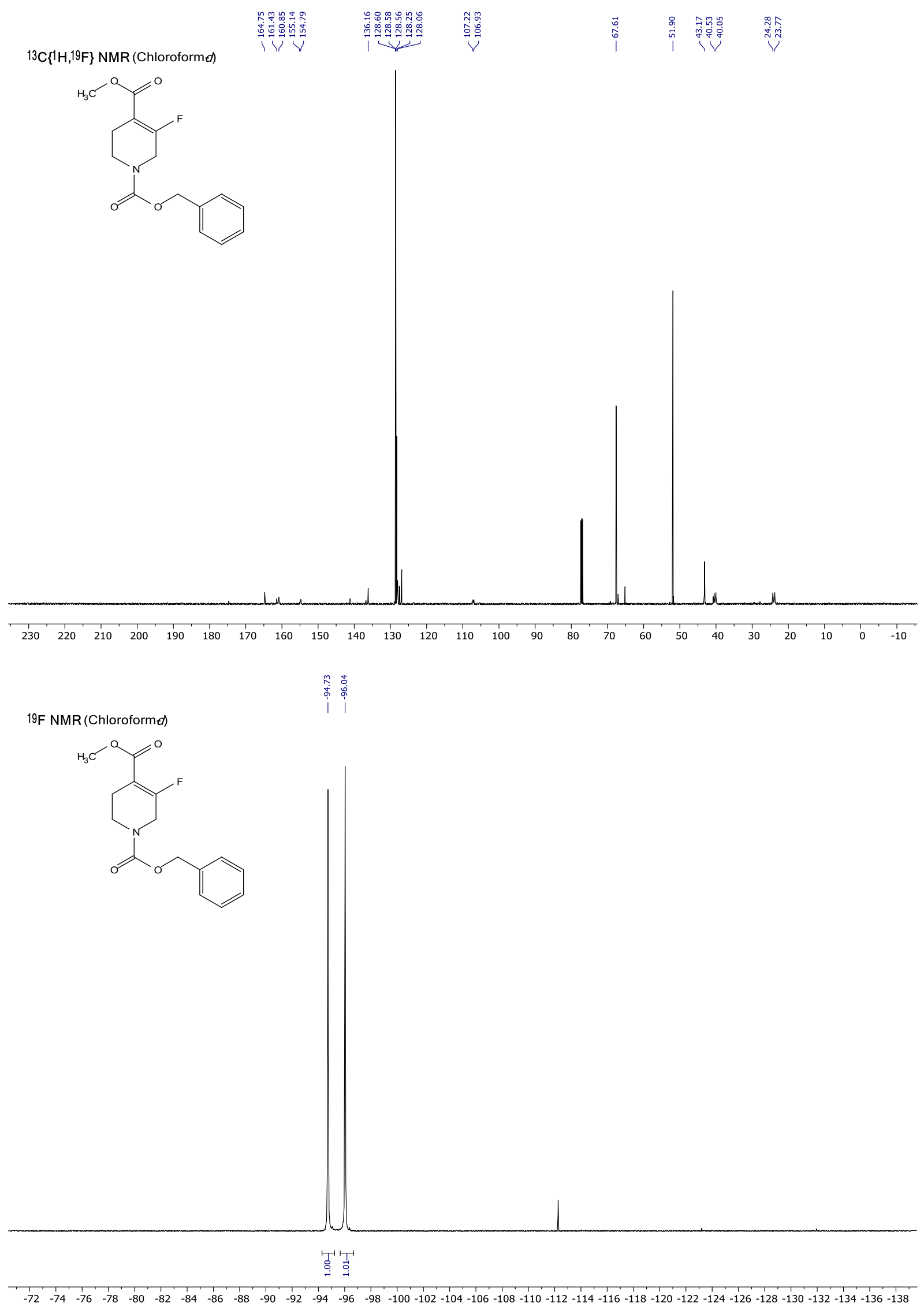

S182 
${ }^{19} \mathrm{~F}\{1 \mathrm{H}\}$ NMR (Chloroforme)

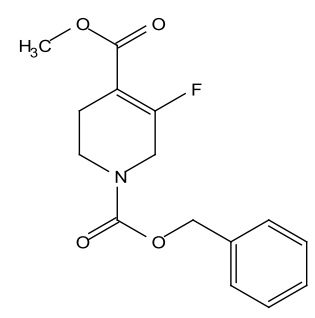

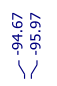

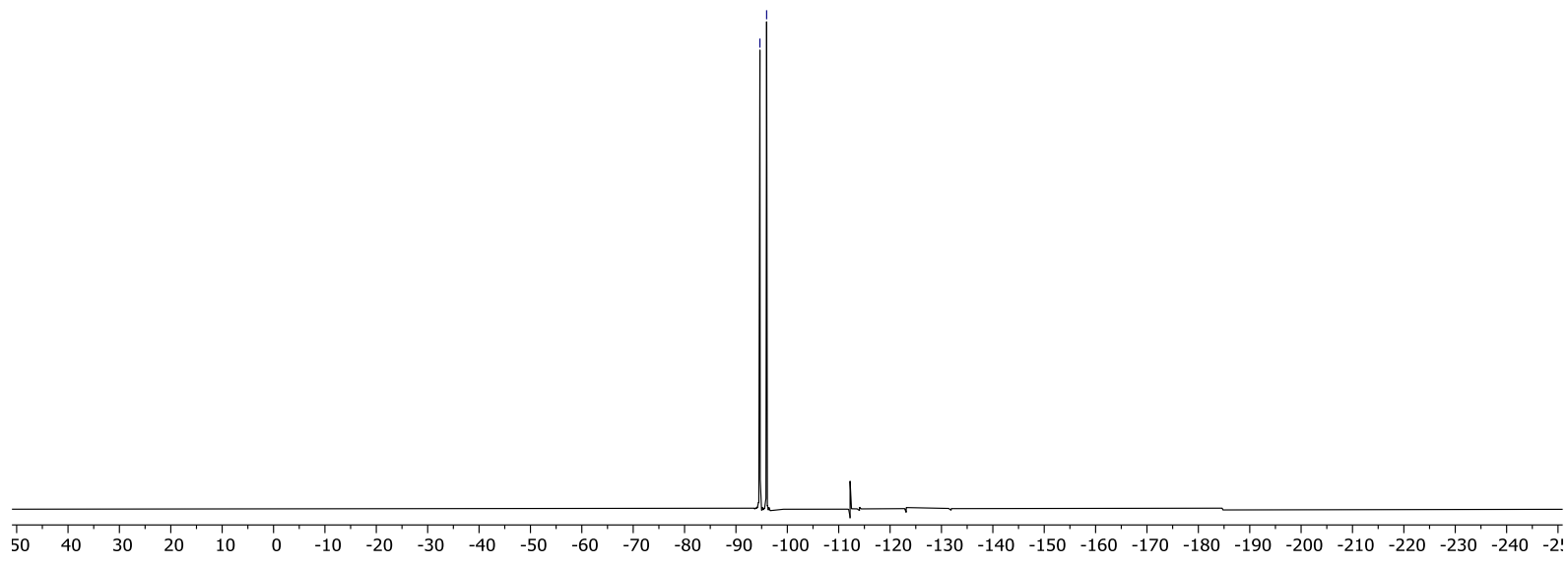

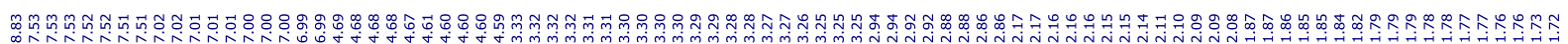

1H NMR (Chloroformel)
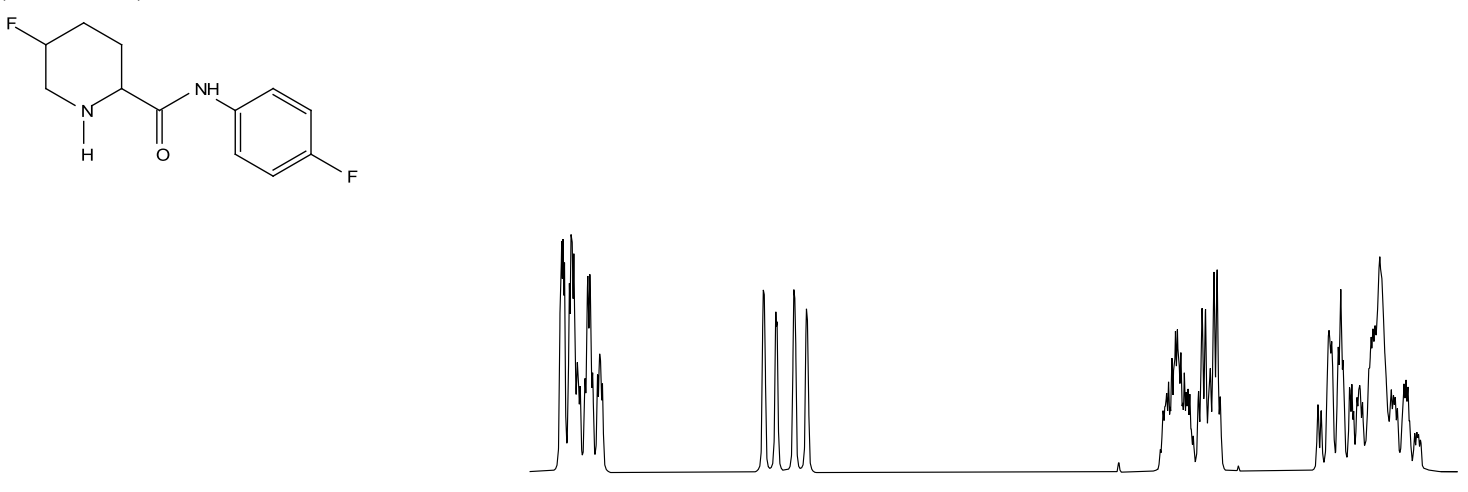

\begin{tabular}{lllllllllllllllllll}
\hline 3.3 & 3.2 & 3.1 & 3.0 & 2.9 & 2.8 & 2.7 & 2.6 & 2.5 & 2.4 & 2.3 & 2.2 & 2.1 & 2.0 & 1.9 & 1.8 & 1.7
\end{tabular}

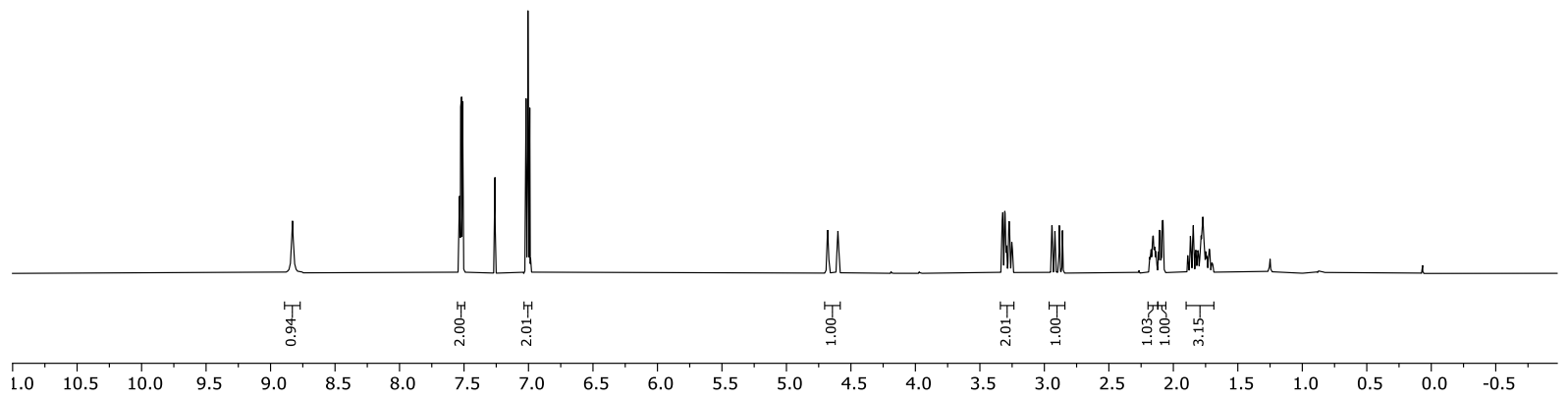

S183 

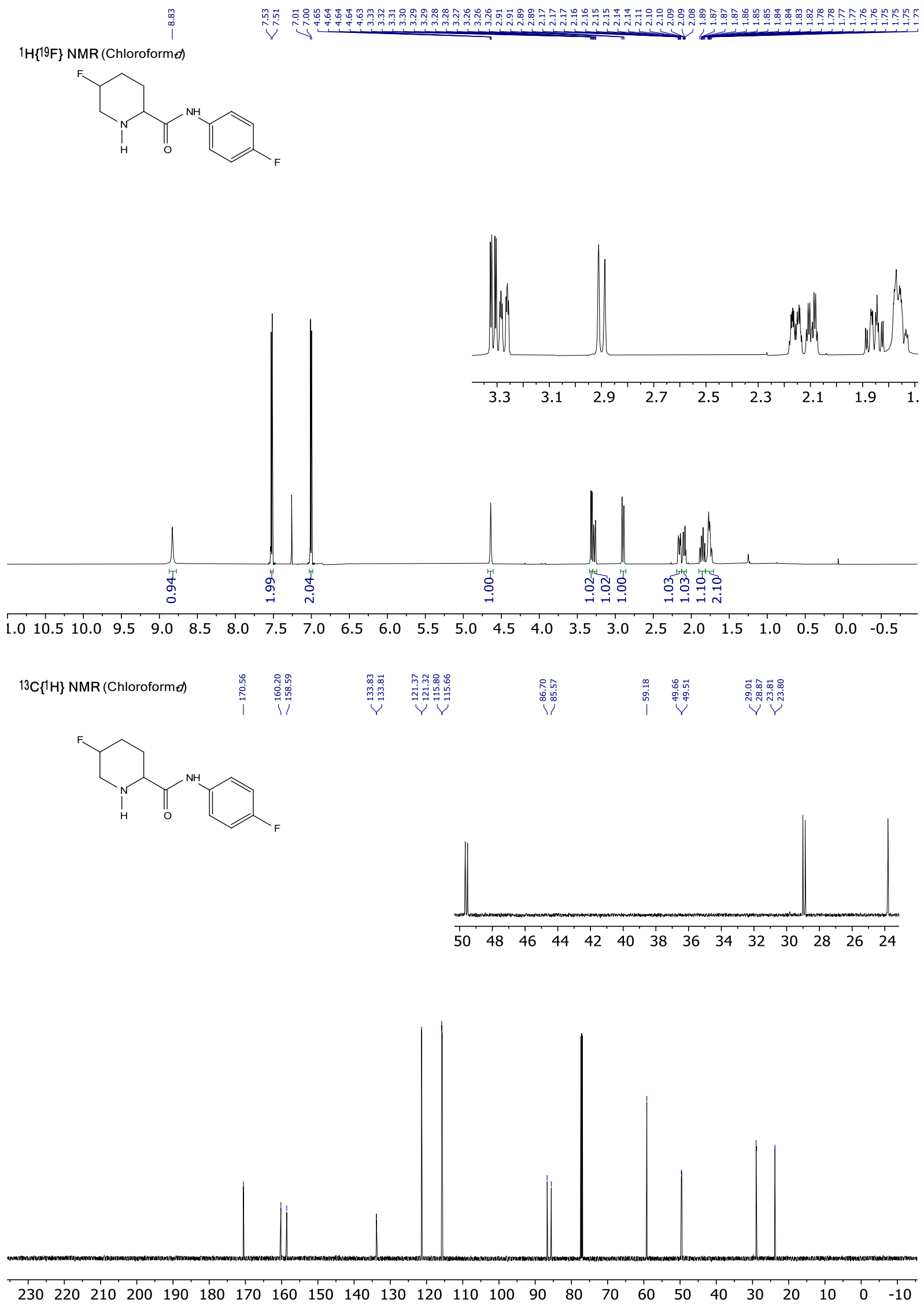

S184 
${ }^{13} \mathrm{C}\left\{{ }^{1} \mathrm{H}, 19 \mathrm{~F}\right\}$ NMR (Chloroforme)

ڤึ.

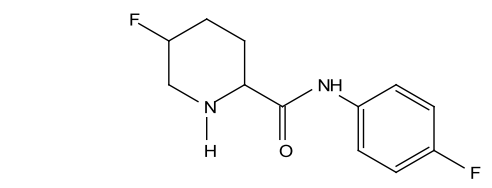

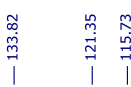

$\underset{\substack{ \pm \dot{0} \\ \dot{0}}}{\mid}$

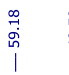

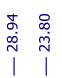

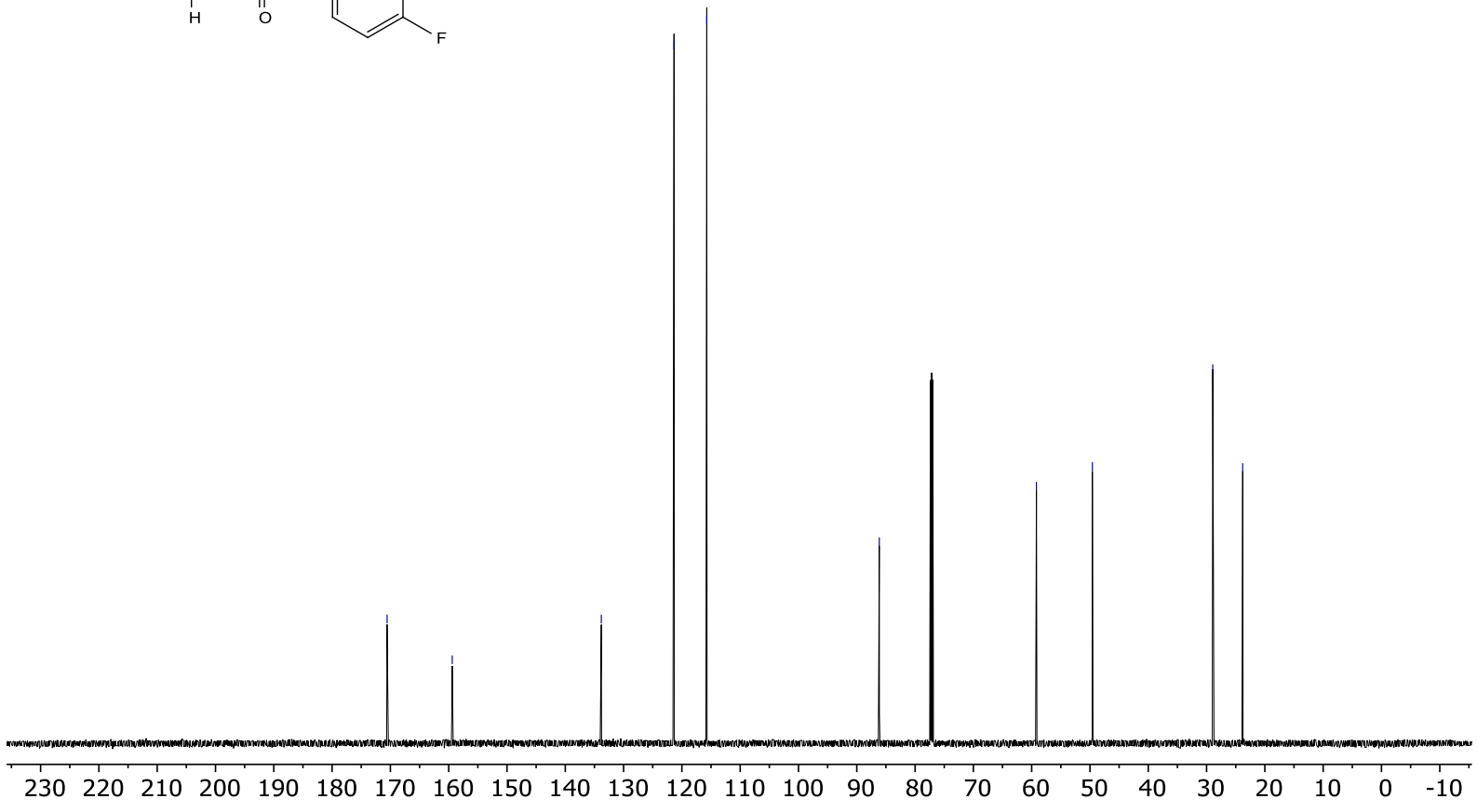

19F NMR (Chloroforme)

1

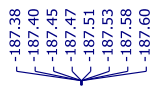
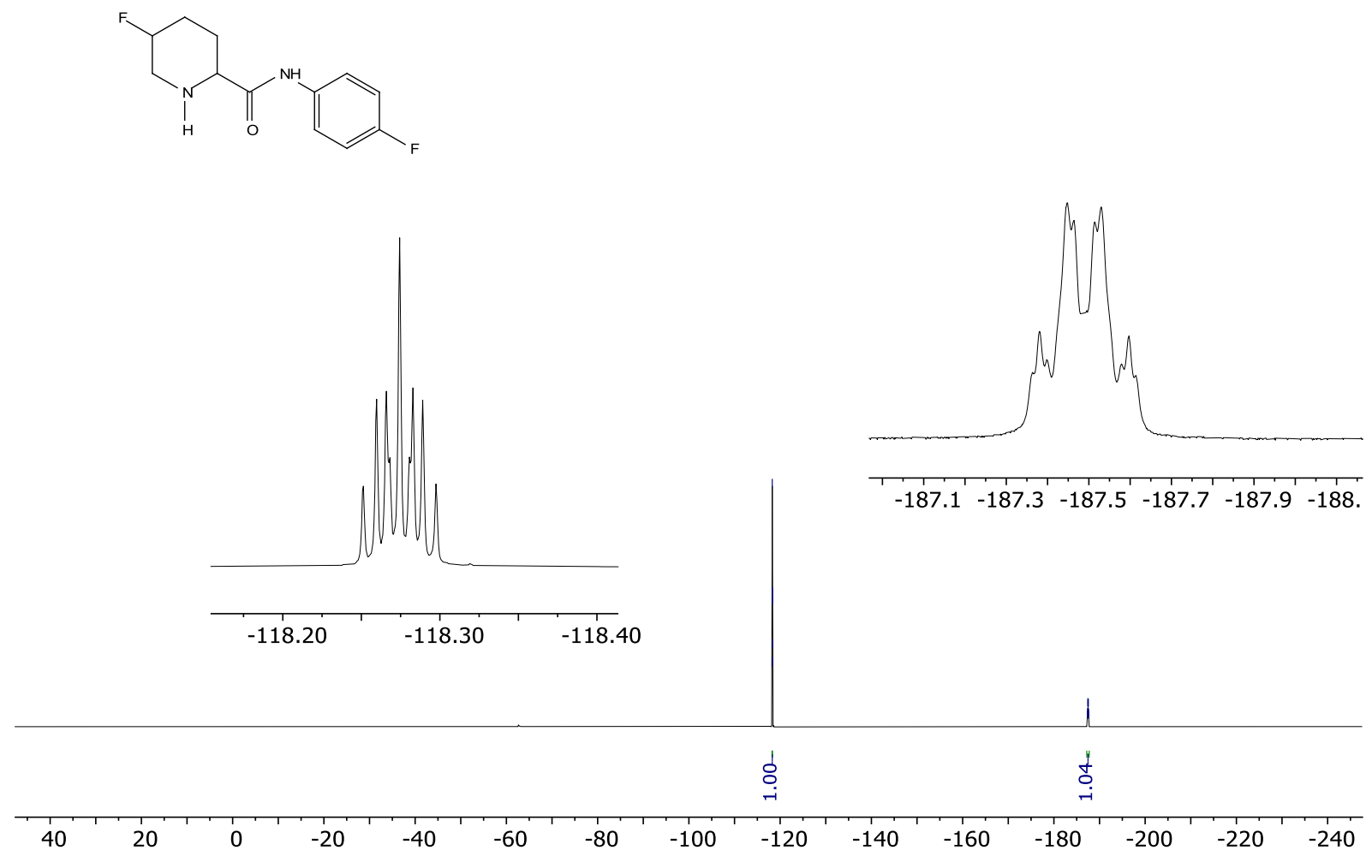

S185 
${ }^{19} \mathrm{~F}\left\{{ }^{1} \mathrm{H}\right\}$ NMR (Chloroform $\left.d\right)$

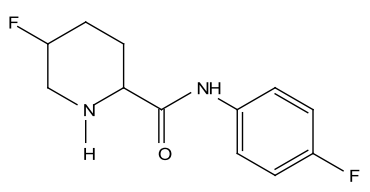

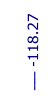

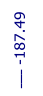

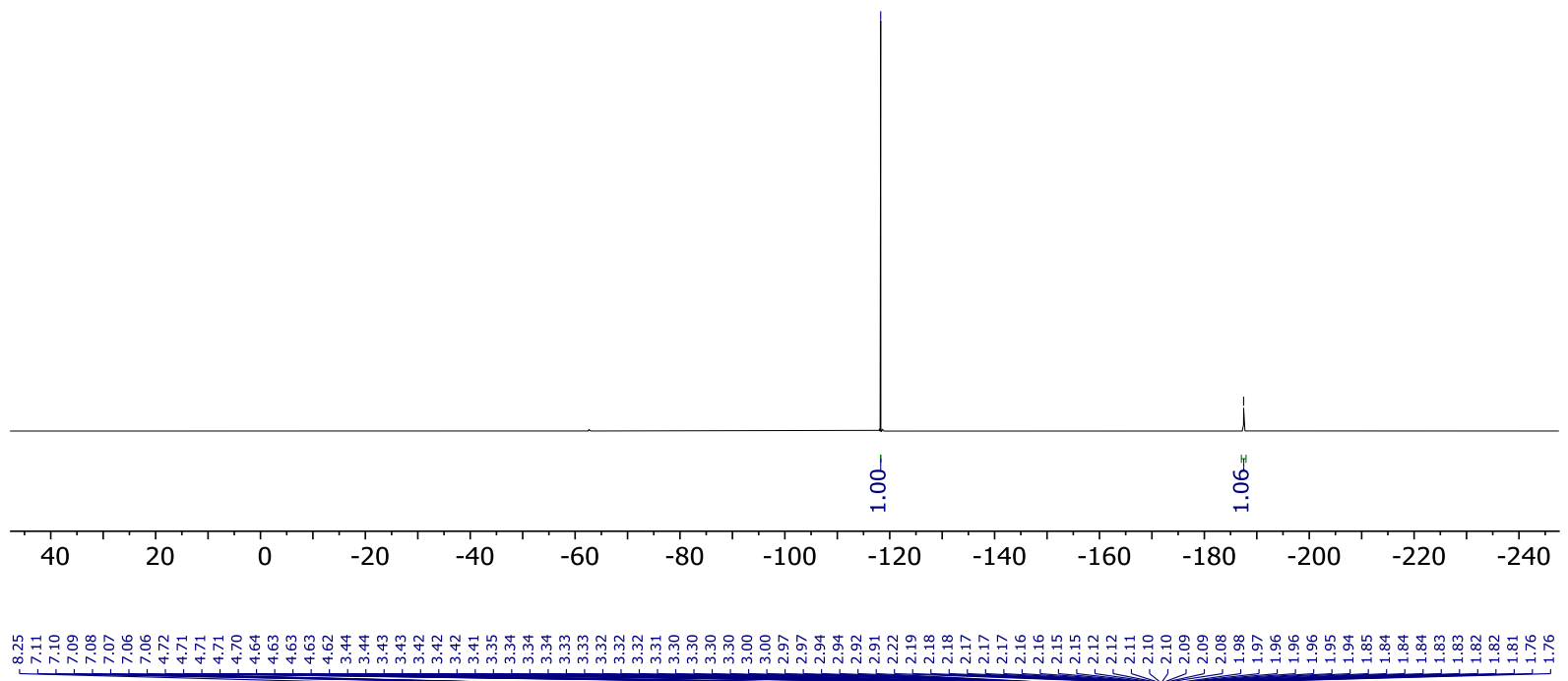

1H NMR (Chlorofor me)
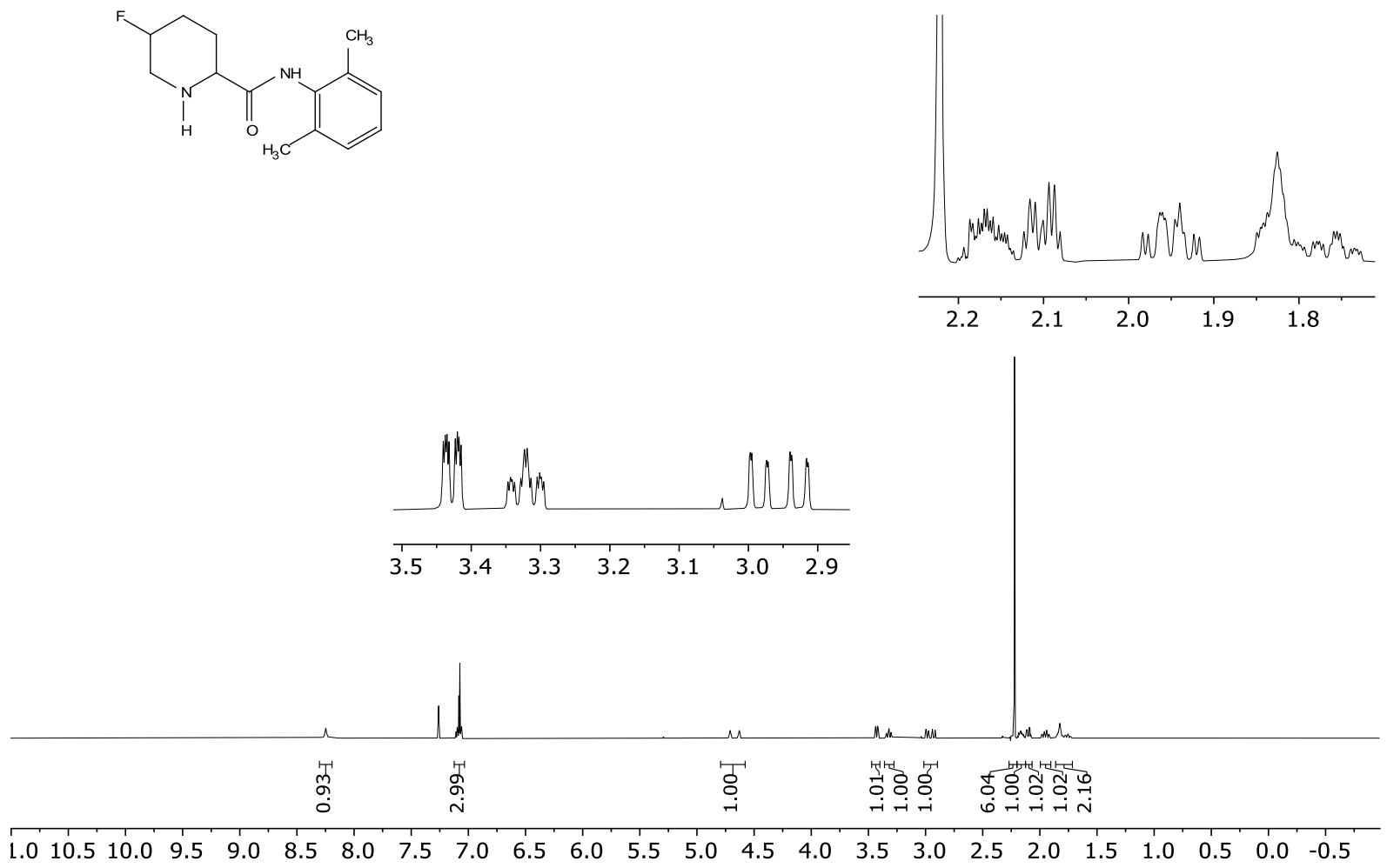

S186 


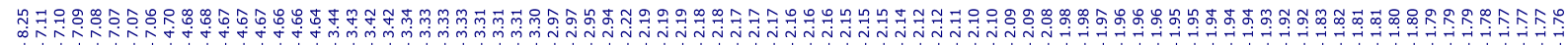
1H $\{19 \mathrm{~F}\}$ NMR (Chloroforme)<smiles>Cc1cccc(C)c1NC(=O)C1CCC(F)CN1</smiles>
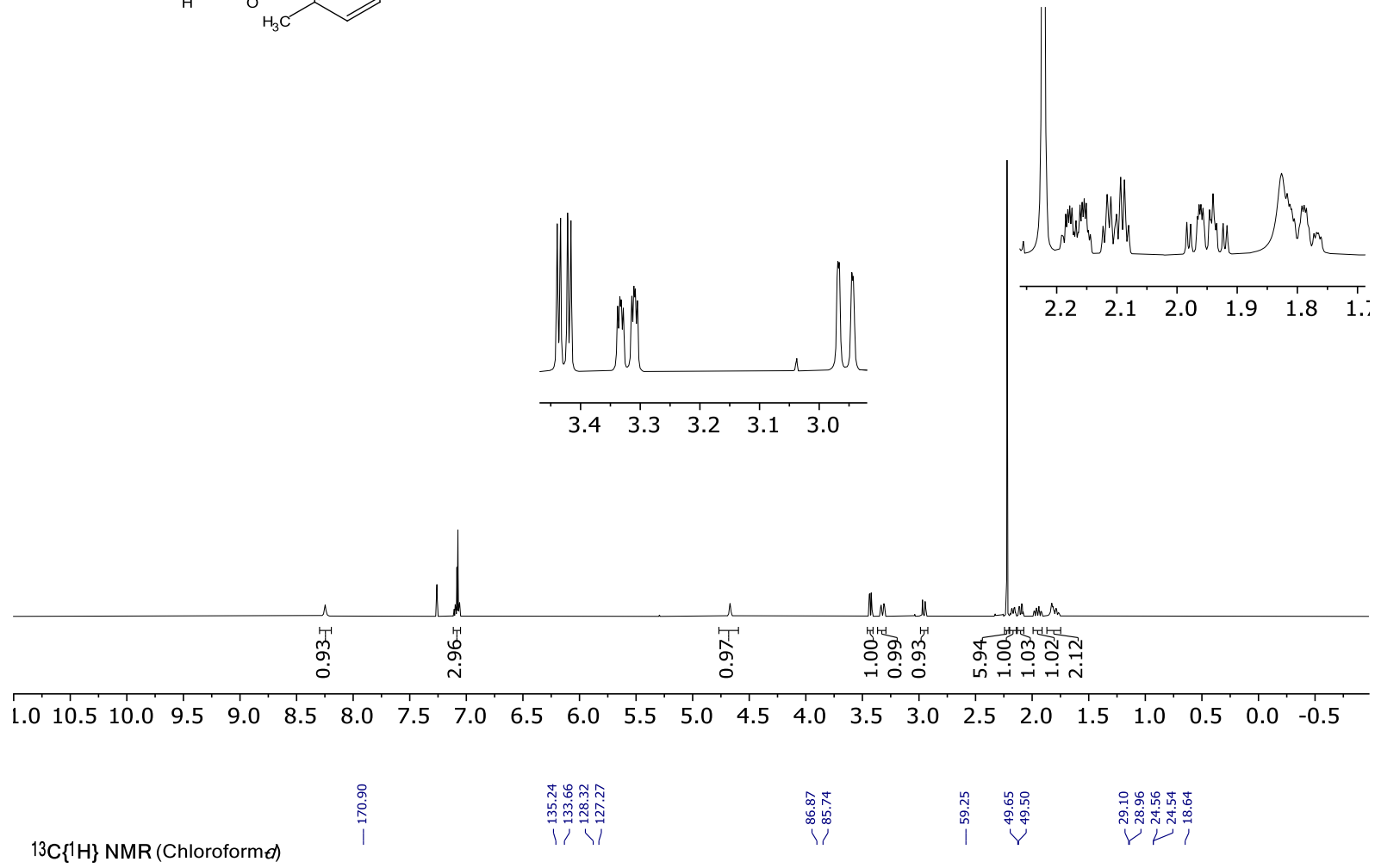<smiles>Cc1cccc(C)c1NC(=O)C1CCC(F)CN1</smiles>

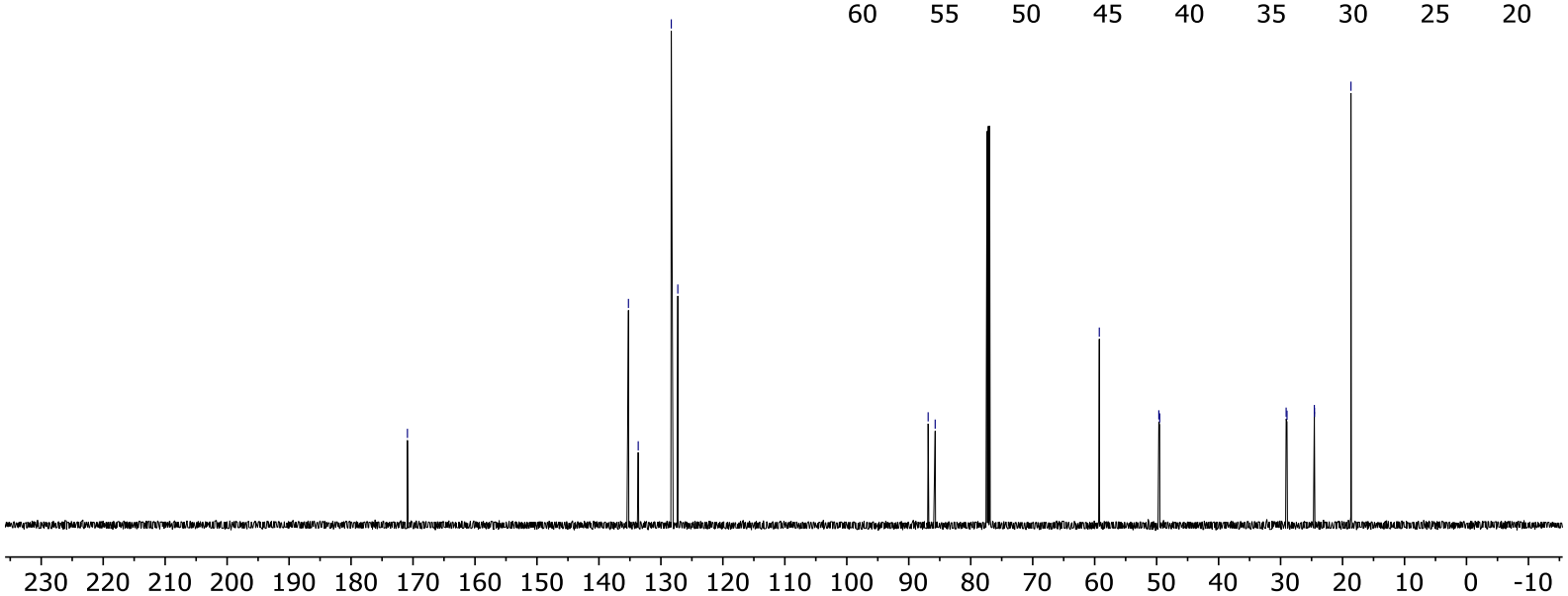




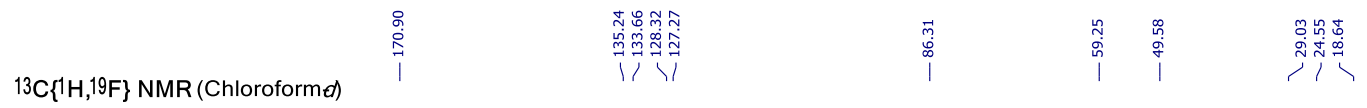<smiles>Cc1cccc(C)c1NC(=O)C1CCC(F)CN1</smiles>

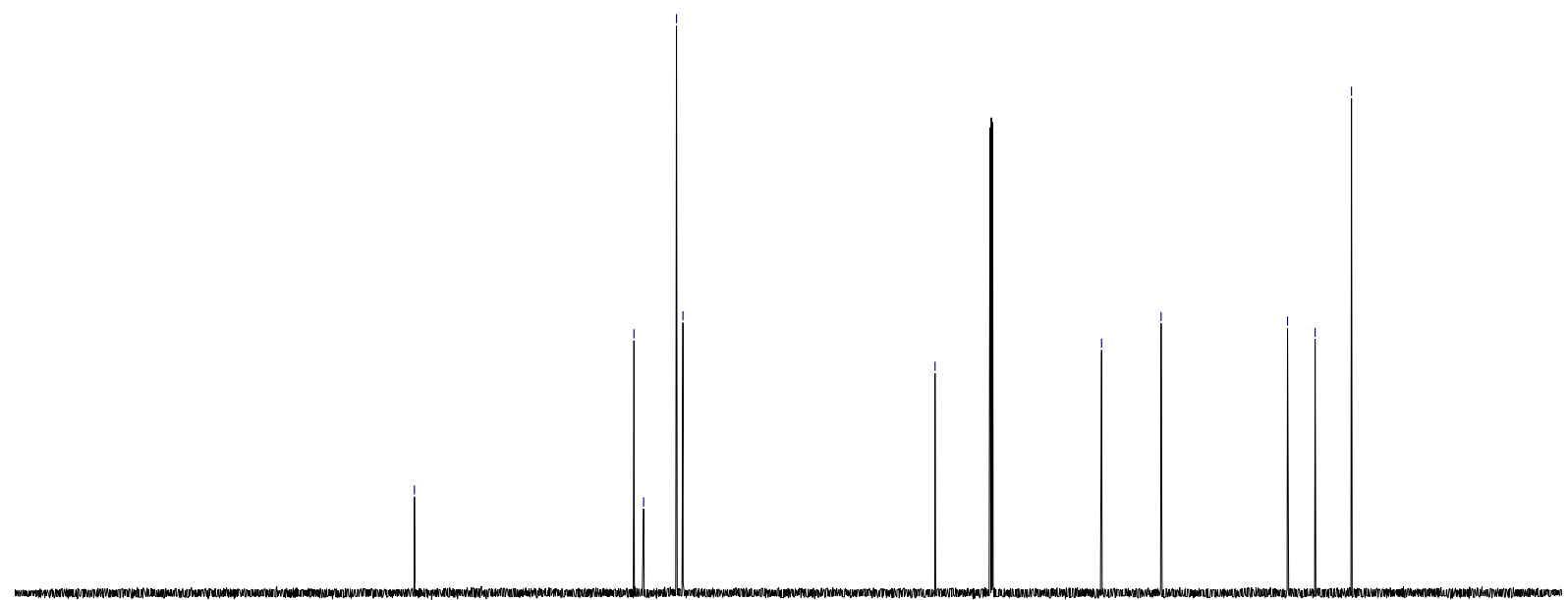

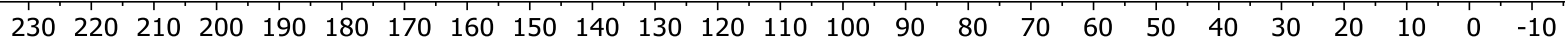

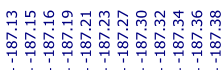

19F NMR (Chloroforme)<smiles>Cc1cccc(C)c1NC(=O)C1CCC(F)CN1</smiles>

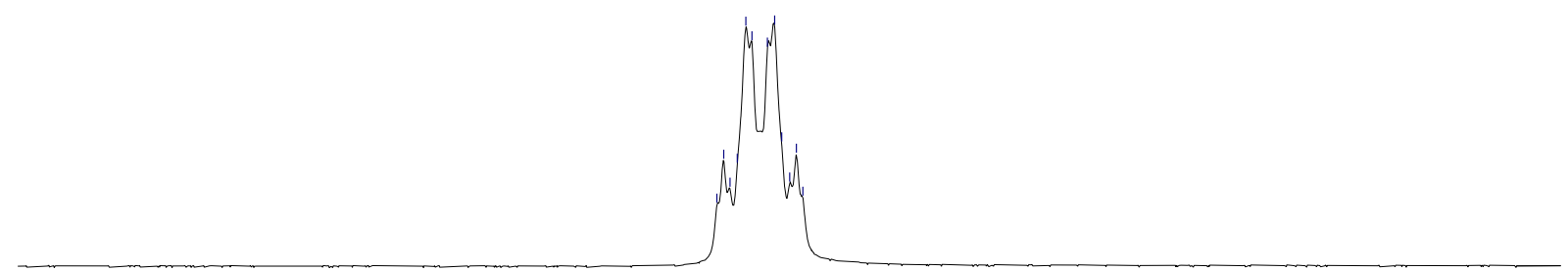

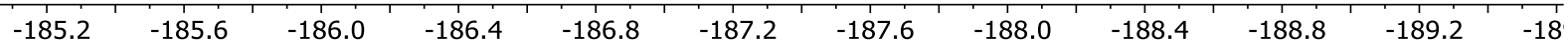


${ }^{19} \mathrm{~F}\{1 \mathrm{H}\}$ NMR (Chloroformed)
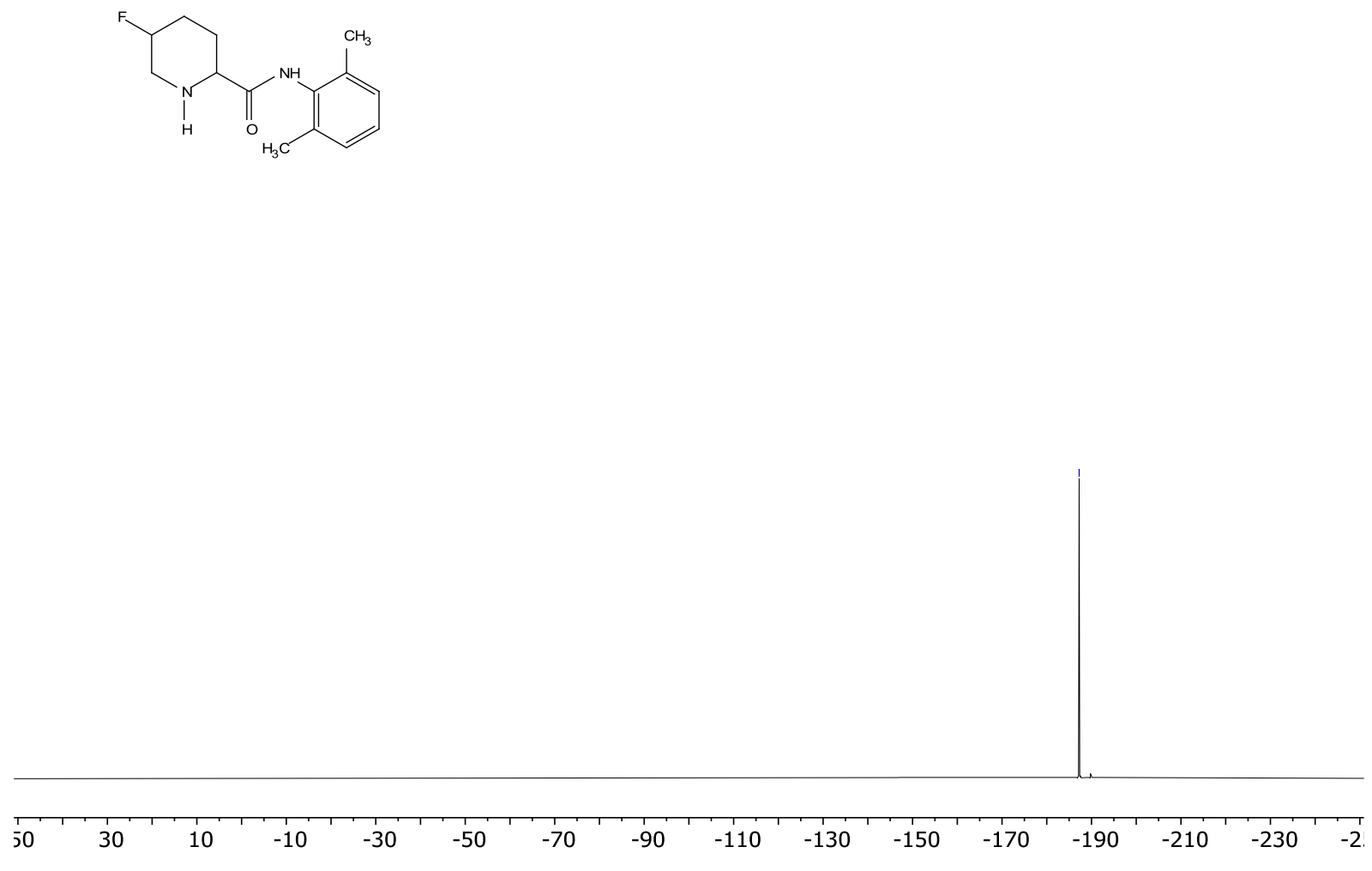

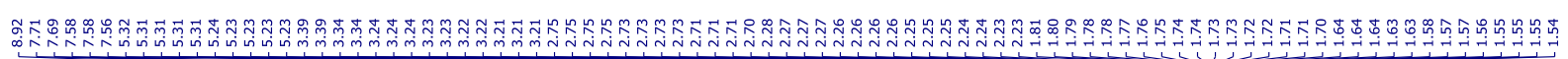

1H NMR (Chloroformed)
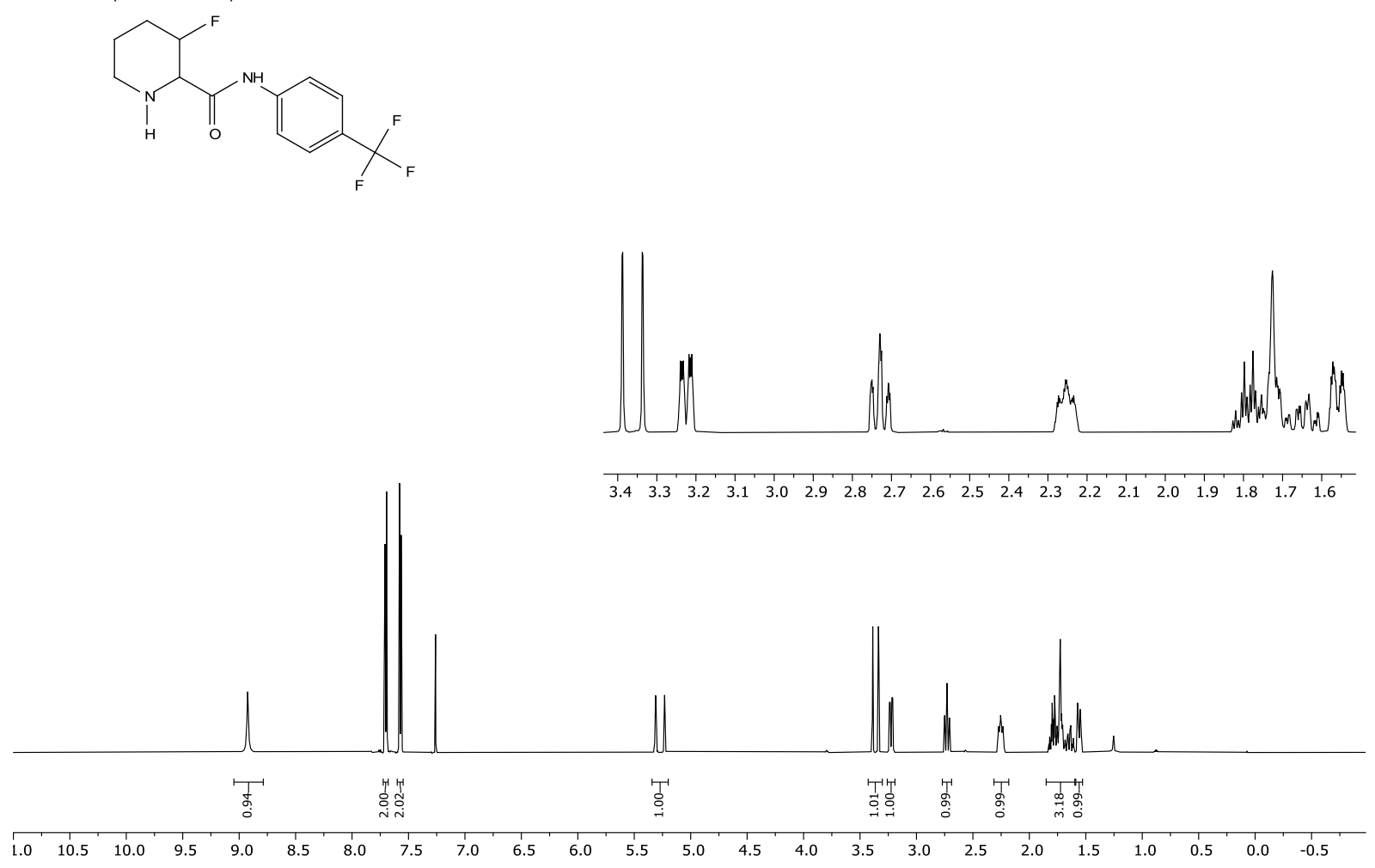

S189 
<smiles>O=C(Nc1ccc(C(F)(F)F)cc1)C1NCCCC1F</smiles>
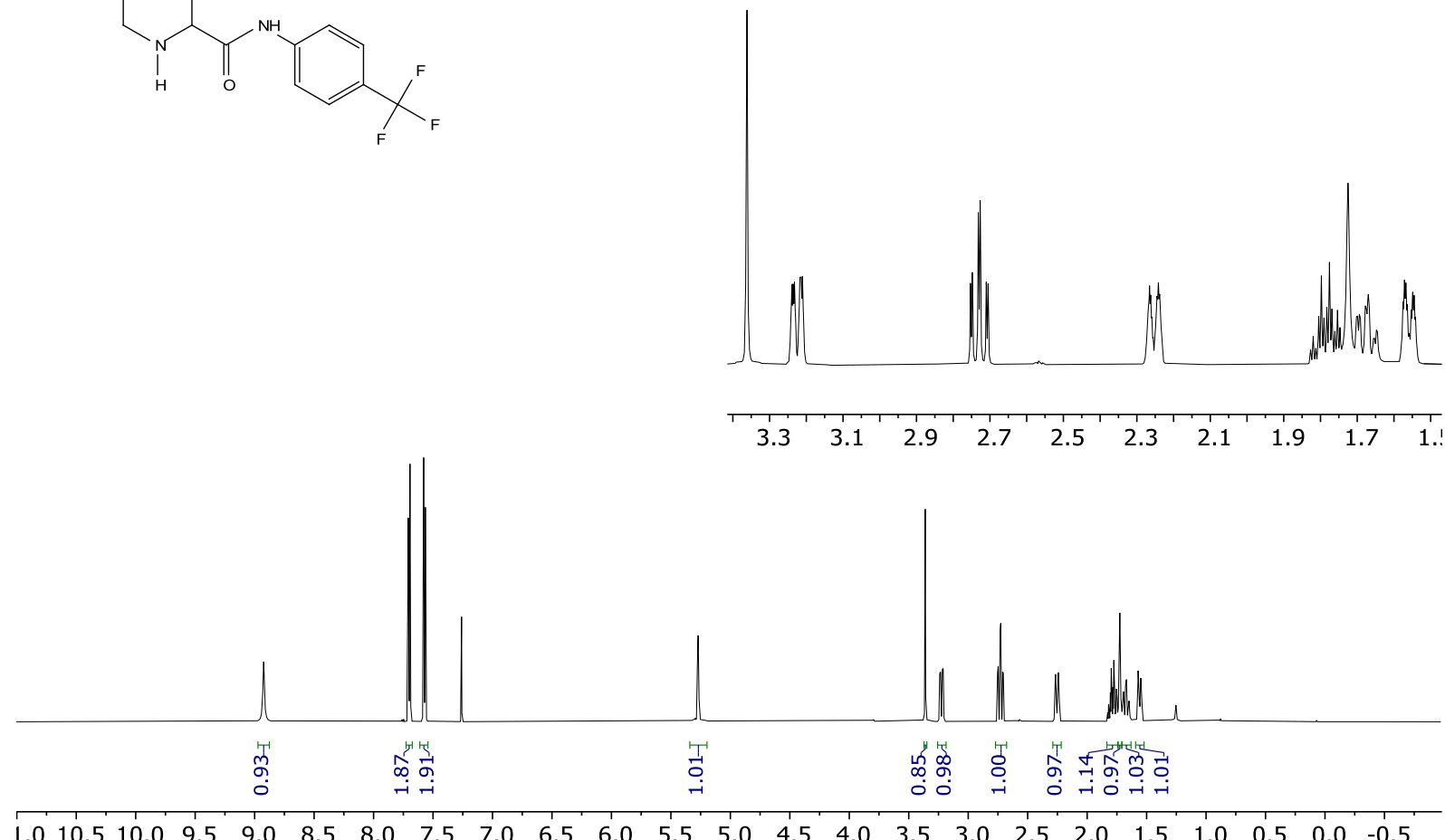

\begin{tabular}{lllllllllllllllllllllllllll}
\hline .0 & 10.5 & 10.0 & 9.5 & 9.0 & 8.5 & 8.0 & 7.5 & 7.0 & 6.5 & 6.0 & 5.5 & 5.0 & 4.5 & 4.0 & 3.5 & 3.0 & 2.5 & 2.0 & 1.5 & 1.0 & 0.5 & 0.0 & -0.5
\end{tabular}

13C\{1H\} NMR (Chloroforme)<smiles>O=C(Nc1ccc(C(F)(F)F)cc1)C1NCCCC1F</smiles>

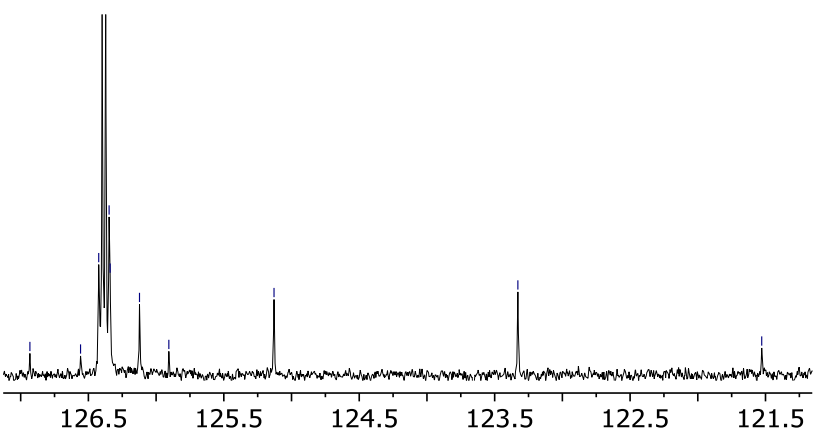

$\begin{array}{lllllllllllllllllllllllllll}230 & 220 & 210 & 200 & 190 & 180 & 170 & 160 & 150 & 140 & 130 & 120 & 110 & 100 & 90 & 80 & 70 & 60 & 50 & 40 & 30 & 20 & 10 & 0 & -10\end{array}$ 


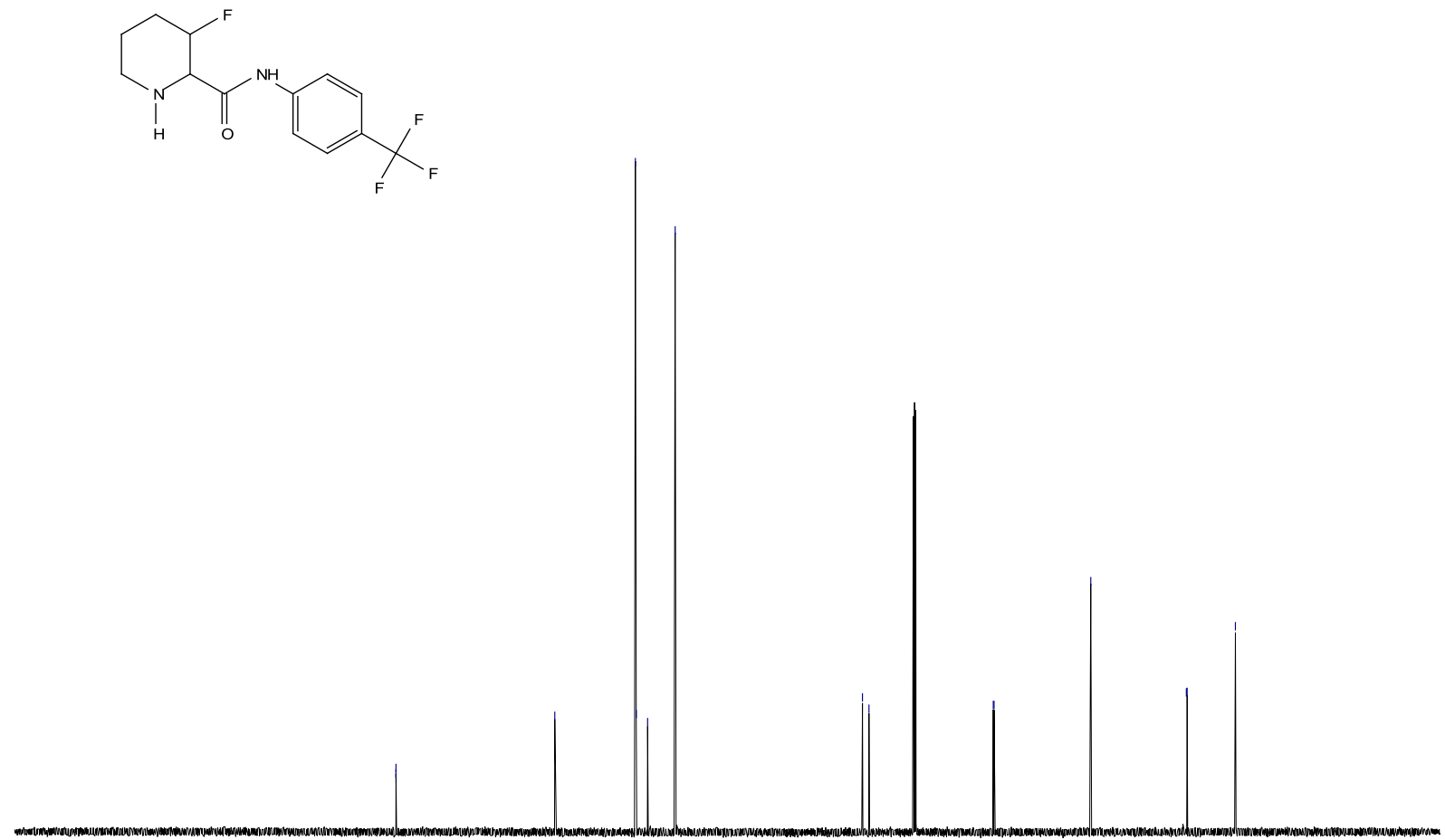

$\begin{array}{lllllllllllllllllllllllllll}230 & 220 & 210 & 200 & 190 & 180 & 170 & 160 & 150 & 140 & 130 & 120 & 110 & 100 & 90 & 80 & 70 & 60 & 50 & 40 & 30 & 20 & 10 & 0 & -10\end{array}$

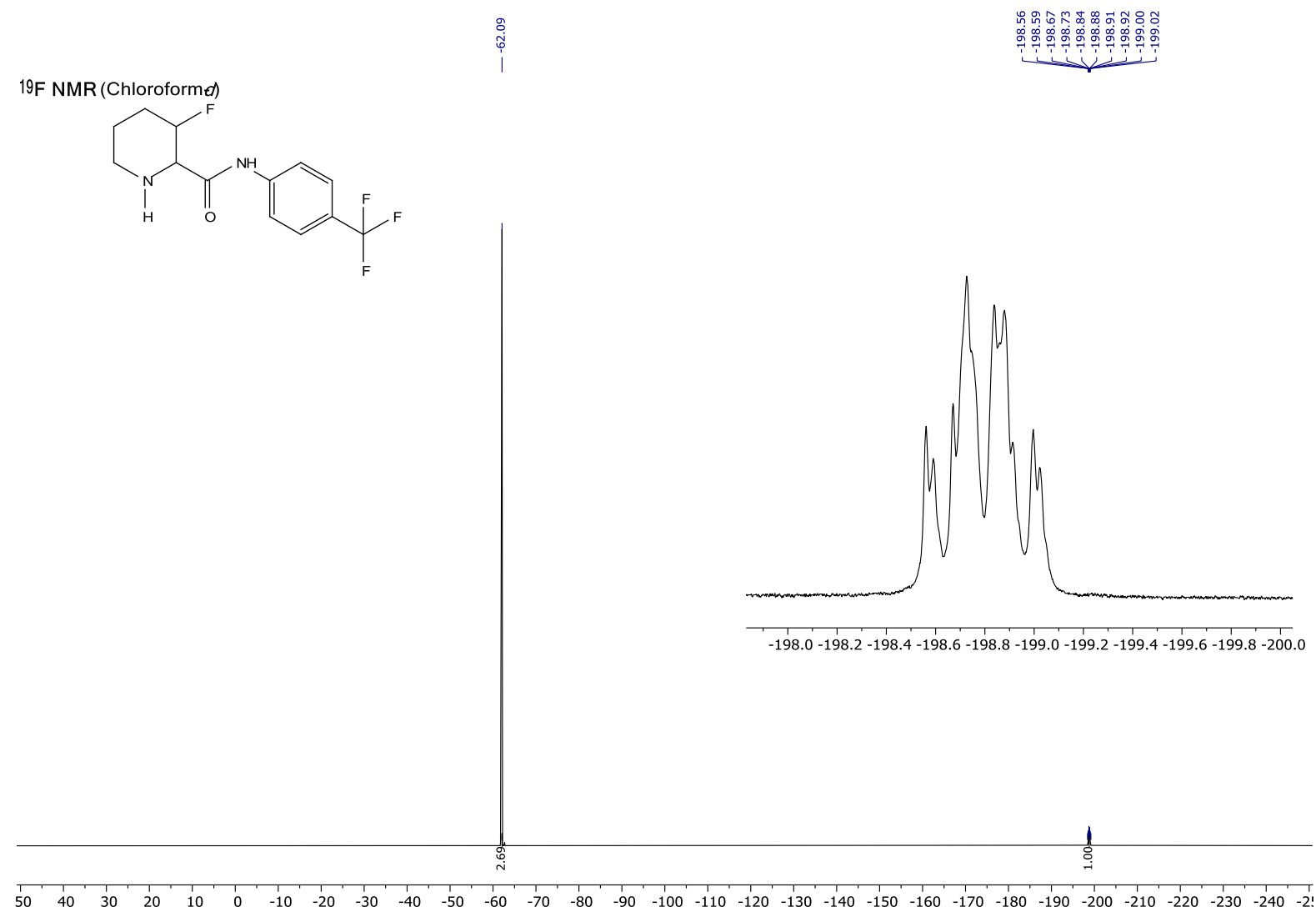


${ }^{19} \mathrm{~F}\{1 \mathrm{H}\}$ NMR (Chloroform $d$ )

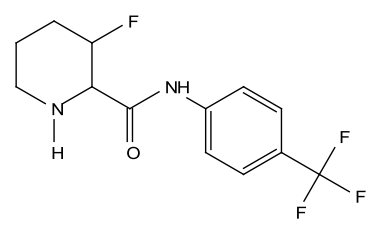

$\stackrel{\text { i }}{\text { îj }}$

$\infty$
$\infty$
$\infty$
$\substack{1 \\ 9}$

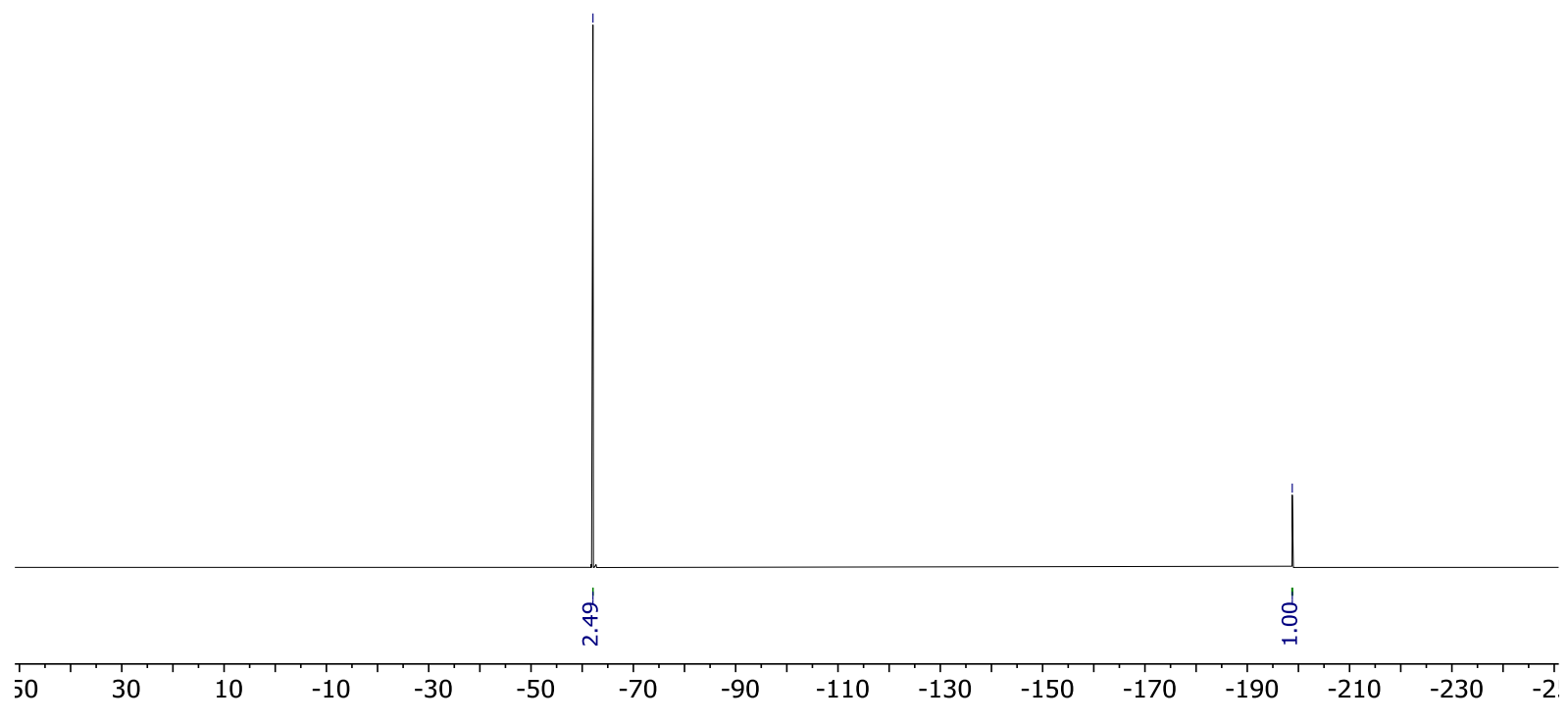

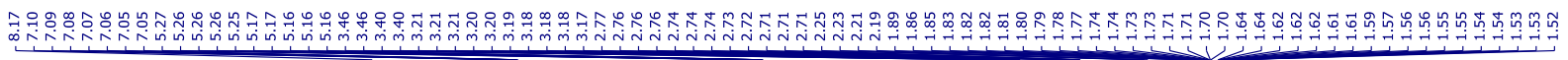

1H NMR (Chloroforme)
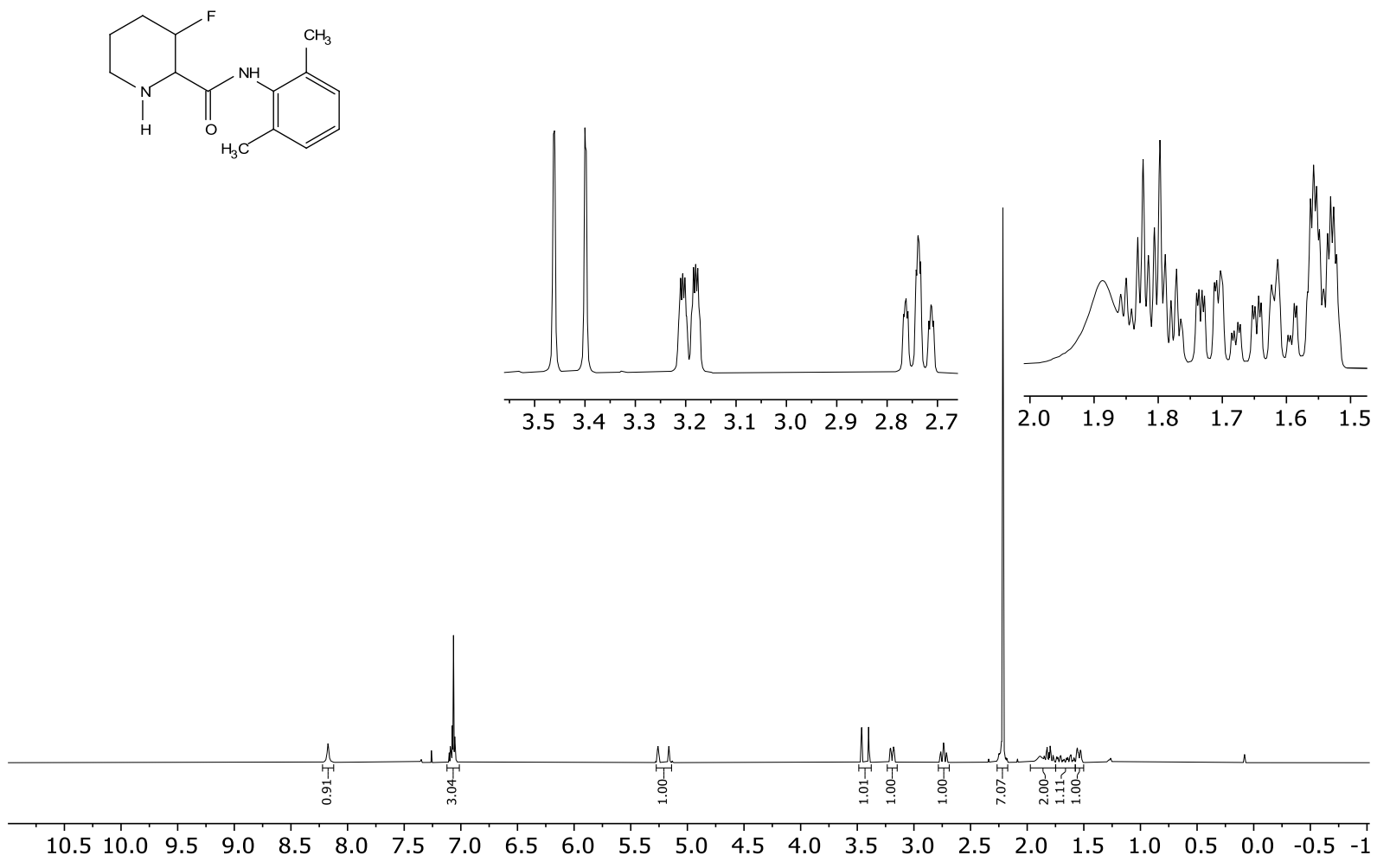

S192 


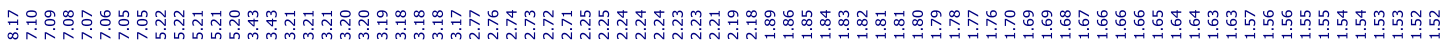

$1 \mathrm{H}\left\{{ }^{19} \mathrm{~F}\right\}$ NMR (Chloroforme)<smiles>Cc1cccc(C)c1NC(=O)C1NCCCC1F</smiles>
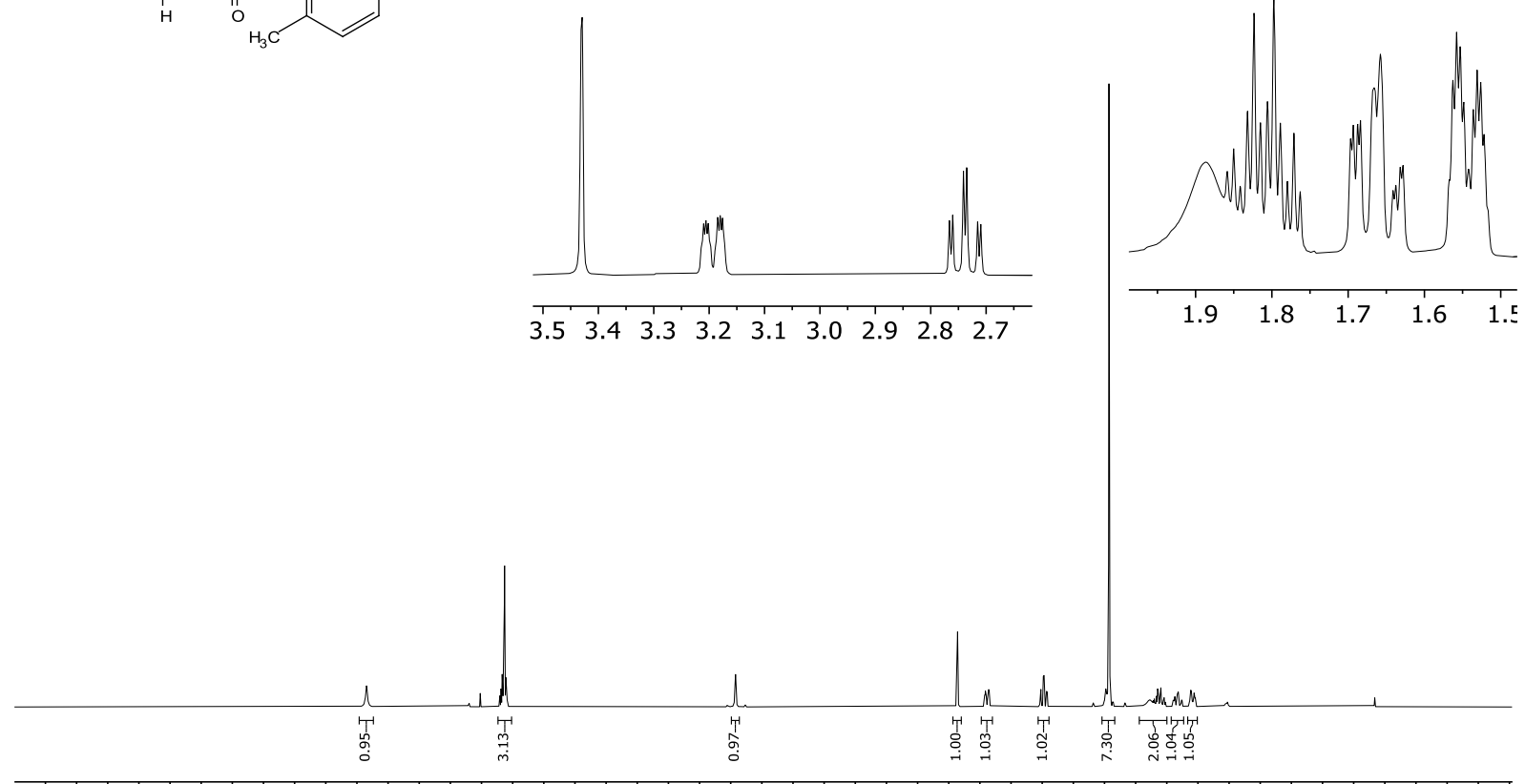

$\begin{array}{llllllllllllllllllllllllllllll}10.5 & 10.0 & 9.5 & 9.0 & 8.5 & 8.0 & 7.5 & 7.0 & 6.5 & 6.0 & 5.5 & 5.0 & 4.5 & 4.0 & 3.5 & 3.0 & 2.5 & 2.0 & 1.5 & 1.0 & 0.5 & 0.0 & -0.5 & -1\end{array}$

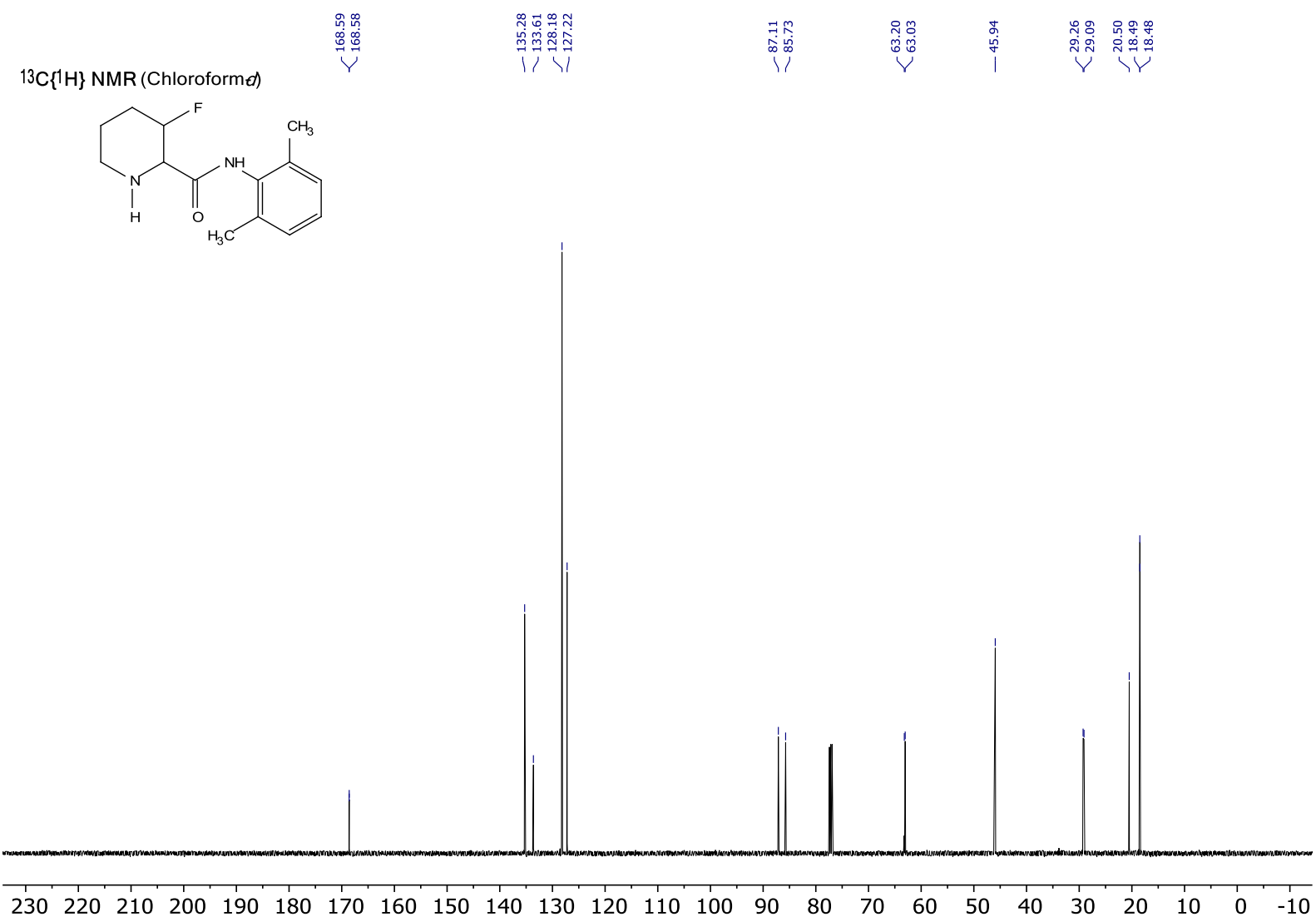




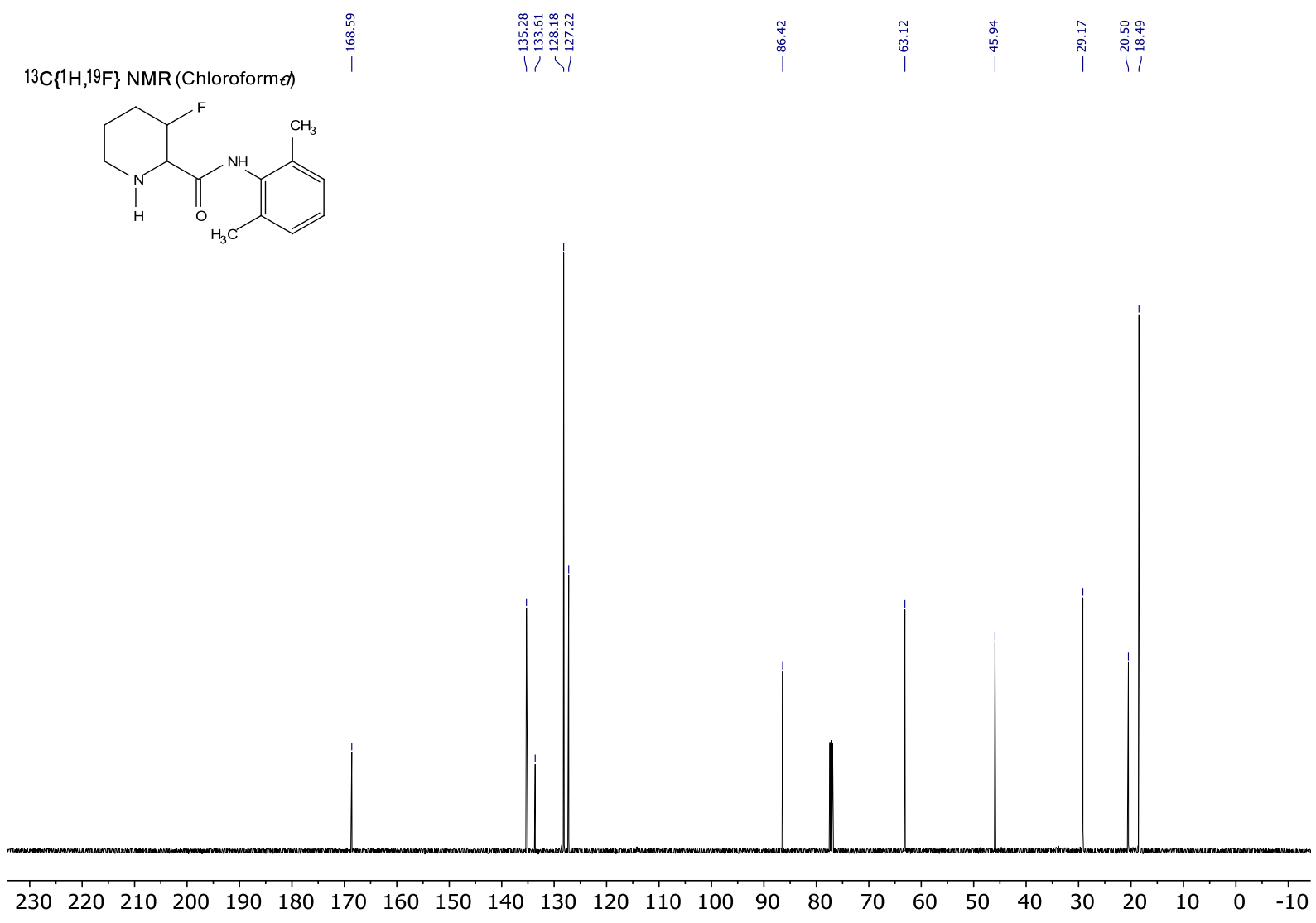

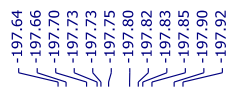

19F NMR (Chloroforme)
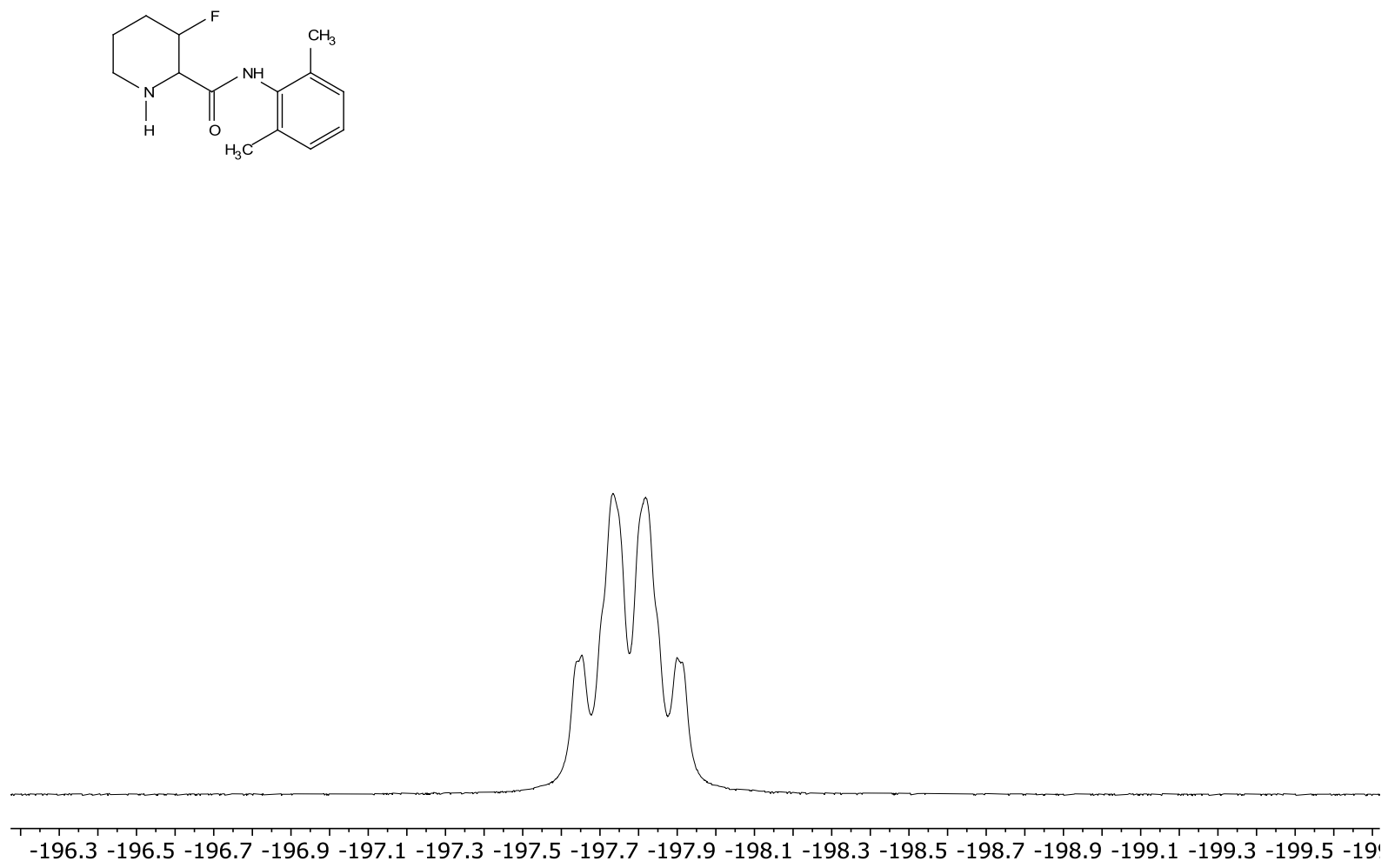

S194 
$19 \mathrm{~F}\{1 \mathrm{H}\}$ NMR (Chloroforme)<smiles>Cc1cccc(C)c1NC(=O)C1NCCCC1F</smiles>

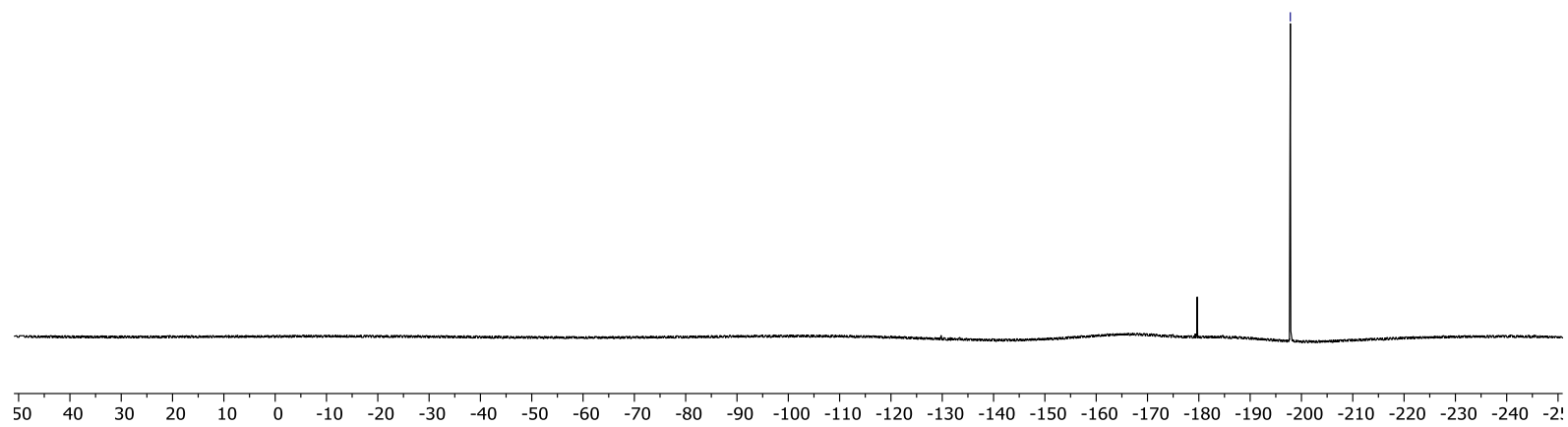

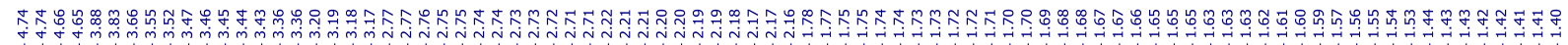

1H NMR (Chloroforme)<smiles>O=C(C1NCCCC1F)N1CCCCC1</smiles>

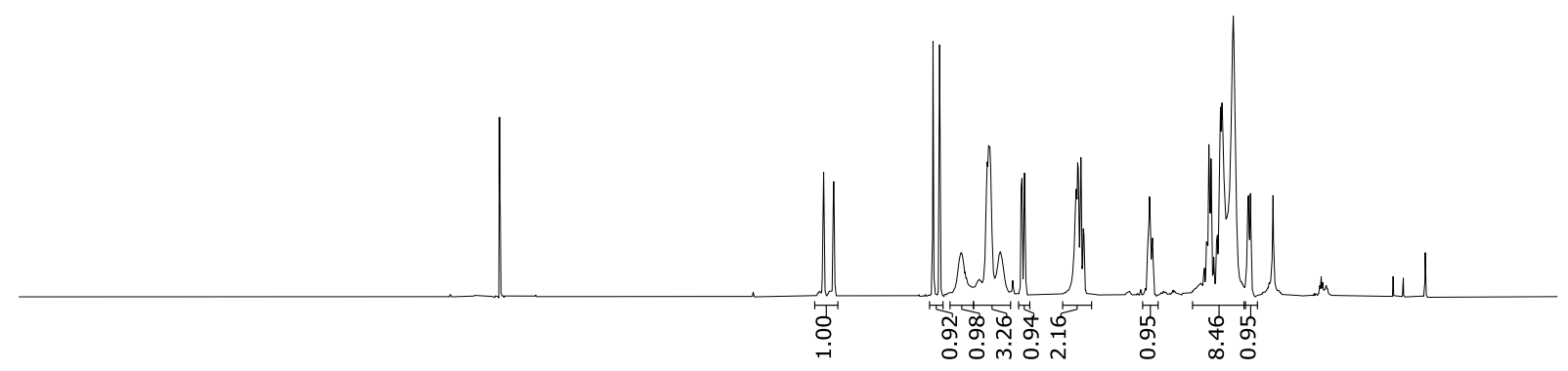

\begin{tabular}{lllllllllllllllllllllllll}
\hline 1.0 & 10.5 & 10.0 & 9.5 & 9.0 & 8.5 & 8.0 & 7.5 & 7.0 & 6.5 & 6.0 & 5.5 & 5.0 & 4.5 & 4.0 & 3.5 & 3.0 & 2.5 & 2.0 & 1.5 & 1.0 & 0.5 & 0.0 & -0.5
\end{tabular} 
$1 \mathrm{H}\{19 \mathrm{~F}\}$ NMR (Chloroformen)<smiles>O=C(C1NCCCC1F)N1CCCCC1</smiles>

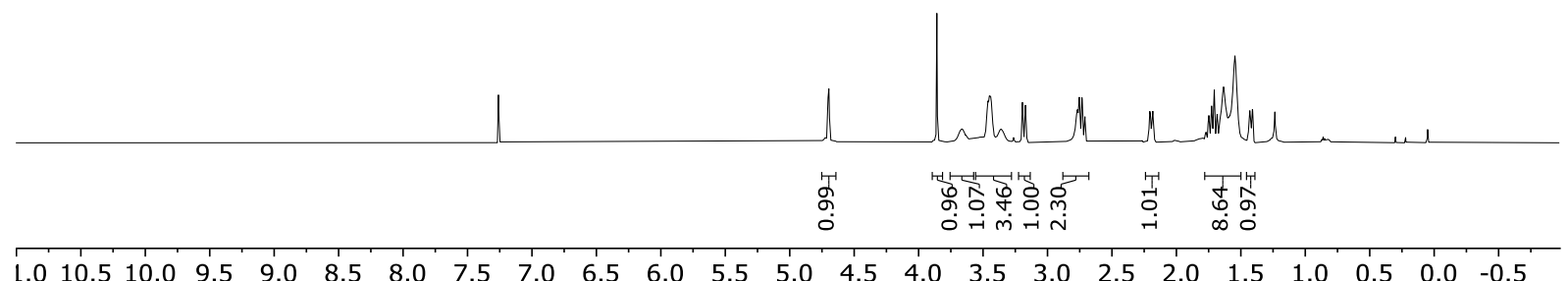

\begin{tabular}{llllllllllllllllllllllll}
\hline .0 & 10.5 & 10.0 & 9.5 & 9.0 & 8.5 & 8.0 & 7.5 & 7.0 & 6.5 & 6.0 & 5.5 & 5.0 & 4.5 & 4.0 & 3.5 & 3.0 & 2.5 & 2.0 & 1.5 & 1.0 & 0.5 & 0.0 & -0.5
\end{tabular}

${ }^{13} \mathrm{C}\{1 \mathrm{H}\}$ NMR (Chloroformet)

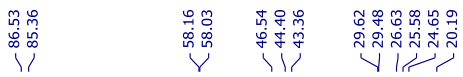<smiles>O=C(C1NCCCC1F)N1CCCCC1</smiles>

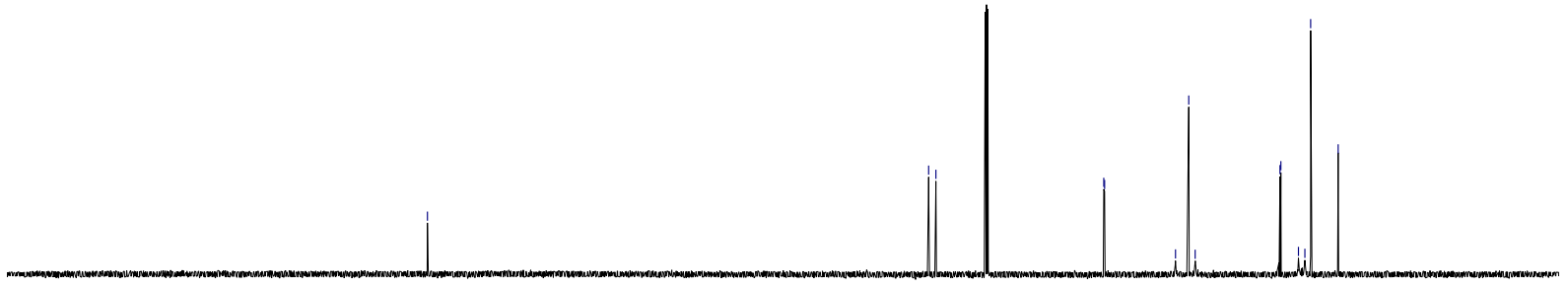

$\begin{array}{lllllllllllllllllllllllllll}230 & 220 & 210 & 200 & 190 & 180 & 170 & 160 & 150 & 140 & 130 & 120 & 110 & 100 & 90 & 80 & 70 & 60 & 50 & 40 & 30 & 20 & 10 & 0 & -10\end{array}$ 
${ }_{13} \mathrm{C}\{1 \mathrm{H}, 19 \mathrm{~F}\}$ NMR (Chloroformel)<smiles>O=C(C1NCCCC1F)N1CCCCC1</smiles>

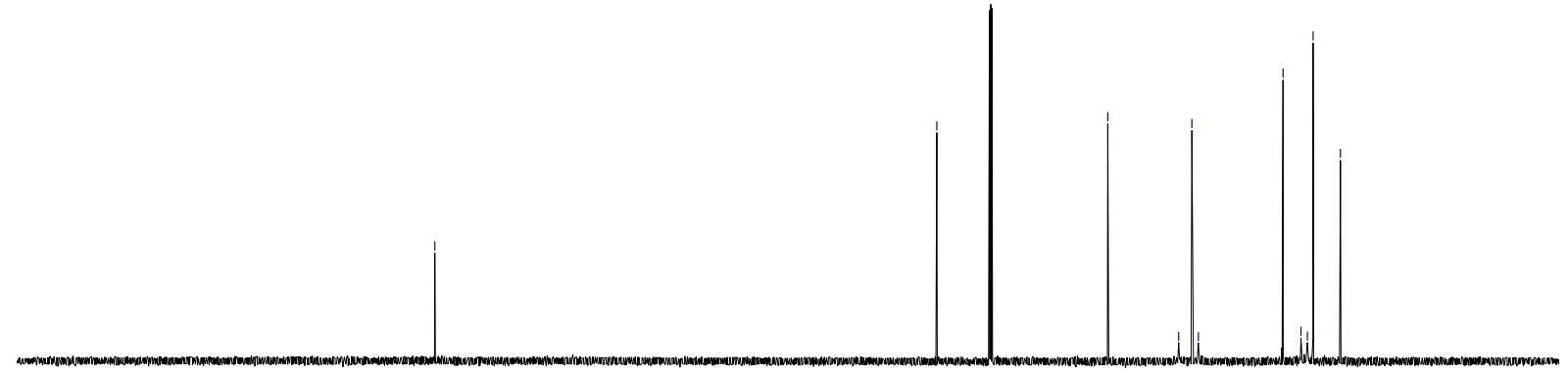

$\begin{array}{lllllllllllllllllllllllll}230 & 220 & 210 & 200 & 190 & 180 & 170 & 160 & 150 & 140 & 130 & 120 & 110 & 100 & 90 & 80 & 70 & 60 & 50 & 40 & 30 & 20 & 10 & 0 & -10\end{array}$<smiles>O=C(C1CCCNC1F)N1CCCCC1</smiles>

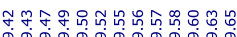 \\ T.}

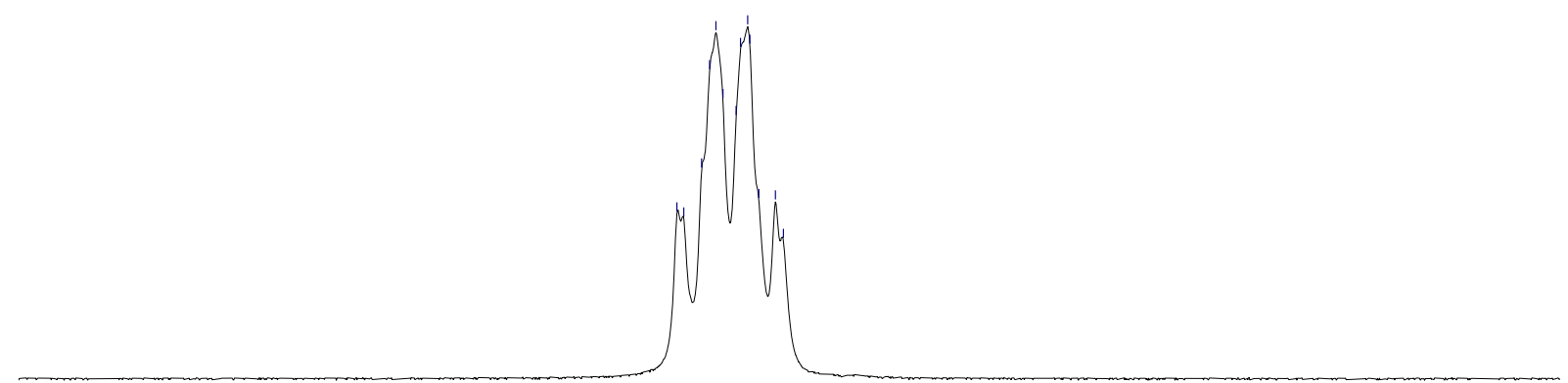

$\begin{array}{lllllllllllllllll}-198.1 & -198.3 & -198.5 & -198.7 & -198.9 & -199.1 & -199.3 & -199.5 & -199.7 & -199.9 & -200.1 & -200.3 & -200.5 & -200.7 & -200.9 & -201.1 & -201\end{array}$ 
${ }^{19} \mathrm{~F}\{1 \mathrm{H}\}$ NMR (Chloroformed)<smiles>O=C(C1NCCCC1F)N1CCCCC1</smiles>

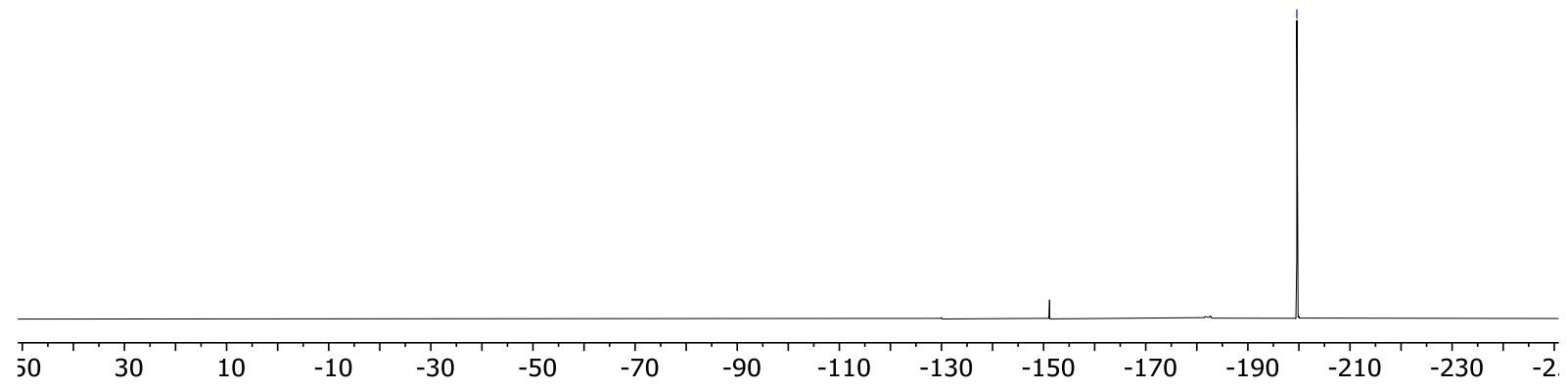

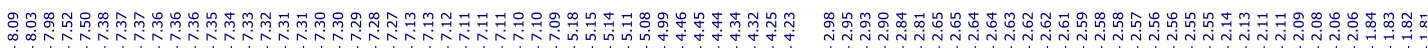
1H NMR (Chloroforme)

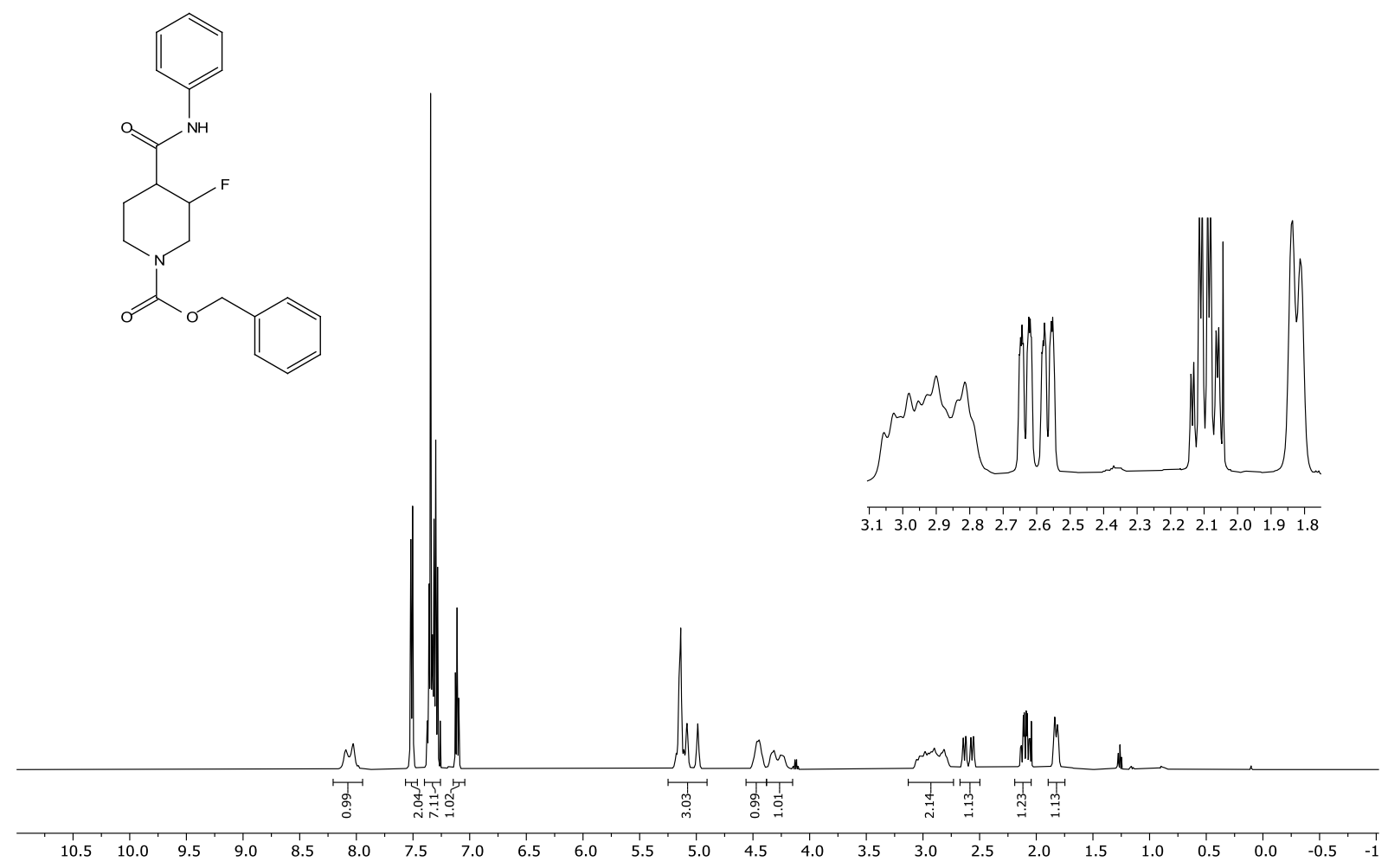




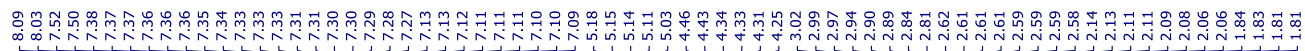
$1 \mathrm{H}\{19 \mathrm{~F}\}$ NMR (Chloroformed)

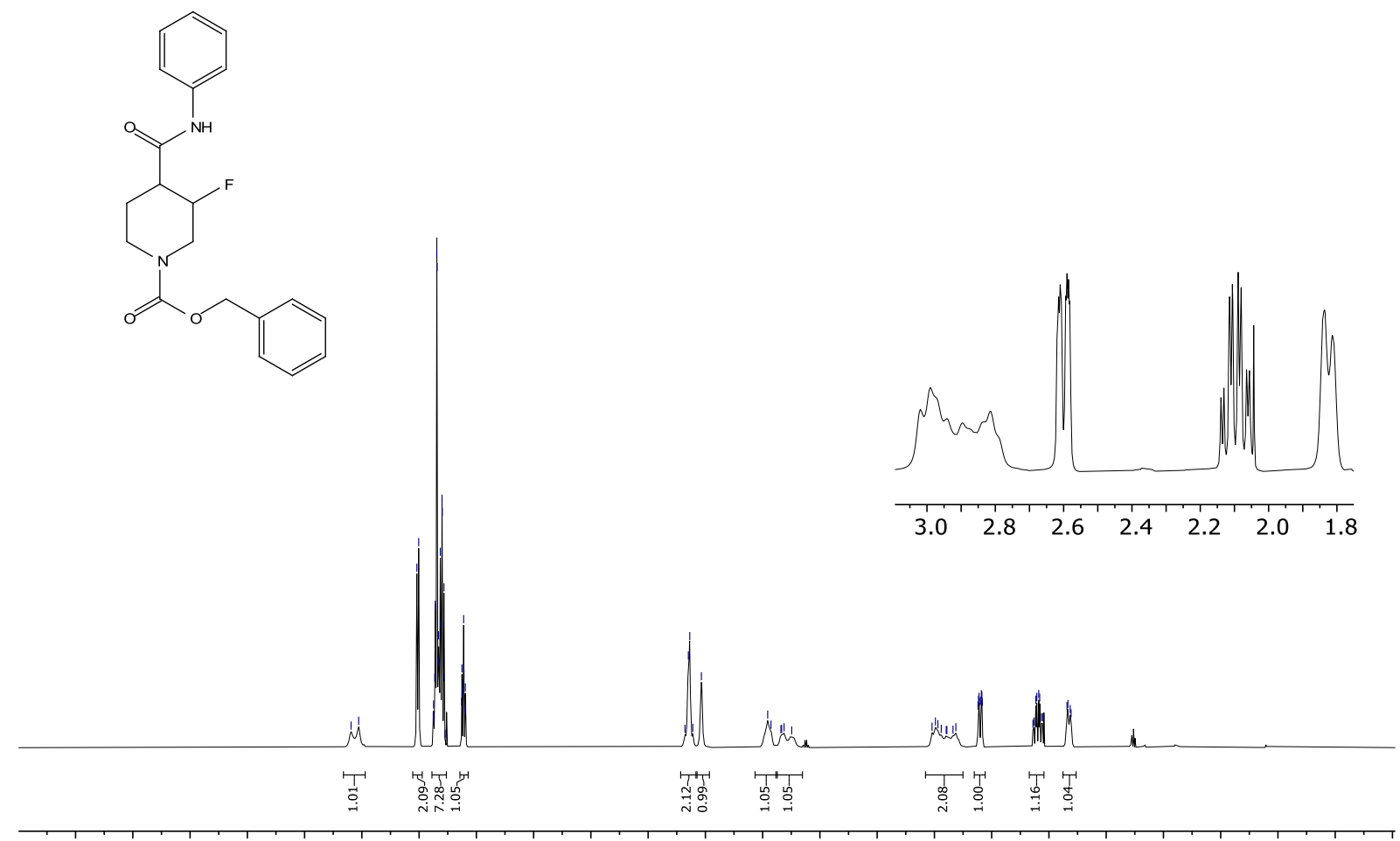

$\begin{array}{lllllllllllllllllllllllllllllll}10.5 & 10.0 & 9.5 & 9.0 & 8.5 & 8.0 & 7.5 & 7.0 & 6.5 & 6.0 & 5.5 & 5.0 & 4.5 & 4.0 & 3.5 & 3.0 & 2.5 & 2.0 & 1.5 & 1.0 & 0.5 & 0.0 & -0.5 & -1\end{array}$ 13C\{1H\} NMR (Chloroformel)<smiles>O=C(Nc1ccccc1)C1CCN(C(=O)OCc2ccccc2)CC1F</smiles>

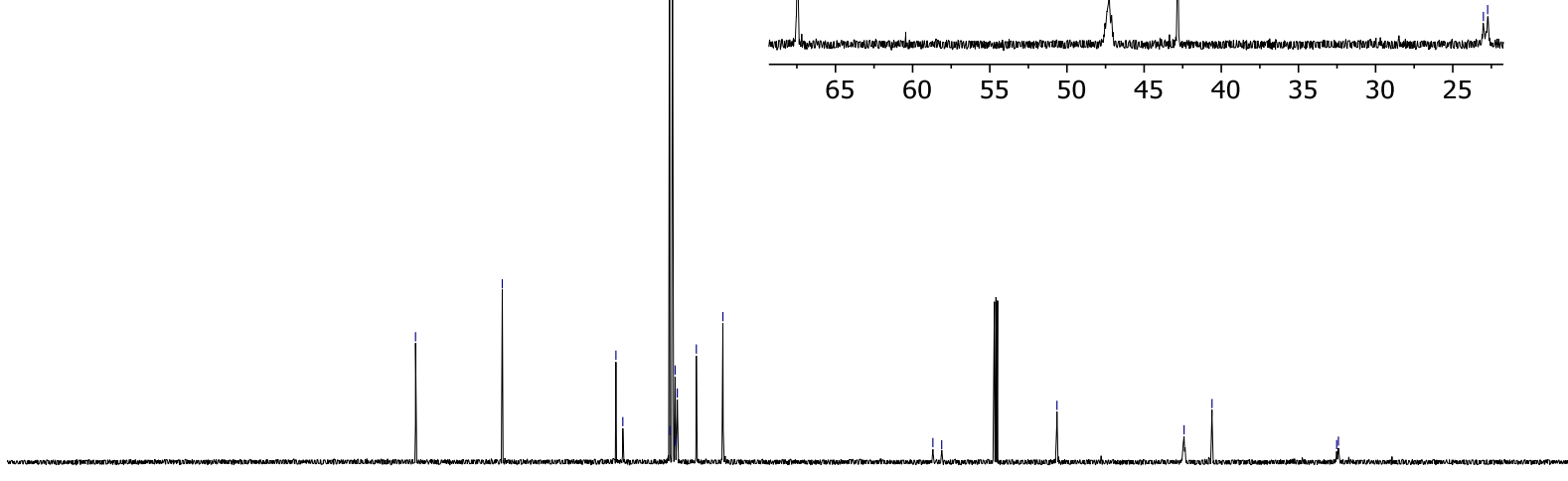

$\begin{array}{lllllllllllllllllllllllllll}230 & 220 & 210 & 200 & 190 & 180 & 170 & 160 & 150 & 140 & 130 & 120 & 110 & 100 & 90 & 80 & 70 & 60 & 50 & 40 & 30 & 20 & 10 & 0 & -10\end{array}$ 


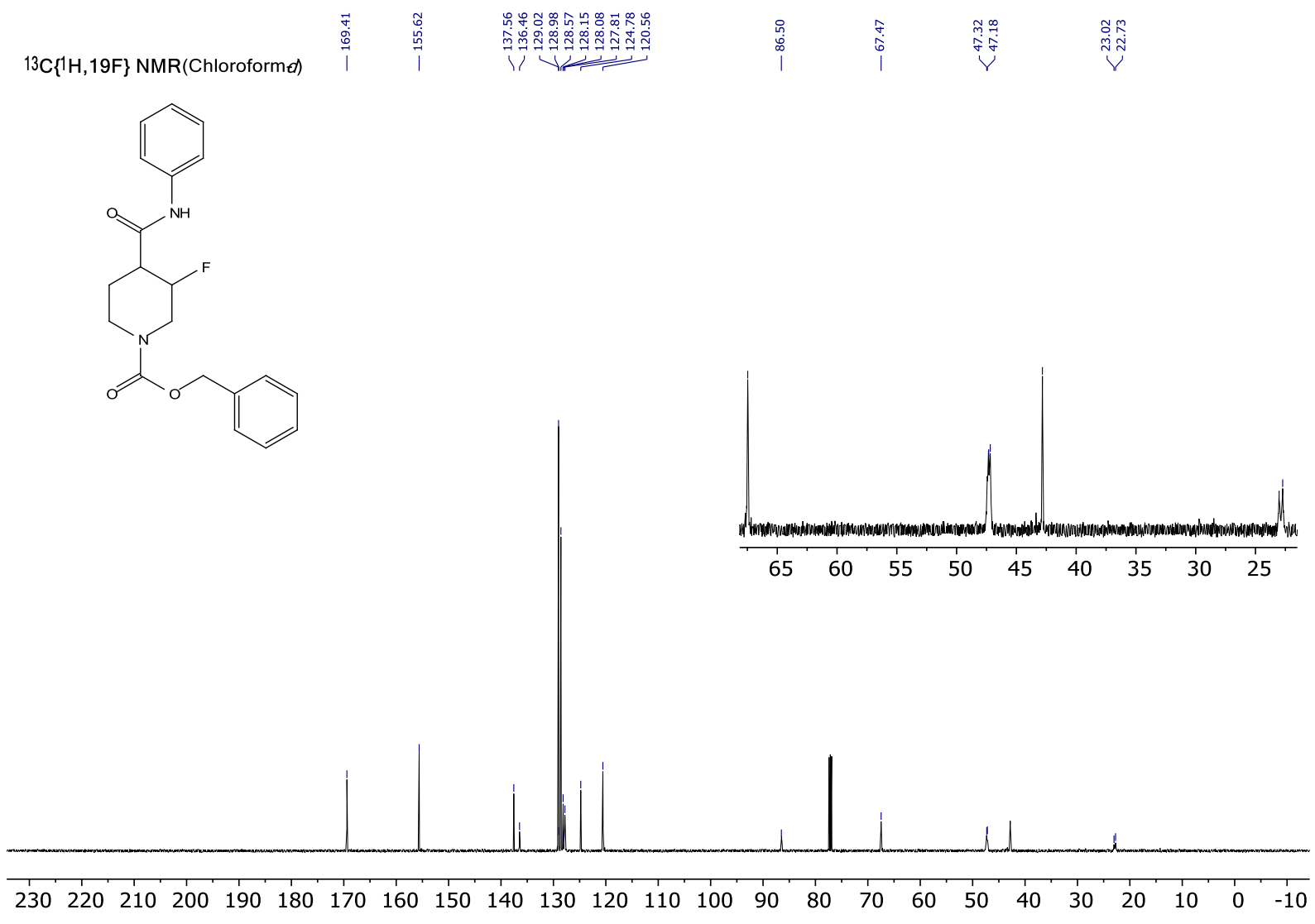

19F NMR (Chloroforme)

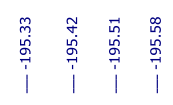
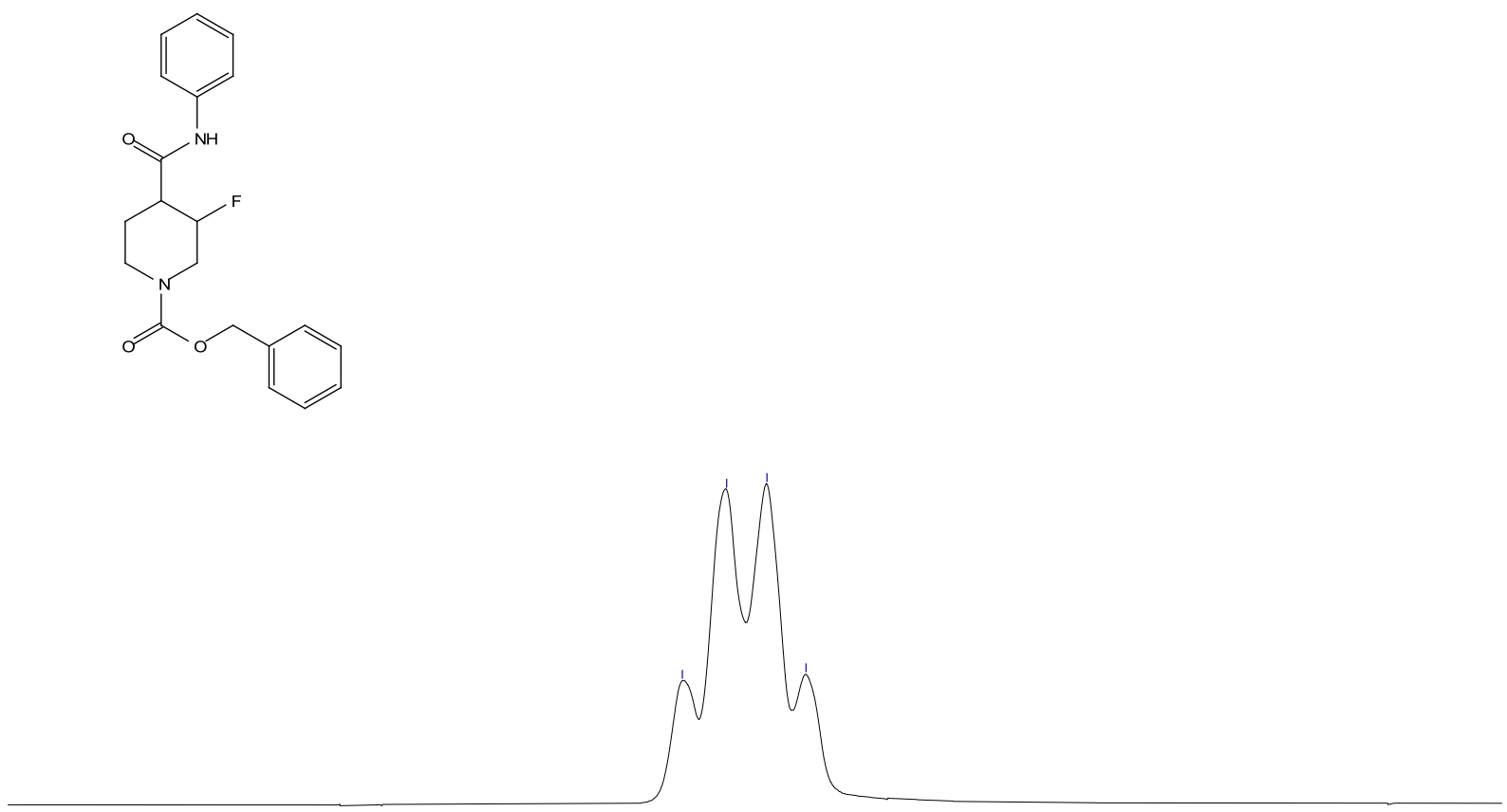

$\begin{array}{llllllllllllllll}-194.1 & -194.3 & -194.5 & -194.7 & -194.9 & -195.1 & -195.3 & -195.5 & -195.7 & -195.9 & -196.1 & -196.3 & -196.5 & -196.7 & -196.9\end{array}$

S200 
${ }^{19} \mathrm{~F}\{1 \mathrm{H}\}$ NMR (Chloroformel)

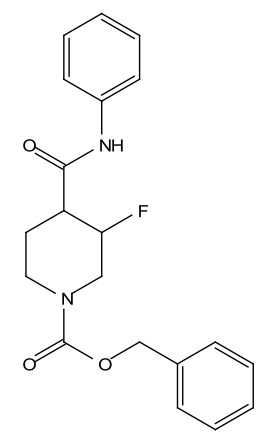

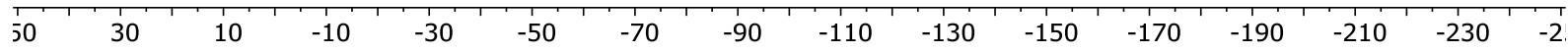

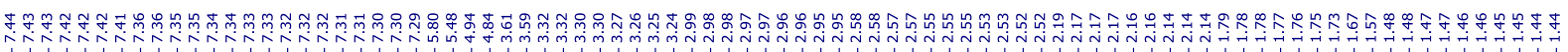
1H NMR (Chloroforme)<smiles>NC(=O)C(c1ccccc1)C1NCCCC1F</smiles>

菓员 作
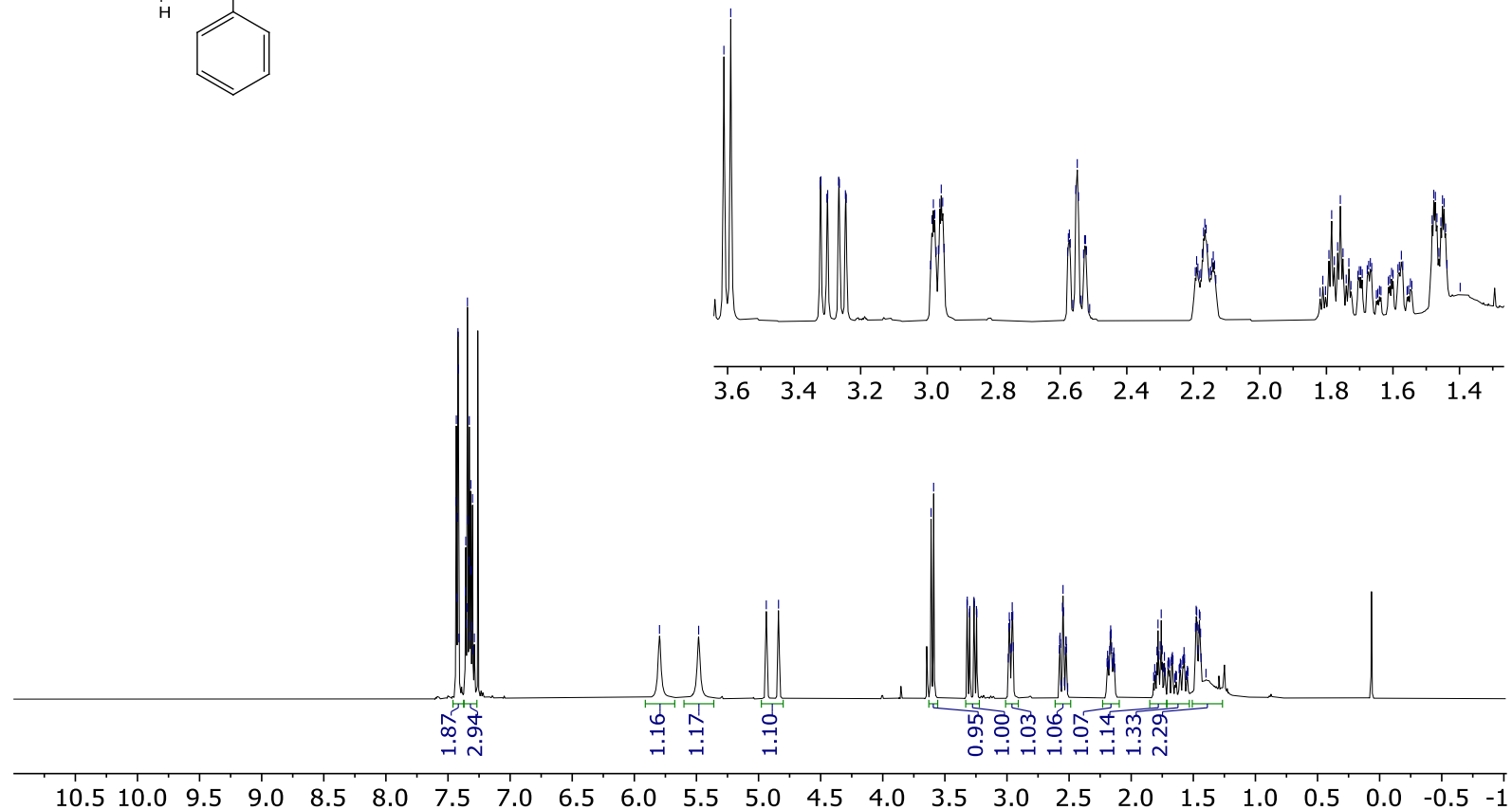


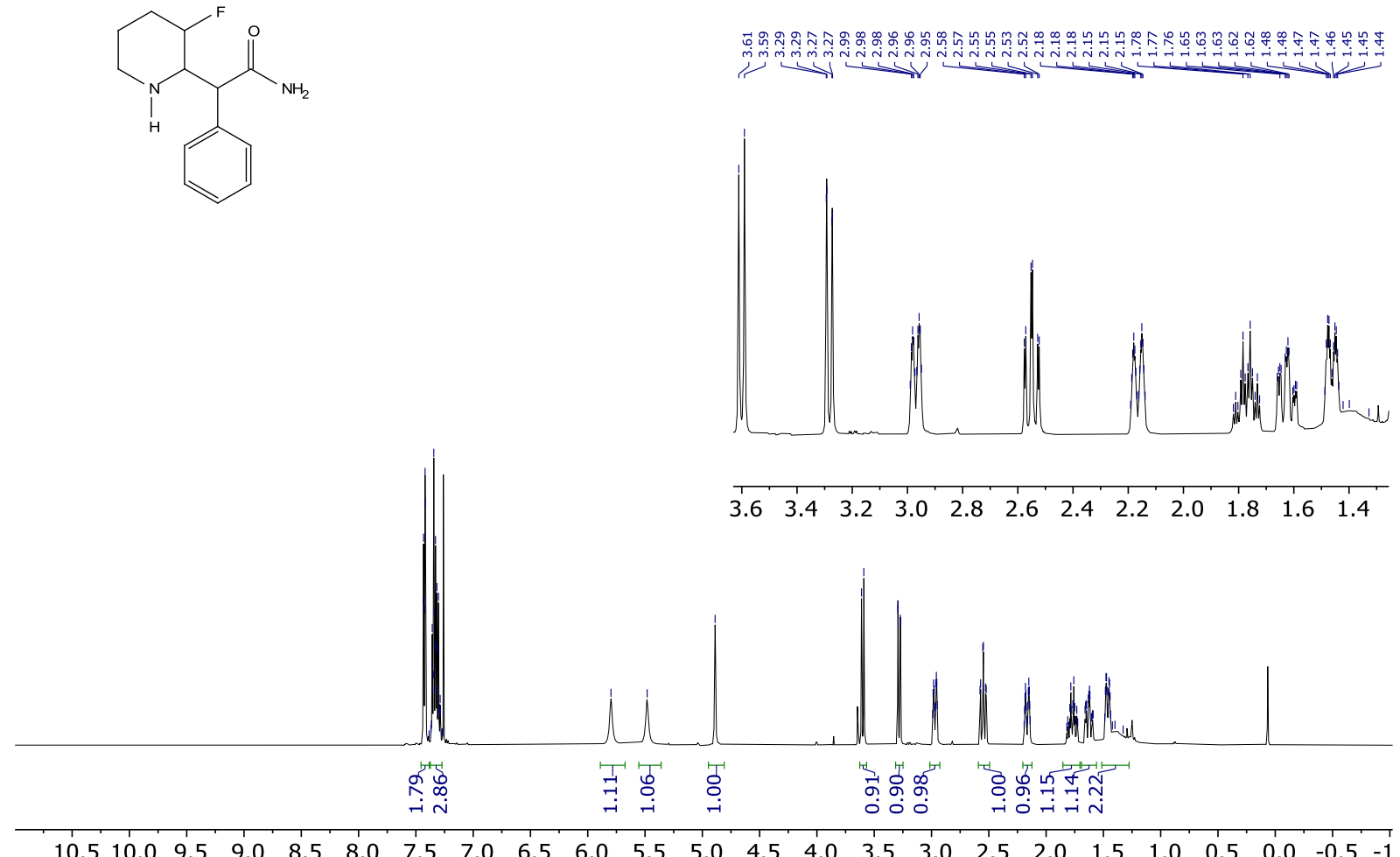
$\begin{array}{lllllllllllllllllllllllllll}10.5 & 10.0 & 9.5 & 9.0 & 8.5 & 8.0 & 7.5 & 7.0 & 6.5 & 6.0 & 5.5 & 5.0 & 4.5 & 4.0 & 3.5 & 3.0 & 2.5 & 2.0 & 1.5 & 1.0 & 0.5 & 0.0 & -0.5 & -1\end{array}$ ${ }^{13} \mathrm{C}\{1 \mathrm{H}\}$ NMR (Chloroforme)

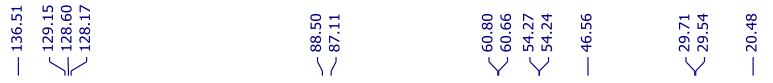<smiles>NC(=O)C(c1ccccc1)C1NCCCC1F</smiles> 


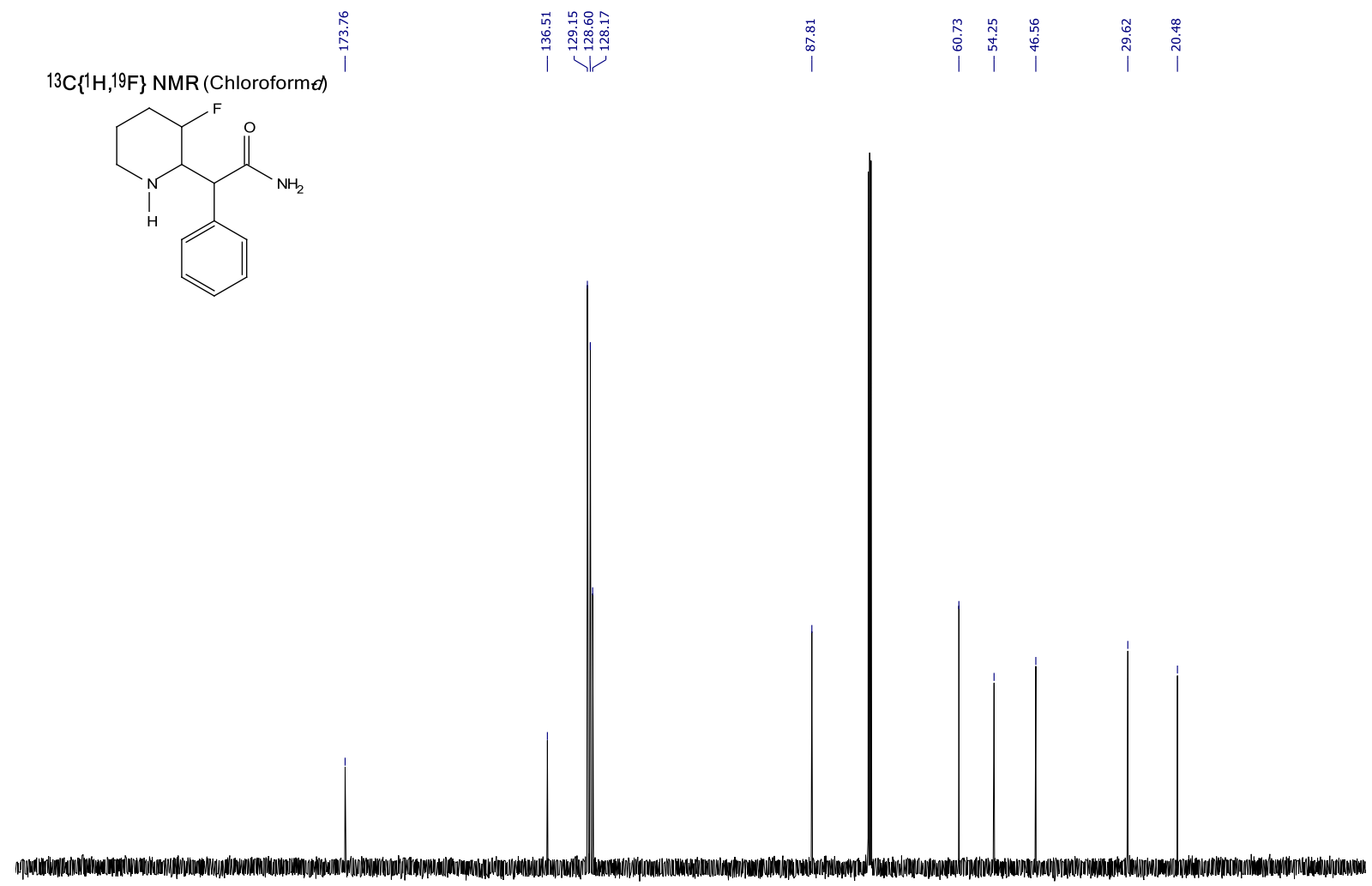

$\begin{array}{lllllllllllllllllllllllll}230 & 220 & 210 & 200 & 190 & 180 & 170 & 160 & 150 & 140 & 130 & 120 & 110 & 100 & 90 & 80 & 70 & 60 & 50 & 40 & 30 & 20 & 10 & 0 & -10\end{array}$

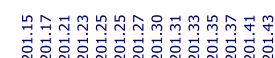

19F NMR (Chloroforme)

iोरोivijil<smiles>NC(=O)C(c1ccccc1)C1NCCCC1F</smiles>

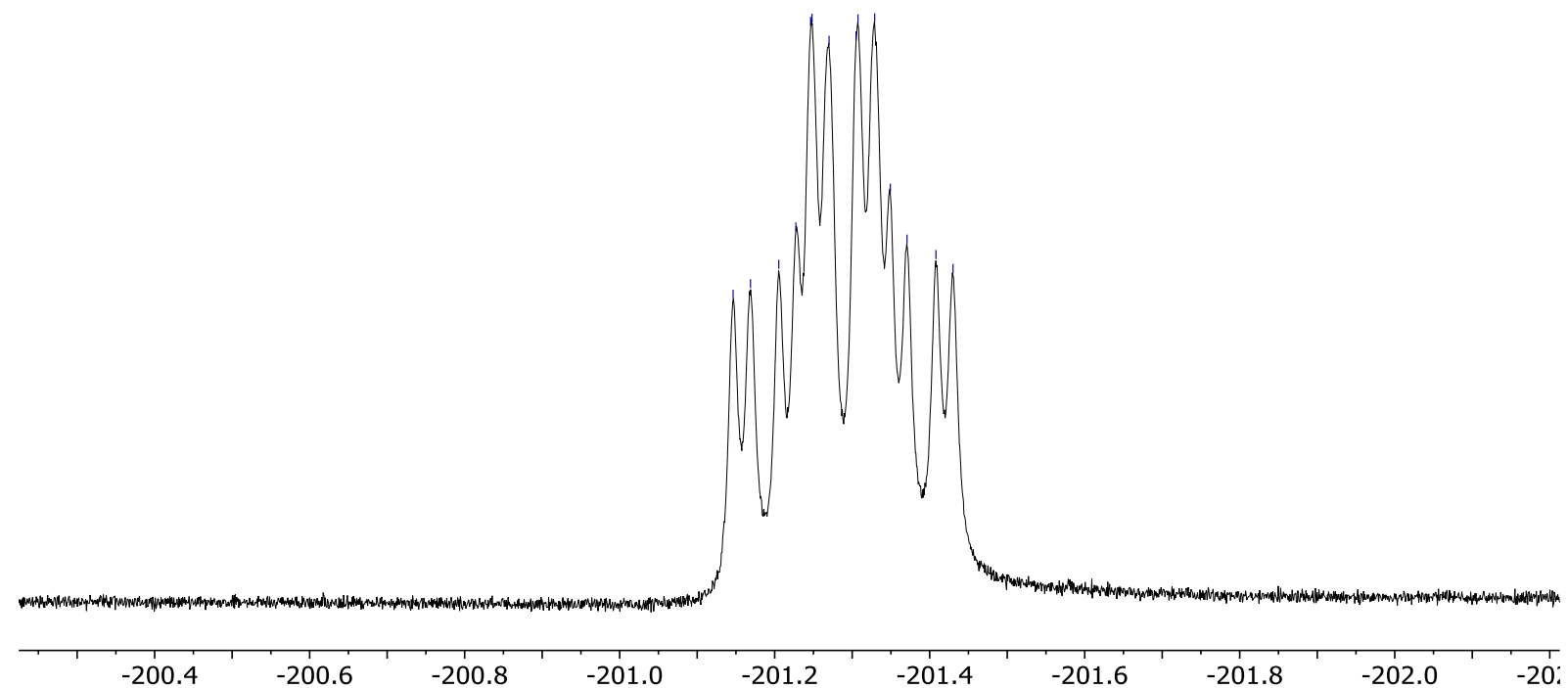


19F $\{1 \mathrm{H}\}$ NMR (Chloroformed)

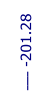
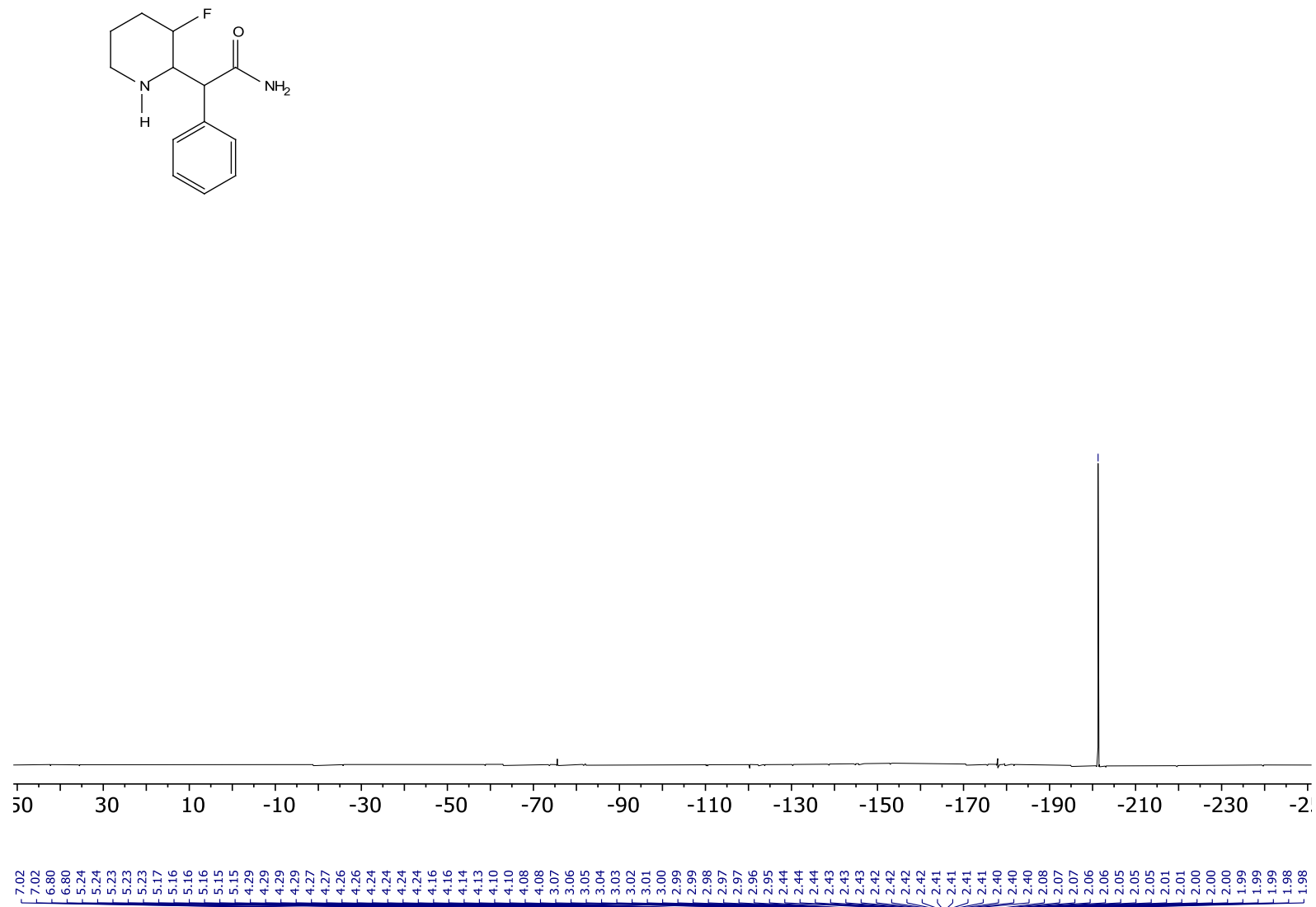

1H NMR (Chloroforme)
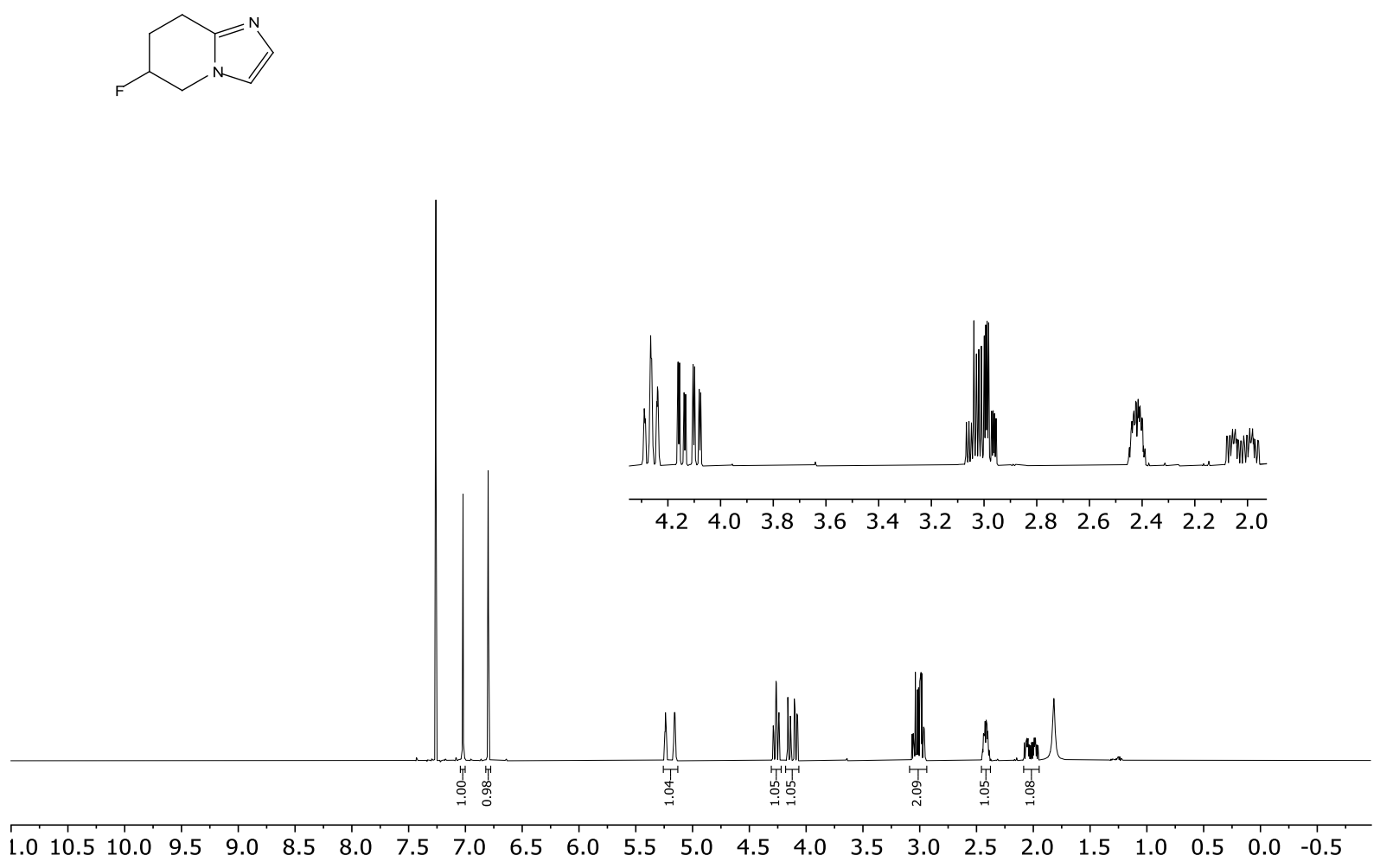

S204 
$1 \mathrm{H}\{19 \mathrm{~F}\}$ NMR (Chloroforme)<smiles>FC1CCc2nccn2C1</smiles>

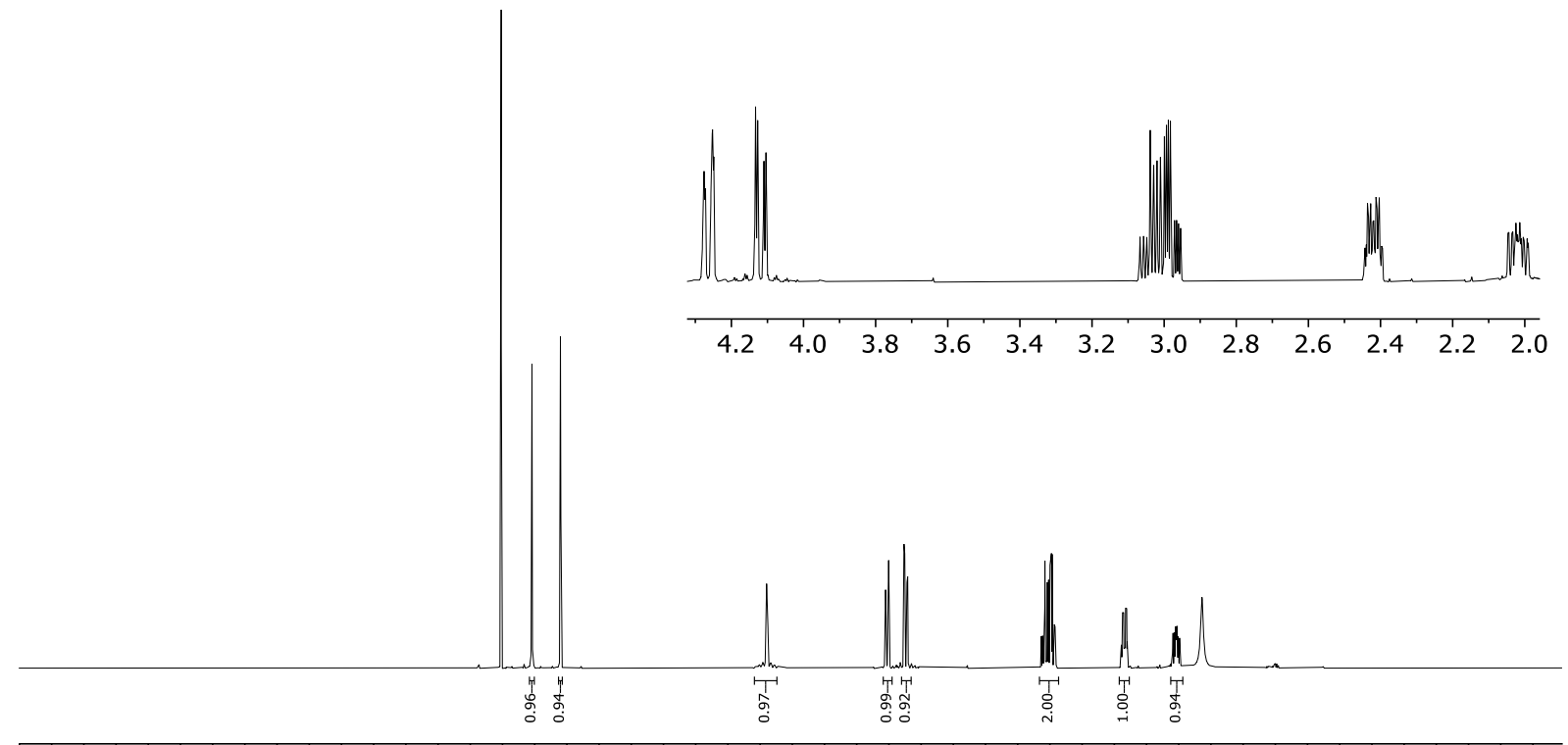

\begin{tabular}{lllllllllllllllllllllllllllll}
\hline .0 & 10.5 & 10.0 & 9.5 & 9.0 & 8.5 & 8.0 & 7.5 & 7.0 & 6.5 & 6.0 & 5.5 & 5.0 & 4.5 & 4.0 & 3.5 & 3.0 & 2.5 & 2.0 & 1.5 & 1.0 & 0.5 & 0.0 & -0.5
\end{tabular}

$13 \mathrm{C}\{1 \mathrm{H}\}$ NMR (Chloroforme)<smiles>FC1CCc2nccn2C1</smiles>
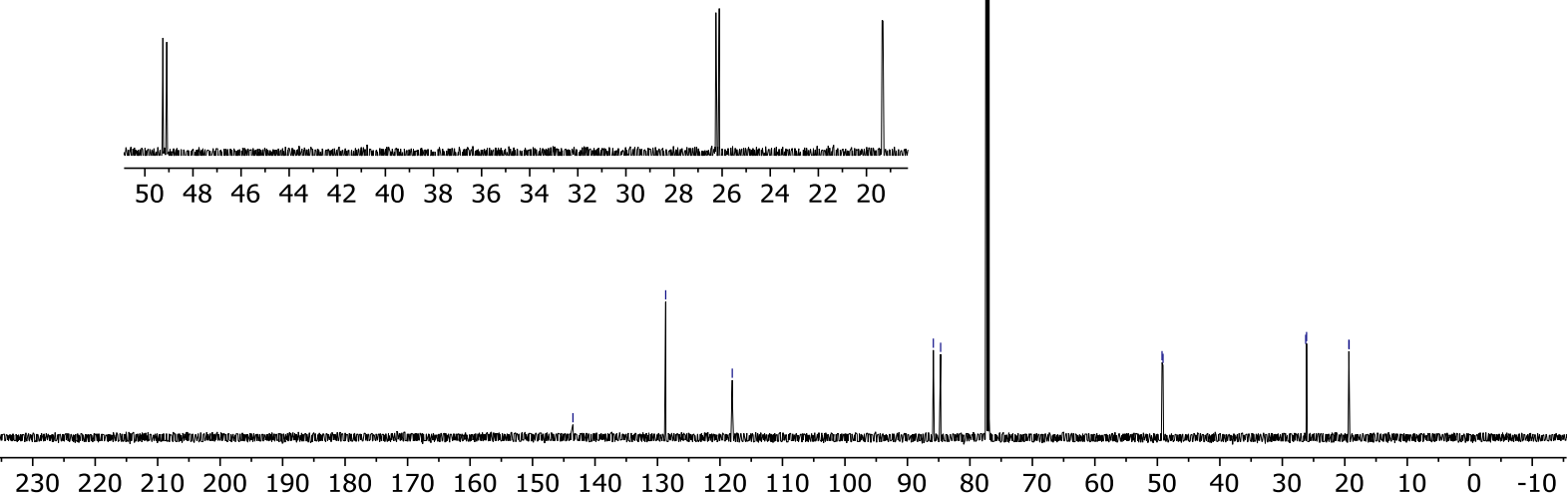
${ }^{13} \mathrm{C}\left\{1 \mathrm{H},{ }^{19} \mathrm{~F}\right\}$ NMR (Chloroforme)

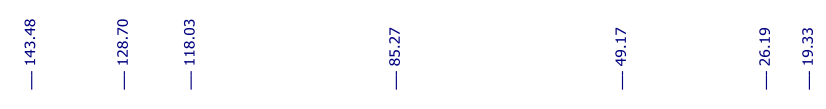<smiles>FC1CCc2nccn2C1</smiles>

19F NMR (Chloroforme)<smiles>FC1CCc2nccn2C1</smiles>

\begin{tabular}{lllllllllll}
\hline-187.60 & -187.70 & -187.80 & -187.90 & -188.00 & -188.10 & -188.20 & -188.30 & -188.40 & -188.50 & -188.60
\end{tabular}


19F $\{1 \mathrm{H}\}$ NMR (Chloroformed)

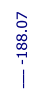
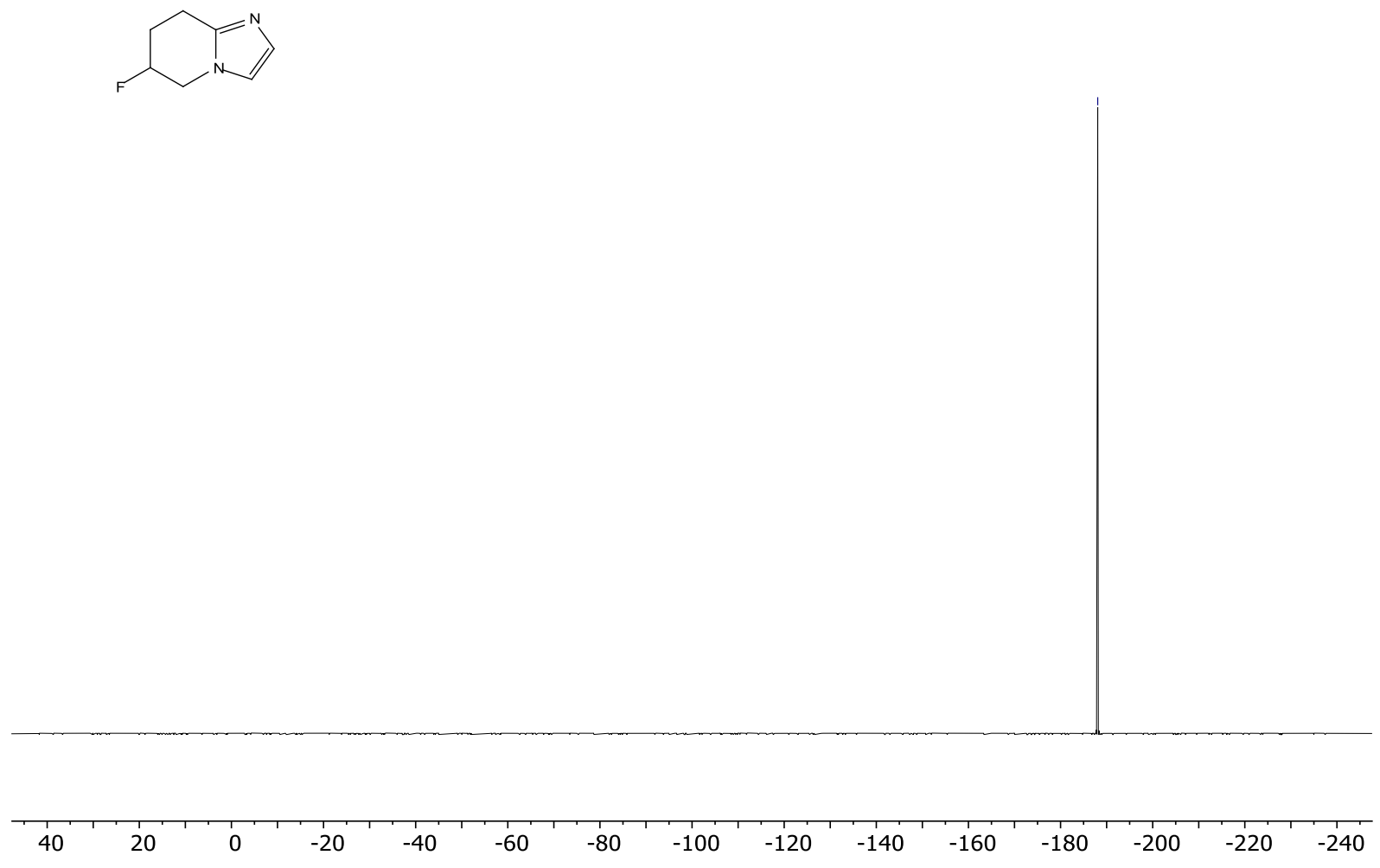

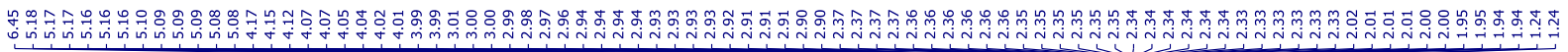

1H NMR (Chloroforme)
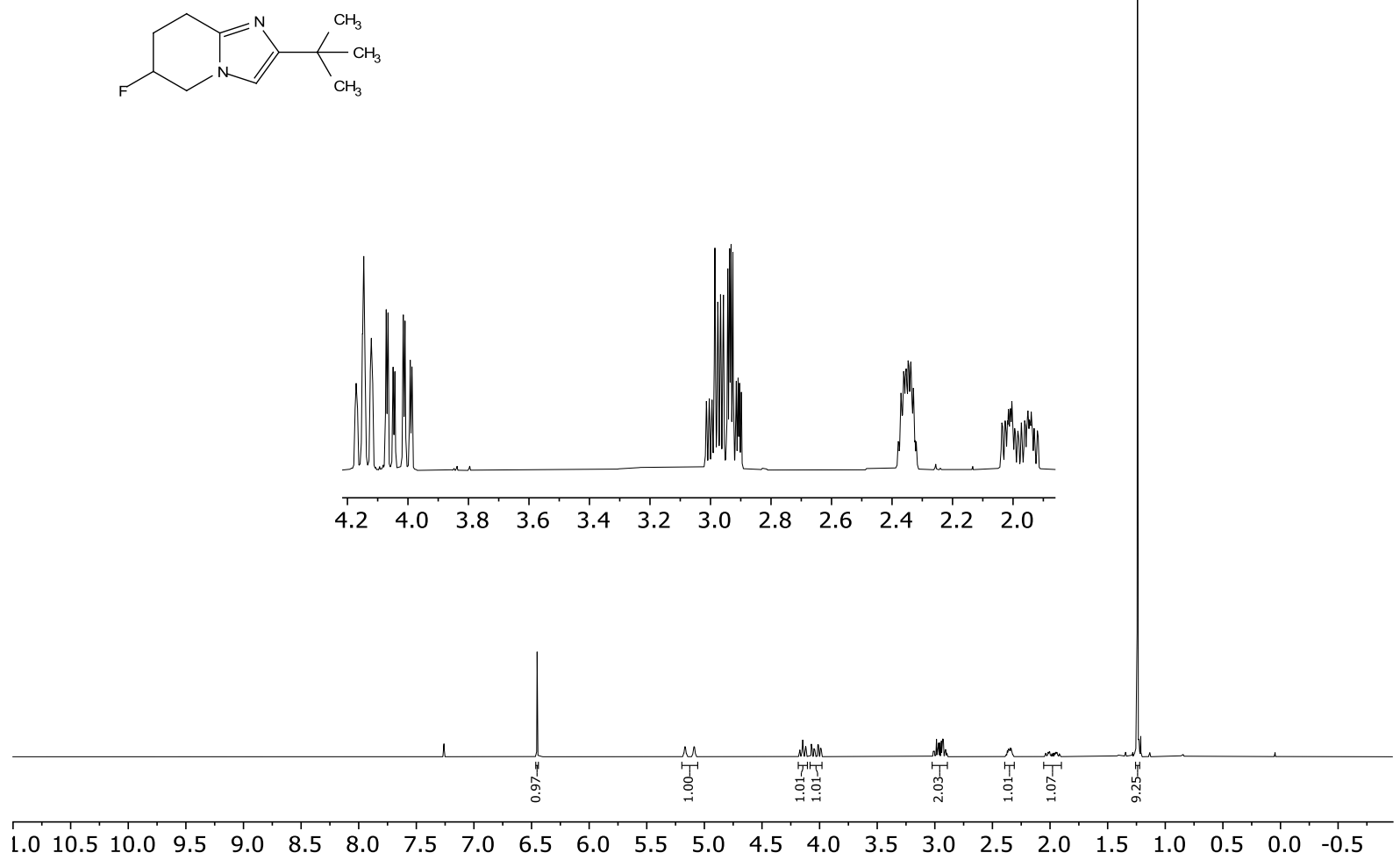

S207 


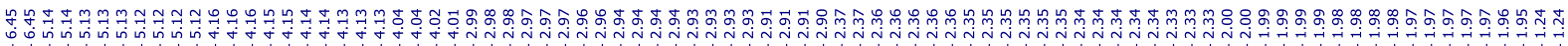
$1 \mathrm{H}\left\{{ }^{19} \mathrm{~F}\right\}$ NMR (Chloroformed)<smiles>CC(C)(C)c1cn2c(n1)CCC(F)C2</smiles>
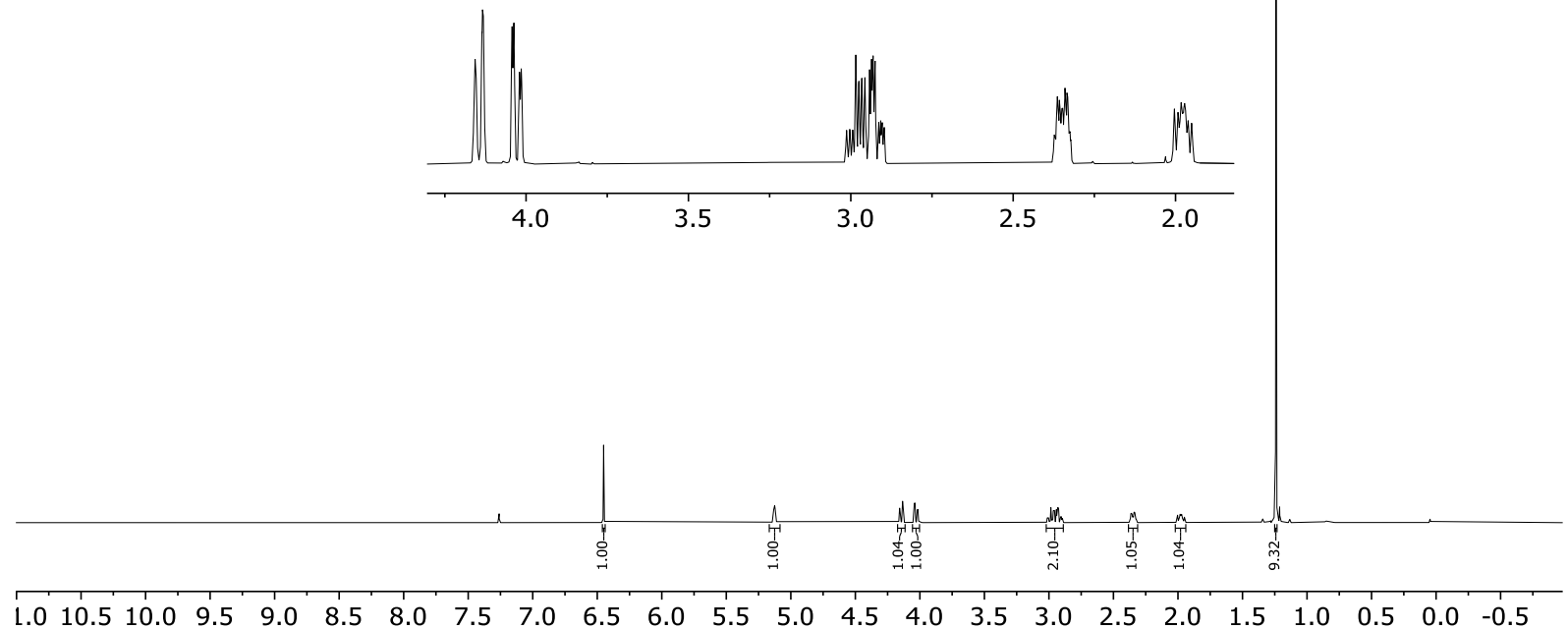

${ }^{13} \mathrm{C}\{1 \mathrm{H}\}$ NMR (Chloroformed)

\begin{tabular}{|c|c|c|c|c|}
\hline 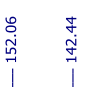 & 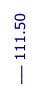 & 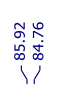 & 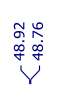 & 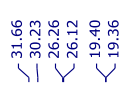 \\
\hline
\end{tabular}<smiles>CC(C)(C)c1cn2c(n1)CCC(F)C2</smiles> 
${ }^{13} \mathrm{C}\{1 \mathrm{H}, 19 \mathrm{~F}\}$ NMR (Chloroformed)

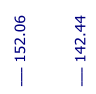

$\stackrel{\substack{n \\-1}}{7}$

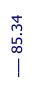

प)
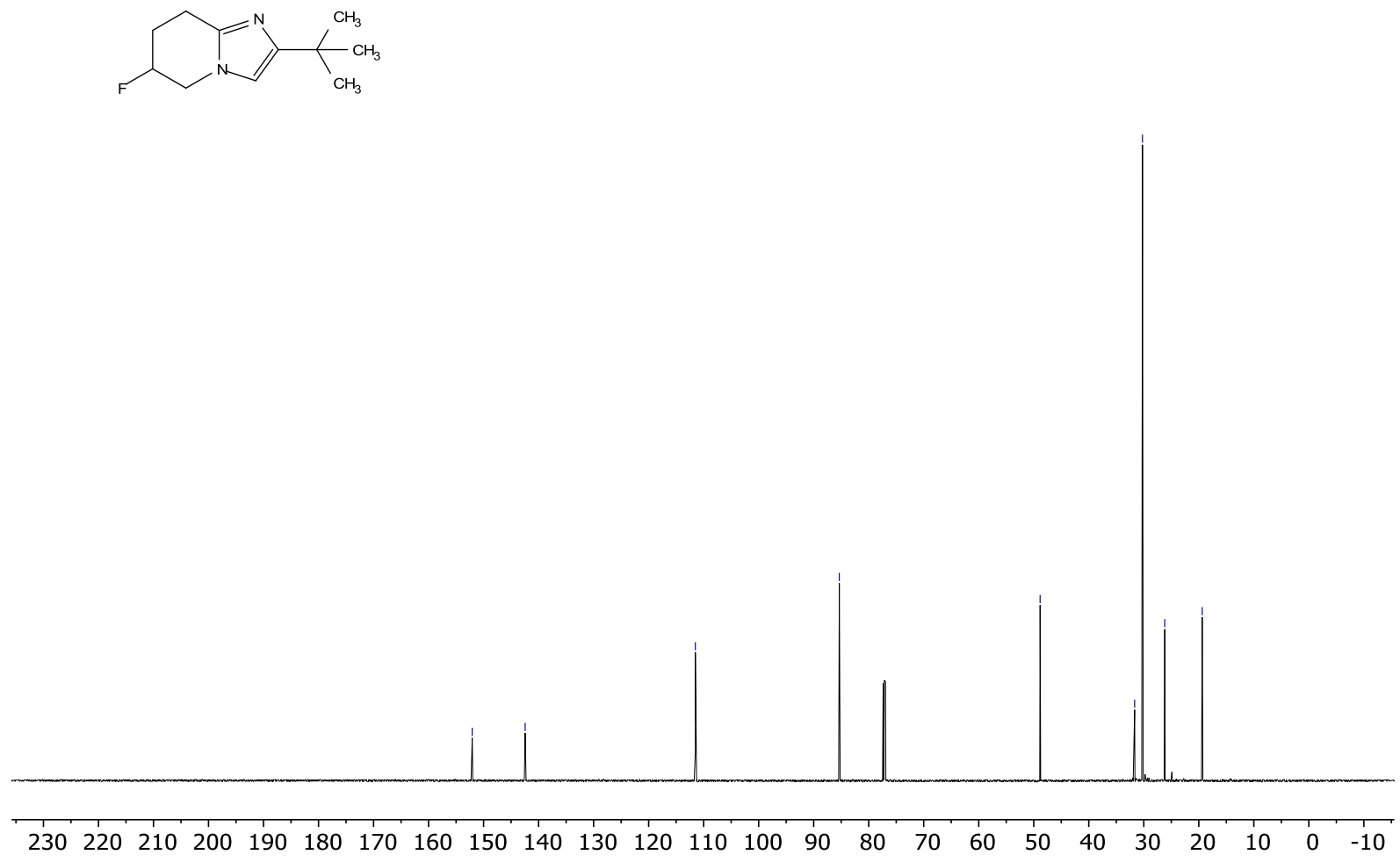

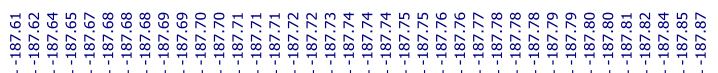

19F NMR (Chloroformed)
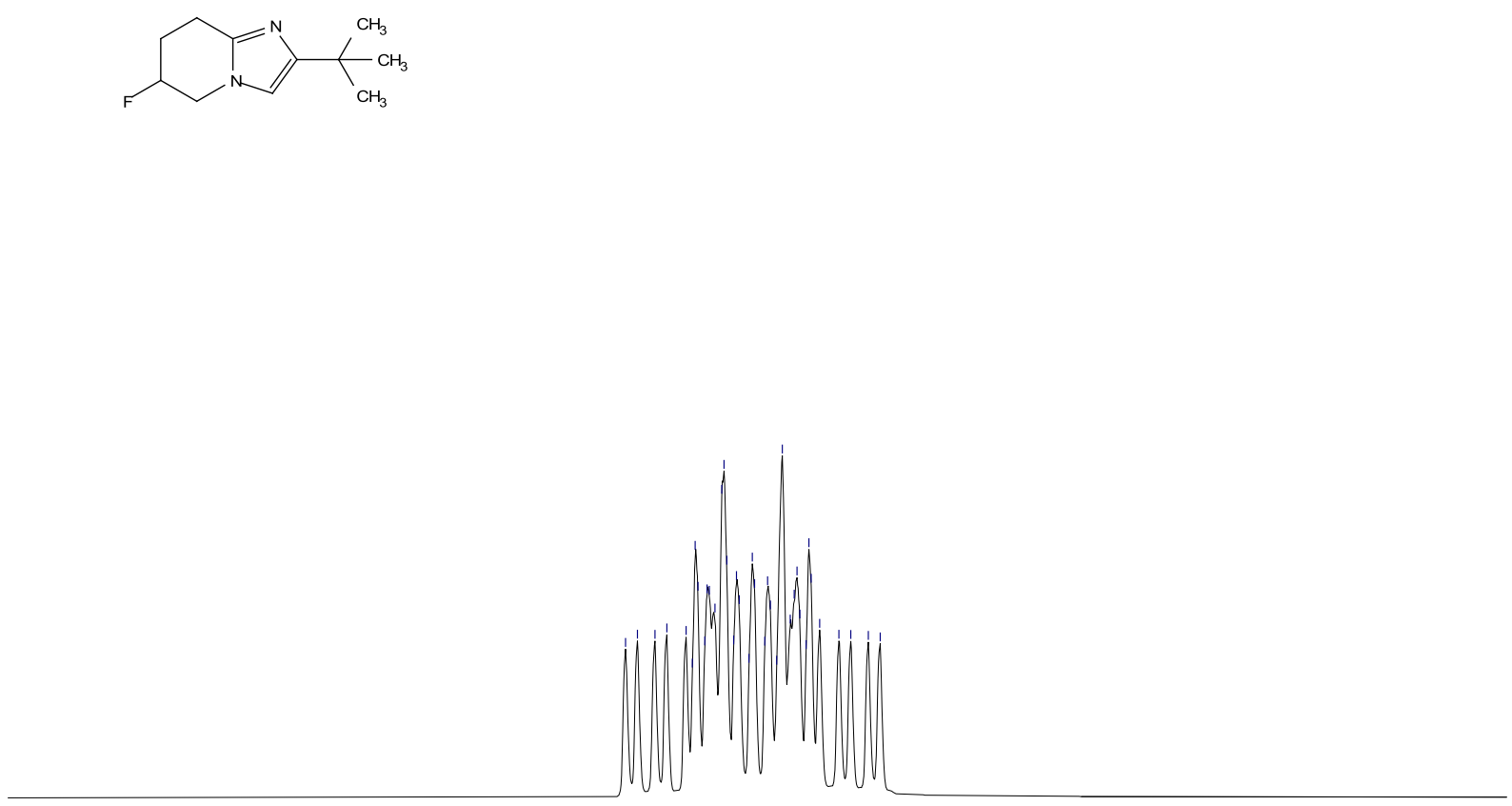

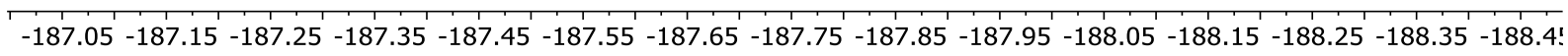

S209 
${ }^{19} \mathrm{~F}\{1 \mathrm{H}\}$ NMR (Chloroformed)

$\stackrel{\substack{\stackrel{\infty}{i} \\ i}}{i}$
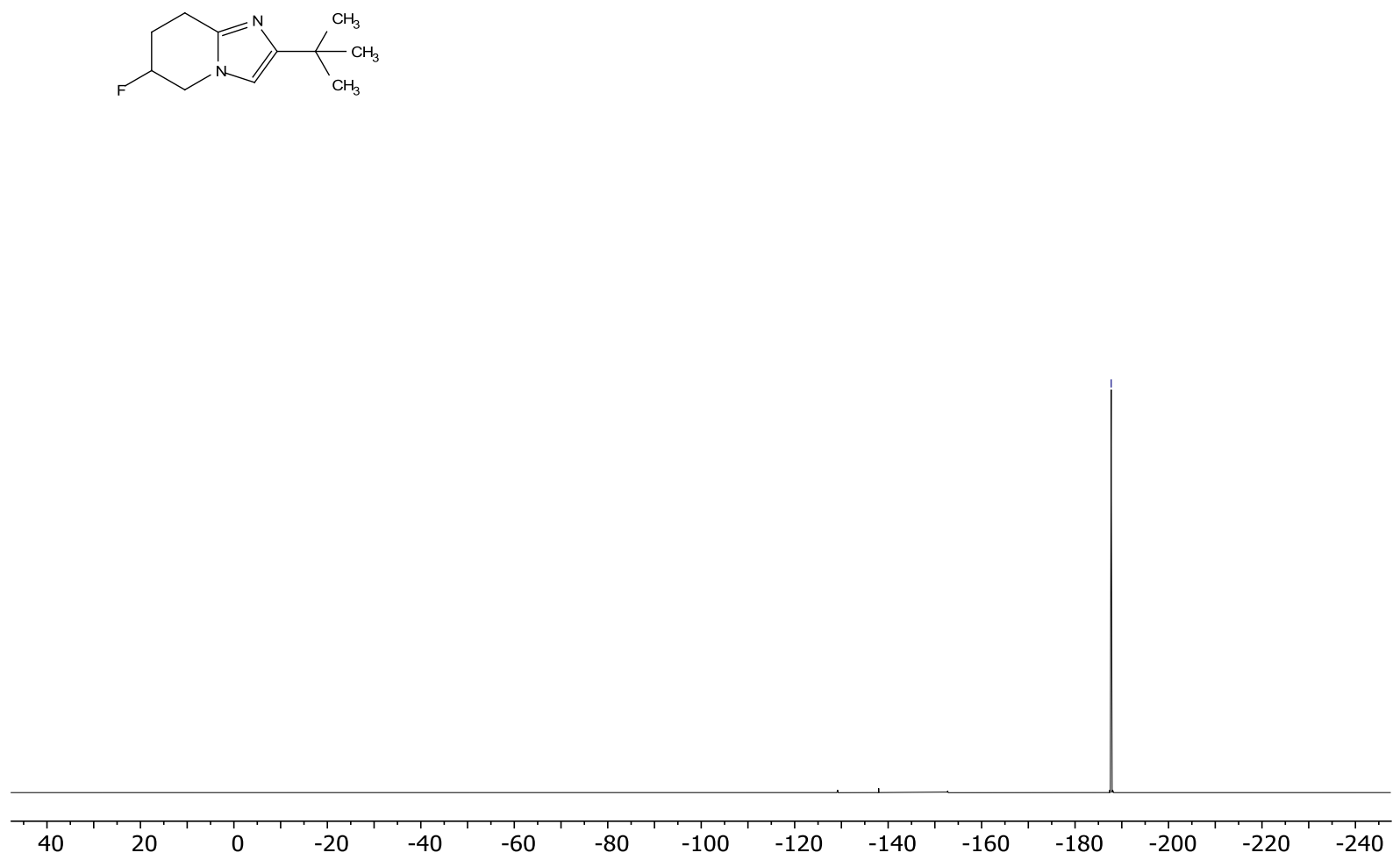

1H NMR (Chloroformed)

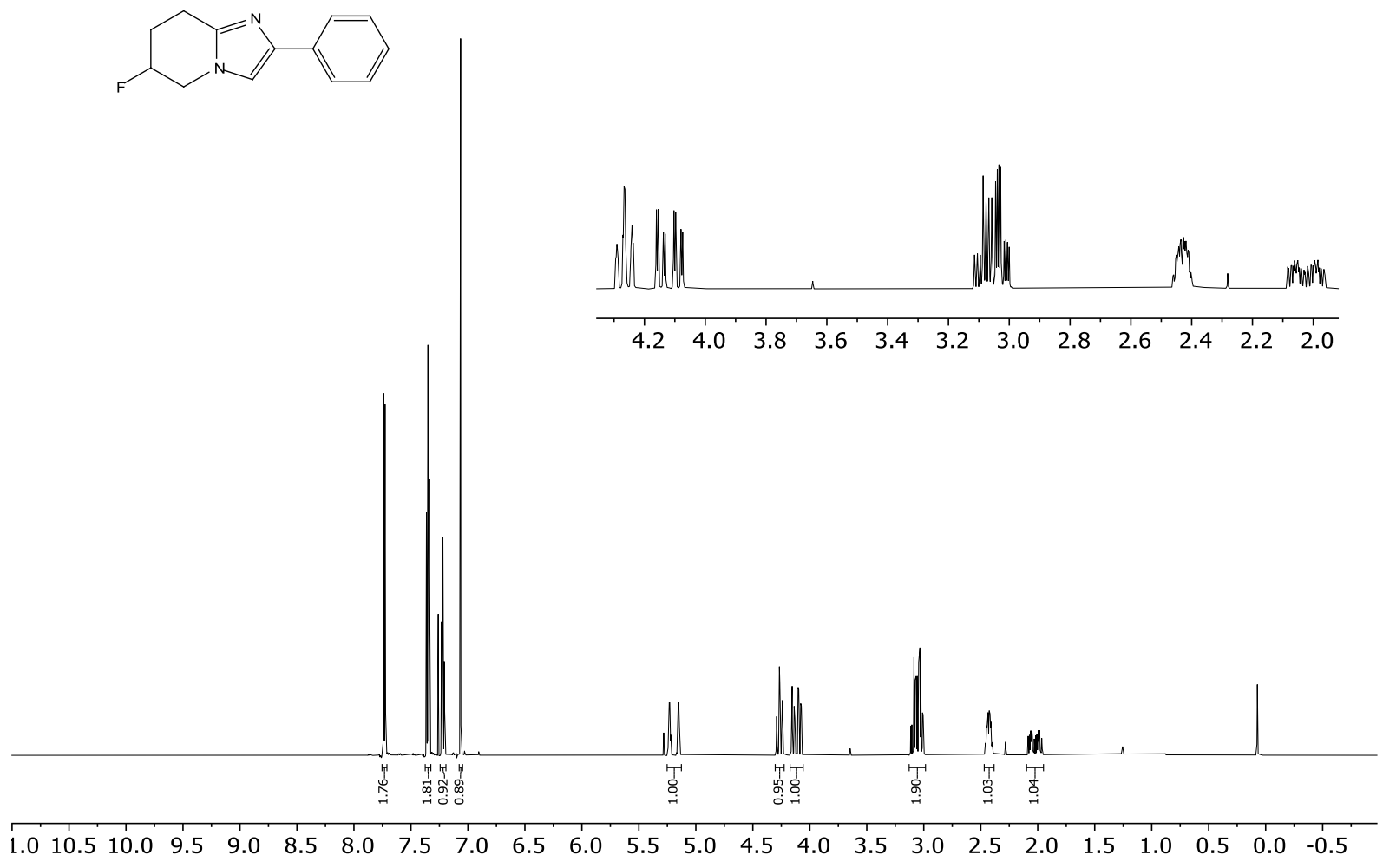

S210 
$1 \mathrm{H}\left\{{ }^{19} \mathrm{~F}\right\}$ NMR (Chloroformed)

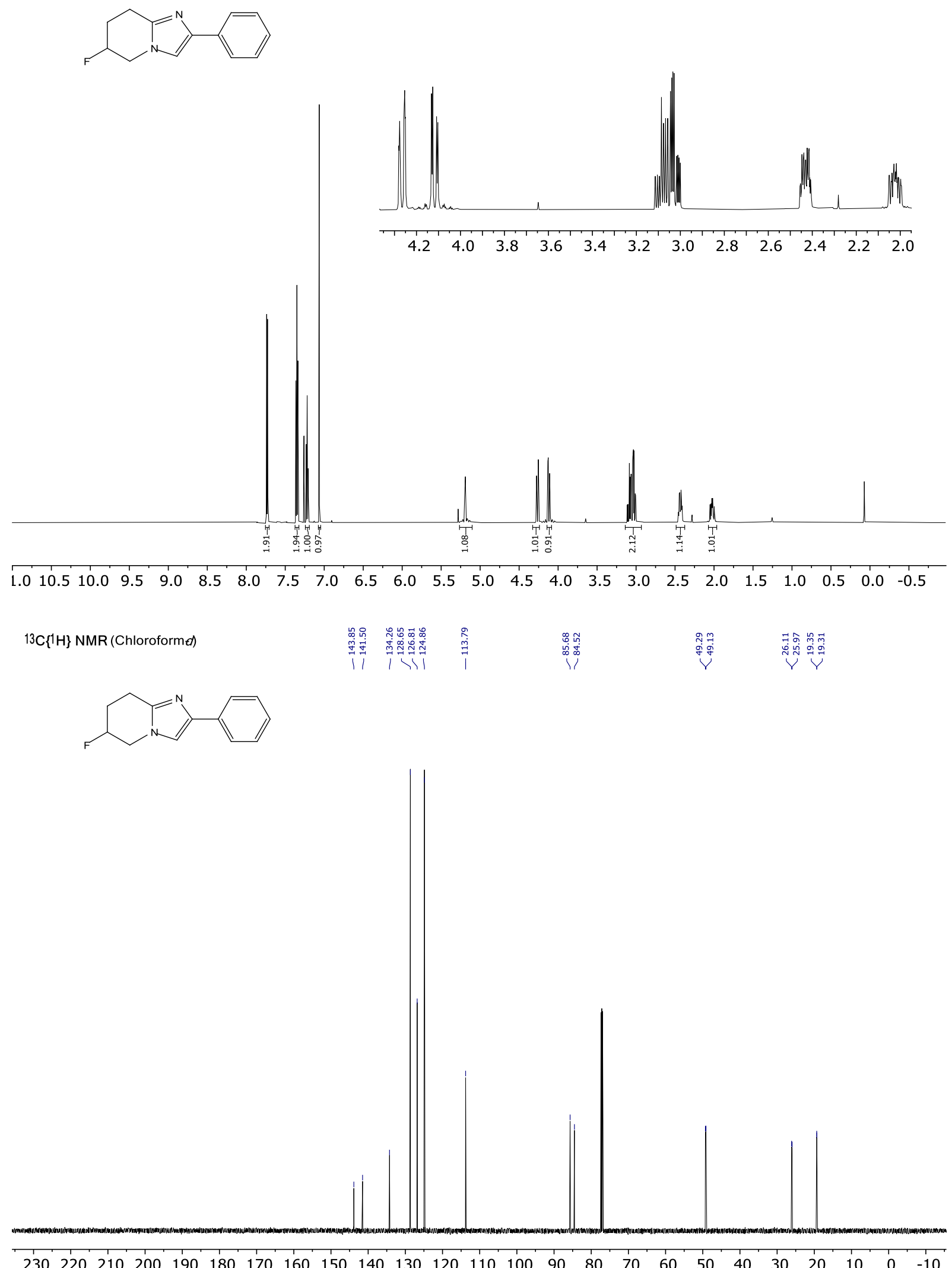




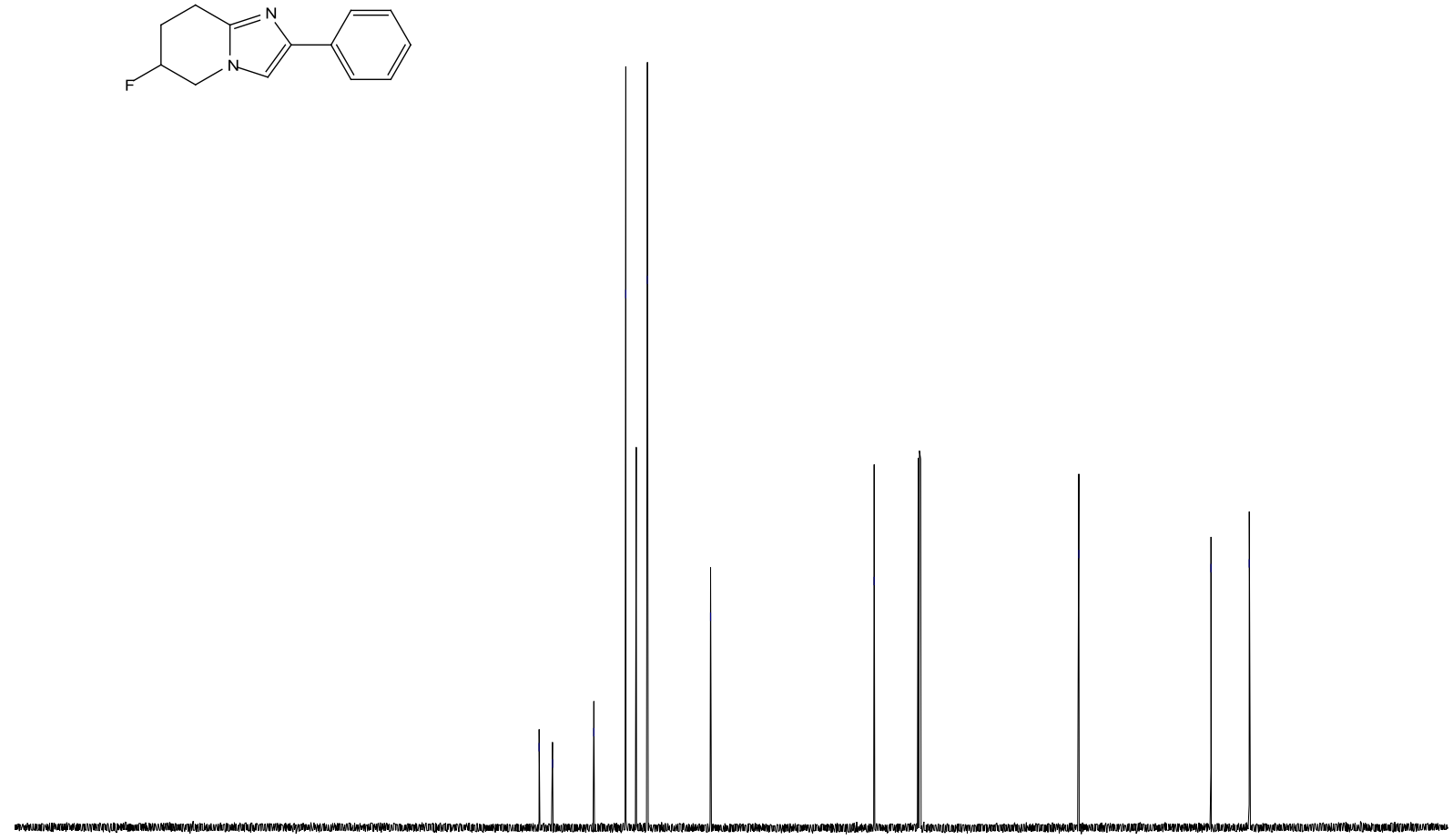

$\begin{array}{lllllllllllllllllllllllll}230 & 220 & 210 & 200 & 190 & 180 & 170 & 160 & 150 & 140 & 130 & 120 & 110 & 100 & 90 & 80 & 70 & 60 & 50 & 40 & 30 & 20 & 10 & 0 & -10\end{array}$ 19 F NMR (Chloroforme)

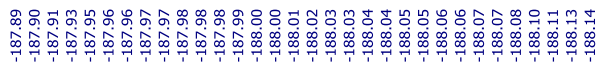<smiles>FC1CCc2nc(-c3ccccc3)cn2C1</smiles> 
${ }^{19} \mathrm{~F}\left\{{ }^{1} \mathrm{H}\right\}$ NMR (Chloroformed)

$m$
$\infty$
$\infty$
$\infty$
1
1
1
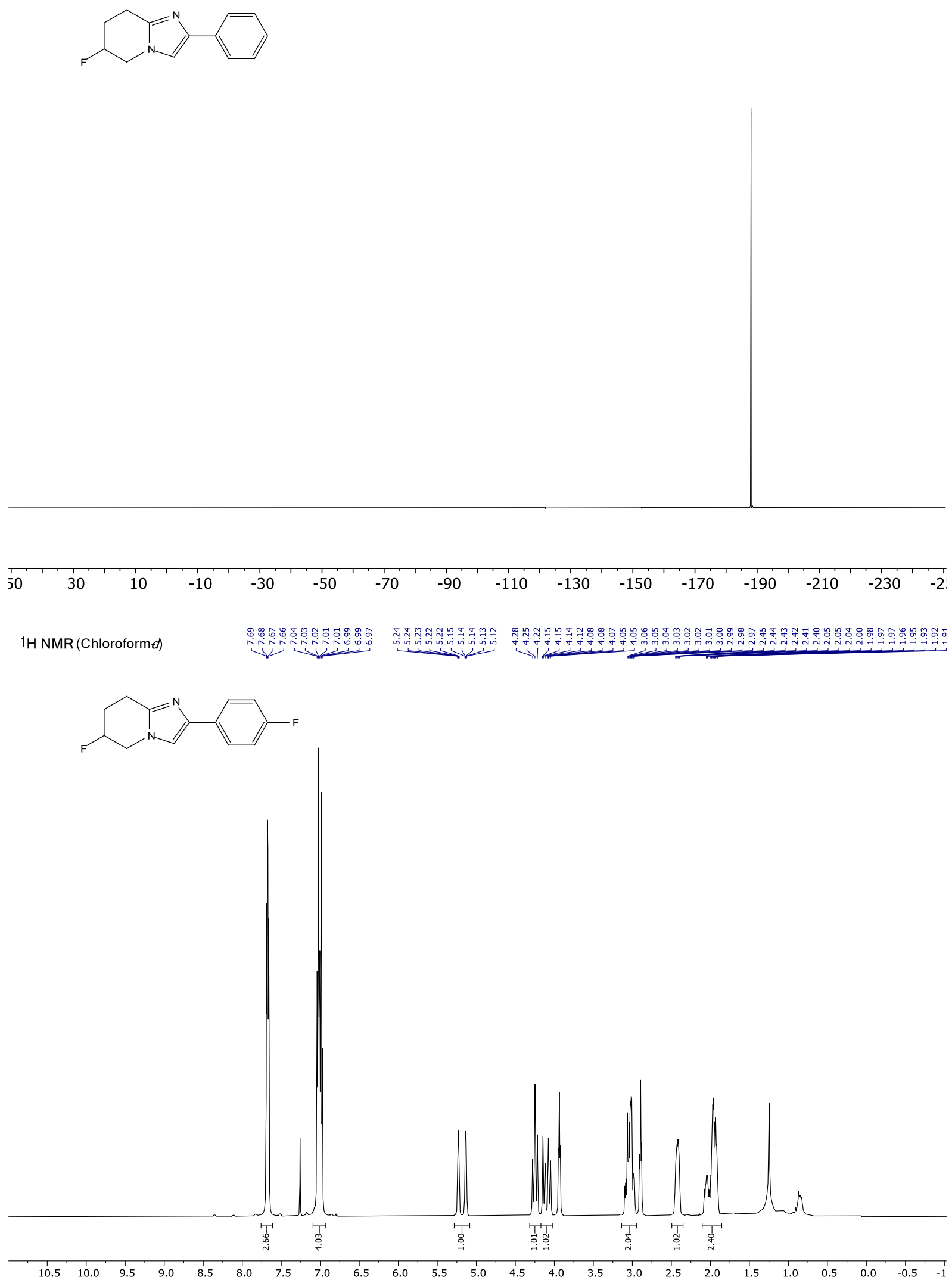

S213 
<smiles>FC1CCc2nc(-c3ccc(I)cc3)cn2C1</smiles>

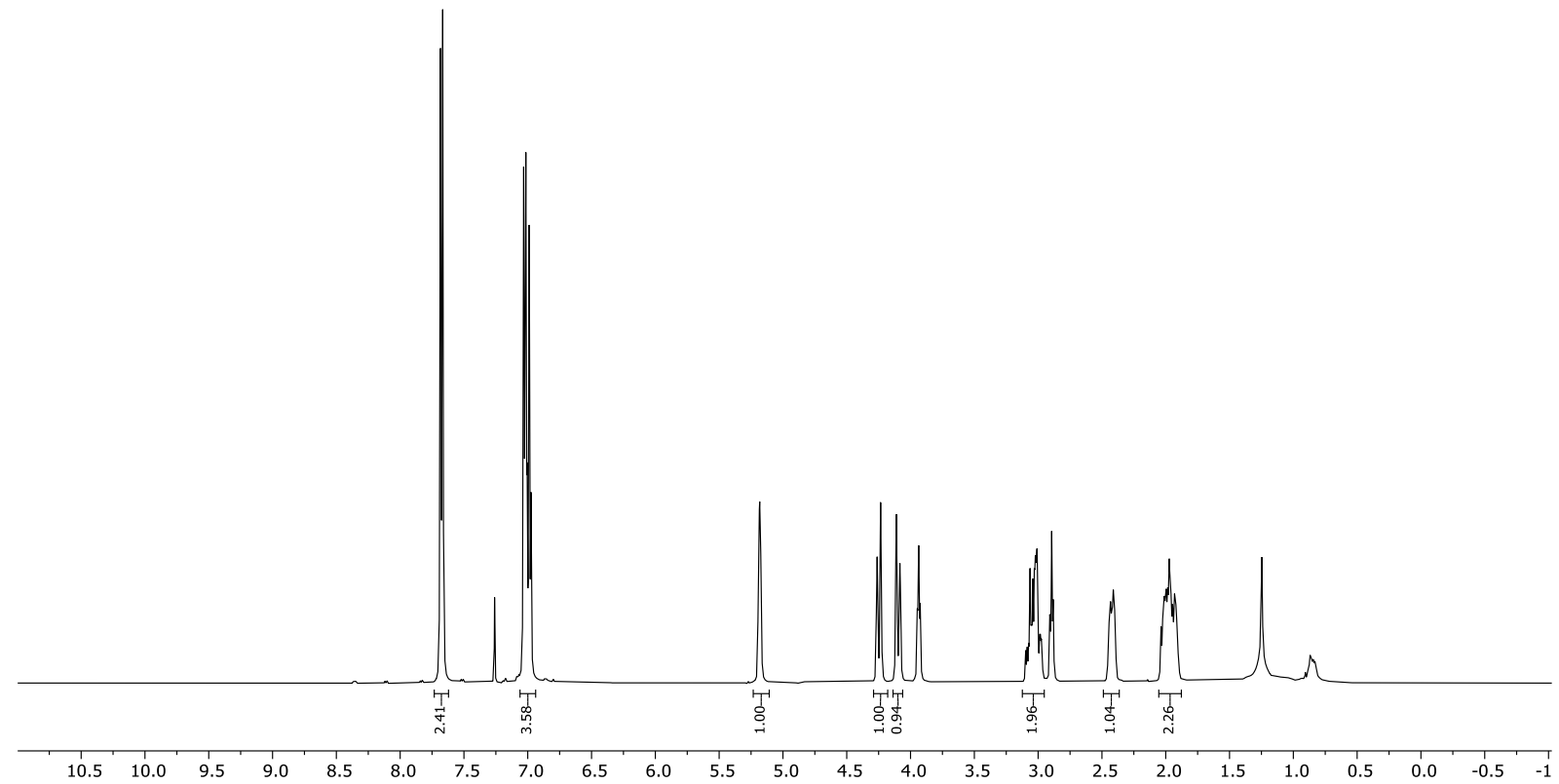

${ }^{13 C}\{1 \mathrm{H}\}$ NMR (Chloroforme)

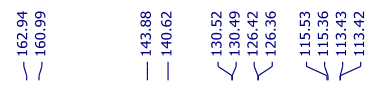

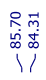

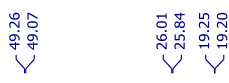
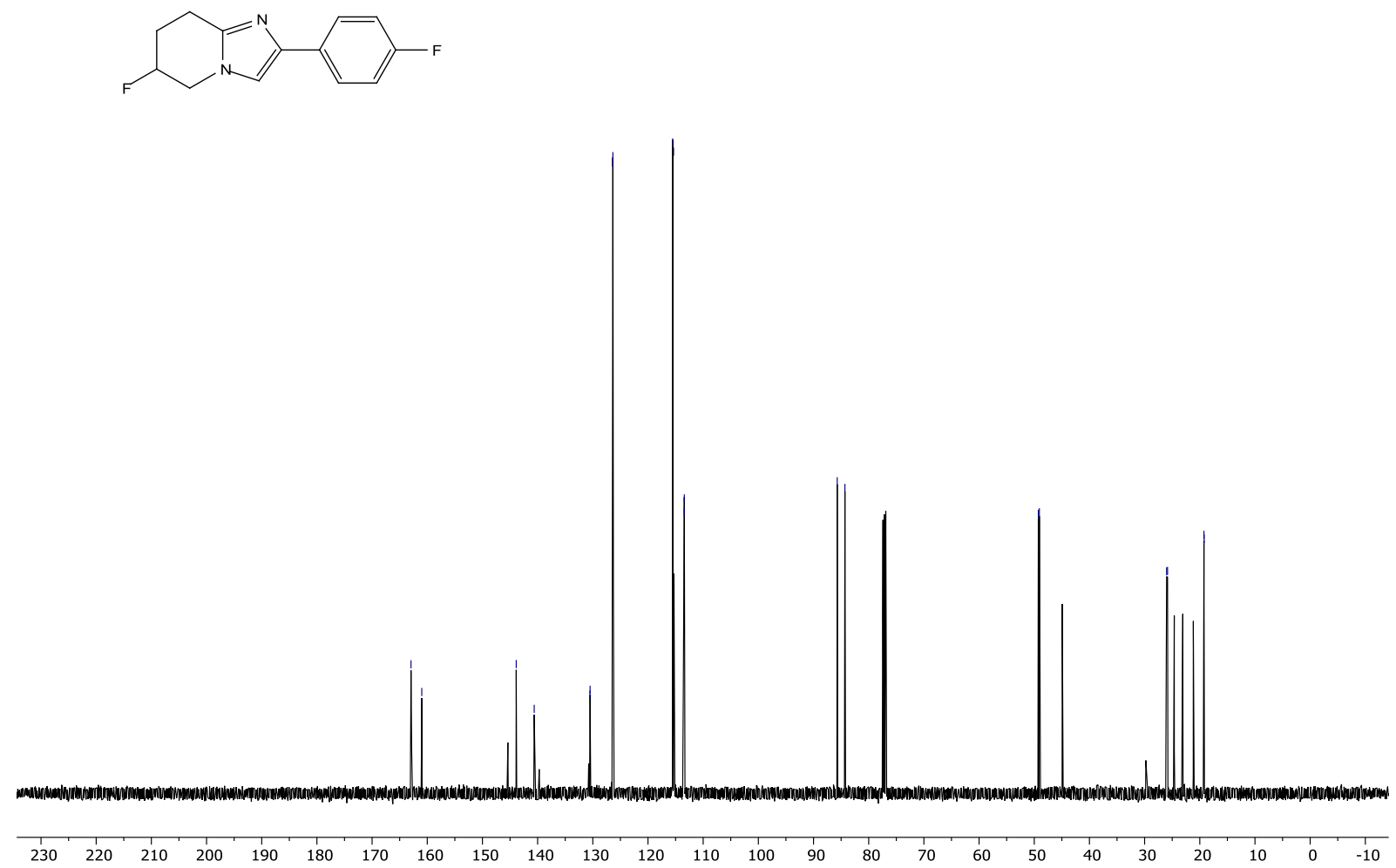


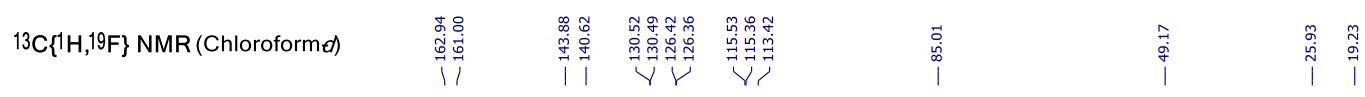<smiles>Fc1ccc(-c2cn3c(n2)CCC(F)C3)cc1</smiles>

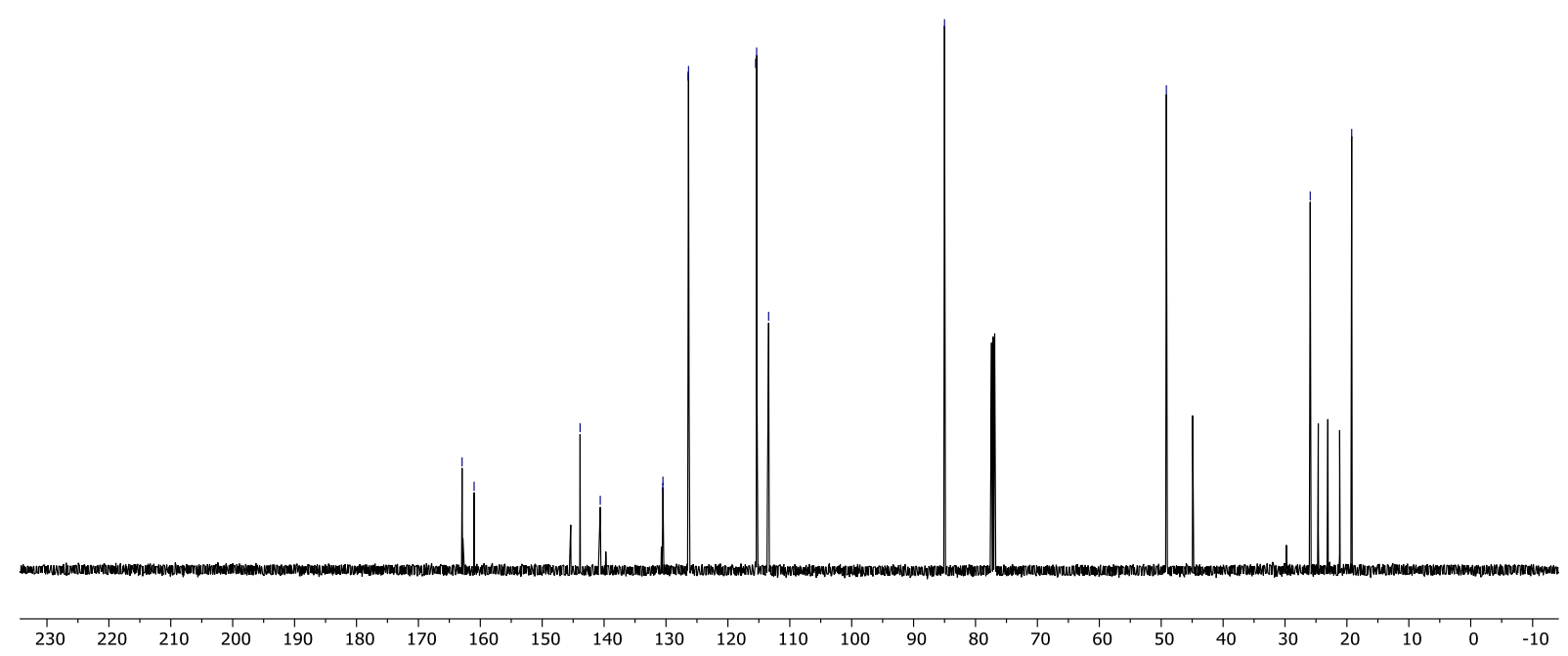

19F NMR (Chloroforme)

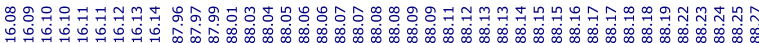

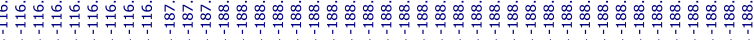<smiles>Fc1ccc(-c2cn3c(n2)CCC(F)C3)cc1</smiles>
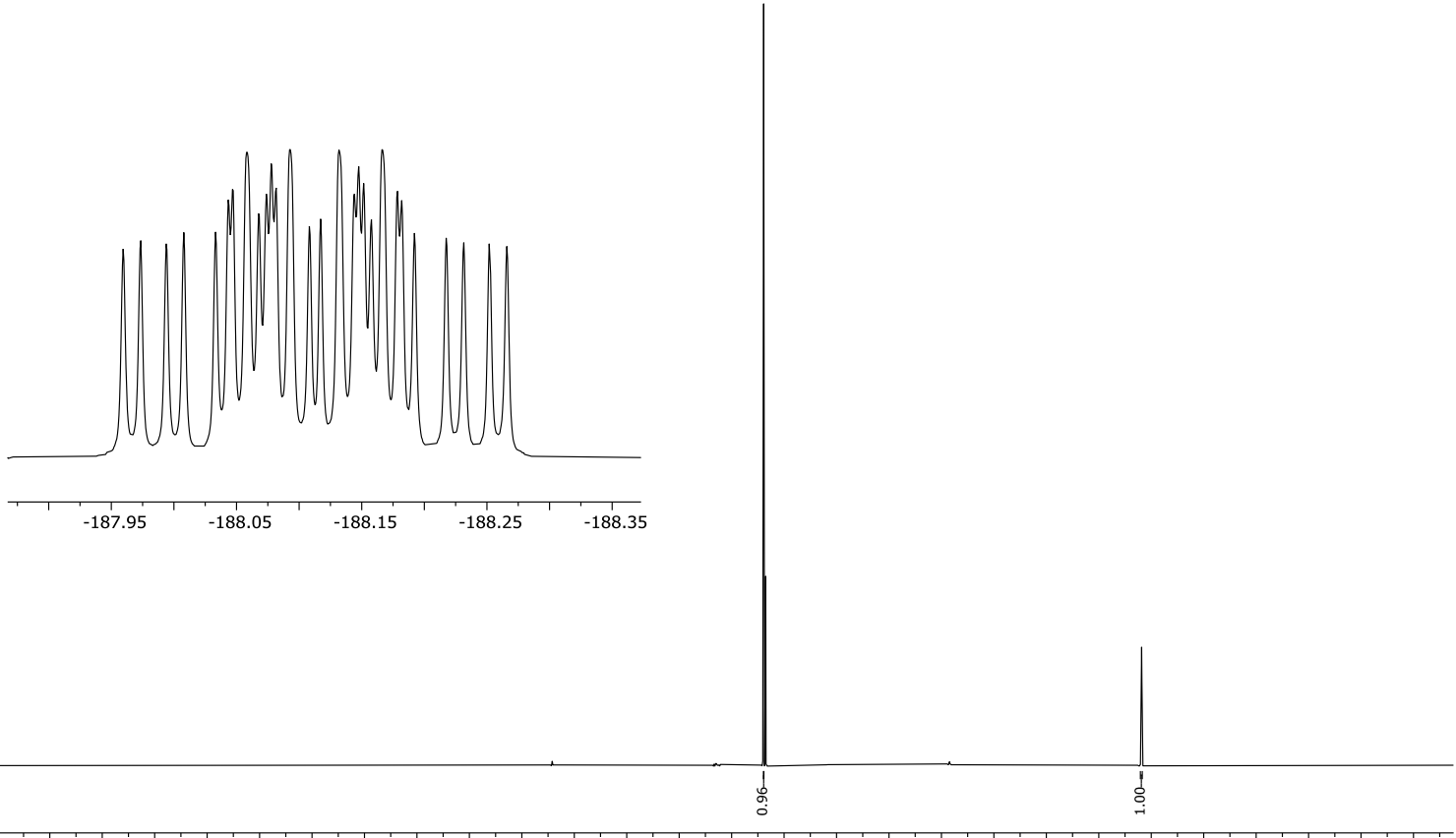

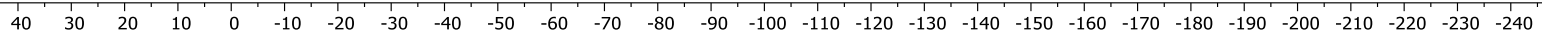


${ }^{19} \mathrm{~F}\{1 \mathrm{H}\}$ NMR (Chloroforme)

$\stackrel{7}{\overrightarrow{0}}$<smiles>Fc1ccc(-c2cn3c(n2)CCC(F)C3)cc1</smiles>

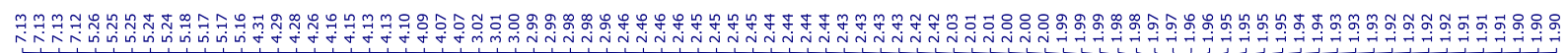
1H NMR (Chloroforme)<smiles>FC1CCc2nc(C(F)(F)F)cn2C1</smiles>

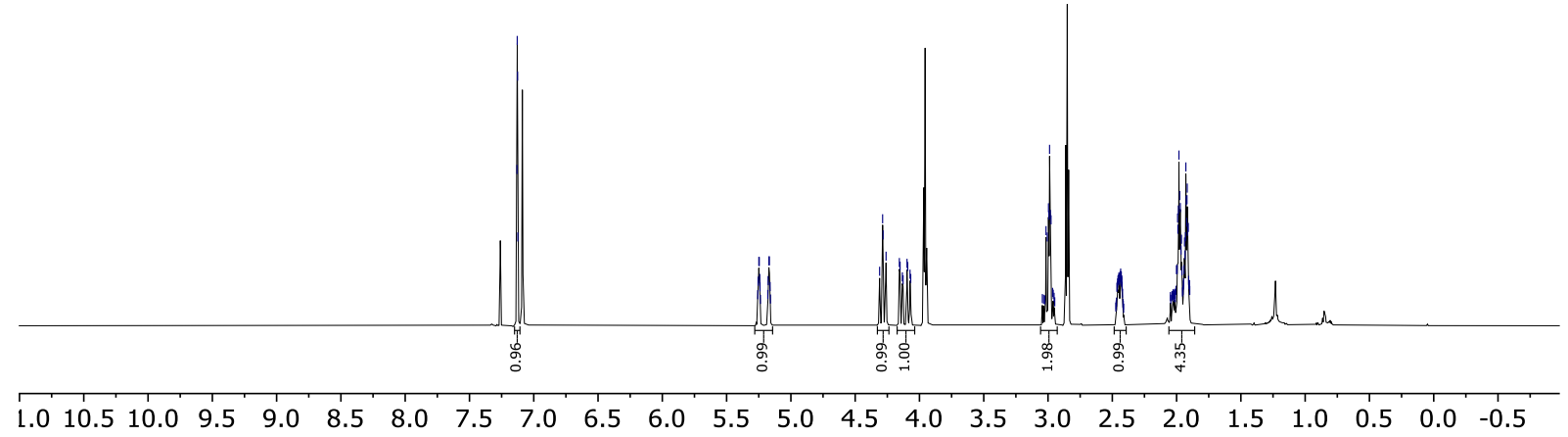




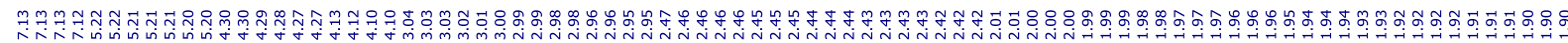
1H $\{19 \mathrm{~F}\}$ NMR (Chloroforme)<smiles>FC1CCc2nc(C(F)(F)F)cn2C1</smiles>

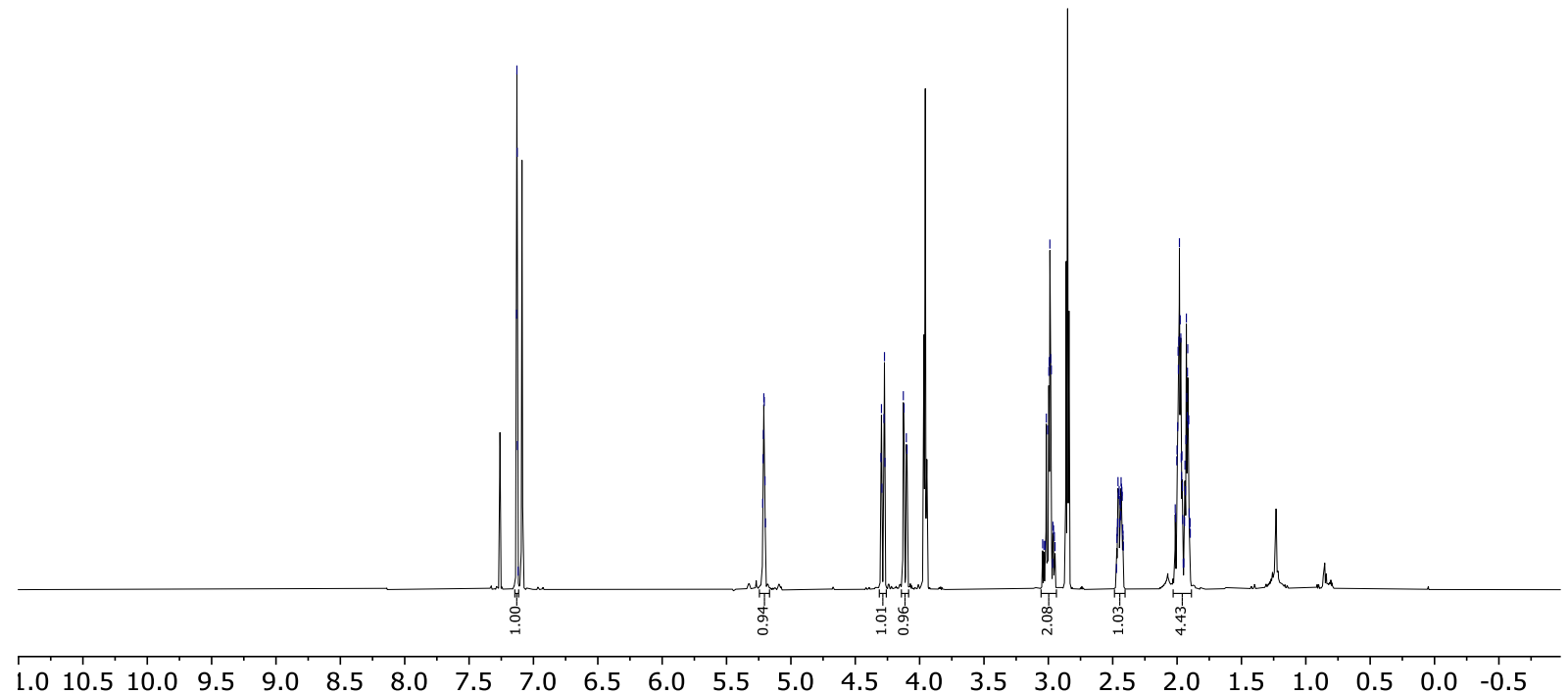

${ }^{13} \mathrm{C}\{1 \mathrm{H}\}$ NMR (Chloroformed)
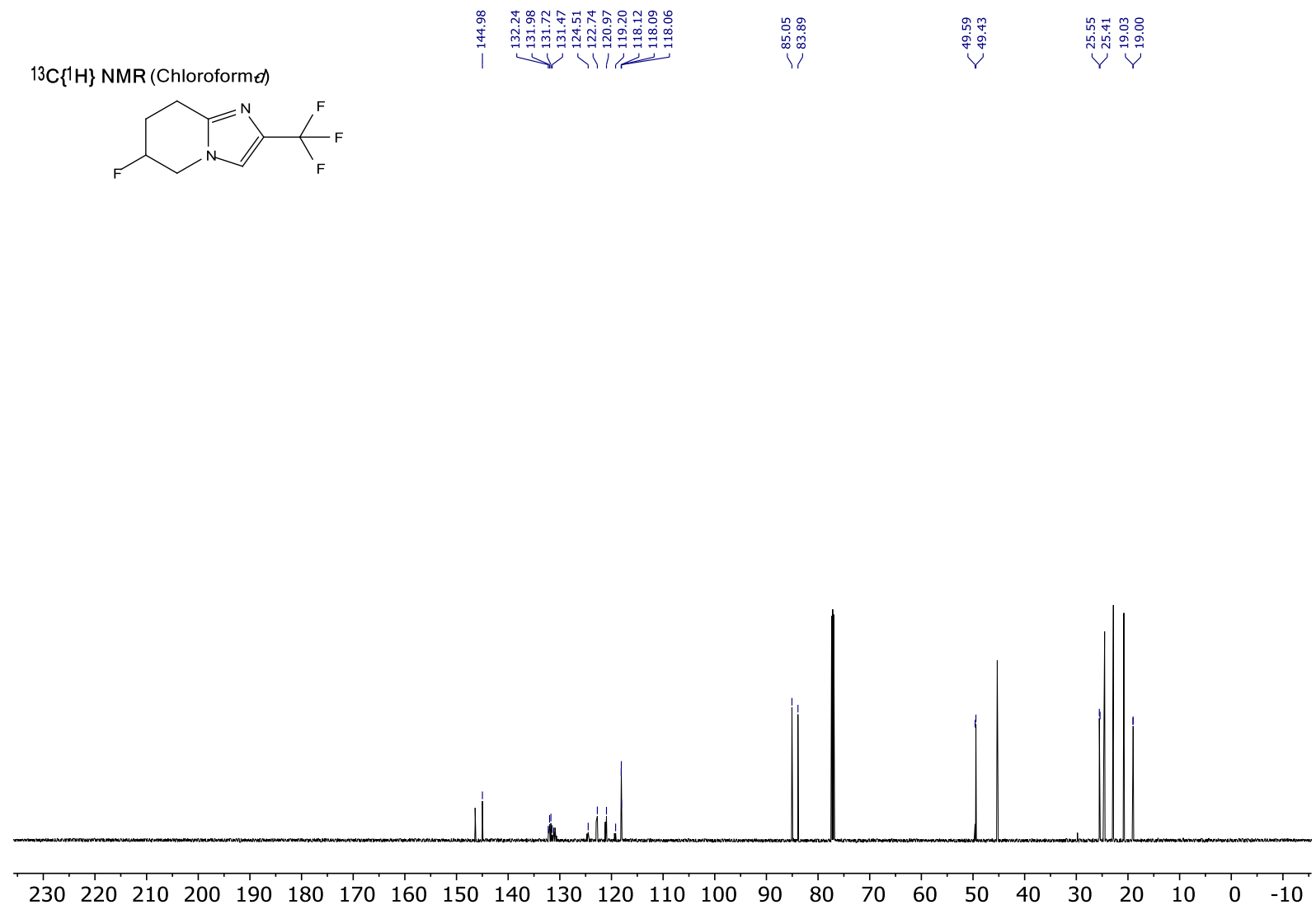
${ }^{13} \mathrm{C}\{1 \mathrm{H}, 19 \mathrm{~F}\}$ NMR (Chloroformet)

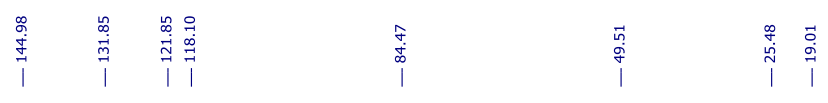<smiles>FC1CCc2nc(C(F)(F)F)cn2C1</smiles>

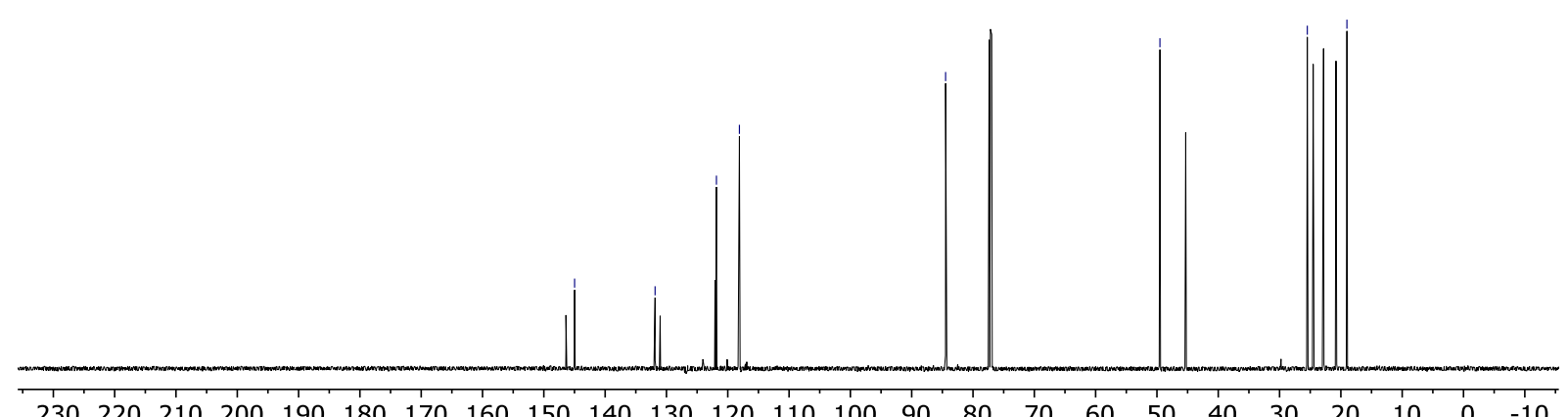

$\begin{array}{llllllllllllllllllllllllll}230 & 220 & 210 & 200 & 190 & 180 & 170 & 160 & 150 & 140 & 130 & 120 & 110 & 100 & 90 & 80 & 70 & 60 & 50 & 40 & 30 & 20 & 10 & 0 & -10\end{array}$ 19F NMR (Chloroforme)

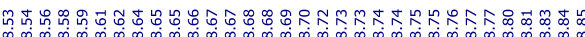

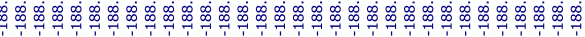<smiles>FC1CCc2nc(C(F)(F)F)cn2C1</smiles>

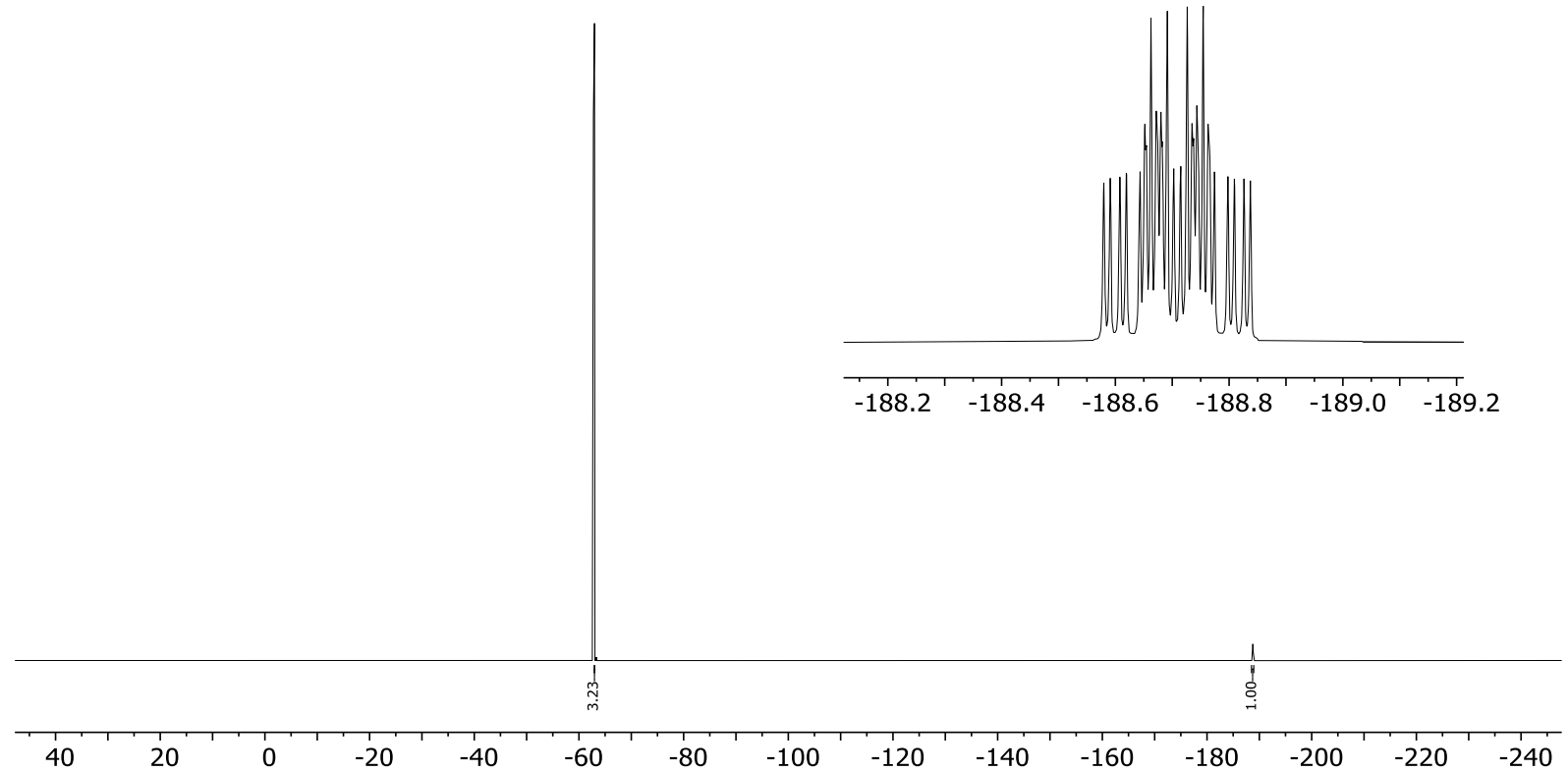


${ }^{19} \mathrm{~F}\{1 \mathrm{H}\}$ NMR (Chloroformed)

ลุ

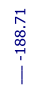
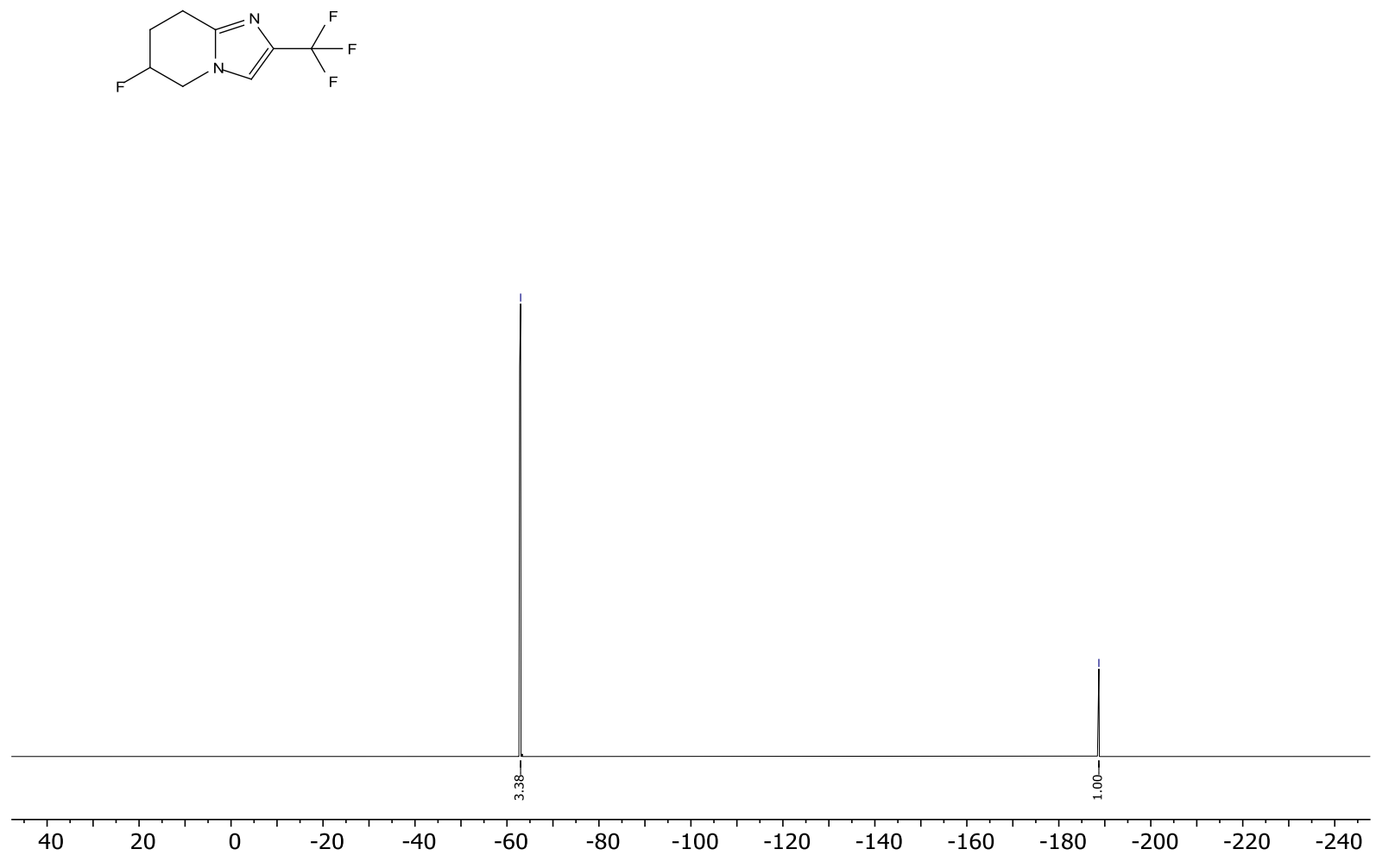

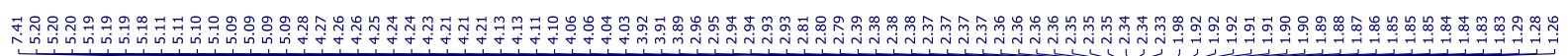

1H NMR (Chloroformed)
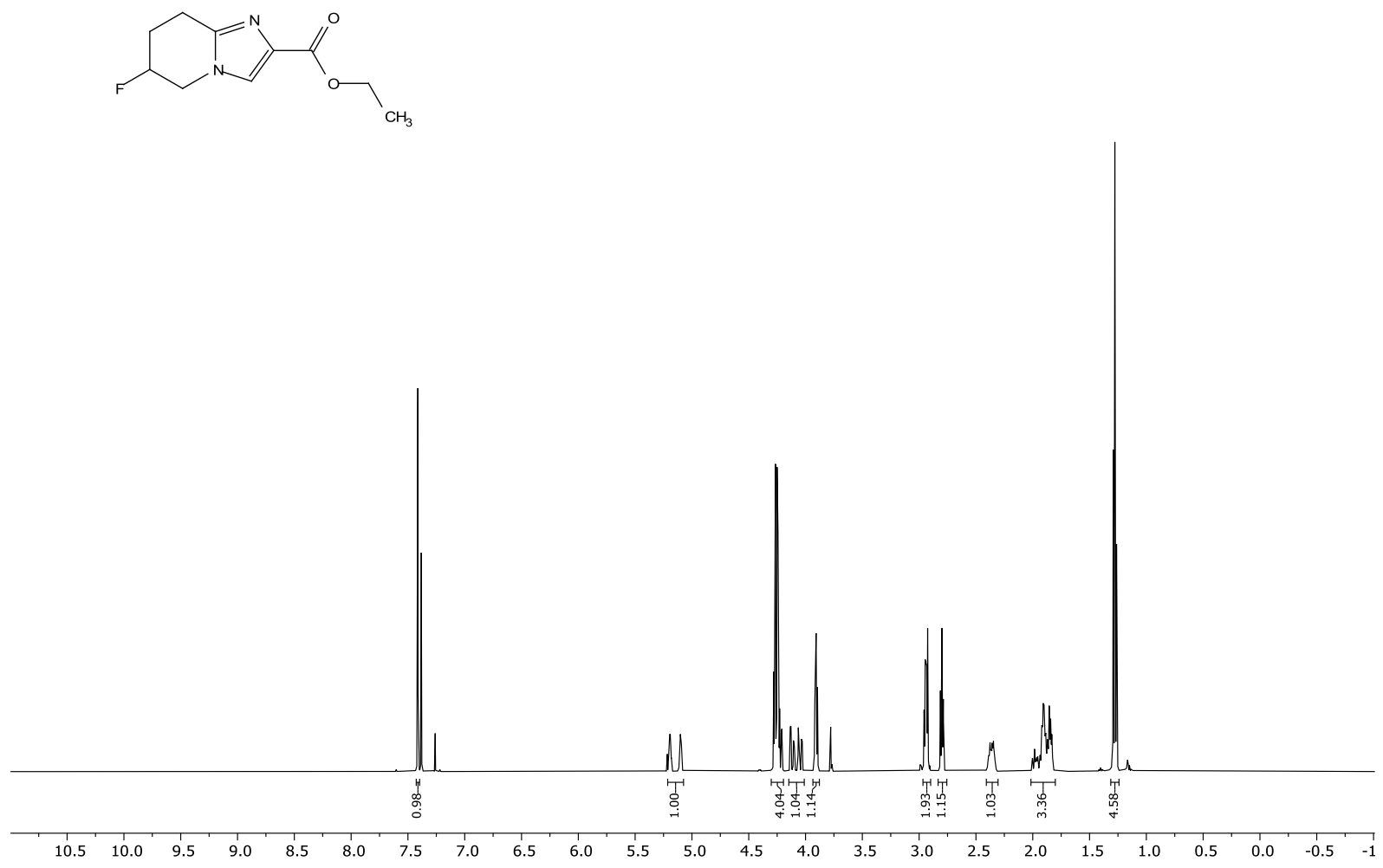

S219 


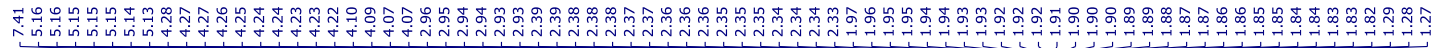
${ }^{1} \mathrm{H}\left\{{ }^{19} \mathrm{~F}\right\}$ NMR (Chloroforme)<smiles>CCOC(=O)c1cn2c(n1)CCC(F)C2</smiles>

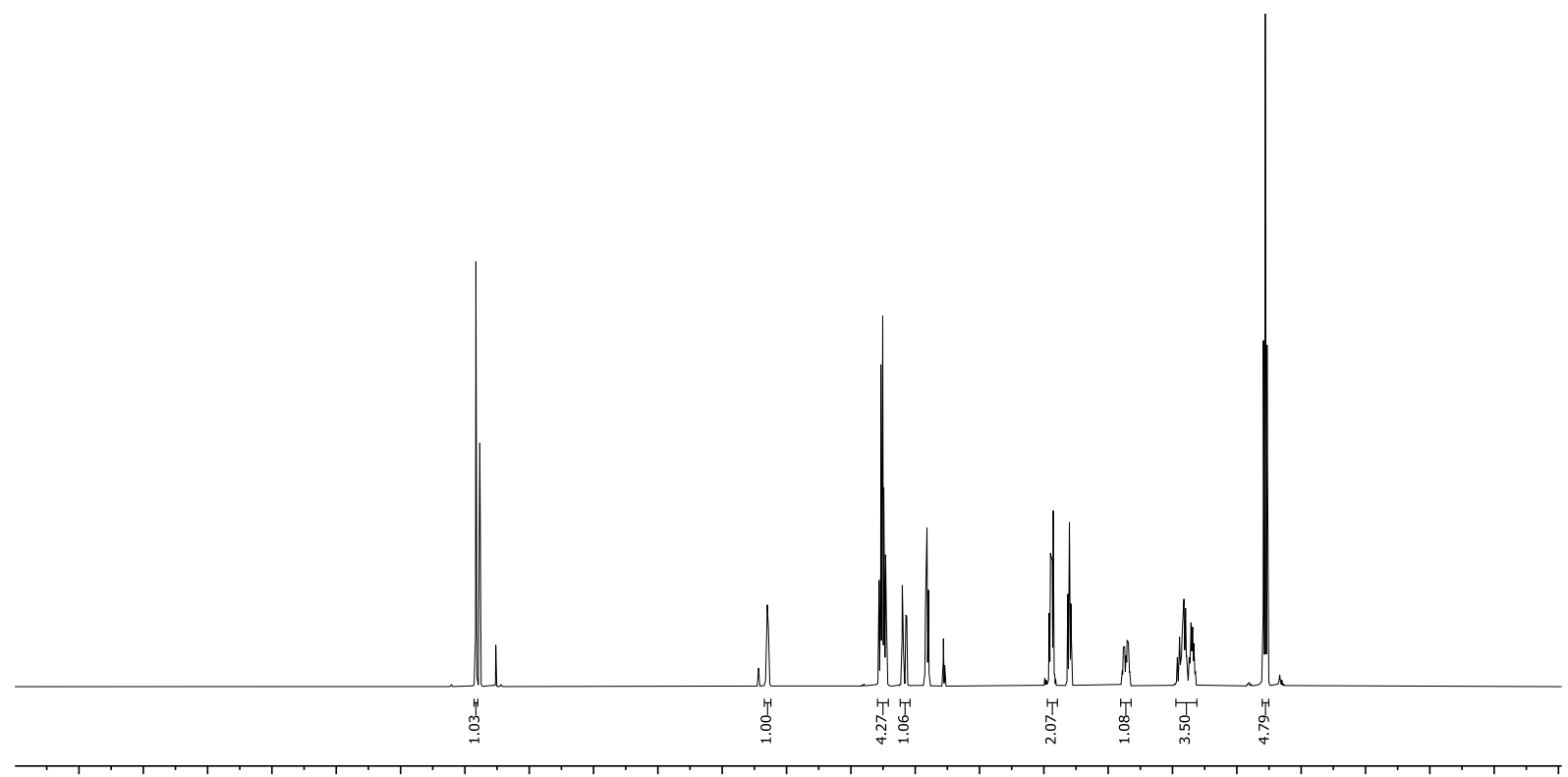
$\begin{array}{lllllllllllllllllllllllllll}10.5 & 10.0 & 9.5 & 9.0 & 8.5 & 8.0 & 7.5 & 7.0 & 6.5 & 6.0 & 5.5 & 5.0 & 4.5 & 4.0 & 3.5 & 3.0 & 2.5 & 2.0 & 1.5 & 1.0 & 0.5 & 0.0 & -0.5 & -1\end{array}$ ${ }^{13} \mathrm{C}\{1 \mathrm{H}\} \mathrm{NMR}$ (Chloroformet)

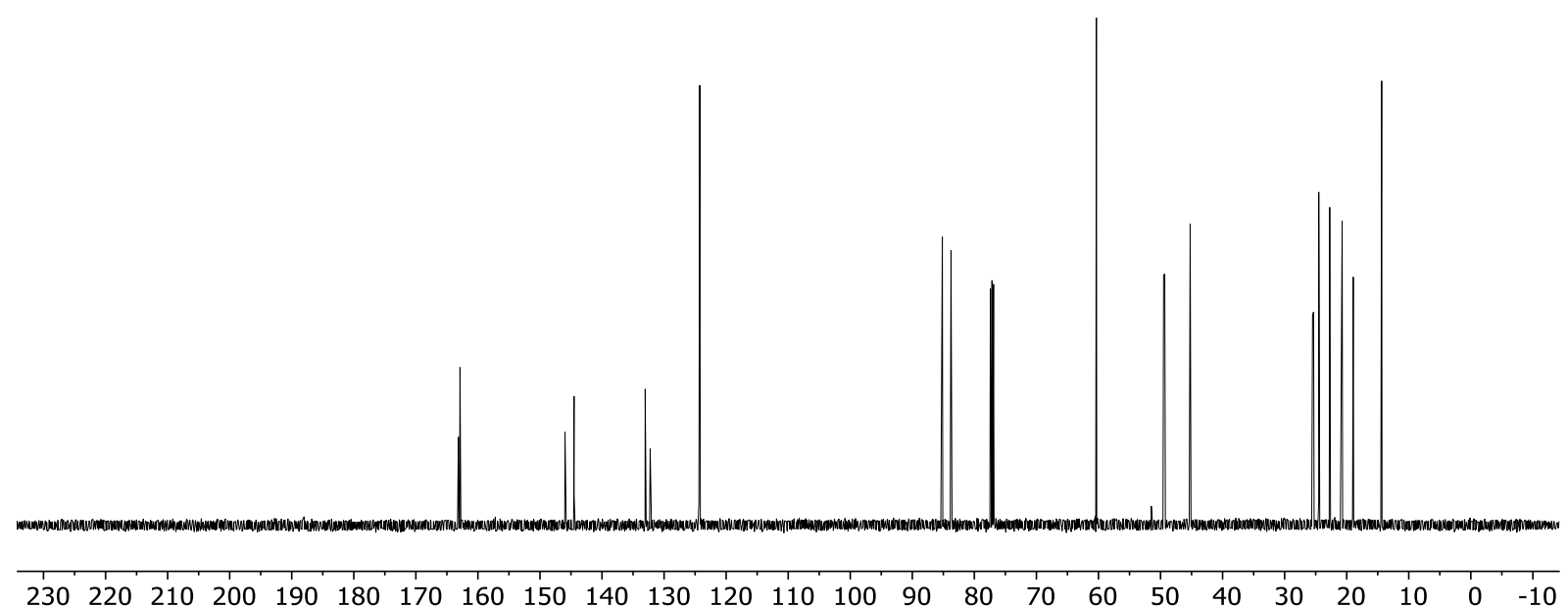




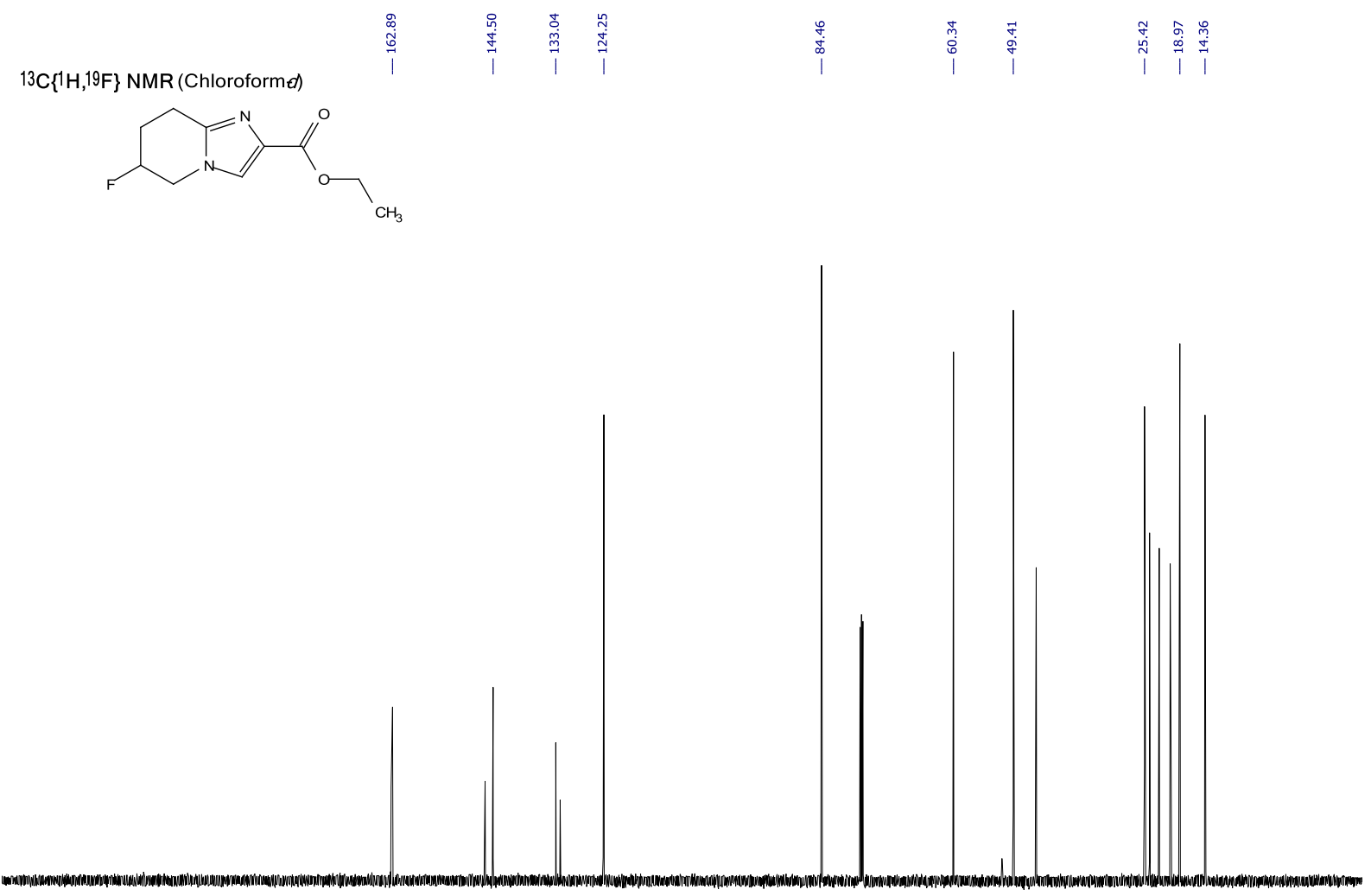

$\begin{array}{llllllllllllllllllllllllll}230 & 220 & 210 & 200 & 190 & 180 & 170 & 160 & 150 & 140 & 130 & 120 & 110 & 100 & 90 & 80 & 70 & 60 & 50 & 40 & 30 & 20 & 10 & 0 & -10\end{array}$
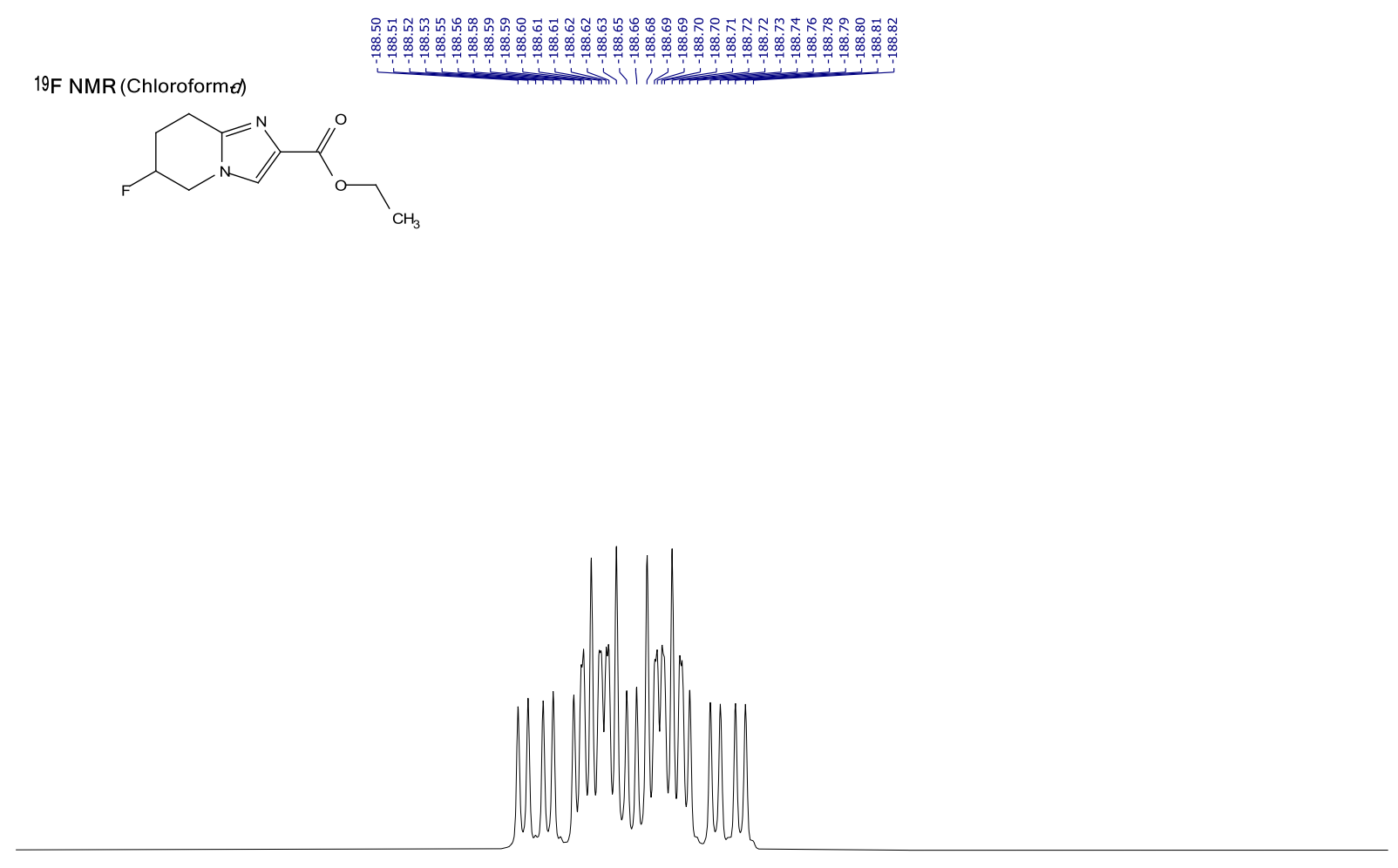

$-187.9-188.0-188.1-188.2-188.3-188.4-188.5-188.6-188.7-188.8-188.9-189.0-189.1-189.2-189.3-189.4-189.5-189.6$ 
${ }^{19} \mathrm{~F}\{1 \mathrm{H}\}$ NMR (Chloroformed)<smiles>CCOC(=O)c1cn2c(n1)CCC(F)C2</smiles>

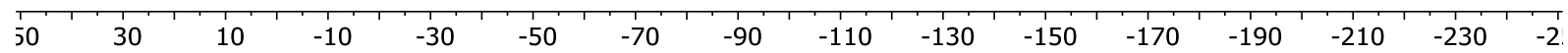

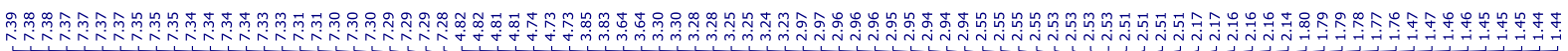
1H NMR (Chloroforme)<smiles>COC(=O)C(c1ccccc1)C1NCCCC1F</smiles>

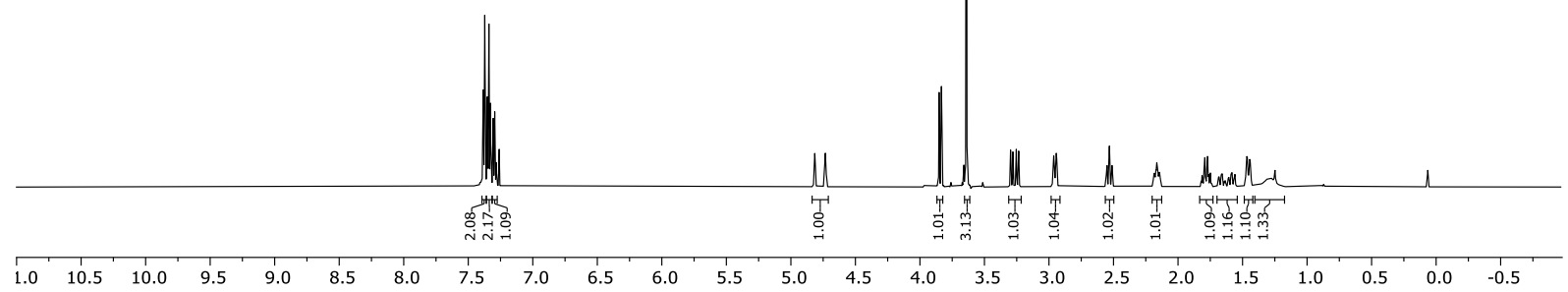




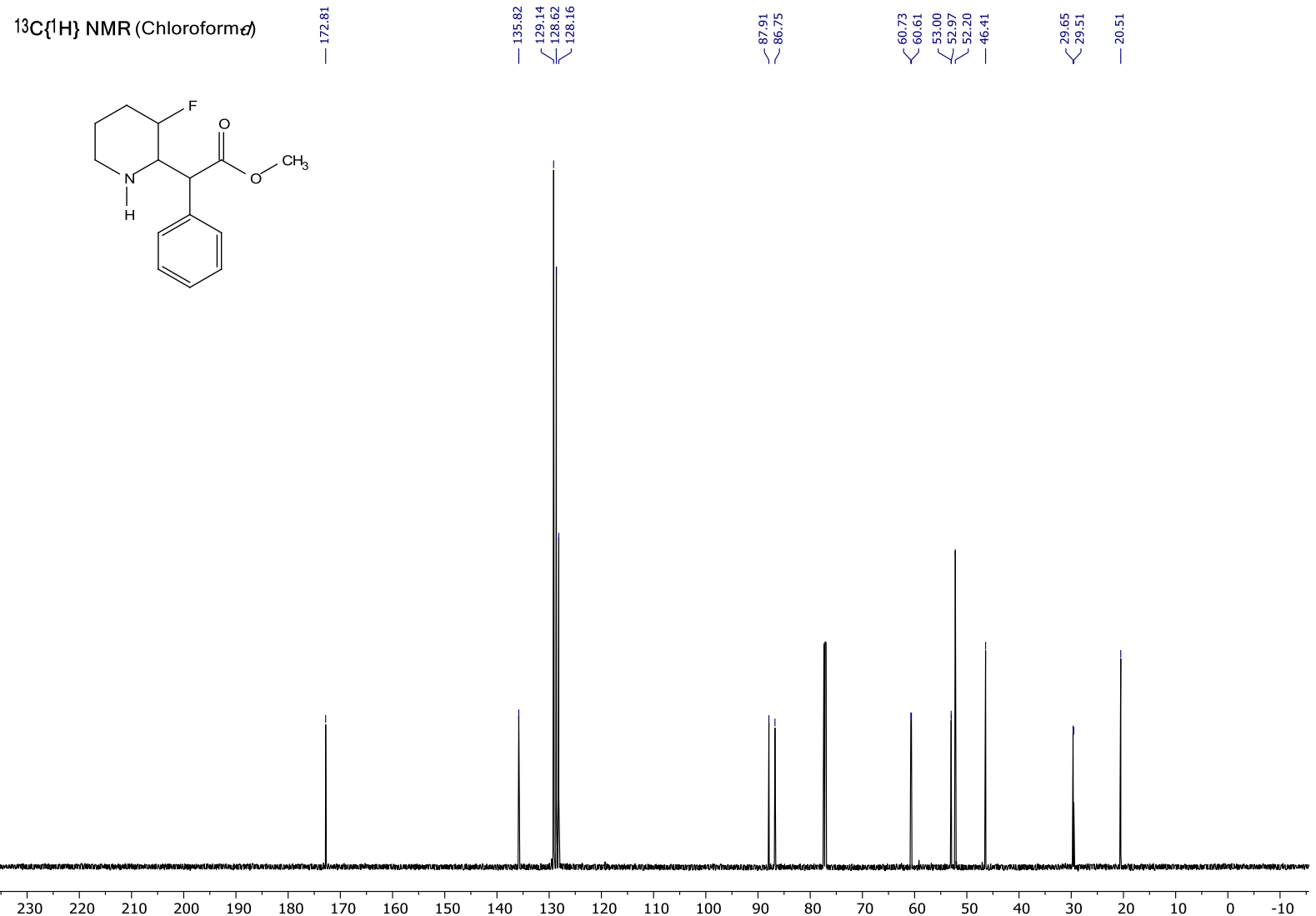

${ }^{19} \mathrm{~F}\{1 \mathrm{H}\}$ NMR (Chloroformed)<smiles>COC(=O)C(c1ccccc1)C1NCCCC1F</smiles>

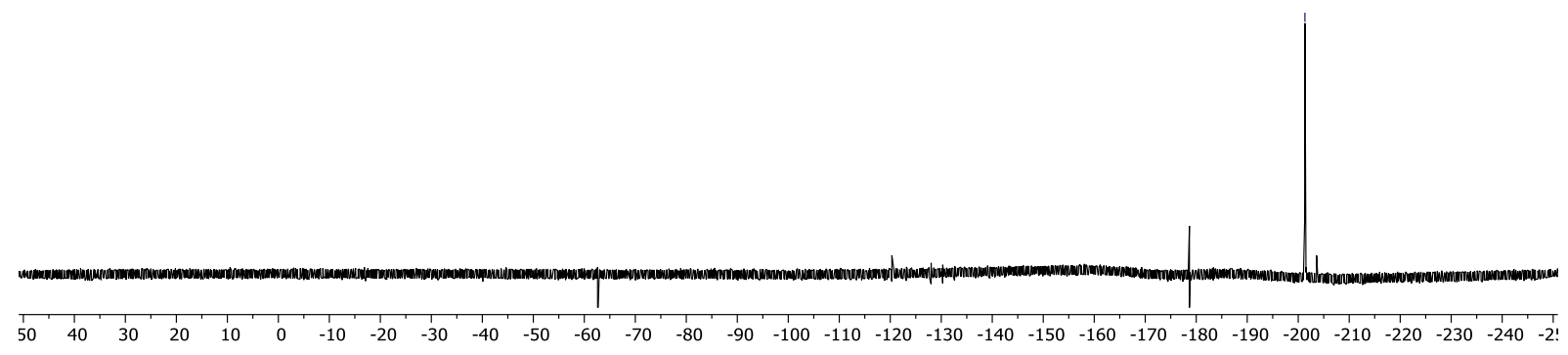




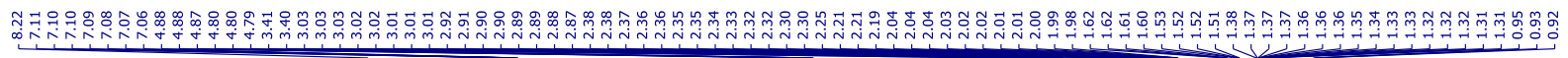

1H NMR (Chloroforme)
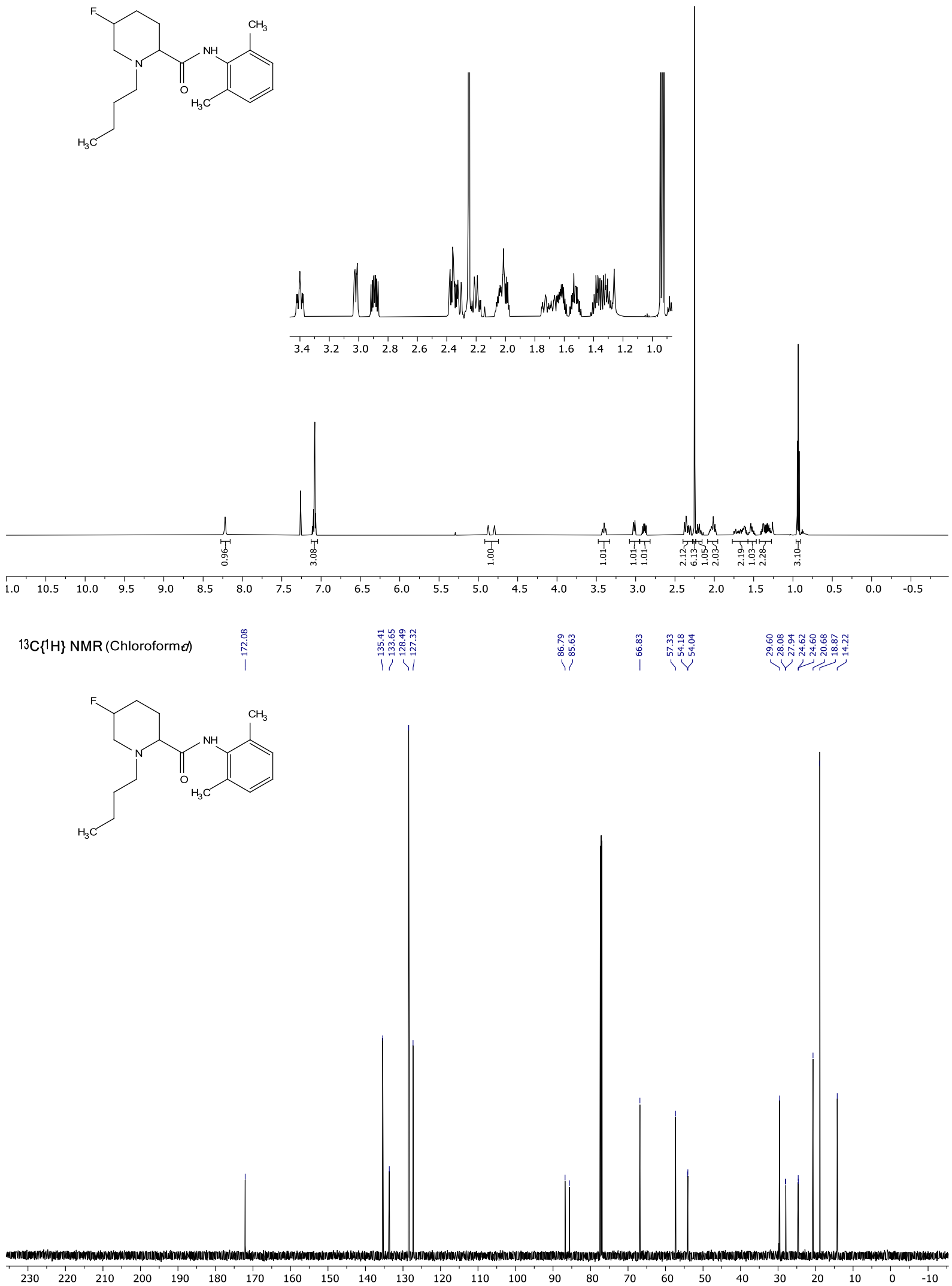

S224 
19F NMR (Chloroforme)

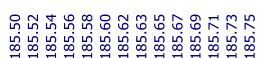

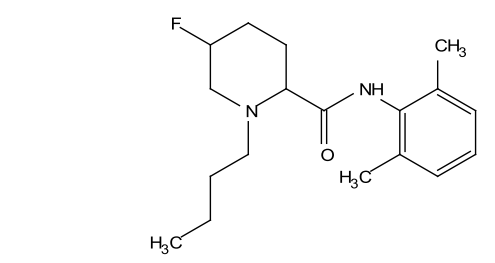

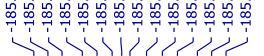
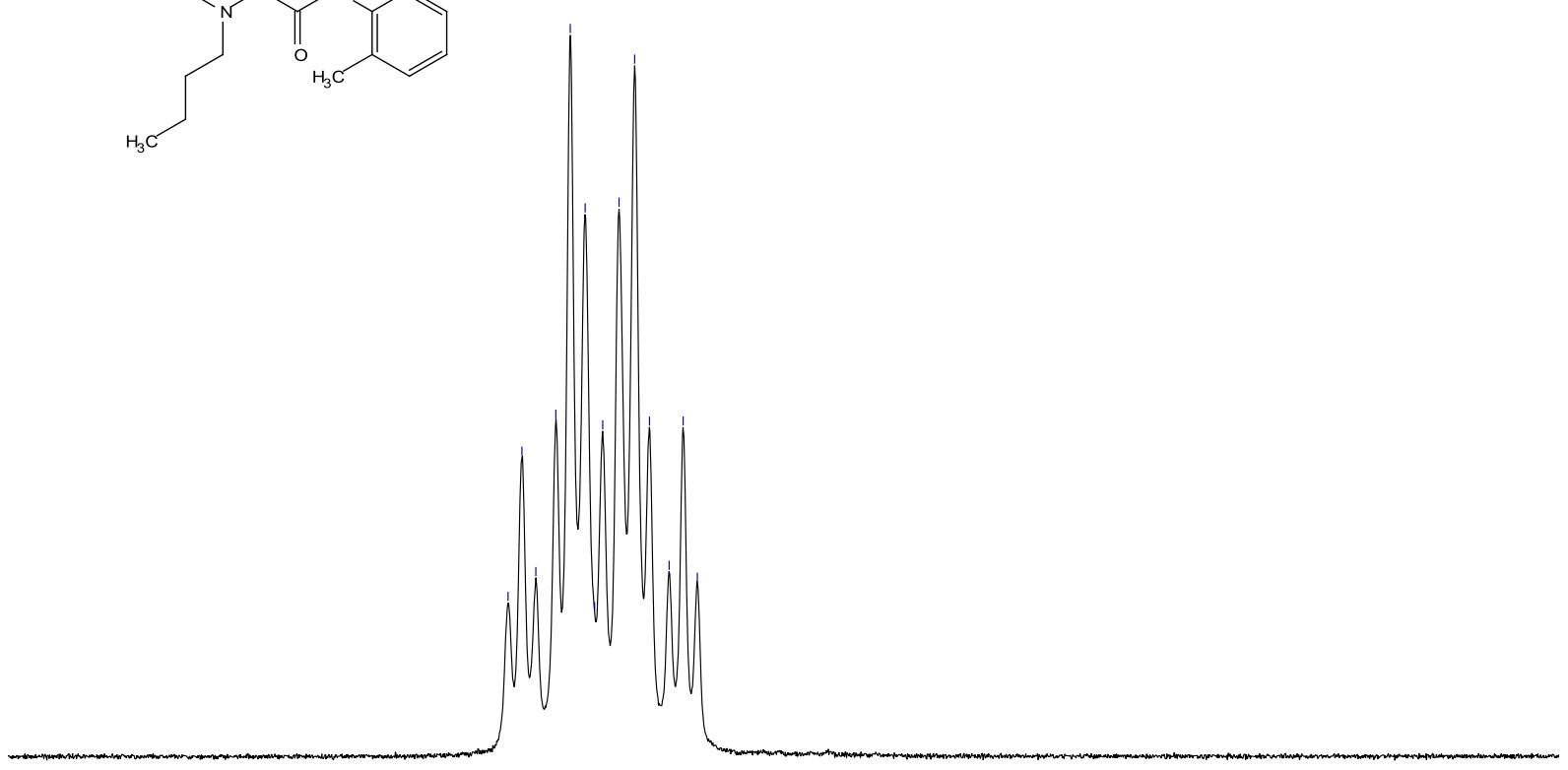

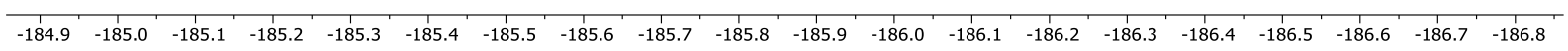

${ }_{19}^{19}\{1 \mathrm{H}\}$ NMR (Chloroformel)

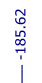

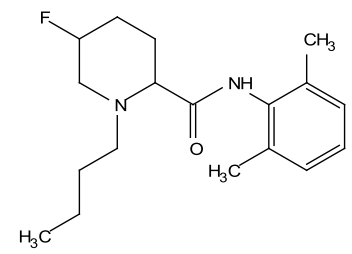

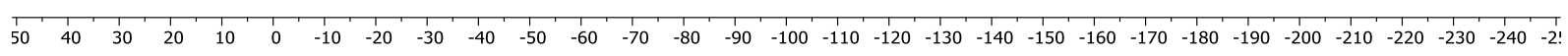

S225 


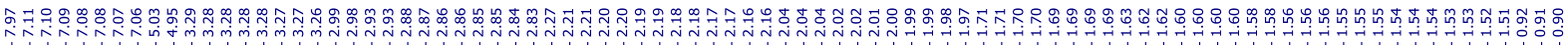

1H NMR (Chloroformed)<smiles>CCCN1CCCC(F)C1C(=O)Nc1c(C)cccc1C</smiles>

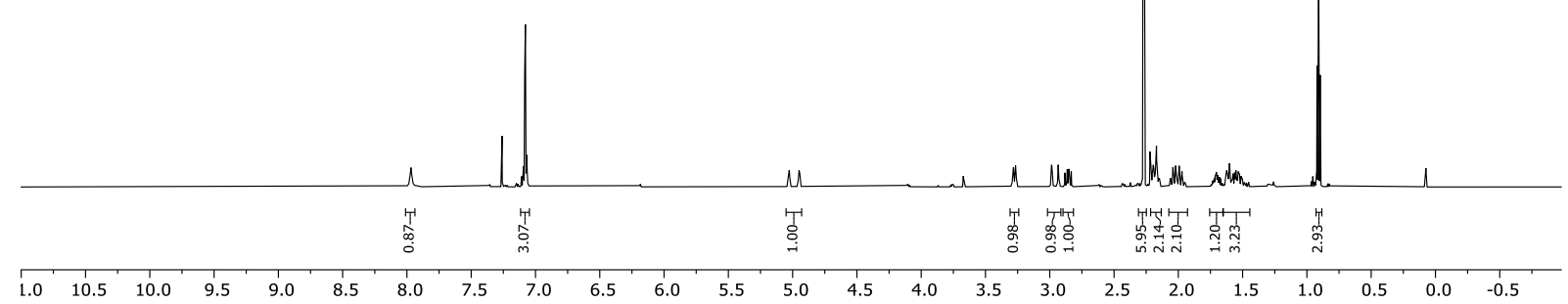

13C\{1H\} NMR (Chloroforme)<smiles>CCCN1CCCC(F)C1C(=O)Nc1c(C)cccc1C</smiles>

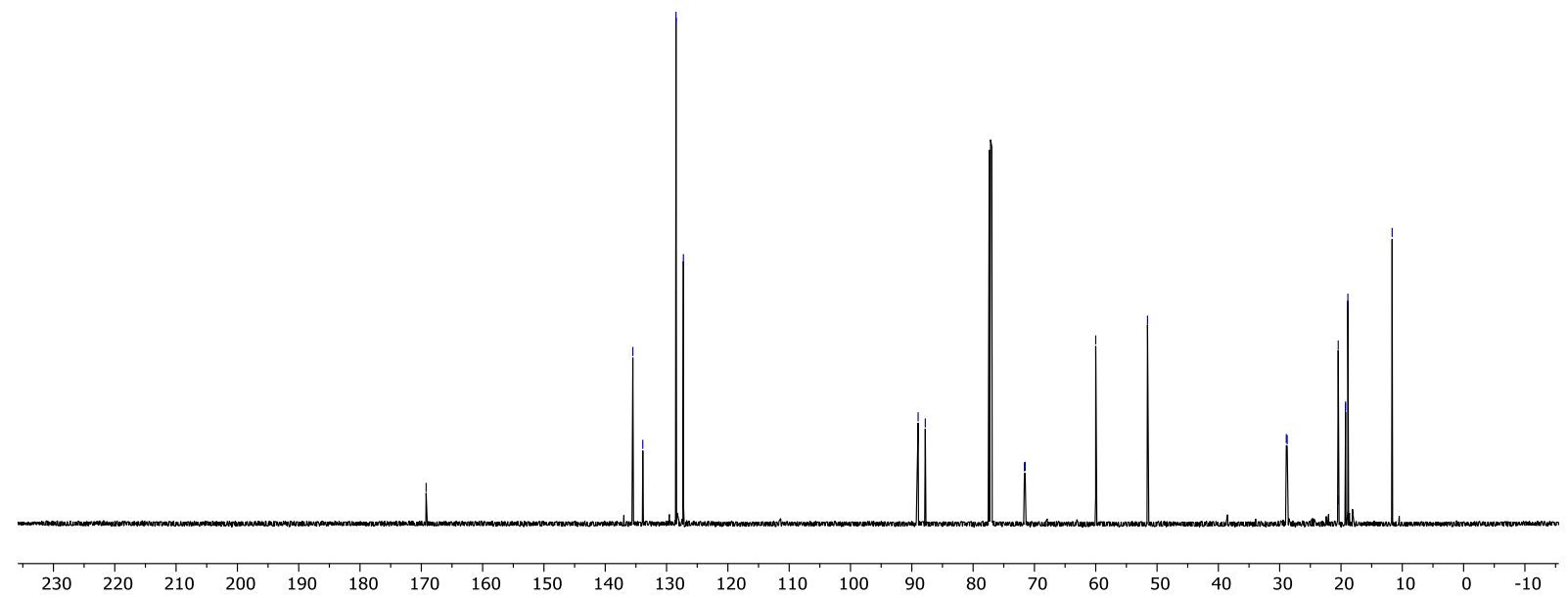


<smiles>CCCN1CCCC(F)C1C(=O)Nc1c(C)cccc1C</smiles>
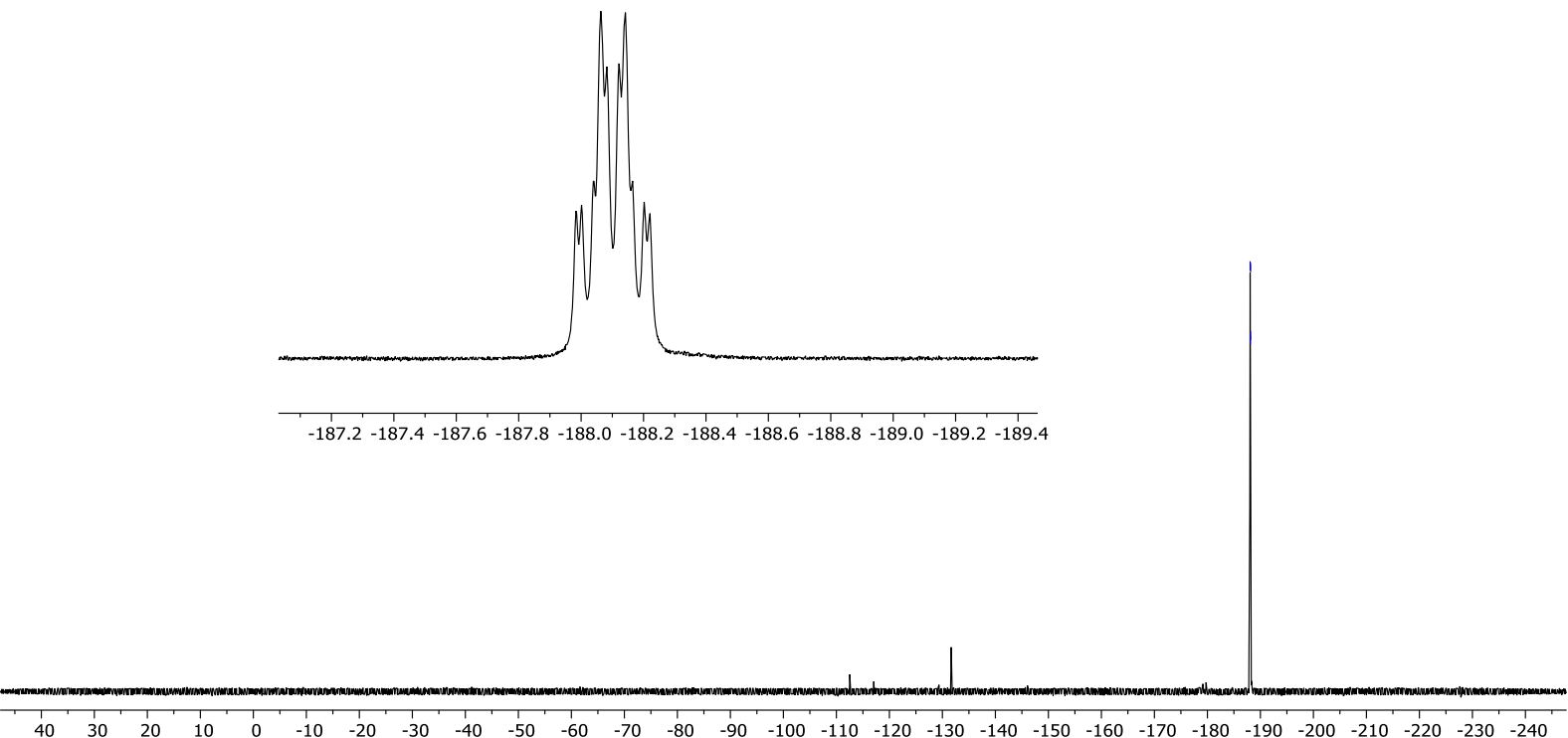

1H NMR (Chloroformd)

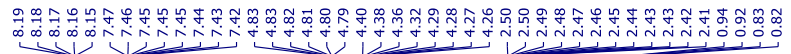<smiles>CC(C)[C@H]1COC(=O)N1c1ccc(F)cn1</smiles><smiles>C1=CCCC=C1</smiles>
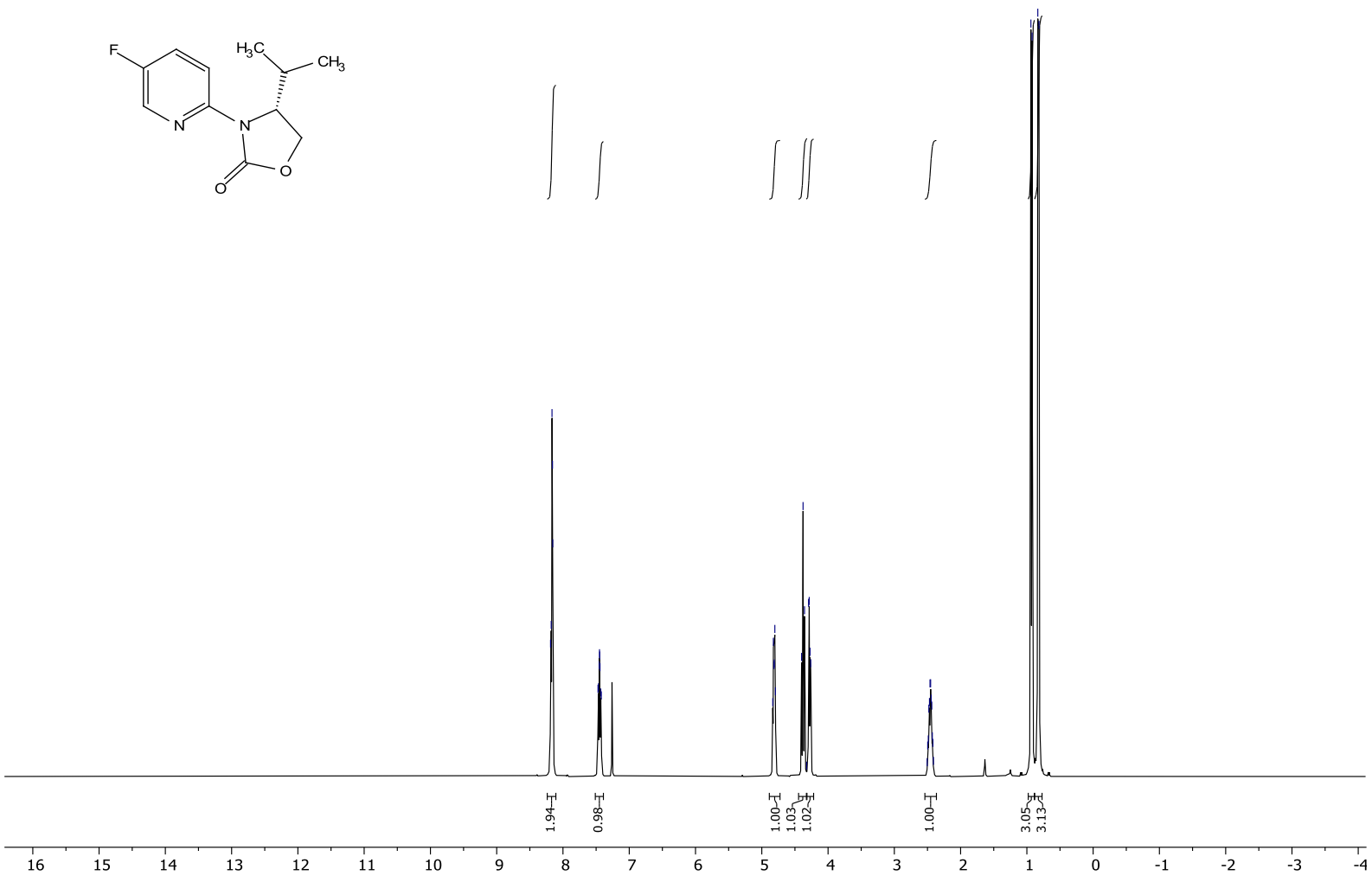
${ }^{13} \mathrm{C}\{1 \mathrm{H}\}$ NMR (Chloroformed)

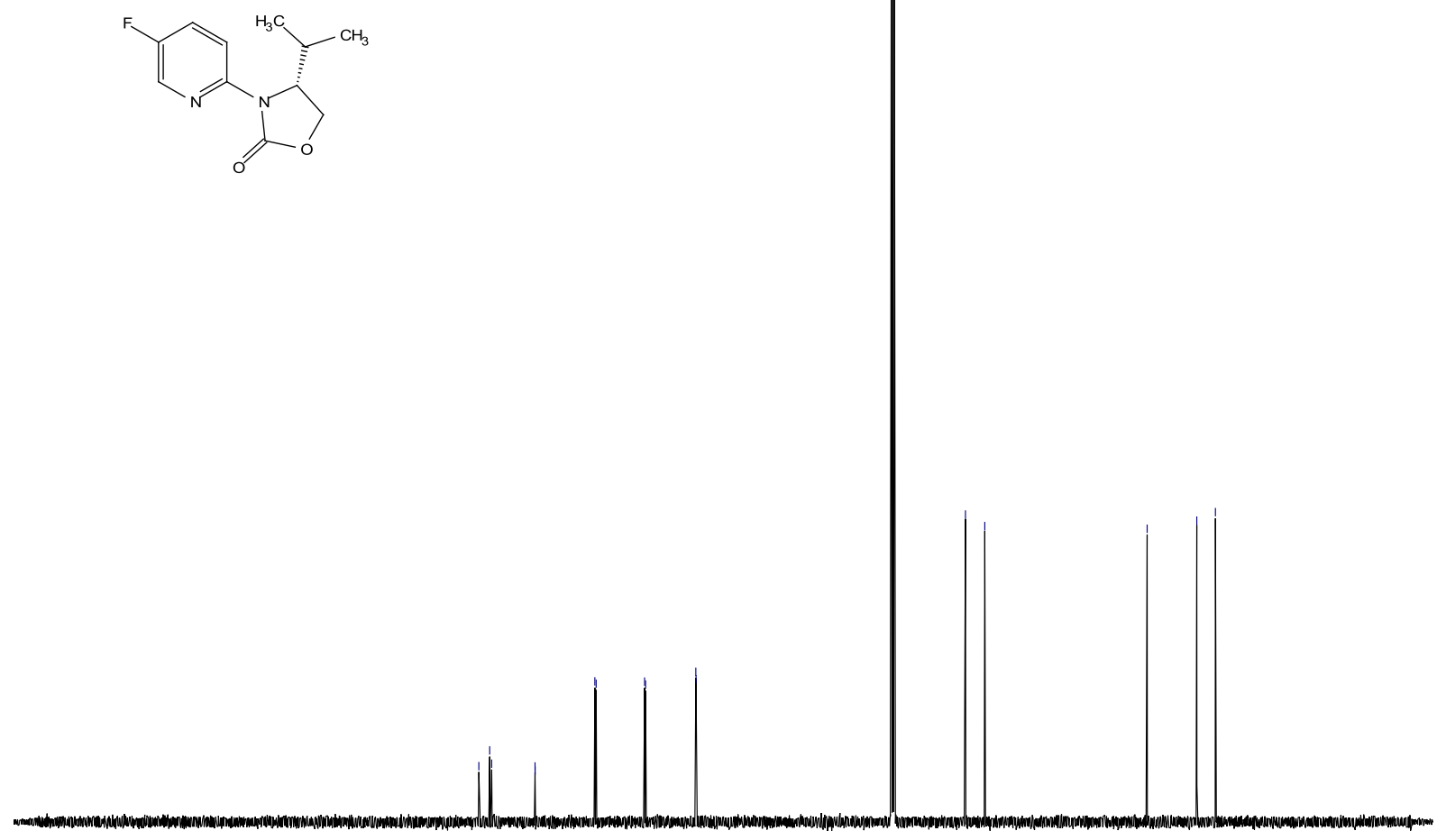

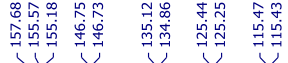

ํ.

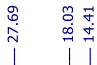

$\begin{array}{lllllllllllllllllllllllllllllll}240 & 230 & 220 & 210 & 200 & 190 & 180 & 170 & 160 & 150 & 140 & 130 & 120 & 110 & 100 & 90 & 80 & 70 & 60 & 50 & 40 & 30 & 20 & 10 & 0 & -10 & -20 & 10\end{array}$

${ }^{19} \mathrm{~F}\{1 \mathrm{H}\}$ NMR (Chloroformed)<smiles>CC(C)[C@H]1COC(=O)N1c1ccc(F)cn1</smiles>

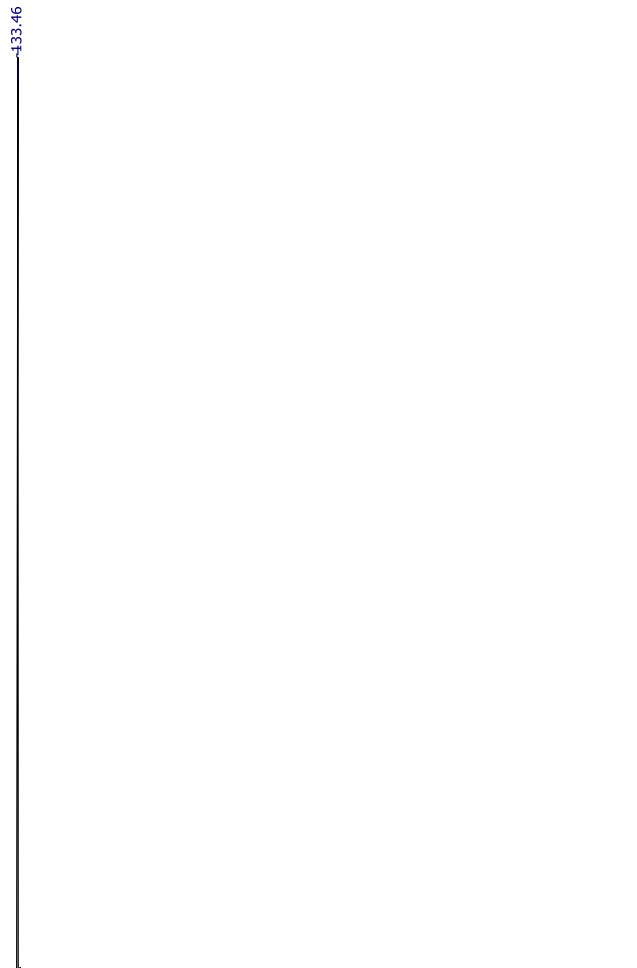

$\begin{array}{lllllllllllllllllllllllllllllllll}50 & 40 & 30 & 20 & 10 & 0 & -10 & -20 & -30 & -40 & -50 & -60 & -70 & -80 & -90 & -100 & -110 & -120 & -130 & -140 & -150 & -160 & -170 & -180 & -190 & -200 & -210 & -220 & -230 & -240 & -2 !\end{array}$ 
1H NMR (Chloroforme)

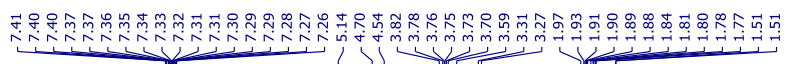
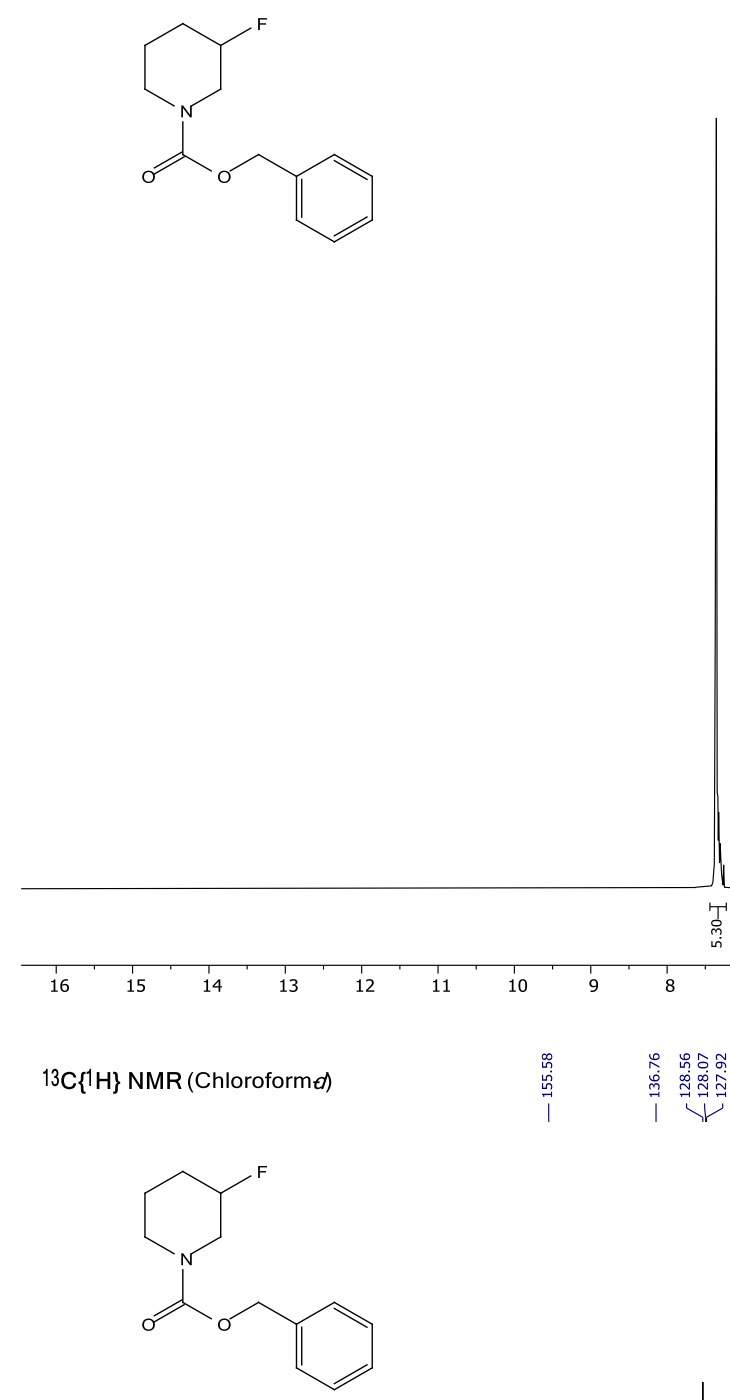

S229 
${ }^{19} \mathrm{~F}\{1 \mathrm{H}\}$ NMR (Chloroform $\left.d\right)$<smiles>O=C(OCc1ccccc1)N1CCCC(F)C1</smiles>

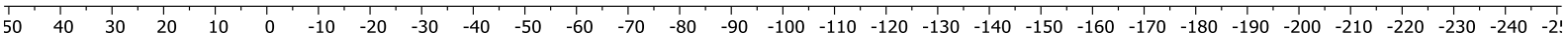

